

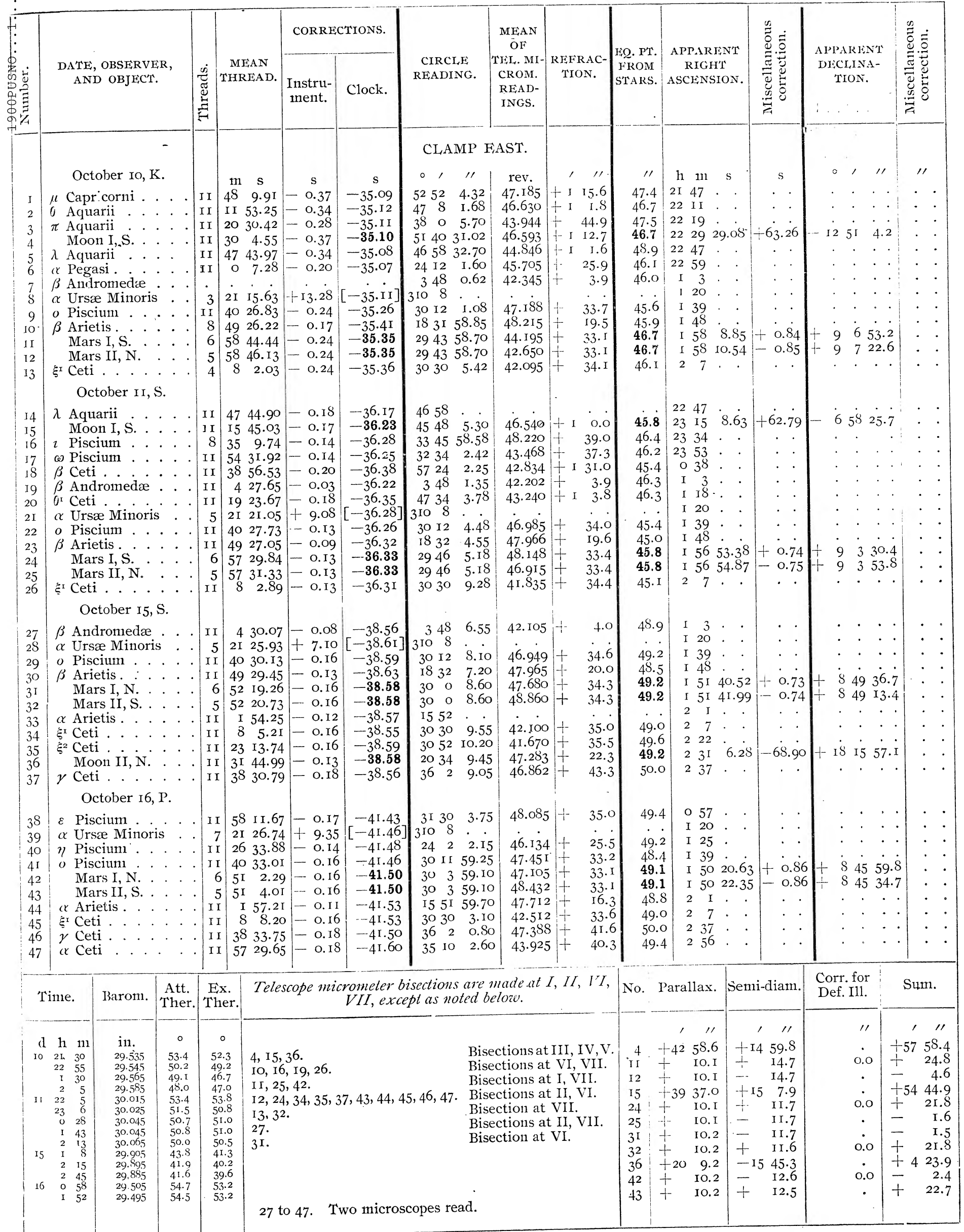




\begin{tabular}{|c|c|c|c|c|c|c|c|c|c|c|c|c|c|c|c|c|c|c|}
\hline \multirow[b]{2}{*}{ 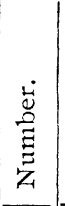 } & \multirow{2}{*}{\multicolumn{3}{|c|}{$\begin{array}{l}\text { DATE, OBSERVER, } \\
\text { AND OBJECT. }\end{array}$}} & \multirow[b]{2}{*}{ 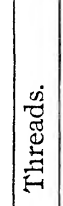 } & \multirow{2}{*}{$\begin{array}{c}\text { MEAN } \\
\text { THREAD. }\end{array}$} & \multicolumn{2}{|c|}{ CORRECTIONS. } & \multirow{2}{*}{$\begin{array}{l}\text { CIRCLE } \\
\text { READING. }\end{array}$} & \multirow{2}{*}{$\begin{array}{c}\text { MEAN } \\
\text { OF } \\
\text { TEL. MI- } \\
\text { CROM. } \\
\text { READ- } \\
\text { INGS. }\end{array}$} & \multirow{2}{*}{$\begin{array}{l}\text { REFRAC- } \\
\text { TION. }\end{array}$} & \multirow{2}{*}{\multicolumn{2}{|c|}{$\begin{array}{l}\text { EQ. PT. } \\
\text { FROM } \\
\text { STARS. }\end{array}$}} & \multirow{2}{*}{\multicolumn{2}{|c|}{$\begin{array}{l}\text { APPARENT } \\
\text { RIGH'T } \\
\text { ASCENSION. }\end{array}$}} & $\frac{\pi}{5}$ & & & \\
\hline & & & & & & $\begin{array}{c}\text { Instru- } \\
\text { ment. }\end{array}$ & Clock. & & & & & & & & 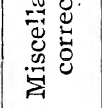 & TI & & 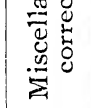 \\
\hline & & 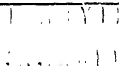 & & & & & & 0.11 & $1 \mathrm{co}$ & & & " & $\mathrm{h} \mathrm{m}$ & $\mathrm{s}$ & $\mathbf{s}$ & $\circ \quad 1$ & & " \\
\hline I & $\zeta$ & retis... & & I I & 933.94 & -0.12 & $-4 \mathrm{I} .6 \mathrm{O}$ & I8 II 59.70 & 43.242 & I8.8 & 48 & 3.9 & & & & 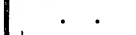 & & . \\
\hline 2 & I & $\begin{array}{l}\text { oon II, N. } \\
\text { ctober I } 7\end{array}$ & . & II & $28 \quad 42.08$ & $-0 . \mathrm{II}$ & -41.60 & I5 $40 \quad 2.35$ & 43.980 & $+\quad I 6 . I$ & 49 & 9.1 & 328 & 0.37 & -71.96 & + 23 II I & I3.I & . \\
\hline 3 & $\alpha$ & Minor & & 4 & $2 \mathrm{I} \quad \mathrm{I} 4.52$ & +22.28 & {$[-42.0 I]$} & 3 IO 8 & & & & & I 20 & • & - & - & $\cdot$ & . \\
\hline 4 & $\eta 1$ & & & 7 & $26 \quad 34.55$ & -0.39 & -41.89 & $\begin{array}{lll}24 & 2 & 1.00\end{array}$ & 46.220 & $+\quad 25.6$ & $5^{\mathrm{I}}$ & .9 & I 25 & te $>$ & 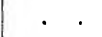 & . & & . \\
\hline 5 & $o 1$ & set & & I I & 4033.82 & -0.44 & -41.98 & $30 \quad 12 \quad 9.35$ & $47.07 \mathrm{I}$ & 33.4 & $5^{1}$ & & I 39 & & $1^{\circ} \cdot{ }^{\circ}$ & 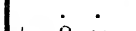 & & . \\
\hline 6 & 1 & $\operatorname{ars} \mathrm{I}, \mathrm{N}$. & & 6 & 4942.53 & -0.44 & -42.03 & $\begin{array}{lll}30 & 8 & 0.95\end{array}$ & 45.950 & $+\quad 33.3$ & 51 & 1.9 & I 49 & 0.06 & +0.99 & +8422 & 24.6 & . \\
\hline 7 & & I, S. & & 5 & $4944.5 \mathrm{I}$ & -0.44 & -42.03 & $\begin{array}{lll}30 & 8 & 0.95\end{array}$ & 46.990 & $33 \cdot 3$ & 51 & 1.9 & I 49 & 2.04 & -0.99 & +842 & 0.4 & . \\
\hline 8 & $\alpha$ & retis. . & 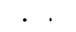 & II & I $5^{8.05}$ & $-0.3 \mathrm{I}$ & -42.16 & I5 $52 \quad 5.90$ & 47.562 & 16.4 & $5^{2}$ & 2.5 & $2 I$ & . . & $\cdot \cdot \cdot$ & . $\cdot$ & . . & . \\
\hline 9 & $\xi^{1}$ & ctober is & s. & II & 89.05 & -0.44 & -42.08 & $\begin{array}{lll}30 & 30 & 7.50\end{array}$ & 42.415 & $+\quad 33.8$ & $5^{1}$ & .8 & 27 & . $\cdot$ & $\cdot$ & . $\cdot$ & .. & . \\
\hline ro & $\varepsilon I$ & scium . & ${ }^{\circ}$ & I I & $5^{8} \quad 13.75$ & -0.26 & $-43.4 \mathrm{I}$ & $\begin{array}{lll}3 \mathrm{I} & 30 & 8.65\end{array}$ & 47.815 & $+\quad 35.7$ & 50 & 2.0 & o 57 & & - & - & & - \\
\hline II & $a^{2}$ & 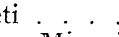 & & II & I9 30.88 & -0.31 & $-43 \cdot 3^{8}$ & $4734 \quad 8.00$ & 43.294 & $+\mathrm{r} 3.6$ & 51 & r.o & I 18 & . & . & . & . . & . \\
\hline I 2 & $<1$ & inor & & 5 & $213 \mathrm{I} .26$ & $+7 . \mathrm{Io}$ & {$[-43.43]$} & 3108. &.$\quad \cdot$ & & . & . & I 20 & 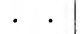 & . & . & - $\cdot$ & . \\
\hline I3 & $o \mathrm{I}$ & $m$ & 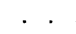 & . II & $40 \quad 35.07$ & -0.26 & -43.40 & $3012 \quad 8.70$ & 47.014 & $+\quad 33.9$ & 50 & 0.4 & I 39 & & $\cdot$ & & . . & . \\
\hline 14 & & S. & . & 6 & $4823.4 \mathrm{I}$ & -0.26 & -43.42 & $3012 \quad 8.60$ & $45 \cdot 390$ & 33.9 & 50 & & I 473 & $39 \cdot 73$ & +0.79 & +8382 & $23 \cdot 3$ & . \\
\hline 15 & & I, N. & . & 5 & $\begin{array}{ll}48 & 24.99\end{array}$ & -0.26 & -43.42 & 30 I2 8.60 & 44.190 & 33.9 & 50 & .2 & I 47 & $4 \mathrm{I} .3 \mathrm{I}$ & -0.79 & 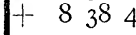 & 46. I & . \\
\hline 16 & $\alpha$ & $\therefore$ & . & I I & I 59.27 & -0.22 & -43.45 & $155^{2} \quad 8.15$ & 47235 & 16.6 & 49 & 6 & $2 \quad I$ &. & . & . & . . & . \\
\hline I 7 & $\xi^{1}$ & ti. . & . & - II I & 8 I0.22 & -0.26 & -43.42 & 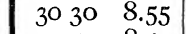 & 42.224 & $34 \cdot 3$ & 49 & 9.8 & 27 & & - & . & - $\cdot$ & - \\
\hline I 8 & $\xi^{2}$ & ti & $\dot{P}$ & I I & $23 \quad \mathrm{I} 8.75$ & -0.26 & -43.46 & $3052 \quad 8.40$ & $4 \mathrm{I} .8 \mathrm{I} 4$ & $+\quad 34.9$ & 50 & D. $I$ & 222 & . . & - & . . & . $\cdot$ & $\cdot$ \\
\hline 19 & $\varepsilon \mathbf{I}$ & um & $\therefore$ & I $\mathrm{r}$ & $5^{8} \quad \mathrm{I} 4.8 \mathrm{I}$ & -0.22 & $-44.5 \mathrm{I}$ & $\begin{array}{lll}31 & 30 & 6.05\end{array}$ & 47.912 & $35 \cdot 5$ & 49 & 9.0 & o 57 & & - & - & & - \\
\hline 20 & $\alpha \mathrm{I}$ & i & is . & 10 & 2I 30.73 & +8.79 & {$[-44.5 \mathrm{I}]$} & 3 10 8 & & & & & I 20 & & . & . & . & . \\
\hline $2 \mathrm{I}$ & $\eta 1$ & 1 & . & II & 2636.95 & -0.19 & -44.47 & $\begin{array}{lll}24 & 2 & 4.02\end{array}$ & 46.005 & 25.9 & 49 & P. I & I 25 & & . & . & 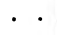 & . \\
\hline 22 & $O \mathrm{H}$ & 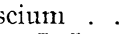 & 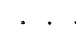 & 11 & 4036.13 & $|-0.2 \mathrm{I}|$ & -44.50 & $\begin{array}{lll}30 & 14 & 4.78\end{array}$ & 40.830 & 33.8 & 48 & 3.7 & I 39 & & $\cdot{ }^{\circ}$ & . & & . \\
\hline 23 & & S. & - & 5 & $\begin{array}{ll}47 & 4.25\end{array}$ & -0.21 & -44.53 & $3014 \quad 4.78$ & 50.462 & 33.8 & 48 & & I 46 & $19.5 \mathrm{I}$ & +0.77 & +8344 & $49 \cdot 3$ & . \\
\hline 24 & & I, N. & . & 6 & $47 \quad 5.79$ & -0.21 & -44.53 & $\begin{array}{lll}30 & 14 & 4.78\end{array}$ & 49.272 & 33.8 & 48 & 3.8 & I 462 & 21.05 & -0.77 & t $835 \mathrm{I}$ & I 2.0 & . \\
\hline 25 & $\xi^{\mathrm{x}}$ & ti . . . & $\cdot \cdot$ & II & 8 II. 35 & $-0.2 \mathrm{I}$ & $-44: 59$ & 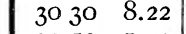 & 42.190 & 34.2 & 48 & 3.7 & 27 &.$\quad \cdot$ & . & . & . . & . \\
\hline 26 & $\xi^{2} c$ & ti & $\dot{L}$ & II & $23 \quad 19.85$ & $-0.2 \mathrm{I}$ & -44.59 & $3052 \quad 1.90$ & 42.099 & $+\quad 34.6$ & 48 & 3.7 & 222 & & . & . . & . . & . \\
\hline 27 & $\varepsilon \mathrm{I}$ & cium . & . . & II & $5^{8} \quad \mathrm{I} 5.85$ & -0.25 & $-45.5 \mathrm{I}$ & 3I 30 II.35 & 47.918 & $+\quad 35 . \mathrm{I}$ & 54 & 1.I & o 57 & & . & . & & - \\
\hline 28 & $g^{2}$ & & & II & 933 & -0. & -45.62 & 4734. & & & & . & I 18 & & . & . & & . \\
\hline 29 & $\alpha \tau$ & ri & is . & 4 & 2 I 3 & +3.48 & {$[-45.55]$} & 3108 & & & . & & I 20 & & . & . . & . . & . \\
\hline 30 & $\eta \mathrm{F}$ & . & . & II & $2637.9^{8}$ & -0.24 & -45.45 & $\begin{array}{lll}24 & 2 & 9.25\end{array}$ & 46.038 & $25 \cdot 5$ & 54 & 1.7 & I 25 & & . & . & . . & . \\
\hline $3 \mathrm{I}$ & o $\mathrm{F}$ & & & II & 4037 & -0.25 & -4 & 30 I2 IO.10 & 47.122 & $33 \cdot 3$ & & & I 39 & & $\cdot . \cdot{ }^{\circ}$ & . & & . \\
\hline 32 & & S. & t & 6 & $45 \quad 44$ & -0.25 & -45.54 & 30 I8 22.85 & $48.4 \mathrm{IO}$ & 33.5 & 53 & & I 445 & 59.20 & +0.70 & $+83 \mathrm{II}$ & I5.6 & . \\
\hline 33 & & $\mathrm{I}, \mathrm{N}$. . & & 5 & 4546 & -0.25 & -45.54 & $30 \quad 18 \quad 22.85$ & 47.100 & 33.5 & 53 & 3.8 & I 45 & 0.60 & -0.70 & $+83^{14}$ & 40.6 & . \\
\hline 34 & $\xi^{1}$ & ti & $\dot{\mathrm{K}}$ & II & 812.36 & -0.25 & -45.55 & $\begin{array}{lll}30 & 30 & \text { 10. } 75\end{array}$ & $42.3^{\mathrm{I} 2}$ & $+\quad 33.7$ & 53 & 3.1 & 27 & $\cdot \cdot$ & $\cdot$. &. & . . & \\
\hline 35 & $\beta A$ & omerl & & II & 440 & +0.06 & -4 & 348 I.95 & 42.508 & & 53 & 3.I & & & • & . & & . \\
\hline 36 & $\alpha \tau$ & & is. . & 5 & $2 \mathrm{I} 3$ & +9.59 & {$[-49.15]$} & 3 IO 8. & & & & & I 20 & & 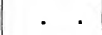 & . & & . \\
\hline 37 & $\eta \mathrm{I}$ & sc & $\cdot \cdot$ & II & 264 & -0.02 & -49.14 & $\begin{array}{lll}24 & 2 & 6.05\end{array}$ & 46.175 & 25.9 & $=$ & 4.8 & I 25 & & & - & & \\
\hline $3^{8}$ & & & 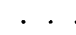 & 4 & $\begin{array}{ll}40 & 33 \\
-1\end{array}$ & -0.6 & -49 & $30324 \mathrm{I} .25$ & 45.235 & 34.2 & 53 & & I 39 & 44.47 & +0.72 & +8175 & 54 & \\
\hline 39 & & N. . & . & 3 & $\begin{array}{ll}40 & 35.08\end{array}$ & -0.0 & -49.13 & $3032 \quad 41.25$ & 43.960 & 34.2 & 53 & 3.9 & I 39 & $45 \cdot 90$ & $-0.7 \mathrm{I}$ & $+818 \mathrm{I}$ & 19.7 & . \\
\hline 40 & $\beta \mathrm{A}$ & & 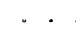 & II & 4939 & 0.0 & -49 & $1832 \quad 7.75$ & 48.218 & I9.5 & 54 & 1.2 & I 48 & & 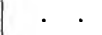 & • & . . & . \\
\hline $4 \mathrm{I}$ & $\xi^{2} \mathrm{C}$ & . & $\cdot \cdot$ & II & 815.78 & -0.05 & -49.13 & $3030 \quad 14.50$ & 42.102 & 34.2 & 53 & 3.5 & 27 & . & . & . & . & . \\
\hline & ime. & Barom. & $\begin{array}{l}\text { Att. } \\
\text { Ther. }\end{array}$ & Ex. & Teles & ppe mi & rometer & ctions are & le at & & No. & Para & allax. & Semi- & -diam. & $\begin{array}{l}\text { Corr. for } \\
\text { Def. I11. }\end{array}$ & & um. \\
\hline d 1 & $\mathrm{~h} \mathrm{~m}$ & & ${ }^{\circ}$ & ० & & & & & & & & & " & & " & " & & \\
\hline 16 & 3 & & 53.0 & I & $\mathrm{I}, \mathrm{I} 4$ & {$[9,21$} & 5,33 . & $x_{2}+2$ & & & 2 & +15 & 34.4 & -15 & 53 & . & - & O I 8.7 \\
\hline & 020 & & & & 2. & & & & IV & V. V. & 0 & & 10.2 & - & 12.1 & 0.0 & - & I.9 \\
\hline & 2 IO & & & & 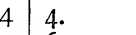 & & & Bise & VI, VII. & & 7 & + & 10.2 & + & I2.I & 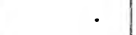 & + & 22.3 \\
\hline 18 & I 2 & & & & 6. & & & Bise & & & & $t$ & 10.2 & + & II. & . & + & 21.6 \\
\hline & I 57 & & .8 & & 7,38 & & & $\mathbf{E}$ & & & & + & I0.2 & - & II & 0.0 & - & I. 2 \\
\hline & $2 \mathrm{I} 6$ & & & & 23. & & & $\mathrm{I}$ & VII. & & & + & I0.2 & + & 11 & . & + & 21.5 \\
\hline 19 & O 59 & & & & 16. & & & & I, VI, VI & & 24 & + & 10.2 & - & II. & 0.0 & 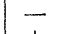 & I. 2 \\
\hline & 224 & & & & 39. & & & Bisection at $\mathrm{V}$ & & & & + & I0.2 & t- & I2. & & + & 22.7 \\
\hline 20 & o 39 & & & & & & & & & & & & I0.2 & - & $\mathrm{I} 2.5$ & 0.0 & 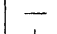 & 2.3 \\
\hline & 2 II & & & & & & & & & & & + & IO. I & + & 12.6 & & + & 22.7 \\
\hline 24 & I IC & & 52.8 & & & & & & & & 39 & + & IO.I & - & 12.5 & 0.0 & - & 2.4 \\
\hline & 2 & 25 & $5^{1.7}$ & & $\mathrm{I}$ to $\mathrm{I} 8$ & 8.\}, & & & & & & & & & & & & \\
\hline $3 I$ & I & 29.735 & 45.2 & $44 \cdot 1$ & 27 to & & & 51 & & & & & & & & & & \\
\hline
\end{tabular}




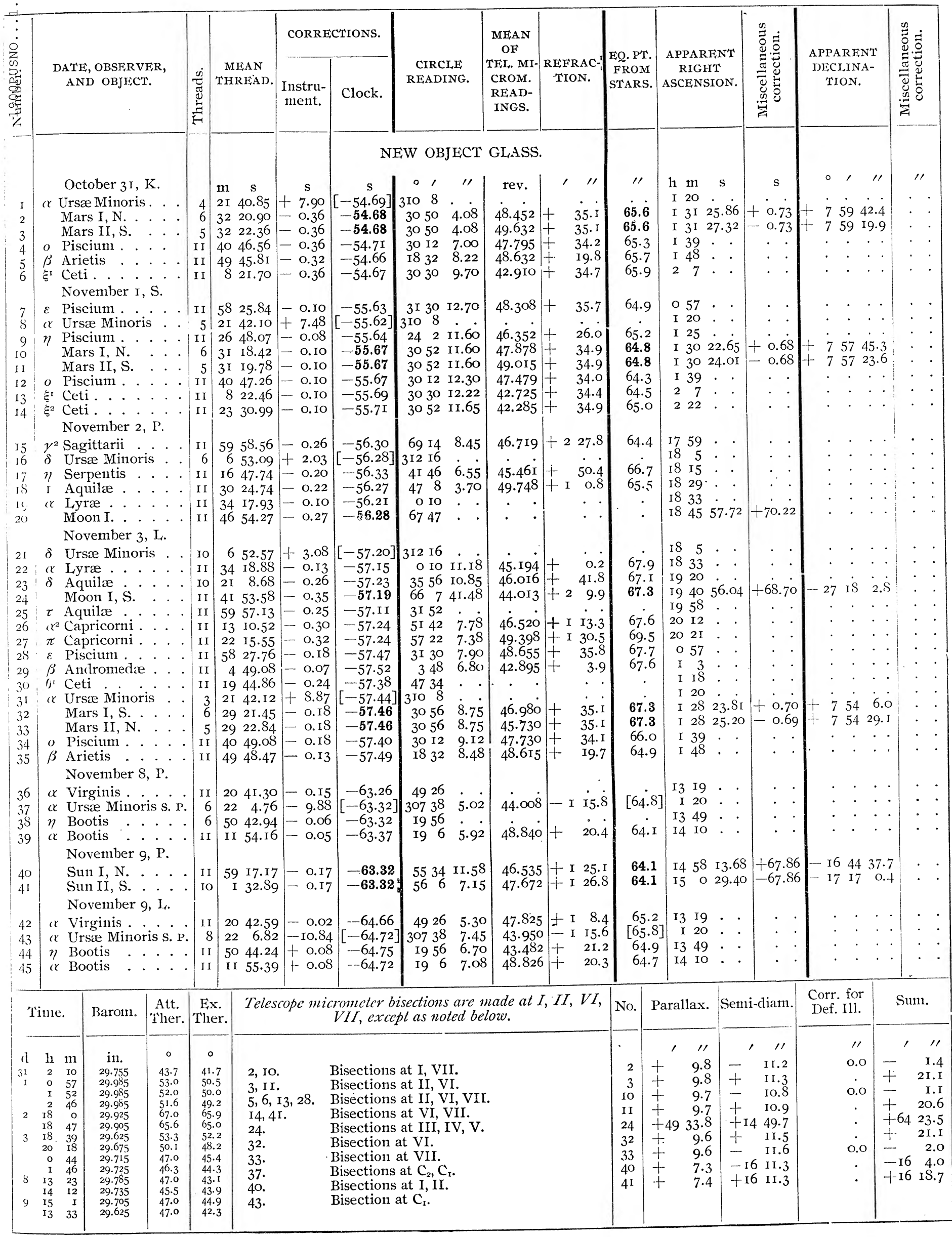




\begin{tabular}{|c|c|c|c|c|c|c|c|c|c|c|c|c|c|c|c|c|c|c|}
\hline \multirow[b]{2}{*}{ 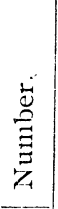 } & \multirow{2}{*}{\multicolumn{3}{|c|}{$\begin{array}{l}\text { DATE, OBSERVER, } \\
\text { AND OBJEC'T. }\end{array}$}} & \multirow[b]{2}{*}{ 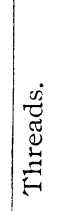 } & \multirow{2}{*}{$\begin{array}{c}\text { MEAN } \\
\text { THREAD. }\end{array}$} & \multicolumn{2}{|c|}{ CORRECTIONS. } & \multirow{2}{*}{$\begin{array}{l}\text { CIRCLE } \\
\text { READING. }\end{array}$} & \multirow{2}{*}{$\begin{array}{c}\text { MEAN } \\
\text { OF } \\
\text { TEL. MI- } \\
\text { CROM. } \\
\text { READ- } \\
\text { INGS. }\end{array}$} & \multirow{2}{*}{$\begin{array}{l}\text { REFRAC- } \\
\text { TION. }\end{array}$} & \multirow{2}{*}{\multicolumn{2}{|c|}{$\mid \begin{array}{c}\text { EQ. PT. } \\
\text { FROM } \\
\text { STARS. }\end{array}$}} & \multirow{2}{*}{\multicolumn{2}{|c|}{$\begin{array}{l}\text { APPARENT } \\
\text { RIGHT } \\
\text { ASCENSION. }\end{array}$}} & \multirow{2}{*}{ 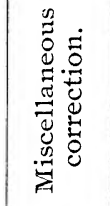 } & & & \\
\hline & & & & & & $\begin{array}{c}\text { Instru- } \\
\text { ment. }\end{array}$ & clock. & & & & & & & & & TION & N. & 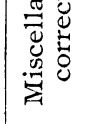 \\
\hline & & & & & $\mathrm{m} \mathrm{s}$ & $\mathrm{s}$ & $\therefore$ & 0111 & rev. & 111 & & "' & $\mathrm{h} \mathrm{m}$ & $\mathrm{s}$ & $\mathrm{s}$ & $\circ \quad 1$ & "I & "I \\
\hline I & $\varepsilon \mathrm{B}$ & botis $:$ & & Io & 4I 27. I 4 & + o.I I & -64.75 & II 206.22 & 45.460 & t $\quad$ Ir.7 & & 55.5 & I 440 & & & & & . \\
\hline 2 & & enus I, N. & & 6 & $455 \mathrm{I} .4 \mathrm{O}$ & -0.03 & -64.75 & $5348 \quad 5.20$ & 50.460 & + I 19.6 & & 65.2 & I4 44 & 46.62 & +0.34 & - I4 59 & $4 \mathrm{I} \cdot 9$ & \\
\hline 3 & i & $\begin{array}{l}\text { enus II, } \\
\text { ovember }\end{array}$ & IO, L. & 5 & $45 \quad 52.08$ & -0.03 & -64.75 & $5348 \quad 5.20$ & 50.850 & + I 19.6 & & 65.2 & I4 44 & 47.30 & -0.34 & - I4 59 & 51.9 & . \\
\hline $\begin{array}{l}4 \\
5\end{array}$ & I & $\begin{array}{l}\text { ercury I, } \\
\text { ercury II }\end{array}$ & C. . & $\begin{array}{l}6 \\
5\end{array}$ & $\begin{array}{ll}5 & 14.68 \\
5 & 15.38\end{array}$ & $\begin{array}{r}-0.04 \\
-0.04\end{array}$ & $\begin{array}{l}-64.77 \\
-64.77\end{array}$ & $\begin{array}{ccc}56 & 8 & 6.00 \\
. & . & .\end{array}$ & $44.28 \mathrm{I}$ & + I 26.6 & & 65.2 & I5 4 & 9.87 & +0.35 & $-17 \quad 17$ & $5 \mathrm{I} . \mathrm{I}$ & - \\
\hline 6 & $\beta \mathrm{H}$ & lis & & II & $26 \quad 45 \cdot 38$ & F 0.04 & $-64.8 \mathrm{I}$ & $\begin{array}{lll}\dot{17} & \dot{8} & 7.30\end{array}$ & $\dot{44.66_{5}}$ & $+\quad$ I8.o & & 55.6 & $\begin{array}{rr}15 & 4 \\
16 & 25\end{array}$ & 10.57 & -0.35 & $\cdot \cdot$ & $\cdot \cdot$ & · \\
\hline 7 & $\kappa 0$ & Dhiuchi & & II & 5345.05 & +0.04 & -64.86 & 29 I $8 \quad 7.42$ & $46.5^{82}$ & $+\quad 32.7$ & & 5.4 & 1652 &. & . & . & $\therefore$ & $\cdot$ \\
\hline s & $\begin{aligned} \alpha^{\mathrm{I}} \mathrm{H} \\
\mathrm{N}\end{aligned}$ & $\begin{array}{l}\text { culis } \\
\text { ember }\end{array}$ & II, S. & I I & IO 54.76 & +0.06 & -64.85 & $2420 \quad 6.52$ & 46.012 & $+\quad 26.4$ & & $65 . \mathrm{I}$ & 179 & . & . & r. & $\therefore$ & $\dot{.}$ \\
\hline $\begin{array}{r}9 \\
10\end{array}$ & $\alpha \mathrm{V}$ & ais & $\therefore \therefore$ & II & $20 \quad 44.21$ & +0.13 & -66.39 & $4926 \quad 8.22$ & 47.540 & + I 10.9 & & 55.0 & I3 I9 & & & • & $\cdot \cdot$ & \\
\hline II & $\begin{array}{ll}\varkappa & \mathrm{U} \\
\eta & \mathrm{B}\end{array}$ & $\begin{array}{l}\text { sæ Mino } \\
\text { otis. . }\end{array}$ & $\begin{array}{l}\text { ris S. P. } \\
\text {. . . . }\end{array}$ & 5 & $\begin{array}{ll}22 & 8.2 \mathrm{I} \\
2 & 85\end{array}$ & -II.06 & {$[-66.40]$} & $\begin{array}{lll}307 & 38 & 3.02\end{array}$ & 44.400 & 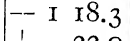 & & $66.8]$ & I 20 & .. &.$\quad$. & . . & $\cdot \cdot$ & $\cdot$. \\
\hline I2 & c $\mathrm{B}$ & otis . & . . & $\begin{array}{l}11 \\
\text { II }\end{array}$ & $\begin{array}{ll}50 & 45.79 \\
\text { I I } & 57.00\end{array}$ & {$\left[\begin{array}{ll}+ & 0.21 \\
+ & 0.2 \mathrm{I}\end{array}\right]$} & $\begin{array}{l}-66.40 \\
-66.44\end{array}$ & $\begin{array}{lrl}\text { I9 } & 56 & 8.50 \\
\text { I9 } & 6 & 8.95\end{array}$ & $\begin{array}{l}43.408 \\
48.709\end{array}$ & $\begin{array}{r}+\quad 22.0 \\
+\quad 21.0\end{array}$ & & 8.5 & $\begin{array}{ll}\text { I3 } & 49 \\
\text { I4 } & \text { IO }\end{array}$ & . $\cdot$ & $\cdot \cdot$ & $\cdot$ & $\cdot \cdot$ & $\cdot \cdot$ \\
\hline I3 & $\varepsilon B$ & otis . & & II & $4 \mathrm{I} 28.7 \mathrm{I}$ & +0.24 & -66.46 & II 20 I 2.38 & 45.120 & $\begin{array}{r}12.2 \\
+\quad 1\end{array}$ & & 5.2 & 1440 & & . & . & . &. \\
\hline I4 & & S I, N. & . & 6 & $\begin{array}{lll}55 & 46.22\end{array}$ & $4-0.12$ & -66.47 & $5442 \quad 7.62$ & $4 \mathrm{I} .45 \mathrm{O}$ & + I 24.9 & & 5.2 & I4 54 & 39.87 & +0.32 & - I5 47 & $\dot{4} 3.0$ & \\
\hline 15 & $\mathrm{~V}$ & vember & I $2, S$ & 5 & 5546.86 & $1+0.12$ & -66.47 & $\begin{array}{lll}54 & 42 & 7.62\end{array}$ & 41.980 & I I 24.9 & & 5.2 & I4 54 & $40.5 \mathrm{I}$ & -0.32 & - I5 47 & $53 \cdot 3$ &. \\
\hline 16 & & $\mathrm{~S}$ & & Io & II 29.47 & +0.12 & -66.49 & $\begin{array}{lll}56 & 56 & 8.28\end{array}$ & 46.672 & + I 32.4 & & 5.2 & I5 IO & 23. IO & +68.40 & - IS 6 & 43.2 & \\
\hline I7 & & & & II & I3 46.26 & -0.12 & -6 & $\begin{array}{lll}56 & 24 & 8.50\end{array}$ & 45.282 & + \pm 30.6 & & 5.2 & $\mathrm{I} 5 \mathrm{I} 2$ & 39.89 & -68.39 & - I7 34 & I 8.6 & . \\
\hline is & $\eta \begin{array}{ll}\eta & \mathrm{H} \\
\gamma^{\mathrm{I}} & \mathrm{H}\end{array}$ & llis & . . . . . & II & $\begin{array}{lll}40 & 22.22\end{array}$ & +0.29 & -66.52 & $\mid \begin{array}{lll}359 & 44 & 7.72\end{array}$ & 44.888 & 0.2 & & 5.2 & I6 39 & . & . & 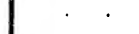 & . & . \\
\hline $\begin{array}{l}19 \\
20\end{array}$ & 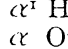 & $\begin{array}{l}\text { trculis } \\
\text { hiuchi }\end{array}$ & $\therefore$ & $S$ & IO 56.43 & 0.19 & -66.66 & $\begin{array}{lll}24 & 20 & 13.82\end{array}$ & 45. & $+\quad 27.1$ & & 5. I & I7 9 & 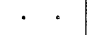 & . & . & . . & . \\
\hline $2 \mathrm{I}$ & $\mu \mathrm{H}$ & erculis & & $\left|\begin{array}{l}\text { II } \\
\text { II }\end{array}\right|$ & $\begin{array}{ll}3 \mathrm{I} & 8.47 \\
4 & \end{array}$ & o.19 & -66.62 & $\begin{array}{ll}26 & \text { I2 } \\
8.22\end{array}$ & 47.058 & $+\quad 29.5$ & & 5.4 & I7 30 & $\cdot \cdot$ & . & . $\cdot$ & $\cdot \cdot$ & · \\
\hline & $\mathrm{N}$ & vember I & $2, \mathrm{P}$ & II & $43 \quad 25.72$ & -0.24 & -66.60 & II $4 \quad 8.82$ & 45.465 & $+\quad$ II.7 & & 5.2 & I7 $42^{\circ}$ & $\cdot \cdot$ & . & - & $\cdot \cdot$ & - \\
\hline 22 & $c r \mathrm{~V}$ & rginis. & & 4 & 2045.23 & -0.02 & -67.24 & 4926 & & - & & & I3 I9 & . & & . & . & \\
\hline 23 & $\alpha \mathrm{U}_{1}$ & sæ Minor & ris s. P. & 5 & $\begin{array}{ll}22 & 8.87\end{array}$ & -Ir.os & {$[-67.27]$} & 30738 & & & & & I 20 & 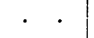 & . . & . . & . & 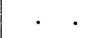 \\
\hline 24 & 7) $\mathrm{B}$ & otis . & $\cdot \cdot \cdot \cdot$ & II & 5046.83 & +0.07 & -67.28 & I9 $56 \quad 7.10$ & $43 \cdot 396$ & $+\quad 21.5$ & & 3.2 & I3 49 & & . & . & . & 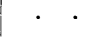 \\
\hline 25 & $\begin{array}{l}\alpha \mathrm{Bc} \\
\mathrm{Nc}\end{array}$ & $\begin{array}{l}\text { otis } \\
\text { vember I }\end{array}$ & $\therefore, P$ & II & II 57.98 & +0.07 & -67.27 & I9 $6 \quad 6.78$ & 48.755 & $+\quad 20.5$ & & 2.5 & 1410 & & 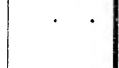 & . & 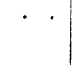 & \\
\hline 26 & & S. & . . . & I I & I5 35.39 & -0.04 & -67.30 & $57 \quad 12 \quad 15.50$ & 46.208 & t I 3I.I & & 3.5 & I5 I4 & 28.05 & +68.40 & - IS 22 & $4 \mathrm{I} \cdot 9$ & \\
\hline 27 & & N. & & II & 1752.19 & -0.04 & -67.30 & $\begin{array}{llll}56 & 40 & 13.05\end{array}$ & $30-$ & + I 29.2 & & 3 & I5 I6 & 44.85 & -68.40 & -1750 & I 8.7 & . \\
\hline 28 & $\eta \mathrm{Se}$ & rpentis & $\cdot \cdot \cdot$ & II & $1658.5 \mathrm{I}$ & 0.00 & -67.39 & $4146 \quad 7.42$ & 45.234 & $+\quad 52.0$ & & 4. I & 1815 & . $\cdot$ & $\cdot \cdot$ & - & . & . \\
\hline 29 & $\begin{array}{ll}\alpha & \mathrm{L}_{\mathrm{j}} \\
\mathrm{No}\end{array}$ & ráe & $3, K$ & II & 3428.65 & +0.16 & -67.39 & O IO 6.92 & 45.316 & $+\quad 0.2$ & & $4 \cdot 3$ & I8 33 & 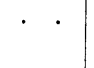 & . & . & 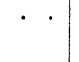 & . \\
\hline 30 & $\alpha \mathrm{Bc}$ & otis . & & IO & II 58.60 & +0.03 & -67.83 & I9 6 & & & & & I4 IO & & & • & & \\
\hline $3 \mathrm{I}$ & & T & & 6 & 546.00 & -0.03 & -6 & 5524.9 . IO & 45.488 & + I 24.2 & & 6.1 & I5 43 & $3^{8.11}$ & +0.39 & - I6 34 & I 4.0 & . \\
\hline 32 & $\begin{array}{l}\mathrm{Tc} \\
\mathrm{NC}\end{array}$ & $\begin{array}{l}\text { nus II, N } \\
\text { vember I }\end{array}$ & I. K. & 5 & 546.78 & -0.03 & -67.86 & $5524 \quad 9.10$ & 44.965 & t I 24.2 & & 6.1 & 1543 & 38.89 & -0.39 & - I6 34 & 4. I & . \\
\hline 33 & & N. & & II & I9 4I.62 & -0.03 & -67.86 & $5656 \quad 9.15$ & 4.08o & H. I 29.2 & & 6 & I5 I8 3 & $33 \cdot 73$ & $\mid-68.67$ & -18 & & \\
\hline 34 & & & & 8 & 2158 & -0.03 & & $\begin{array}{lll}57 & 28 & 3.40\end{array}$ & 45.660 & t I 3I.I & & 1 & I5 20 & 51.07 & -68.67 & - Is $35=$ & 20.4 & \\
\hline 35 & $\alpha O_{1}$ & ichi & & II & $\begin{array}{ll}3 \mathrm{I} & 9.93\end{array}$ & +0.02 & -6 & $26 \mathrm{I2} 6.70$ & $47.19^{6}$ & $+\quad 28.8$ & & & 1730 & . . &.$\quad$. & . . & . & \\
\hline 36 & $\delta \mathrm{Ur}$ & se Minor & ris & 5 & 659.68 & +3.04 & {$[-67.94]$} & $31216 \quad 16.25$ & $43 \cdot 325$ & - I 4.0 & {$[66$} & 6.0] & IS 5 & & . & . & & \\
\hline 37 & 7) Se & rpentis. & $\cdot \cdot \cdot$ & II & 1659.10 & -0.01 & -67.97 & $4146 \quad 7.68$ & $45 \cdot 394$ & $+\quad 52.3$ & & 7.6 & 18 I5 & . & . & . & & \\
\hline $3^{8}$ & $\begin{array}{l}x \text { LI } \\
\text { No }\end{array}$ & re & $\dot{A}, \mathrm{~L}$ & II & 3429.25 & +0.09 & -67.93 & $0 \quad 10 \quad 10.92$ & 45.162 & $+\quad 0.2$ & & 5. I & I8 33 & . & . . & . . & . & . \\
\hline 39 & $\propto \mathrm{Vi}$ & is & . . & II & I9 45.48 & +0.05 & 52 & 1926 I0.90 & 47.415 & + I 9.6 & & & 1319 & & . & . & & \\
\hline 40 & $\alpha \mathrm{Ur}$ & & ris s. P. & 7 & & -8.66 & $-7.56]$ & $30738 \quad 10.80$ & 44.050 & - I I6.8 & & I] & I 20 & & & . & & \\
\hline $\begin{array}{l}4 \mathrm{I} \\
42\end{array}$ & $\begin{array}{ll}\eta & \mathrm{BO} \\
c x & \mathrm{Bo}\end{array}$ & $\begin{array}{l}\text { otis. . . } \\
\text { otis. . }\end{array}$ & $\begin{array}{ll}\cdots \\
.\end{array}$ & $\begin{array}{ll}\text { II } \\
\text { II }\end{array}$ & $\mid \begin{array}{ll}49 & 46.97 \\
\text { IO } & 58.25\end{array}$ & {$\left[\begin{array}{ll}+ & \text { O.II }\end{array}\right.$} & -7.42 & I9 56 III.42 & 43.201 & $+\quad 21.5$ & & $3 \cdot 3$ & I3 49 & & & . & - & \\
\hline 42 & & & . & II & IO 58.25 & O.I I & -7.54 & I9 $6 \quad 9.52$ & 48.662 & $+\quad 20.5$ & & 2.9 & I4 IO & & . & . & & \\
\hline & me. & Barom. & $\begin{array}{l}\text { Att. } \\
\text { Ther. }\end{array}$ & $\begin{array}{l}\text { Fix. } \\
\text { Then }\end{array}$ & Tel & pe m & ieter 1 & $\begin{array}{l}\text { sections are? } \\
\text { t as noted be }\end{array}$ & $\begin{array}{l}\text { ade at } I, \\
\text { wee. }\end{array}$ & $I I$, & No. & Par & rallax. & Semi- & i-diam. & $\begin{array}{l}\text { Corr. for } \\
\text { Def. Ill. }\end{array}$ & & sum. \\
\hline$d$ & $\mathrm{~h} \quad \mathrm{~m}$ & & ${ }^{\circ}$ & $\circ$ & & & & & & & & ' & 11 & 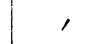 & 'I & "I & 1 & "I \\
\hline & $\begin{array}{ll}15 & 9 \\
16 & 35\end{array}$ & 26 & 47. & & $2,15,3$ & & $\mathrm{Bi}$ & II & & & 2 & + & 4.2 & - & 5.0 & 0.0 & - & 0.8 \\
\hline & $\begin{array}{ll}7 & 15\end{array}$ & 29.555 & 47.0 & & & & & 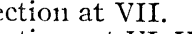 & & & 3 & & s & + & 5.0 & & & 0 \\
\hline & 330 & & & & 6,17 & I 9 & Bis & is at VI & VII. & & 4 & 1 & IO. & & & & $T$ & 10.8 \\
\hline & $\begin{array}{lr}14 & 7 \\
15 & 13\end{array}$ & $\begin{array}{l}30.075 \\
30.055\end{array}$ & 39 & & 8. & & Bise & ons at I, II, & VI. & & I4 & + & 4. & 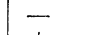 & $5 . \mathrm{I}$ & 0.0 & - & 0.9 \\
\hline & $17 \quad 14$ & & & & IO. & & Bise & $11 \mathrm{~s}$ at $\mathrm{C}_{2}$, & & & & t & 4. & 干 & 5.2 & . & & 9. \\
\hline & 1747 & & & & 14,3 & & & & & & 16 & + & 7 . & +56 & $6 \times 2.3$ & . & -16 & 619.8 \\
\hline $\mathrm{I}_{3}$ & {$\left[\begin{array}{ll}3 & 51 \\
17 & 17\end{array}\right]$} & & & & $14,15$. & & Z. D & hread B use & & & 1 & + & 7 & -16 & $6 \quad 12.2$ & . & -16 & $\begin{array}{ll}6 & 4.8\end{array}$ \\
\hline 13 & $\begin{array}{ll}17 \\
87\end{array}$ & & 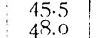 & & I6, 2 & & Bise & at I, I & & & 26 & $t$ & 7 & +16 & 6 II.6 & r. & +16 & 6 Ig. I \\
\hline & is 3.5 & & 48.5 & & 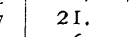 & & Bisec & at II, VI & II, VII. & & 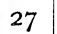 & $-t$ & 7. & --16 & 6 II.6 & 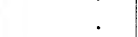 & -16 & 64.1 \\
\hline & & & $4^{4}$ & & 36. & & Bisec & t IV. & & & J & + & $4 \cdot 3$ & + & 4.9 & . & + & 9.2 \\
\hline$I_{4}$ & $\begin{array}{ll}15 & 5 \\
25\end{array}$ & & $\begin{array}{l}47 \\
48\end{array}$ & $\frac{4}{5}$ & 40. & & Bisec & ction at III. & & & 32 & + & 4.3 & - & 5.0 & 0.0 & - & 0.7 \\
\hline & $17 \quad 25$ & & & & & & & & & & 33 & & 7.5 & -16 & 615.0 & . & -16 & $7 \cdot 5$ \\
\hline & $\begin{array}{ll}\text { Is } & 40 \\
\text { I3 } & 34\end{array}$ & .725 & 46 & 40.3 & $4,5.0$ & Observe & aring tra & ansit over su & ''s disk. & & 34 & + & $7 \cdot 5$ & +16 & 6 I5.I & 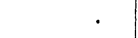 & +16 & 622.6 \\
\hline
\end{tabular}




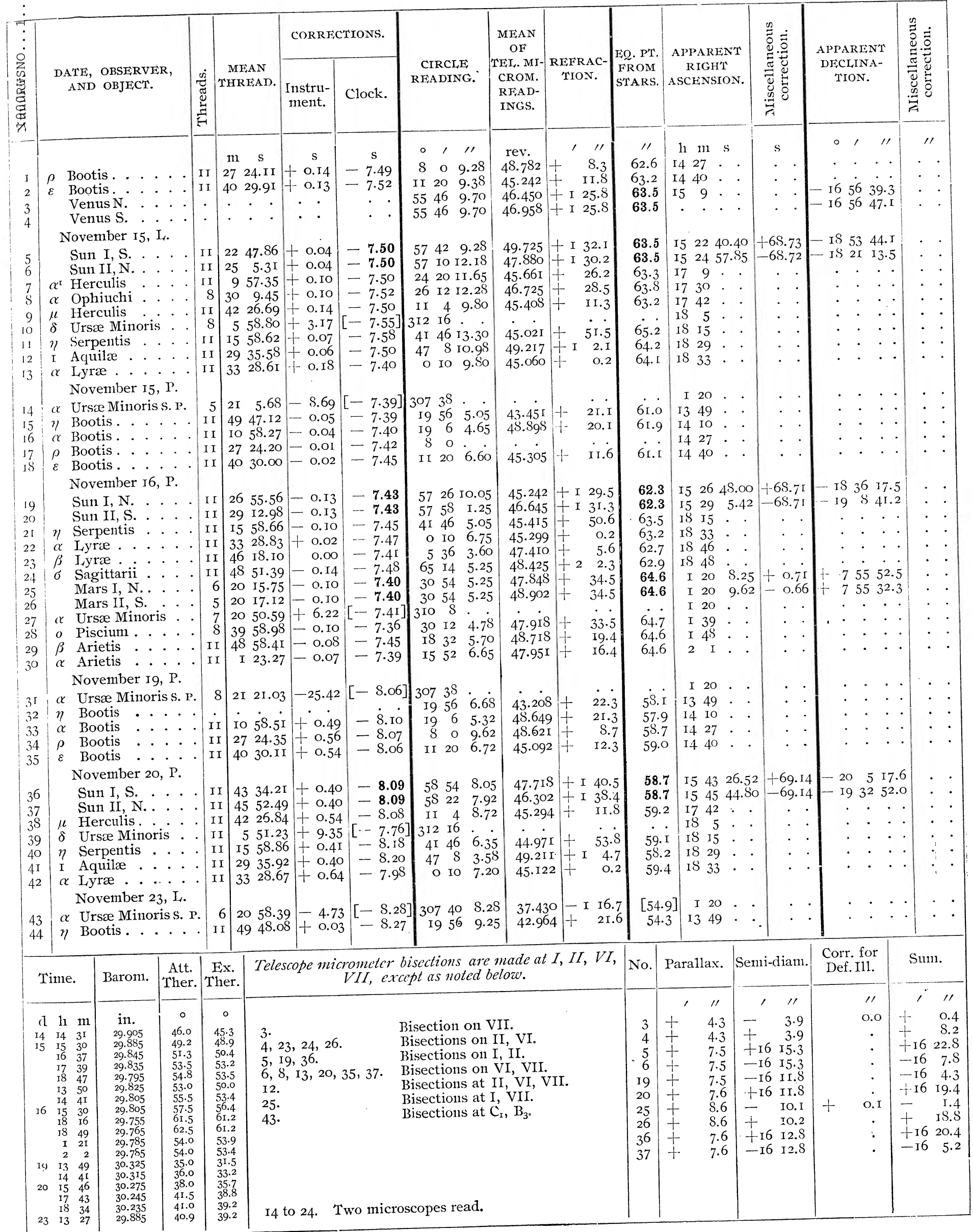




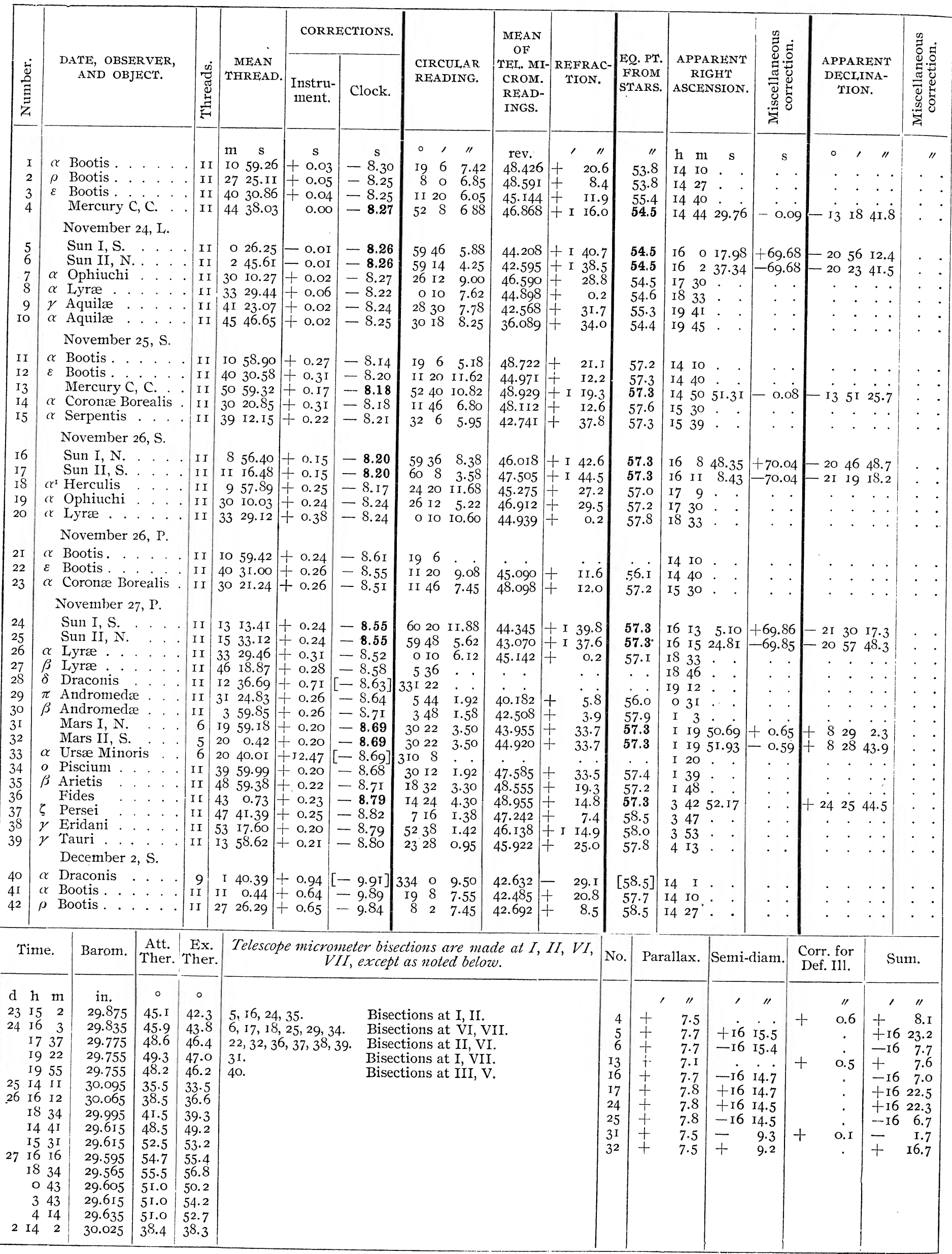




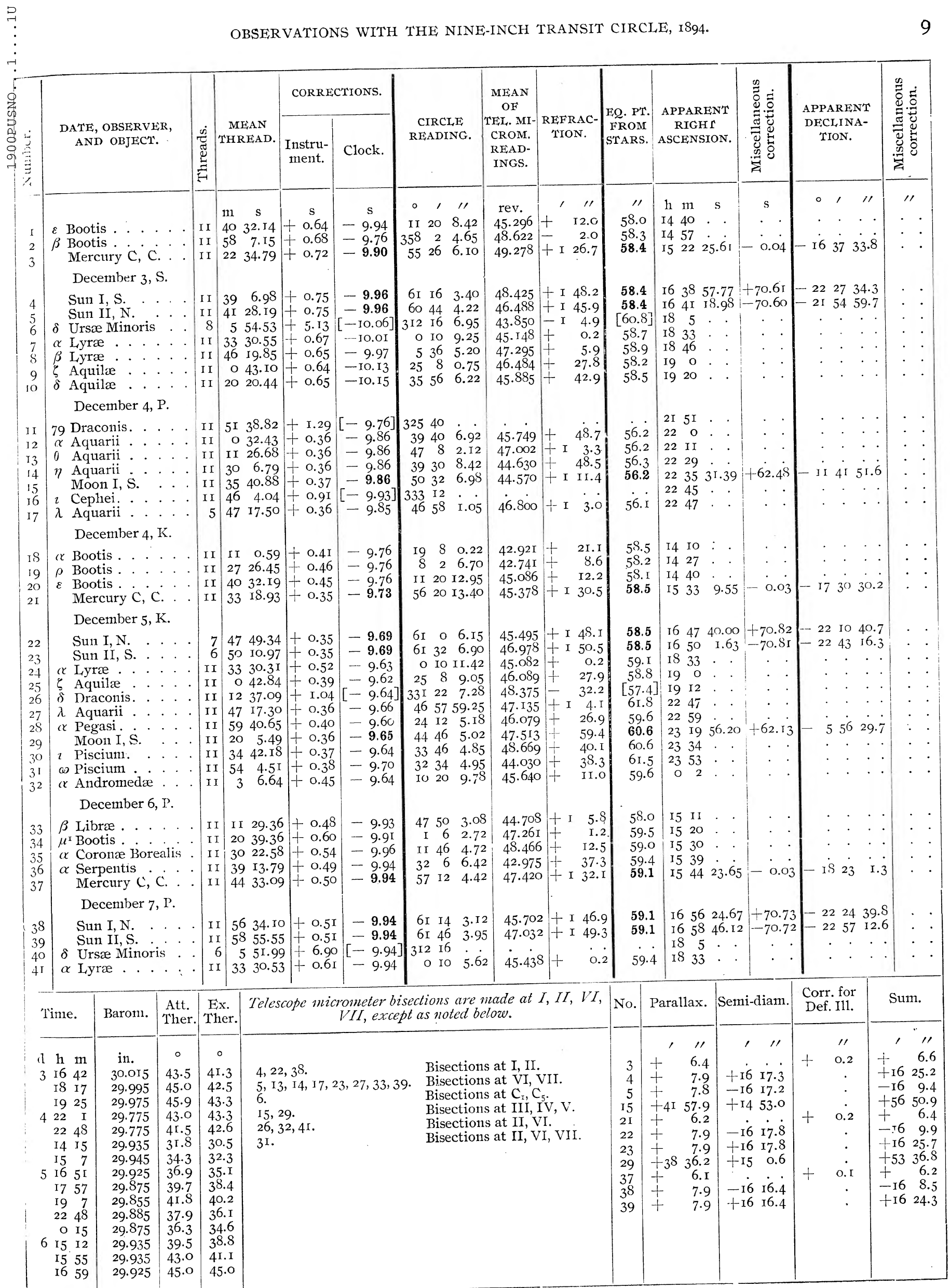




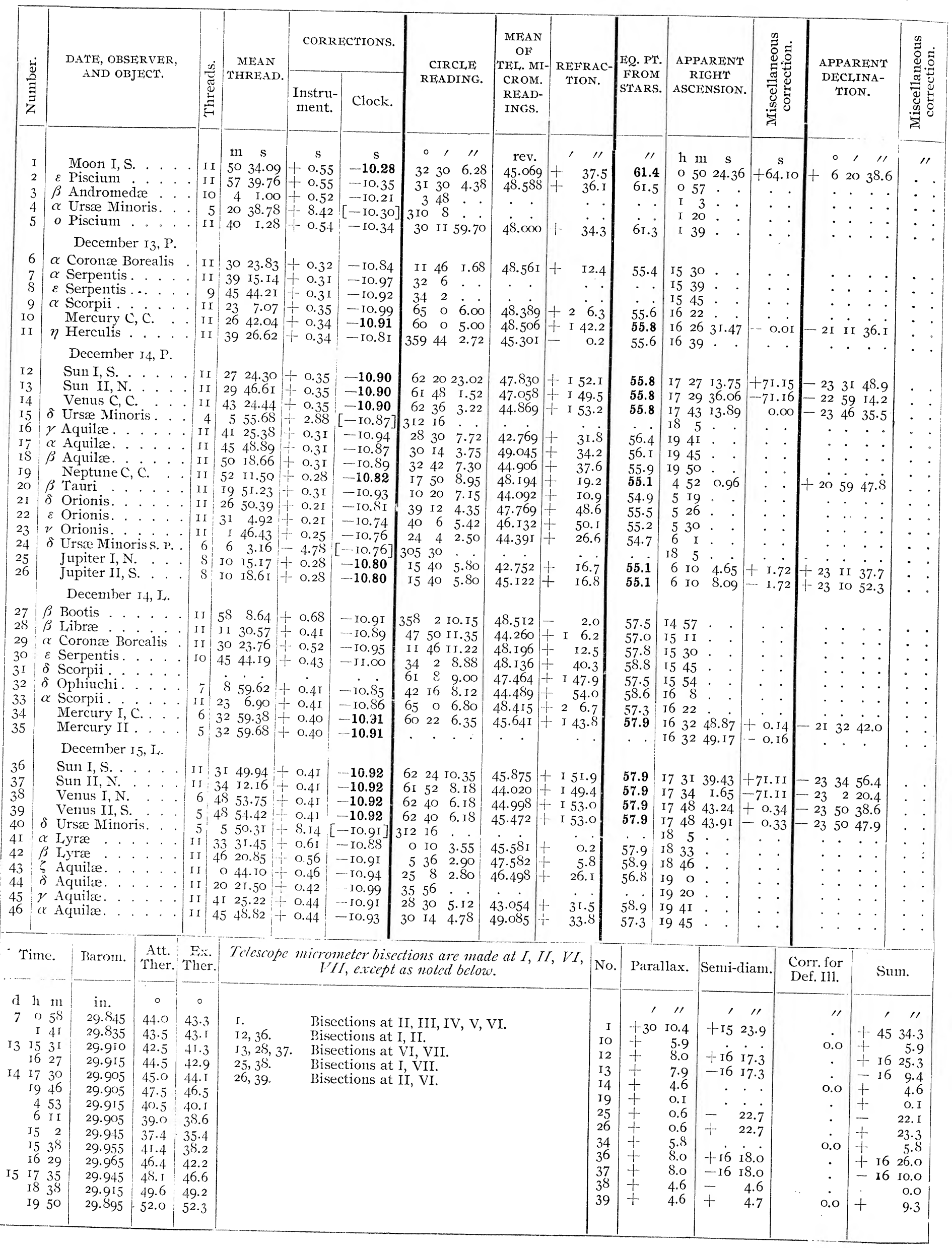




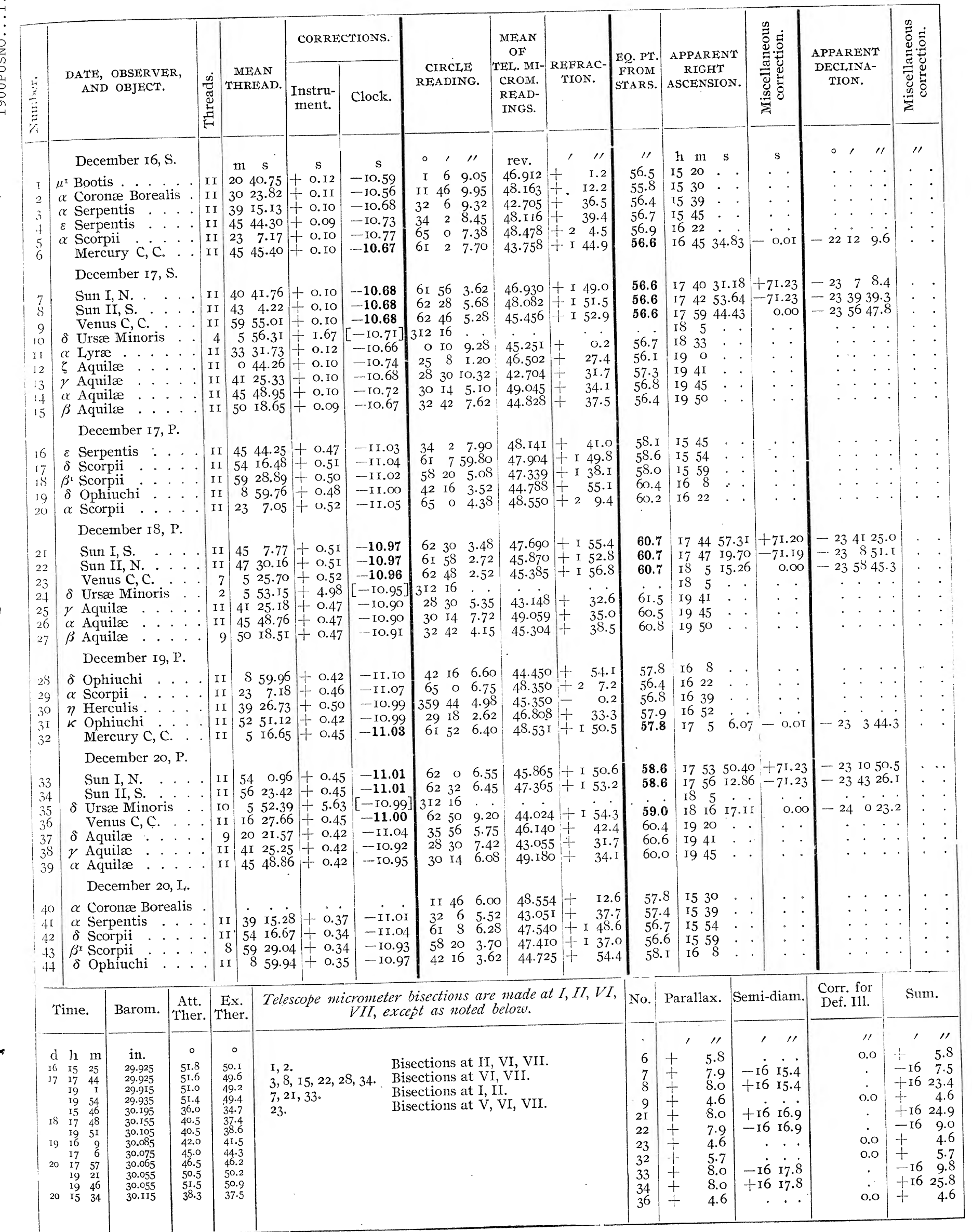




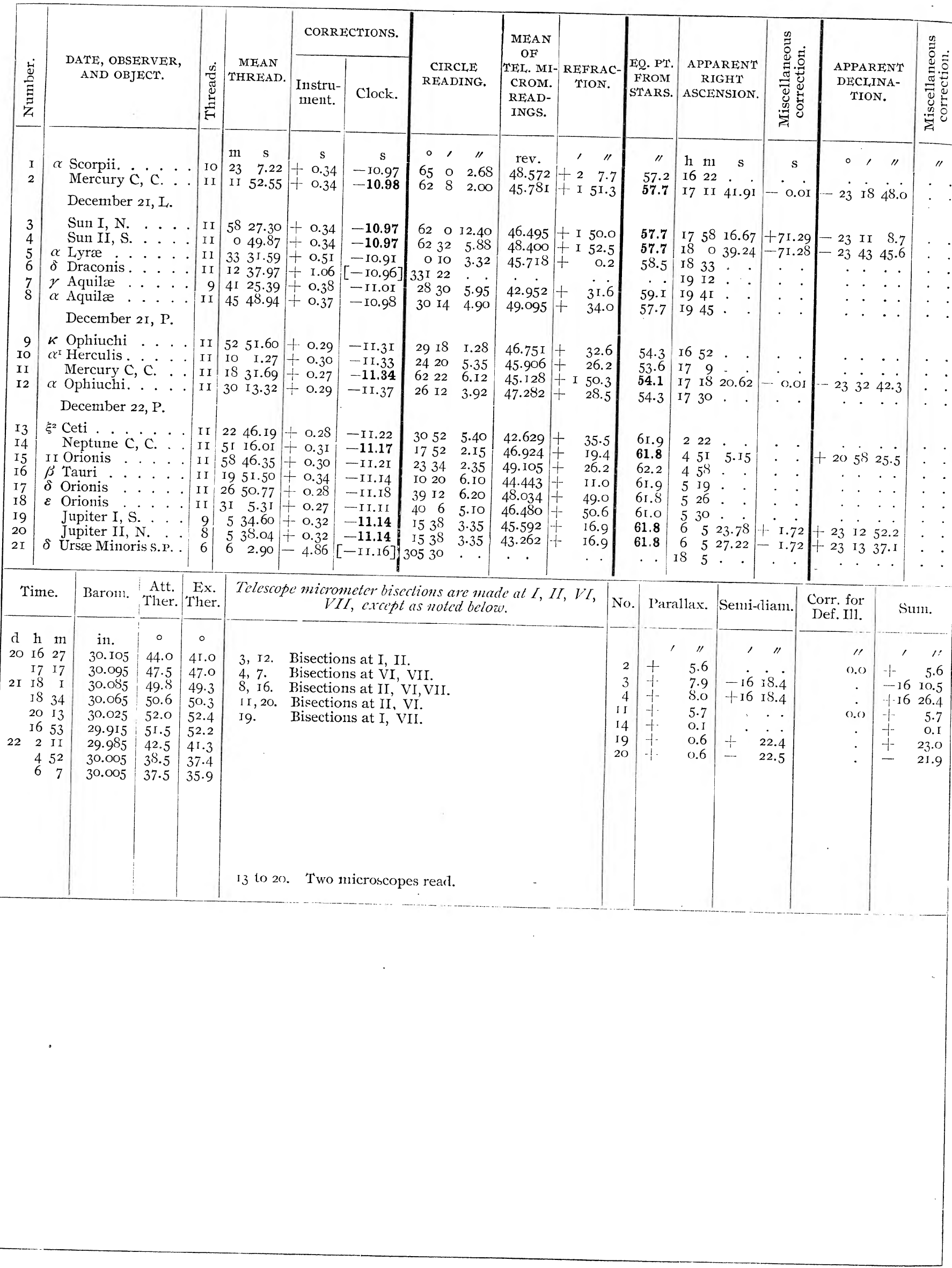




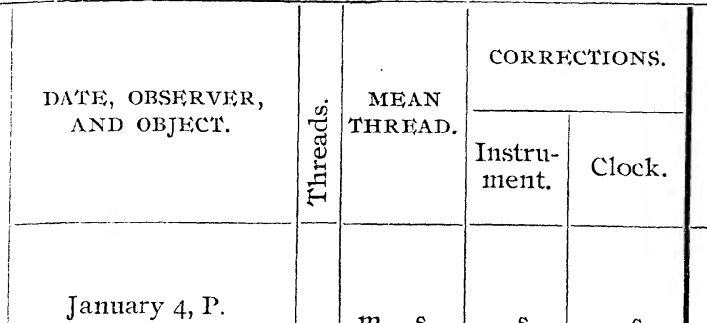

$\mid \begin{array}{ll} & \text { January 4, P. } \\ \delta & \text { Piscium . . } \\ \varepsilon & \text { Piscium . }\end{array}$

II $5743.57+0.79-\mathrm{I} 4.65$

Moon I, S. . . . II I6 $39.25+0.80-14.66$

$\alpha$ Ursæ Minoris : : $320 \mathrm{I2.5}$ I $+15.27[$ [-14.65]

January $22, \mathrm{~K}$.

5 « Lyyre . . . . I I 334 41.52 + $0.66-20.57$

6 \& Lyra . . . . . I I $4630.89+0.69-20.70$

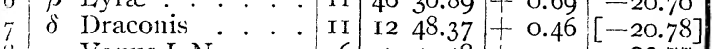

Venus I, N. . . . 6 I7 $52.48+$ I.00 -20.77

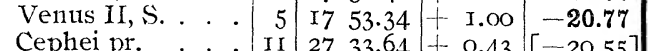

$\beta$ Cephei pr. . . . II $2733.64+0.43[-20.55]$

January $26, I_{1}$.

$12 \varepsilon$ Tauri ...... II 225 I.I6 + $0.94-2$ I.9I

13 \& $\alpha$ Tauri . . . . . 10 30 $15.68+0.95-21.81$

Neptune C, C. . . II 48 I $4.75+0.94-21.80$

15 II Orionis ..... II $5856.23+0.95-2$ I. 76

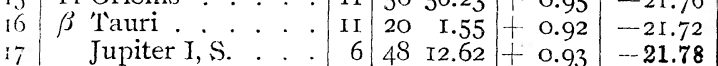

\begin{tabular}{l|l|r|rr|r|r|} 
is & & Jupiter II, N. . . & 4 & 48 & 16.15 & + \\
I9 & $v$ & Orionis . . . . . . & II & I & 56.98 & -21.78 \\
\hline
\end{tabular}

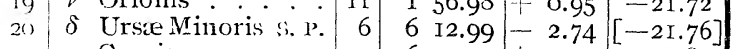

21 $\mu$ Geminorum... II I6 59.I4 $+0.93-2$ I. 83

Hebruary $26, \mathrm{~L}$.

$22 \gamma$ Aquilie .... I I 4I $49.65+0.27-34.23$

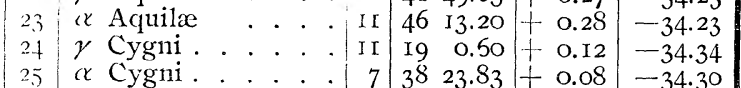

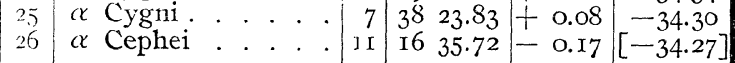

February $27, \mathrm{~L}$.

27 Sun I, N. . . . . II 4 I $29.17+0.34-34.37$

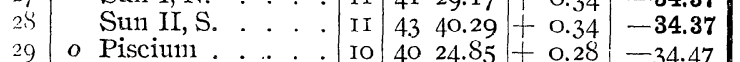

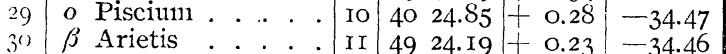

31 50 Cassiopea ... . $85459.89-0.50$ [-34.47]

$.32 \ll$ Arietis ..... II I I $49.16+0.22[-34.50$

February 28, I.

33 Neptune C, C. . . I I 4742.34 - 0.08 - 34.64

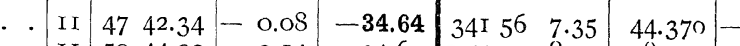

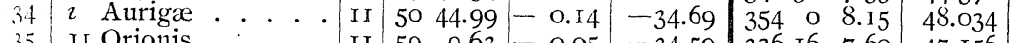

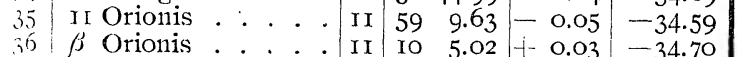

i7 Jupiter I, S. . . . $644450.17-0.09-34.64$

\begin{tabular}{rl|r|r|r|r|} 
Jupiter I, S. . . . & 6 & 44 & 50.17 & -0.09 & $\mathbf{- 3 4 . 6 4}$ \\
Jupiter II, N. . . & 5 & 44 & 53.30 & -0.09 & $\mathbf{3 4 . 6 4}$
\end{tabular}

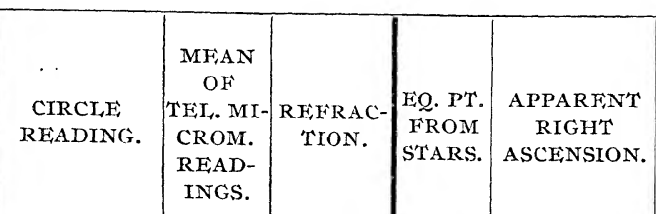

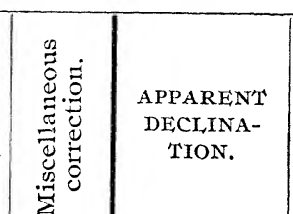

CLAMP EAST.

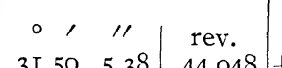

3I. $30 \quad 4.82$

48.202

3108 .

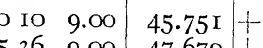

$536 \quad 9.00$

$\begin{array}{rrr}56 & 8 & 8.90 \\ 5 & 8 & 8.90\end{array}$

$\begin{array}{lll}56 & 8 & 8.90\end{array}$

$47.679+$

$49.010-32.1$

32846 .

$45.810+1$
1

$48.340+$

I9 $54 \quad 7.50 \quad 44.34 \mathrm{I}+$

\begin{tabular}{lll|l}
22 & 32 & 8.40 & 47.022 \\
I 7 & 56 & 9.00 & 46.498
\end{tabular}

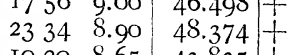

IO $20 \quad 8.65 \quad 43.835$ +

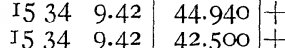

\begin{tabular}{rrr|r}
24 & 4 & 9.45 & 44.068
\end{tabular}

$\begin{array}{lll}16 & \text { I } 6 \text { I0. } 85 & 46.948\end{array}$

\section{CLAMP WEST.}

\begin{tabular}{lll|l}
33 I 22 & 10. 50 & 46.045 & -
\end{tabular}

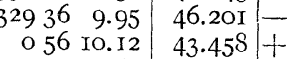

55553.62 41.280 +

238 .

$41.280+$
.

\begin{tabular}{lll}
3 I 3 & 4 & 3.48 \\
\hline I & 3.43 & 4.20
\end{tabular}

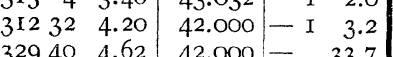

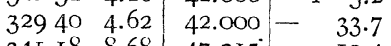

$\begin{array}{llll}341 \text { I8 } & 8.68 & 47.215 & -\end{array}$

I 9.4

21.2

$2 \mathrm{I} .3$

20.4 I 49

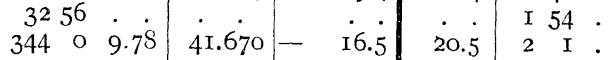

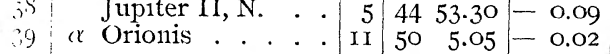

336 I6 7.60

$\begin{array}{lll}312 & 42 & 8.10 \\ 344 & 22 & 10.50\end{array}$

$47.156-\quad 5.8$
45.914

\begin{tabular}{l|l|lll} 
& & & & \\
I8.2 & 24.5 & 4 & 47 & 7.62
\end{tabular}

\begin{tabular}{l|l|lll}
5.8 & 23.5 & 4 & 50 \\
24.6 & 24.8 & 4 & 58
\end{tabular}

\begin{tabular}{l|l}
24.8 & 458
\end{tabular}

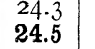

$\begin{array}{rrr}4 & 58 \\ 5 & 9 & .\end{array}$

\begin{tabular}{l|lll|}
-34.67 & 344.2 .2 & 0.50 \\
328 & 24 & 9.00
\end{tabular}

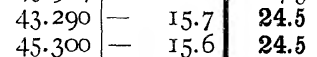

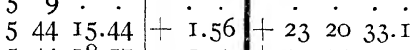

$25 \cdot 3$

$5 \quad 49$.

\begin{tabular}{|c|c|c|c|c|c|c|c|c|c|c|}
\hline 'Time. & Baront. & Att. & $\left|\begin{array}{c}\text { Ex. } \\
\text { Ther. }\end{array}\right|$ & Telescop & $\begin{array}{l}\text { icrometer bisections are made at } I, I I, V I, \\
\text { VII, except as noted below. }\end{array}$ & No. & Parallax. & Semi-diam. & $\begin{array}{l}\text { Corr. for } \\
\text { Def. I11. }\end{array}$ & Sum. \\
\hline $\begin{array}{rrr}1 & h & 111 \\
4 & 0 & 44 \\
& 1 & 17 \\
2 & 18 & 30 \\
19 & 30 \\
21 & 10 \\
& 22 & 0 \\
31 & 5 & 1 \\
& 6 & 11 \\
4 & 19 & 54 \\
& 20 & 44 \\
27 & 22 & 43 \\
& 1 & 46 \\
& 2 & 20 \\
28 & 4 & 51 \\
& 5 & 53\end{array}$ & $\begin{array}{c}\text { in. } \\
30.015 \\
30.015 \\
29.455 \\
29.445 \\
29.395 \\
29.395 \\
29.485 \\
29.515 \\
29.980 \\
29.964 \\
29.930 \\
29.850 \\
29.830 \\
29.402 \\
29.396\end{array}$ & $\begin{array}{l}\circ \\
25.5 \\
25.0 \\
36.0 \\
36.9 \\
38.8 \\
40.1 \\
31.0 \\
30.5 \\
37.5 \\
41.6 \\
49.5 \\
52.0 \\
53.2 \\
60.0 \\
58.2\end{array}$ & $\begin{array}{r}0 \\
24.5 \\
23.7 \\
33.3 \\
34.7 \\
37.3 \\
37.7 \\
30.5 \\
29.6 \\
41.2 \\
44.9 \\
50.2 \\
53.2 \\
54.2 \\
59.2 \\
58.1\end{array}$ & $\begin{array}{l}\text { I, } 29,39 \\
3 . \\
7,9,38 \\
8,37 \\
13,25 \\
\text { I7, } 28 \\
\text { I8. } \\
27 . \\
\\
33 \text { to } 39 .\end{array}$ & $\begin{array}{l}\text { Bisections at II, VI, VII. } \\
\text { Bisections at III, IV, V. } \\
\text { Bisections at II, VI. } \\
\text { Bisections at I, VII. } \\
\text { Bisections at VI, VII. } \\
\text { Bisection at VI. } \\
\text { Bisection at VII. } \\
\text { Bisections at I, II. } \\
\\
\text { Two microscopes read. }\end{array}$ & $\begin{array}{r}3 \\
8 \\
9 \\
14 \\
17 \\
18 \\
27 \\
28 \\
33 \\
37 \\
38\end{array}$ & $\begin{array}{rr} & 11 \\
+26 & 58.8 \\
+ & 4.5 \\
+ & 4.5 \\
+ & 0.1 \\
+ & 0.5 \\
+ & 0.5 \\
+ & 6.5 \\
+ & 6.5 \\
+ & 0.1 \\
+ & 0.5 \\
+ & 0.5\end{array}$ & 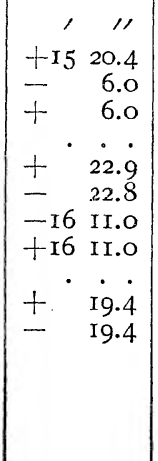 & 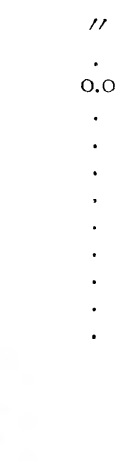 & $\begin{array}{rr} & 11 \\
+42 & 19.2 \\
+ & 1.5 \\
+ & 10.5 \\
+ & 0.1 \\
+ & 23.4 \\
- & 22.3 \\
-16 & 4.5 \\
+16 & 17.5 \\
+ & 0.1 \\
+ & 19.9 \\
- & 18.9\end{array}$ \\
\hline
\end{tabular}




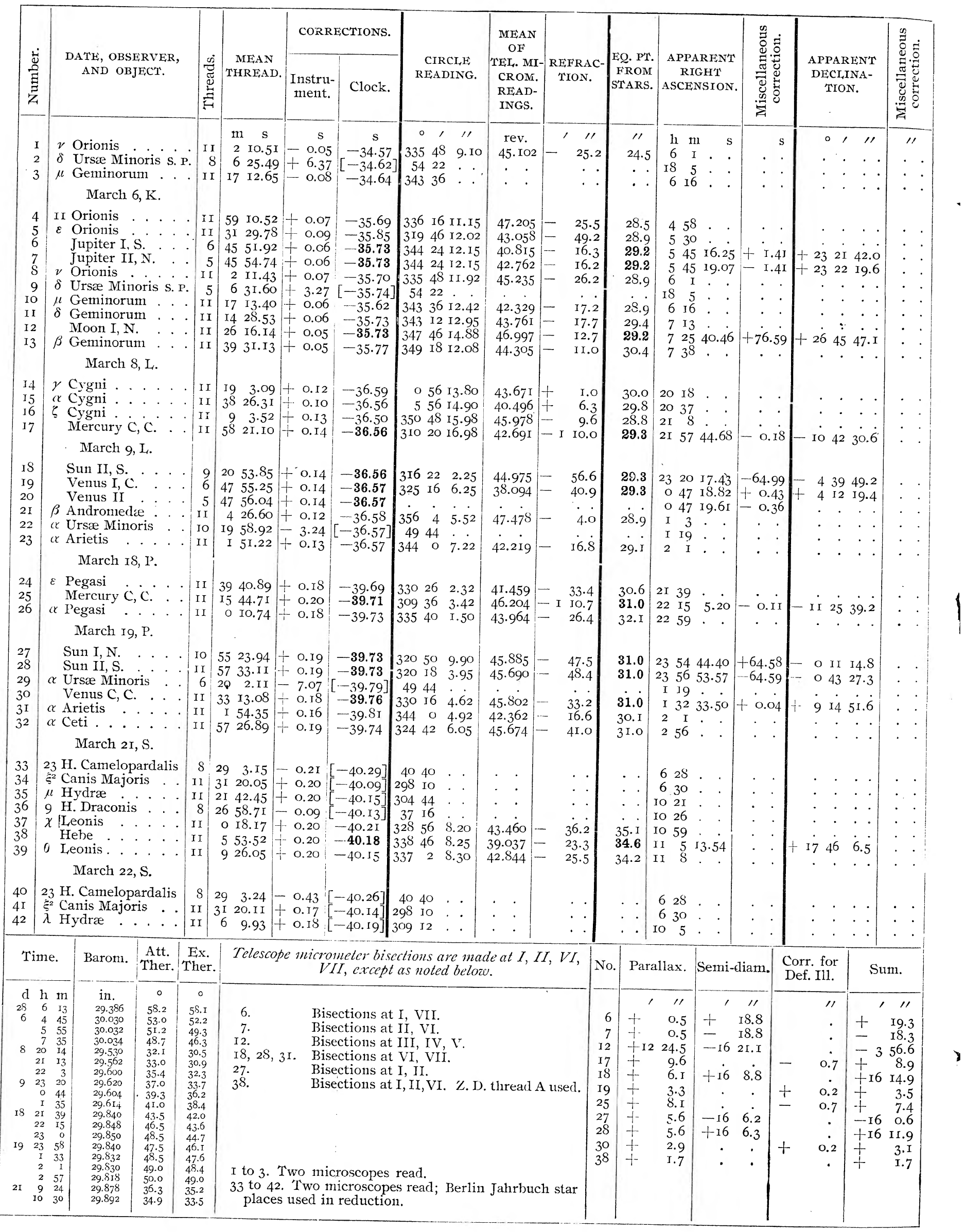




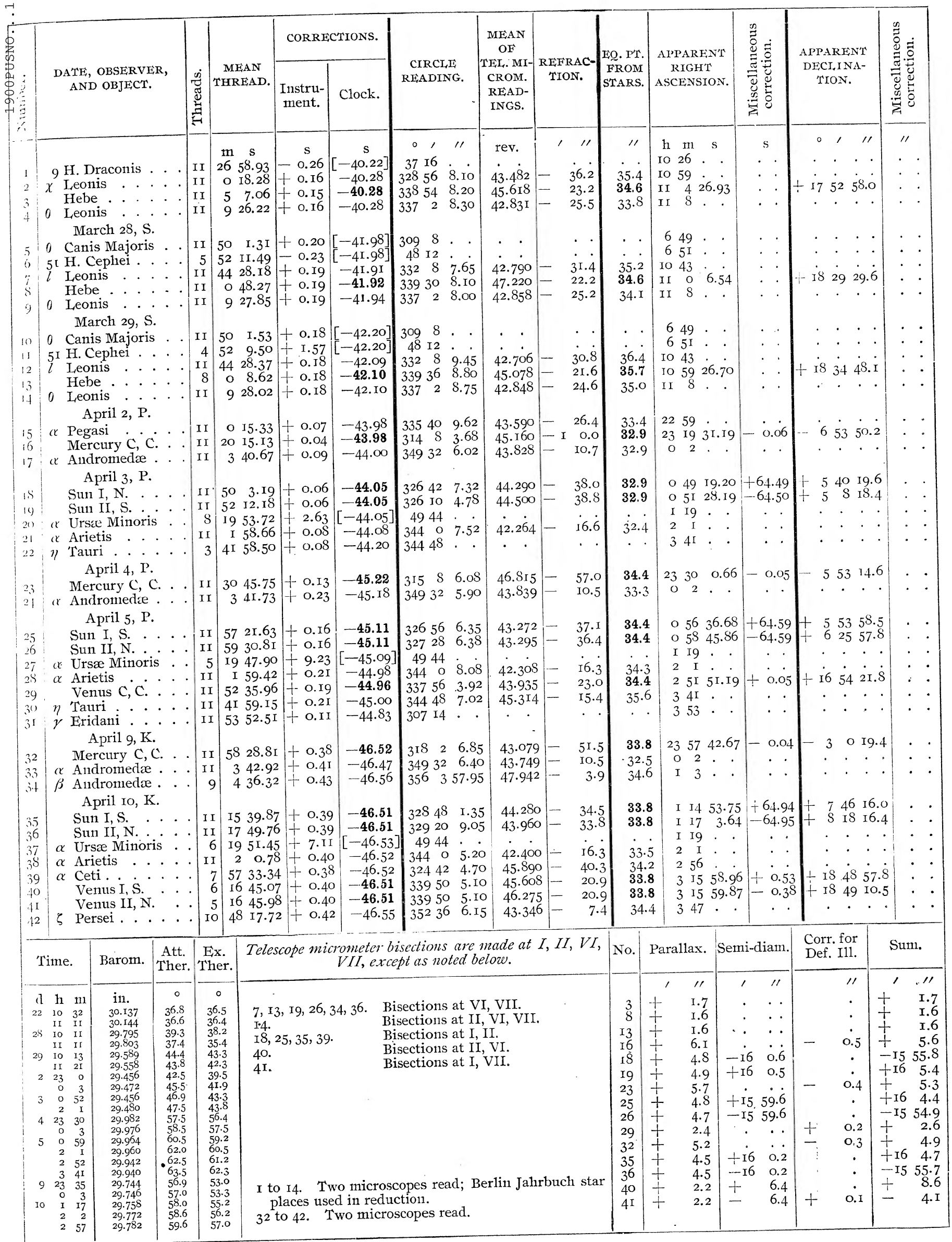




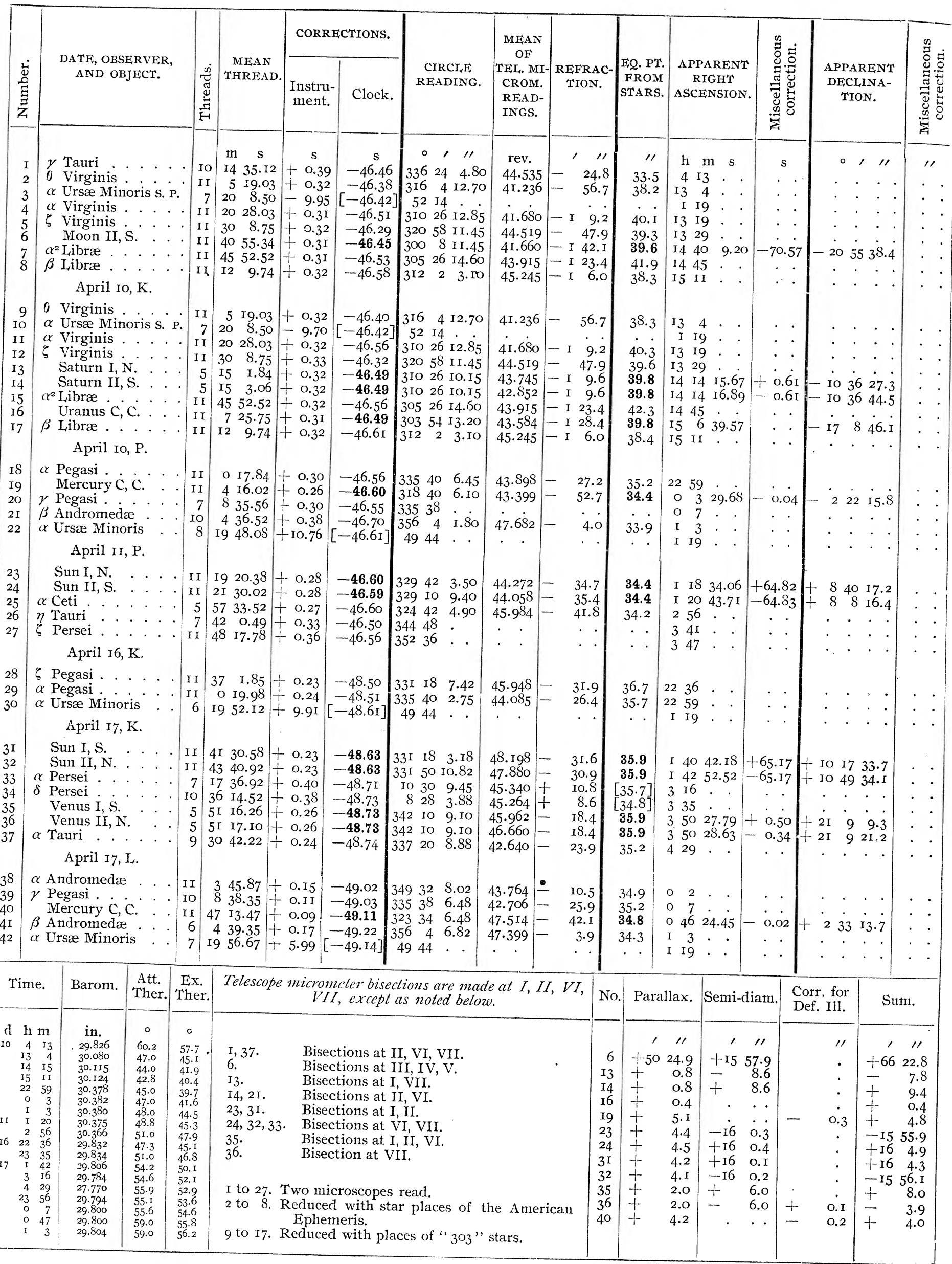




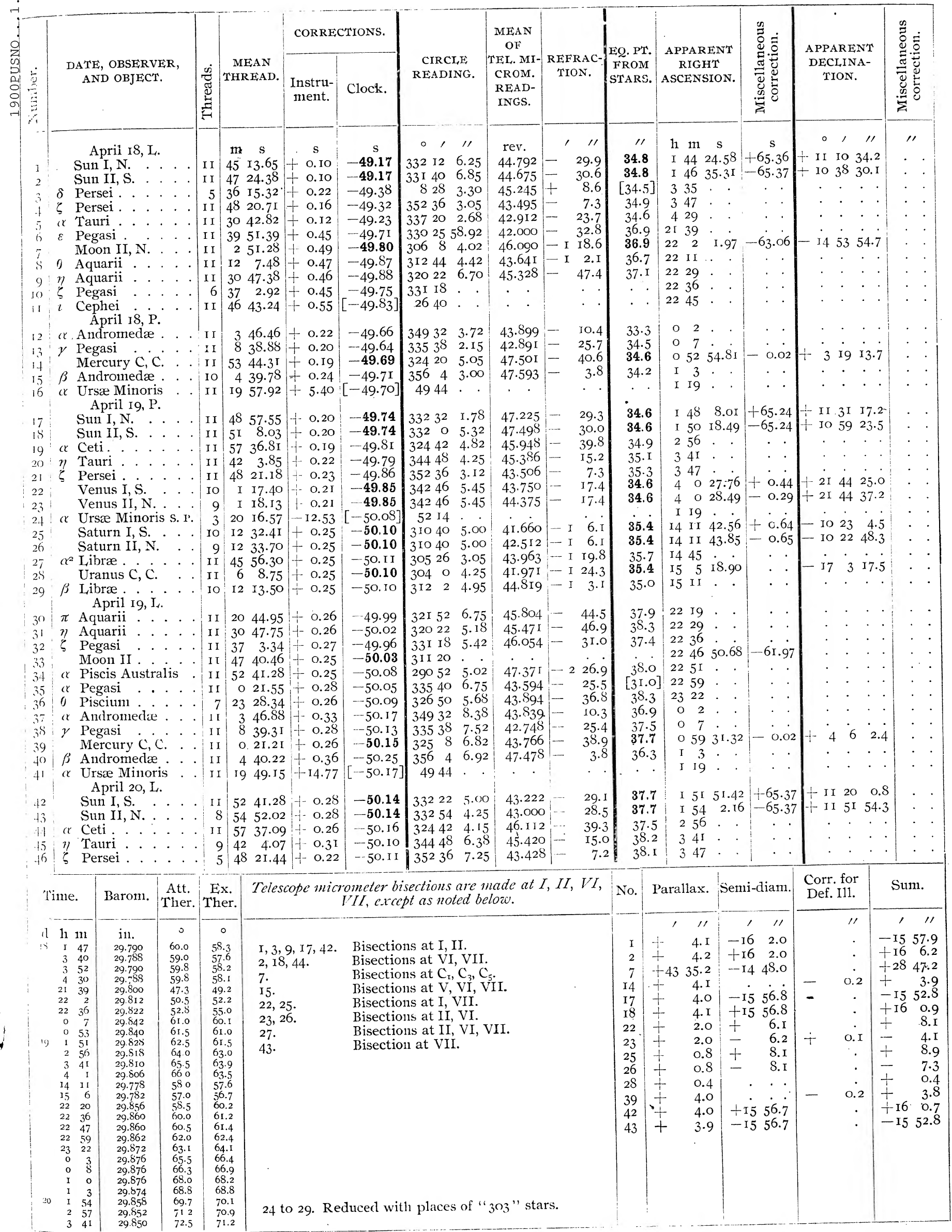




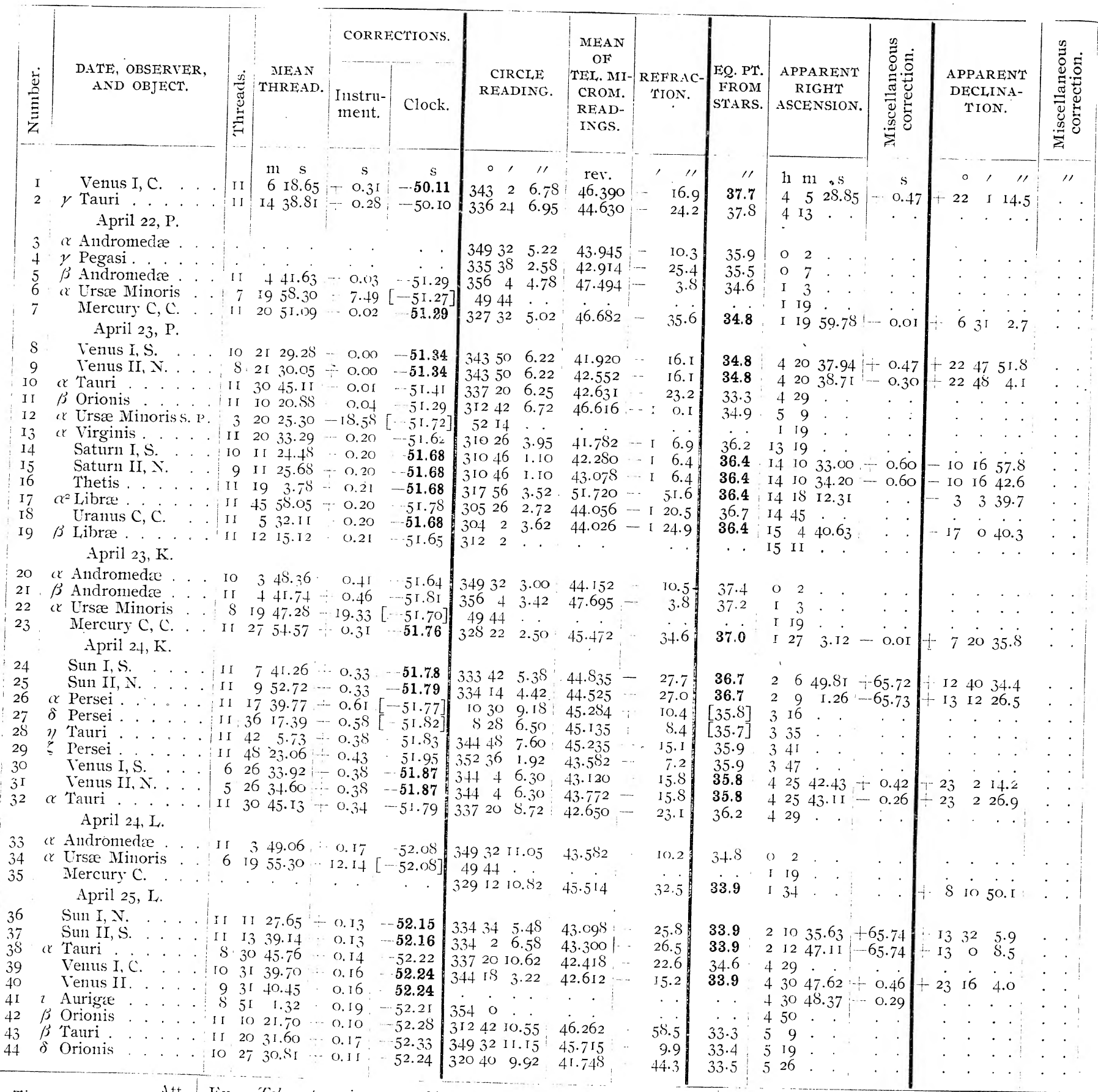

Time. Barom, Att. Ex. Telescope micrometer bisections are made at $I, I I, I \%$

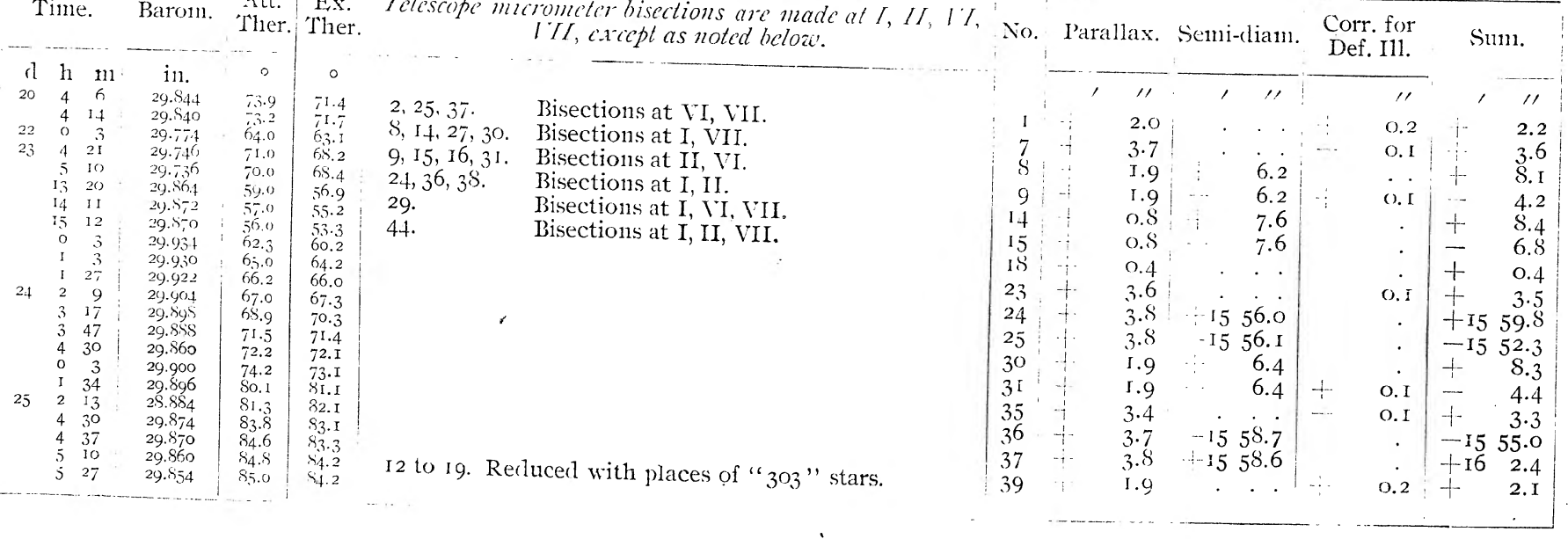




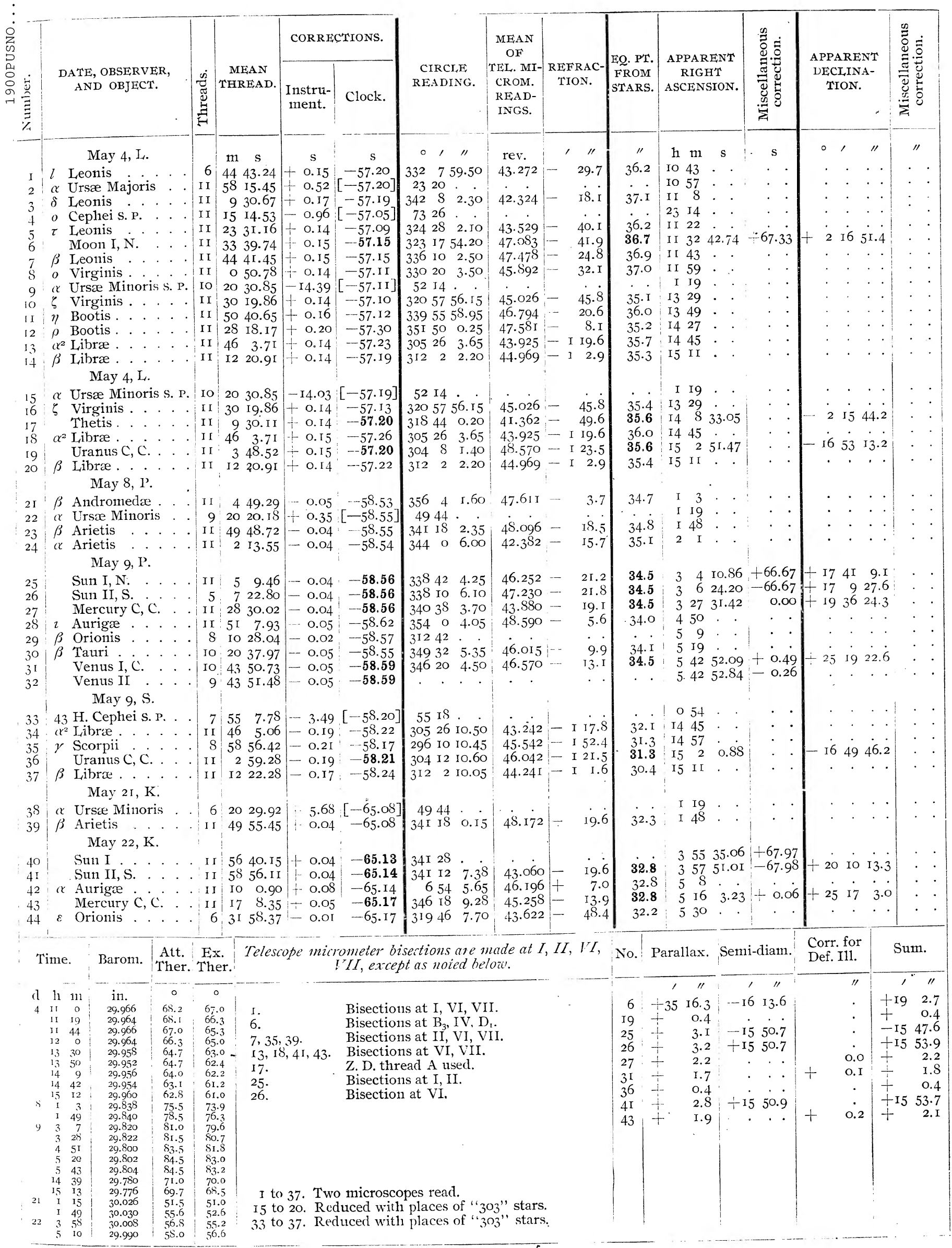




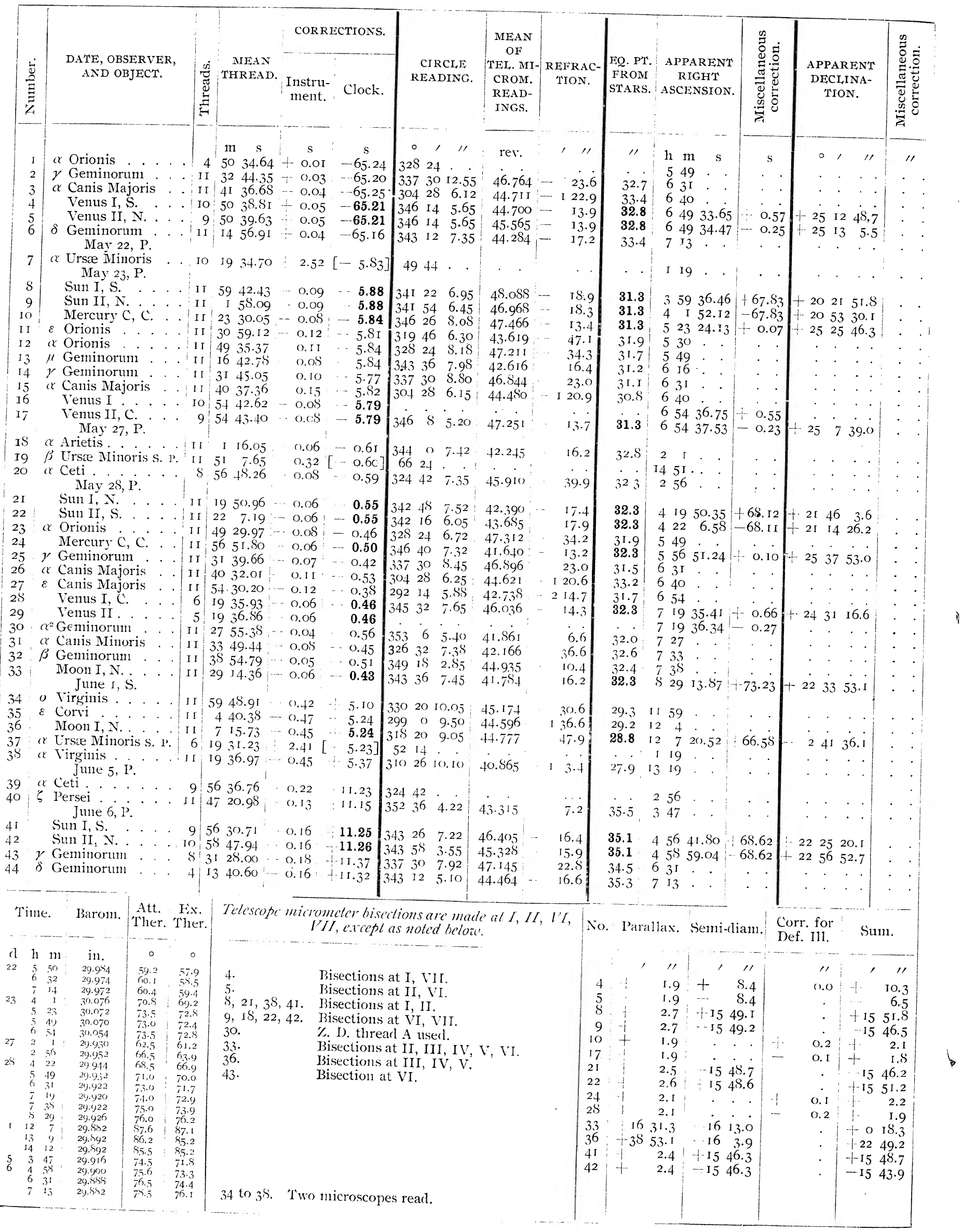




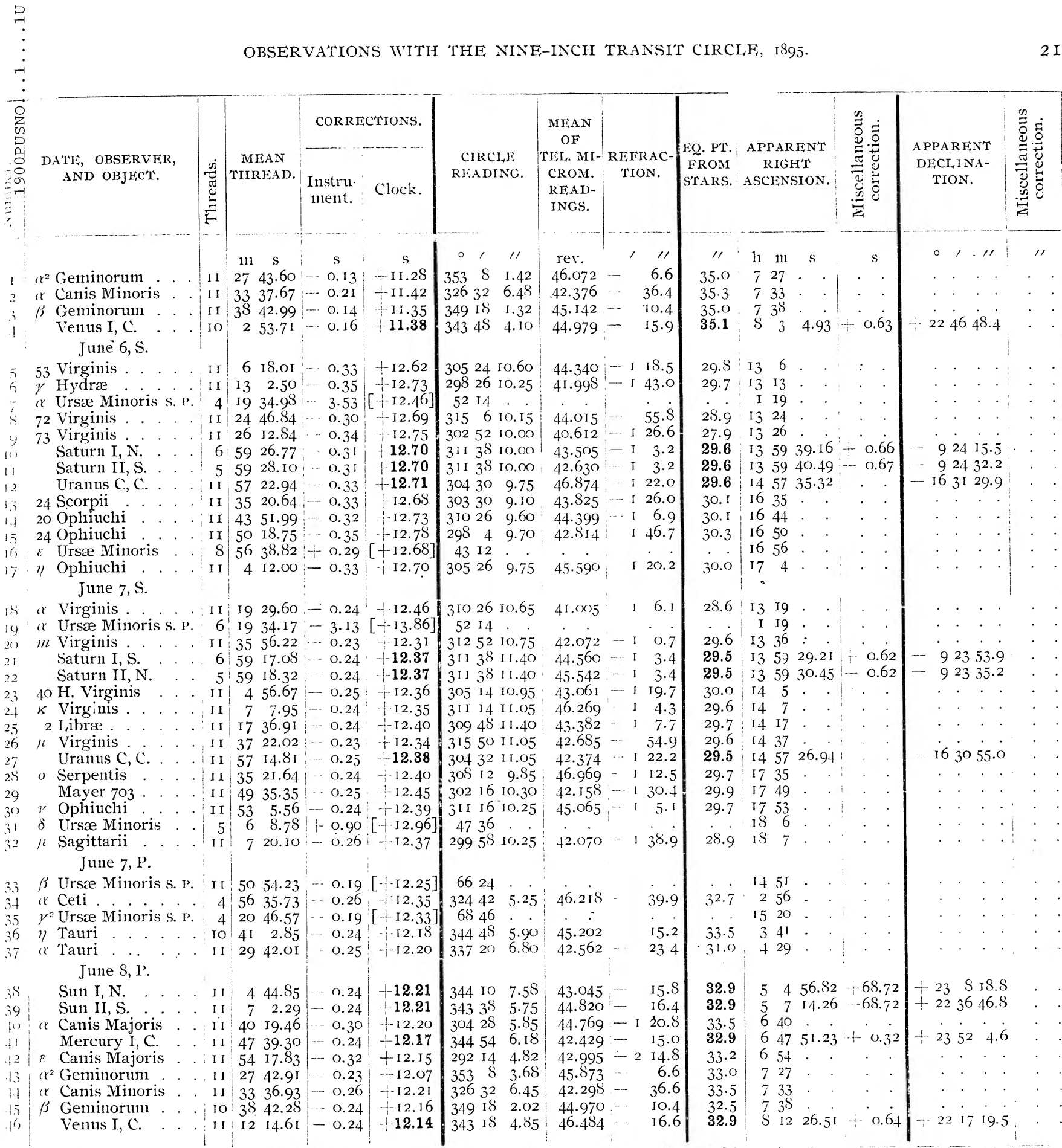

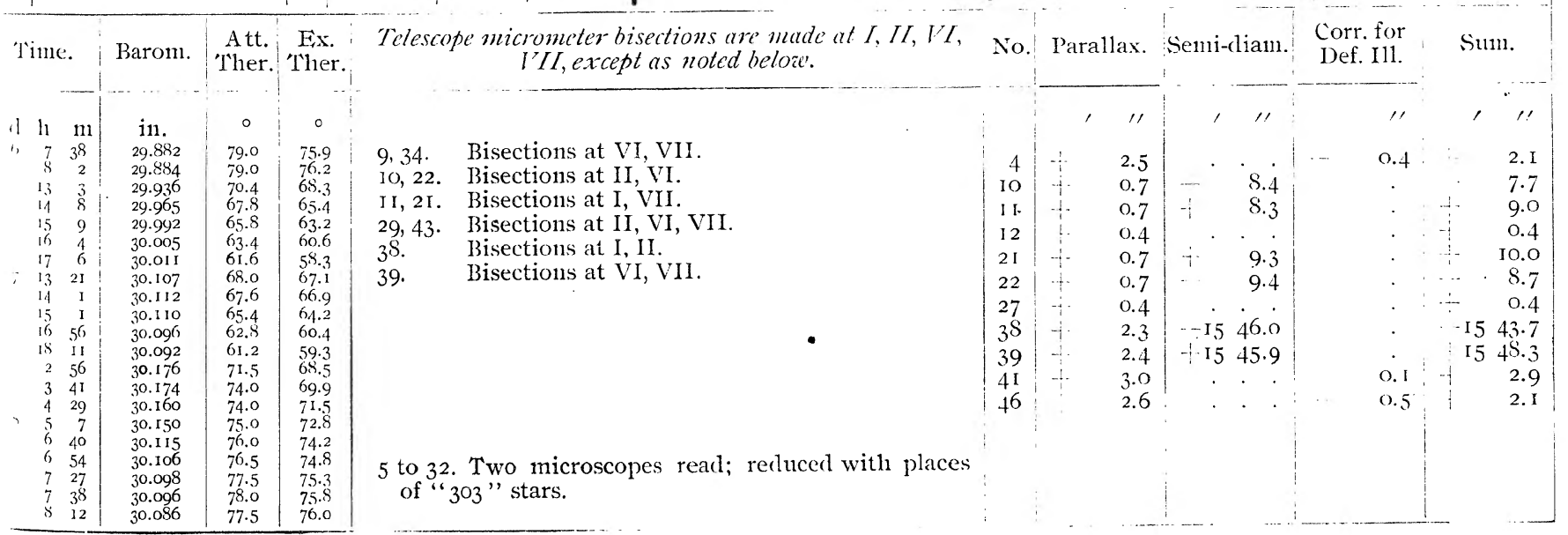




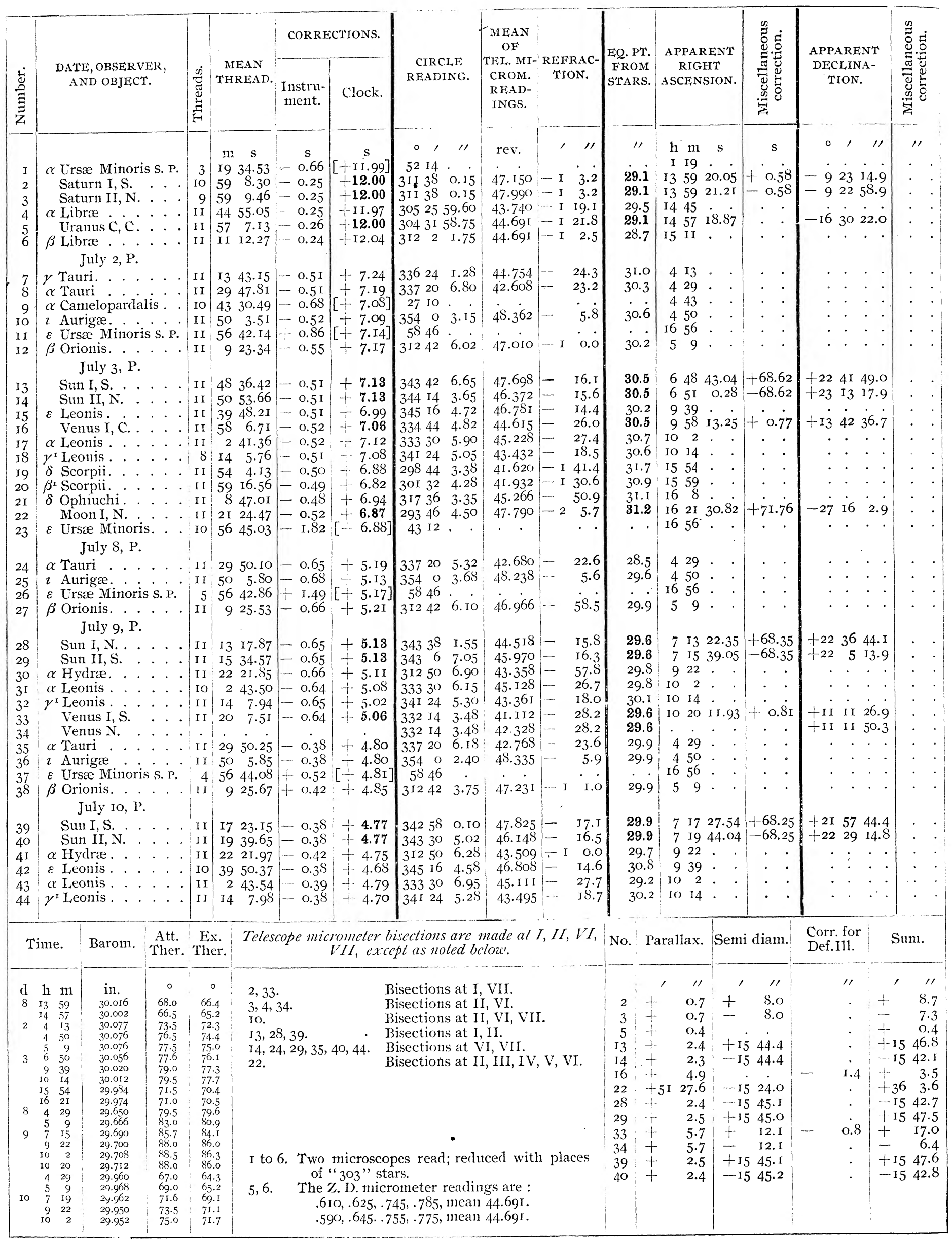




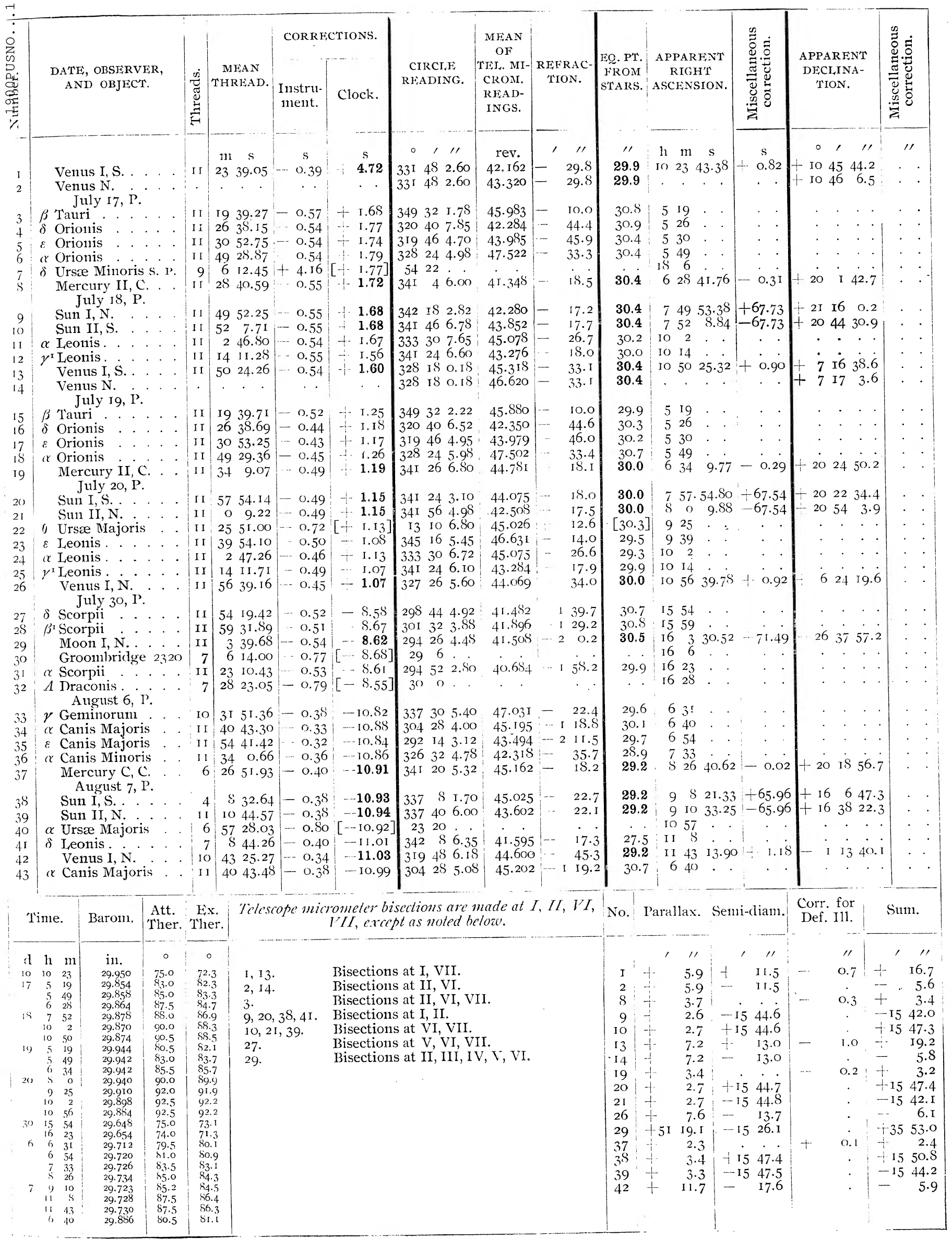




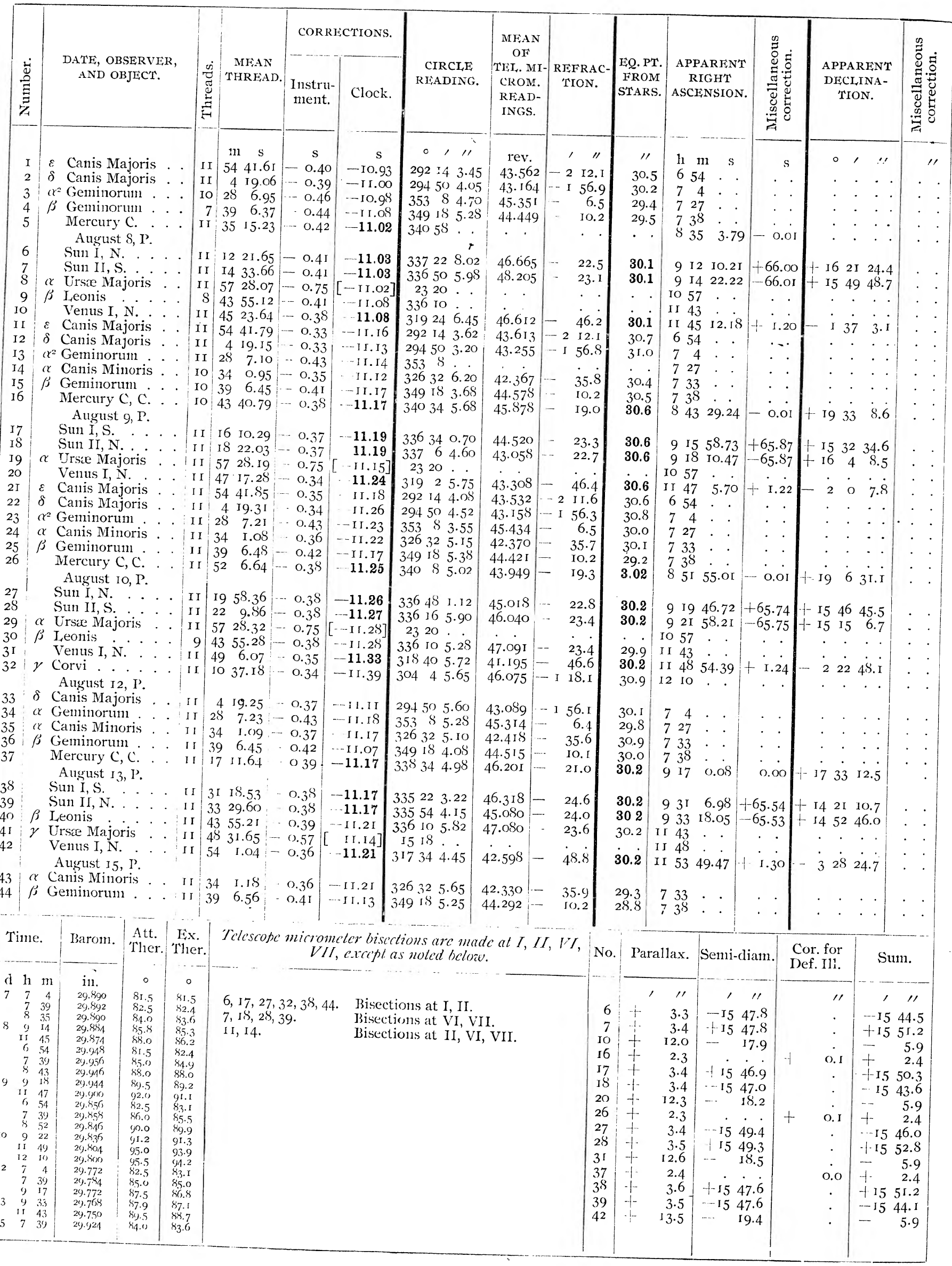




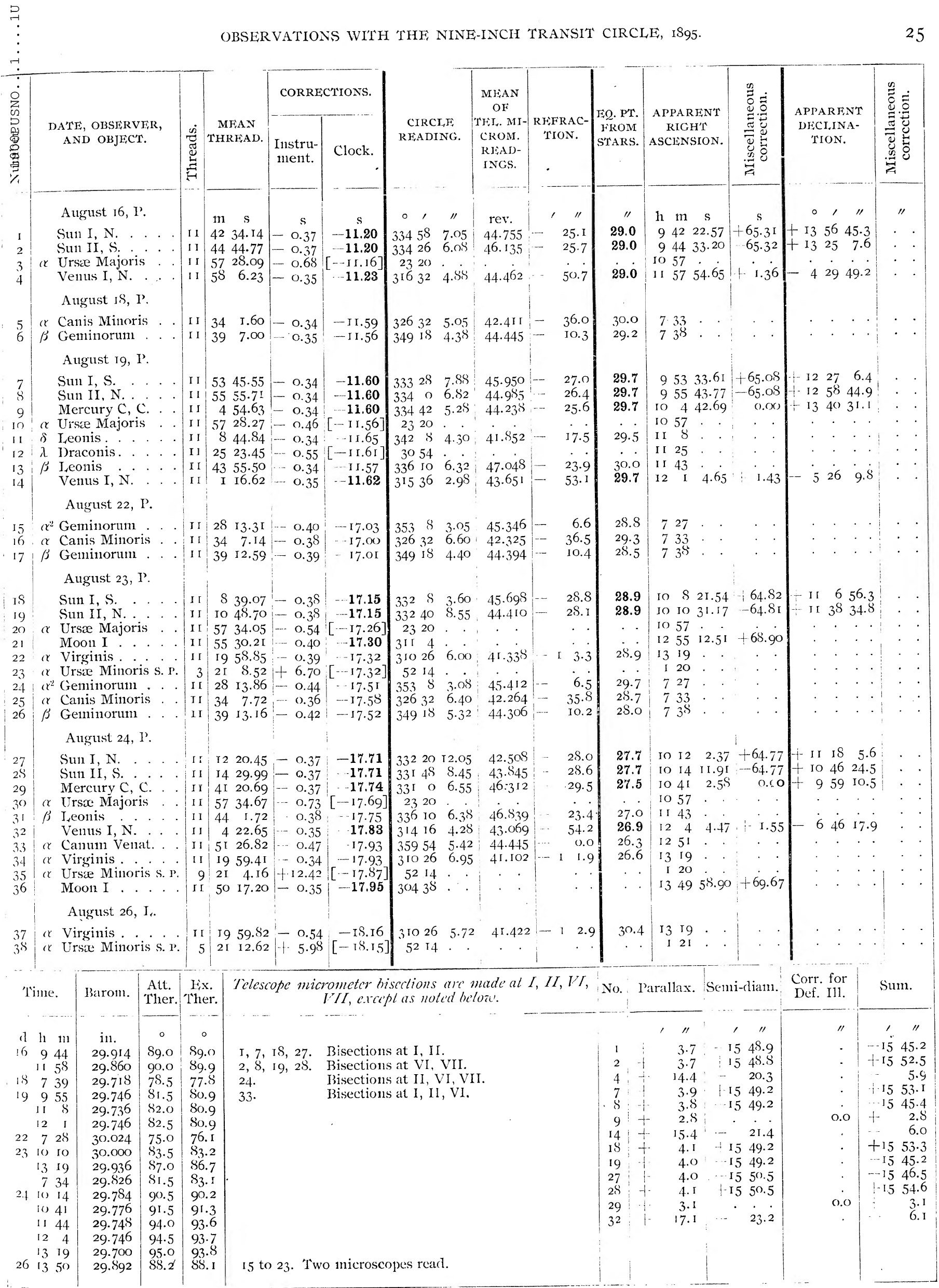




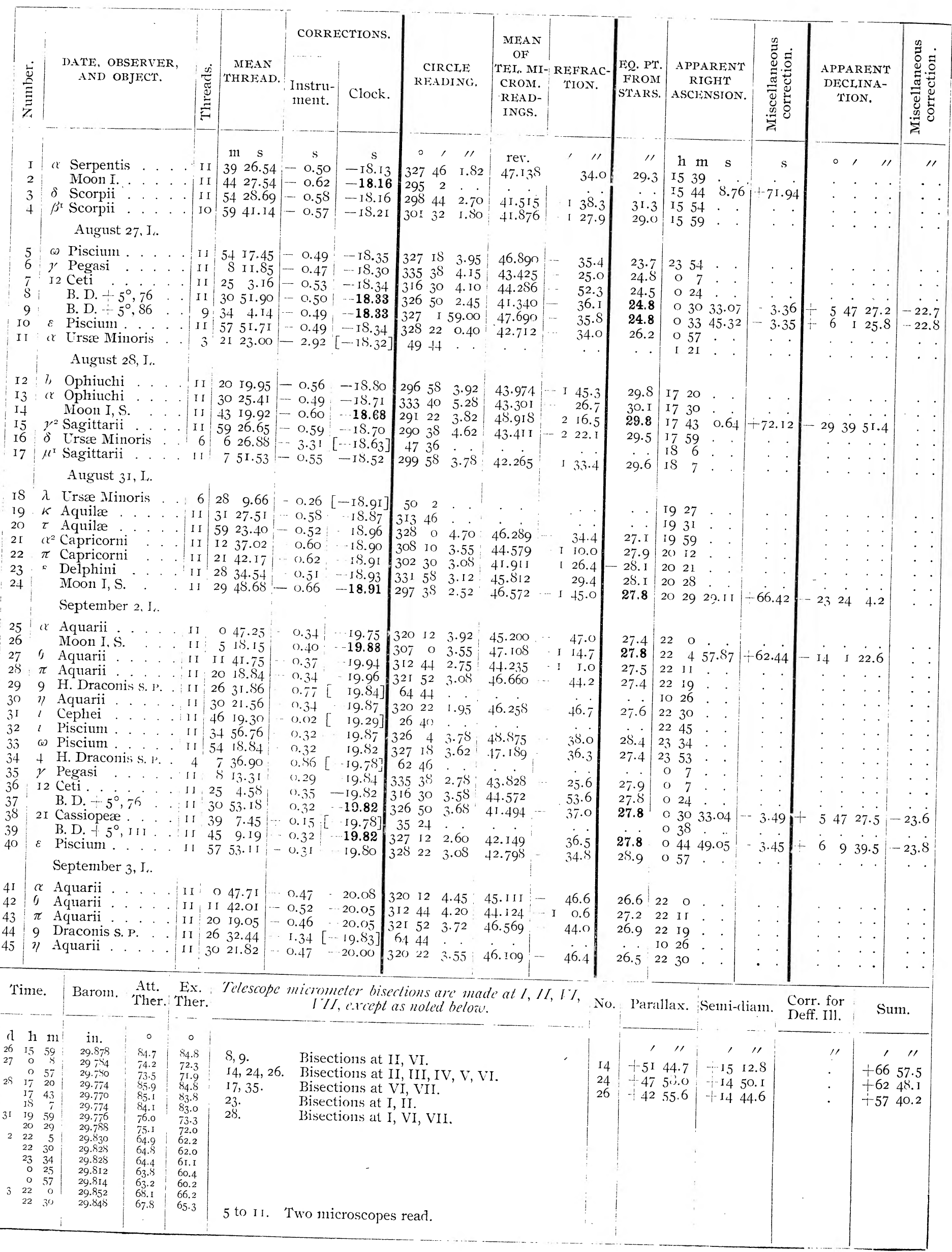




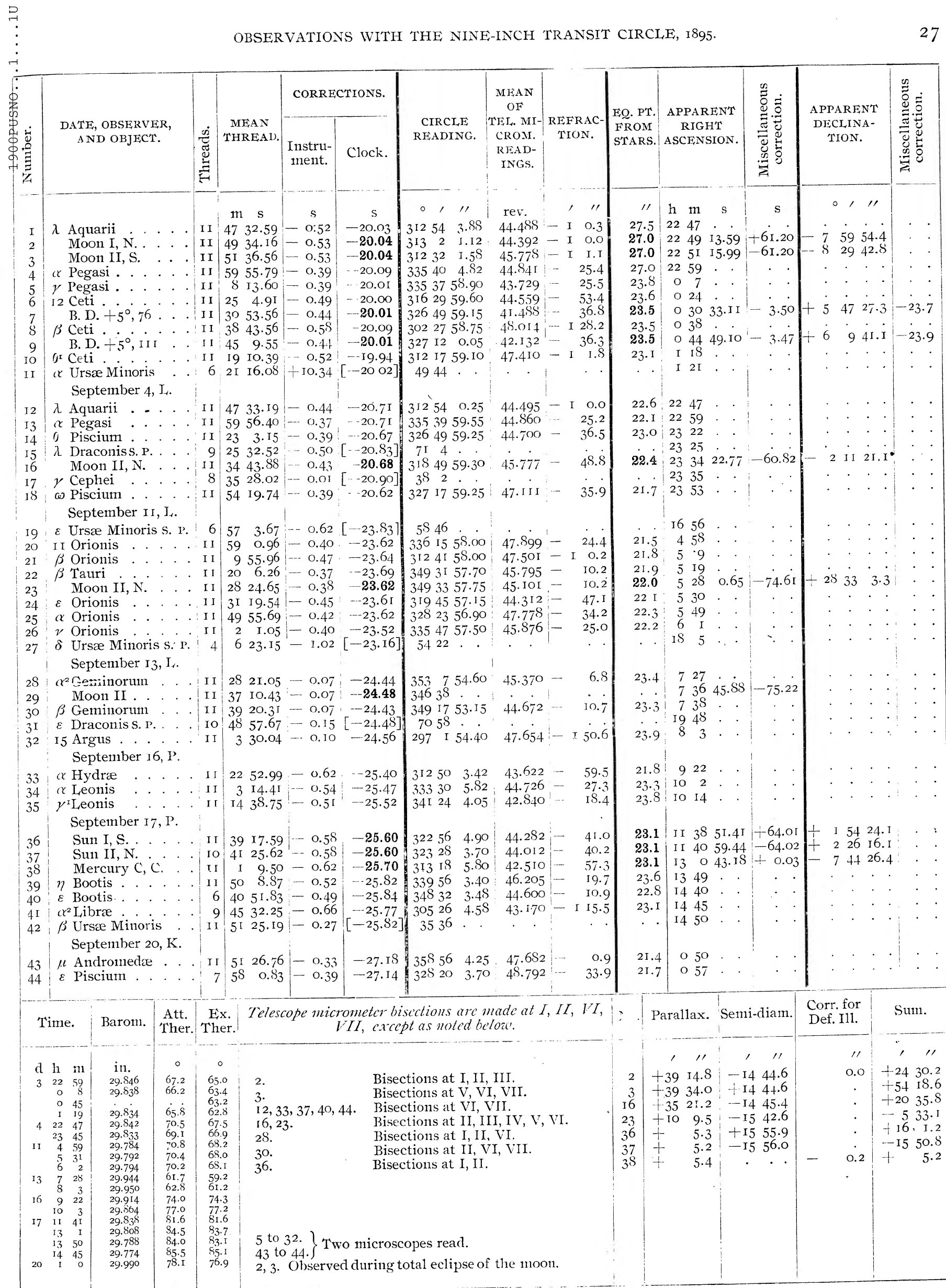




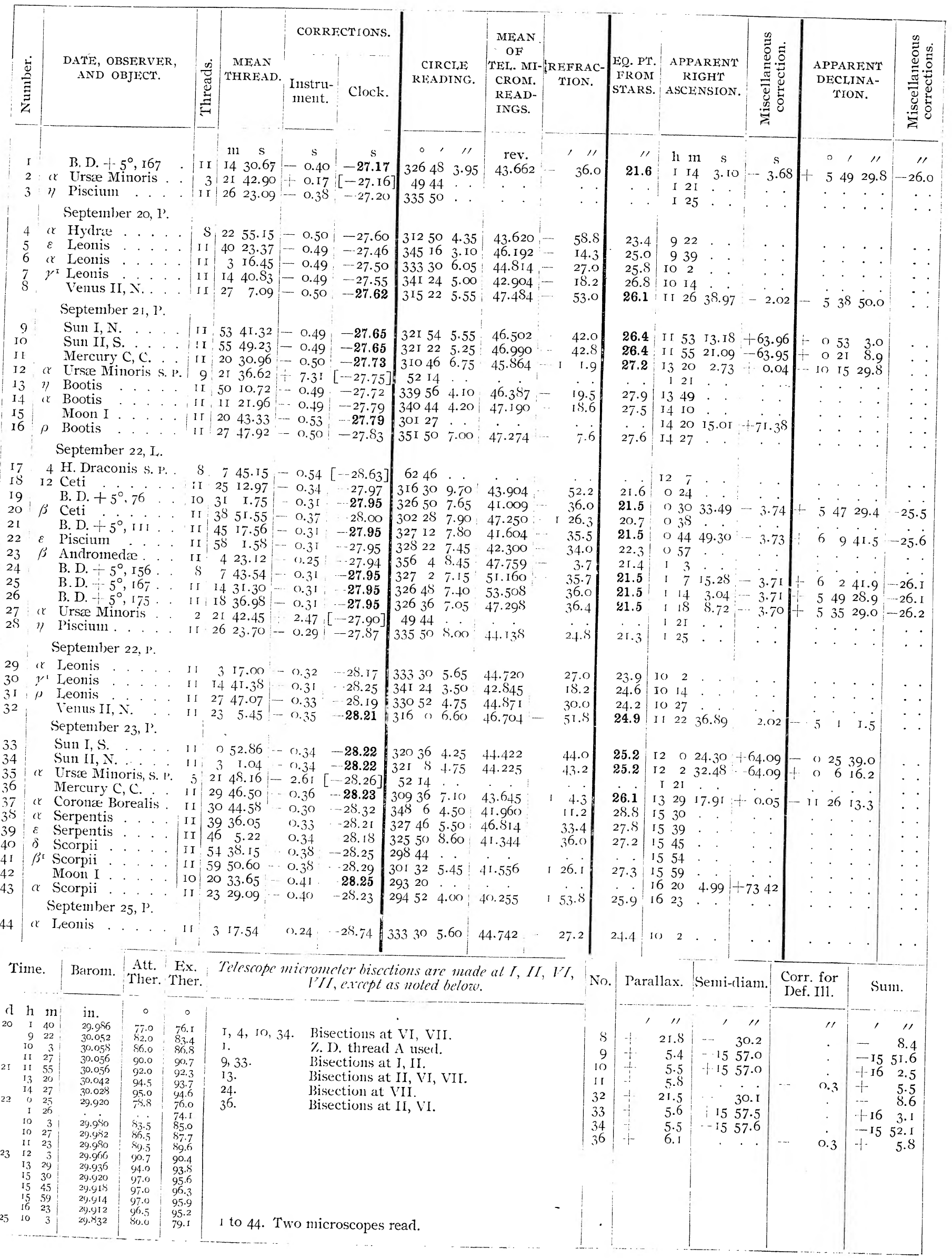




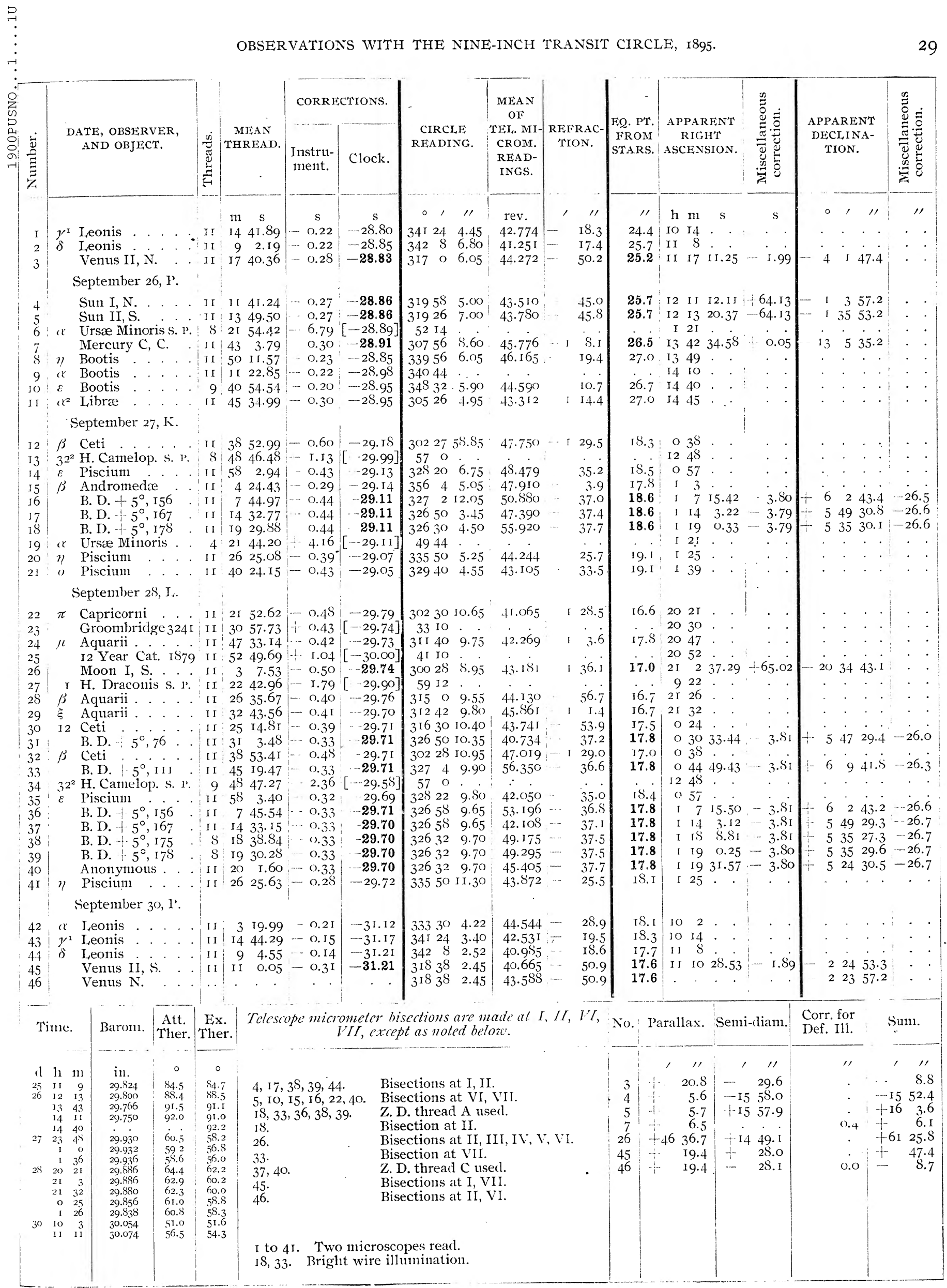




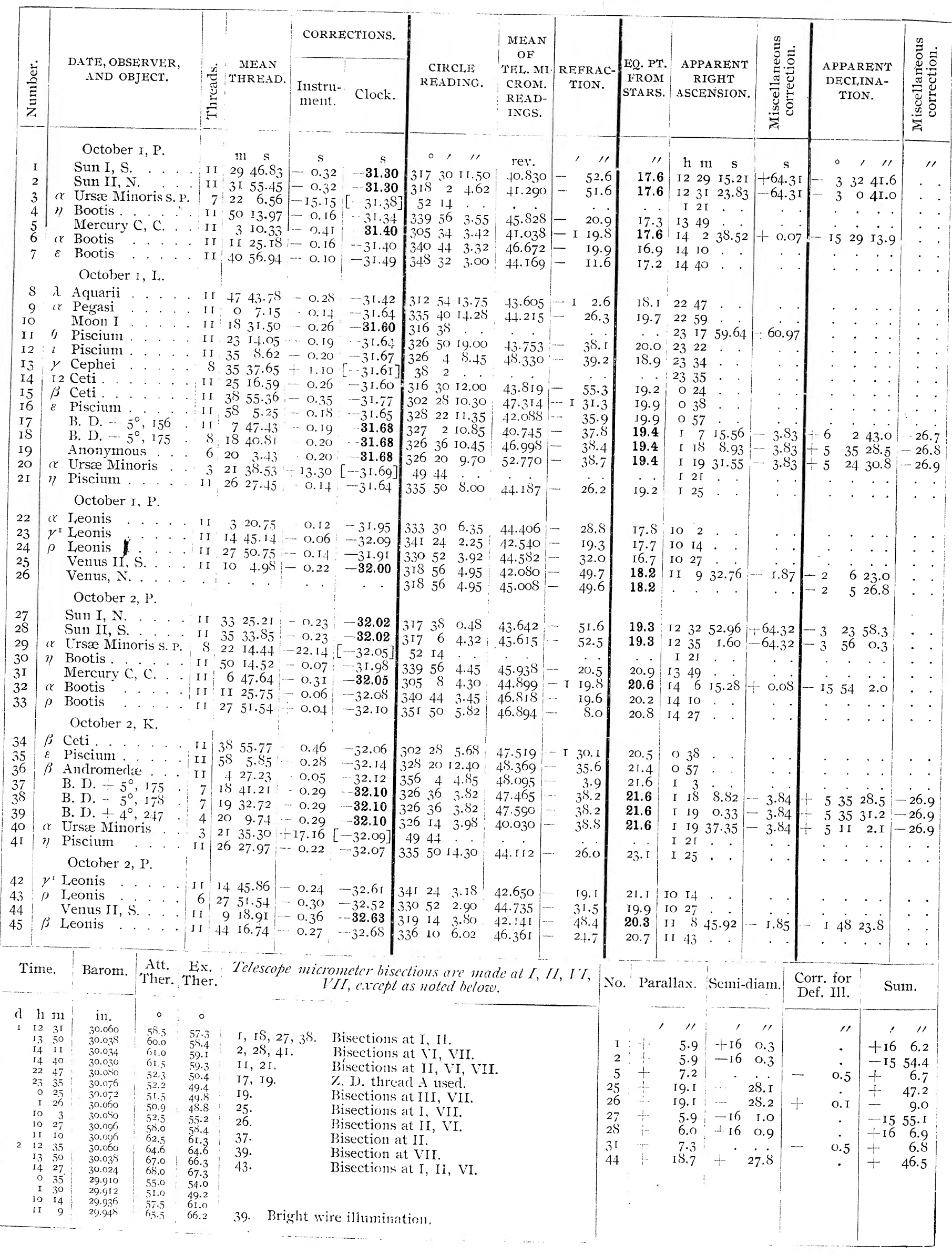




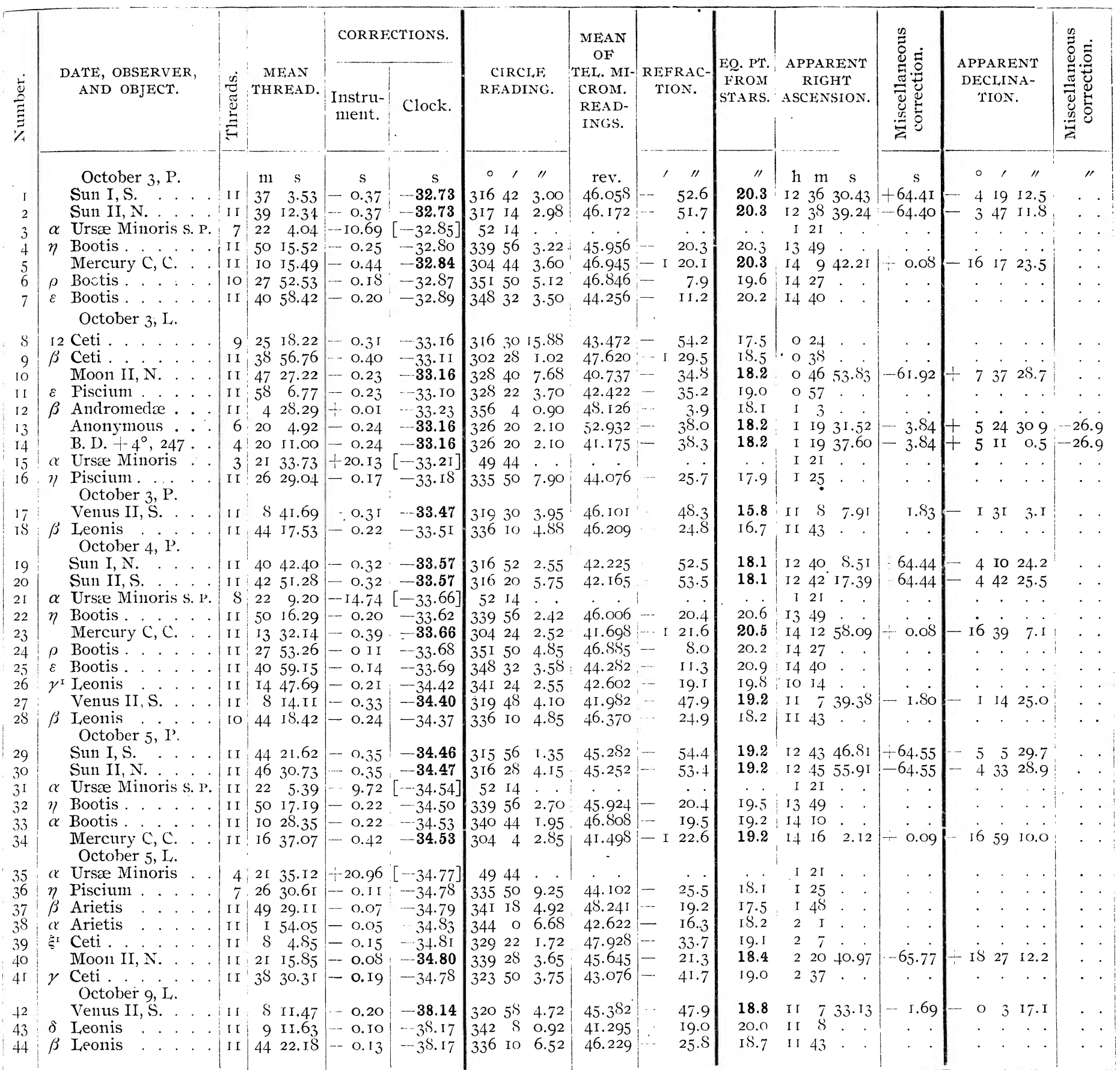

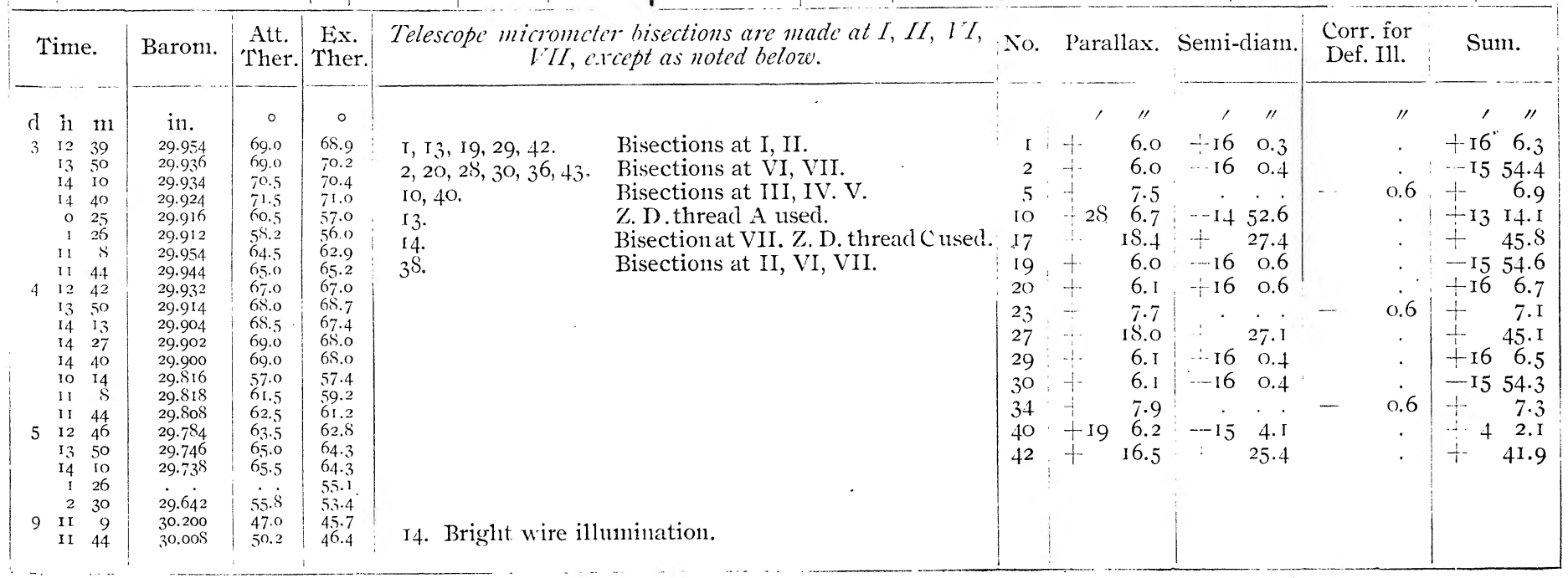




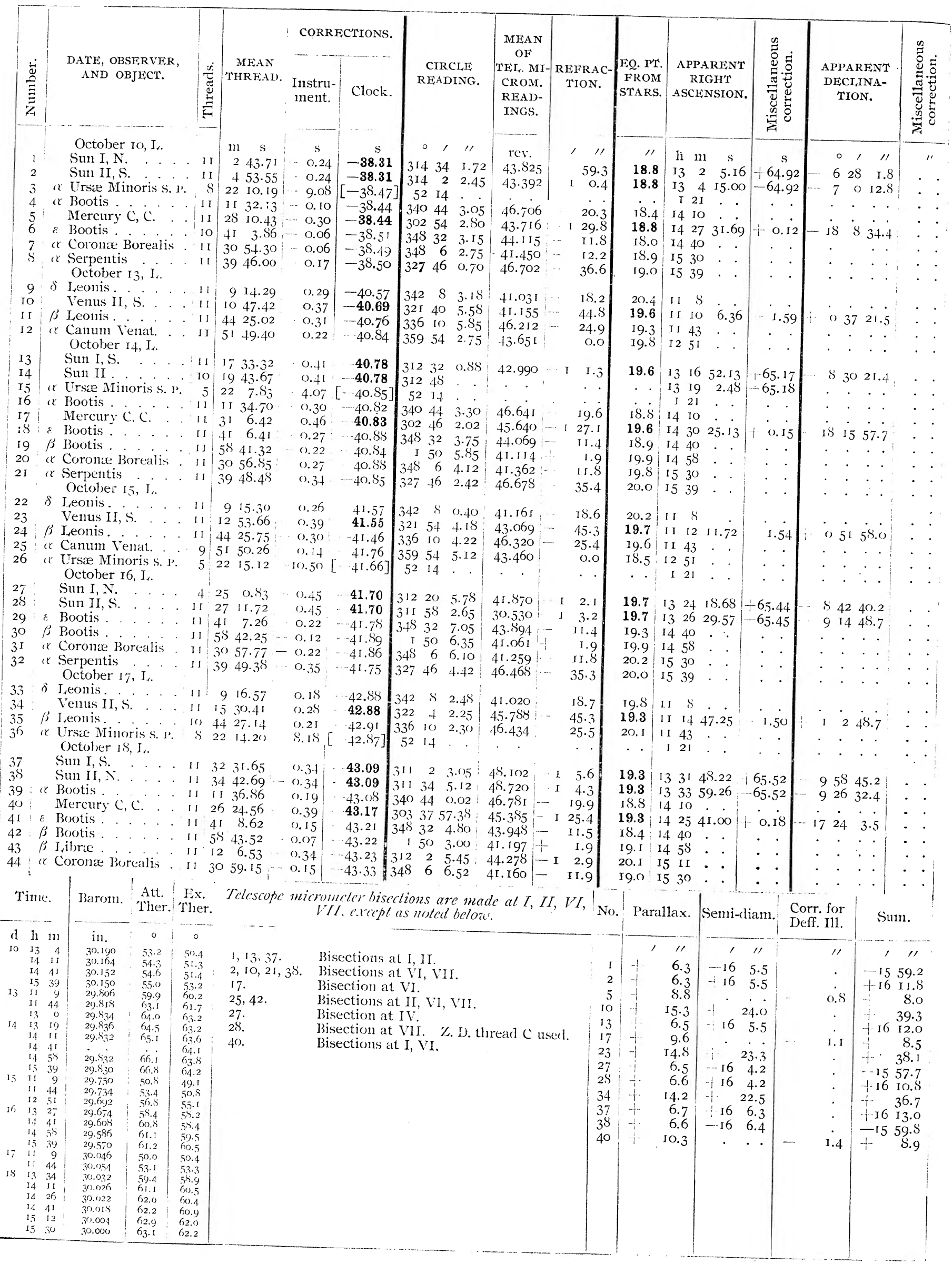




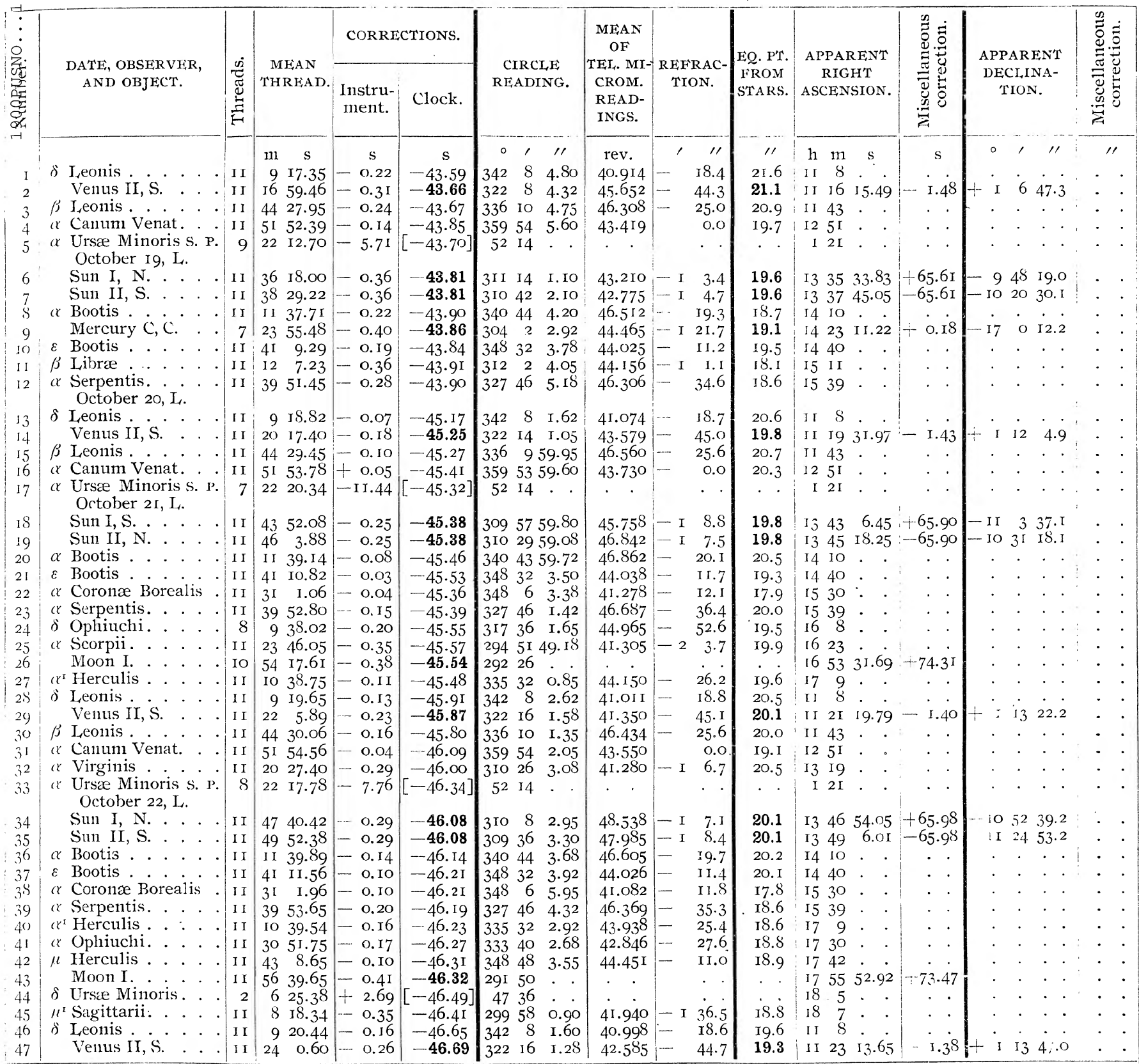

\begin{tabular}{|c|c|c|c|c|c|c|c|c|c|c|}
\hline 'Time. & Baronl. & $\begin{array}{l}\text { Att. } \\
\text { Ther. }\end{array}$ & $\begin{array}{l}\text { Ex. } \\
\text { Ther. }\end{array}$ & $\begin{array}{c}\text { Telescope micrometer bisections are made at } I, I I, V I, \\
\text { VII, except as noted below. }\end{array}$ & No. & Parallax. & Semi-diam. & & for & Sum. \\
\hline $\mathrm{d} h \mathrm{hl}$ & in. & o & $\circ$ & & & "I & 111 & & 11 & 111 \\
\hline Is II 9 & 29.748 & 53.9 & 55.0 & Bisections at I, II. & 2 & $+\quad 14.0$ & † $\quad 22.2$ & & . & $+\quad 36.2$ \\
\hline II 44 & $\begin{array}{l}29.732 \\
20662\end{array}$ & 56.4 & 57.3 & 7, I 9,35 . Bisections at VI, VII. & 6 & 6.6 & $-16 \quad 5.5$ & & & - I5 58.9 \\
\hline $\begin{array}{lll}19 & 13 & 38 \\
14 & \text { II }\end{array}$ & $\begin{array}{l}29.002 \\
29.634\end{array}$ & $\begin{array}{l}04.3 \\
65.5\end{array}$ & $\begin{array}{c}60.2 \\
67.9\end{array}$ & $9,36,45$. Bisections at II, VI. & 7 & 6.7 & $+16 \quad 5.5$ & & & +1612.2 \\
\hline $144 \mathrm{I}$ & 29.612 & 67.1 & 689 & $22,23,3 \mathrm{I}$. Bisections at II, VI, VII. & 9 & I0.4 & . & - & I.5 & $+\quad 8.9$ \\
\hline $\begin{array}{rrr}15 & 12 \\
15 & 39\end{array}$ & $\begin{array}{l}29.600 \\
29.592\end{array}$ & 68.8 & $\begin{array}{ll}70.1 \\
71.3\end{array}$ & & I4 & $\mathrm{I} 3.5$ & $+\quad 2 \mathrm{I} .5$ & & . & +35.0 \\
\hline $\begin{array}{ccc}15 & 39 \\
20 & \text { II } & 9\end{array}$ & $\begin{array}{l}29.592 \\
29.796\end{array}$ & $\begin{array}{l}7 \mathrm{~T} .4 \\
47 . \mathrm{I}\end{array}$ & $\begin{array}{ll}71.3 \\
47.2\end{array}$ & & I8 & 6.8 & $+\mathrm{I} 6 \quad 9.4$ & & . & +1616.2 \\
\hline II 44 & 29.820 & $49 \cdot 3$ & 48.6 & & 19 & 6.7 & $-16 \quad 9.5$ & & . & $\begin{array}{ll}-16 & 2.8\end{array}$ \\
\hline $\begin{array}{lll}21 & 13 & 46\end{array}$ & $\begin{array}{l}29.852 \\
20.858\end{array}$ & 52.5 & 50.6 & & 29 & 13.3 & $2 \mathrm{I} . \mathrm{I}$ & & . & $+\quad 34.4$ \\
\hline $\begin{array}{ll}14 & 11 \\
14\end{array}$ & 29.862 & 53.5 & $5^{1.2}$ & & 34 & 6.8 & $-16 \quad 7.0$ & & . & $\begin{array}{ll}-\mathrm{I} 6 & 0.2\end{array}$ \\
\hline $\begin{array}{l}1531 \\
15 \\
15\end{array}$ & & & & & 35 & +6.8 & $+16 \quad 7.0$ & & . & +1613.8 \\
\hline 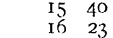 & $\begin{array}{l}29.858 \\
29.874\end{array}$ & $\begin{array}{l}54.6 \\
55.2\end{array}$ & $\begin{array}{l}51.3 \\
52.2\end{array}$ & & 47 & $+\quad \mathrm{I} 3 . \mathrm{I}$ & $+\quad 20.8$ & & & $+\quad 33.9$ \\
\hline 1710 & 29.872 & 55.2 & 52.4 & & & & & & & \\
\hline 11 9 & 29.890 & 45.2 & 46.1 & & & & & & & \\
\hline II 44 & 29.896 & 47 & 48.8 & & & & . & & & \\
\hline $\begin{array}{ll}12 & 5 \mathrm{I} \\
13 & 20\end{array}$ & $\begin{array}{l}29.8 \\
29.8\end{array}$ & 54.2 & & & & & & & & \\
\hline $\begin{array}{lll}13 & 20 \\
22 & 13 & 50\end{array}$ & $\begin{array}{l}29.872 \\
29.866\end{array}$ & $\begin{array}{l}57.2 \\
59 \cdot 3\end{array}$ & 60.5 & & & & & & & \\
\hline $\begin{array}{ll}\mathrm{I} & \mathrm{I} \\
\mathrm{I} & \mathrm{I}\end{array}$ & $\dot{29} \cdot \dot{8} 8$ & & $\begin{array}{l}61 \\
62\end{array}$ & & & & & & & \\
\hline $\begin{array}{ll}4 & 41 \\
: 5 & 39\end{array}$ & 29.8 & 66.3 & 66.1 & & & & & & & \\
\hline 1710 & $\begin{aligned} 29.798 \\
20.76\end{aligned}$ & 68.2 & 67.1 & & & & & & & \\
\hline $\begin{array}{ll}17 & 43 \\
18 & 8\end{array}$ & $\begin{array}{l}\begin{array}{l}29.796 \\
29.792\end{array}\end{array}$ & $\begin{array}{l}68.2 \\
68.0\end{array}$ & $\begin{array}{l}67.0 \\
66.7\end{array}$ & & & & & & & \\
\hline 11 & 29.920 & 51.9 & 52.0 & & & & & & & \\
\hline
\end{tabular}




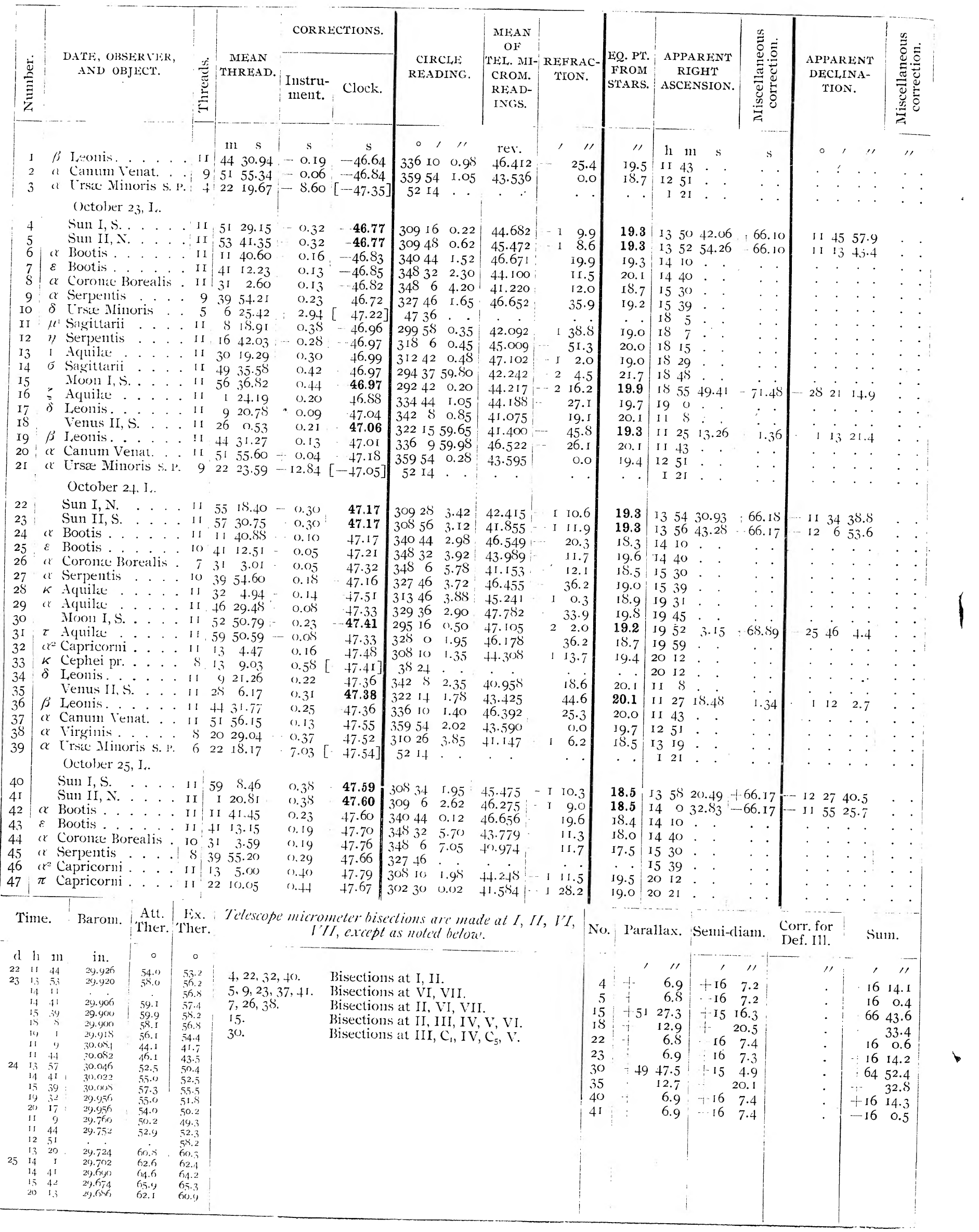




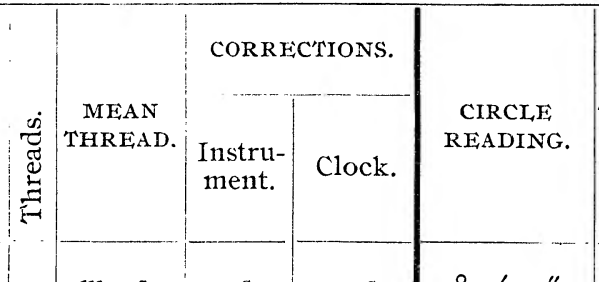

\& Delphin Moon I, S.

$\mu$ Aquarii I2 Year Cat. is 79

$\sigma^{2}$ Ursæ Majoris S.P.

$6 \mathrm{I}^{\mathrm{I}}$ Cygni

$\delta$ Leonis

Venus II, $\mathrm{S}$.

$\beta$ Leonis

S.

. . .

Ursa Minoris s.

October 26, L.

\begin{tabular}{|l|l|l|l|l|l|lll|} 
Sun I, N. . . . . . & Io & 2 & 59.23 & 0.32 & $\mathbf{- 4 8 . 1 4}$ & 308 & 46 & 1.28
\end{tabular}

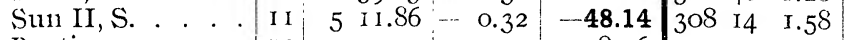

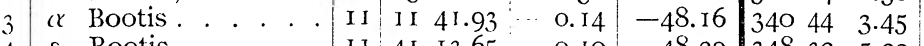

$\varepsilon$ Bootis.

(7) Coron $x$ Borealis

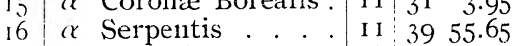

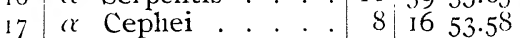

Is' I Pegasi

19 I H. Draconis S. P. .

is Aquarii

Moon I, S. .

$22 \varepsilon$ Pegasi

$\mu$ Capricorni October $27, \mathrm{~J}_{4}$.

24 if Leonis

25

26

Venus II, s. .

is I,eonis

Canum Venat

I I I 18 4.8 I

\begin{tabular}{lll}
0.10 & -48.29 \\
\hline
\end{tabular}

$\begin{array}{lllll}0.10 & -48.29 & 348 & 32 & 5.22\end{array}$

\begin{tabular}{r|r|rrr|}
0.21 & -48.19 & 348 & 6 & 5.62 \\
327 & 46 & 4.02
\end{tabular}

$\left.\begin{array}{l|l|rrr}0.21 & -48.19 \\ 0.15 & {[-48.19}\end{array}\right] \begin{array}{rrr}327 & 46 & 4.02 \\ 23 & 8 & .8 \\ 0.18 & -48.11 & -80\end{array}$

\begin{tabular}{r|rr:rr}
4 & 23 & 5.13 & - & 1.41 \\
9 & 26 & 53.80 & - & 0.30
\end{tabular}$\left[\begin{array}{ll}-48.18 \\
-48.32\end{array}\right] \begin{array}{rrrr}34 & 12 & . & . \\
514 & 59 & 59.95\end{array}$

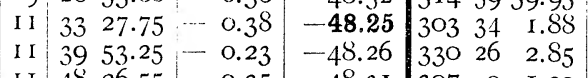

I I 3953.25

\begin{tabular}{ll}
-48.26 \\
-48.31 \\
\hline
\end{tabular}

307 O 1.90

\begin{tabular}{l|l|lll|} 
& & & & \\
29 & -48.75 & 342 & 8 & 1.72 \\
0.36 & -48.82 & 322 & 6 & 0.58
\end{tabular}

\begin{tabular}{|r|rr|r|r|rrr|} 
II & 9 & 22.79 & -0.29 & -48.75 & 342 & 8 & I.72 \\
I & 34 & 54.77 & -0.36 & -488.82 & 322 & 6 & 0.58 \\
I & 44 & 33.31 & -0.31 & -48.78 & 336 & 10 & 0.25
\end{tabular}

\begin{tabular}{r|rrr|}
-48.94 & 359 & 53 & 59.30 \\
-48.90 & 3 I0 & 26 & I.75 \\
-48.91 & 5 & 14 &.
\end{tabular}

\begin{tabular}{l|ll:l} 
I & 51 & 57.70 & -0.24 \\
I & 20 & 30.49 & -0.40
\end{tabular}

$x$ Ursie Minoris October $28, \mathrm{~L}$.

$3 \mathrm{3}$

32

$32 \&$ Bootis.

Sun I, S.

Sun II, N.
Bootis.

a Coronz Borealis

$\alpha$ Serpentis

35

36

37

39

39 Leonis . . . . i I $44.33 .30=0.09$

40 a Canum Venat. 8 5 $57.56+0.07-49.09$

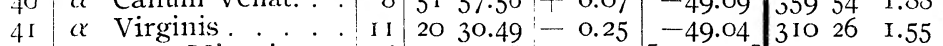

$42 \alpha$ Ursæ̉ Minoriss.

October 29, L.

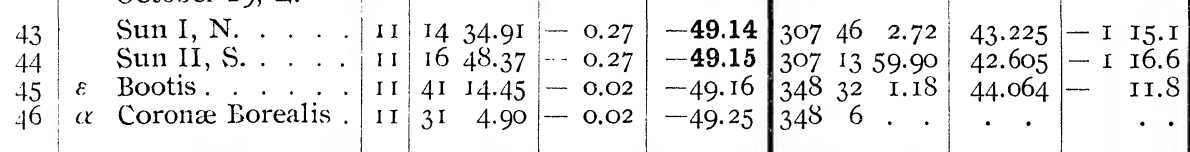

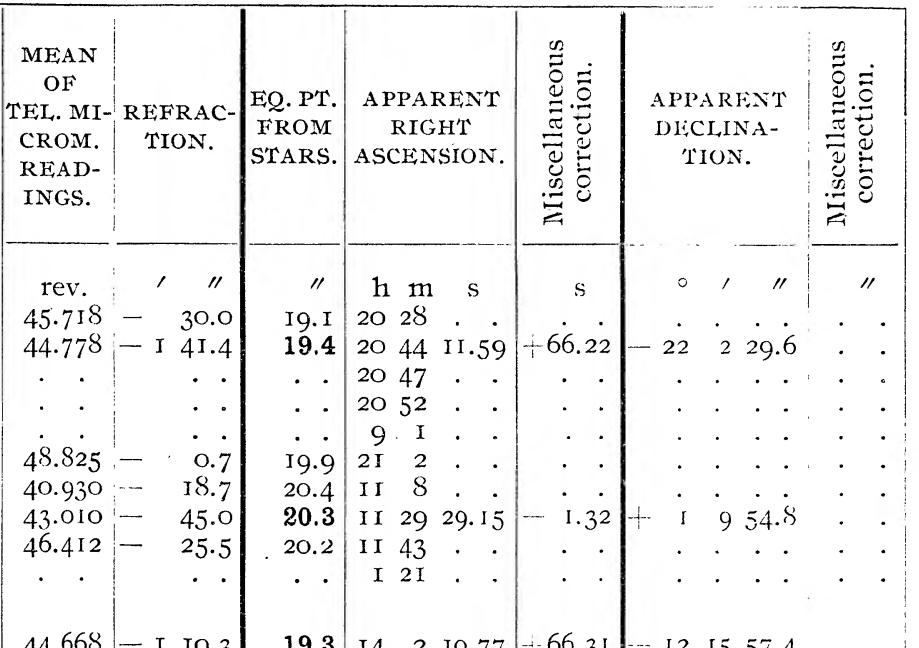

\begin{tabular}{l|lll|llllll}
19.3 & I4 & 2 & I0.77 & -66.31 & I 2 & I 5 & 57.4
\end{tabular} \begin{tabular}{l|llllllllll}
19.3 & I4 & 4 & 23.40 & -66.32 & - I 2 & 48 & I 5.0
\end{tabular}

I9. I I4 IO

I9. I I 4 40

I8.7 1530

$18.8 \quad 1539$

$.2 \mathrm{I} 16$

19.6 2I I7

I8 $\dot{6} \quad 922$

19.3 21 $3239.12+63.91-172746.7$

I9.8 2I 39

I9.3 2I 47

I9.4 II $S$.

19.0 II $345.59-$ i. 28 i 3 i 4.8

I8. 8 I2 51

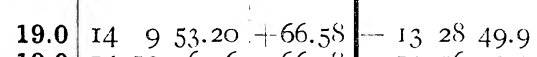

19.0 I4 I2 $6.36-66.5^{8}-$ I 25631.2

I9. I I4 40

IS.3 I5 30

\begin{tabular}{ll|ll}
18.9 & 15 & 39
\end{tabular}

\begin{tabular}{l|lll}
18.7 & 16 & 8
\end{tabular}

I8.2 I6 23

2 I.O II I4

19.7 in 36 3i. i $4-$ i. $26+$ o 5843.3

I9.7 If 43

I8.8 I $25 \mathrm{I}$

20.3 I3 19

19.7 I4 I3 $45.50+66.72-$ I 3 I6 28.9

$\begin{array}{llllllll}19.7 & \text { I4 } & \text { I5 } & 58.95 & -66.73 & -13 & 45 & +7.5\end{array}$

I9.4 I4 40 .

\begin{tabular}{|c|c|c|c|c|c|}
\hline Time. & Barom. & $\begin{array}{l}\text { Att. } \\
\text { Ther. }\end{array}$ & $\begin{array}{l}\text { Ex. } \\
\text { Ther. }\end{array}$ & \multicolumn{2}{|c|}{$\begin{array}{c}\text { Telescope micrometer bisections are made at } I \\
V I I, \text { except as noted below. }\end{array}$} \\
\hline d $\mathrm{h} \mathrm{m}$ & 11 & $\circ$ & $\circ$ & & \\
\hline $25 \quad 21$ & 29.708 & 59.8 & 59.1 & & Bisections at II, III, \\
\hline $\begin{array}{l}\text { II } \\
\text { II }\end{array}$ & $\begin{array}{l}29.772 \\
20.72\end{array}$ & 48.1 & 46.0 & $6,13$. & Bisections at II, VI, VII. \\
\hline $\begin{array}{rrr}26 & \text { I4 } & 54\end{array}$ & 29.760 & 61.1 & 60.0 & II $, 30,43$. & Bisections at I, II. \\
\hline $\begin{array}{l}14 \\
15\end{array}$ & $\begin{array}{l}29.736 \\
29.720\end{array}$ & $\begin{array}{l}62.8 \\
65.3\end{array}$ & $\begin{array}{l}63.2 \\
65.6\end{array}$ & I 2, 3I, 40, 44. & Bisections at VI, VII. \\
\hline 21 is & 29.708 & 60.1 & 57.8 & & $\mathrm{Bi}$ \\
\hline 21 & 29.706 & 59.2 & & & \\
\hline
\end{tabular}

$I, I I, V I$ . . 1530 .

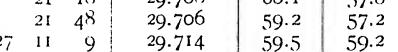

\begin{tabular}{rr|r|r|r}
\hline 11 & 9 & 29.714 & 59.5 & 59.2 \\
11 & 44 & 29.718 & 60.9 & 61.1 \\
\hline 13 & 20 & 9.738 & 65.4 & 64.3
\end{tabular}

\begin{tabular}{ll|l}
13 & 20 & 29.718
\end{tabular}

\begin{tabular}{ll|l}
13 & 20 & 29.738 \\
14 & 12 & 29.734
\end{tabular}

\begin{tabular}{ll|l}
14 & 12 & 29.734 \\
14 & 41 & 29.736
\end{tabular}

\begin{tabular}{l|l|l}
29.736 & 65.9 & 64.4 \\
\hline
\end{tabular}

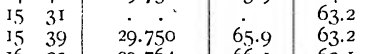

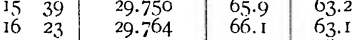

II $44 \quad 30.046$

$\begin{array}{ll}13 & 20 \\ 14 & 30.036\end{array}$

14 I6

$14 \quad 41$

\begin{tabular}{|c|c|c|c|c|c|}
\hline No. & Para & 11 ax. & Semi-diam. & $\begin{array}{l}\text { Corr. for } \\
\text { Def. Ill. }\end{array}$ & Sumin. \\
\hline & 1 & " & , II & " & , II \\
\hline 2 & +47 & 40.5 & TI4 56. I & . & 6236.6 \\
\hline$S$ & + & I 2.5 & 19.8 & . & 32.3 \\
\hline II & + & 6.9 & - I6 8.8 & . & $\begin{array}{ll}16 & 1.9\end{array}$ \\
\hline 12 & + & 7.0 & +168.7 & . & 1615.7 \\
\hline $2 \mathrm{I}$ & -45 & 5.5 & +1450.3 & - & +5956.1 \\
\hline 25 & 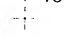 & 12.2 & $\quad 19.2$ & . & 31.4 \\
\hline 30 & & 7.0 & $\begin{array}{ll}16 & 9.3\end{array}$ & . & 16 I6.3 \\
\hline 31 & + & 7.0 & $-16 \quad 9.4$ & . & $\begin{array}{ll}-16 & 2.4\end{array}$ \\
\hline $3 \mathrm{~s}$ & - & 12.0 & 18.9 & . & 30.9 \\
\hline 43 & 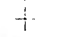 & 7.0 & $\begin{array}{ll}\cdots & 9.3\end{array}$ & . & $\begin{array}{ll}-16 & 2.3\end{array}$ \\
\hline 44 & & 7.1 & I6 9.2 & - & $-16 \quad 16.3$ \\
\hline & & & & & \\
\hline & & & & & \\
\hline & & & & & \\
\hline
\end{tabular}




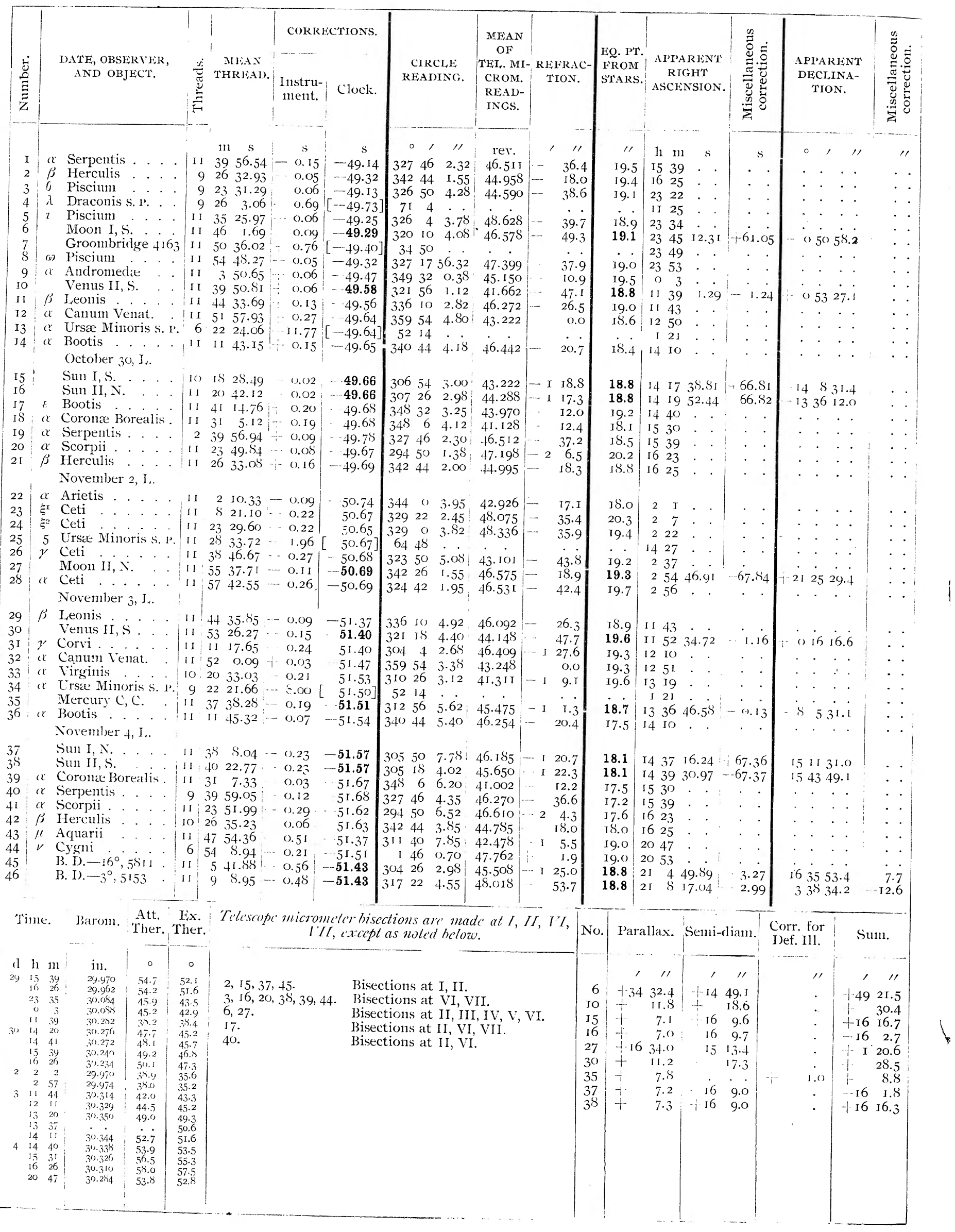




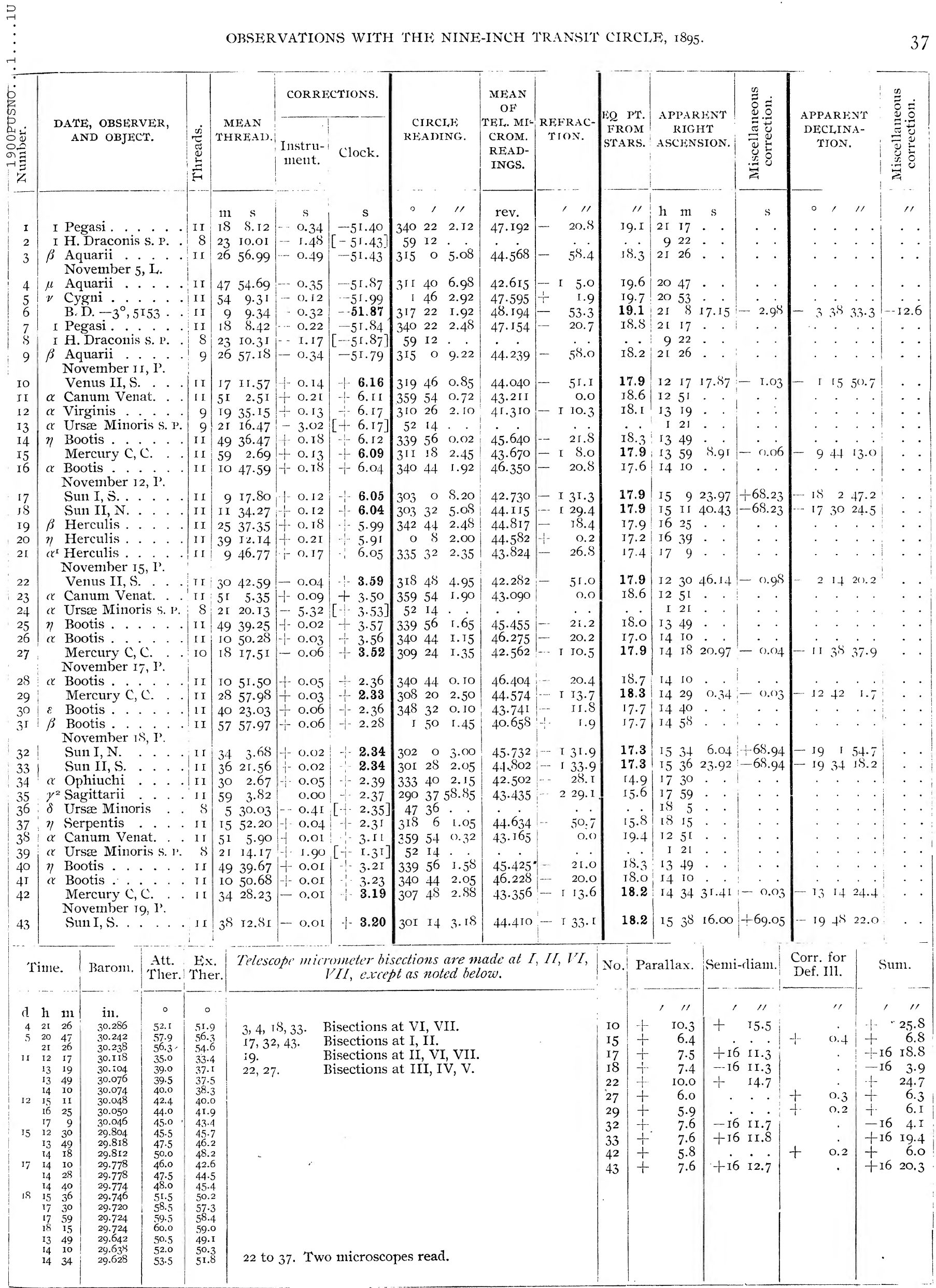

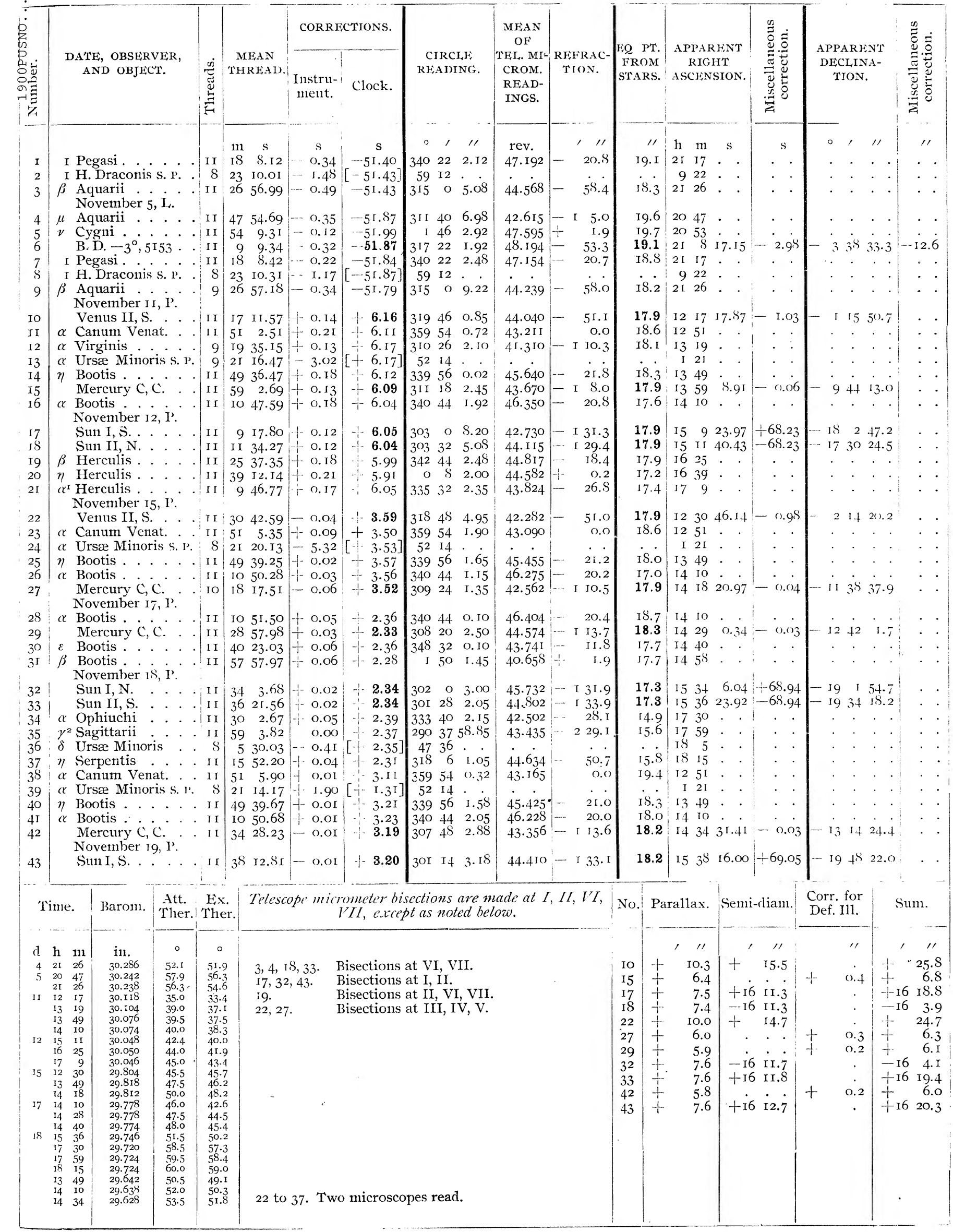




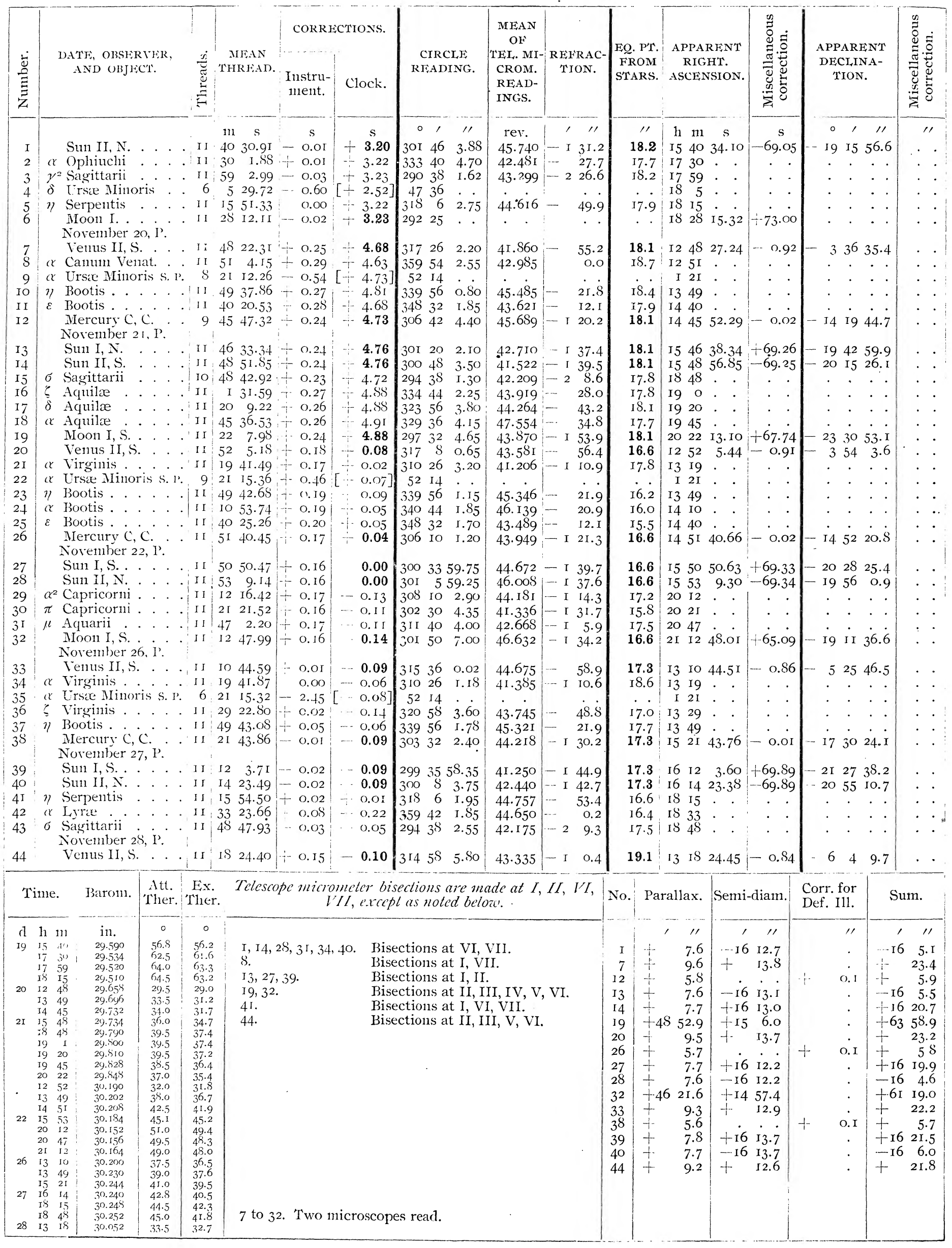




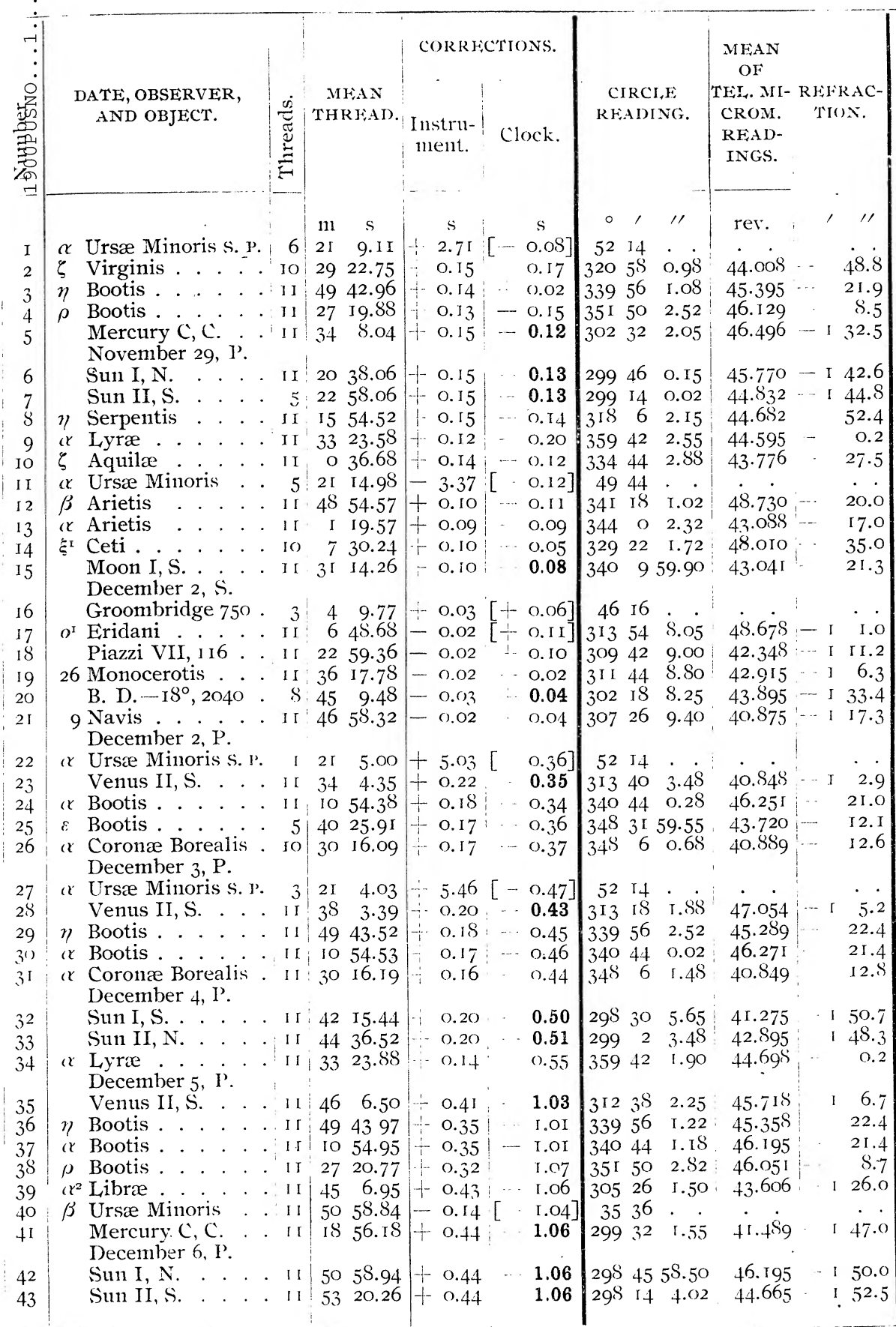

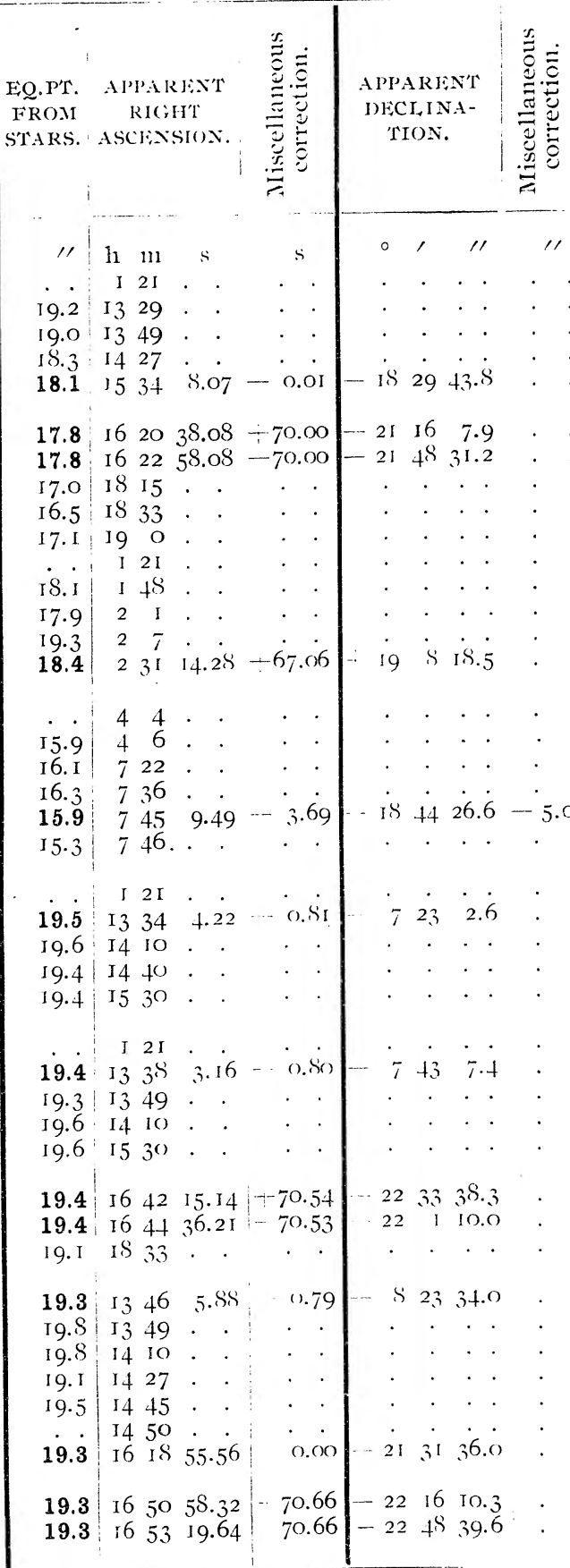

Time Att. Ex. Telescope micrometer bisections are made at I, II, ITI, VII, ercept as noted beloai.

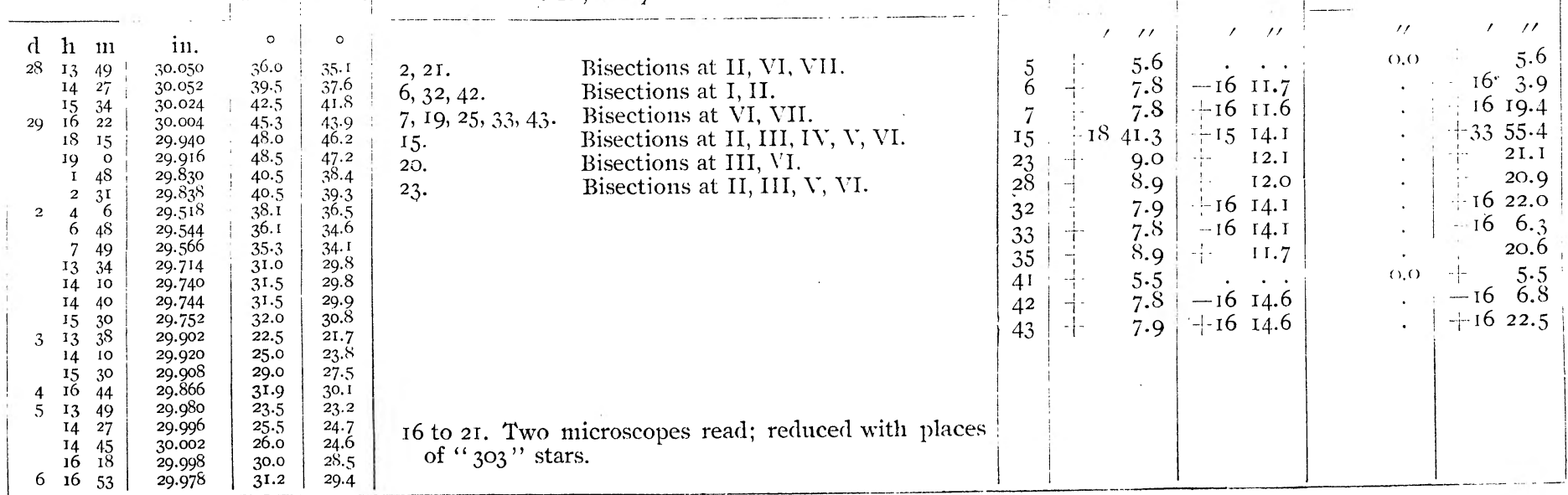




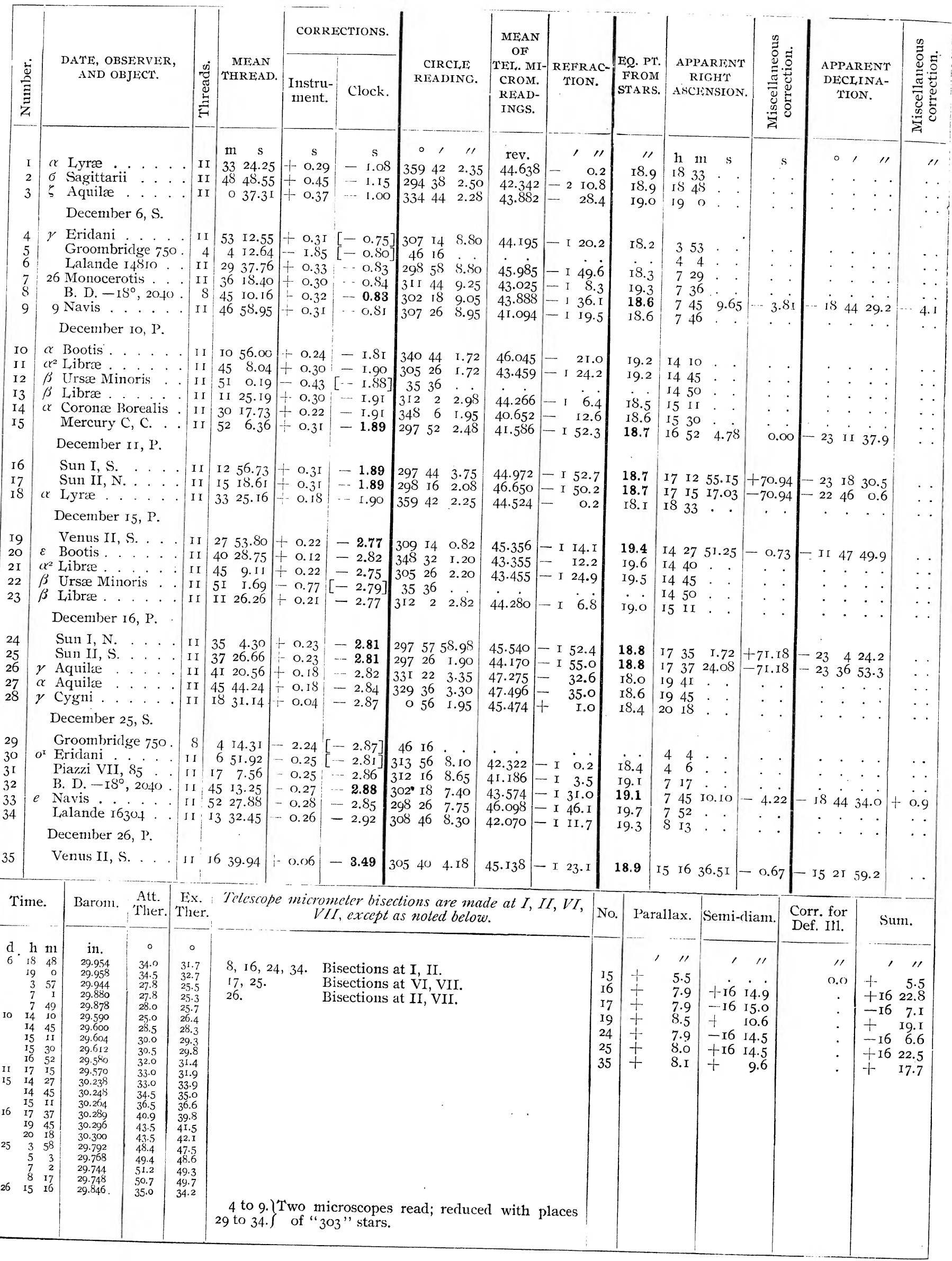




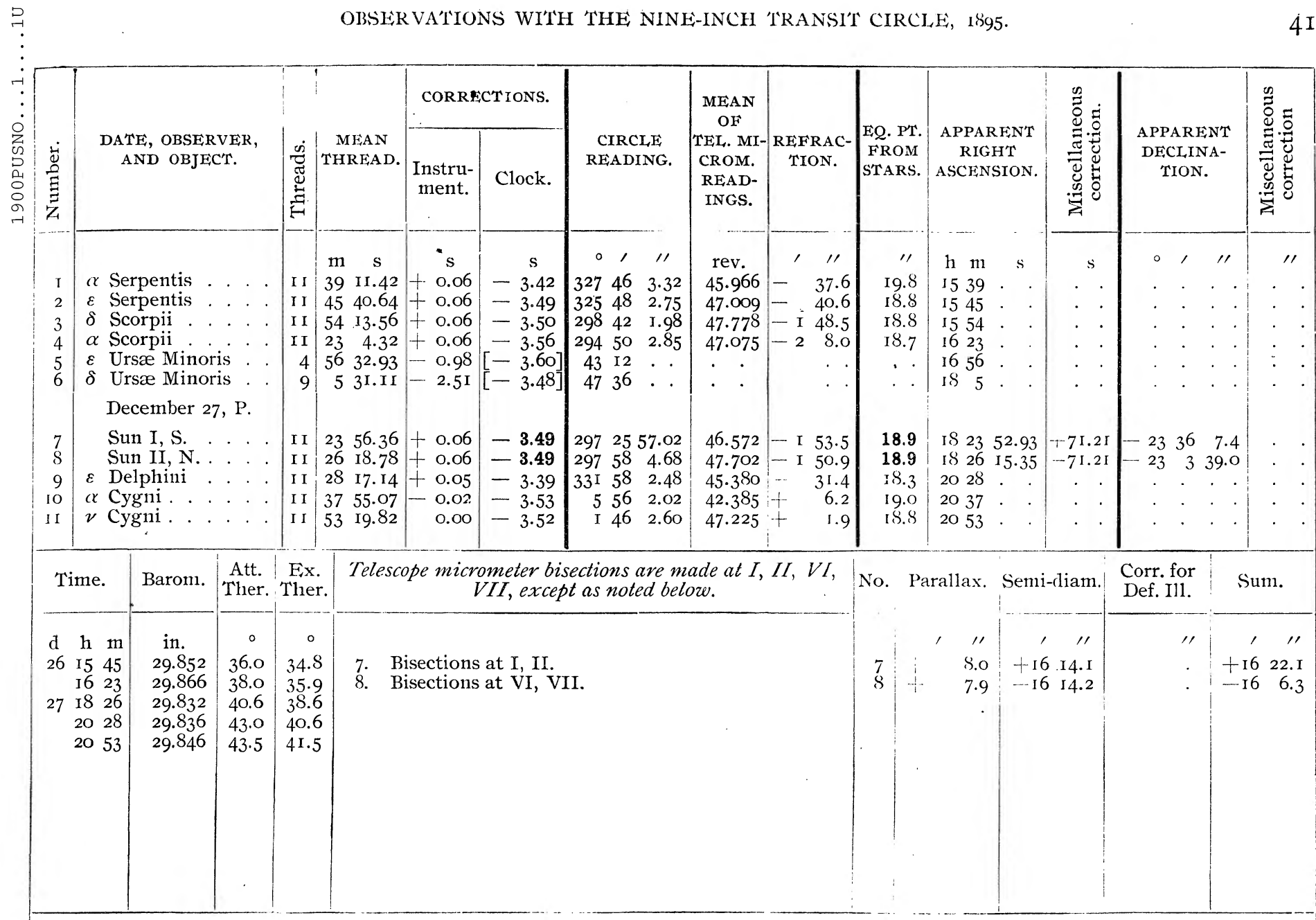




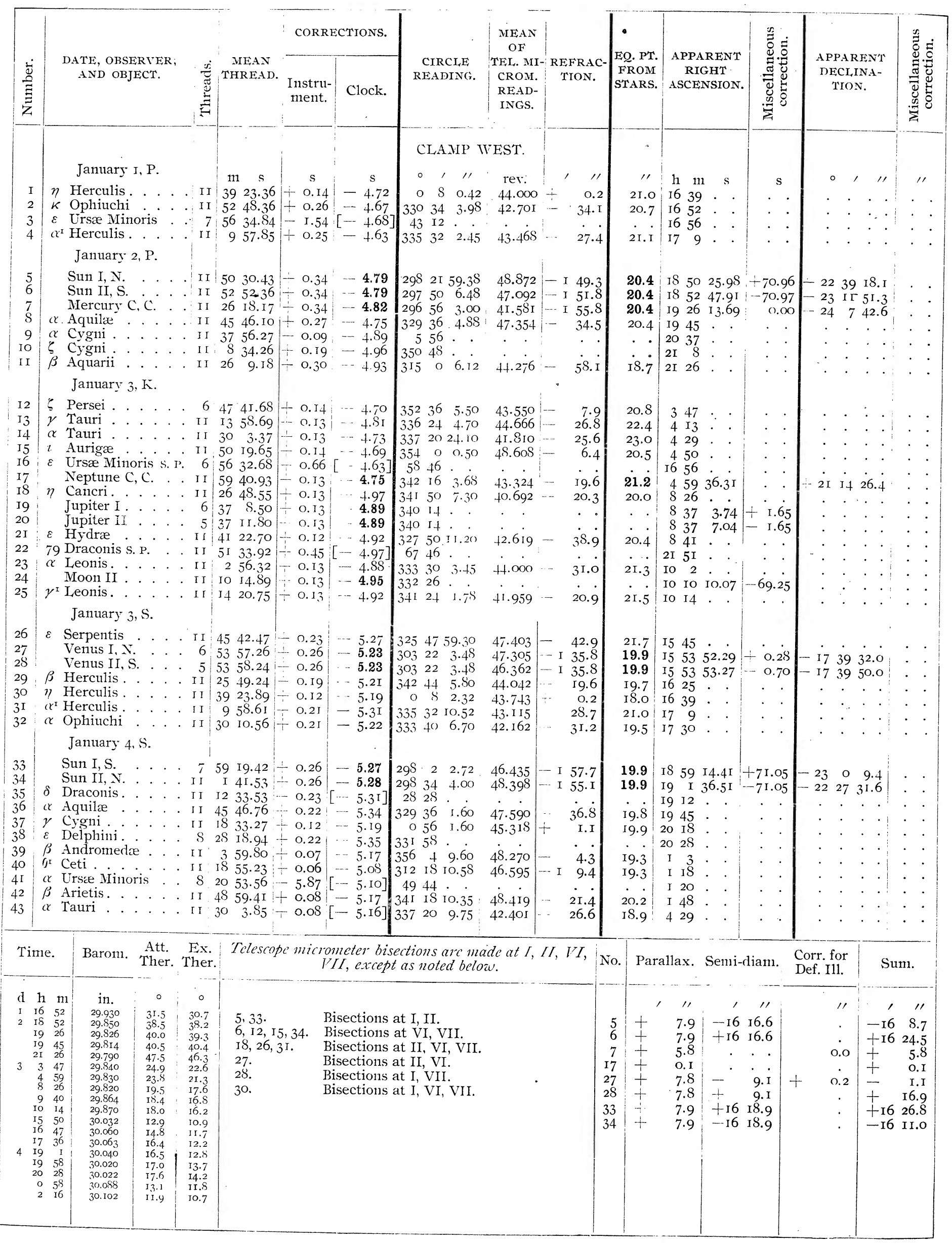




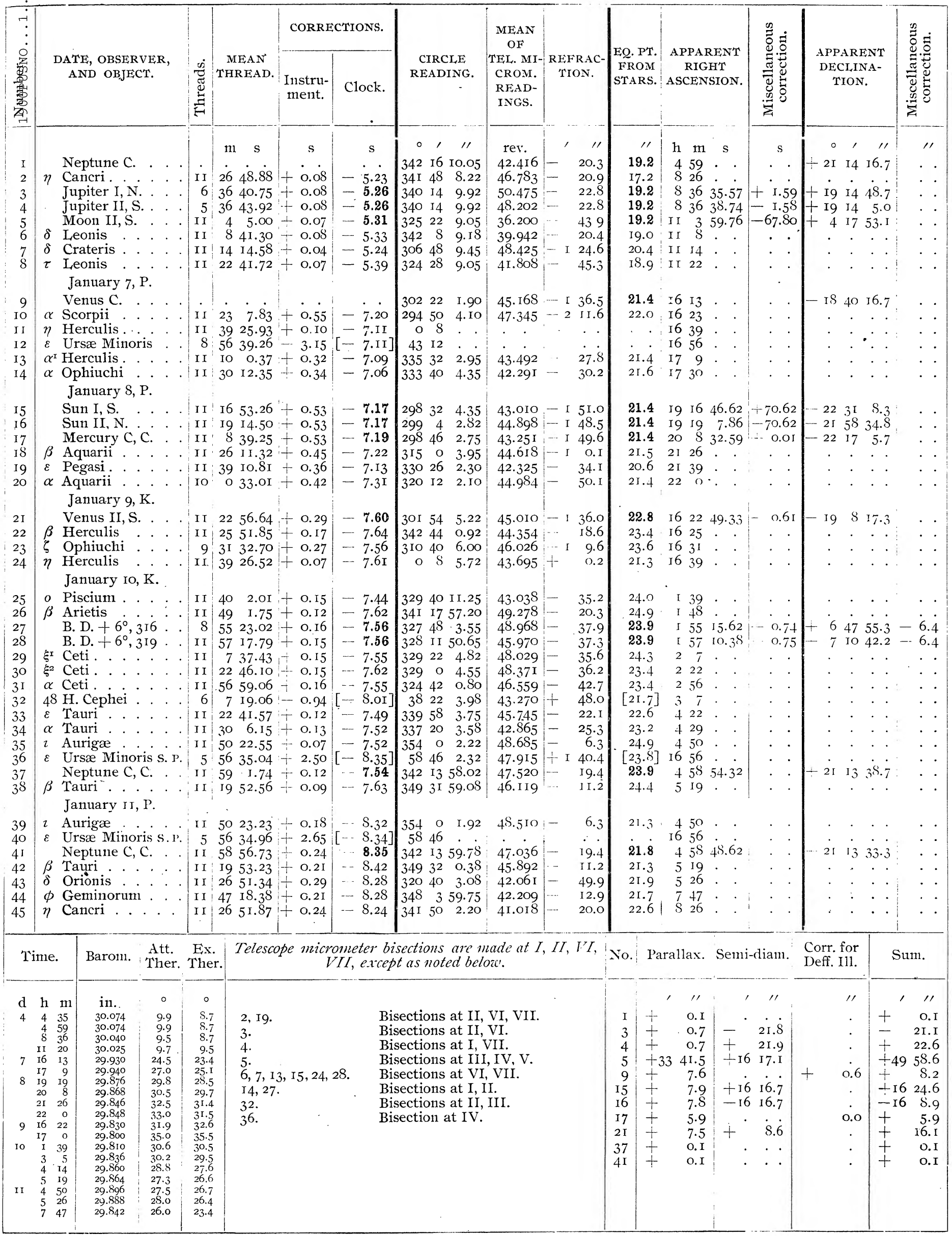




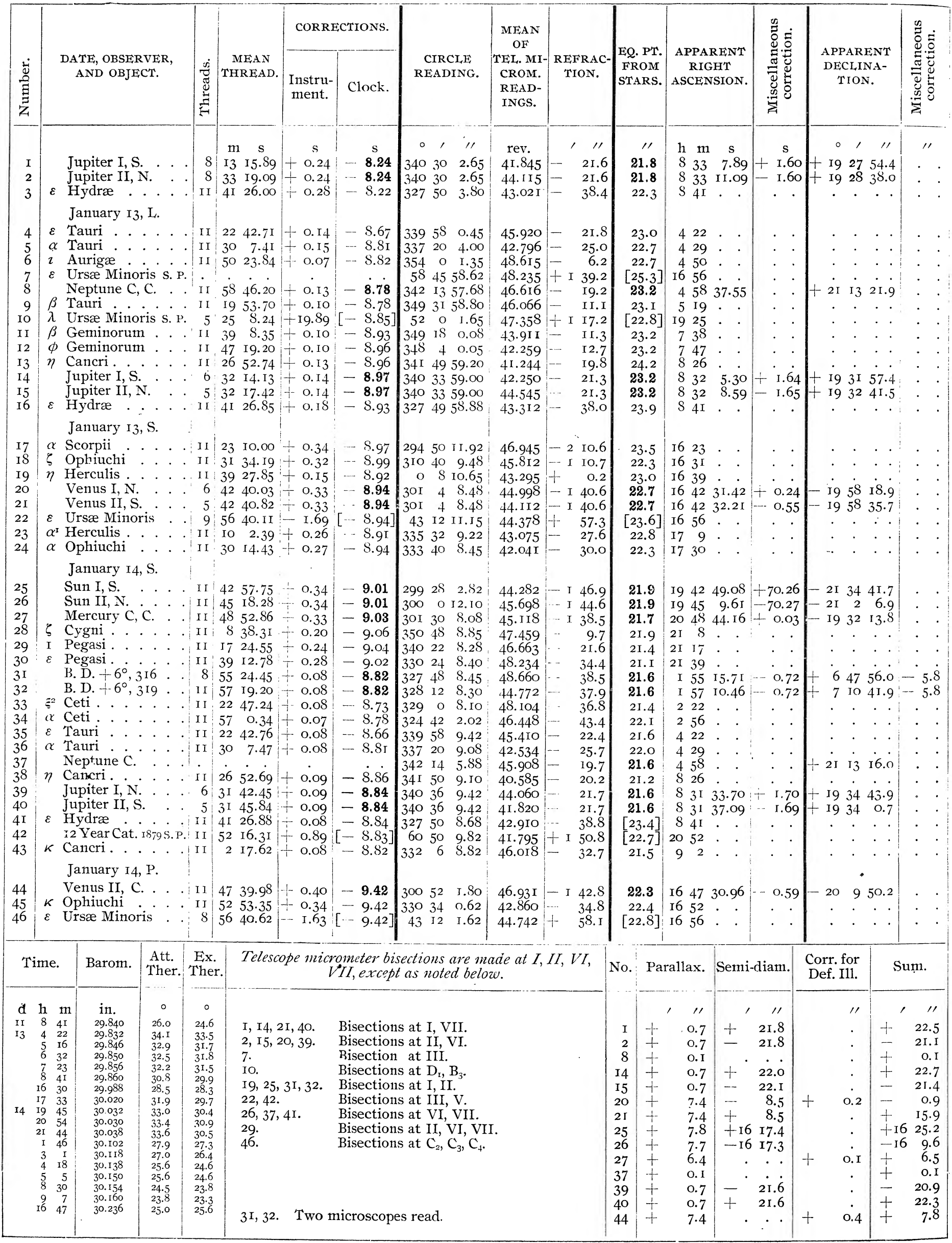




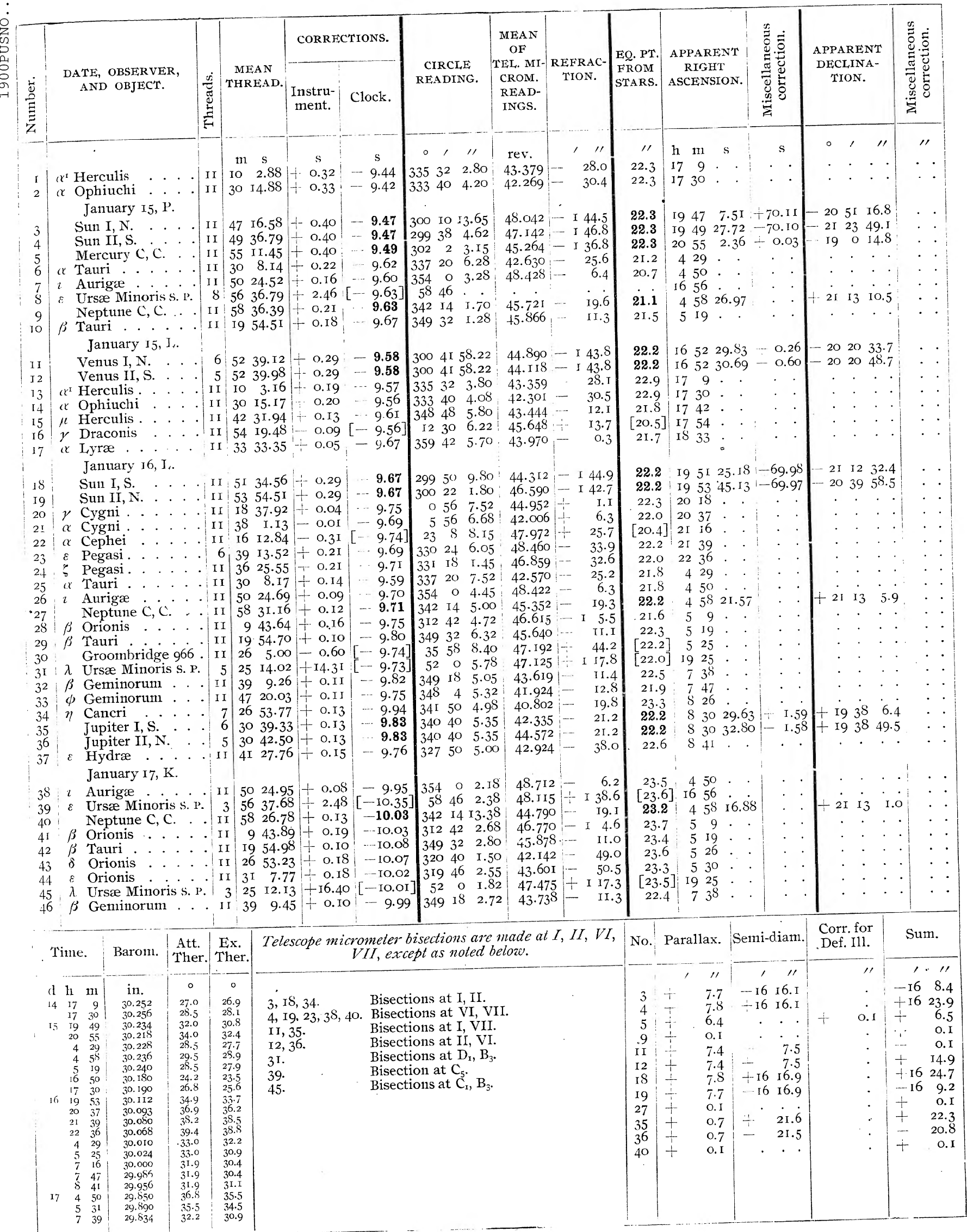




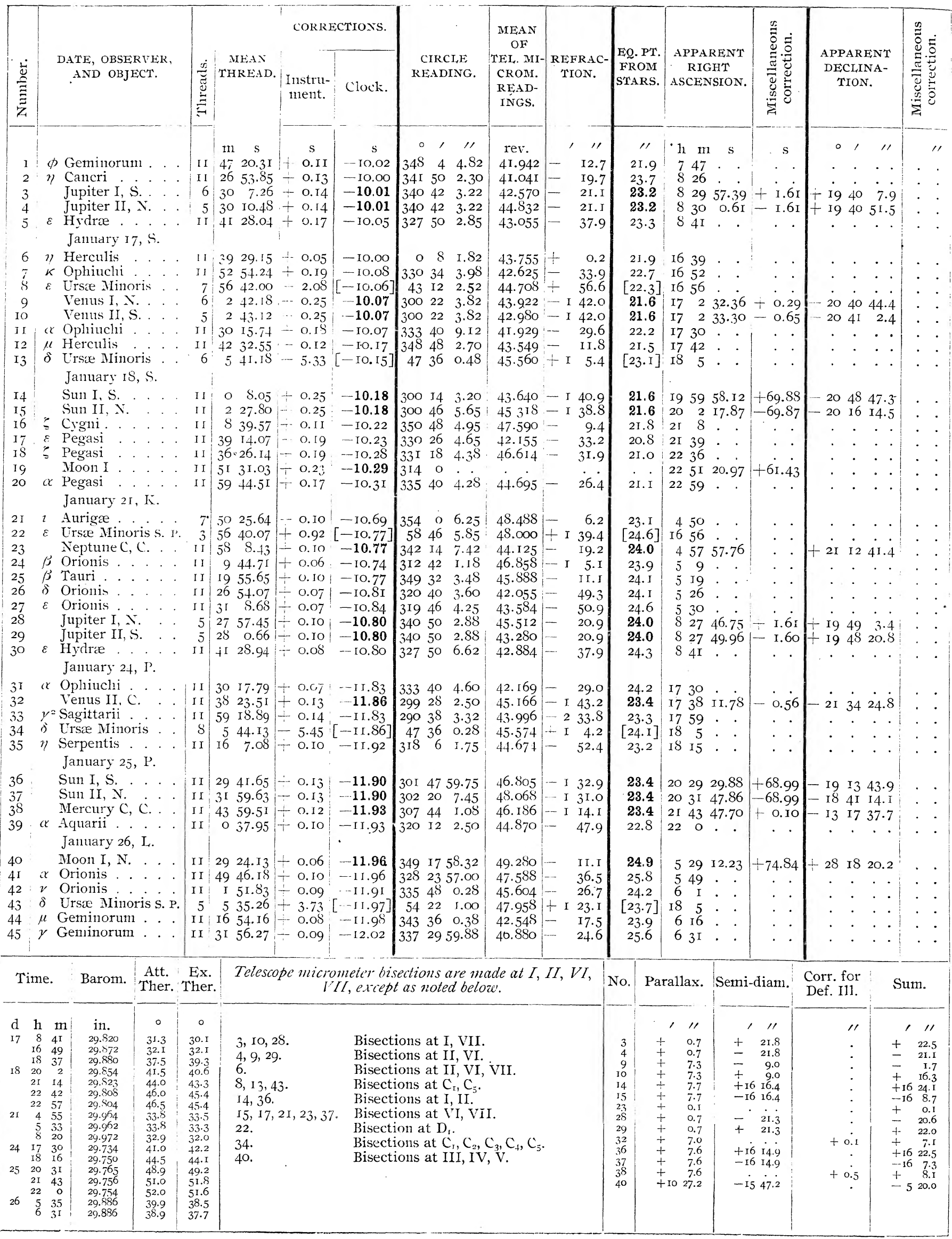




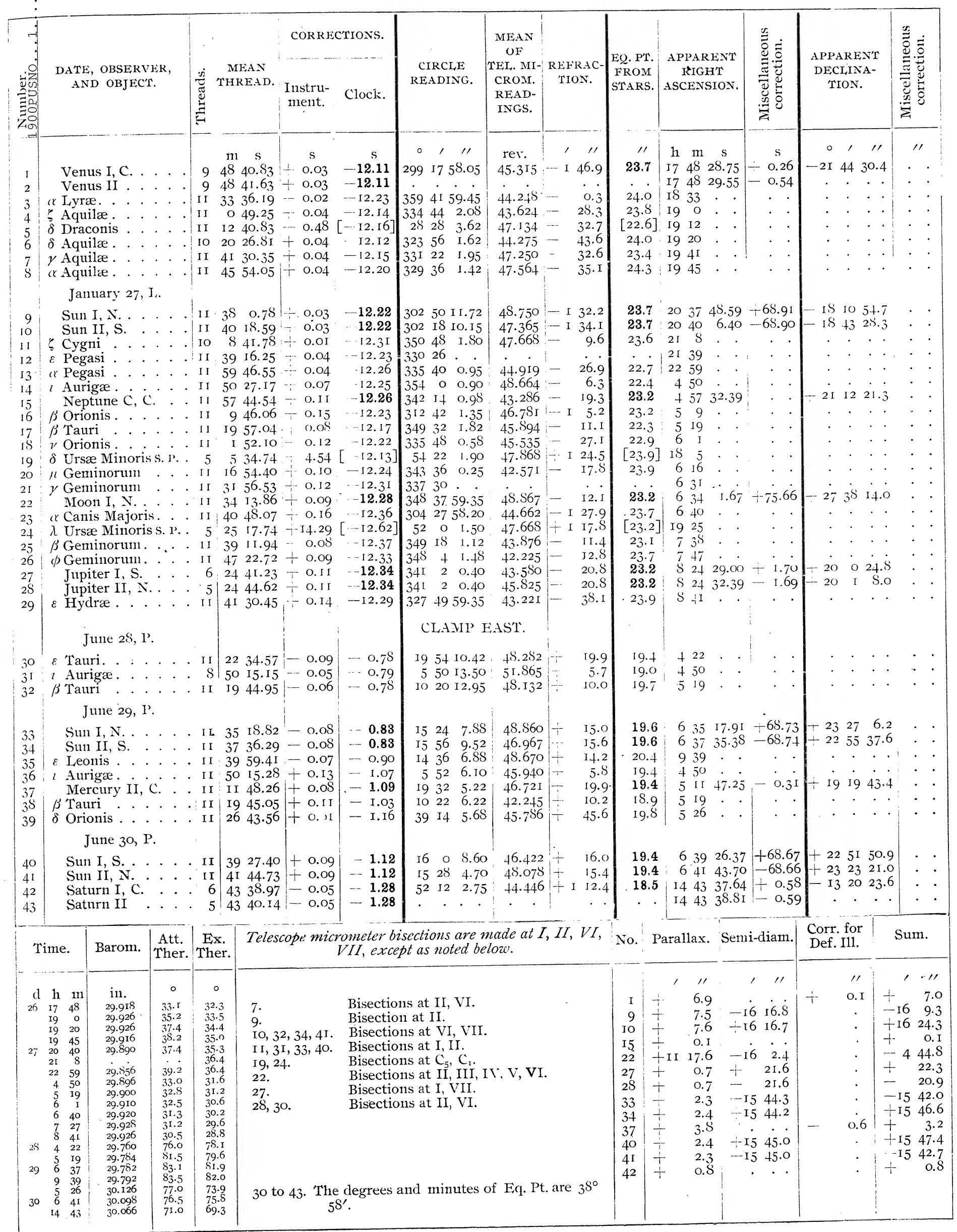




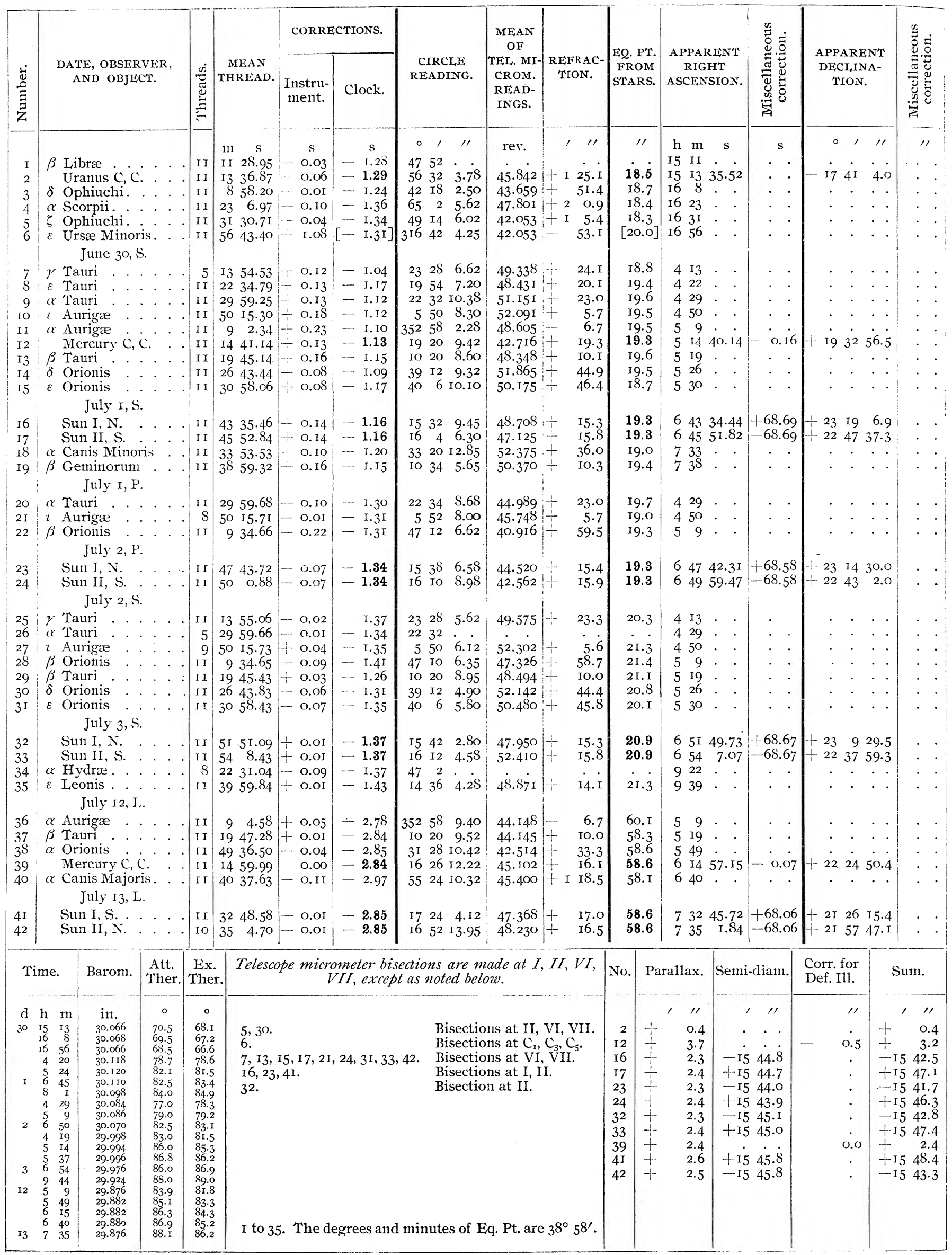




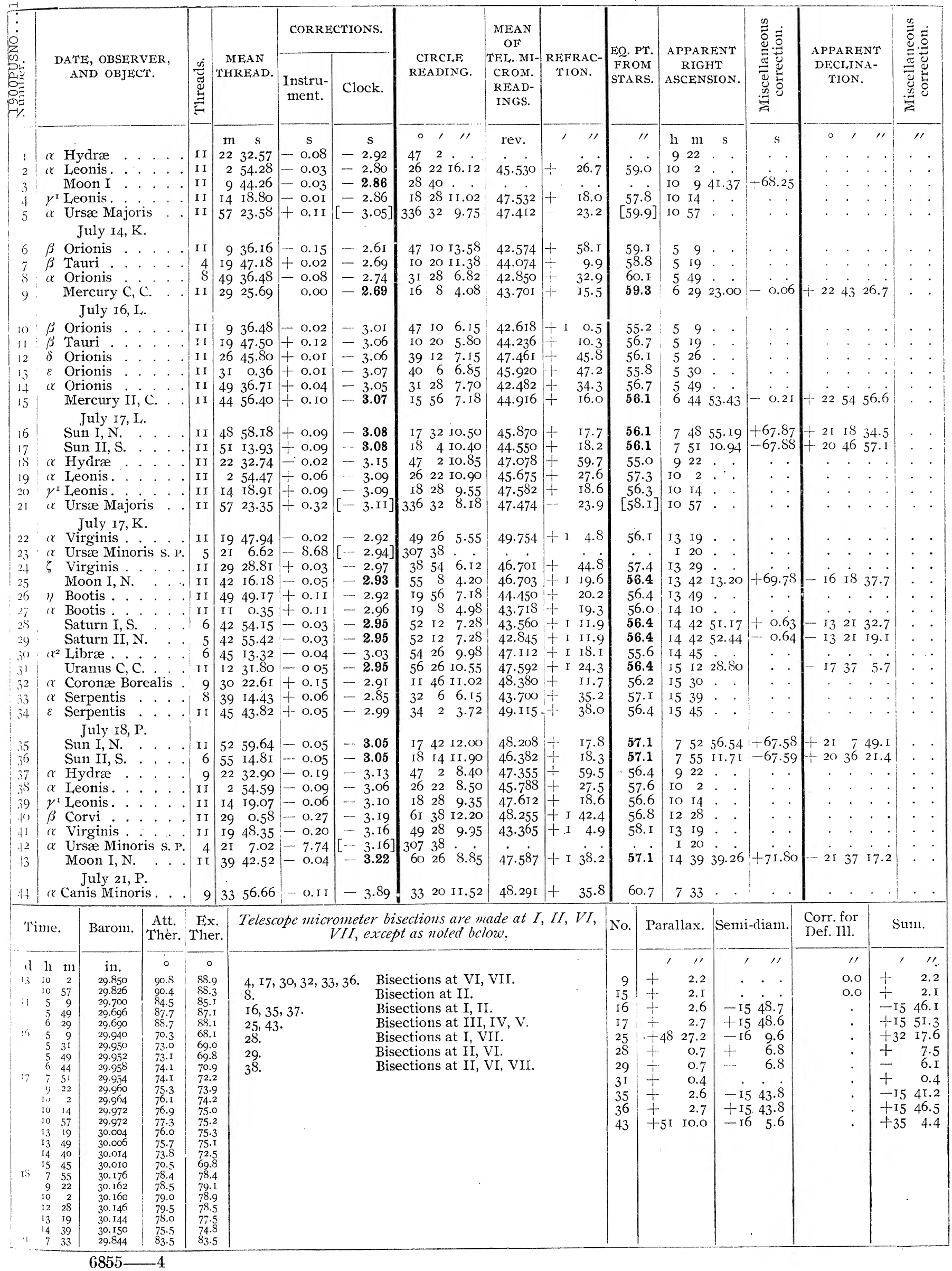




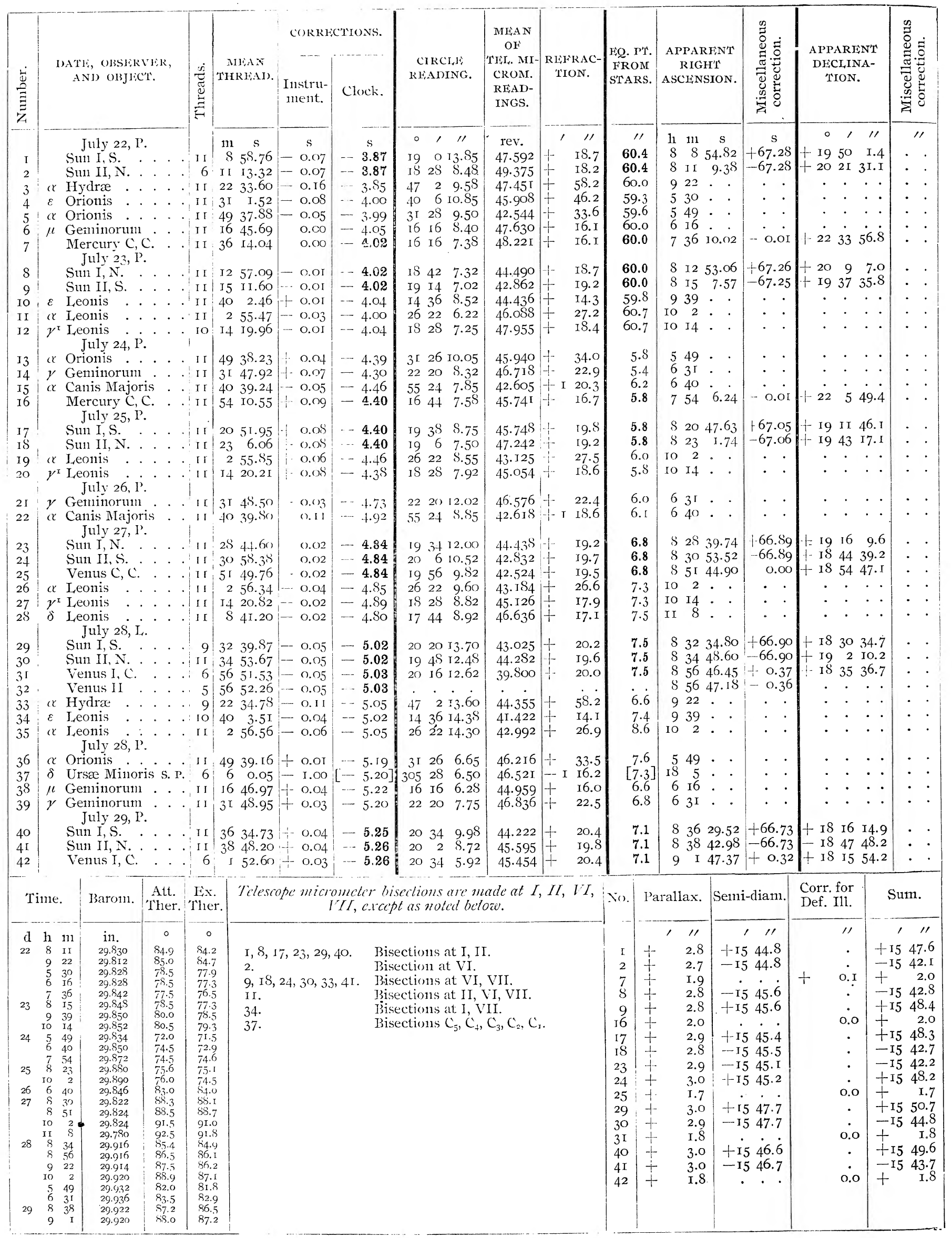




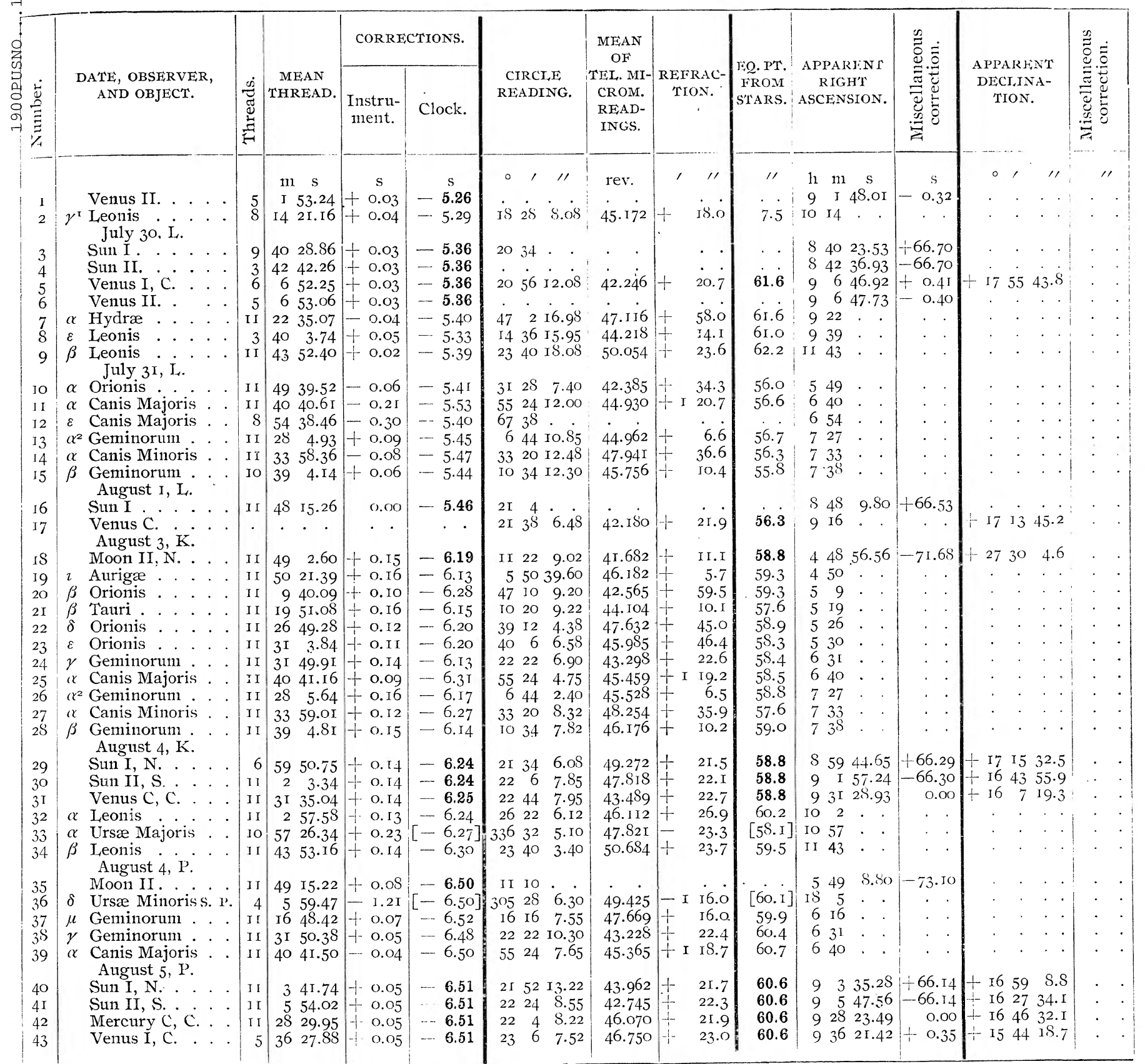

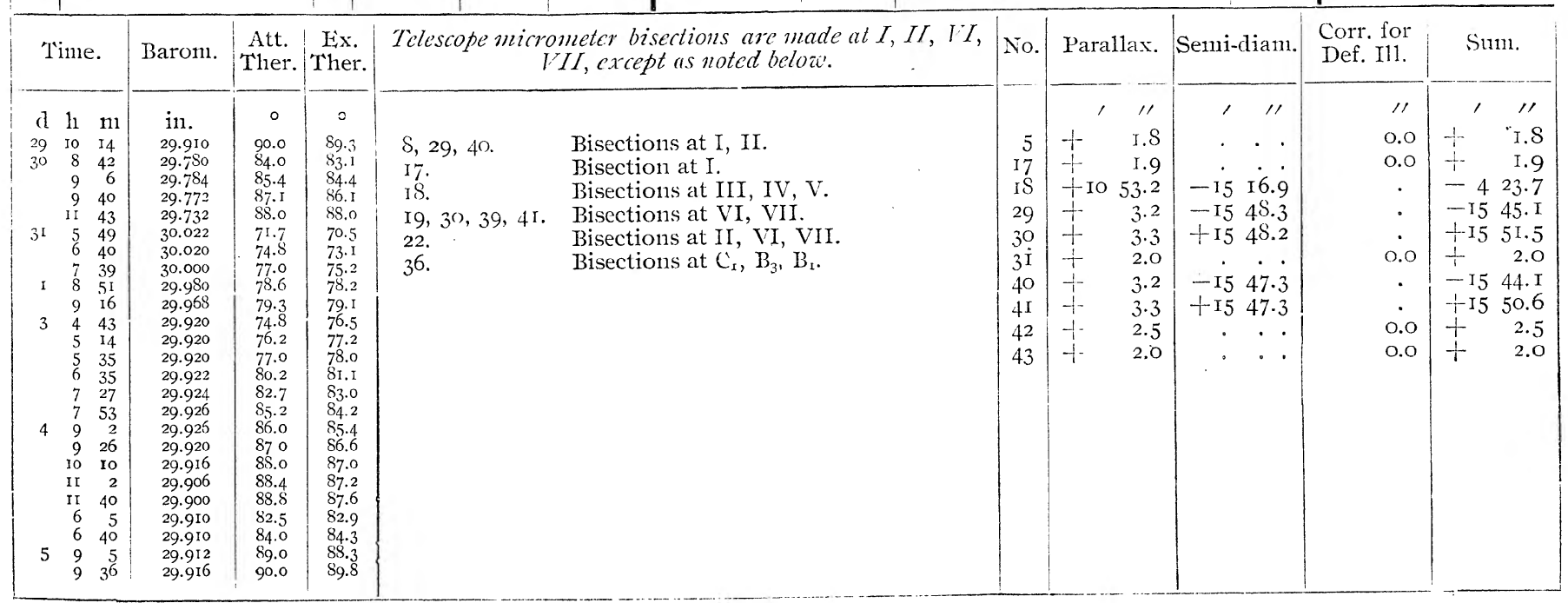




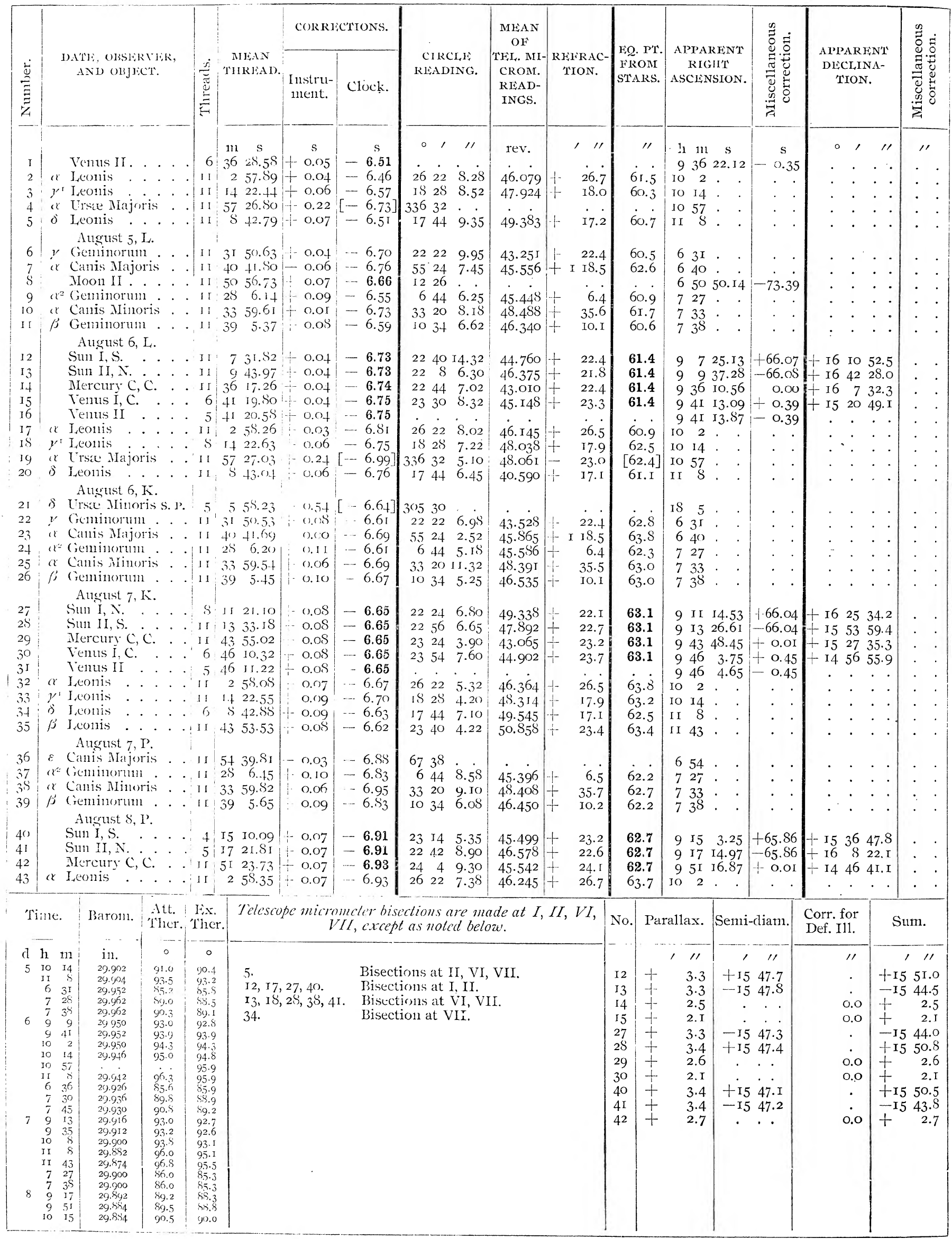




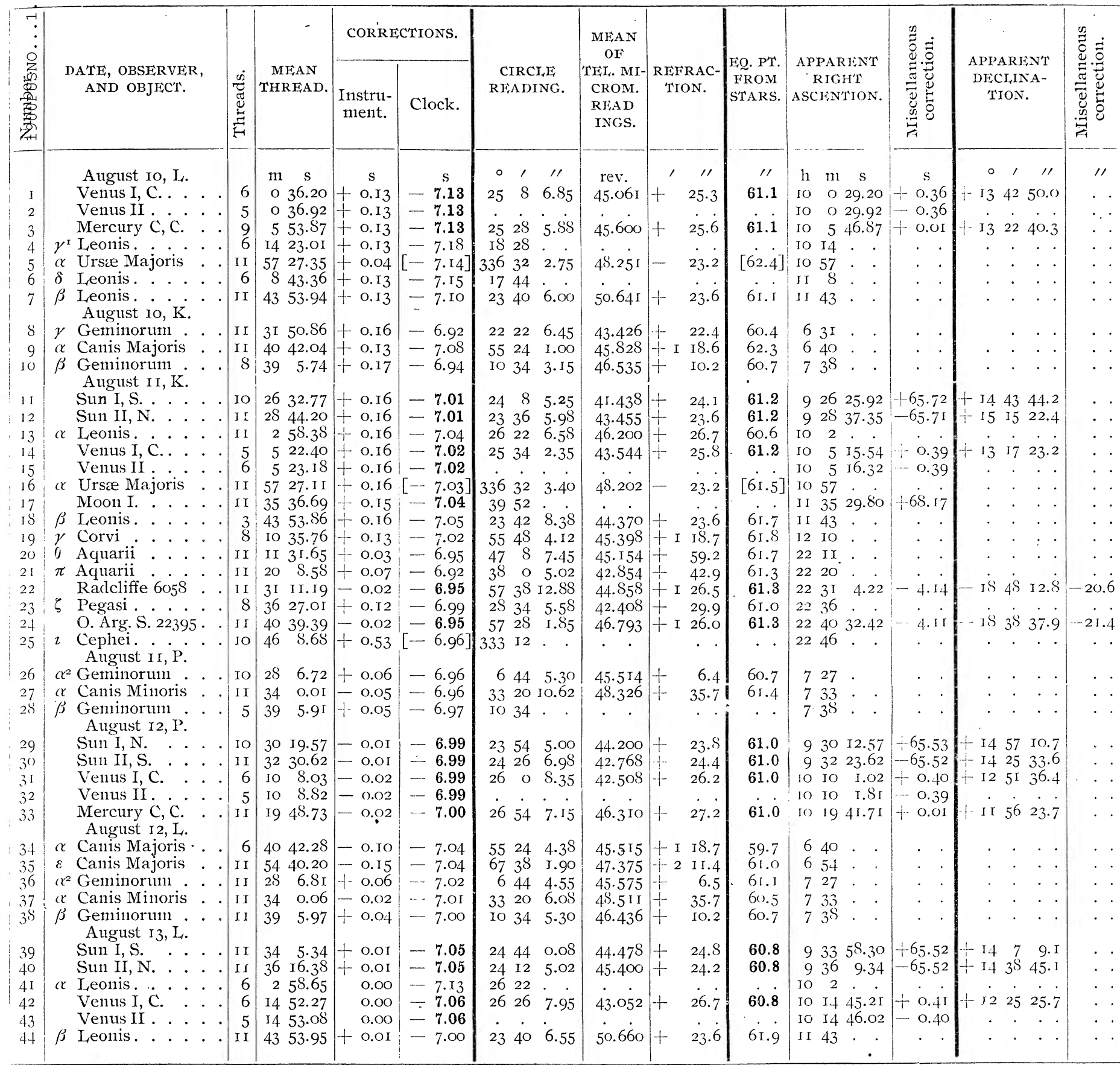

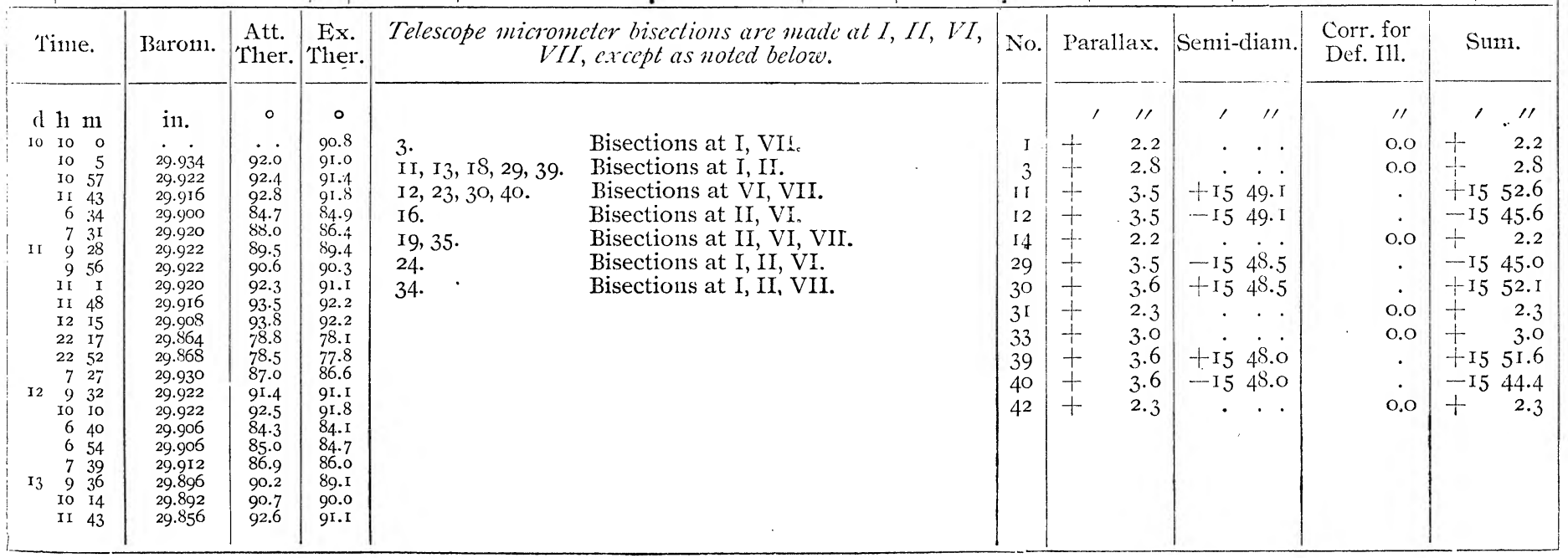




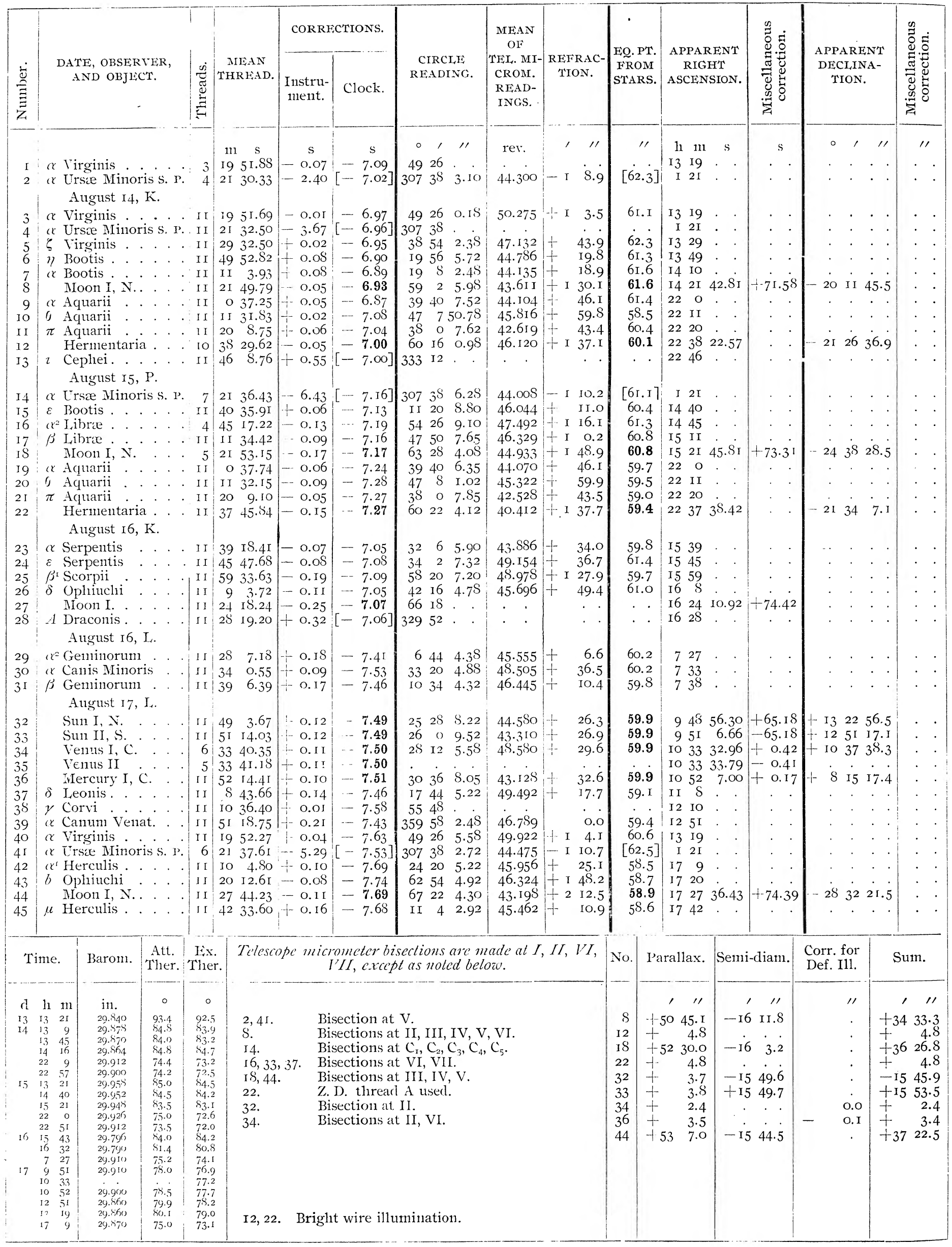




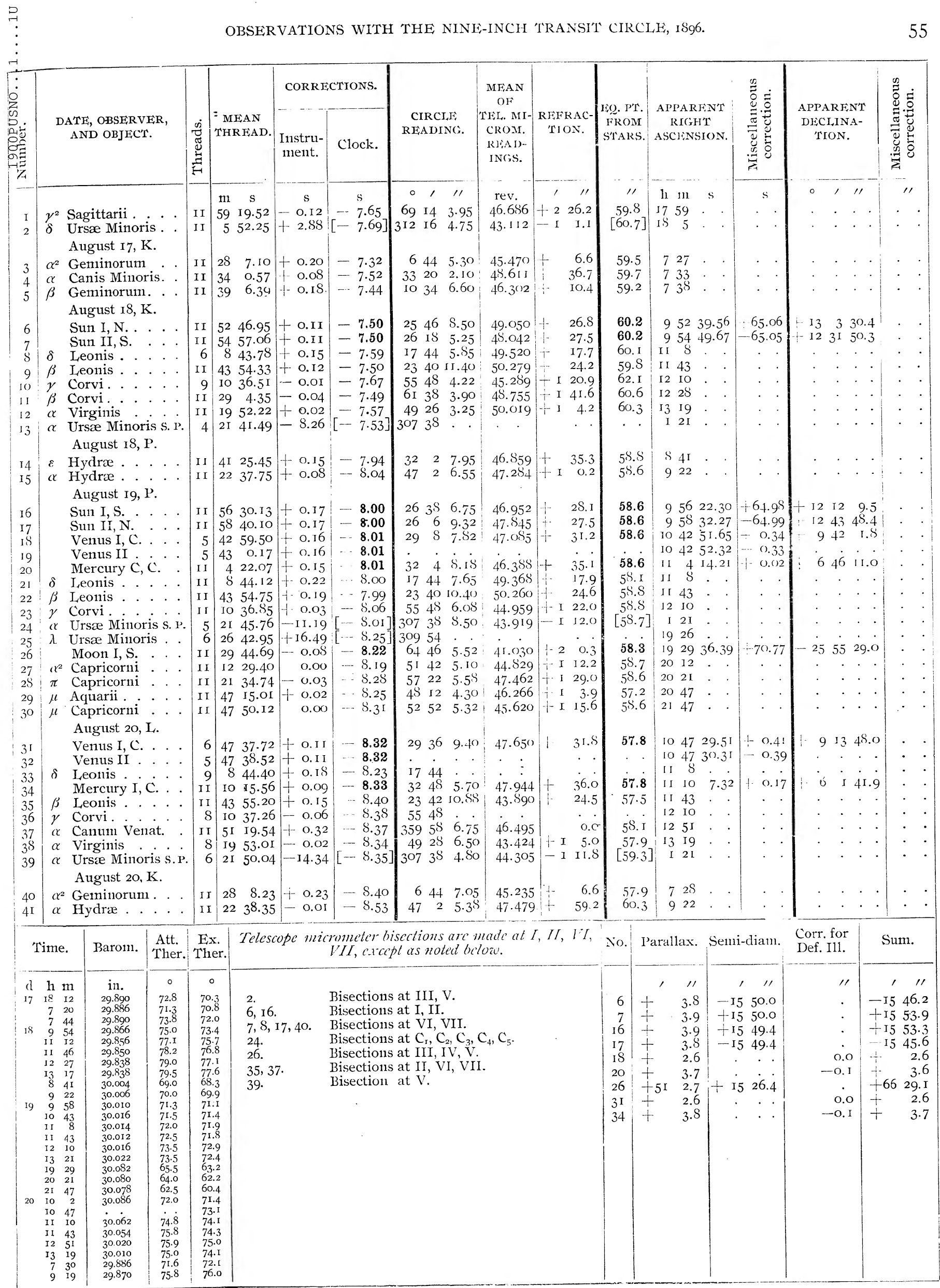




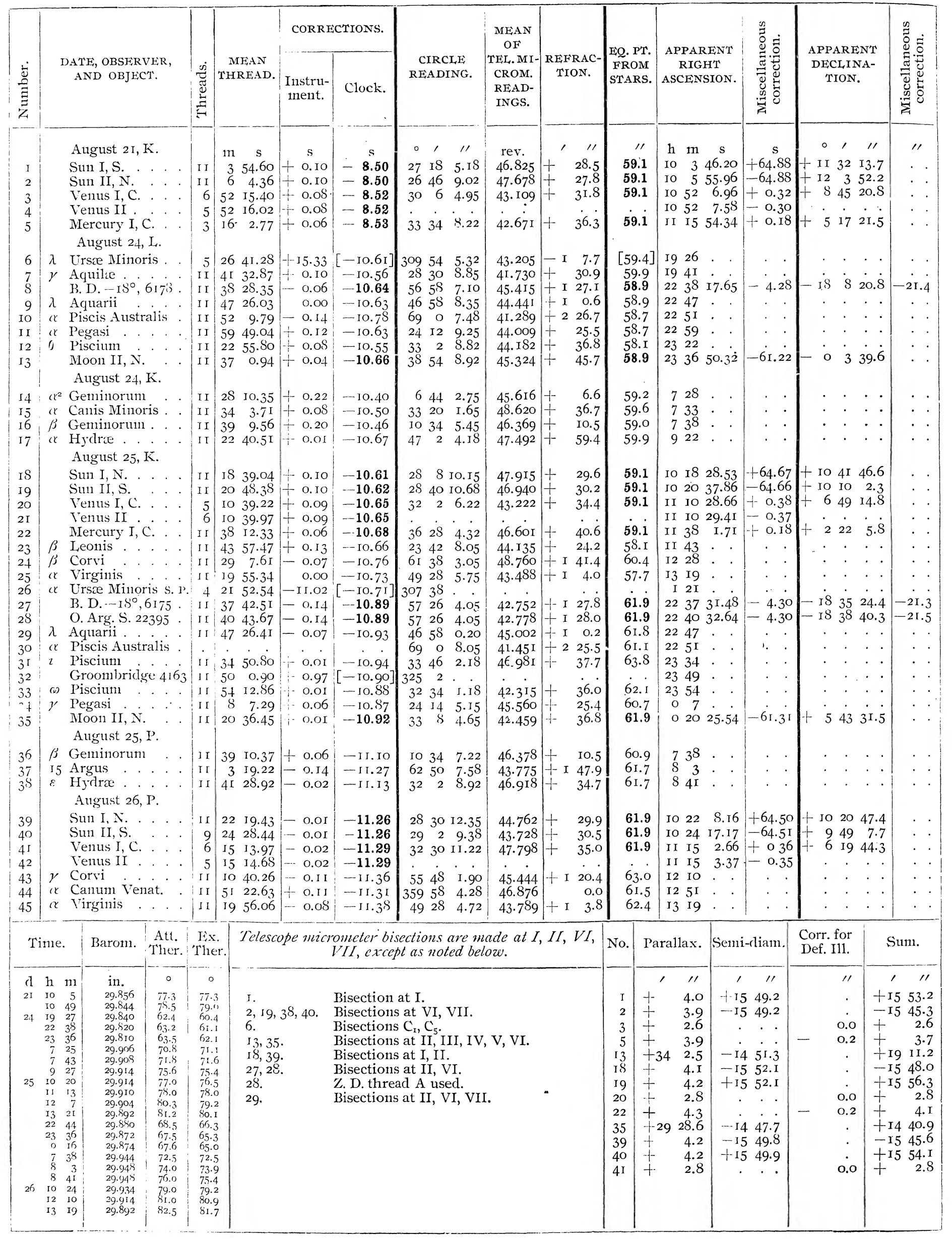




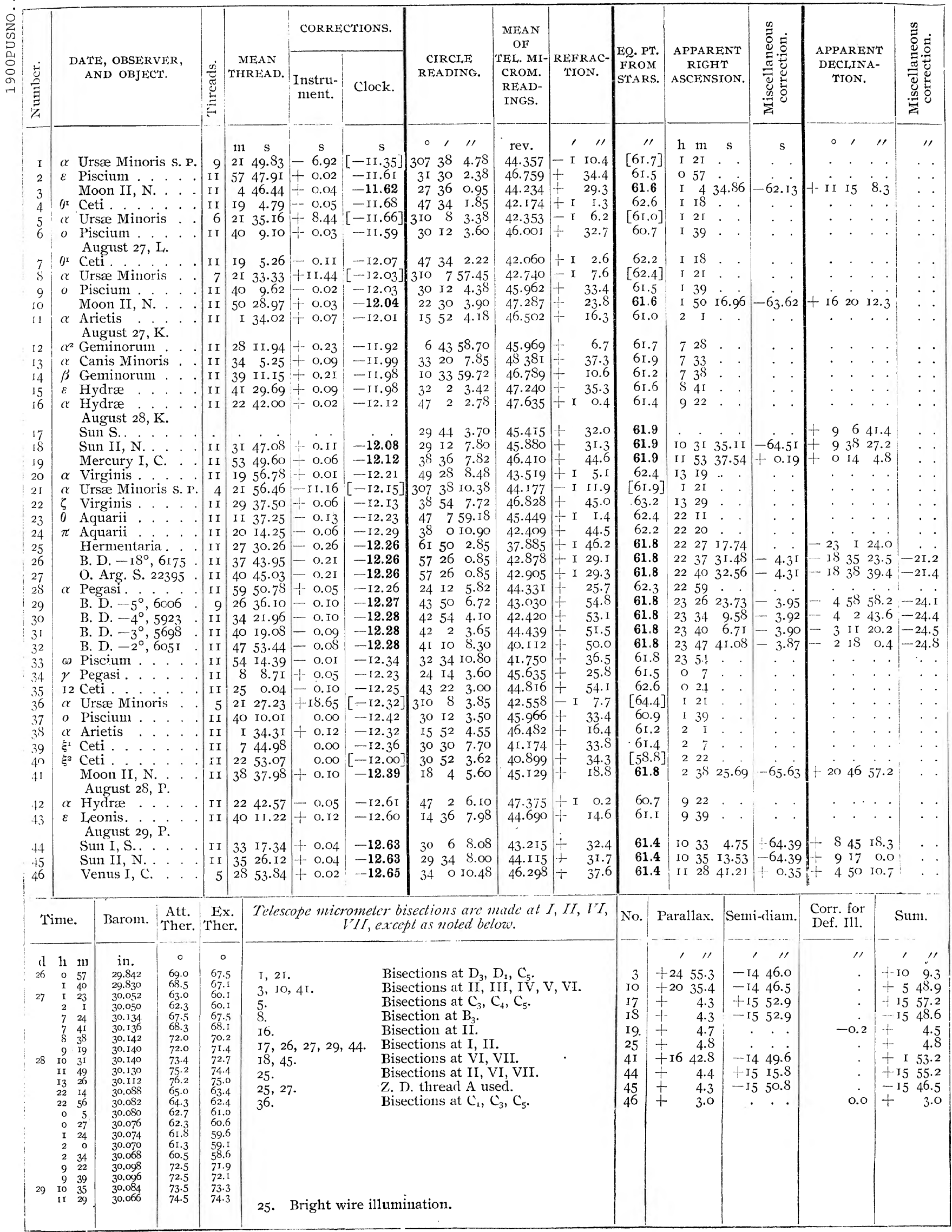




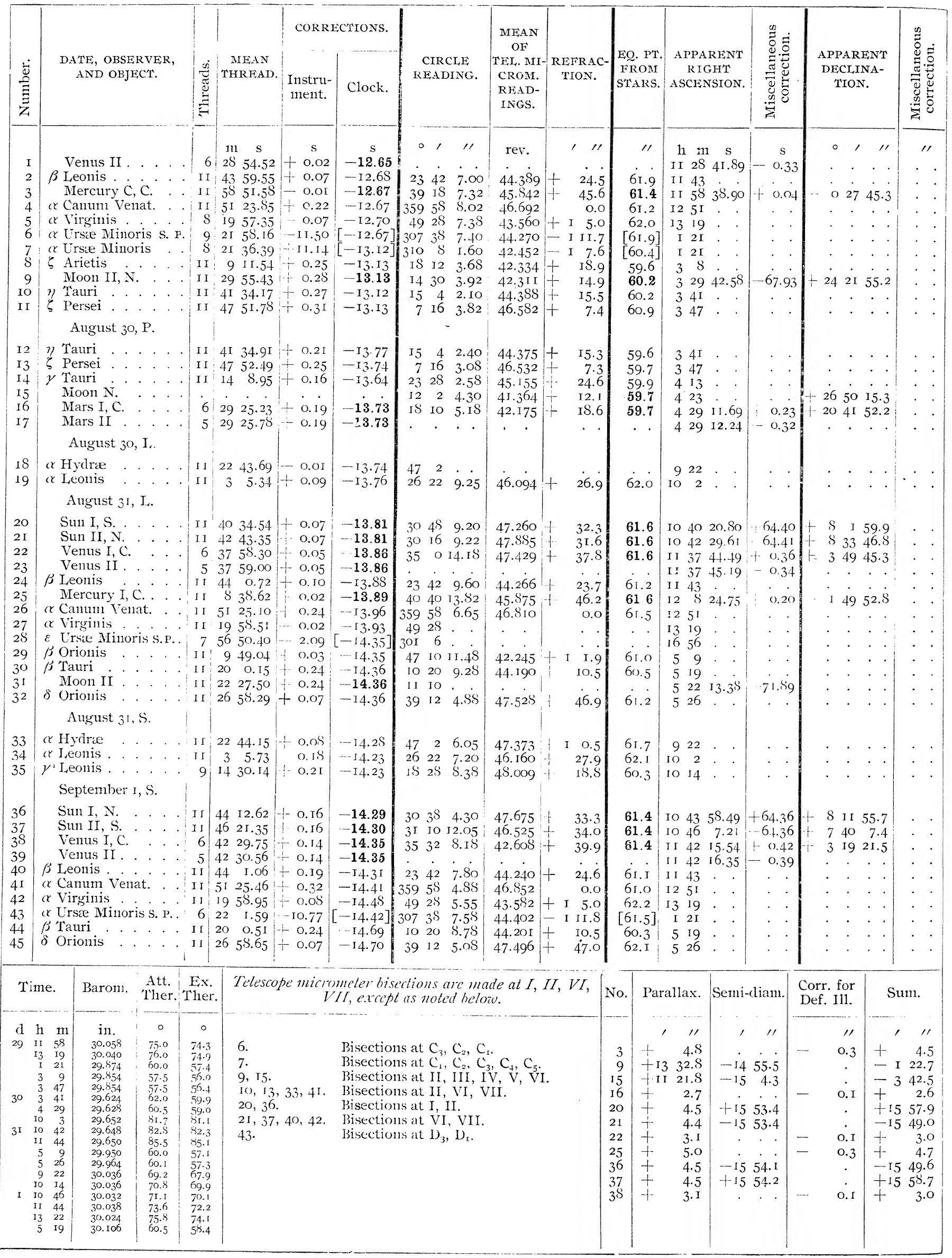




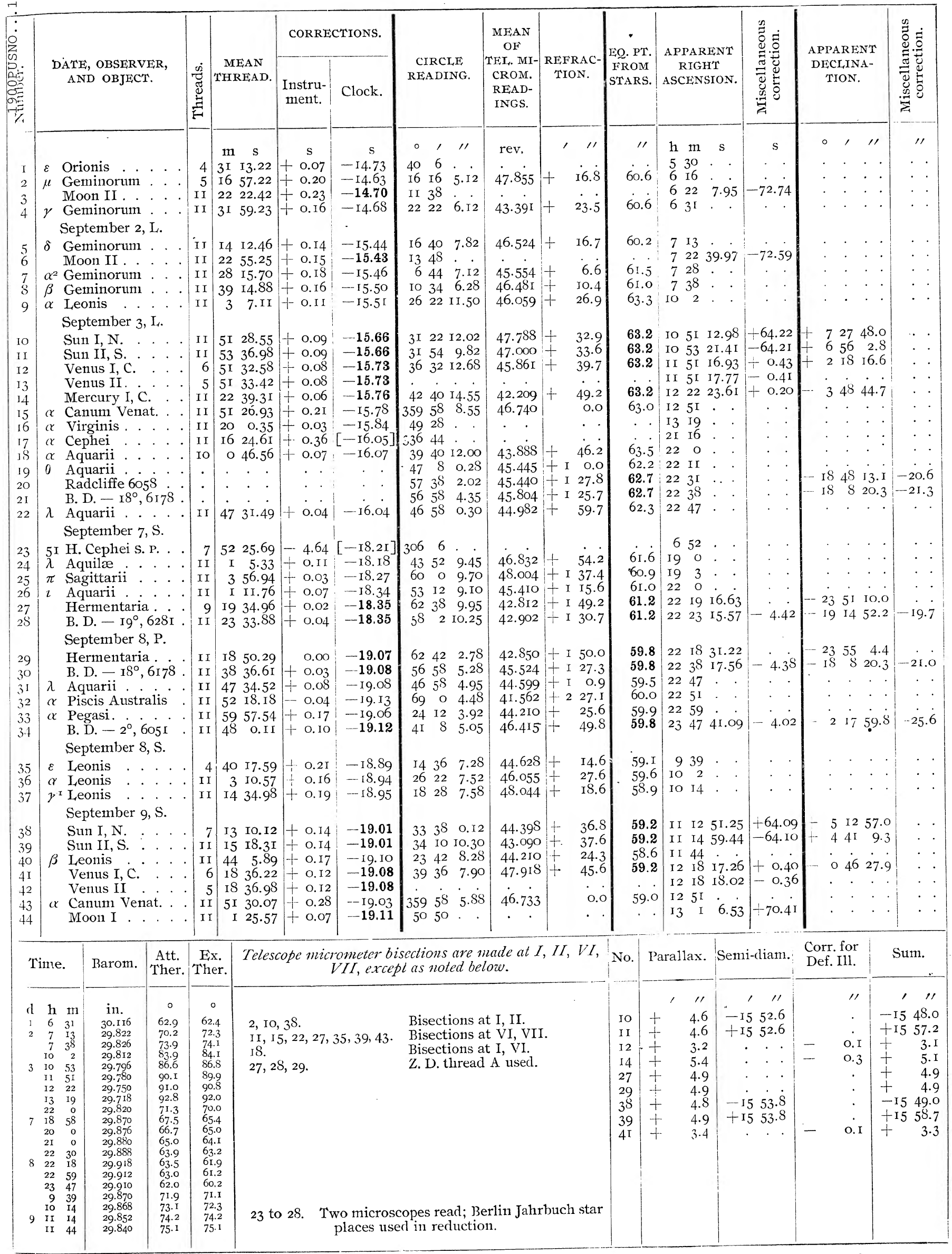




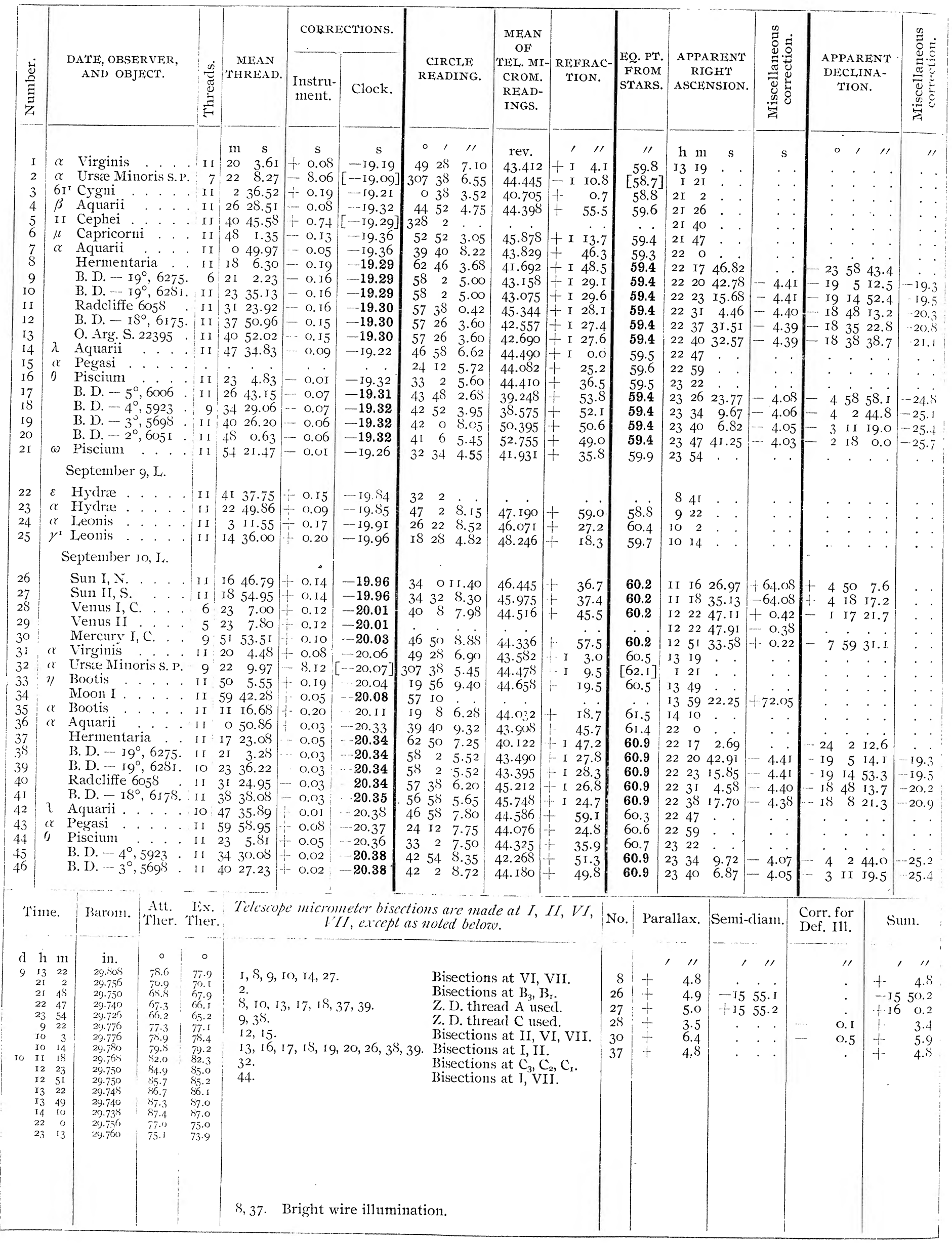




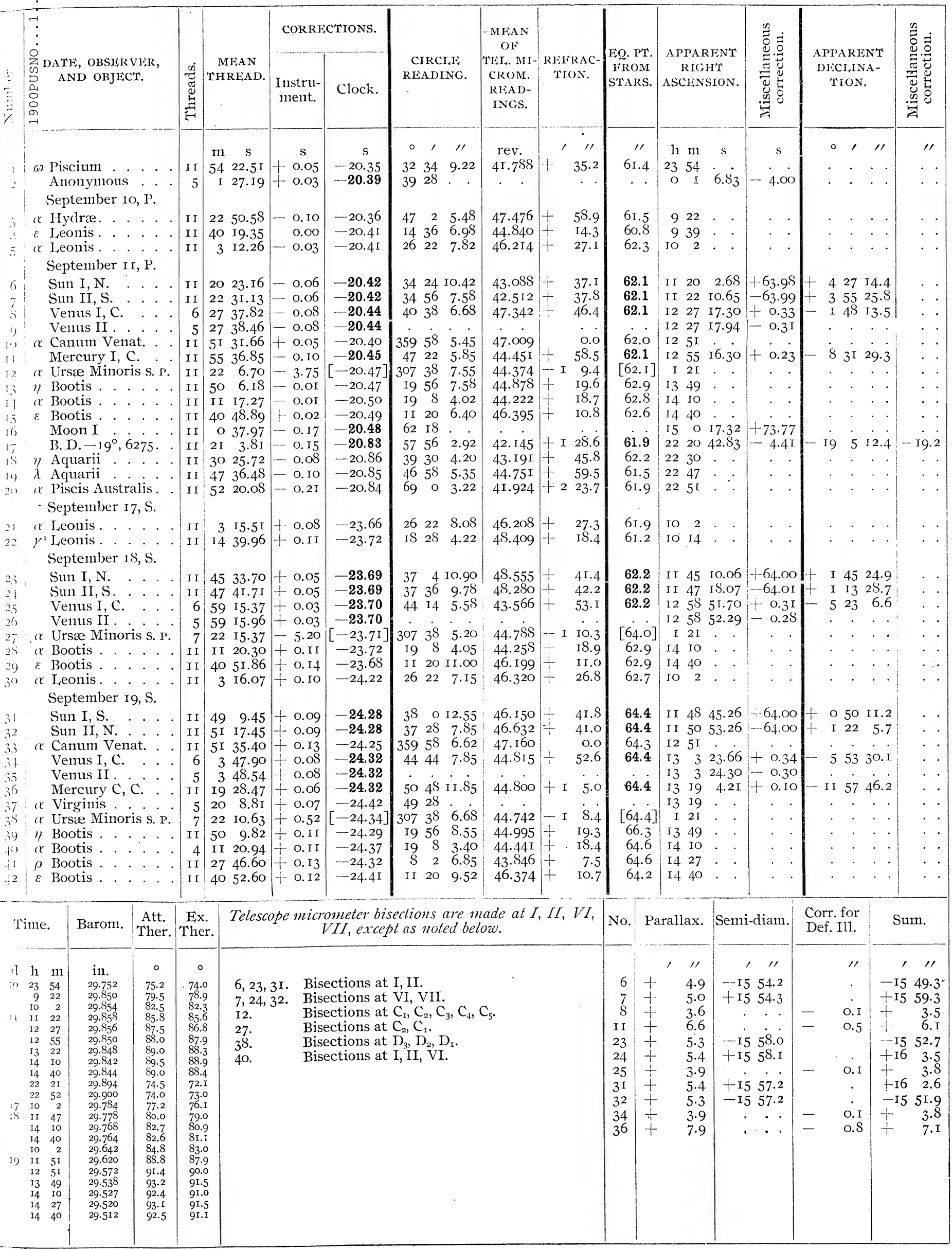




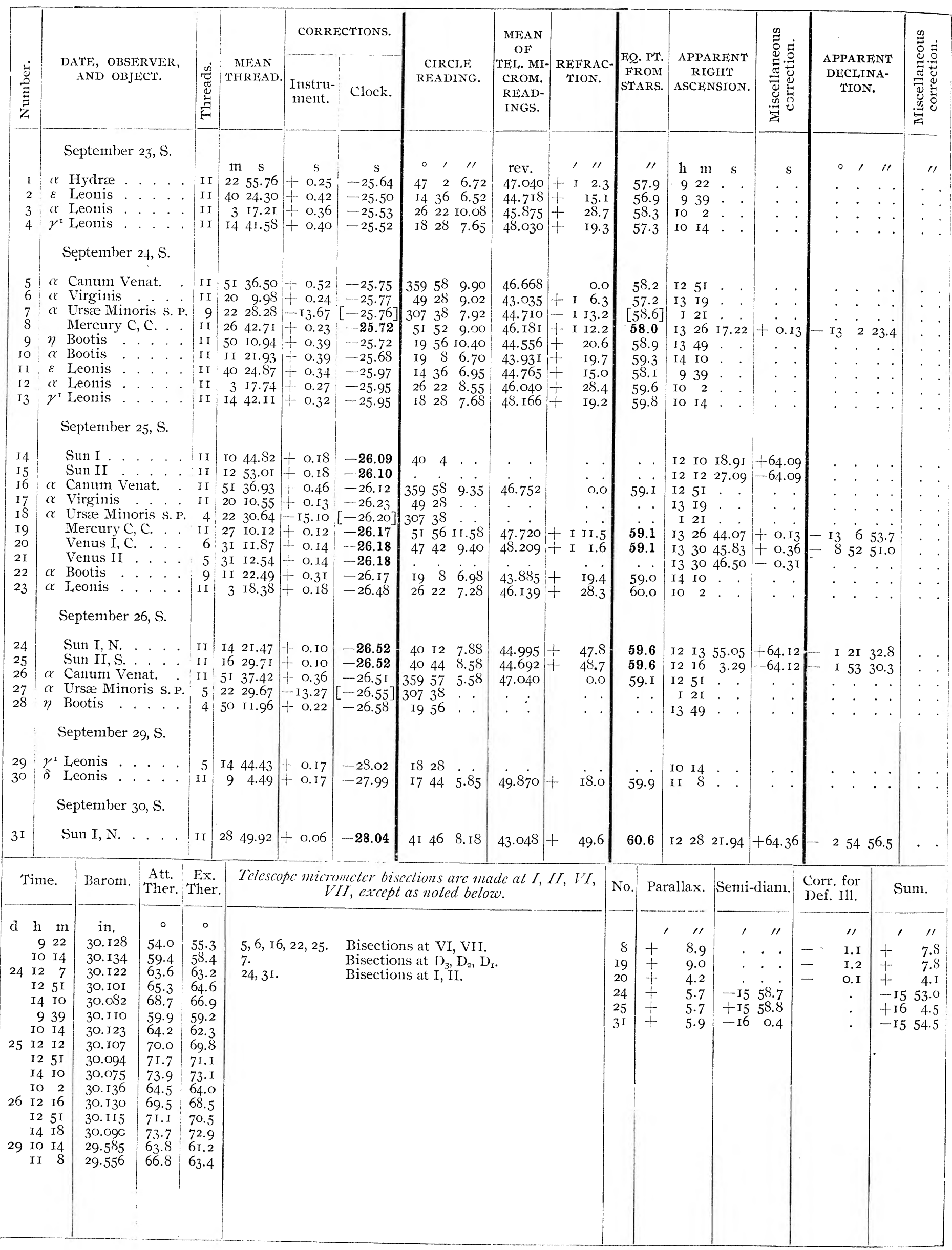




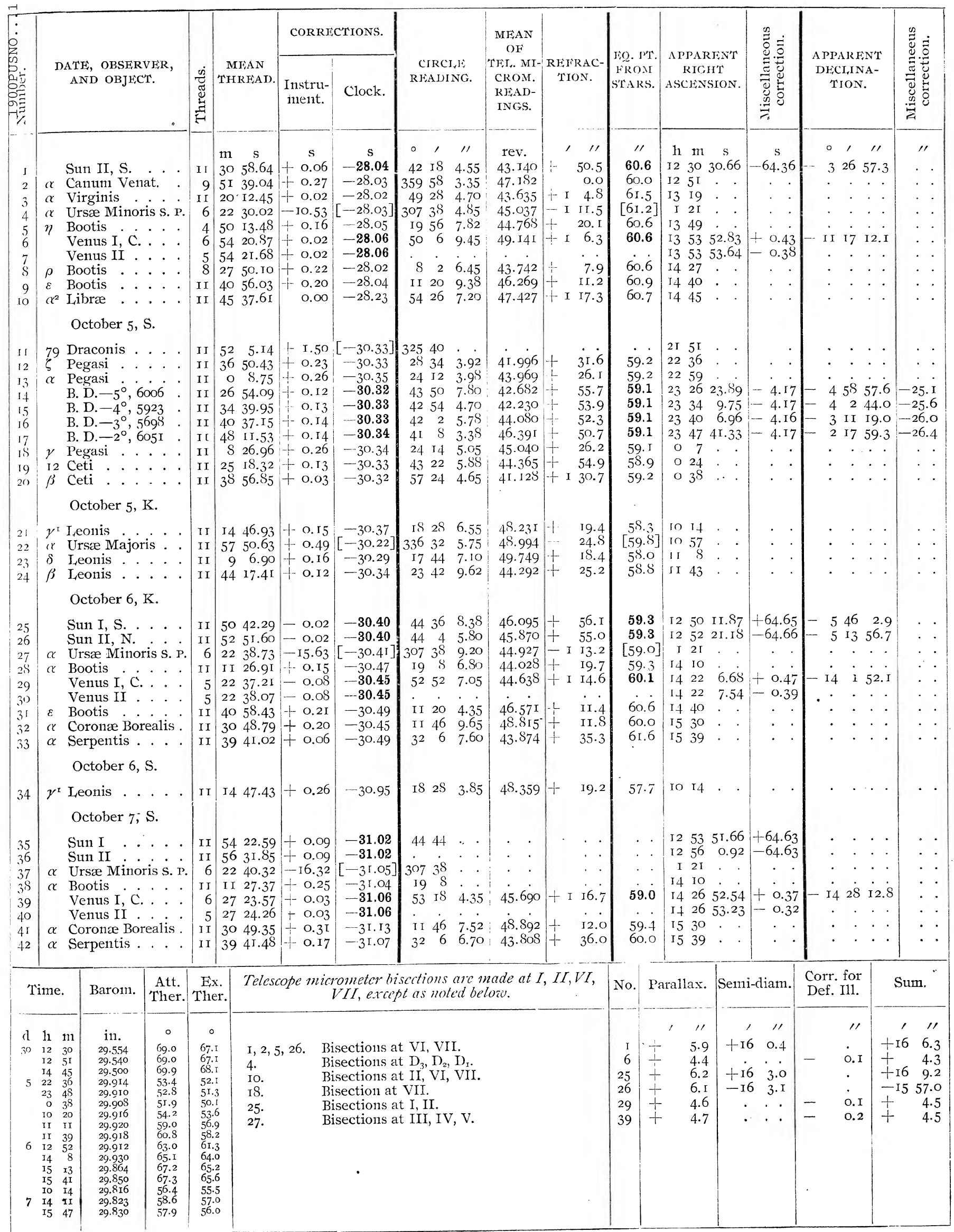




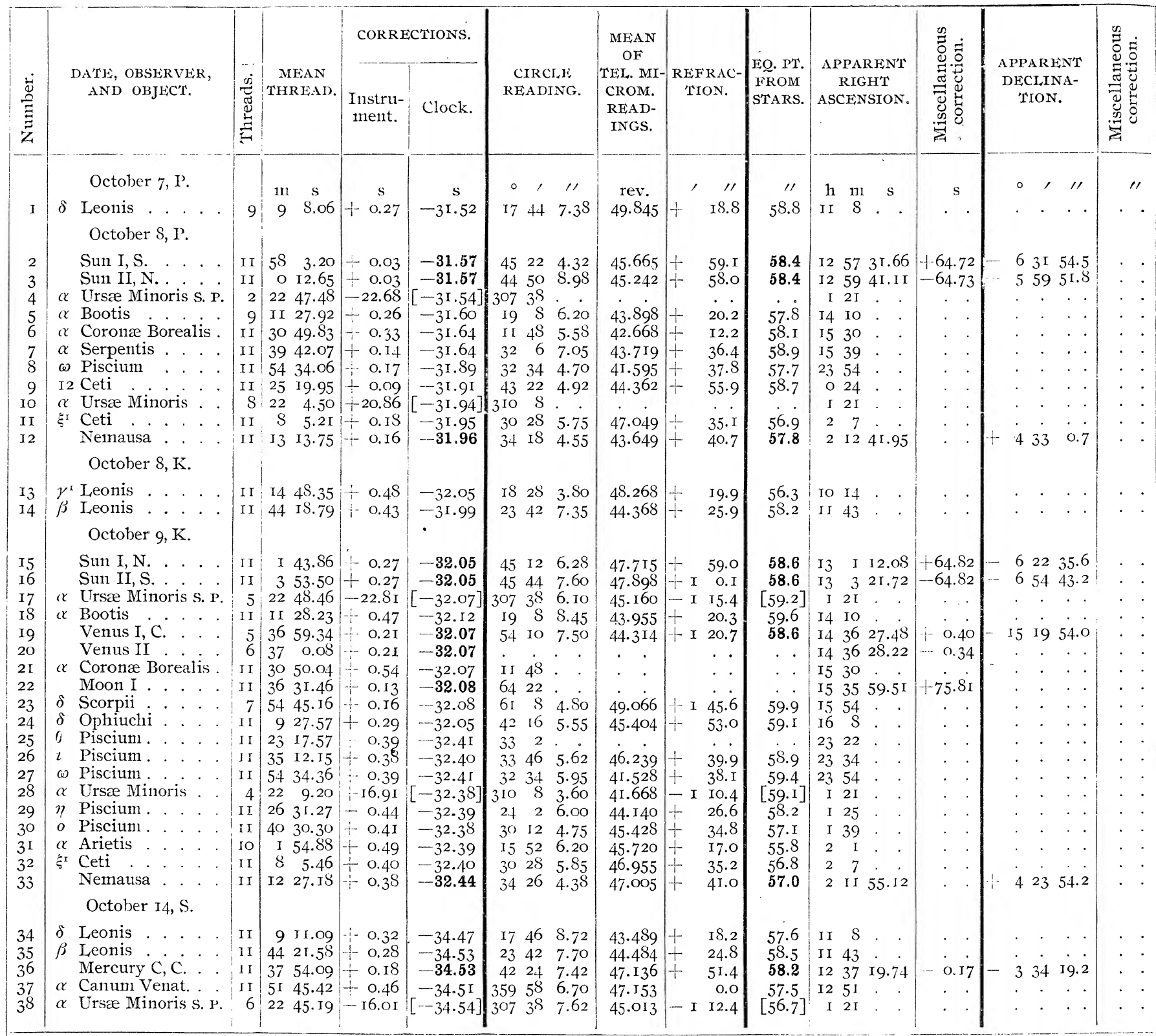

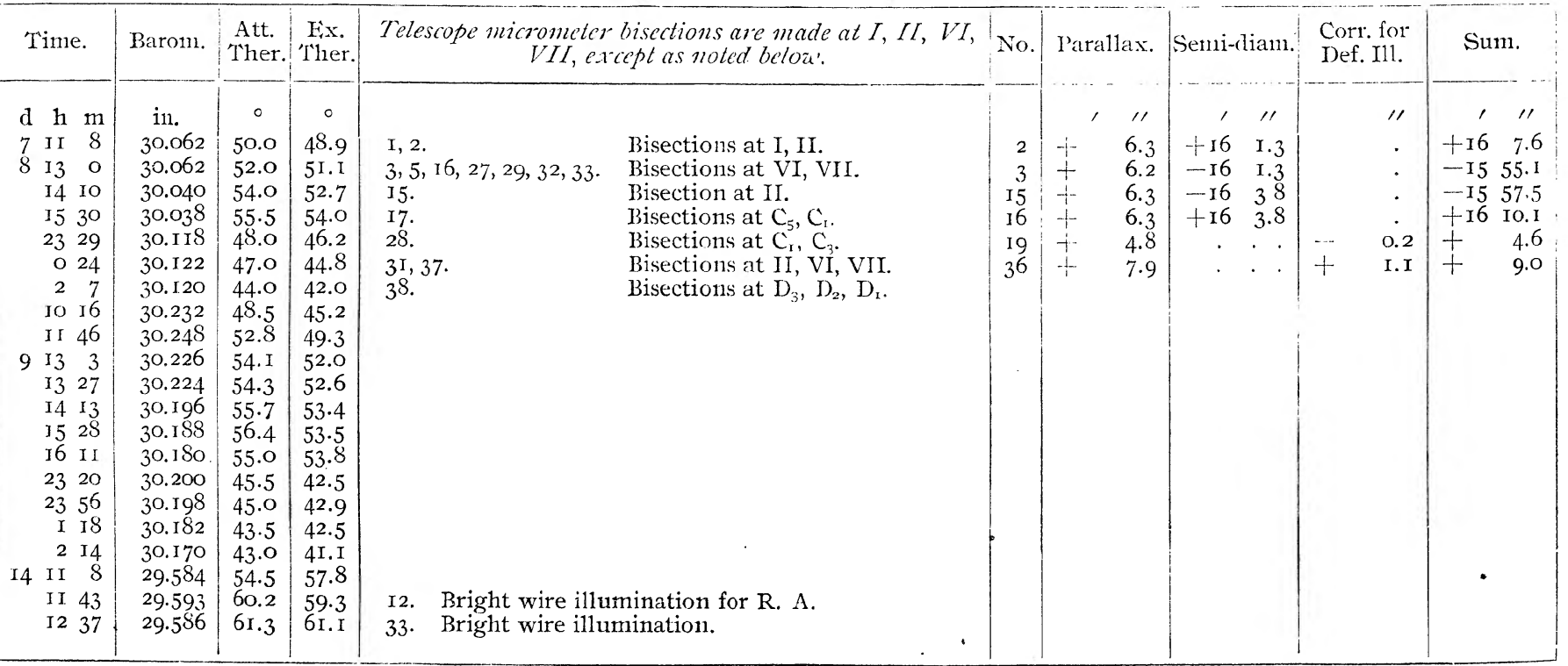




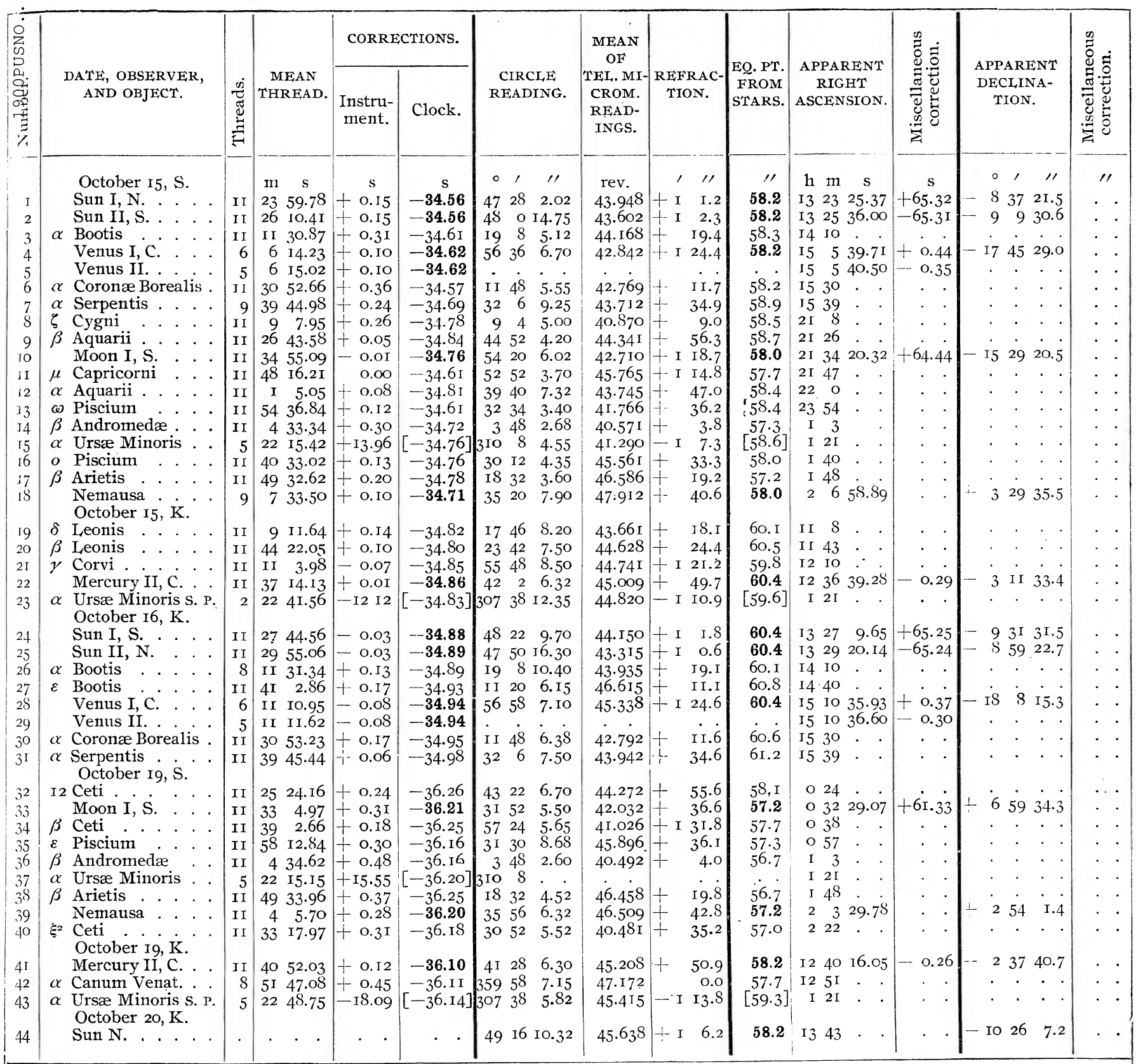

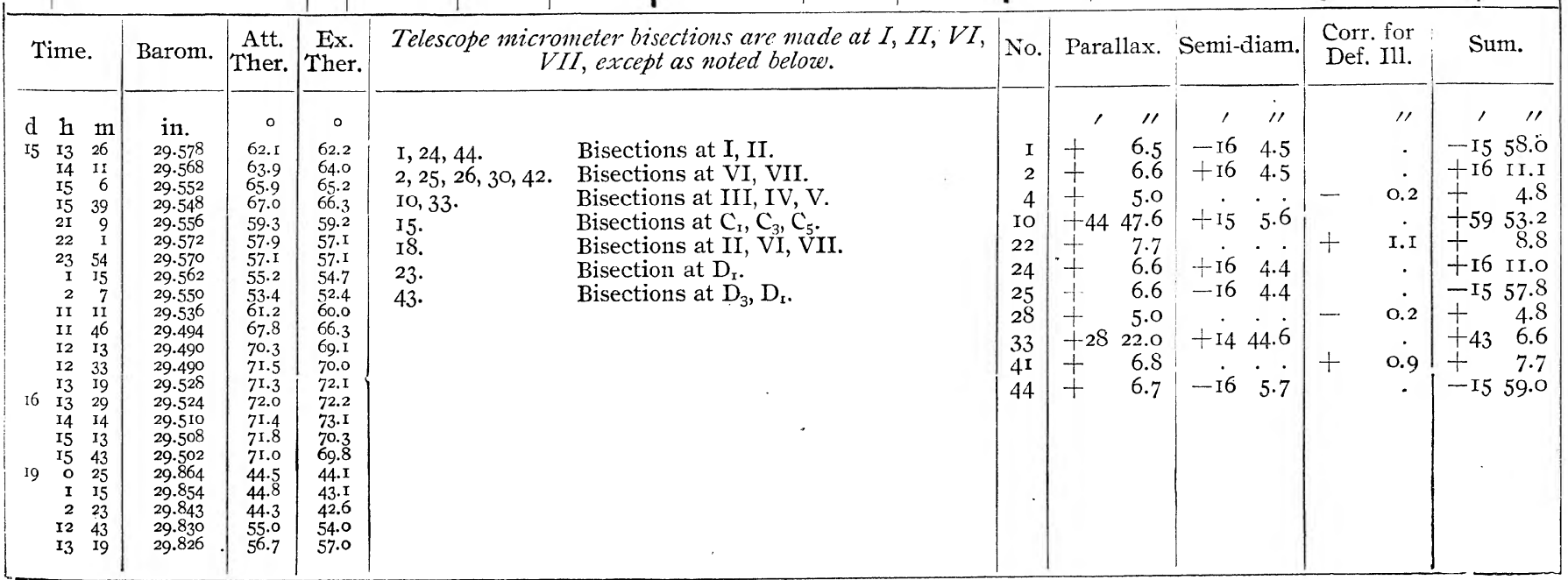




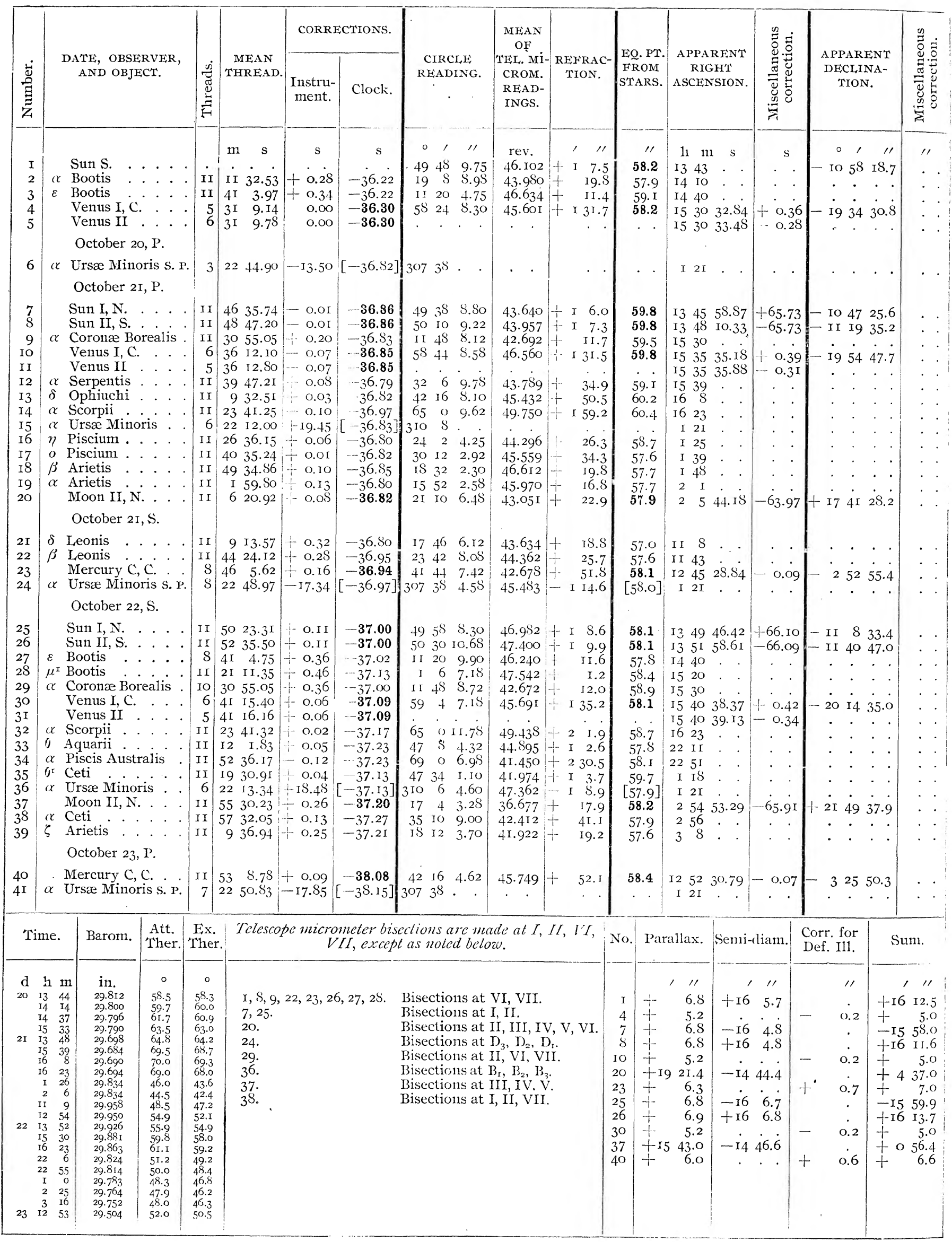




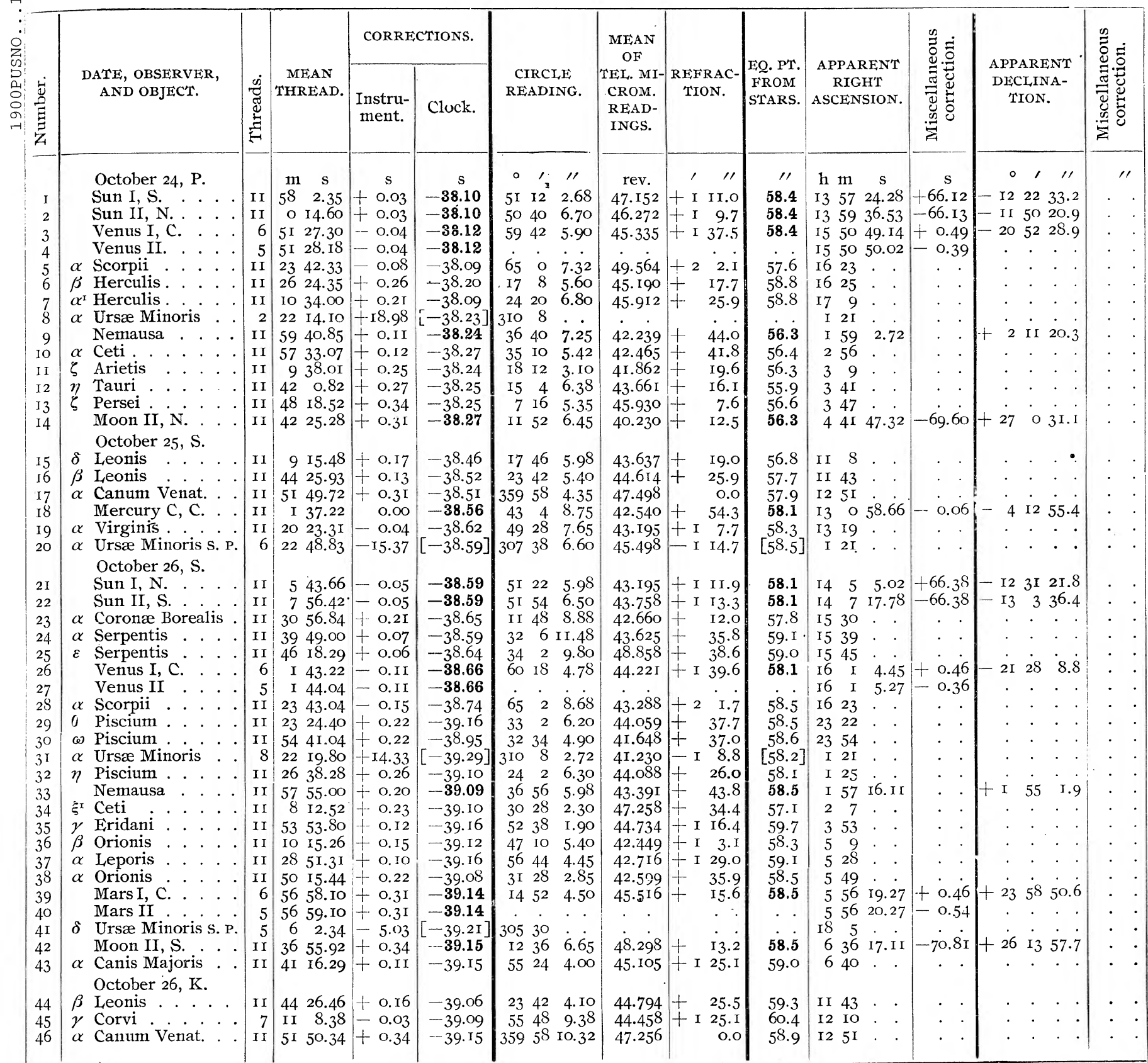

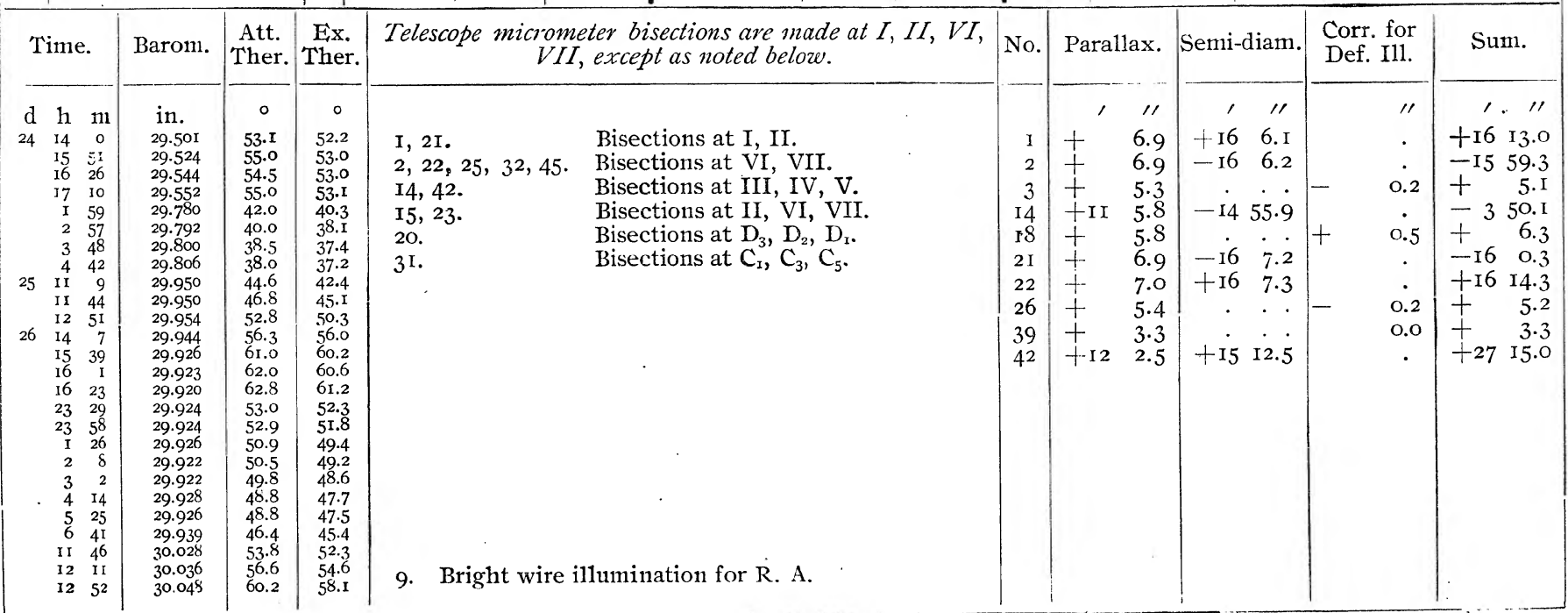




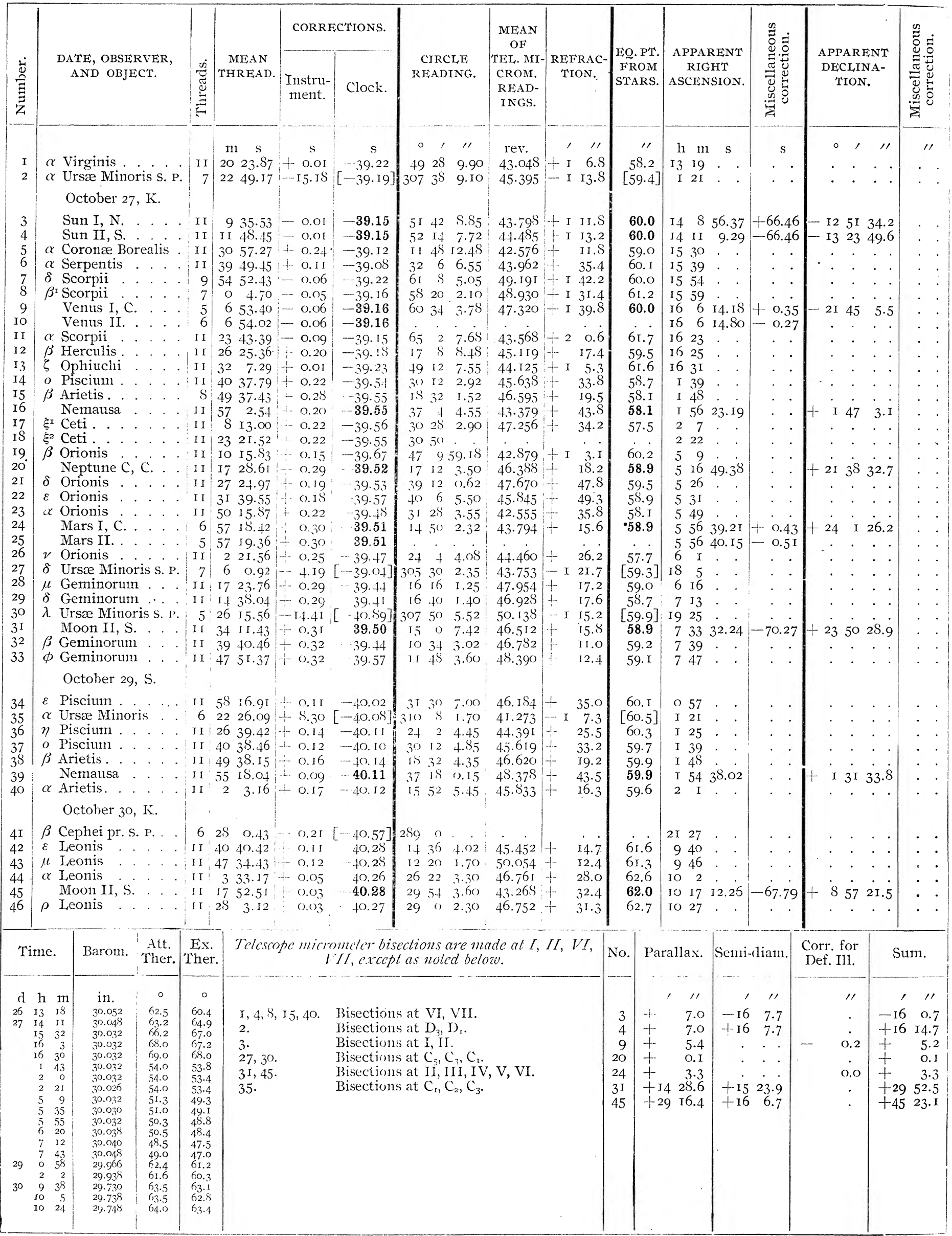




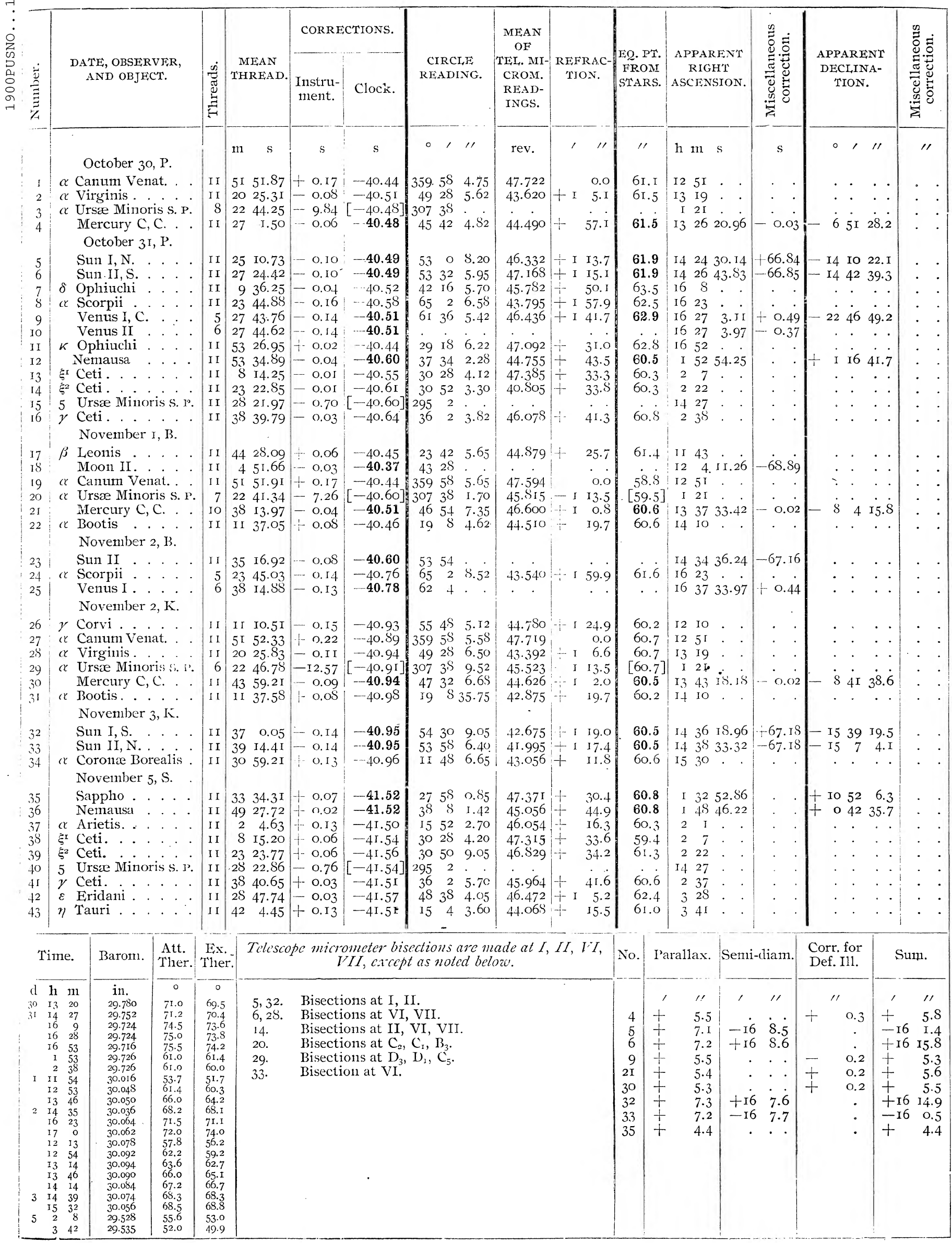




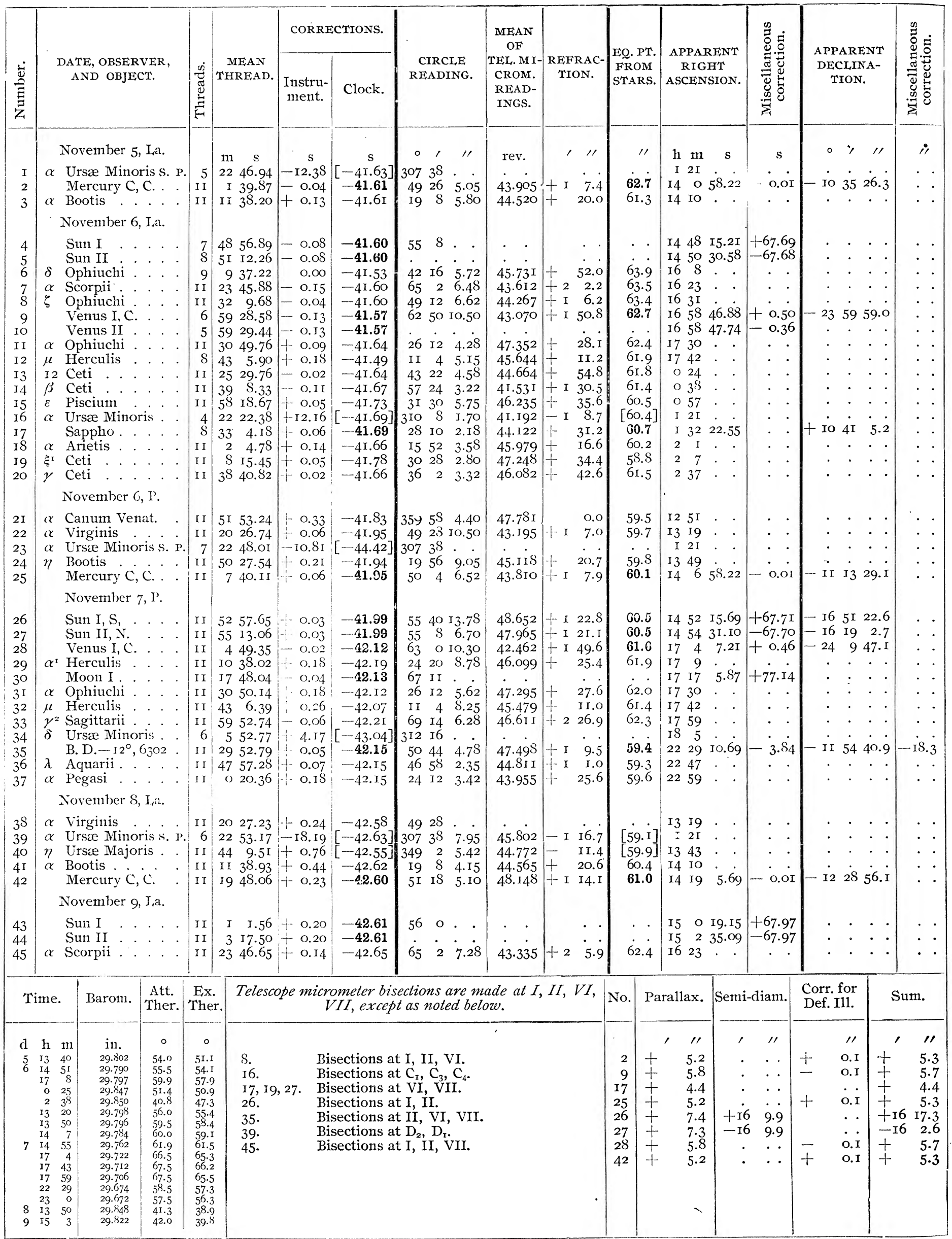


(20)
DATE, OBSERVER, AND OBJECT.

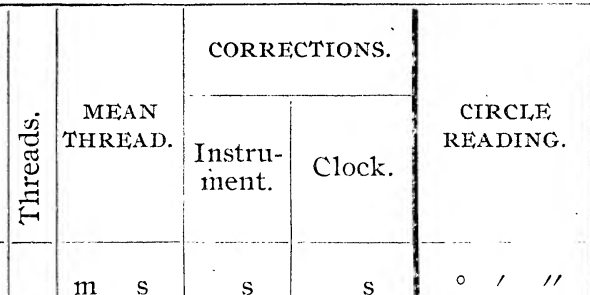

Venus I, C.

Venus II

( $\ell$ Ophiuchi

Lyra. $\cdot$.

Canum Venat.

$x$ Ursce Minoris . . II $2027.55+0.22-42.86 \quad 4928 \quad 5.90$

$\alpha$ Bootis. . . . . I I I $39.32+0.39-42.94$ I9 86.98

November Io, S.

Moon I, S. . . . I I 2457. II $+0.23-43.18$ 60 366.30

$\alpha$ Cygni. . . . . . II $3837 . \mathrm{I} 4+0.5^{8}[-43.06] 35.3566 .70$

11 Aquarii . . . . . I I $4748.92+0.28-43.27$ I8 I2 5.30

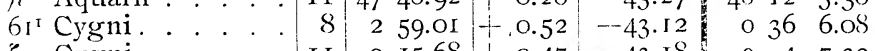

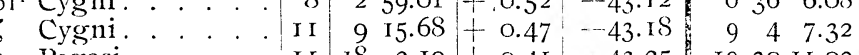

Pegasi

1) Ceti

( $)$ Ursae Minoris

Nemausa

(x Arietis

है Ceti.

November I2, K.

o) $a$ Canum Venat.

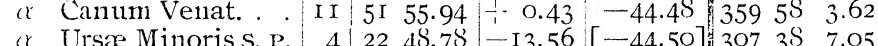

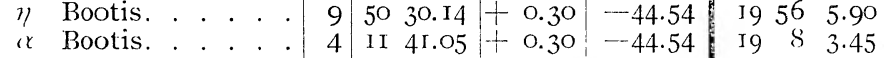

November I $_{3}, \mathrm{~K}$.

Sun I, S.

Sun II, N.

$\kappa$ Oplinuchi

$\left(\gamma^{\mathrm{I}}\right.$ Herculis.

(r Ophiuchi

Venus I, C.

Venus II

$\iota$ Herculis.

B. D. $-19^{\circ}, 6303$.

C Pegasi

$\lambda$ Aquarii

Moon I, s.

(x Pegasi

() Piscium

Piscium .

gr Ceti.

(r Urse Minoris

Sappho

Piscium.

Nemausa

$\beta$ Arietis

November I3, B.

15

Virginis. \begin{tabular}{l|rr|rrrrrr} 
II & 18 & 2.19 & 0.41 & -43.25 & I & 30 & 1.02 \\
I I & I9 & 36.96 & + & 0.28 & 43.39 & 47 & 34 & 4.30
\end{tabular}

$62222.45+12.8$ I $[-43.4$ I $]$ 3 IO 8 . 2.80

\begin{tabular}{|r|rr|rr|r|rrr} 
II & 45 & 42.49 & -0.32 & -43.37 & 3 & 36 & 5.38 \\
I I & 2 & 6.23 & - & 0.43 & --43.38 & I5 & 52 & 3.88
\end{tabular}

\begin{tabular}{l|ll|ll|l|lll} 
II & 8 & 16.74 & -0.73 & -43.3 & 15 & 52 & 3.88 \\
30 & 28 & 4.18
\end{tabular}

II 1720.47 †

I I I9 $37.37+0.10-\mathbf{4 4 . 5 7} 5^{6} 48 \quad 6.78$

I I $5330.8 \mathrm{~S}-0.24 \quad-44.63 \quad 29$ is 6.68

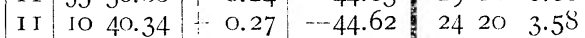

\begin{tabular}{r|rr|r|r|lll} 
I I & 30 & 52.56 \\
6 & 37 & 6.25 & 0.26 & -44.65 & 26 & I 2 & 3.55 \\
& 5.06 & -44.63 & 63 & 42 & 3.00
\end{tabular}

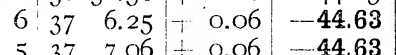

I I $43 \quad 8.77-0.35 \quad-44.59$

\begin{tabular}{rrr|rr|r} 
II & 43 & 8.77 & + & 0.35 & -44.59 \\
\hline & 30.85 & + & 0.02 & -44.60
\end{tabular}

I I $\quad$\begin{tabular}{lll|l|l|l}
37 & 4.23 & $-0.2 \mathrm{I}$ & -44.49
\end{tabular}

II 0 22.68 $+0.24 \quad-44.60 \quad 24$ I 2

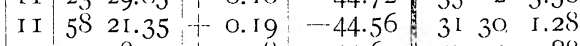

I I $1938.43+0.08-44.67 \quad 4734$ I.88

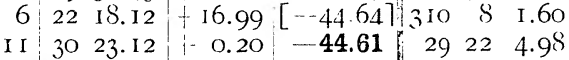

II $40 \begin{array}{llllllllll} & 43.02 & \text { f } & 0.20 & - & 44.69 & 30 & \text { i2 } & 4.52\end{array}$

\begin{tabular}{l|ll|ll|llll} 
I I & 43 & 46.17 & + & 0.14 & -44.61 & 38 & 54 & 4.75 \\
I & 49 & 42.58 & + & 0.28 & -44.53 & I & 32 & 3.82
\end{tabular}

\begin{tabular}{l|ll|l|l} 
& & & & \\
I I & 20 & $29.8 I$ & $+0.3 I$ & -45.13
\end{tabular}

$\begin{array}{lll}49 & 2 \mathrm{~S} & 2.52\end{array}$
I I $\begin{array}{lll}5 \mathrm{I} & 4.3 \mathrm{O}\end{array}$

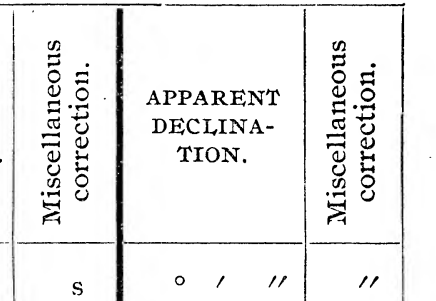

Telescope micromeler bisections are made at I, II, VI,

in. $\left|\frac{\text { Ther. Ther. }}{0}\right| \frac{\text { VII, except as noted below. }}{0} \mid-$

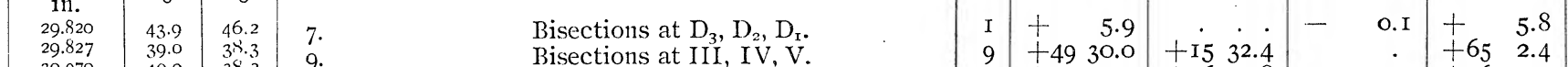

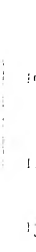

19 19 40 29.82

\begin{tabular}{ll|l}
12 & $5:$ & 30.079 \\
14 & i1 & 30.076
\end{tabular}

\begin{tabular}{ll|l}
14 & i1 & 30.07 \\
20 & 24 & 30.04
\end{tabular}

\begin{tabular}{rr|r}
20 & 24 & 30.044 \\
21 & 30 & 30034 \\
1 & 45 & 29.970 \\
2 & 8 & 29.968 \\
12 & 51 & 29.792
\end{tabular}

\begin{tabular}{ll|r}
12 & 51 & 29.792 \\
13 & 39 & 29.792
\end{tabular}

\begin{tabular}{ll|l}
13 & 38 & 29.792 \\
I4 & 15 & 29.800
\end{tabular}

\begin{tabular}{ll|l}
15 & 19 & 29.762 \\
16 & 29 & 29.750 \\
16 & 56 & 29.750
\end{tabular}

$\begin{array}{ll}17 & 56 \\ 17 & 29\end{array}$

\begin{tabular}{ll|l}
17 & 29 & 29.748 \\
17 & 58 & 29.762 \\
22 & 33 & 29.830
\end{tabular}

$\begin{array}{rr}22 & 33 \\ 23 & 3 \\ 23 & 20\end{array}$

\begin{tabular}{rr|r}
23 & 3 & 29.846 \\
23 & 20 & 29.850
\end{tabular}

$\begin{array}{rr}\text { I } & 0 \\ \text { I } & 52\end{array}$

I3 10

\begin{tabular}{l|l|l}
40.0 & 38.2 & 9. \\
46.5 & 45.2 & 15.
\end{tabular}

\begin{tabular}{l|l|l}
48.1 & 45.2 & 15.2 \\
47.4 & 46.4 & 16.
\end{tabular}

\begin{tabular}{l|l|l}
47.4 & 46.4 & 16. \\
43.7 & 42.7 & 20.
\end{tabular}

\begin{tabular}{l|l|l}
43.7 & 42.7 & $2 \mathrm{O}$. \\
43.4 & 42.5 & $2 \mathrm{I}, 4 \mathrm{O}$.
\end{tabular}

\begin{tabular}{l|l|l}
43.4 & 42.5 & $21,40$. \\
43.8 & 43.0 & $22,25,27,37$.
\end{tabular}

\begin{tabular}{l|l|l}
46.8 & 45.1 & 22, \\
48.8 & 46.4 & 23.
\end{tabular}

\begin{tabular}{l|l|l}
48.8 & 46.4 & 23 \\
48.9 & 46.2 & 35.
\end{tabular}

\begin{tabular}{l|l|l}
48.9 & 46.5 & $4 \mathrm{I}$. \\
48.5 & 45.5 &
\end{tabular}

\begin{tabular}{l|l}
48.5 & 45.5 \\
46.3 & 43.4 \\
45.0 & 43.3
\end{tabular}

\begin{tabular}{l|l|}
\hline 45.0 & 43.3 \\
36.8 & 34.6 \\
\hline 3.5 & 34.4
\end{tabular}

\begin{tabular}{l|l}
35.8 & 34.4 \\
35.5 & 34.1
\end{tabular}

\begin{tabular}{l|l|}
35.5 & 34.1 \\
33.8 & 32.7
\end{tabular}

\begin{tabular}{l|l}
33.8 & 32.7 \\
34.0 & 32.8 \\
36.8 & 35.4
\end{tabular}

Bisections at $B_{1}, B_{2}, B_{3}$.

Bisections at I, II, VI.

Bisections at $B_{3}, B_{I}$.

Bisections at VI, VII.

Bisection at II.

Bisections at II, III, IV, V, VI.

Bisections at II, VI. Z. D. thread $B$ used. 


\begin{tabular}{|c|c|c|c|c|c|c|c|c|c|c|c|c|c|c|c|c|c|c|}
\hline \multirow[b]{2}{*}{$\frac{\stackrel{D}{\Xi}}{\stackrel{\Xi}{\Xi}}$} & \multirow{2}{*}{\multicolumn{3}{|c|}{$\begin{array}{l}\text { DATE, OBSERVER, } \\
\text { AND OBJECT. }\end{array}$}} & \multirow[b]{2}{*}{ 总 } & \multirow{2}{*}{$\begin{array}{c}\text { MEAN } \\
\text { THREAD. }\end{array}$} & \multicolumn{2}{|c|}{ CORRECTIONS. } & \multirow{2}{*}{$\begin{array}{l}\text { CIRCLE } \\
\text { READING. }\end{array}$} & \multirow{2}{*}{$\begin{array}{c}\text { MEAN } \\
\text { OF } \\
\text { TEL. MI- } \\
\text { CROM. } \\
\text { READ- } \\
\text { INGS. }\end{array}$} & \multirow{2}{*}{$\begin{array}{l}\text { REFRAC- } \\
\text { TION. }\end{array}$} & & & & & & & & \\
\hline & & & & & & $\begin{array}{c}\text { Instru- } \\
\text { ment. }\end{array}$ & Clock. & & & & & $\begin{array}{l}\text { ROM } \\
\text { ARS. }\end{array}$ & $\begin{array}{r}\text { RIG } \\
\text { ASCEN }\end{array}$ & $\begin{array}{l}\text { H'T } \\
\text { isron. }\end{array}$ & 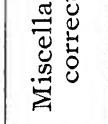 & $\begin{array}{l}\text { DECLIN } \\
\text { TION. }\end{array}$ & $\begin{array}{l}\text { NA- } \\
\text { N. }\end{array}$ & 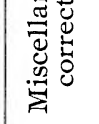 \\
\hline $\mathrm{I}$ & $\because \mathrm{U}$ & sæe Minor & ris S. P. & 8 & $\mathrm{~m}_{22} \mathrm{~s}$ & s & 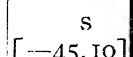 & $\begin{array}{ccc}0 & 1 & 11 \\
207 & 28 & \\
58\end{array}$ & rev. & 111 & & 11 & $\mathrm{~h} \mathrm{~m}$ & s & $\mathrm{s}$ & $\circ \quad 1$ & "I & 11 \\
\hline 2 & $\eta \mathrm{B}$ & otis.. & . . . & 9 & $\left|\begin{array}{ll}22 & 40.30 \\
50 & 30.63\end{array}\right|$ & $\mid \begin{array}{l}-13.04 \\
+\quad 0.44\end{array}$ & {$\left[\begin{array}{l}{[} \\
-45.15\end{array}\right]$} & $\mid \begin{array}{rrr}307 & 38 & \text { I.58 } \\
19 & 56 & .\end{array}$ & 46.228 & - I 17.7 & & $9 \cdot 4]$ & $\begin{array}{rr}\text { I } & 2 \text { I } \\
\text { I3 } & 49\end{array}$ & $\dot{.}$ & & . & - & . \\
\hline 3 & (x) $\mathrm{B}$ & otis. . . & ... . & II & II $4 \mathrm{I} .4 \mathrm{O}$ & +0.45 & -45.02 & 198. & & & & & I4 IO & $\cdot$ & . &. & $: \dot{0}$ & : \\
\hline 4 & $\rho \mathrm{B}$ & otis . . . & . . & II & $28 \quad 6.90$ & $+0.5 \mathrm{I}$ & --45.00 & $\begin{array}{lll}5 & 2 & 6.35\end{array}$ & 44.310 & 8.5 & & 0.3 & I4 27 & & & . & . & . \\
\hline 5 & $\varepsilon \quad \mathrm{B}$ & otis. . . & & II & $4 \mathrm{I} \quad \mathrm{I} 2.7 \mathrm{O}$ & +0.49 & -44.94 & II $20 \quad 8.25$ & 46.789 & I2. I & & 9.6 & 1440 & & & . . & 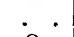 & . \\
\hline 6 & $\begin{array}{l}\mathrm{M} \\
\mathrm{Nc}\end{array}$ & $\begin{array}{l}\text { rcury } C \text {, } \\
\text { rember I }\end{array}$ & C. & 6 & 5043.09 & +0.29 & -45.05 & $\begin{array}{lll}54 & 20 & 7.85\end{array}$ & 44.959 & + I 23.5 & & 0.1 & I4 49 & 58.33 & 0.00 & - I5 30 & 8.0 & \\
\hline 7 & & $\mathrm{n} I, \mathrm{~S}$. & . . . & II & 2I 26.94 & +0.28 & -45.06 & $\begin{array}{lll}57 & 36 & 6.32\end{array}$ & 45.IIO & t I 34.2 & & 0.1 & I 520 & 42.16 & +68.55 & - I8 46 & I9.3 & . \\
\hline 8 & $\mathrm{~S}$ & n II, N. . & . . . & II & 2344.04 & $+0.2 S$ & -45.06 & 5746.80 & 43.910 & t I $3^{2.3}$ & & 0.1 & I5 22 & 59.26 & -68.55 & $-18 \times 3$ & 56.7 & . \\
\hline 9 & $\alpha \mathrm{Ss}$ & orpii. . . & . . . & II & 2349.06 & +0.24 & -45.14 & $\begin{array}{lll}65 & 2 & 4.72\end{array}$ & $43.32 \mathrm{I}$ & $+2 \quad 7.7$ & & I. 9 & I6 23 & . . & . & . . & $\cdot \cdot$ & \\
\hline IO & $\eta \mathrm{H}$ & rculis. . & . . . & I0 & $40 \quad 5.03$ & +0.57 & $-45 . \mathrm{II}$ & $\begin{array}{lll}359 & 44 & 4.70\end{array}$ & 45.572 & $-\quad 0.2$ & & 8.9 & 1639 & . . & . & . . & . & \\
\hline II & $\kappa O$ & hiuchi. & . . . & II & 533 I.I 5 & +0.40 & -45.06 & 29 Is 0.80 & 47.235 & $+\quad 33.5$ & & 0.5 & I 652 & . . & . & . & . & \\
\hline I2 & $\alpha \mathrm{O}$ & hiuchi . & . . & II & $3^{\circ} 52.82$ & +0.42 & -45.07 & $\begin{array}{lll}26 & 12 & 4.52\end{array}$ & 47.231 & $+\quad 29.4$ & & 0.4 & I7 30 & . . & . & . & . & 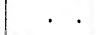 \\
\hline I3 & V & nus C. & . . & . &.$\quad \cdot \quad \cdot$ & . & . & $63 \quad 48 \quad 1.40$ & 44.022 & +20.7 & & 0.1 & $174 \mathrm{I}$ & 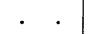 & 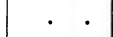 & $-245^{8}$ & 20.7 & \\
\hline I4 & $\ll L_{-}$ & $\mathrm{r} \ldots$ & . . . & II & $34 \quad 10.32$ & +0.57 & -44.98 & 0 io 7.50 & 44.555 & 0.2 & & 9.6 & is 33 & . & . & . . & . & 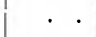 \\
\hline 15 & $\varepsilon \mathrm{P}$ & gasi.. & . . . & II & 3952.69 & -0.42 & -45.37 & $\begin{array}{lll}29 & 26 & 5.72\end{array}$ & 46.415 & f- 33.8 & & 0.2 & 2 I 39 & • & . & . & - & \\
\hline I6 & $\begin{array}{ll}\alpha & \mathrm{A} \\
& \end{array}$ & uarii & & II & I $\quad$ I $4.8_{4}$ & +0.39 & -45.27 & $\begin{array}{llll}39 & 40 & 6.40\end{array}$ & 43.736 & +49.8 & & 9.3 & 220 & & • & . & . & \\
\hline I7 & $0 \mathrm{~A}$ & uarii & & II & I2 9.33 & $+0.3^{6}$ & $-45.3 \mathrm{I}$ & $47 \quad 890.80$ & 45.152 & + I 4.7 & & O. I & 22 II & & & & & \\
\hline is & B. & D. $-19^{\circ}$, &, 6303. & II & $302 \mathrm{I} .5 \mathrm{I}$ & +0.32 & -45.32 & $\begin{array}{lll}57 & 46 & 4.98\end{array}$ & 47.169 & $+\mathrm{I} 35.2$ & & 9.9 & 2229 & $36.5 \mathrm{I}$ & -3.91 & $-185^{6}:$ & 59.4 & -15.4 \\
\hline I9 & & on I. & . . . & 9 & 3455.07 & +0.40 & -45.33 & 390. & & & & & 2334 & Io.14 & +6 I.15 & . & $\cdot \cdot$ & . . \\
\hline 20 & $\omega \mathrm{Pi}$ & cium & . . . & 9 & $5+47.10$ & $+0.4 \mathrm{I}$ & -45.32 & $\begin{array}{llll}3^{2} & 34 & 4.82\end{array}$ & $4 \mathrm{I} .658$ & $3^{8.5}$ & & 0.0 & 2354 & . . & . $\cdot$ & - & - & . \\
\hline $2 \mathrm{I}$ & $\gamma \mathrm{P}$ & gasi & . . & IO & $84 \mathrm{I} .67$ & +0.44 & -45.34 & $24 \mathrm{I} 4 \quad 4.70$ & $45.01 \mathrm{I}$ & 727.2 & & 9.6 & 07 & . & . . & . & . & . \\
\hline 22 & $\begin{array}{l}\mathrm{I} 2 \mathrm{Ce} \\
\alpha \mathrm{U}\end{array}$ & si. irinor & ris : & $\mid \begin{array}{r}\text { II } \\
8\end{array}$ & $\left|\begin{array}{ll}25 & 33.05 \\
22 & 23.3 \mathrm{I}\end{array}\right|$ & $\begin{array}{r}1+0.37 \\
-12.01\end{array}$ & {$\left[\begin{array}{c}-45.36 \\
{[-45.35]}\end{array}\right.$} & $\begin{array}{rrr}43 & 22 & 8.5^{\circ} \\
3 & 8 & 8\end{array}$ & $44.28 r$ & t 57.0 & & 0.0 & O 24 & 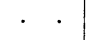 & $\cdot \cdot$ & . $\cdot$ & - & . $\cdot$ \\
\hline $\begin{array}{l}23 \\
24\end{array}$ & $\mathrm{~S}$ & pho. . & . . & $\begin{array}{l}0 \\
8\end{array}$ & $\left|\begin{array}{rr}22 & 23.31 \\
30 & 7.32\end{array}\right|$ & $\begin{array}{r}12.04 \\
+\quad 0.42\end{array}$ & $\left|\begin{array}{r}{[-45.35]} \\
-45.35\end{array}\right|$ & $\mid \begin{array}{rrr}310 & 8 & . \\
29 & 32 & 3.23\end{array}$ & $55.792:$ & $+\quad .3 .3$ & & & $\begin{array}{ll}\text { I } & 2 \mathrm{I} \\
\mathrm{I} & 20\end{array}$ & 22. & $\cdot \cdot$ & + i & $\ddot{\dot{4}} \dot{4}$ & $\cdot \cdot$ \\
\hline & & mber Is & $5, \mathrm{~B}$. & & & & & $1293^{2} 3.20$ & $53 \cdot 19^{2}:$ & & & 9.9 & & 22.39 & & $+92 \mathrm{I}$ & 42.4 & $\cdot \cdot$ \\
\hline 25 & $\mu \mathrm{Ca}$ & pricorni . & & II & \begin{tabular}{|ll}
48 & 26.88
\end{tabular} & +0.20 & -45.89 & $\begin{array}{lll}52 & 52 \quad 3.60\end{array}$ & 45.990 & $\begin{array}{lll}\text { t- I } & \text { I } 6.4\end{array}$ & & I.9 & 2147 & $\cdot$ & & & & \\
\hline 26 & $\pi$ A & harii & & II & 2046.95 & +0.26 & -45.80 & $3^{8}$ o 10.18 & $42.22 \mathrm{I}$ & $+\quad 45.3$ & & 0.2 & 2220 & 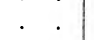 & & $\therefore$ & & \\
\hline 27 & B. & D. $-\mathrm{I} 2^{\circ}$, & , 6302. & II & $2956.1 \mathrm{II}$ & $+0.2 \mathrm{I}$ & -45.83 & $5044 \quad 2.82$ & $47.5^{8 \mathrm{r}}$ & I I II.O & & 0.6 & $22 \quad 29$ & I0.49- & -3.74 & - I I 54 & 40.3 & -I 7.7 \\
\hline 28 & $\alpha \mathrm{Pi}$ & cis Austr & ralis & II & 5244.19 & 0.13 & $-45.8 \mathrm{I}$ & 69 o IO.IO & $4 \mathrm{I} .542$ & +230.3 & & 0.2 & 22. $5 \mathrm{I}$ & . . & 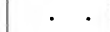 & . . & . & . \\
\hline 29 & $\propto \mathrm{U}$ & sæ Major & ris S. P. & II & $\begin{array}{ll}58 & 8.65\end{array}$ & -0.15 & $[-45.84]]$ & $\begin{array}{lll}28 \text { I } & \text { I4 } & 0.5^{8}\end{array}$ & 45.768 & -442.4 & {$\left[5^{8}\right.$} & $8.2]$ & 10 57 & . & . & . . & . & . \\
\hline 30 & $\Leftrightarrow \mathrm{Pi}$ & scium . . & . . . . & II & 5447.68 & +0.28 & $-45.7 \mathrm{~S}$ & $\begin{array}{lll}32 & 34 & 6.12\end{array}$ & $4 \mathrm{r} .67 \mathrm{I}$ & $+\quad 37.2$ & & 0.2 & 2354 & & & - & . & . \\
\hline $3^{I}$ & $\mathrm{M}$ & on I . . & $\cdots$ & II & IS 22.29 & +0.28 & -45.85 & $3320 \cdot$ & & & & & O I7 ? & 36.72 & +61.22 & . . & . . & \\
\hline $3^{2}$ & $\begin{array}{r}\beta \mathrm{Ce} \\
\mathrm{No}\end{array}$ & ti. . . & · $\dot{\text { La. }}$ & II & 39 โ2.23 & -0.18 & $-45.9 \mathrm{I}$ & $\begin{array}{lll}57 & 24 & 6.00\end{array}$ & $4 \mathrm{I} .359$ & t I 3I.I & & 0.4 & O $3^{8}$ & . & . & 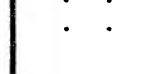 & . & . \\
\hline 33 & $\alpha \mathrm{C}$ & num Ven & 1at. . . & II & 5157.76 & +0.37 & -46.17 & $3595^{8} \quad 4.98$ & 47.868 & & & 8.9 & $125^{\mathrm{I}}$ & . & . & • & . & . \\
\hline 34 & $(\varkappa \mathrm{Vi}$ & is . &... & II & $203 \mathrm{I} . \mathrm{I} 3$ & $\mid \begin{array}{l}1 \\
-\end{array}$ & $-46.20^{8}$ & $4928 \quad$ I. 42 & 43.6 I I & H I 8.4 & 59 & 9.0 & I3 19 & . & & . & . & \\
\hline 35 & $\ll \mathrm{Ur}$ & sæe Minor & ris s. P. & 6 & 2246.70 & -II.I9 & $=-46.18]$ & $\begin{array}{lll}307 & 38 & 7.30\end{array}$ & 45.912 & - I 15.5 & {$[5 s$} & S.8] & I 21 & & & . & . & . \\
\hline $3^{6}$ & $\eta \mathrm{Ur}_{1}$ & sæe Major & ris . . & II & $44 \quad \mathrm{I} 3.52$ & +0.46 & {$[-46.11]$} & $\begin{array}{lll}349 & 2 & 6.35\end{array}$ & 44.820 & $-\quad$ II.2 & {$[59$} & $9.4]$ & I3 43 & . & & - & . & - \\
\hline 37 & $\begin{array}{l}\qquad \mathrm{BC} \\
\mathrm{No}\end{array}$ & $\begin{array}{l}\text { otis. } \\
\text { ember } 6\end{array}$ & 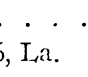 & II & II 42.84 & 0.25 & -46.23 & I9 $8 \quad 5.32$ & 44.550 & $+\quad 20.2$ & 59 & 9.1 & 14 10 & 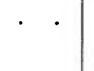 & . & . & . & . . \\
\hline $3^{8}$ & $\mathrm{~S}$ & n I, N. . & & II & 2943.56 & $-1-0.06$ & -46.28 & $\begin{array}{lll}57 & 34 & 7.40\end{array}$ & 43.990 & t- I 29.7 & 60 & 0.0 & I5 28 & $57 \cdot 34$ & +68.72 & - IS 43 & 54.5 & \\
\hline 39 & $\mathrm{Su}$ & iil, S. . & . . . . & II & 32 I.OI & +0.06 & -46.28 & $\begin{array}{lll}58 & 6 & 7.30\end{array}$ & 45. I 88 & $+\mathrm{I} 3 \mathrm{I} .5$ & 60 & 0.0 & 1531 & I4.79- & -68.73 & -1916 & $2 \mathrm{I} . \mathrm{O}$ & . \\
\hline 40 & $\kappa O_{1}$ & hiuchi . & . . . & II & $\begin{array}{ll}53 & 32.67\end{array}$ & 0.20 & -46.38 & 29 I8 7.90 & 46.965 & $+\quad 3 \mathrm{r} .7$ & & 0.4 & I6 52 & . . & & .. & & . \\
\hline $4 \mathrm{I}$ & $\prec \mathrm{O}_{\mathrm{H}}$ & hiuchi . & $\cdots$ & II & 3054.29 & +0.22 & -46.35 & 26 I2 8.12 & 47.165 & $+\quad 27.8$ & 60 & 0.8 & 1730 & & & . & . . & . \\
\hline 42 & Ve & nus I, C. & . . & 6 & $\begin{array}{ll}53 & 20.53\end{array}$ & -0.03 & -46.36 & $63 \quad 54 \quad 8.75$ & 47.930 & I I 54.5 & 61 & 1.2 & 1752 & 34.20 & $1-0.46$ & $-25 \quad 5$ & 37.0 & . \\
\hline 43 & $\mathrm{Ve}$ & nus II . & $\cdot \cdot \cdot$ & 5 & $532 \mathrm{I} .3 \mathrm{O}$ & $\div 0.03$ & --46.36 & . $\therefore$. & & & & & 1752 & 34.97 & $-0.3 \mathrm{I}$ & 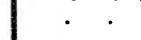 & & \\
\hline 44 & $\eta \mathrm{Se}$ & rpentis. & . $\cdot$ & I I & I6 44. I9 & +0.14 & -46.37 & $41 \quad 46^{\circ} 7.92$ & 44.999 & 50.3 & & I.9 & IS I5 & . . & . . & . & & . \\
\hline 45 & $\begin{array}{ll}\alpha & \mathrm{Ly} \\
\alpha & \mathrm{Ac}\end{array}$ & ræ & $\cdot \cdot$ & I I & 34 II. 85 & $\div 0.37$ & -46.34 & o I0 9.95 & 44.568 & 0.2 & & I. 4 & I8 33 & . & - & . & & 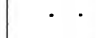 \\
\hline 46 & & uaril . & & I I & I 16.25 & +0.18 & -46.49 & $3940 \quad 8.48$ & 43.828 & $+\quad 47.5$ & & 0.8 & 220 & . & 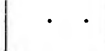 & . & & \\
\hline & me. & Barom. & Ther. & Ex. & r. Telesio & ope micr & $\begin{array}{l}\text { oneter b } \\
V I I, \text { exce }\end{array}$ & $\begin{array}{l}\text { sections are } \\
\text { t as noted be }\end{array}$ & de at & $I I, V I$ & No. & Par: & rallax. & Semi- & i-diam. & $\begin{array}{l}\text { Corr. for } \\
\text { Def. Ill. }\end{array}$ & & Sum. \\
\hline$d$ & & in & $\circ$ & o & & & & & & & & & & & il & 11 & & \\
\hline & & & $3^{8.2}$ & 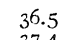 & I. & & icestiot & $\mathrm{C}_{3}, \mathrm{C}_{2}, \mathrm{C}_{\mathrm{I}}$. & & & 6 & + & & & & 0.0 & & 5.2 \\
\hline & $\begin{array}{ll}14 & 3.5 \\
15 & 23\end{array}$ & & & & $7,38$. & & 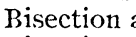 & 11. & & & & & & +16 & 5 II. 3 & . & & I 8.8 \\
\hline & 16 30 & $\begin{array}{l}038 \\
038\end{array}$ & $\begin{array}{r}39.9 \\
41.0\end{array}$ & & $8,24,3$ & 39,42 . & Bisec & t VI, VII & & & $\$$ & + & 7.5 & -16 & II. 3 & & $-I$ & 3.8 \\
\hline & 1720 & & $42 . \mathrm{I}$ & & I4, I 5 . & & Bisection & II, VI, VII. & & & I3 & + & 6.0 & & . & O.I & & \\
\hline & $\begin{array}{ll}18 & 40 \\
21 & 50\end{array}$ & & $\begin{array}{l}43.0 \\
39.0\end{array}$ & $\begin{array}{l}41.3 \\
37.9\end{array}-9$ & 24 . & & D. $\mathrm{t}$ & ad c used. & & & 24 & + & & & & & & \\
\hline & 2240 & & $\begin{array}{l}38.5 \\
38.5\end{array}$ & 36.4 & 35. & & Bisections & at $\mathrm{D}_{3}, \mathrm{D}_{2}, \mathrm{D}_{\mathrm{1}}$. & & & 38 & + & 7.5 & $-I 6$ & 13.2 & & $-I t$ & I6 5.7 \\
\hline & $\begin{array}{rr}23 & 45 \\
0 & 40\end{array}$ & & $\begin{array}{l}37.0 \\
36.0\end{array}$ & & & & & & & & 39 & + & 7.6 & +16 & $\mathrm{I} 3.2$ & & & 20.8 \\
\hline & $\begin{array}{cc}0 & 40 \\
22 & 0\end{array}$ & & & 5 & & & & & & & 42 & + & & . & $\therefore$ & O.I & + & 6.0 \\
\hline & $\begin{array}{ll}22 & 45 \\
23 & 45\end{array}$ & & & & & & & & & & & & & & & & & \\
\hline & $\begin{array}{ll}23 & 45 \\
0 & 30\end{array}$ & $\begin{array}{l}29.972 \\
29.972\end{array}$ & $\begin{array}{l}51.5 \\
5^{0.0}\end{array}$ & & & & & & & & & & & & & & & \\
\hline 16 & $\begin{array}{ll}13 & 35 \\
15 & 32\end{array}$ & $\begin{array}{l}30.059 \\
30.046\end{array}$ & $\begin{array}{l}52 . \\
59 .\end{array}$ & $\begin{array}{l}50 \\
62\end{array}$ & & & & & & & & & & & & & & \\
\hline & $\begin{array}{lll}19 & 32 \\
16 & 58\end{array}$ & & & & & & & & & & & & & & & & & \\
\hline & $\begin{array}{ll}17 & 53 \\
18 & 37\end{array}$ & 043 & $\begin{array}{l}67 . \\
69 .\end{array}$ & & & & & & & & & & & & & & & \\
\hline & & $.04 \mathrm{I}$ & & & & & & & & & & & & & & & & \\
\hline
\end{tabular}




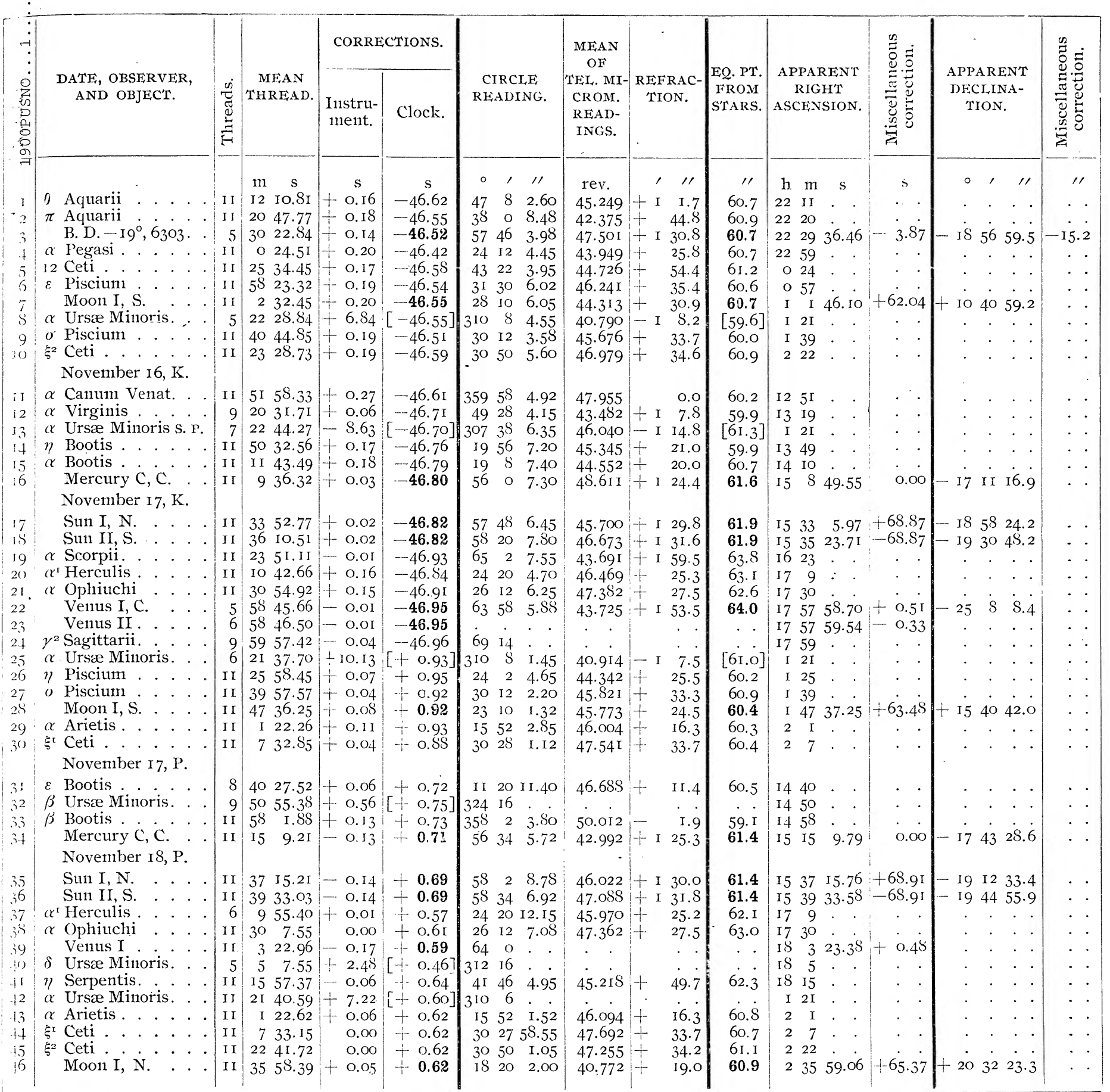

\begin{tabular}{|c|c|c|c|c|c|c|c|c|c|c|}
\hline 'Time. & Barom. & Att. & $\begin{array}{l}\text { Eix. } \\
\text { Ther. }\end{array}$ & Telescope & $\begin{array}{l}\text { nicrometer bisections are made at } I, I I, I T, \\
\text { III, except as noted below. }\end{array}$ & No. & Parallax. & Semi-cliam. & $\begin{array}{l}\text { Corr. for. } \\
\text { Def. Ill. }\end{array}$ & Sum. \\
\hline 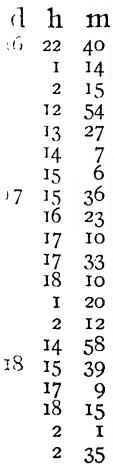 & $\begin{array}{c}\text { in. } \\
30.040 \\
30.035 \\
30.022 \\
30.056 \\
30.056 \\
30.062 \\
30.046 \\
30.034 \\
30.022 \\
30.020 \\
30.018 \\
30.014 \\
30.012 \\
30.000 \\
29.996 \\
29.974 \\
29.924 \\
29.924 \\
29.862 \\
29.864\end{array}$ & $\begin{array}{l}\circ \\
60.1 \\
56.9 \\
55.9 \\
53.0 \\
56.5 \\
59.6 \\
63.0 \\
65.0 \\
68.5 \\
72.3 \\
72.8 \\
73.8 \\
6.5 \\
60.0 \\
65.5 \\
67.8 \\
72.5 \\
74.5 \\
58.5 \\
58.0\end{array}$ & $\begin{array}{c}\circ \\
59.2 \\
5.8 \\
54.3 \\
51.6 \\
54.2 \\
57.2 \\
6.6 \\
65.6 \\
72.3 \\
73.3 \\
73.9 \\
74.4 \\
60.4 \\
59.3 \\
6.3 \\
68.3 \\
71.3 \\
72.9 \\
57.5 \\
57.1\end{array}$ & $\begin{array}{l}7,46 . \\
8 . \\
\text { I2, } 31 \text {. } \\
\text { I3. } \\
\text { I } 7,35 . \\
\text { I8, } 36,37 . \\
25 . \\
28 .\end{array}$ & $\begin{array}{l}\text { Bisections at III, IV, V. } \\
\text { Bisections at } \mathrm{C}_{\mathrm{T}}, \mathrm{C}_{2}, \mathrm{C}_{3} . \\
\text { Bisections at VI, VII. } \\
\text { Bisections at } \mathrm{D}_{3}, \mathrm{D}_{1}, \mathrm{C}_{5} . \\
\text { Bisections at I, II. } \\
\text { Bisections at VI, VII. } \\
\text { Bisections at } \mathrm{B}_{\mathrm{I}}, \mathrm{B}_{3}, \mathrm{C}_{\mathrm{r}}, \mathrm{C}_{3} \text {. } \\
\text { Bisections at II, III, IV, V, VI. }\end{array}$ & $\begin{array}{r}7 \\
\text { I6 } \\
\text { I } 7 \\
\text { I8 } \\
22 \\
28 \\
34 \\
35 \\
36 \\
46\end{array}$ & \begin{tabular}{rr} 
& \multicolumn{1}{r}{11} \\
+25 & 21.7 \\
+ & 5.2 \\
+ & 7.6 \\
+ & 7.6 \\
+ & 6.1 \\
+21 & 7.8 \\
+ & 5.2 \\
+ & 7.6 \\
+ & 7.6 \\
+ & \\
+ &
\end{tabular} & $\begin{array}{rr}\prime & \prime \prime \\
+\mathrm{I} 4 & 44.8 \\
- & . \\
-\mathrm{I} 6 & \mathrm{I} 2.0 \\
+\mathrm{I} 6 & \mathrm{I} 2.0 \\
. & . \\
+\mathrm{I} 4 & 45.2 \\
- & . \\
-\mathrm{I} 6 & \mathrm{II} .2 \\
+\mathrm{I} 6 & \mathrm{II} .3 \\
-\mathrm{I} 4 & 47.3\end{array}$ & $\begin{array}{c}\prime \\
\dot{0.0} \\
\dot{0} \\
\dot{0.1} \\
\dot{0.0} \\
\dot{.} \\
\dot{ }\end{array}$ & \begin{tabular}{rr}
$\prime$ & \multicolumn{1}{l}{} \\
+40 & 6.5 \\
+ & 5.2 \\
+16 & 4.4 \\
+16 & 19.6 \\
+ & 6.0 \\
+35 & 53.0 \\
+ & 5.2 \\
-16 & 3.6 \\
+16 & 18.9 \\
+2 & 6.0
\end{tabular} \\
\hline
\end{tabular}




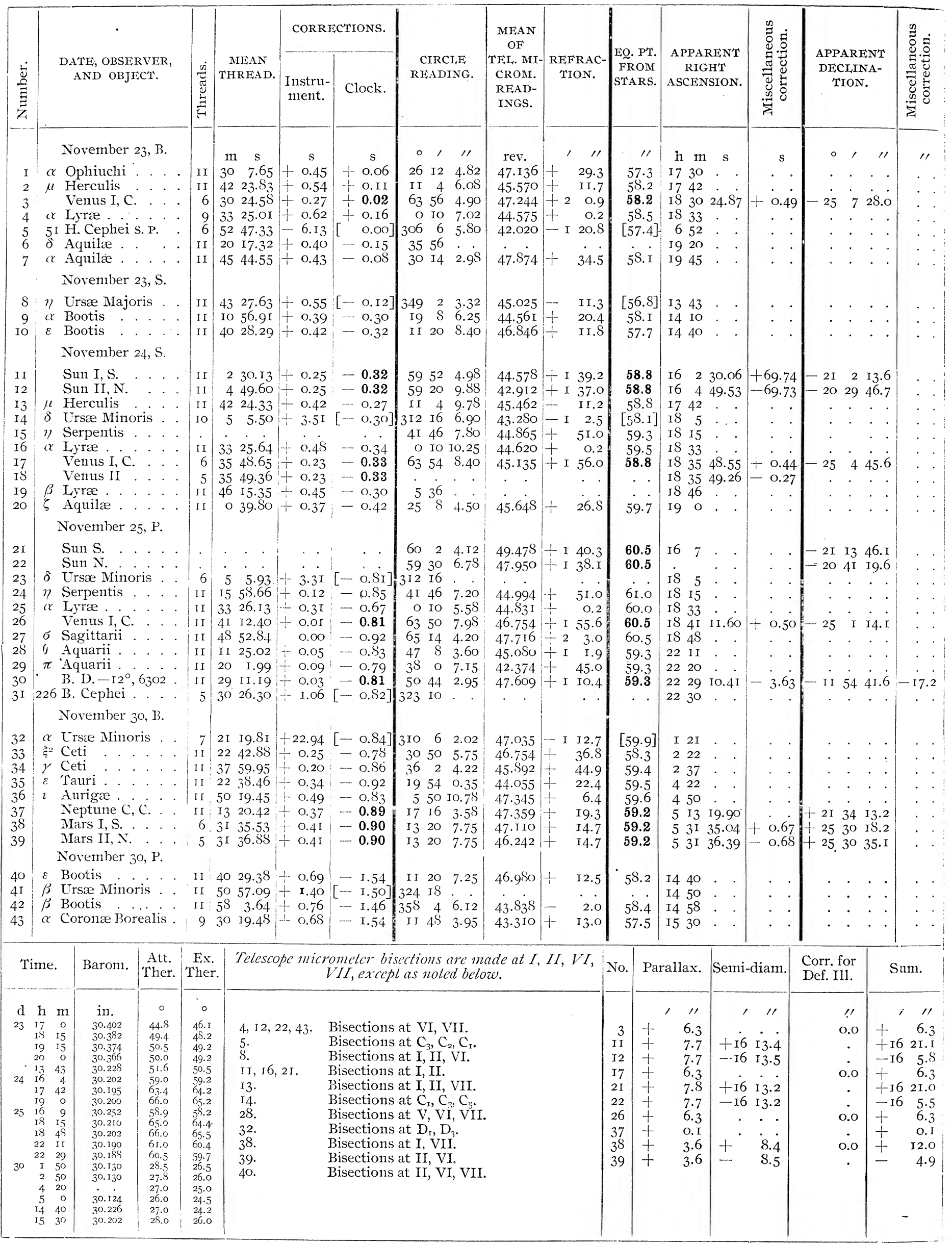




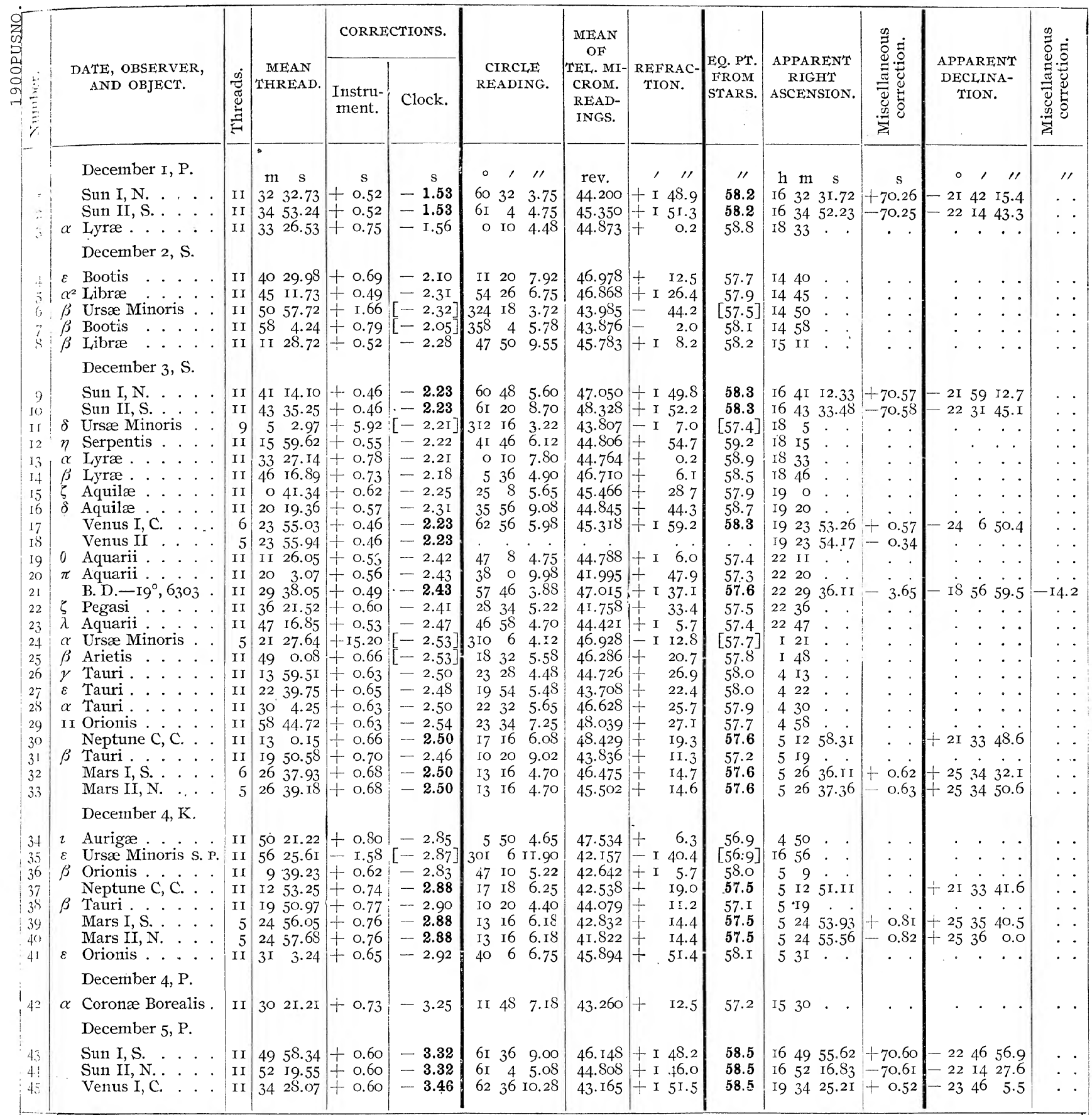

\begin{tabular}{|c|c|c|c|c|c|c|c|c|c|c|}
\hline Time. & Barom. & \begin{tabular}{|l|} 
Att. \\
Ther.
\end{tabular} & $\begin{array}{l}\text { Ex. } \\
\text { Ther. }\end{array}$ & $\begin{array}{c}\text { Telescope micromeler bisections are made at } I, I I, V I, \\
V I I, \text { except as noted below. }\end{array}$ & No. & Parallax. & Semi-diam. & $\begin{array}{l}\text { Cor } \\
\text { Def }\end{array}$ & $\begin{array}{l}\text { for } \\
\text { I11. }\end{array}$ & Sum. \\
\hline 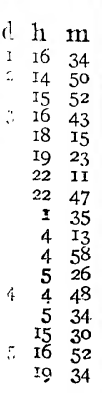 & $\begin{array}{c}\text { in. } \\
\text { 30.I8I } \\
30.050 \\
30.048 \\
30.042 \\
30.028 \\
30.031 \\
30.060 \\
30.068 \\
30.090 \\
30.097 \\
30.097 \\
30.094 \\
29.854 \\
29.838 \\
29.930 \\
29.916 \\
29.898\end{array}$ & $\begin{array}{c}\circ \\
28.4 \\
26.3 \\
26.6 \\
29.6 \\
29.5 \\
31.4 \\
28.3 \\
28.9 \\
26.8 \\
23.0 \\
23.6 \\
23.4 \\
23.0 \\
28.7 \\
27.5 \\
39.0 \\
45.6 \\
52.0\end{array}$ & $\begin{array}{c}\circ \\
26.5 \\
23.4 \\
24.6 \\
25.8 \\
27.8 \\
28.6 \\
27.5 \\
27.5 \\
25.5 \\
23.5 \\
23.9 \\
23.6 \\
26.7 \\
26.7 \\
36.5 \\
36.8 \\
5.2 \\
5.5\end{array}$ & $\begin{array}{ll}\text { I, 9, I6, 43. } & \text { Bisections at I, II. } \\
2,5, \text { Io, 44. } & \text { Bisections at VI, VII. } \\
3,8,42 . & \text { Bisections at II, VI, VII. } \\
6,35 . & \text { Bisections at III, IV, V. } \\
\text { II, 24. } & \text { Bisections at C. C, C. C. } \\
32,40 . & \text { Bisections at II, VI. } \\
33,39 . & \text { Bisections at I, VII. } \\
34 . & \text { Bisections at I, II, VII. }\end{array}$ & $\left\{\begin{array}{r}\text { I } \\
2 \\
9 \\
\text { IO } \\
17 \\
30 \\
32 \\
33 \\
37 \\
39 \\
40 \\
43 \\
44 \\
45\end{array}\right.$ & 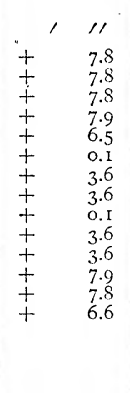 & 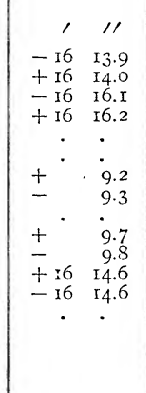 & + & $\begin{array}{c}0.0 \\
: \\
0.2\end{array}$ & $\begin{array}{rr} & 1 \\
-16 & 6.1 \\
-16 & 21.5 \\
+16 & 21.5 \\
-16 & 8.3 \\
+16 & 24.1 \\
+ & 6.6 \\
+ & 0.1 \\
+ & 12.8 \\
\pm & 5.7 \\
+ & 0.1 \\
+ & 13.3 \\
+ & 6.2 \\
+16 & 22.5 \\
+16 & 6.8 \\
+ & 6.8\end{array}$ \\
\hline
\end{tabular}




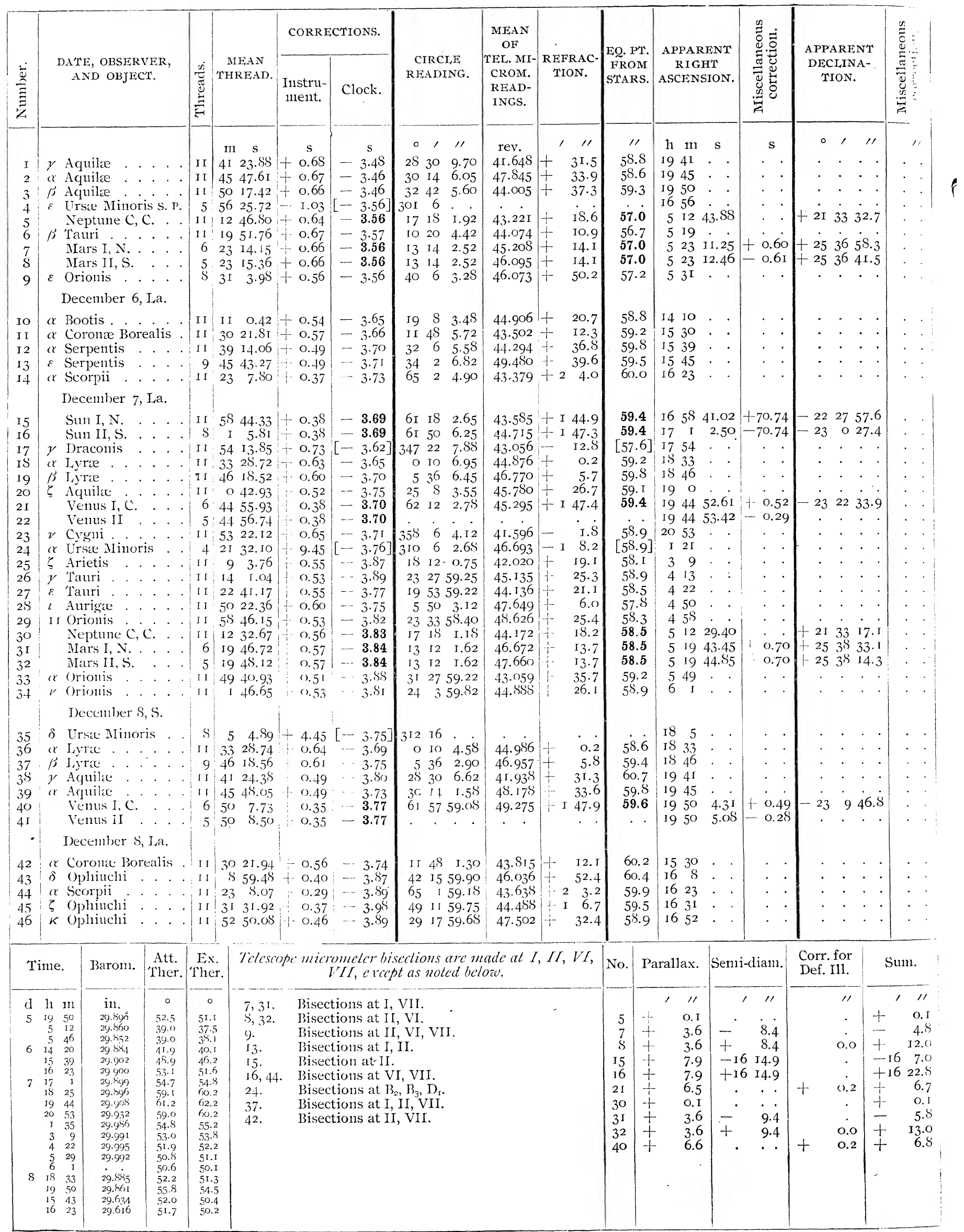




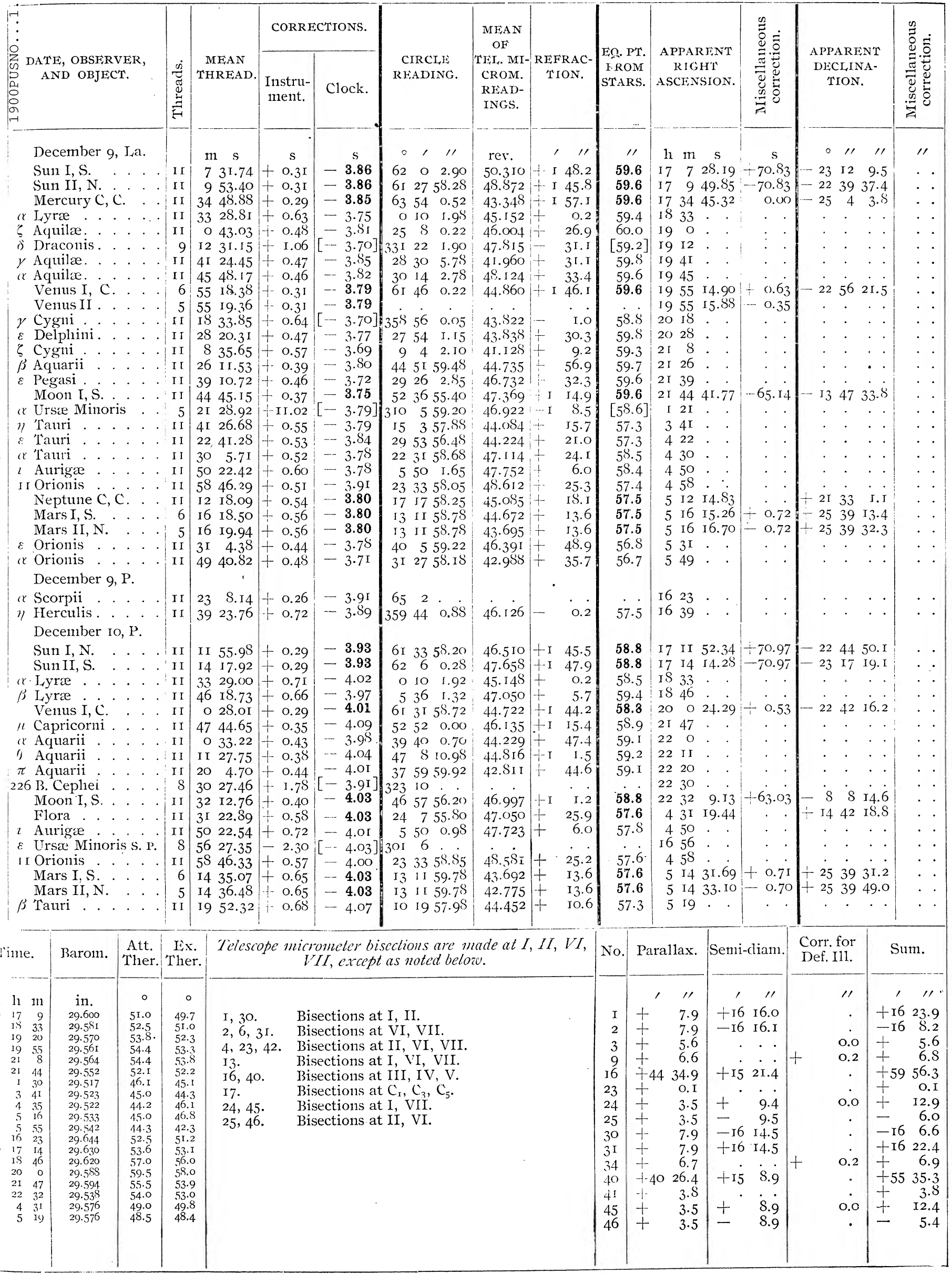




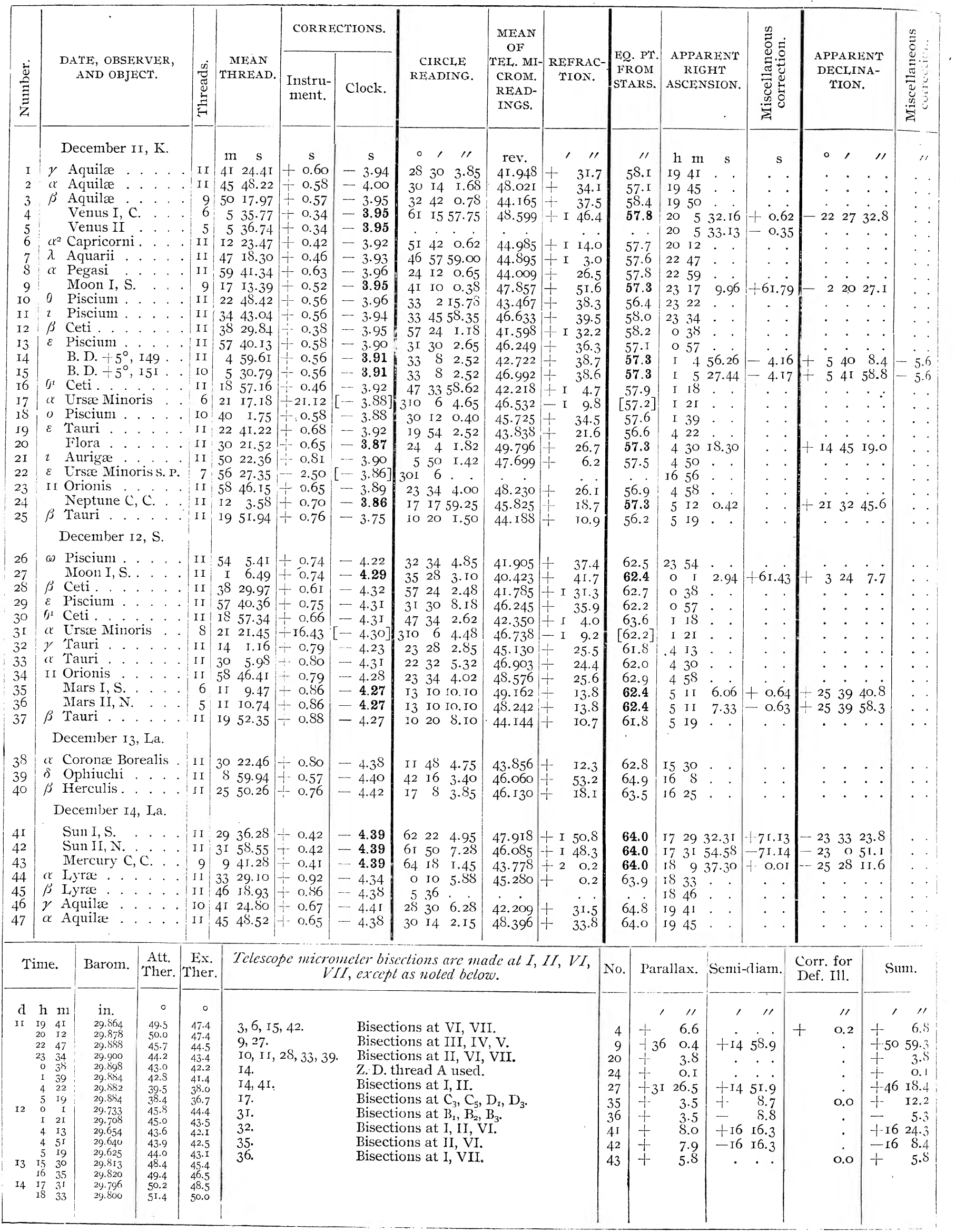




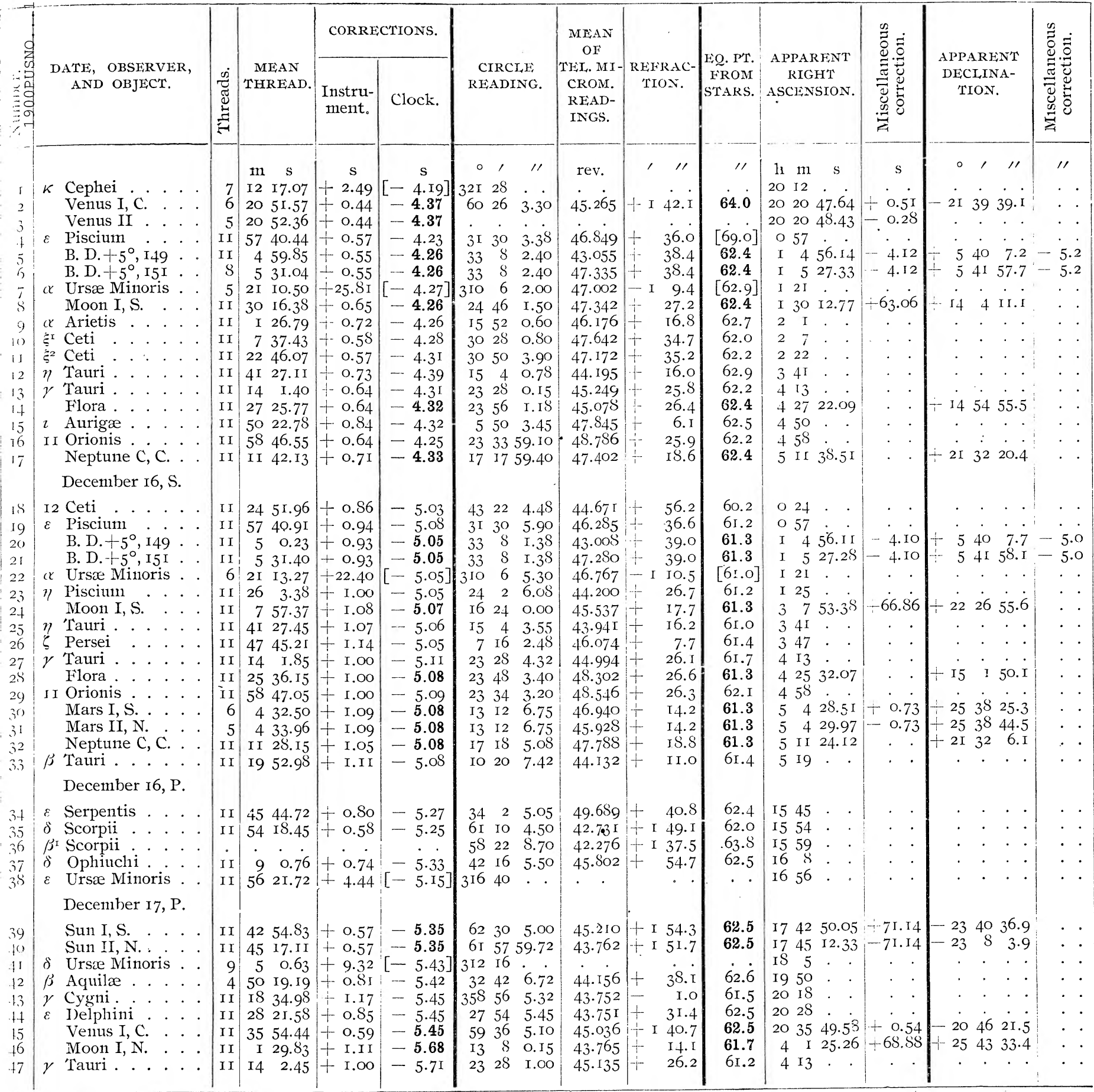

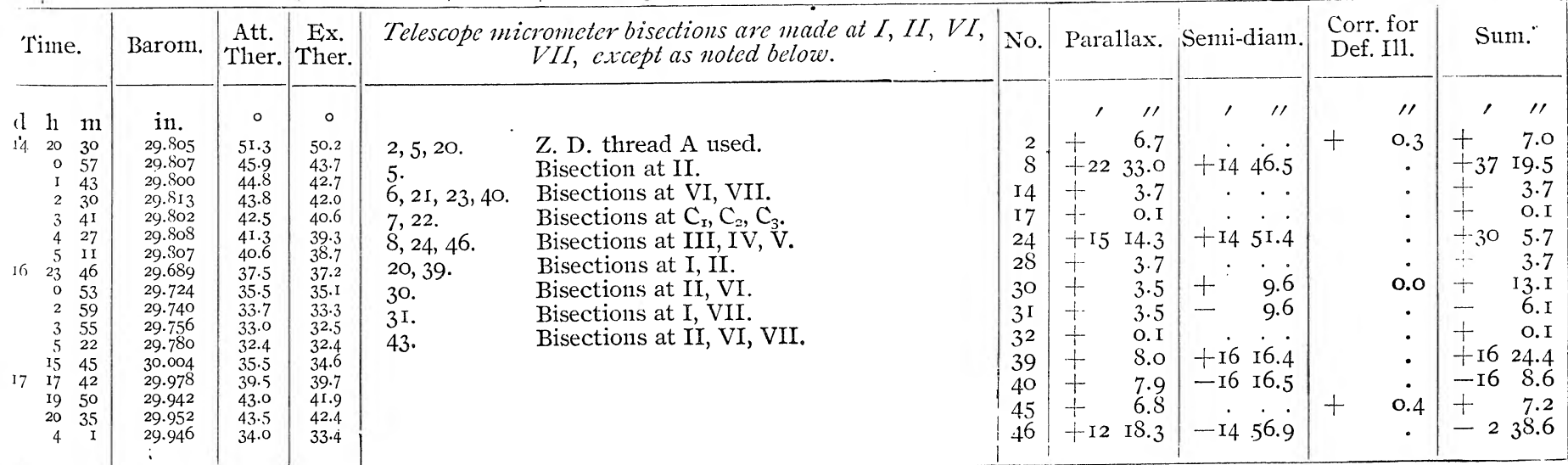




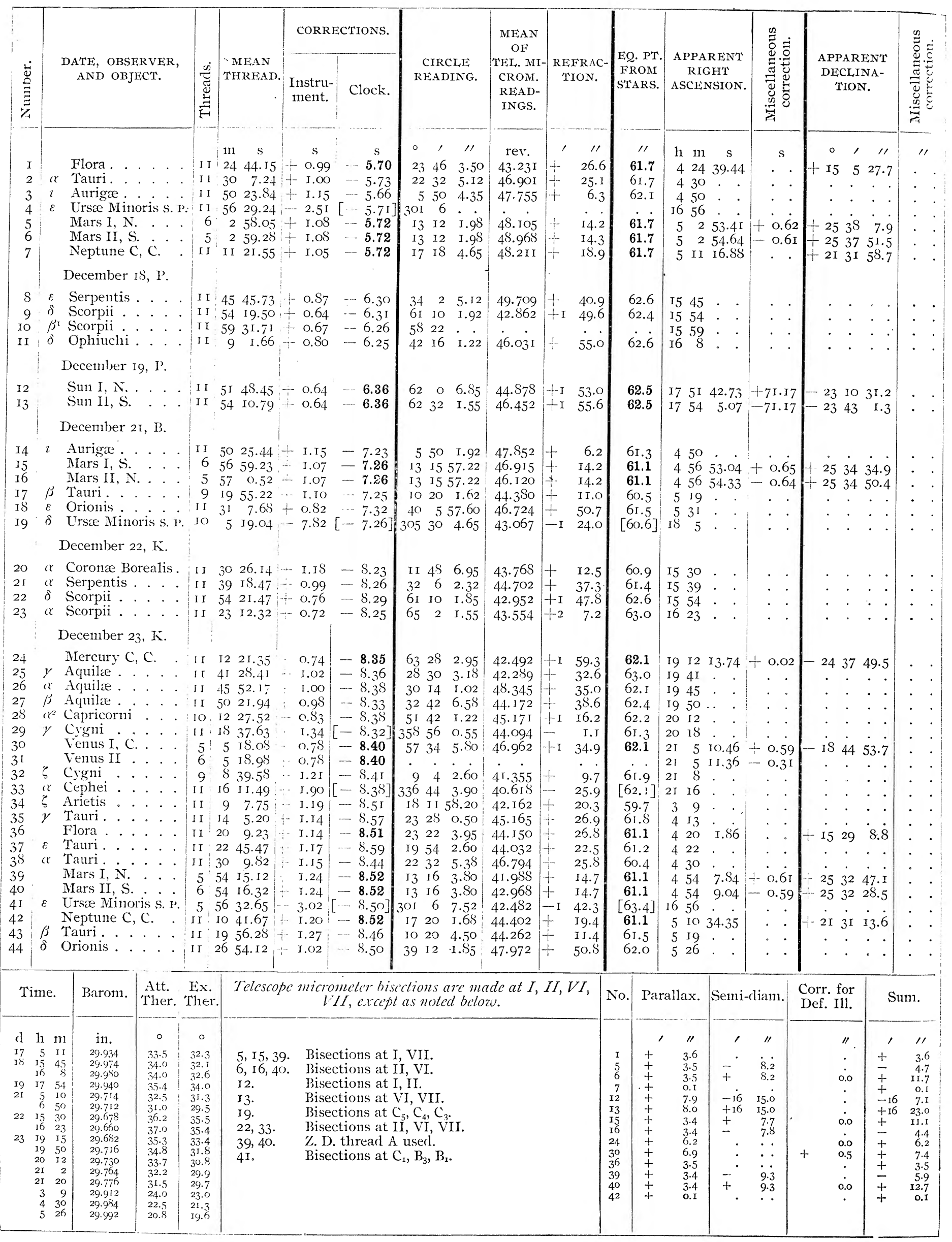




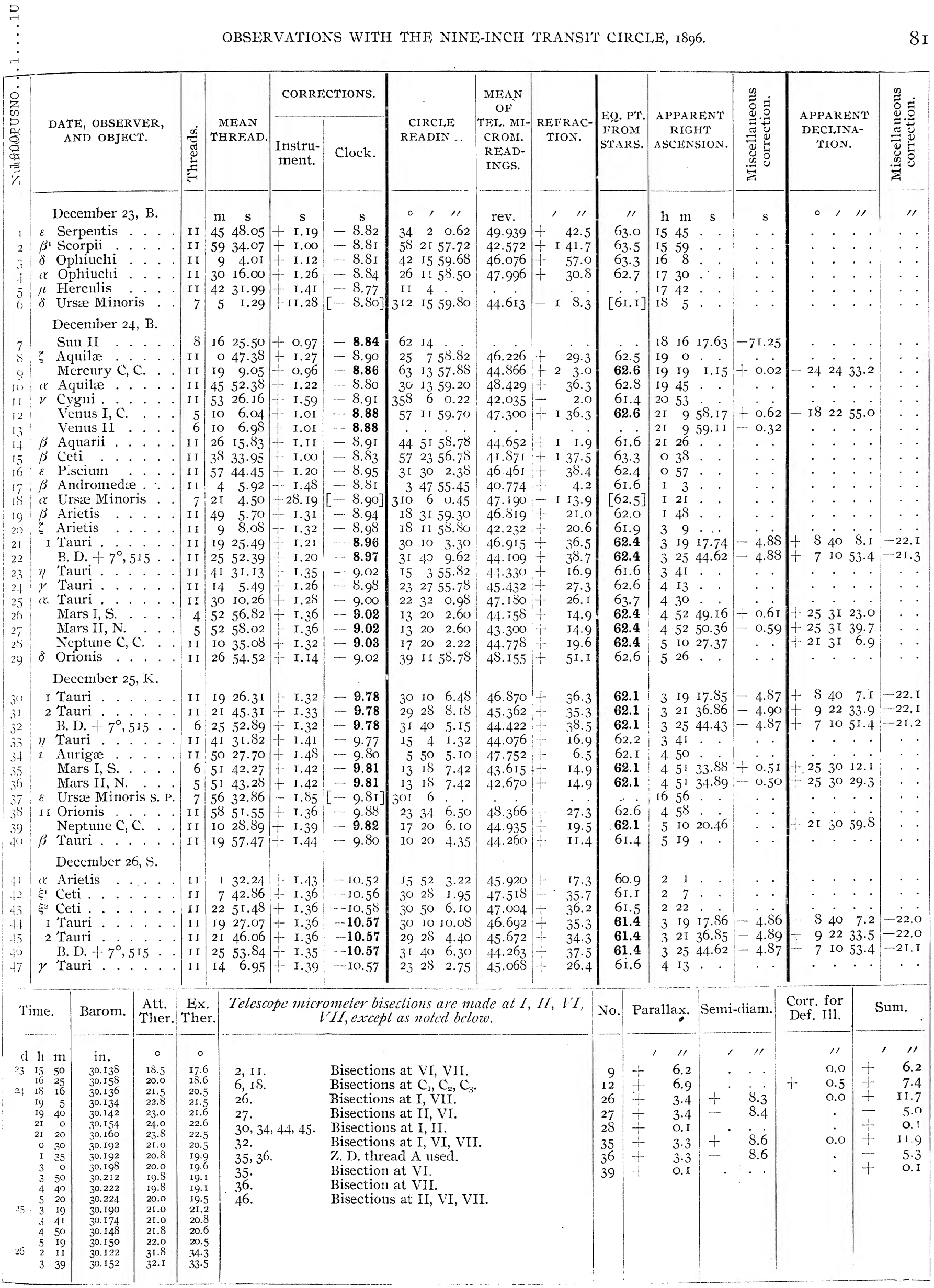
$68.55-6$ 


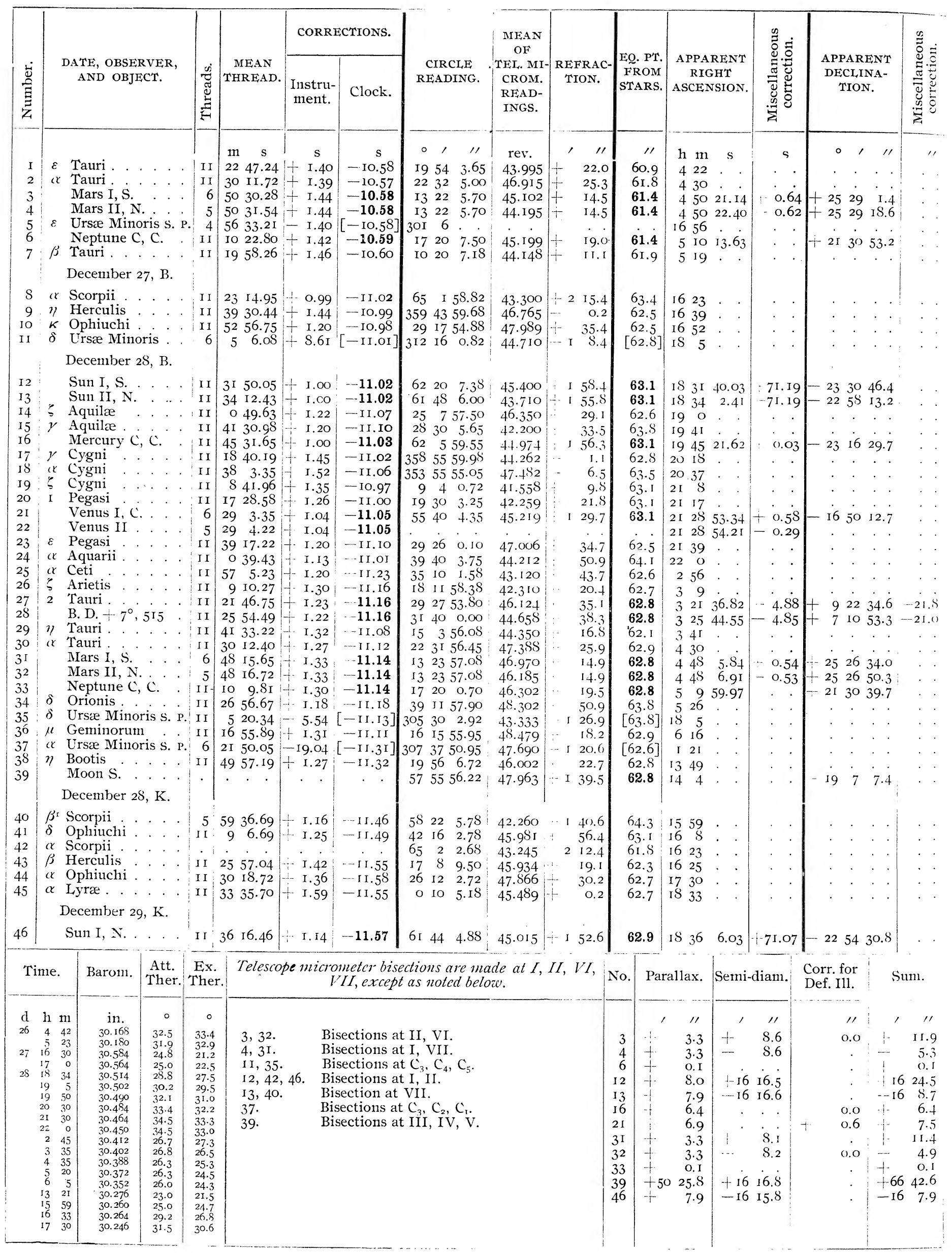




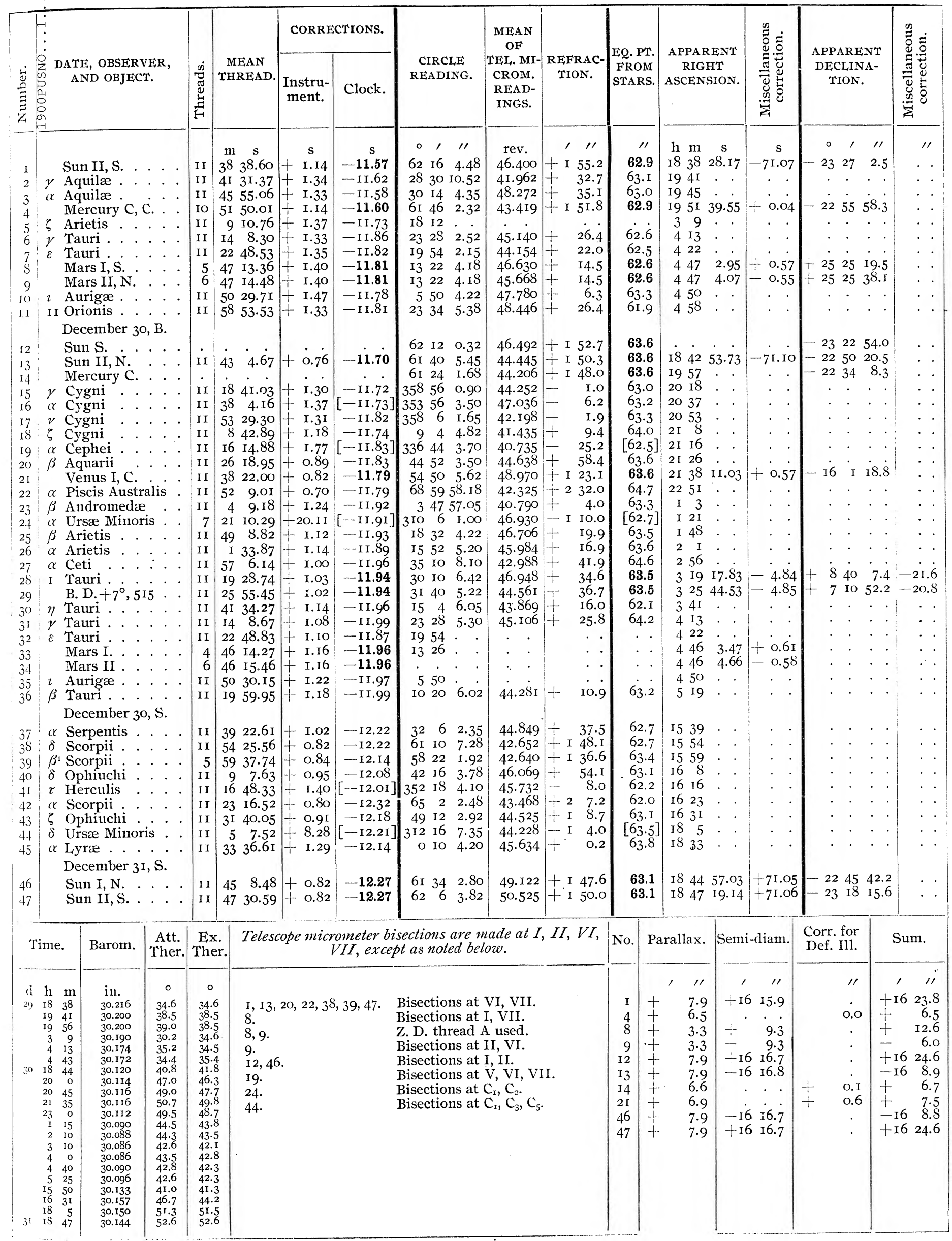




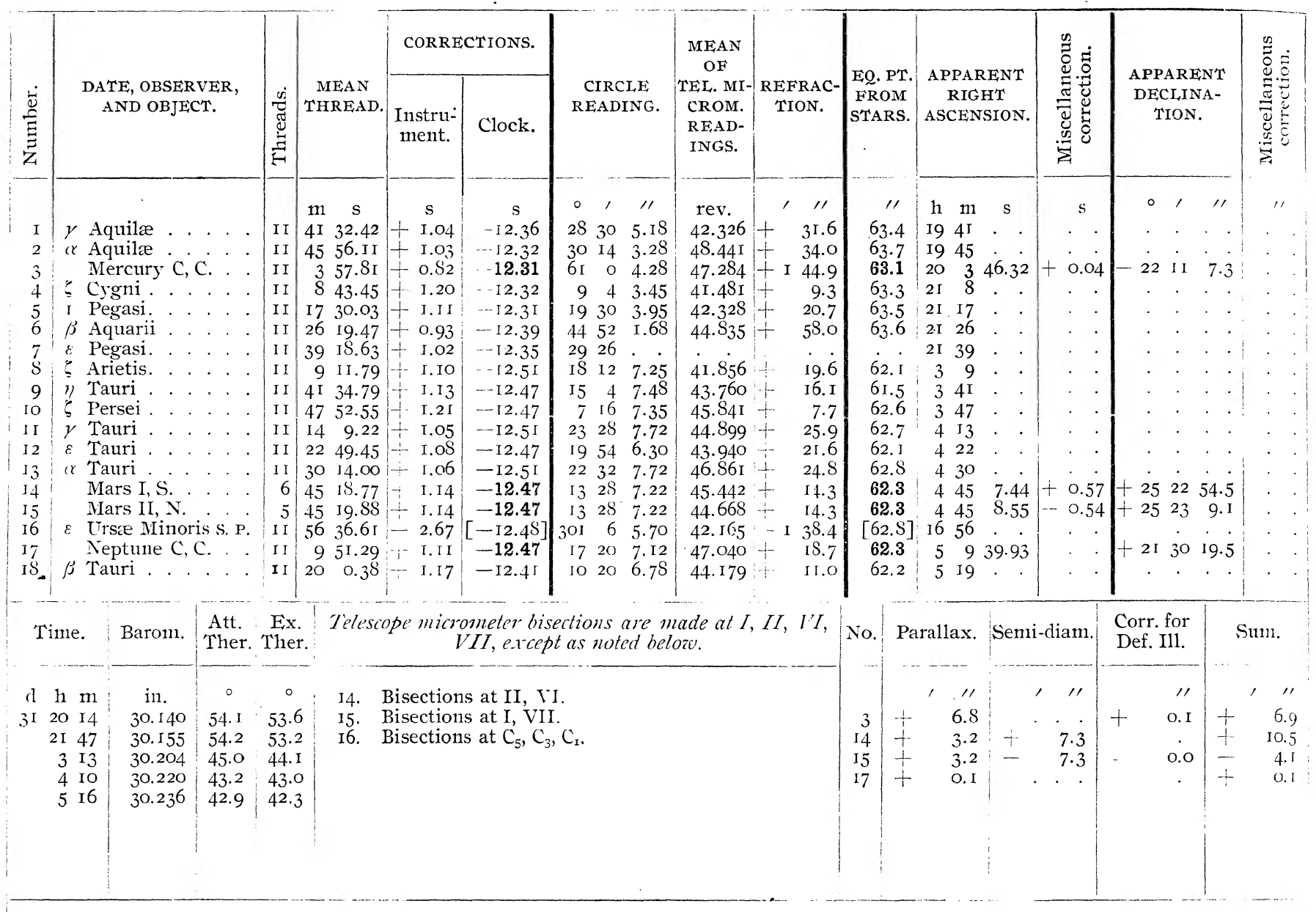




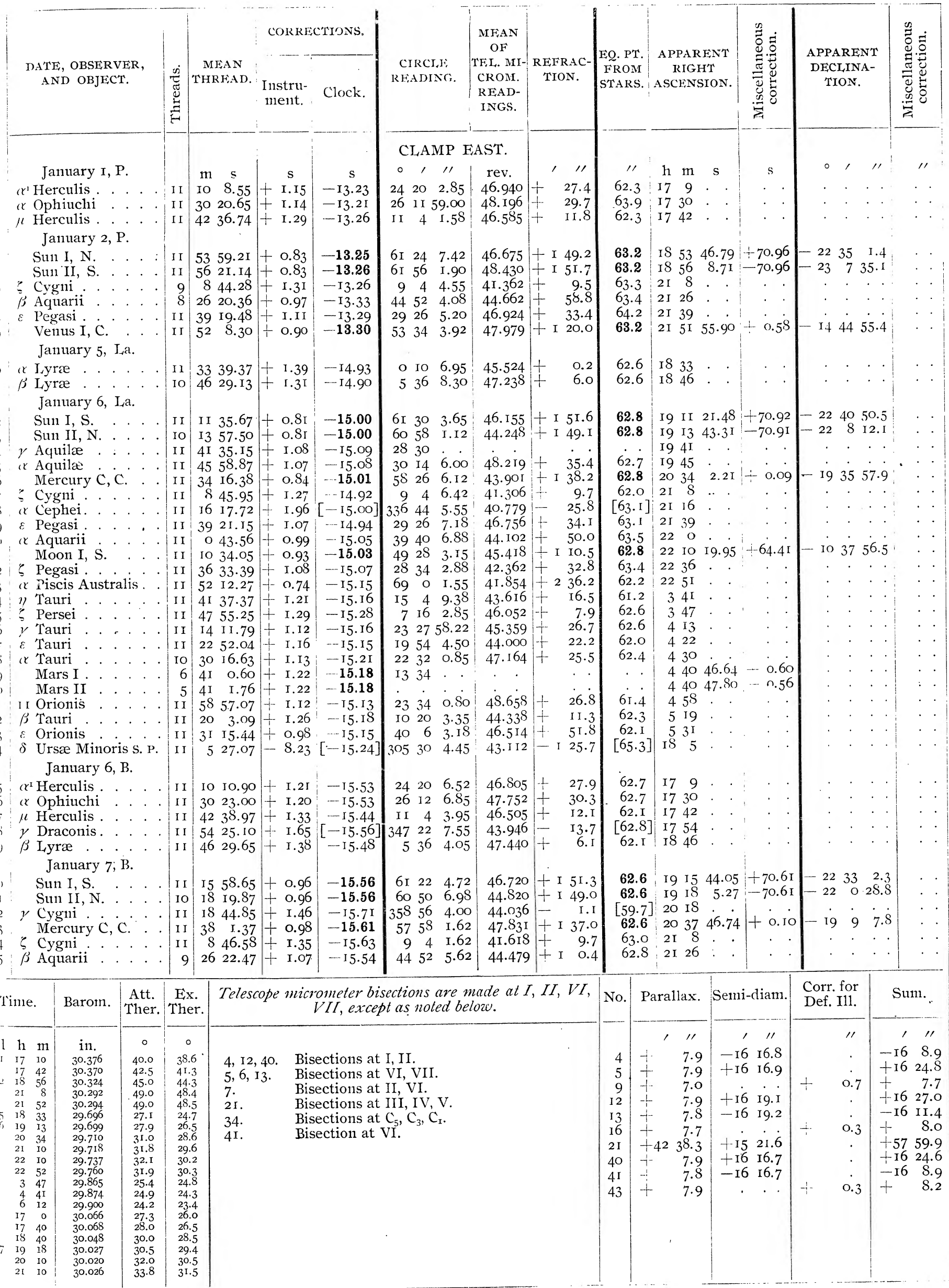




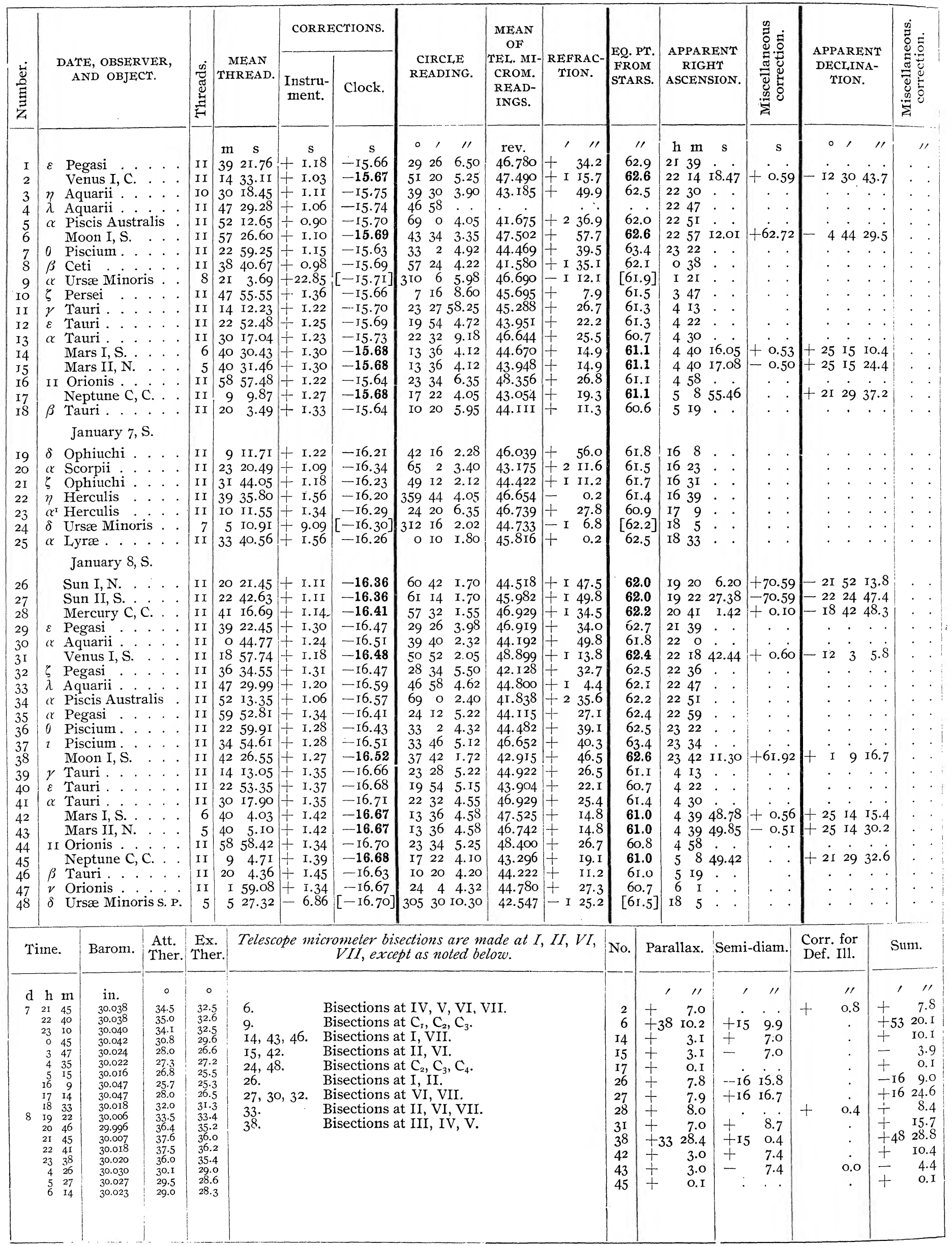




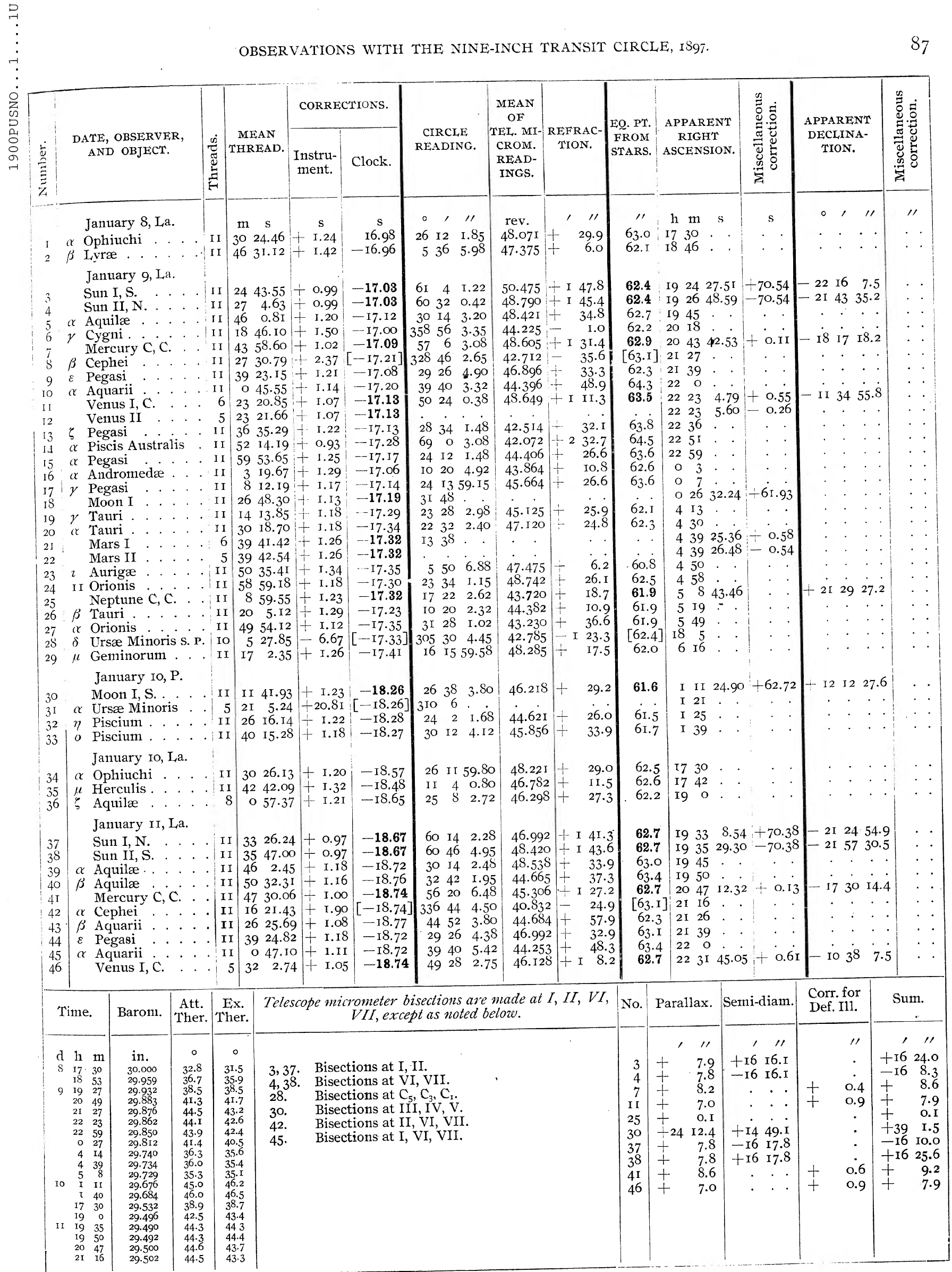




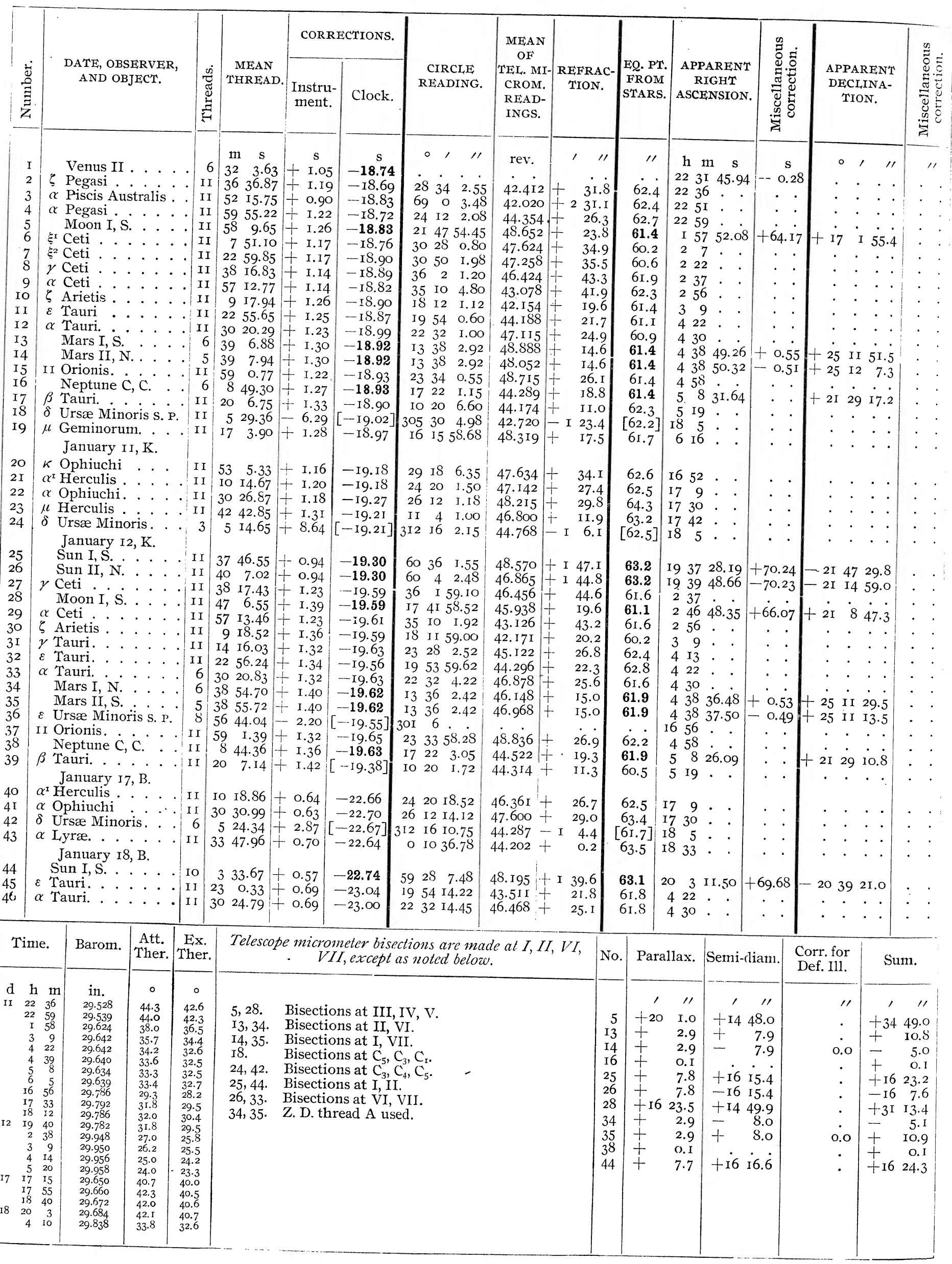




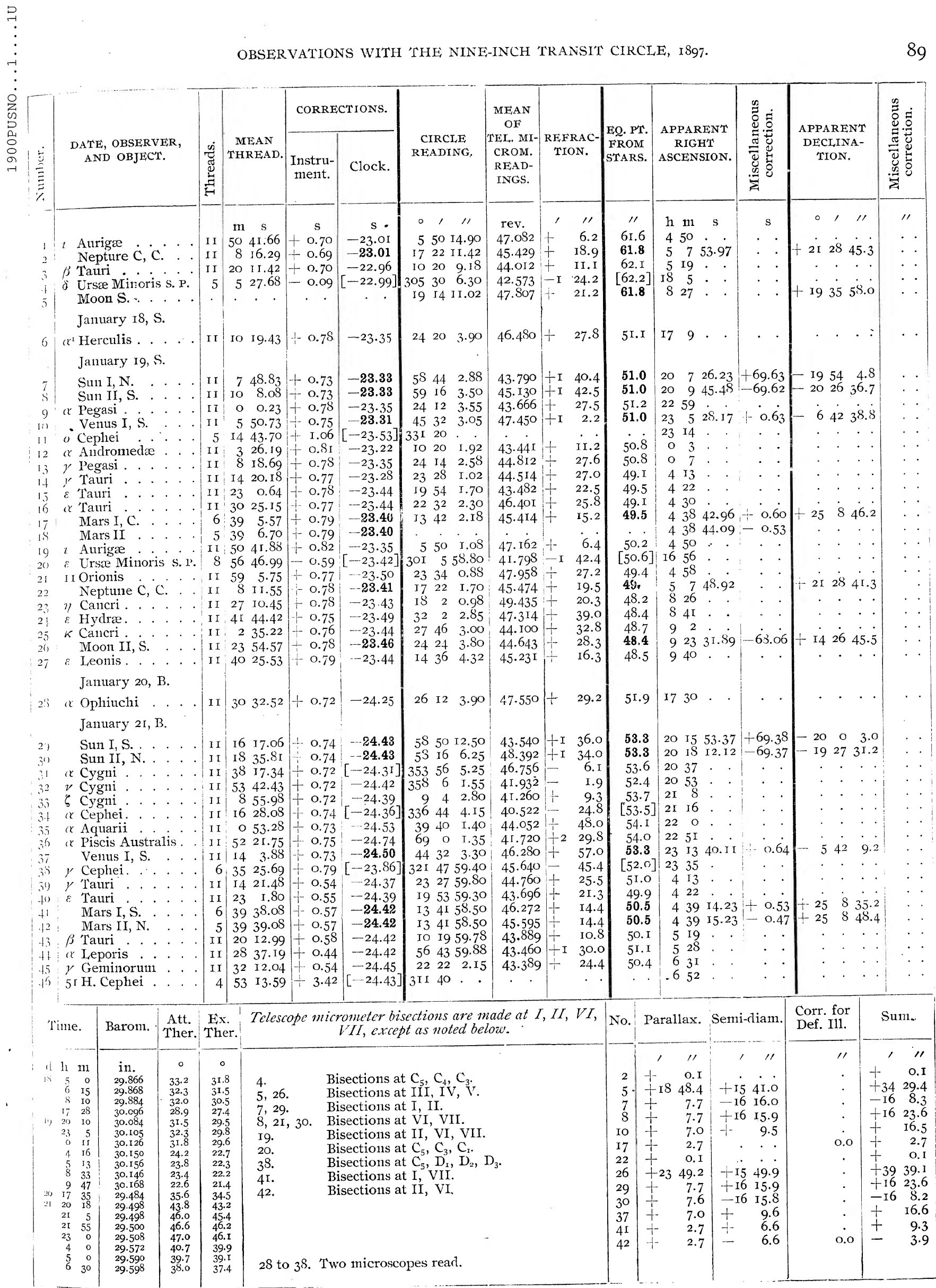




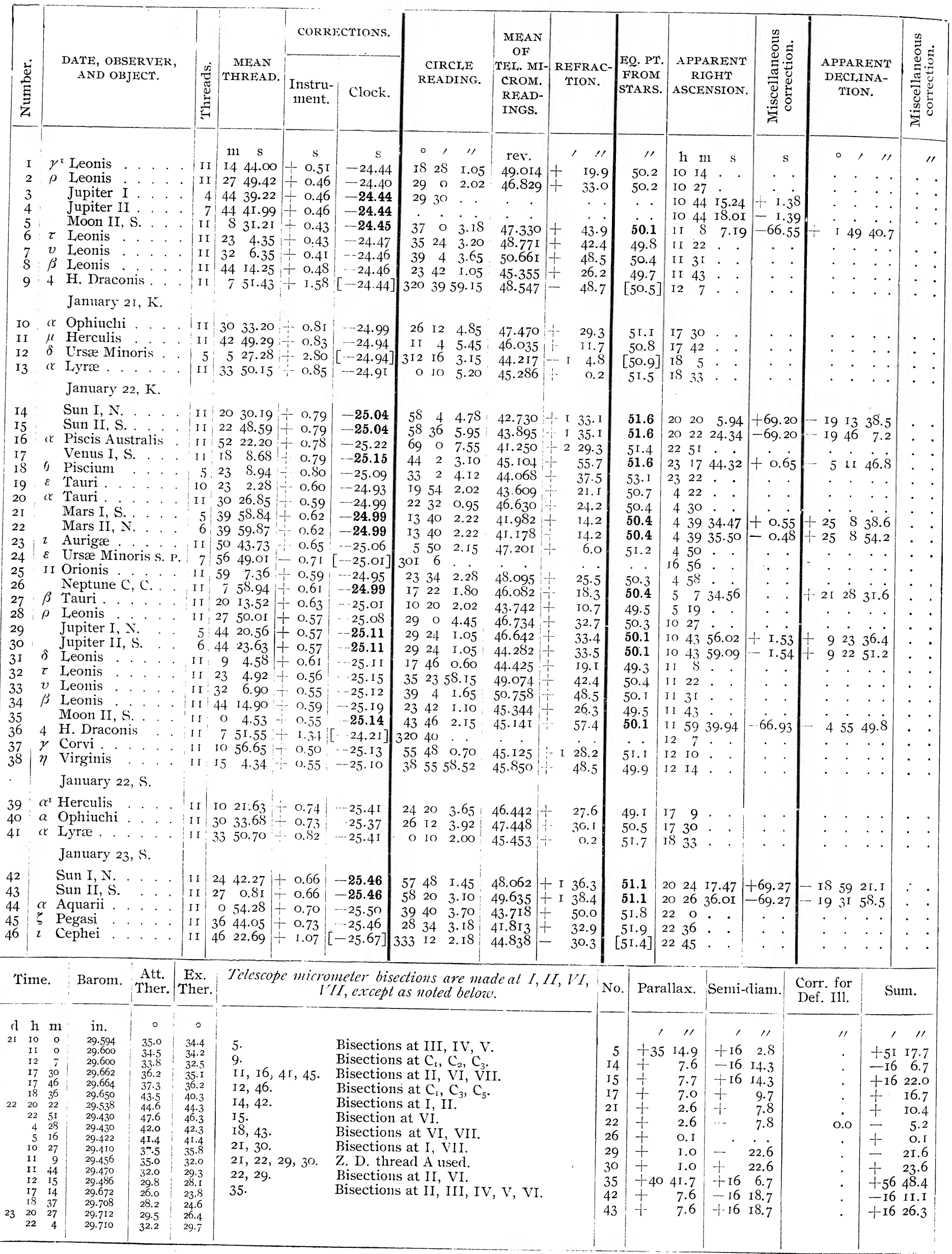




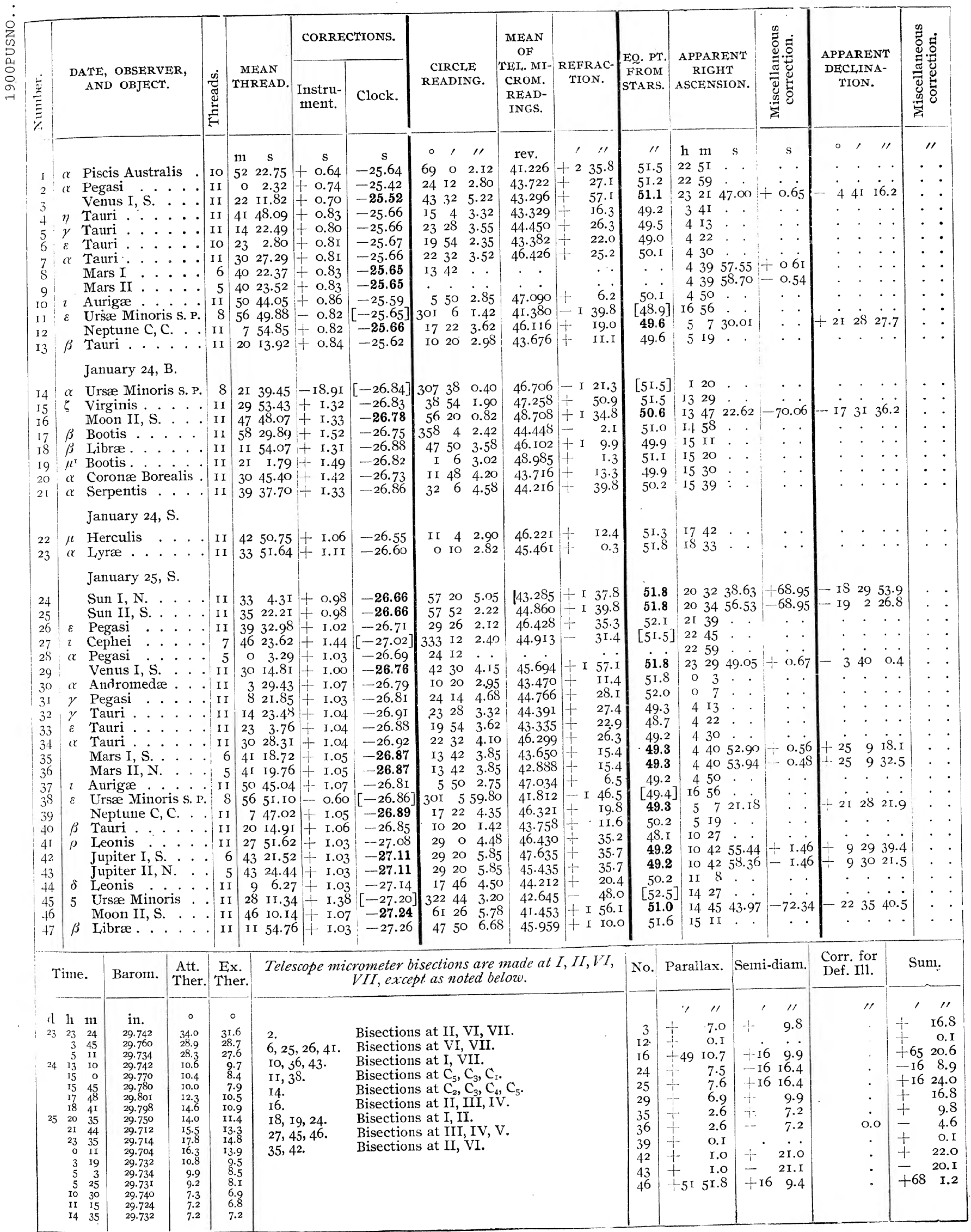




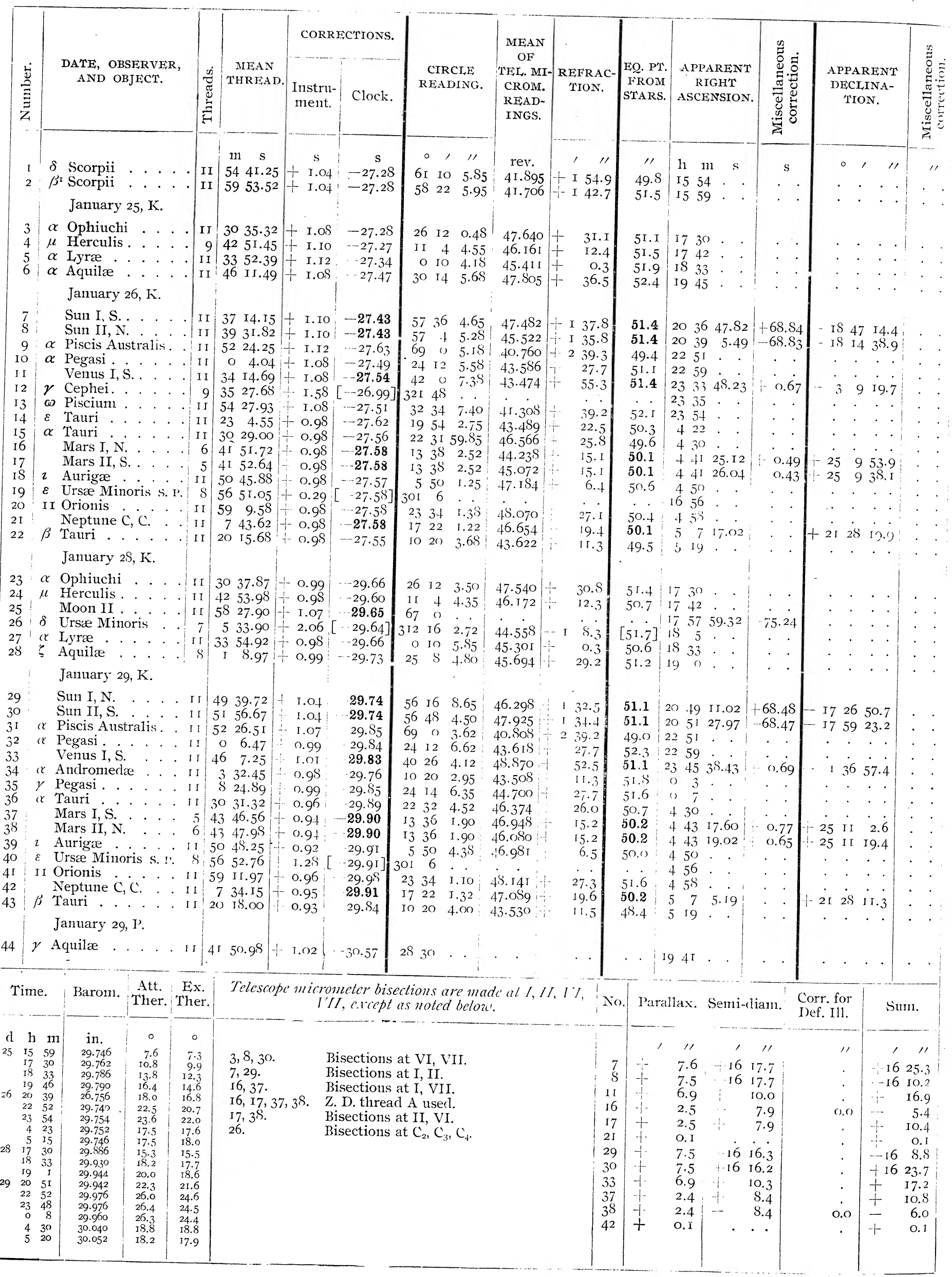




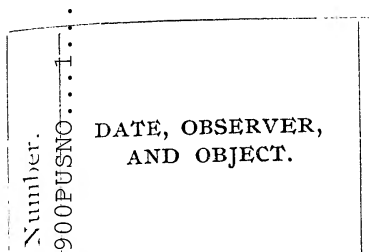

January $30, P$.

Sun I, S.

Sun II, N.

$\alpha$ Piscis Australis

is Pegasi

6) Piscium

$\imath$ Piscium

Venus I, S.

$\rho$ Leonis

Jupiter I, N.

Jupiter II, s.

l. Leonis

$\delta$ Leonis

$13, \tau$ Leonis

February $3 \mathrm{P}$.

1.t $\rho$ Leonis

15 Jupiter I, S.

Jupiter II, N.

$l$ Leonis

is $\delta$ Leonis

$19 \tau$ Leonis

February 3,13

21) $\mu$ Herculis

$21 \delta$ Urse Minoris

22 ( $)$ Lyræe.

23 3 Lyræ.

$21 \div \zeta$ Aquilæ

Mercury II, C.
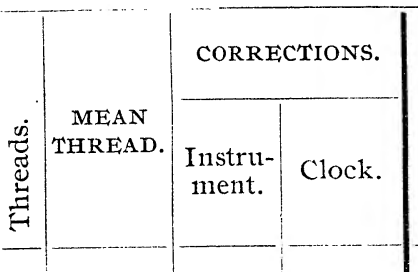

CIRCLE
READING.

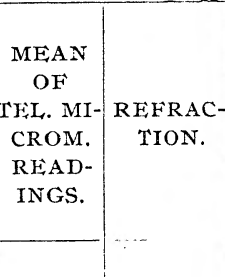

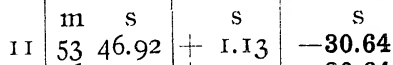

II $56 \quad 3.5 \mathrm{I}+$ I.I 3 - 30.64

II $5227.32+$ I.2O -30.79

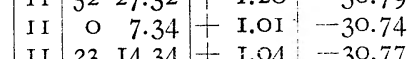

I I 23 14.34 + $1.04 \quad-30.77$

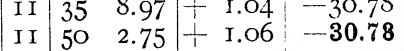

\begin{tabular}{r|rr|r|r|} 
& II & 27 & $55.69+$ I.O2 & -31.04 \\
\hline & 4 & 33.6
\end{tabular}

\begin{tabular}{l|ll|l|l|}
8 & $4 \mathrm{I}$ & $33.6 \mathrm{I}$ & $+\mathrm{I} . \mathrm{O} 2$ & $\mathbf{- 3 1 . 0 3}$ \\
8 & $4 \mathrm{I}$ & $36.57+\mathrm{I} . \mathrm{O} 2$ & $\mathbf{- 3 1 . 0 3}$
\end{tabular}

IO $4422.96+$ I.OI -3 I.OI

II $910.33+0.98-3 \mathrm{I} .02$

\begin{tabular}{l|ll|} 
II & 23 & 10.55
\end{tabular}

\begin{tabular}{rrr|}
56 & 32 & 3.28 \\
56 & 0 & 4.50 \\
6 & 0 & 2.62
\end{tabular}

rev.

$\begin{array}{llll}69 & 0 & 2.62\end{array}$

\begin{tabular}{rrr|}
24 & 12 & 7.12 \\
33 & 2 & 6.38
\end{tabular}

$\begin{array}{llll}33 & 46 & 7.98\end{array}$

$\begin{array}{lll}39 & 56 & 3.92 \\ 2 & 4.98\end{array}$

$\begin{array}{lll}29 & 0 & 3.25 \\ 29 & 8 & 5.70\end{array}$

$\begin{array}{lll}29 & 8 & 5.70\end{array}$

$\begin{array}{lll}27 & 44 & 7.18\end{array}$

1746 I 8.68

$\begin{array}{llll}35 & 26 & 2.65\end{array}$

$46.378+$ I 33.8

$44.775+$ I 31.9

$40.89 \mathrm{I}+239.7$

$43.020+\quad 40 . \mathrm{I}$
$45.914+\quad 4 \mathrm{I} .2$

$46.206+$

$46.748+$

$44.550+35.2$

$46.788+$

$49.065+$

$43.514-$

$42.569+$

\section{2}

33.2
20.3

20.3
45.0

II \begin{tabular}{lll|l|l|} 
& 27 & 58.43 & 0.73 & -33.42 \\
\hline
\end{tabular}

\begin{tabular}{|l|ll|l|l|lll|l|l}
8 & 39 & 58.14 & 0.72 & -33.43 & 28 & 58 & $2.5^{2}$ & 46.814 & -
\end{tabular}

\begin{tabular}{l|lr|r|r|rrr|r|r|}
7 & 40 & I.06 & 0.72 & -33.43 & 28 & 58 & 2.80 & 42.960 \\
\hline & 44 & 25.77 & 0.73 & -33.46 & 27 & 44 & 2.55 & 49.404
\end{tabular}

\begin{tabular}{l|ll|l|l|lll|l|l|} 
I I & 44 & 25.77 & 0.73 & -33.46 & 27 & 44 & 2.55 & 49.404 \\
\hline
\end{tabular}

\begin{tabular}{|l|rr|r|r|rrr|r|r|}
\hline II & 9 & 13.03 & 0.74 & -33.39 & I & 46 & 2.40 & 44.359 \\
\hline
\end{tabular}

\begin{tabular}{rrr:r|r|r}
11 & 23 & 13.34 & 0.72 & -33.45 \\
\hline
\end{tabular}

\begin{tabular}{lll|l|l|}
35 & 26 & 4.12 & 42.554
\end{tabular}

$$
\text { II } 42 \quad 58.09
$$

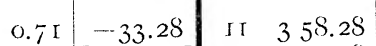

I. $45[-33.26]$

Aquilæ

February 4,13 .

27
25
29
3
3
3
33
3
35
36
3
3
3
4
4
4
4

Sun I, S.

Sun II, N.

Moon I, S.

$\alpha$ Andromedæe

Venus I, S.

$\varepsilon$ Piscium

$\beta$ Andromedie

or Ceti

$\gamma$ Tauri .

$\varepsilon$ Tauri .

Tauri .

Mars I, C.

Mars II

Neptune C, C.

$\beta$ Tauri.

$\mid \begin{array}{ll}\alpha & \text { Leporis } \\ \alpha & \text { Orionis }\end{array}$

$\delta$ Ursæ Minoris S. P.

59 Draconis s. P. .

a Leonis.

$\gamma^{\mathrm{i}}$ Leonis \begin{tabular}{l|ll}
8 & 5 & 39.35
\end{tabular}

$0.72 \mid-33.23$

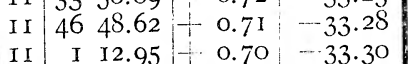

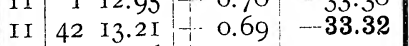

\section{46 I 7.96}

\begin{abstract}
\begin{tabular}{l|l}
.70 & -33.42 \\
\hline
\end{tabular}
\end{abstract}
I 2 I 559.08

o 958.68

$\begin{array}{llll}5 & 35 & 58.72 \\ 25 & 8 & 4\end{array}$

\begin{tabular}{lll|l|l|l|}
57 & 46 & 0.48 & 47.849 & I & 35.6 \\
30 & 14 & 1.95 & 48.088 & t & 35.2
\end{tabular}

\begin{tabular}{l|ll|ll|l|}
. & II & I4 & 8.29 & -0.69 & -33.34 \\
\hline
\end{tabular}

\begin{tabular}{l|ll|l|l|} 
II & I6 & 23.93 & 0.69 & -33.34 \\
II & 22 & $4 \mathrm{I} . \mathrm{IO}$ & $0.7 \mathrm{O}$ & -33.37
\end{tabular}

\begin{tabular}{r|rr|rrr|r} 
II & 22 & 4 I. IO & 0.70 & -33.37 \\
II & 3 & 36.30 & -1 & 0.71 & -33.40
\end{tabular}

II 920.56 - $0.70-33.38$

\begin{tabular}{r|rr|rrr} 
II & 58 & 8.95 & 0.70 & -33.40 \\
IO & 4 & 30.59 & 0.7 I & -33.34 \\
\hline & 9 & 25.86 & -0.69 & 33.44
\end{tabular}

II I9 $25.86+0.69-33.44$

II I4 $30.25+0.73-33.48$

\begin{tabular}{l|ll|l|l|} 
II & 23 & 10.58 & +0.74 & -33.5 \\
& &
\end{tabular}

II $3035.08+0.74 \quad-33.5$ I

$64847.30+0.74$

$54848.18+0.74:-33.50$

$97 \quad 7 \quad 17.35+0.74-\mathbf{3 3 . 5 0}$

II $2021.72+0.75--33.44$

\begin{tabular}{l|lll|ll|l} 
II & 28 & 45.87 & - & 0.71 & -33.5 I \\
II & 50 & 10.63 & + & 0.73 & -33.56
\end{tabular}

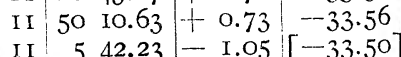

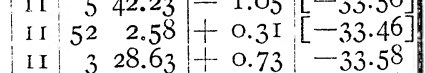

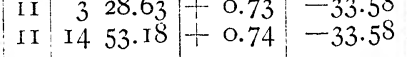

$\begin{array}{lll}55 & 6 & 2.02\end{array}$

\begin{tabular}{lll|l|ll}
54 & 34 & 2.28 & $4 \mathrm{I} .07 \mathrm{O}$ & $\mathrm{I}$ & 25.9 \\
\hline
\end{tabular}

$\begin{array}{lll:l}40 & 16 & 2.05 & 45.517\end{array}$

IO 20 I.7O $43.57 \mathrm{O}$

\begin{tabular}{lll|l|l}
37 & 22 & 3.22 & 46.804
\end{tabular}

\begin{tabular}{lll|l}
31 & 30 & 2.35 & 46.122
\end{tabular}

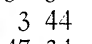

$\begin{array}{lll}23 & 28 & 1.45\end{array}$

\begin{tabular}{lll|l}
22 & 32 & 8.82 & 46.115 \\
13 & 36 & 1.95 & 43.360
\end{tabular}

\begin{tabular}{lll|l} 
I7 & 22 & 0.62 & 47.5 II
\end{tabular}

10 $1959.55 \quad 43.819$

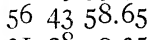

$\begin{array}{lll}3 \mathrm{I} & 28 & 0.35 \\ 305 & 30 & 0.62\end{array}$

$\begin{array}{rrr}305 & 30 & 0.62 \\ 292 & 5 & 58.95\end{array}$

262159.05 $\begin{array}{rrr}47 & 34 & 2.00\end{array}$

$43.350 \quad$ II. 2
IS 2757.05
$42.674+37.4$

$42.025-125.3$

$47.394-230.0$

$47.014+30.6$
$49.168+20.7$

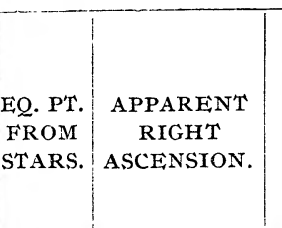

旔

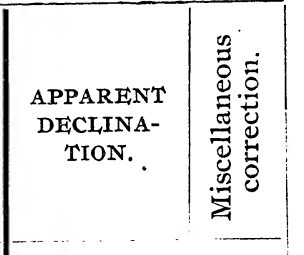

h $\mathrm{m} \mathrm{s}$

50.8

\begin{tabular}{l|llll}
$\mathbf{5 0 . 8}$ & 20 & 55 & 34.00
\end{tabular}

$\begin{array}{l:lll}50.2 & 22 & 5 \mathrm{I}\end{array}$

$50.7: 22 \quad 59$

\begin{tabular}{l:ll}
5 I. I & 2322 \\
\hline I. & 23 & 34
\end{tabular}

\begin{tabular}{l|lll}
$5 \mathrm{I} .3$ & 23 & 34
\end{tabular}.

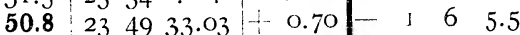

\begin{tabular}{l|l|l}
50.7 & IO 27
\end{tabular}

50.8 Io 4 I 3.60 I. 18 + 94240.7

50.8 Io 4 I $6.56-1.48$ - 9 4I 57.9

$\begin{array}{l:l:}50.4 & \text { IO } 43 \\ 50.6 & \text { If }\end{array}$

50.6 I I 8

51.6 if 22

49.4 : $10 \quad 27$

49.5 Io 3925.43 - $1.46 \div 95232.5$

49.5 Io $3928.35-1.46-953$ 1 9.6

49. I IO 43

49.4 II 8

$\begin{array}{l:ll}50.2 & \text { If } 22\end{array}$

5I.I I I 42

[49.7] is 5

5I.I 1833

\begin{tabular}{l|ll}
5 SI.I & I & 0 \\
5 & 0
\end{tabular}

$\begin{array}{llllllllll}51.3 & 19 & 4 \mathrm{I} & 40.58 & -0.3 \mathrm{r} & - & \text { is } & 57 & 16.9\end{array}$ $\begin{array}{l:ll}5 \text { I.6 } & 1945\end{array}$

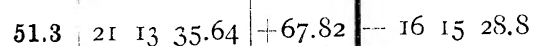

\begin{tabular}{l|l|lll|l|lll}
51.3 & $2 \mathrm{I}$ & $\mathrm{I}$ & 5 & $5 \mathrm{I} .28$ & -67.82 & $-\mathrm{I} 5$ & 42 & 59.2
\end{tabular}

\begin{tabular}{l|llll|ll}
51.3 & 23 & 22 & $8.43+62.37-$ & I & 25 & 49 . I
\end{tabular}

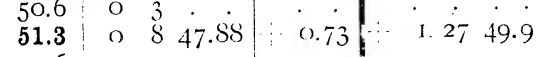

\begin{tabular}{ll|ll}
51.6 & 0 & $5 i$
\end{tabular}

$\begin{array}{llll}5 & 0.0 & 1 & 3 \\ 5 & 1\end{array}$

\begin{tabular}{l|c:ccc}
5.7 & 52.0 & 1 & 15 \\
.50 .1 & 4 & 13
\end{tabular}

22. I 49.8 + 422

\begin{tabular}{l|l|ll}
25.3 & 49.1 & 4 & 30
\end{tabular}

\begin{tabular}{l|l|lllllllll} 
& I4. & 49.6 & 4 & 48 & 14.54 & 0.48 & 25 & 15 & 26.4
\end{tabular}

\begin{tabular}{lllll|ll}
- & - & 4 & 48 & 15.42 & -0.40
\end{tabular}

\begin{tabular}{l|lll}
499.6 & 5 & 6 & 44.59
\end{tabular}

\begin{tabular}{l|ll}
49.4 & 5 & 19
\end{tabular}

\begin{tabular}{l|ll}
49.9 & 5 & 28 \\
49.6 & 5 & 49
\end{tabular}

$[49.7]$ I $\left[\begin{array}{rr}5 & 4 \\ {[5} & \end{array}\right.$

$[5 \mathrm{I} .5]$ 2I $5 \mathrm{I}$

\begin{tabular}{l|ll}
50.2 & IO 2 \\
49.4 & IO & 14
\end{tabular}

49.4 IO 14

\begin{tabular}{|c|c|c|c|c|c|}
\hline Time. & Bảrom. & $\begin{array}{l}\text { Att. } \\
\text { Ther. }\end{array}$ & $\begin{array}{l}\text { Ex. } \\
\text { Ther. }\end{array}$ & Tele & $\begin{array}{l}\text { iicrometer bisections are m } \\
\quad V I I, \text { except as noted beld }\end{array}$ \\
\hline & & ○ & $\circ$ & & \\
\hline $30 \quad 20$ & & 25.0 & 24.5 & & Bisections at I, II. \\
\hline & & 28 & 27 & $2, \mathrm{II}, 28$. & Bisections a \\
\hline 23 & & 29 & 27.8 & $9,15$. & at I, VII. \\
\hline $\begin{array}{ll}10 & 27 \\
11 & 23\end{array}$ & & 18 & $\begin{array}{l}17.6 \\
17.6\end{array}$ & I0, 16. & Bisections at II, VI. \\
\hline $\begin{array}{lll}3 & 10 & 28 \\
3\end{array}$ & & & 30.1 & & Bisections at $C_{1}, C_{2}, C$ \\
\hline II 23 & & & $\begin{array}{l}28.9 \\
28.7\end{array}$ & 29. & Bisections at III, IV, V. \\
\hline is & & 32.0 & $3 \mathrm{r} .5$ & 42 & Bisections at V, VI, VII. \\
\hline $\begin{array}{ll}19 & 30 \\
20 & 30\end{array}$ & $\begin{array}{l}29.934 \\
29.936\end{array}$ & $\begin{array}{l}34.5 \\
36.2\end{array}$ & $\begin{array}{l}32.5 \\
34.4\end{array}$ & 44 & Bisections at $\mathrm{C}_{2}, \mathrm{C}_{3}, \mathrm{C}_{4}, \mathrm{C}_{5}$. \\
\hline
\end{tabular}

de at $I, I I, I T$,

\begin{tabular}{|c|c|c|c|c|}
\hline No. & Parallax. & Seni-diann. & $\begin{array}{l}\text { Corr. for } \\
\text { Def. Ill. }\end{array}$ & Sum. \\
\hline & 111 & 111 & 11 & 111 \\
\hline I & 7.5 & +1613.9 & . & +16 21.4 \\
\hline 2. & $7 \cdot 4$ & $-16 \quad 13.8$ & . & $\begin{array}{ll}-16 & 6.4\end{array}$ \\
\hline 7 & 6.9 & IO. 4 & . & I 7.3 \\
\hline 9 & 1.0 & $2 \mathrm{I} .4$ & . & 20.4 \\
\hline 10 & I.O & $2 \mathrm{I} .4$ & . & 22.4 \\
\hline I5 & I.O & 21.0 & . & 22.0 \\
\hline 16 & 1.0 & ... 2 I.I & & 20. I \\
\hline 25 & 9.5 & & 0.1 & + \\
\hline 27 & $7 \cdot 3$ & $1.16 \quad 14.8$ & $\cdot$ & +1622.1 \\
\hline 28 & $7 \cdot 3$ & $-16 \quad 14.8$ & . & $-16 \quad 7.5$ \\
\hline 29 & +3539.8 & $15 \quad 7.0$ & . & 5046.8 \\
\hline $3 \mathrm{I}$ & 6.8 & $\because \quad 10.9$ & & I 7.7 \\
\hline 38 & 2.3 & 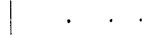 & 0.0 & 2.3 \\
\hline 40 & + & 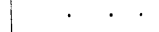 & . & + \\
\hline
\end{tabular}




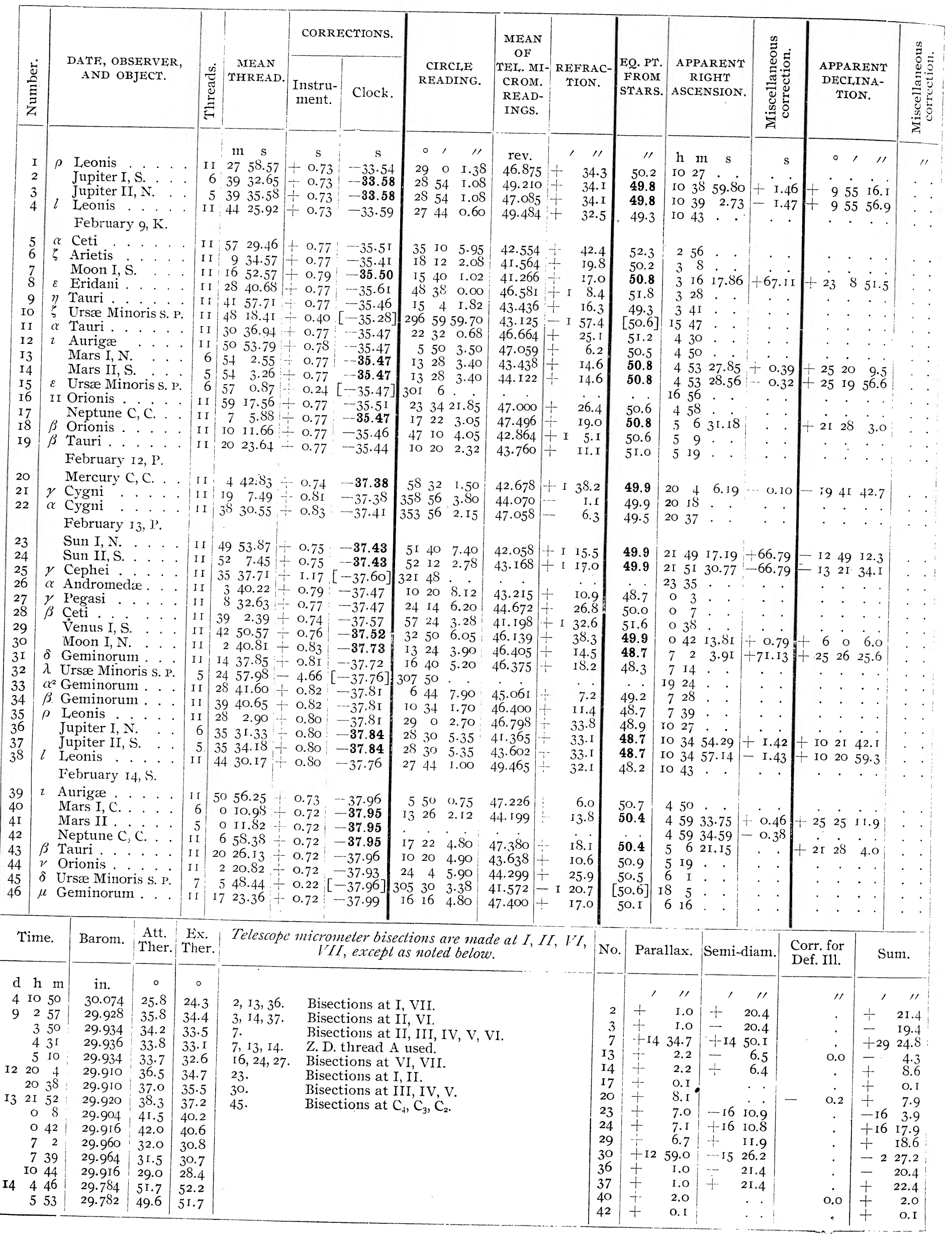




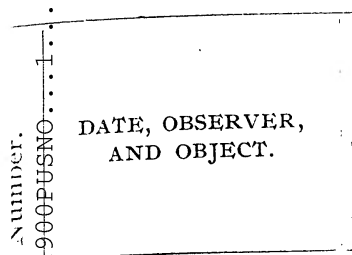

$\beta$ Geminorum . . II $3940.97+0.72-38.04$ $\varphi$ Geminorum I I $475 \mathrm{I} .78$ - 0.72 - 38.00 Moon I, N. . . . II $\quad$ o $33.79+0.74-\mathbf{3 8 . 0 1}$ Cancri.... . II 2725.28 - $0.72-38.02$ Ir Leonis .... II $333.24: 0.72 \quad-38.06$ $\gamma^{\mathrm{i}}$ Leonis . . . . . II I4 57.74 + $0.72-37.99$ $\rho$ Leonis . . . . . I I 28 3.25 $-0.72 \quad-38.07$ Jupiter I, s. . . $6 \quad 35 \quad 2.82 \div 0.72:-38.06$ Jupiter II, N. . $5355.88+0.72-\mathbf{3 8 . 0 6}$

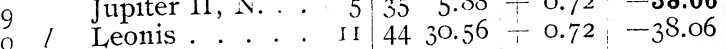
February $16, \mathrm{~K}$.

$50-2.00 \quad 47.18 S$ $23342.7048 .176+25.0$ I3 $22 \quad 3.00$ I7 $226.5 \mathrm{~S}$ IO $20 \quad 2.05$ $\begin{array}{rrr}305 & 30 & 5.38 \\ 16 & 16 & 3.82\end{array}$ $\begin{array}{lll}26 & 22 & 4.95\end{array}$ IS 2 S 3.00 $290 \quad 4.70$ $2 \mathrm{~S}$ IS $5 . \mathrm{I} 2$ 28 IS $5.12 \quad 42.090$ $\begin{array}{llll}34 & 13 & 57.65\end{array}$ $\begin{array}{lll}7 & 46 & 3.68\end{array}$ $\begin{array}{lll}35 & 26 & 4.20\end{array}$

46.920

$47.08 \mathrm{r}$

\begin{tabular}{l|l} 
MEAN & \\
OF & \\
TEL. MI- & REFRA \\
CROM. & TION. \\
READ- & \\
INGS. & \\
& \\
&
\end{tabular}

rev 48.018 45.298 49.472 46.865 48.872 46.840 47.108 44.925 $49.382 \div$

$4 \mathrm{r} .39 \mathrm{~S} ;$ I $3 \mathrm{I} . \mathrm{I}$ $42.020+35.6$ 40.749 46.072 47.110

48.240 40.340
39.775 47.244

43.659 47.889 45.320 41.856 46.834 48.854 46.896

4 I. 190 43.515

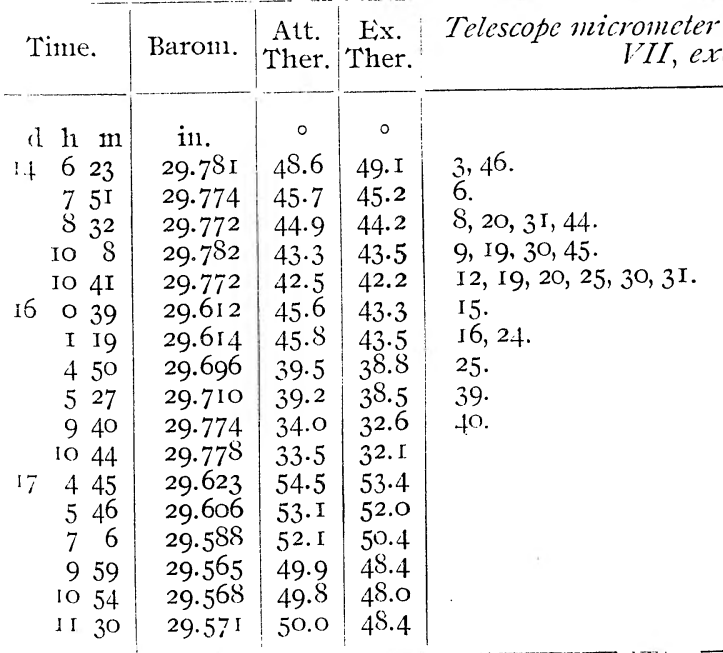

Bisections at III, IV, V. Bisections at II, VI, VII Bisections at II, VI. Bisections at I, VII.

Z. D. thread A used. Bisections at $\mathrm{C}_{3}, \mathrm{C}_{4}, \mathrm{D}_{\mathrm{I}}, \mathrm{D}_{3}$ Bisections at VI, VII. Bisections at II, III, IV, V, VI. Bisections at $\mathrm{C}_{5}, \mathrm{C}_{3}, \mathrm{C}_{1}$. Bisections at I, II. 


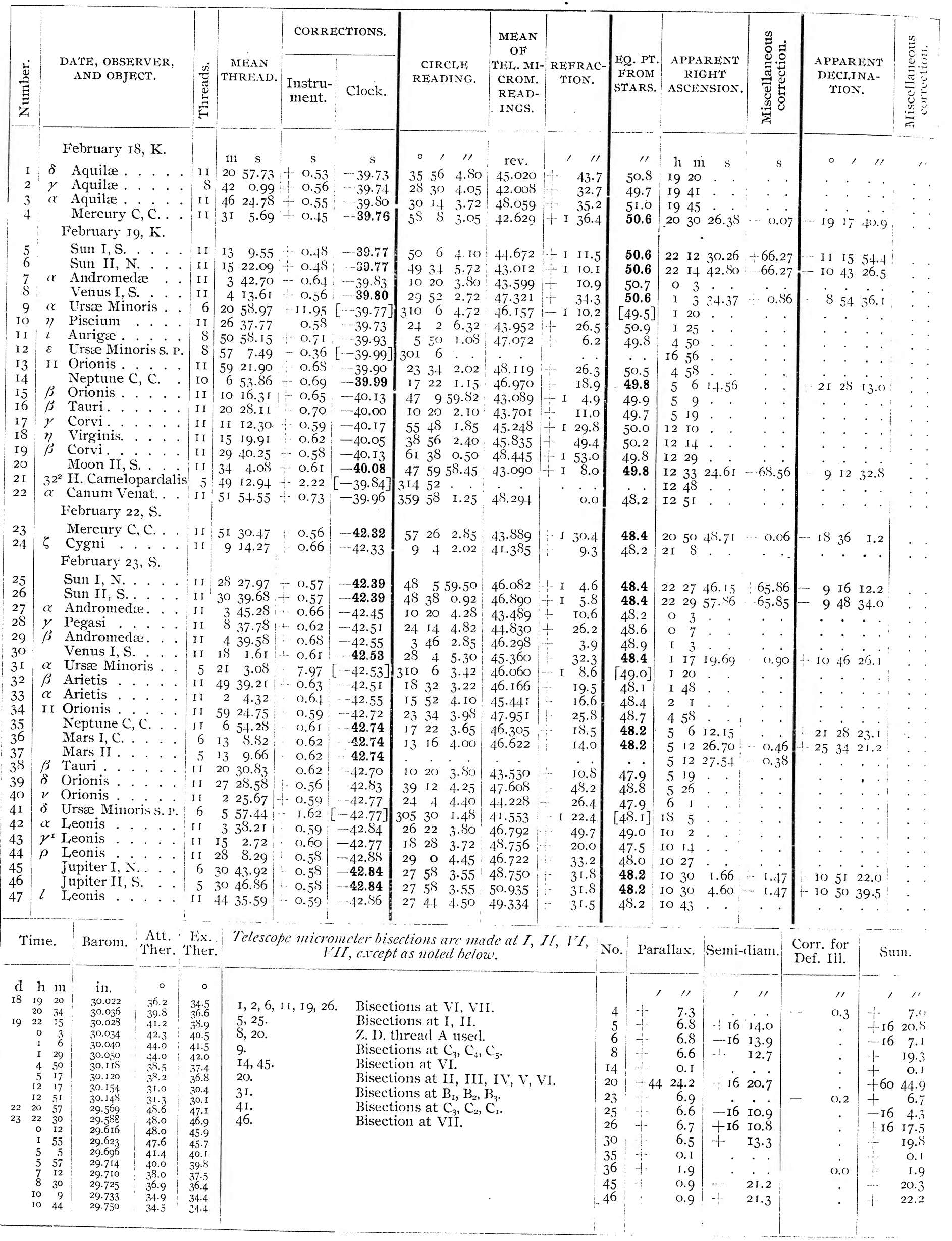




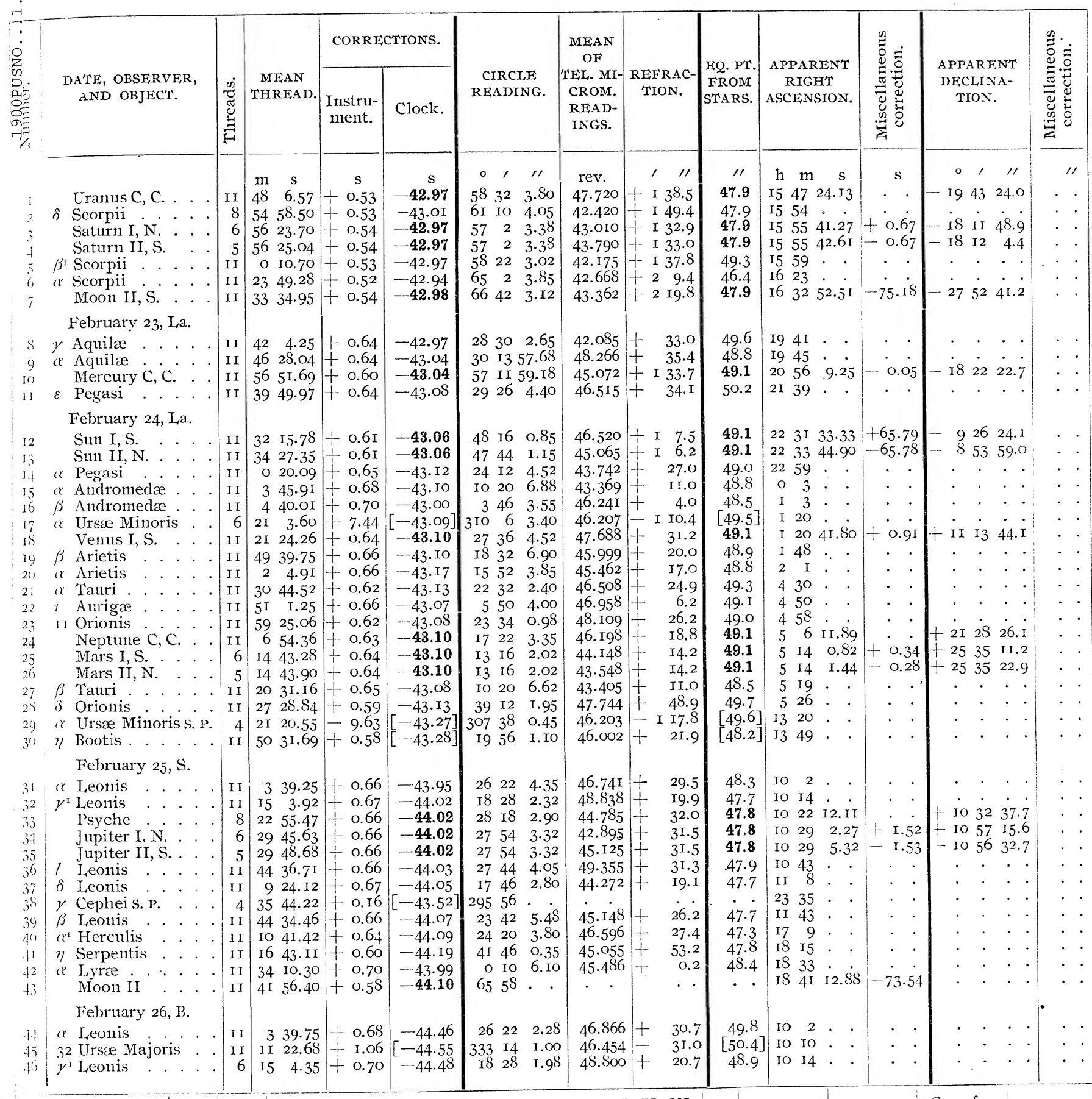

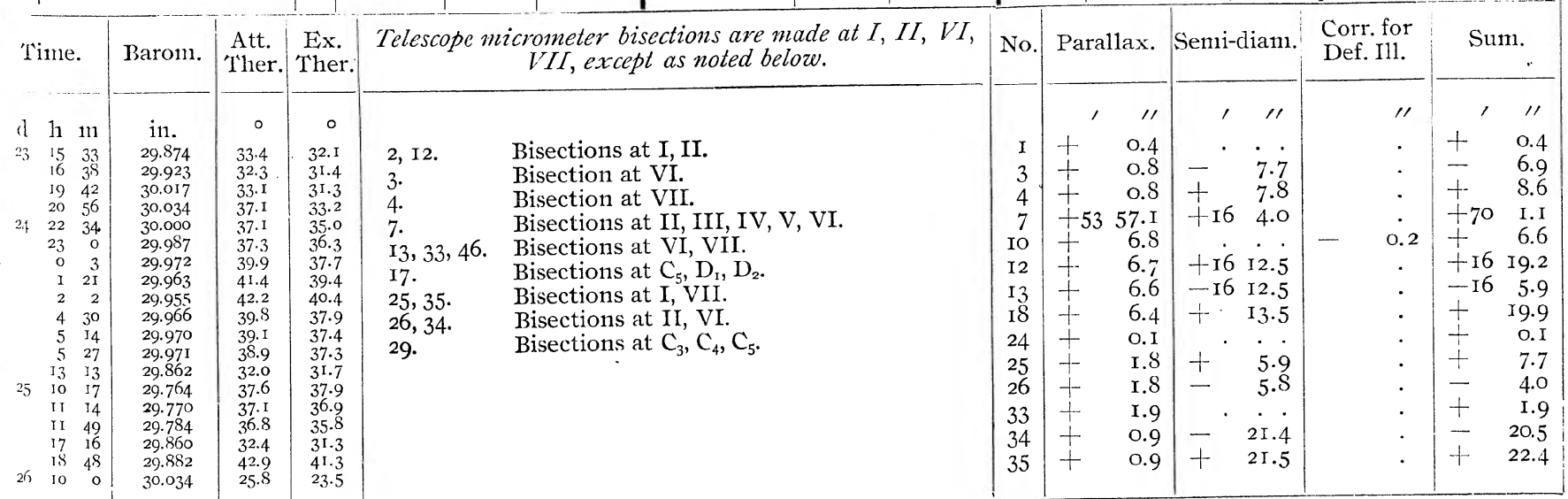

$6855-7$ 


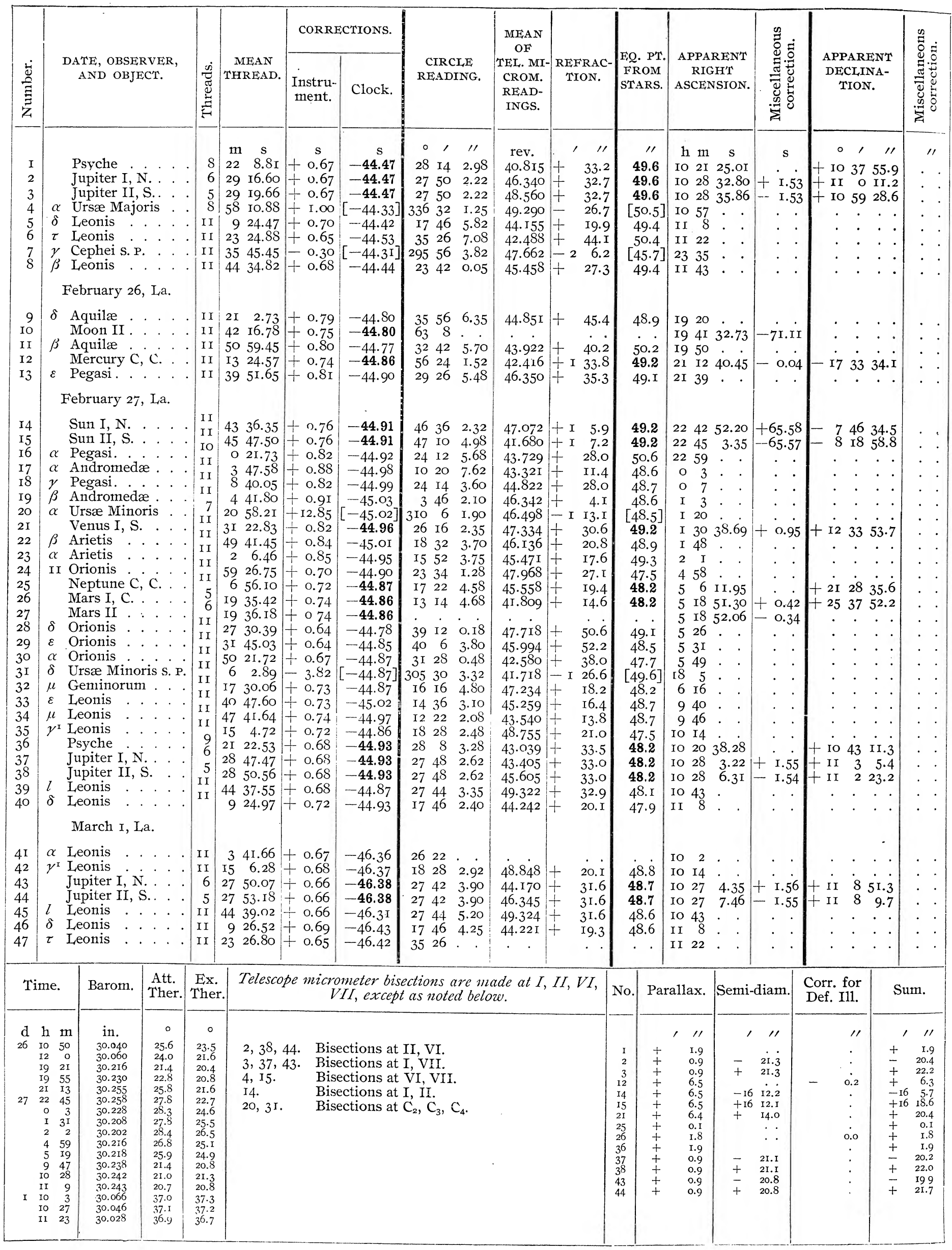




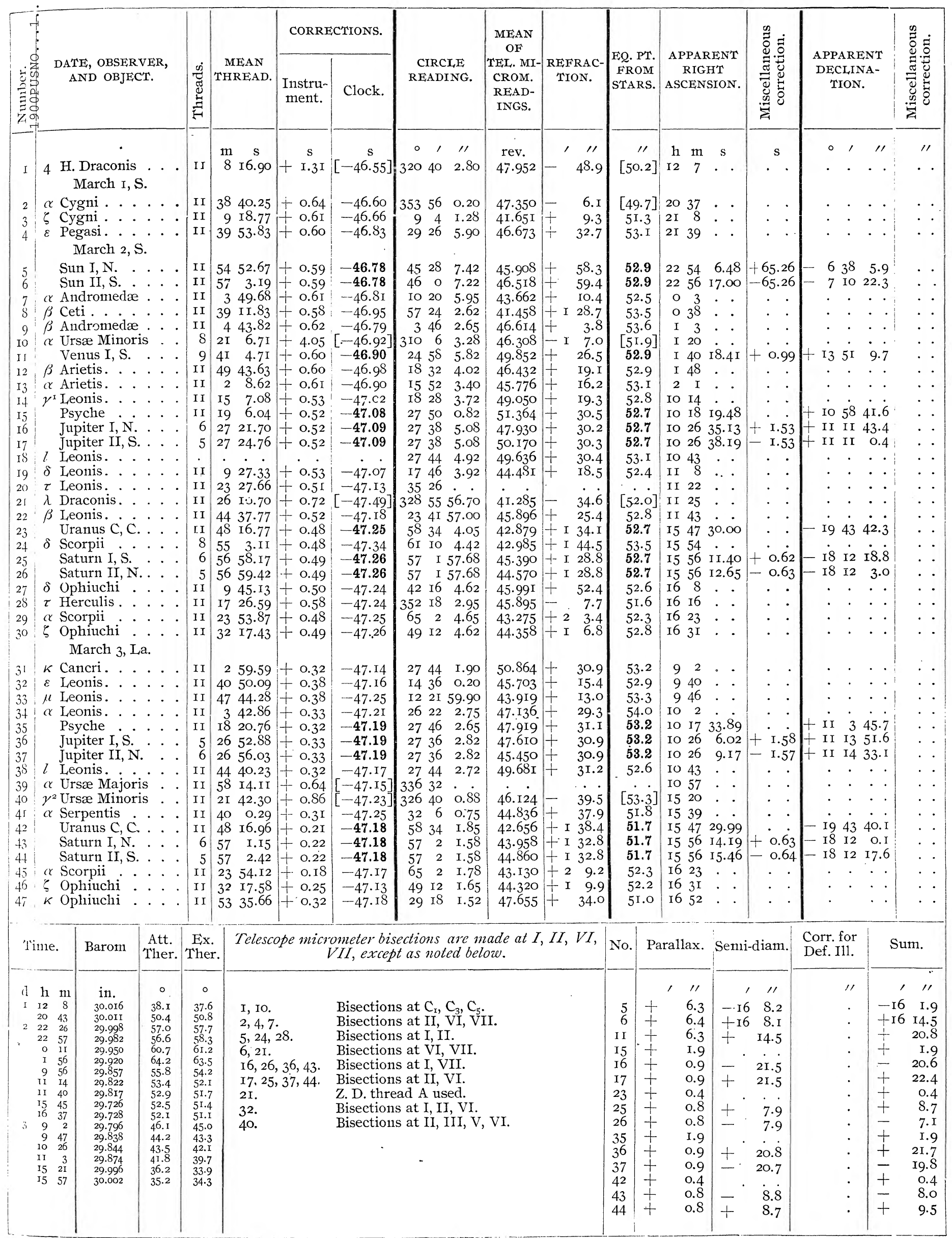




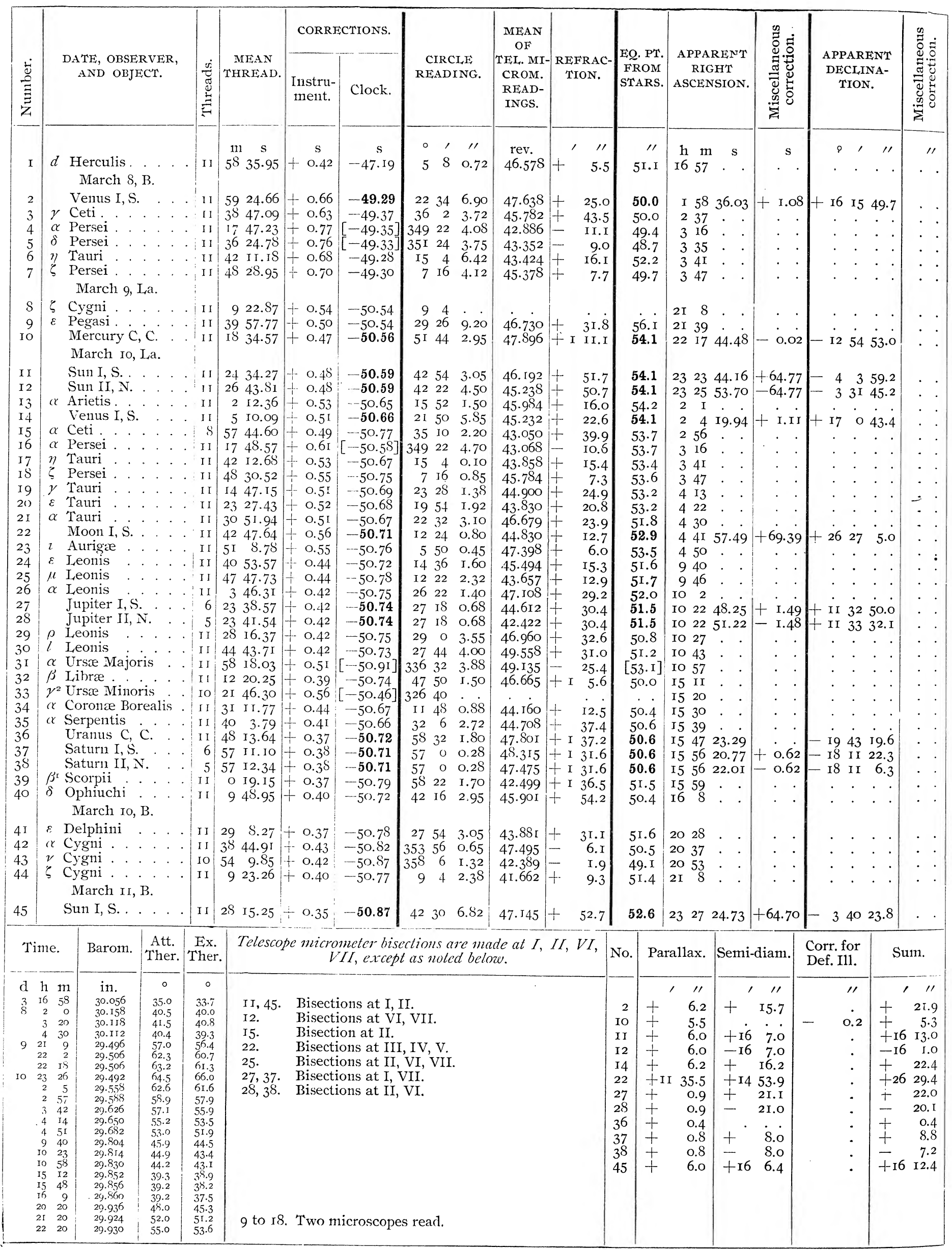




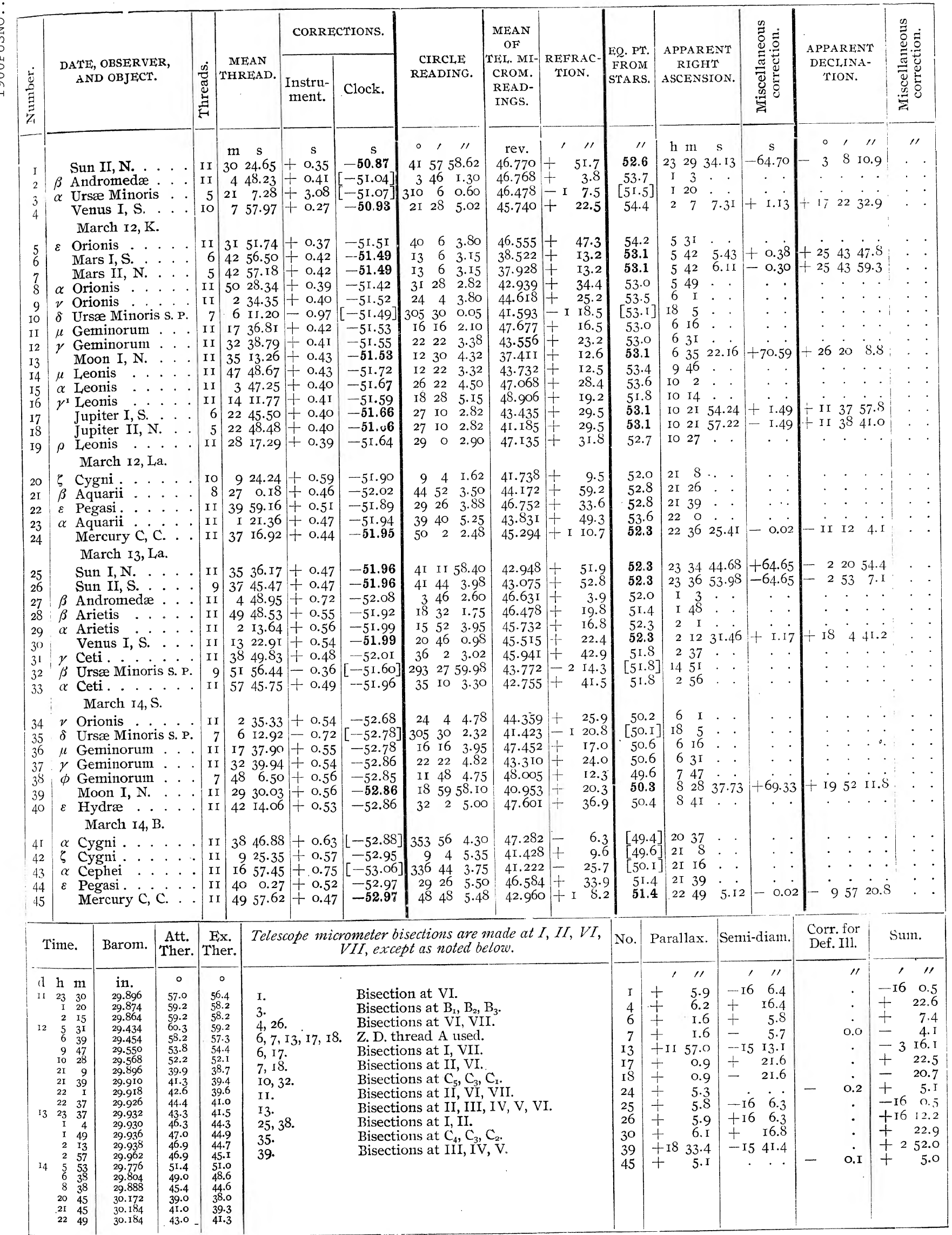




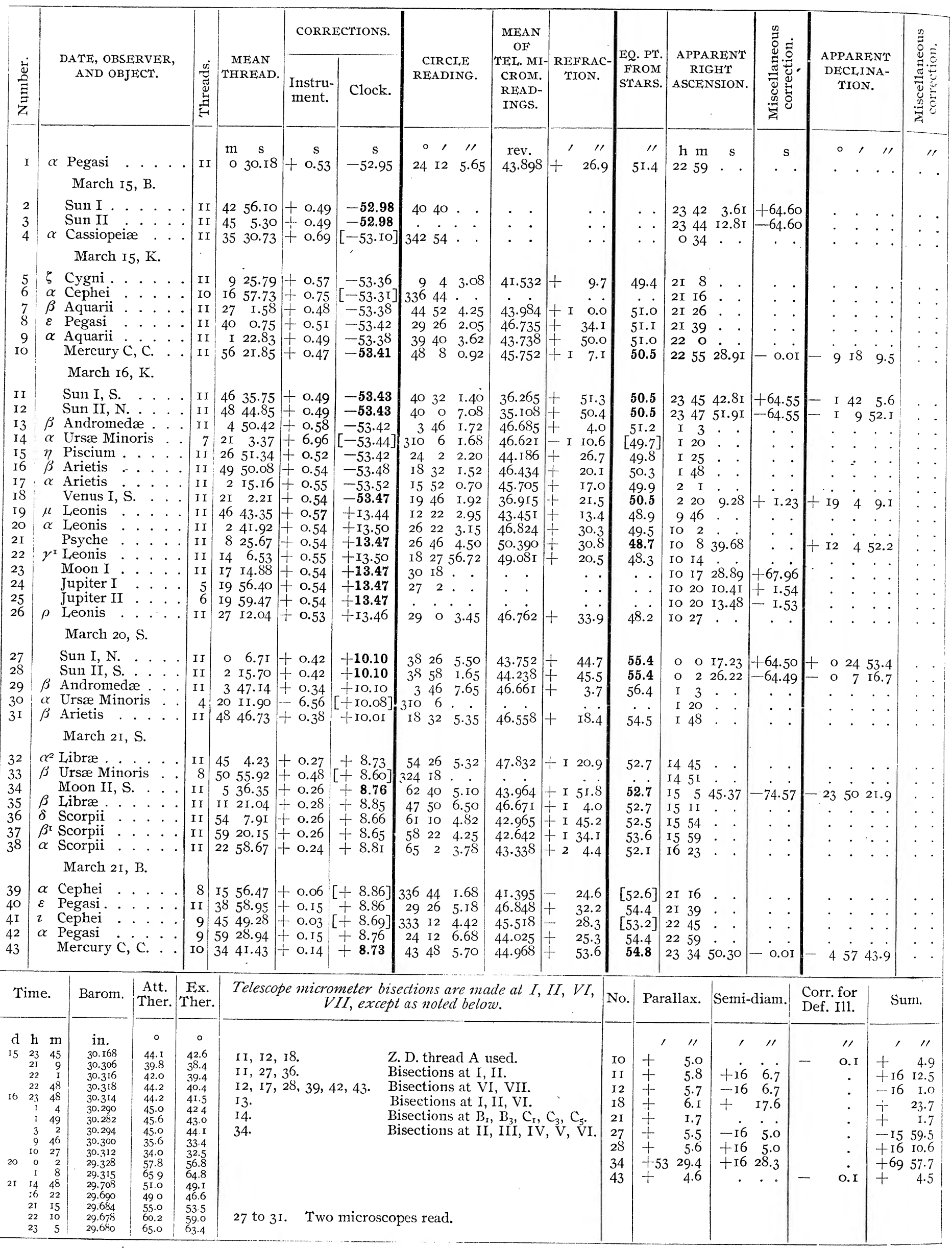




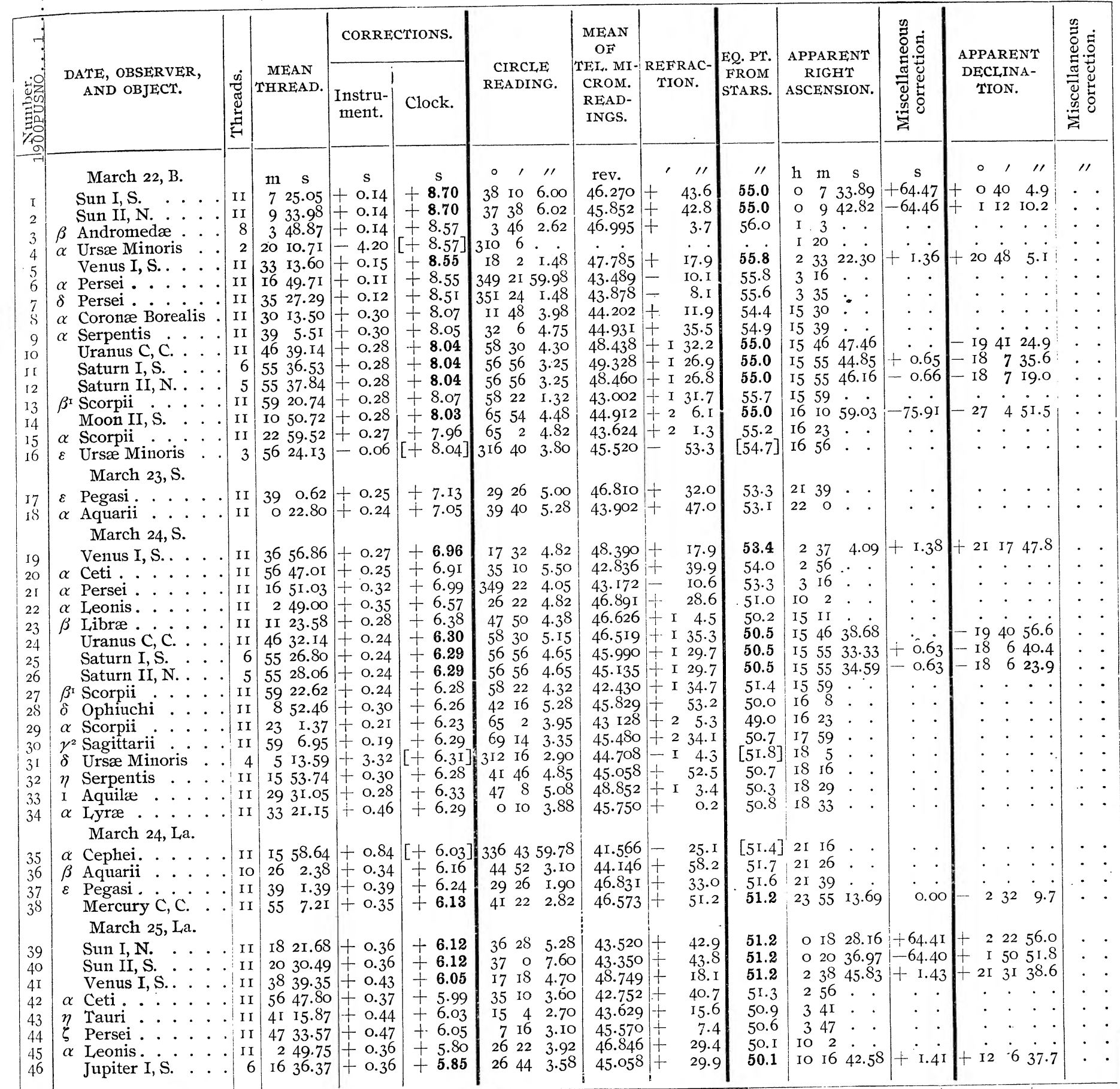

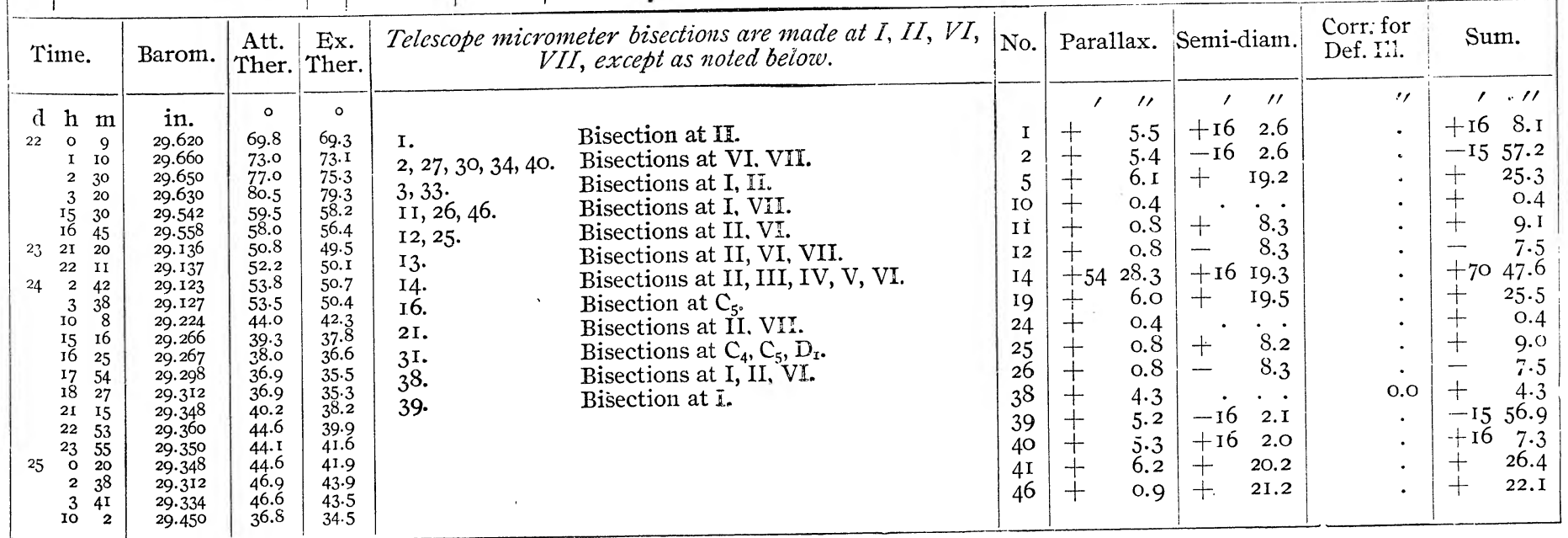




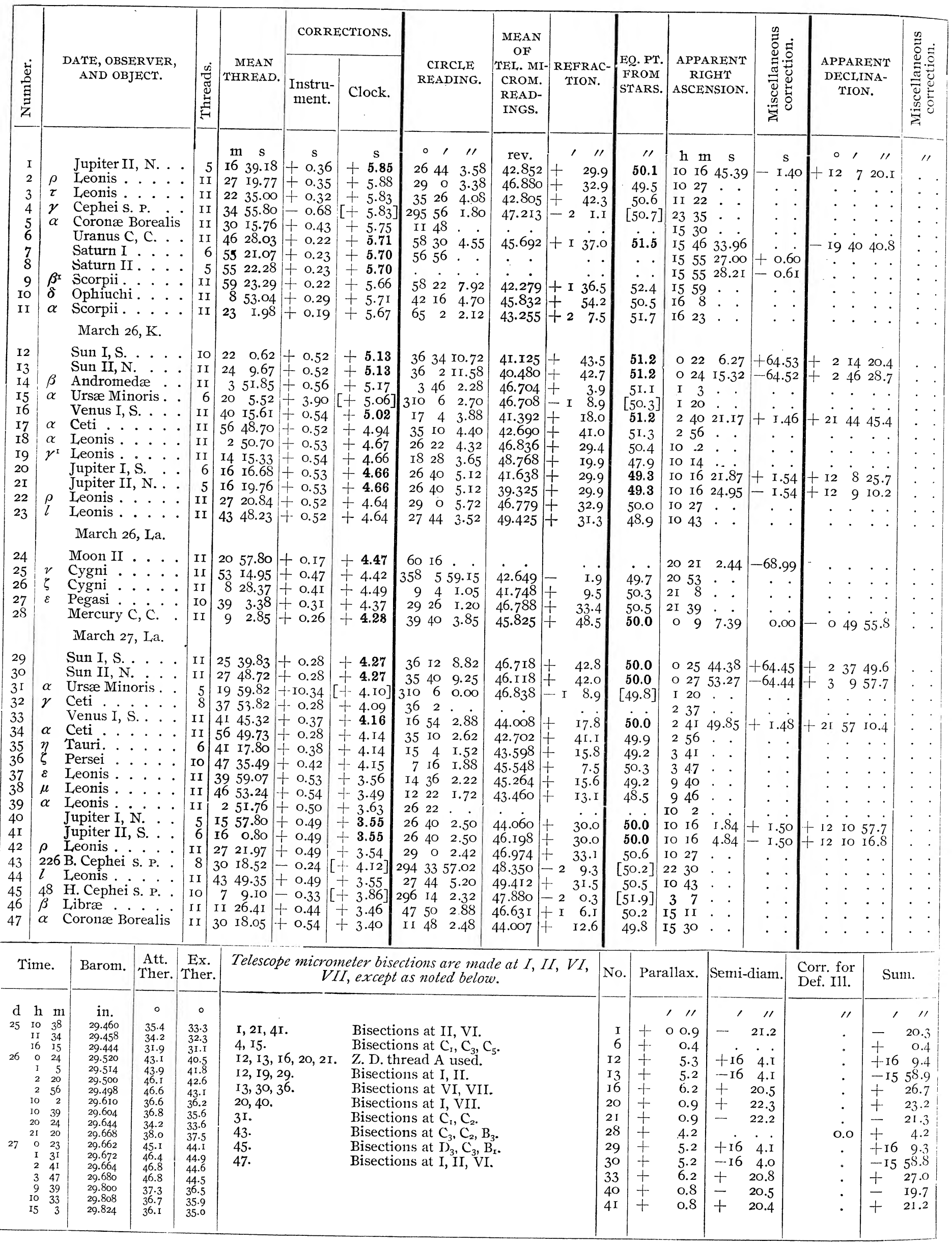




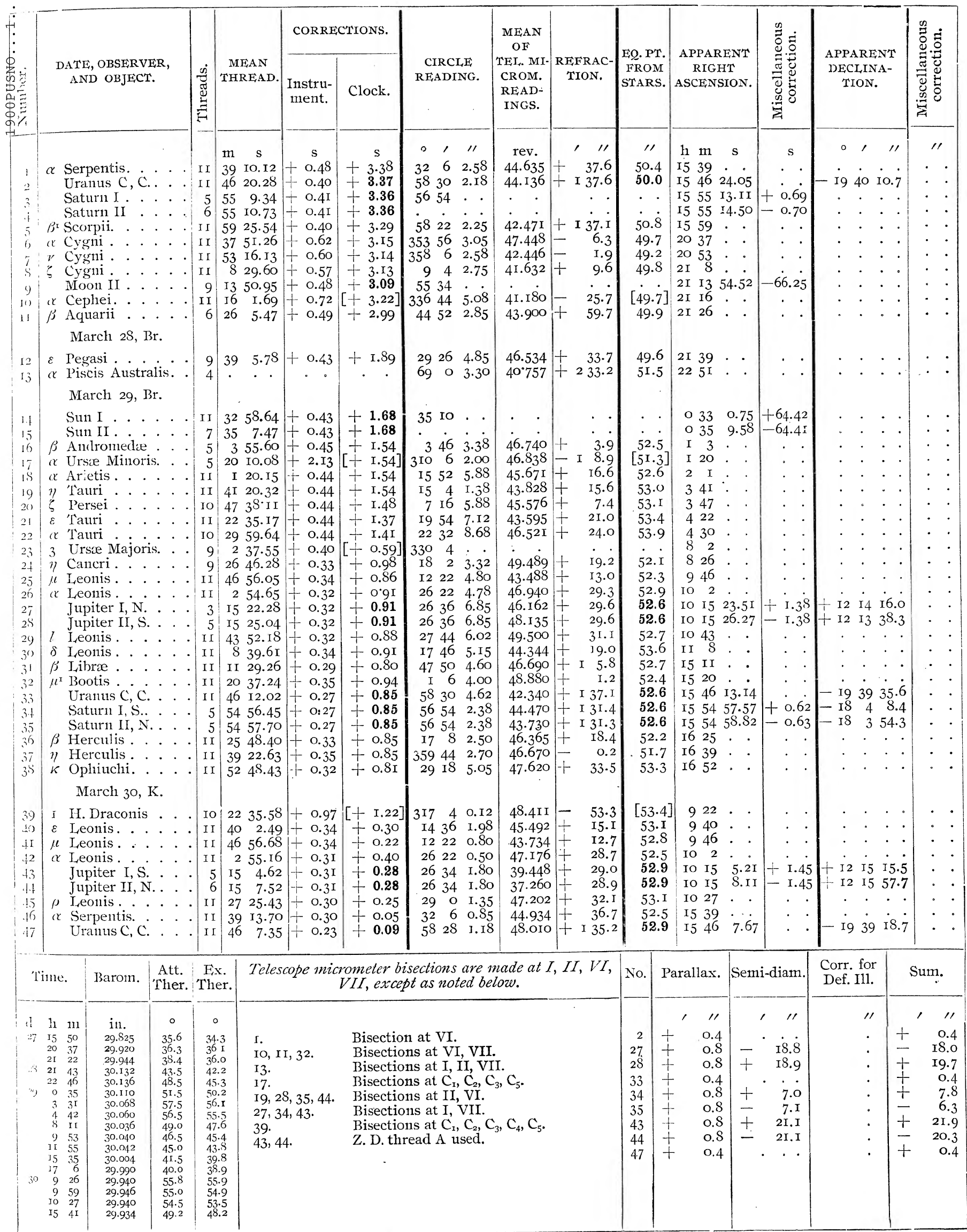




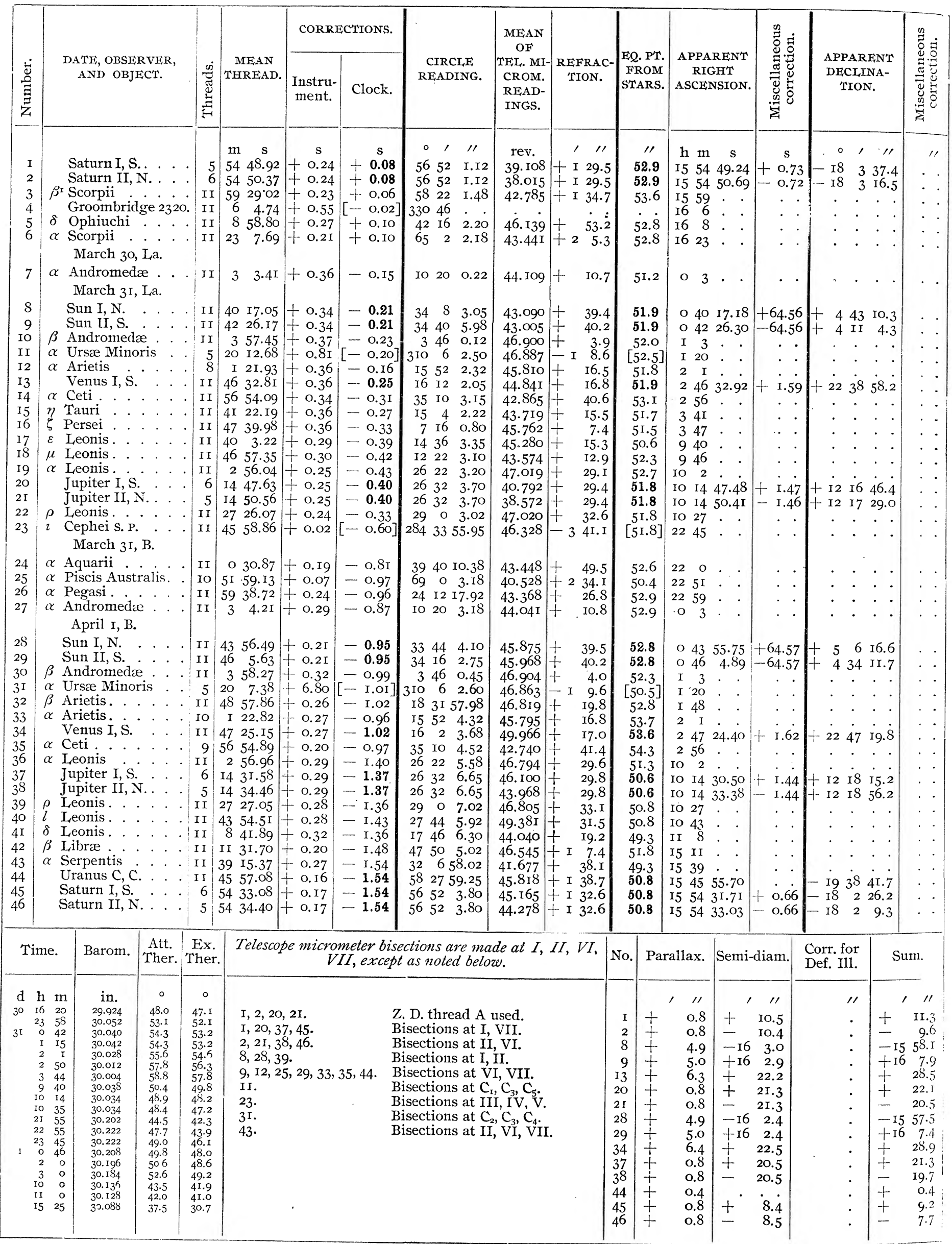




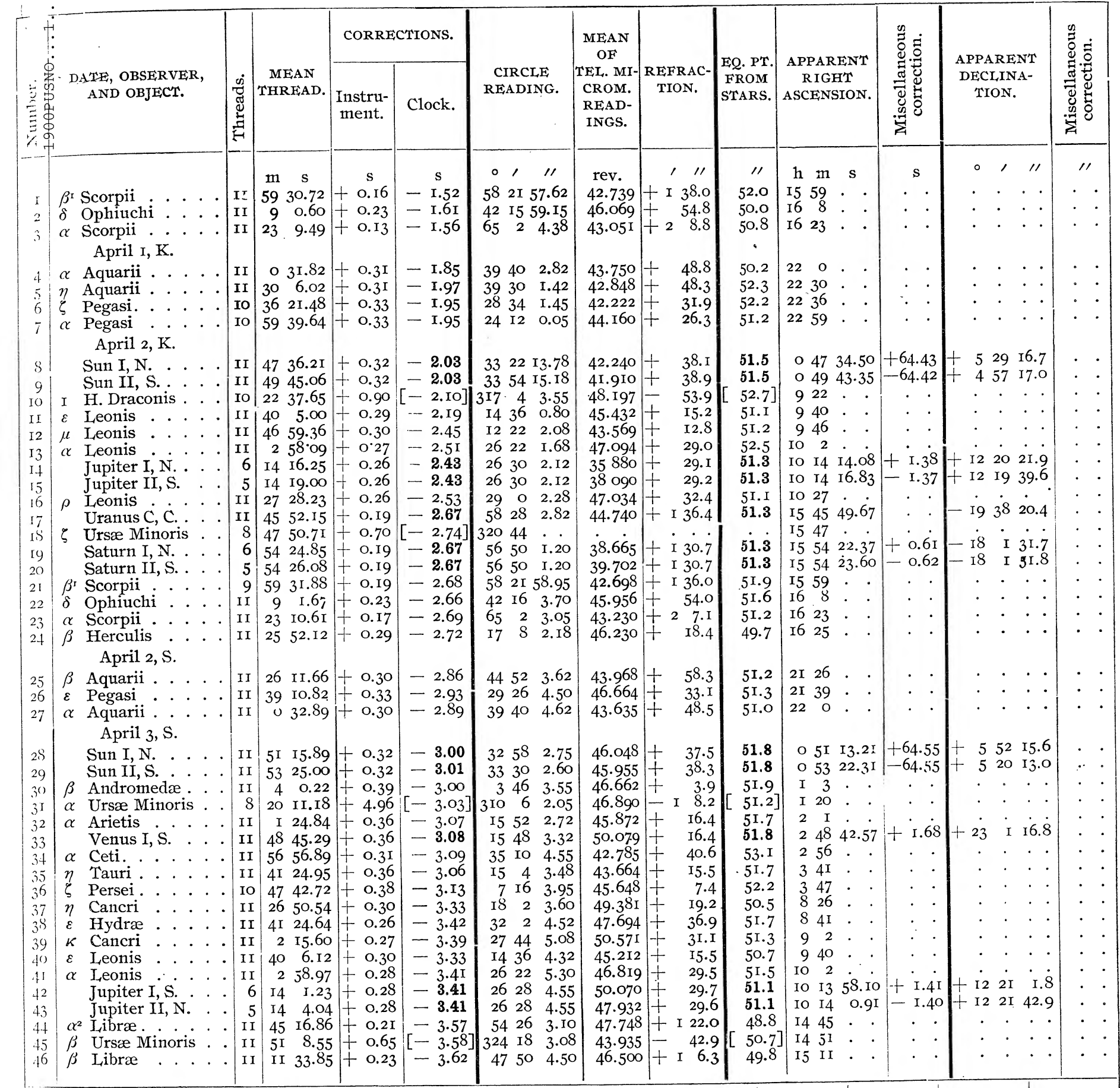

\begin{tabular}{|c|c|c|c|c|c|c|c|c|c|c|c|c|c|}
\hline \multicolumn{3}{|c|}{ 'Time. } & \multirow{2}{*}{$\frac{\text { Barom. }}{\text { in. }}$} & \multirow{2}{*}{$\frac{\text { Att. }}{\text { Ther. }}$} & \multirow{2}{*}{$\begin{array}{c}\text { Ex. } \\
\text { Ther. } \\
\circ\end{array}$} & \multicolumn{2}{|c|}{$\begin{array}{c}\text { Telescope micrometer bisections are made at } I, I I, V I, \\
V I I, \text { except as noted below. }\end{array}$} & \multirow{3}{*}{ No. } & \multicolumn{2}{|c|}{ Parallax. } & Semi-diam. & \multirow{2}{*}{$\begin{array}{l}\text { Corr. for } \\
\text { Def. Iil. } \\
/ \prime\end{array}$} & Sum. \\
\hline d & $\mathrm{h}$ & & & & & & & & ' & & , II & & 111 \\
\hline & 16 & 30 & & 37.8 & 37.2 & $5,7,9,2 \mathrm{I}, 25,27,29,3 \mathrm{O}$. & Bisections at VI, VII. & & + & 4.8 & -I5 59.8 & . & -I5 55.0 \\
\hline & 22 & 0 & 30.088 & 47.6 & $47 \cdot 3$ & 8,28 . & Bisections at I, II. & 9 & + & 4.9 & +r5 59.8 & - & tI6 4.7 \\
\hline & 22 & $\begin{array}{r}35 \\
2\end{array}$ & $\begin{array}{l}30.088 \\
30.086\end{array}$ & $\begin{array}{l}50.4 \\
53.3\end{array}$ & $\begin{array}{l}49.7 \\
51.0\end{array}$ & IO, 3I. & Bisections at $\mathrm{C}_{\mathrm{I}}, \mathrm{C}_{3}, \mathrm{C}_{5}$. & 14 & + & 0.8 & $2 \mathrm{I} .2$ & - & 20.4 \\
\hline 2 & 0 & 49 & 30.032 & 55.6 & 55.1 & I4, I5, I9, 20. & Z. D. thread A used. & 15 & + & 0.8 & 2I.I & - & 21.9 \\
\hline & & 25 & 29.940 & $5^{1 \cdot 7}$ & 50.4 & I4, I9, 43 . & Bisections at I, VII. & I7 & + & 0.4 & . . & . & 0.4 \\
\hline & 10 & 25 & 29.930 & 50.7 & 48.6 & I $5,20,42$. & Bisections at II, VI. & I9 & + & 0.8 & IO.O & . & 9.2 \\
\hline & $\begin{array}{l}15 \\
16\end{array}$ & $\begin{array}{l}43 \\
20\end{array}$ & $\begin{array}{l}29.914 \\
29.920\end{array}$ & $\begin{array}{l}42.0 \\
4 \mathrm{I} .8\end{array}$ & $\begin{array}{l}4 \mathrm{I} \cdot 4 \\
40.3\end{array}$ & 45 & Bisections at III, IV, V. & 20 & + & 0.8 & + IO.I & . & 10.9 \\
\hline & $2 \mathrm{I}$ & 17 & 30.052 & 49.6 & 49.0 & 46. & Bisections at II, VI, VII. & 28 & + & 4.8 & - I6 $\mathrm{I} .3$ & . & - I5 56.5 \\
\hline & 23 & 26 & 30.089 & $55 \cdot 7$ & $\begin{array}{l}53.6 \\
56.2\end{array}$ & & & 29 & + & 4.9 & $+16 \quad$ I.2 & . & +166.1 \\
\hline 3 & I & $\begin{array}{l}53 \\
44\end{array}$ & $\begin{array}{l}30.086 \\
30.07 \mathrm{~J}\end{array}$ & $\begin{array}{l}57.0 \\
58.1\end{array}$ & $\begin{array}{l}50.2 \\
57.2\end{array}$ & & & 33 & + & 6.5 & $+\quad 23.2$ & . & 29.7 \\
\hline & & $\begin{aligned} 17 \\
2\end{aligned}$ & 30.057 & 58.9 & 58.0 & - & & 42 & + & $0 . \check{8}$ & 20.6 & • & 21.4 \\
\hline & 3 & 54 & 30.063 & $\begin{array}{l}59.1 \\
50.0\end{array}$ & $\begin{array}{l}58.1 \\
48.4\end{array}$ & & & 43 & + & 0.8 & $-\quad 20.5$ & - & I9.7 \\
\hline & 9 & 35 & $\begin{array}{l}30.128 \\
30.136\end{array}$ & 47.4 & $45 . \mathrm{I}$ & & & & & & & & \\
\hline & & 40 & 30.108 & 40.3 & $3^{8} .4$ & & & & & & & & \\
\hline
\end{tabular}




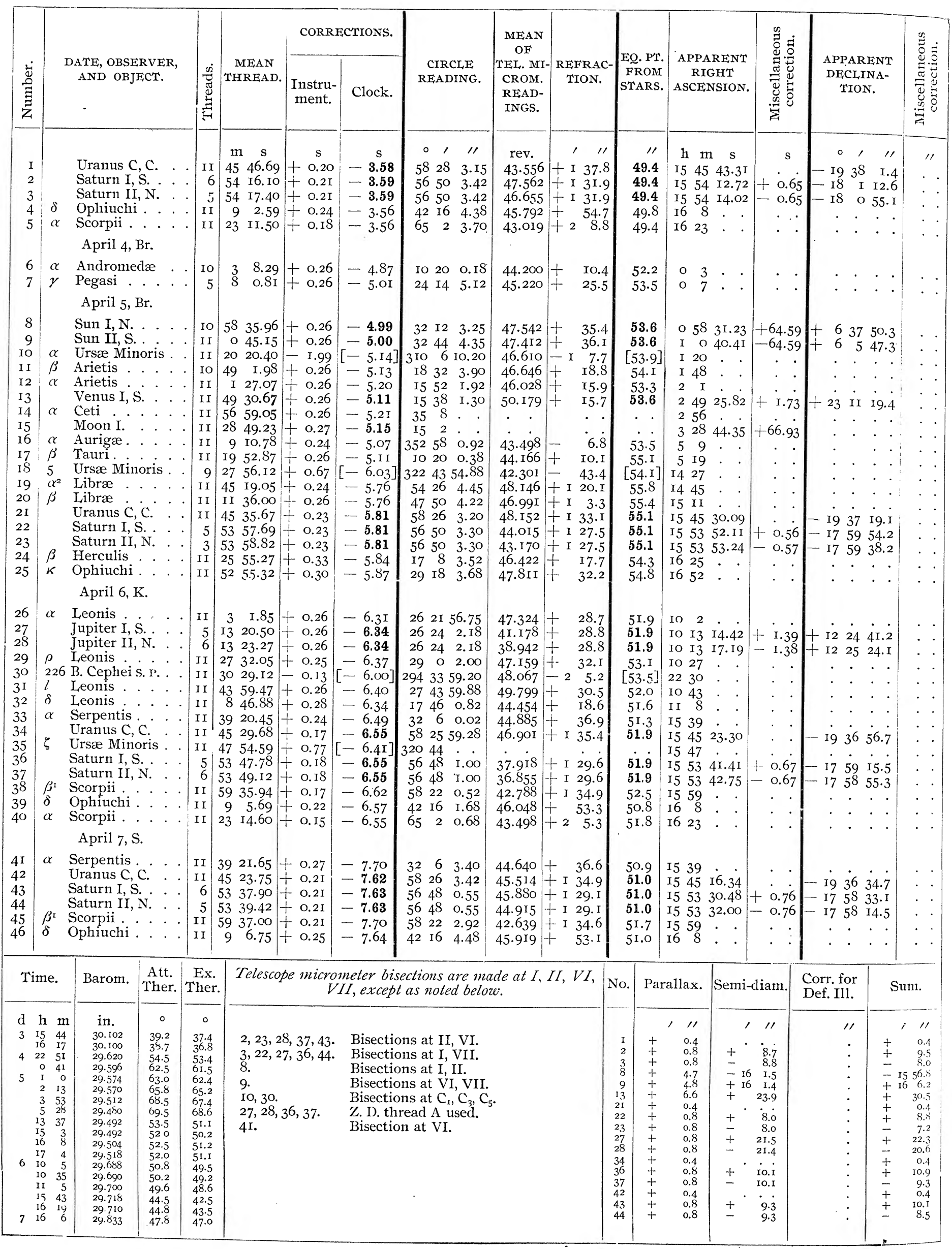




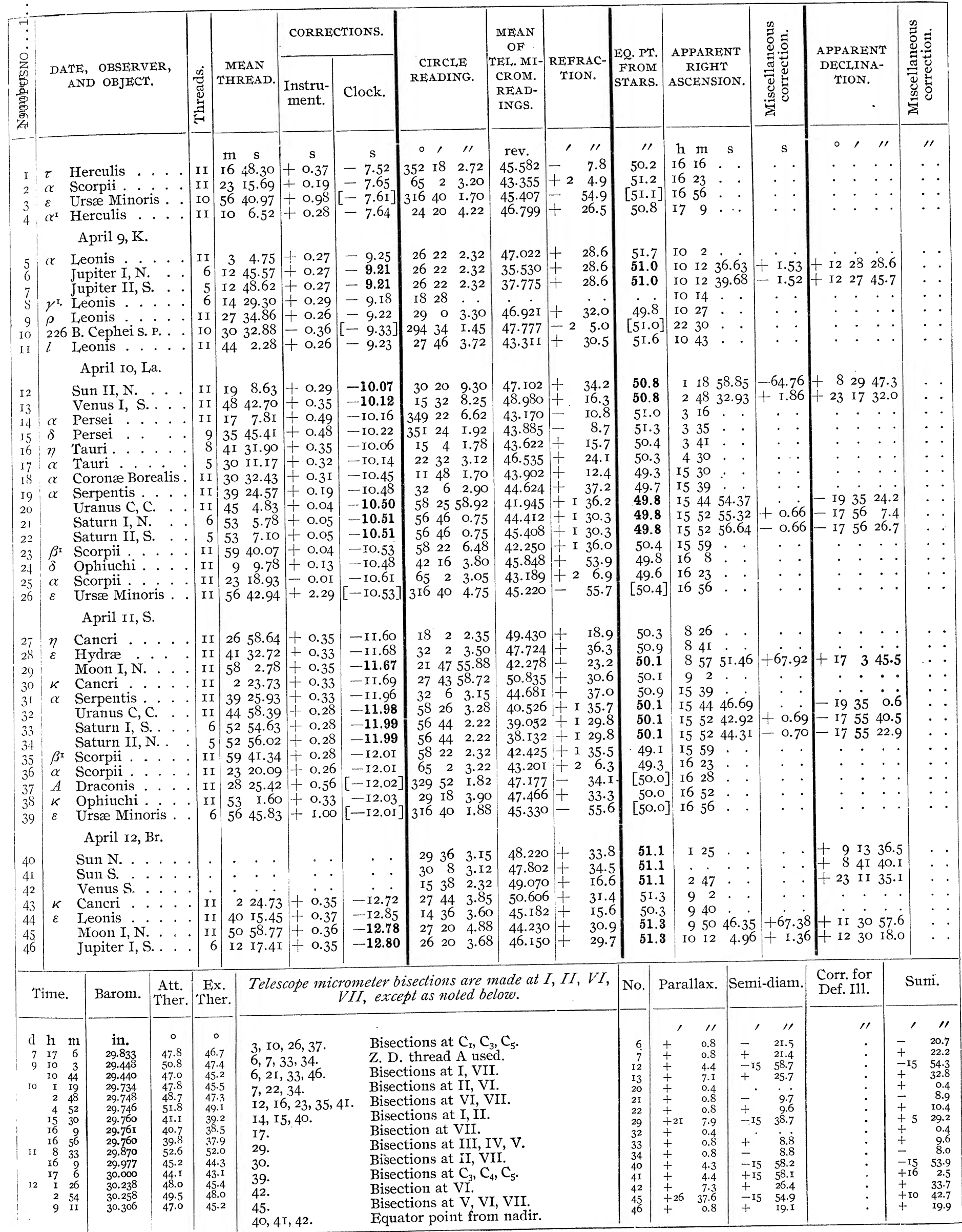




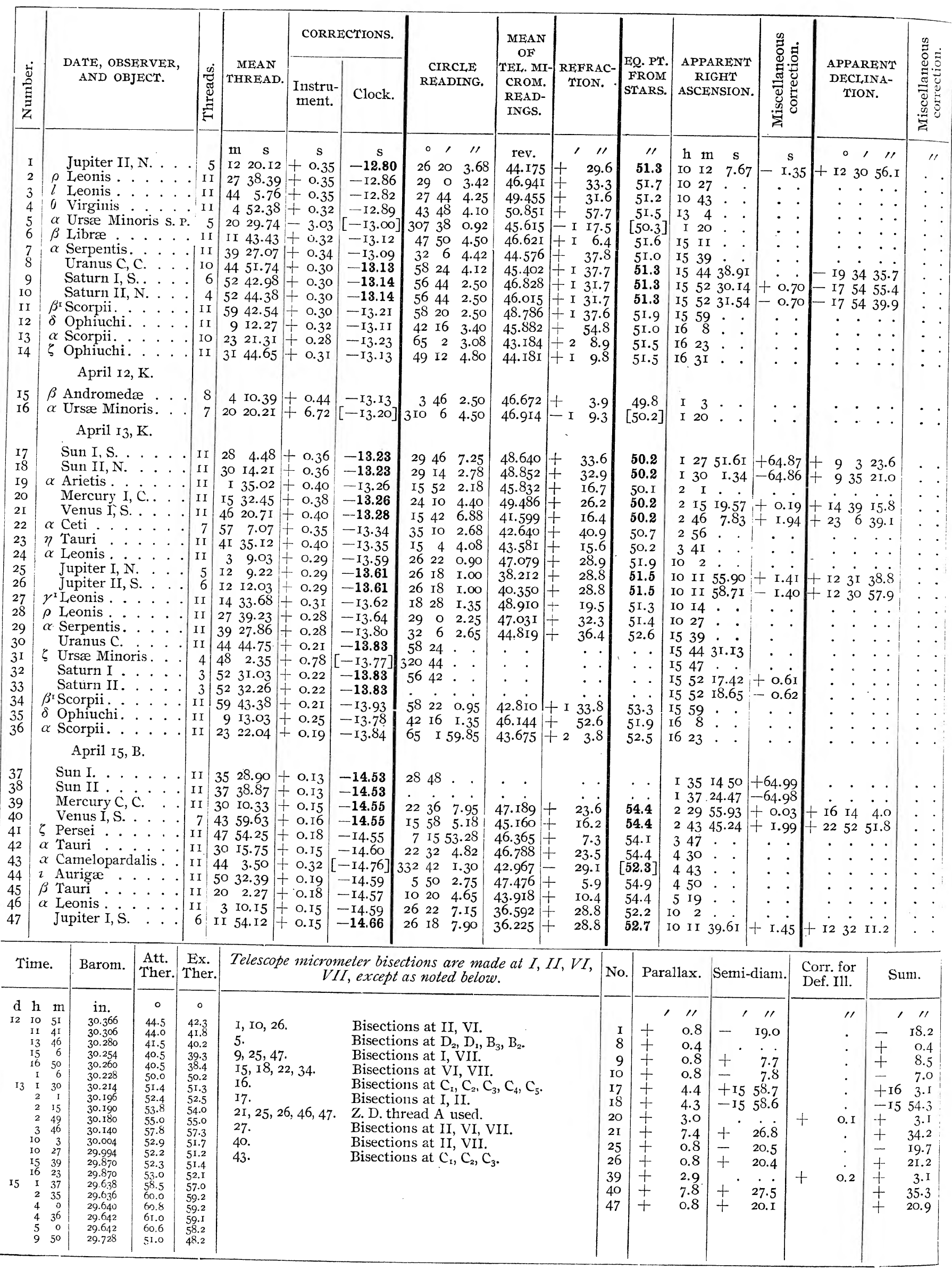




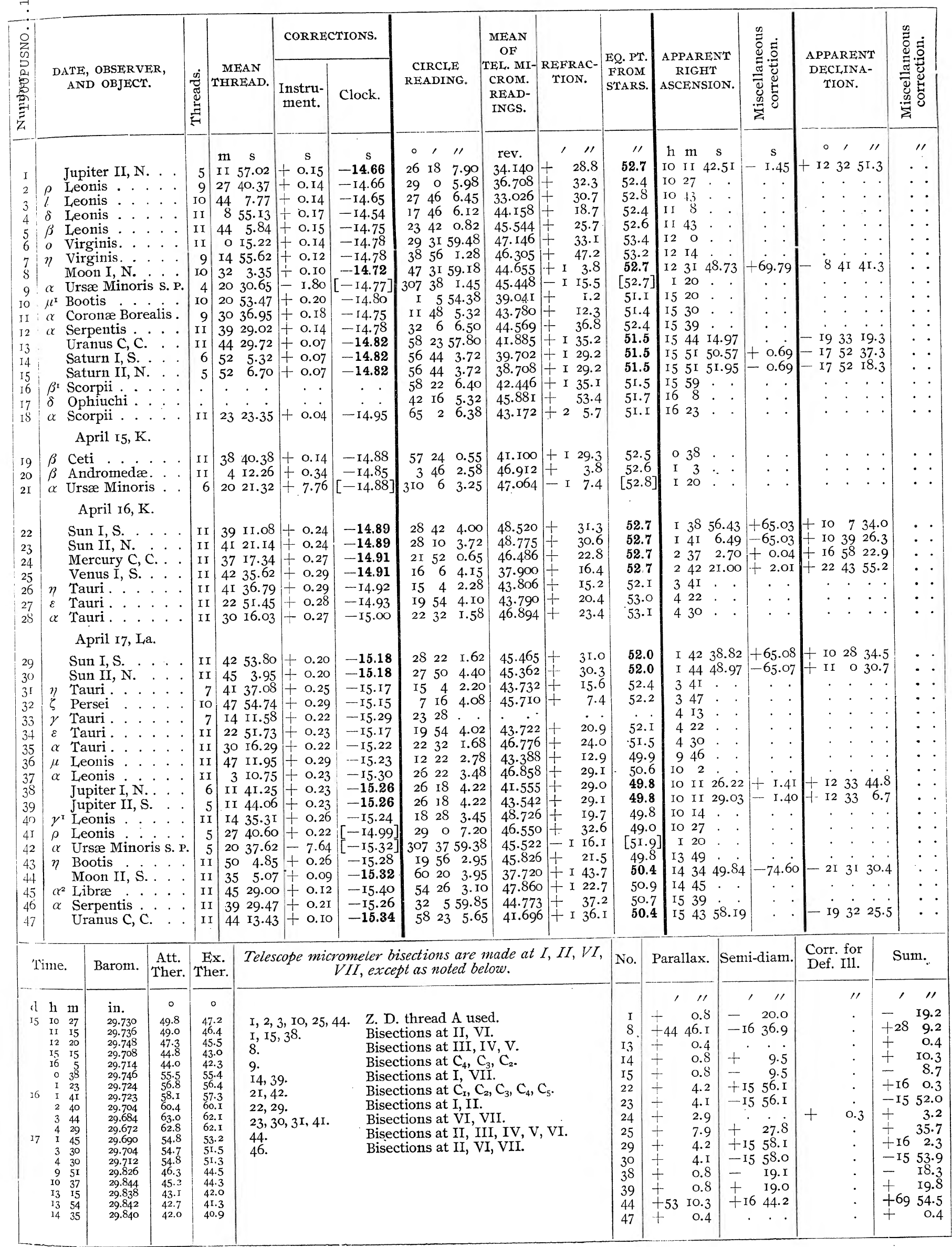




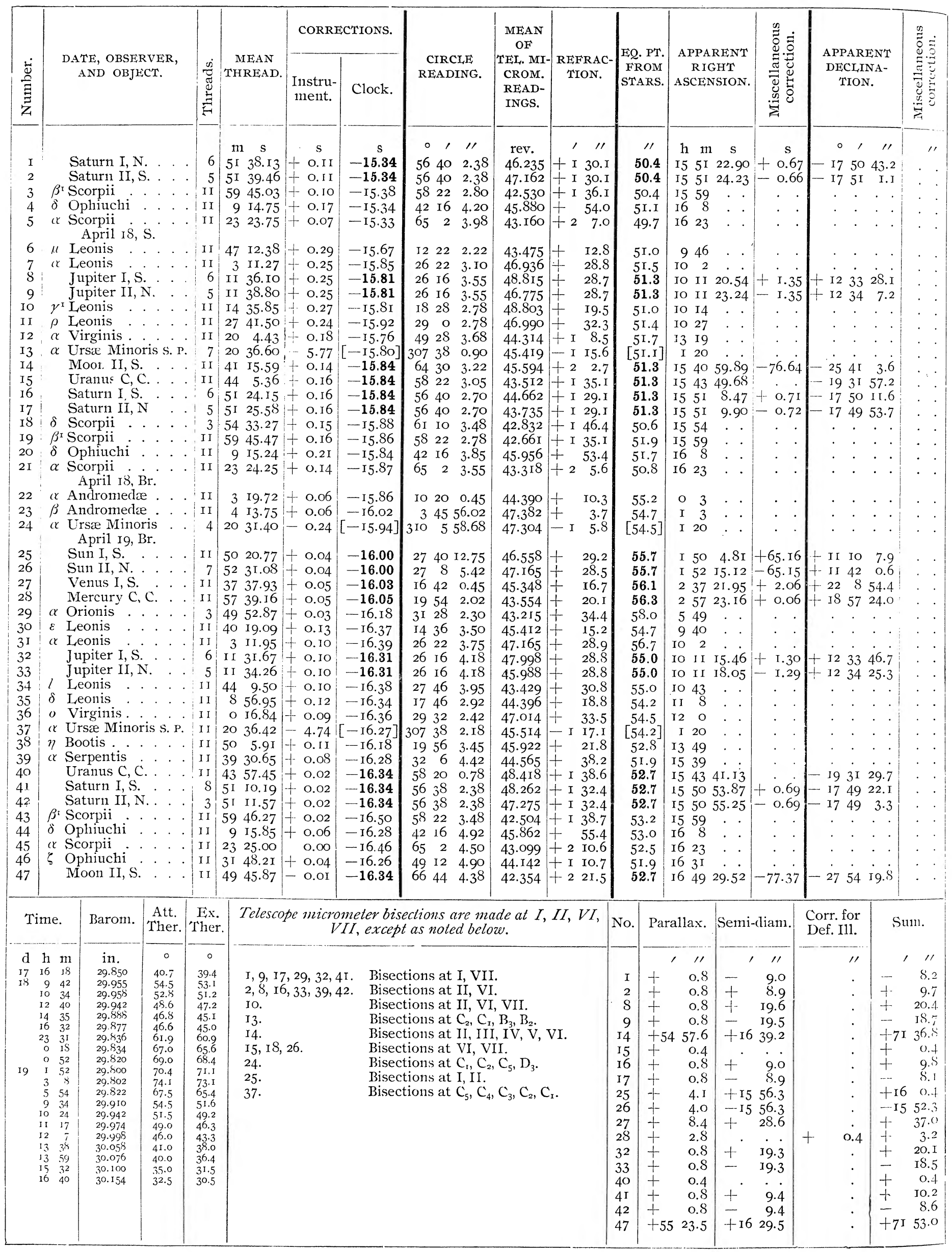




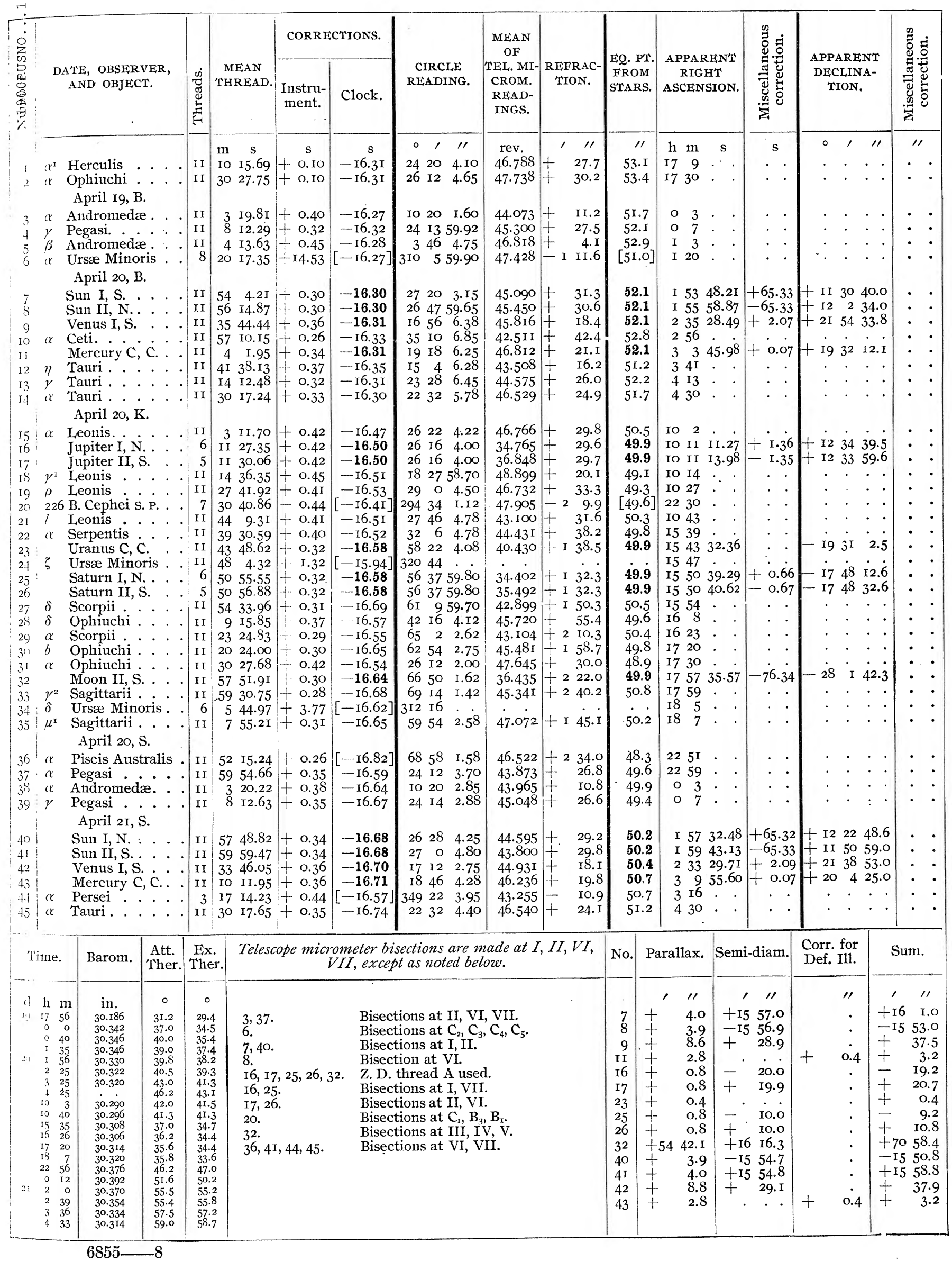




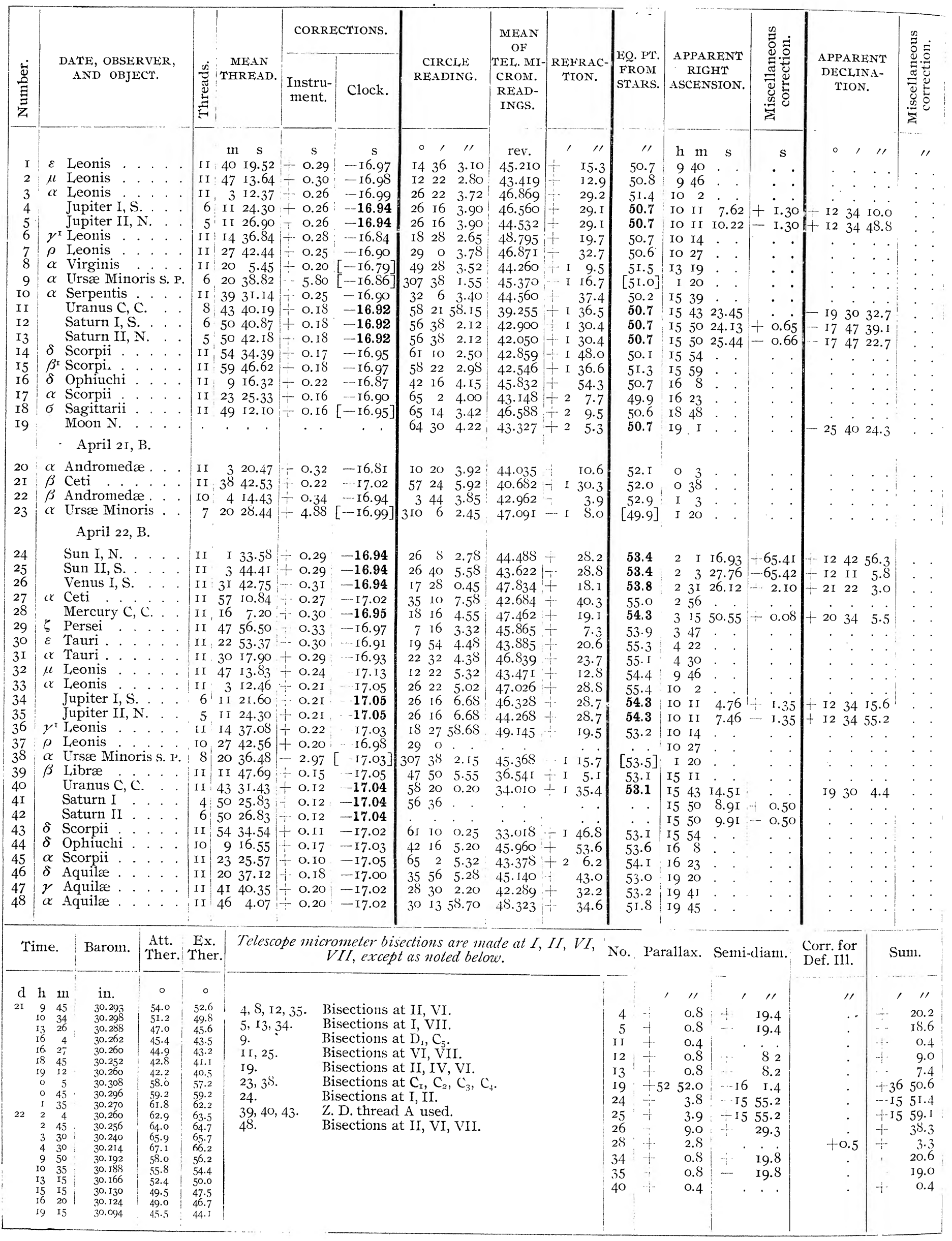




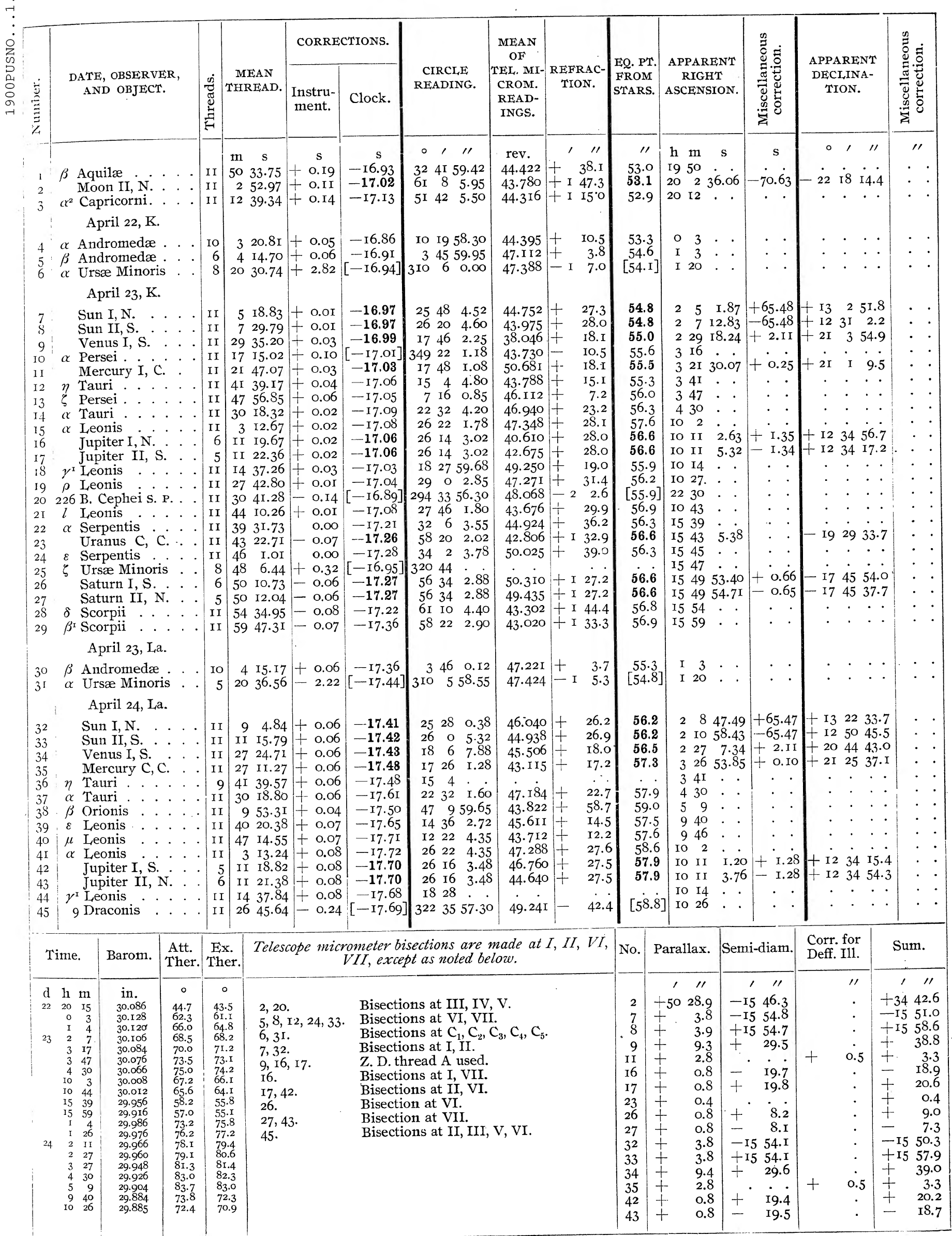




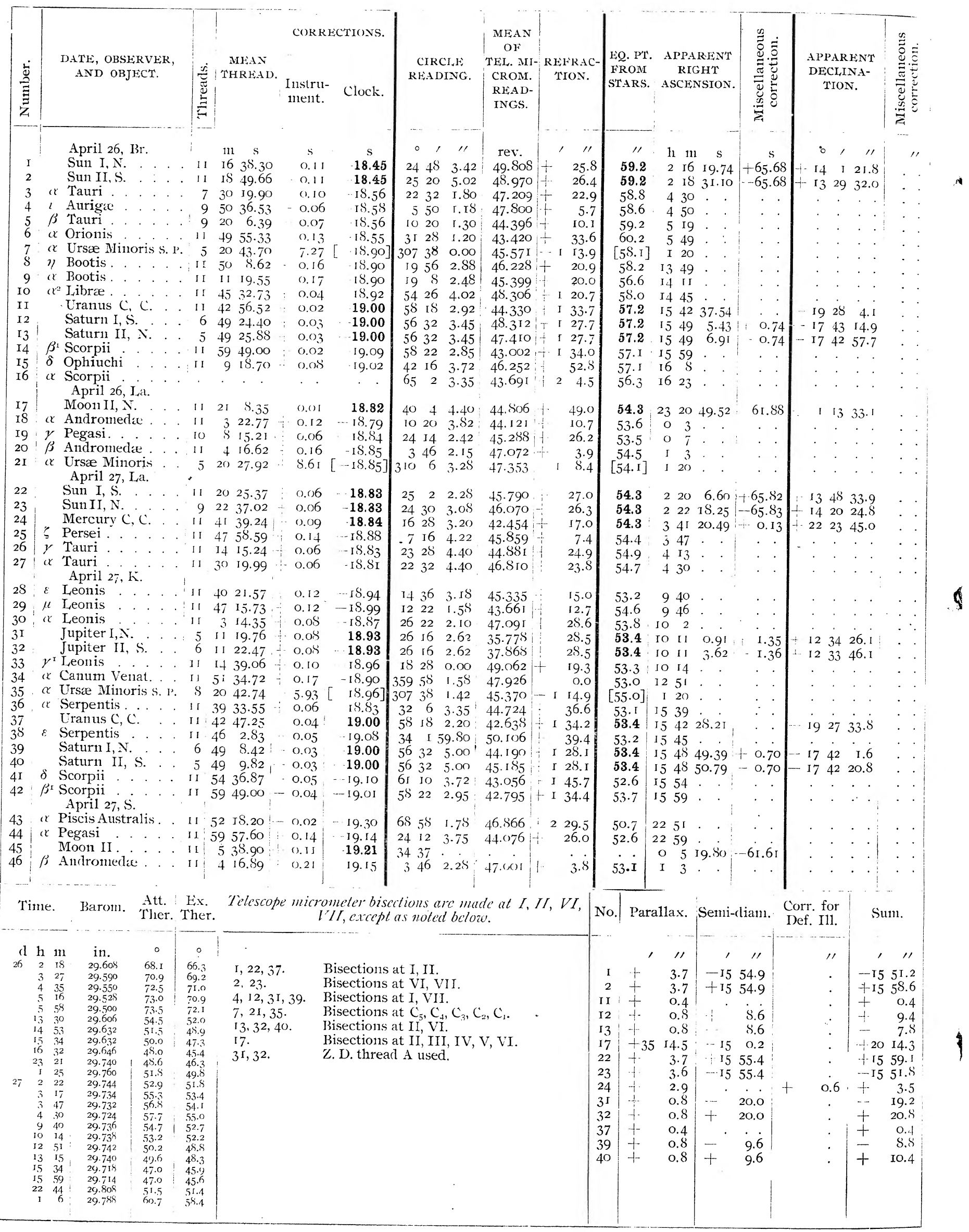




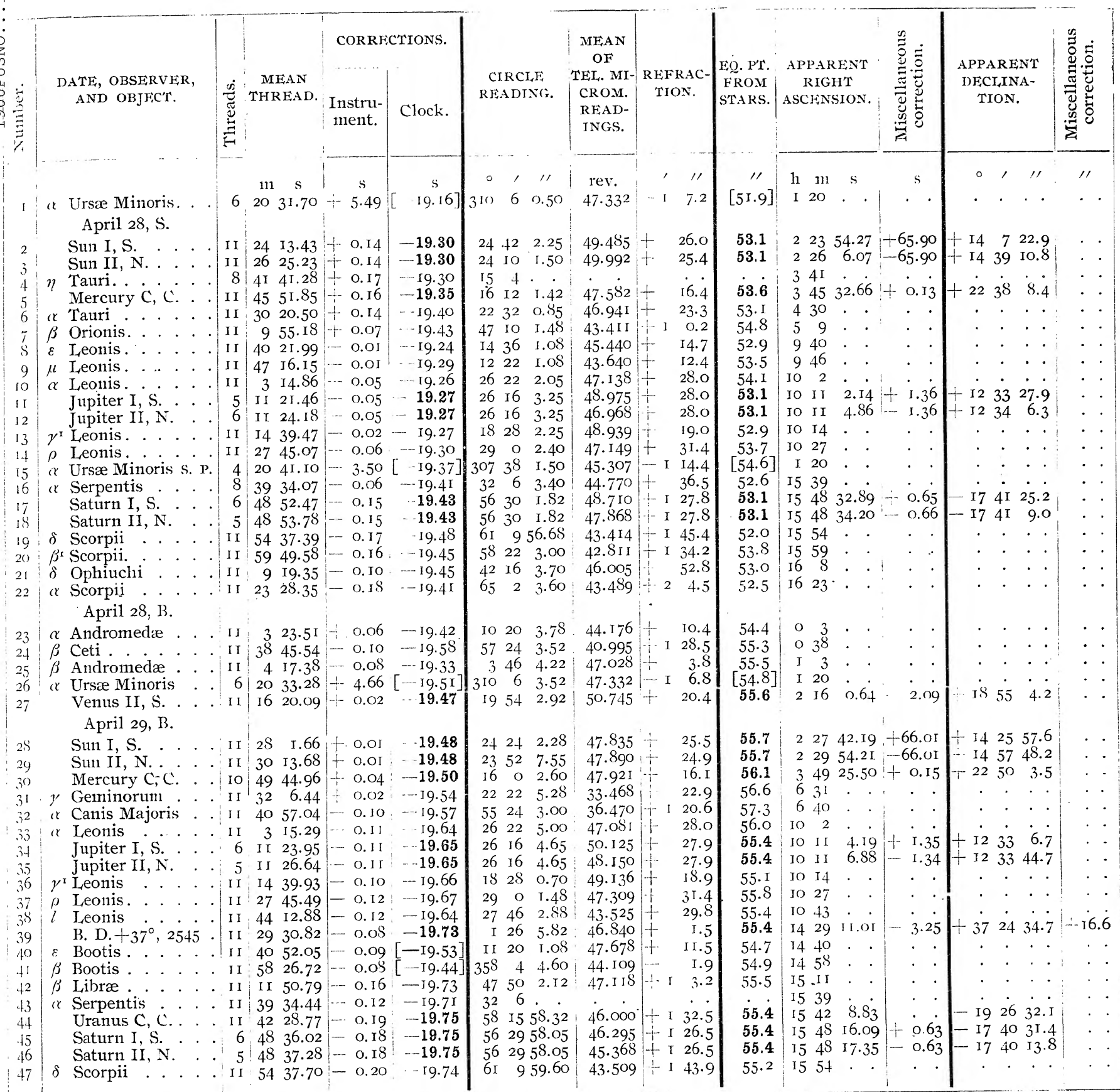

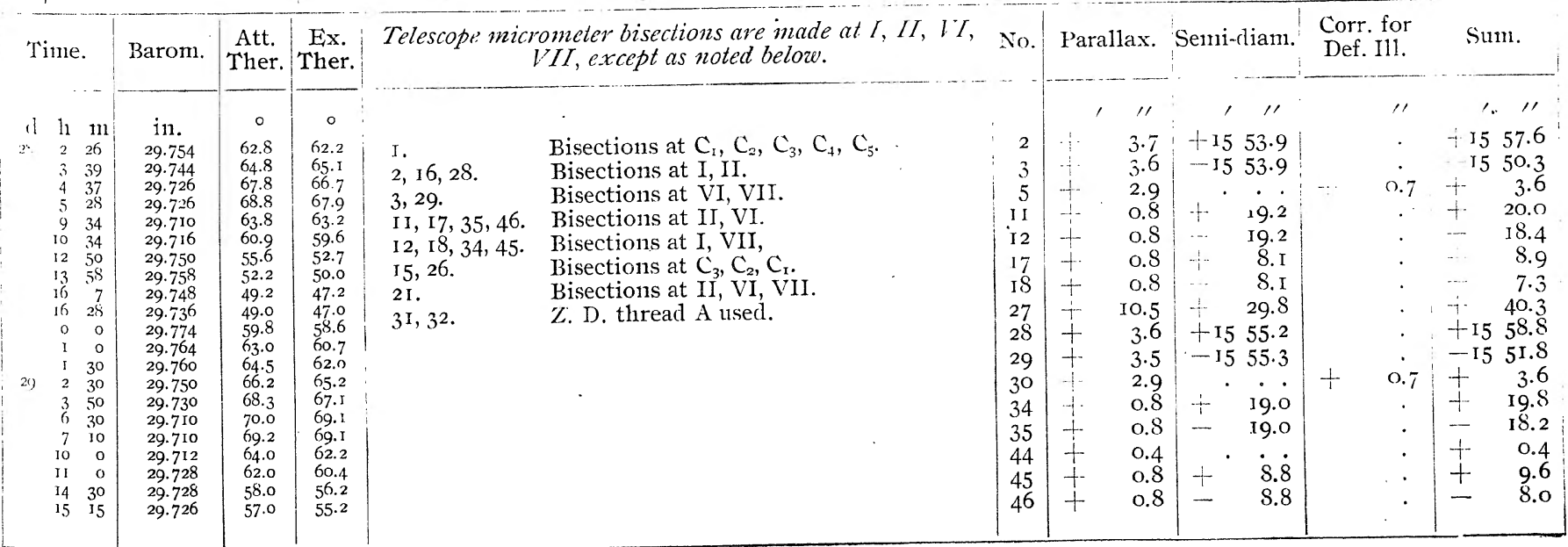




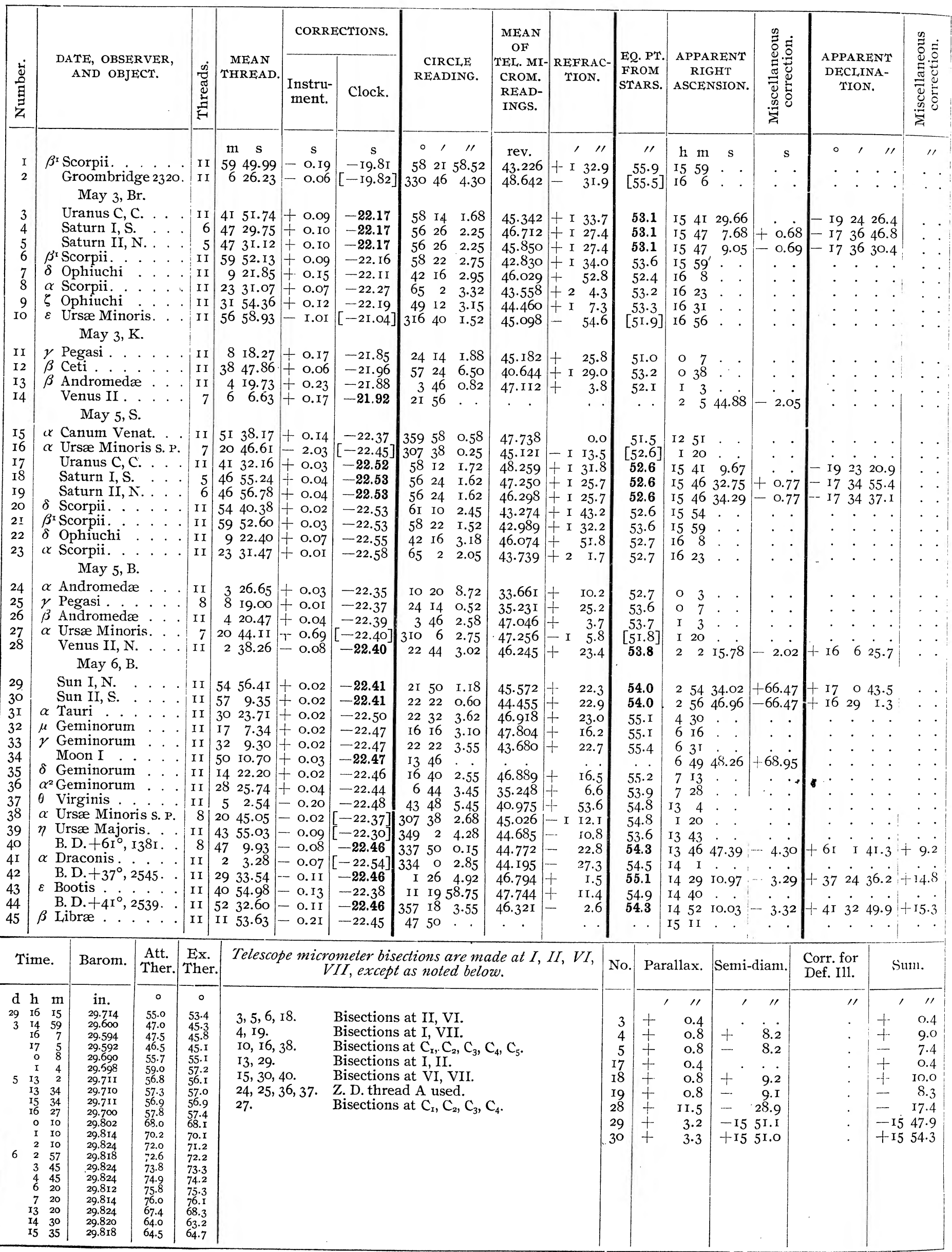




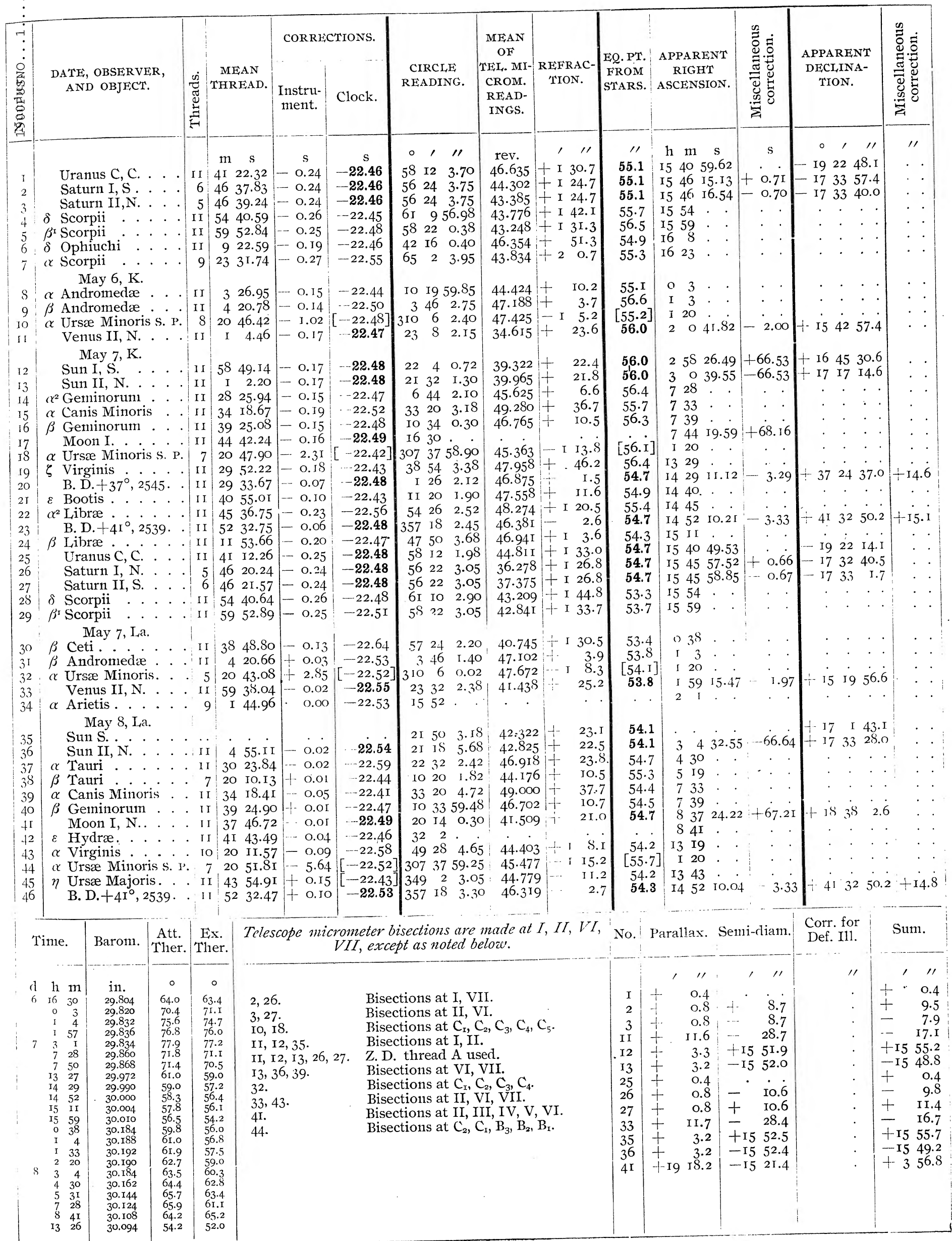




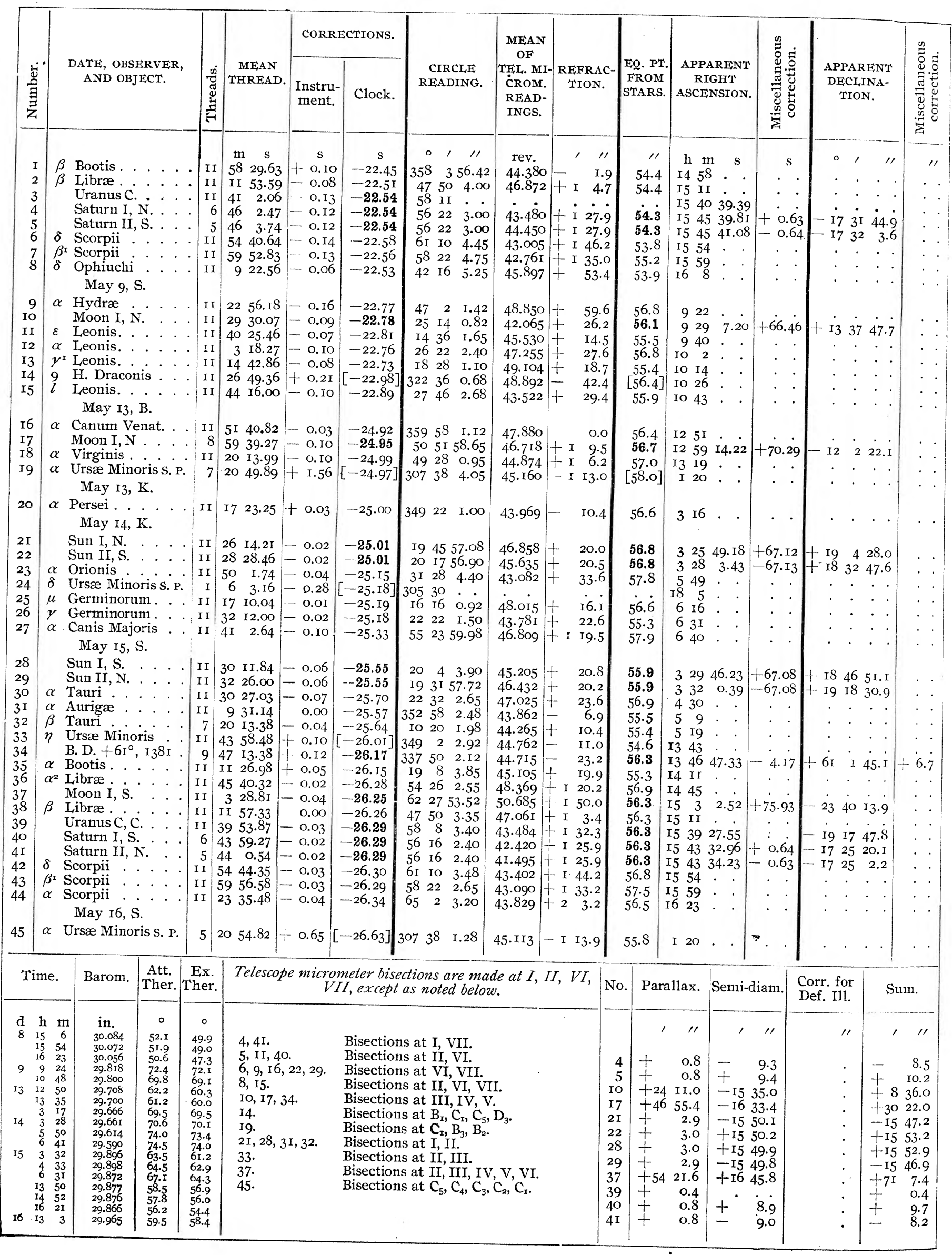




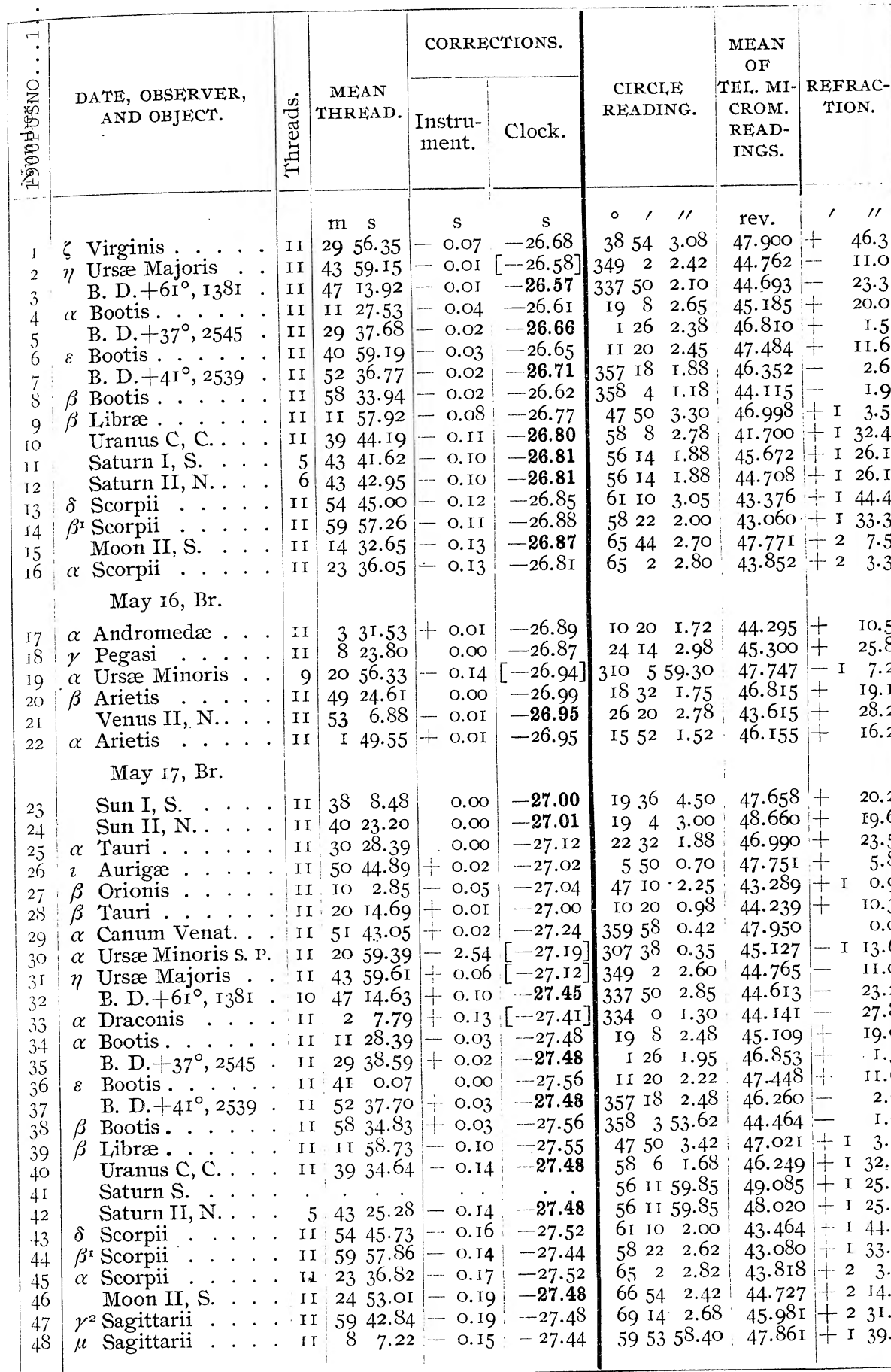

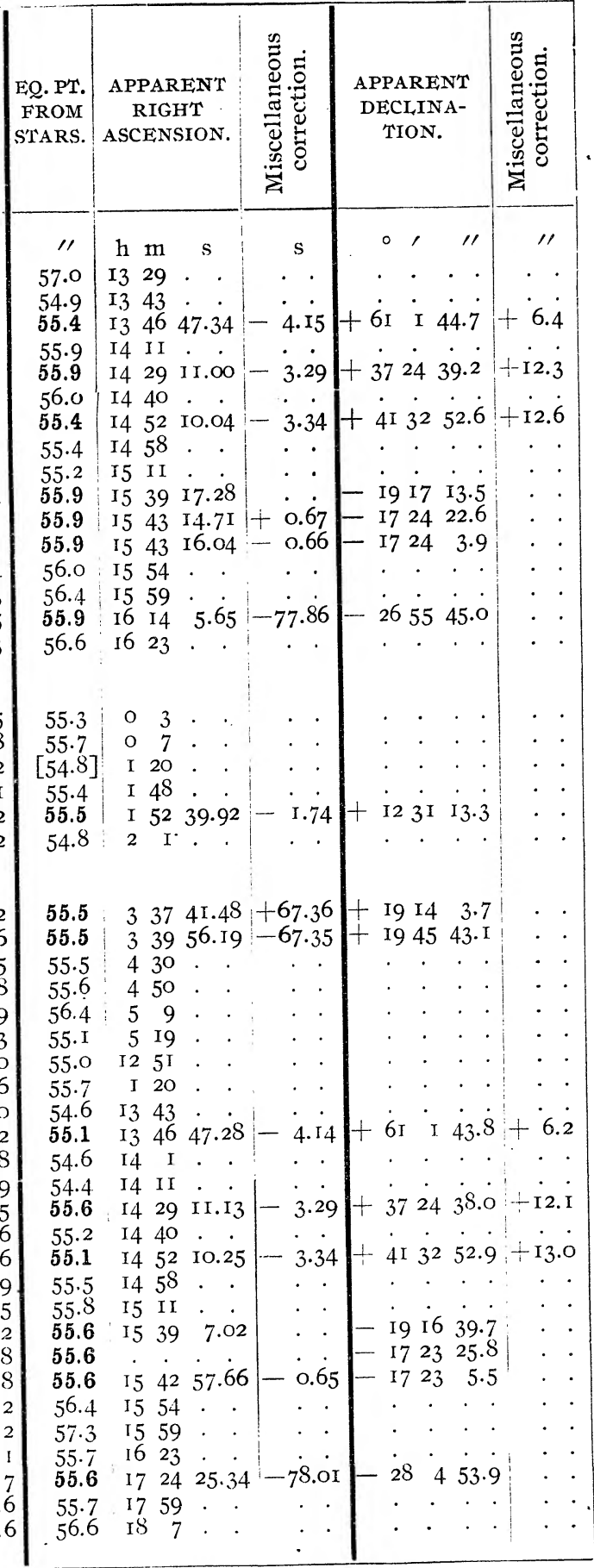

\begin{tabular}{|c|c|c|c|c|c|c|c|c|c|}
\hline Time. & Baronı. & $\begin{array}{l}\text { Alt. } \\
\text { Ther. }\end{array}$ & $\begin{array}{l}\text { Ex. } \\
\text { Ther. }\end{array}$ & $\begin{array}{c}\text { Telescope micrometer bisections are made at I, II, IT, } \\
\text { VII, except as noted below. }\end{array}$ & No. & Parallax. & Semi-diam. & $\begin{array}{l}\text { Corr. for } \\
\text { Def. I11. }\end{array}$ & Sum. \\
\hline 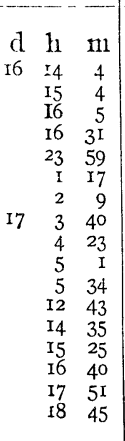 & $\begin{array}{l}\text { i11. } \\
29.963 \\
29.964 \\
29.963 \\
29.961 \\
30.044 \\
30.052 \\
30.056 \\
30.042 \\
30.034 \\
30.030 \\
30.032 \\
30.018 \\
30.010 \\
30.002 \\
30.000 \\
30.004 \\
29.998\end{array}$ & \begin{tabular}{c|}
$\circ$ \\
58.8 \\
57.8 \\
56.8 \\
56.8 \\
61.0 \\
65.8 \\
66.8 \\
67.1 \\
67.5 \\
68.5 \\
69.0 \\
63.0 \\
59.0 \\
58.5 \\
58.0 \\
56.8 \\
55.5
\end{tabular} & $\begin{array}{c}0 \\
57.6 \\
56.4 \\
55.4 \\
55.5 \\
61.2 \\
63.4 \\
64.5 \\
66.2 \\
66.2 \\
67.0 \\
67.1 \\
63.2 \\
57.7 \\
57.5 \\
56.4 \\
55.2 \\
53.9\end{array}$ & $\begin{array}{ll}\text { I, 24. } & \text { Bisections at VI, VII. } \\
2,3,46 . & \text { Bisections at III, IV, V. } \\
7,8 . & \text { Bisections at I, II, VI. } \\
\text { II, 42. } & \text { Bisections at II, VI. } \\
\text { I2, 4I. } & \text { Bisections at I, VII. } \\
\text { I5. } & \text { Bisections at II, III, IV, V, VI. } \\
\text { I9. } & \text { Bisections at } C_{3}, C_{4}, C_{5}, D_{I}, D_{2 .} . \\
23,29,3 \text { r. } & \text { Bisections at I, II. } \\
\text { 30. } & \text { Bisections at } C_{5}, \mathrm{C}_{4}, \mathrm{C}_{3}, \mathrm{C}_{2}, \mathrm{C}_{\mathrm{r}} . \\
32,35 . & \text { Bisections at II, VI, VII. }\end{array}$ & \begin{tabular}{l|} 
IO \\
II \\
I2 \\
I5 \\
2 I \\
23 \\
24 \\
40 \\
41 \\
42 \\
46
\end{tabular} & 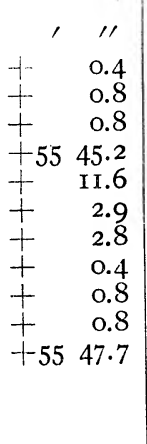 & 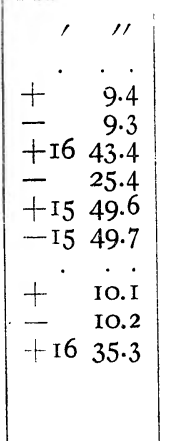 & \begin{tabular}{l|}
$\prime \prime$ \\
$\cdot$ \\
$\cdot$ \\
$\cdot$ \\
$\cdot$ \\
$:$ \\
$:$ \\
$:$ \\
$:$ \\
$:$
\end{tabular} & 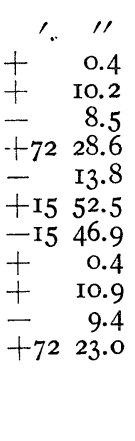 \\
\hline
\end{tabular}




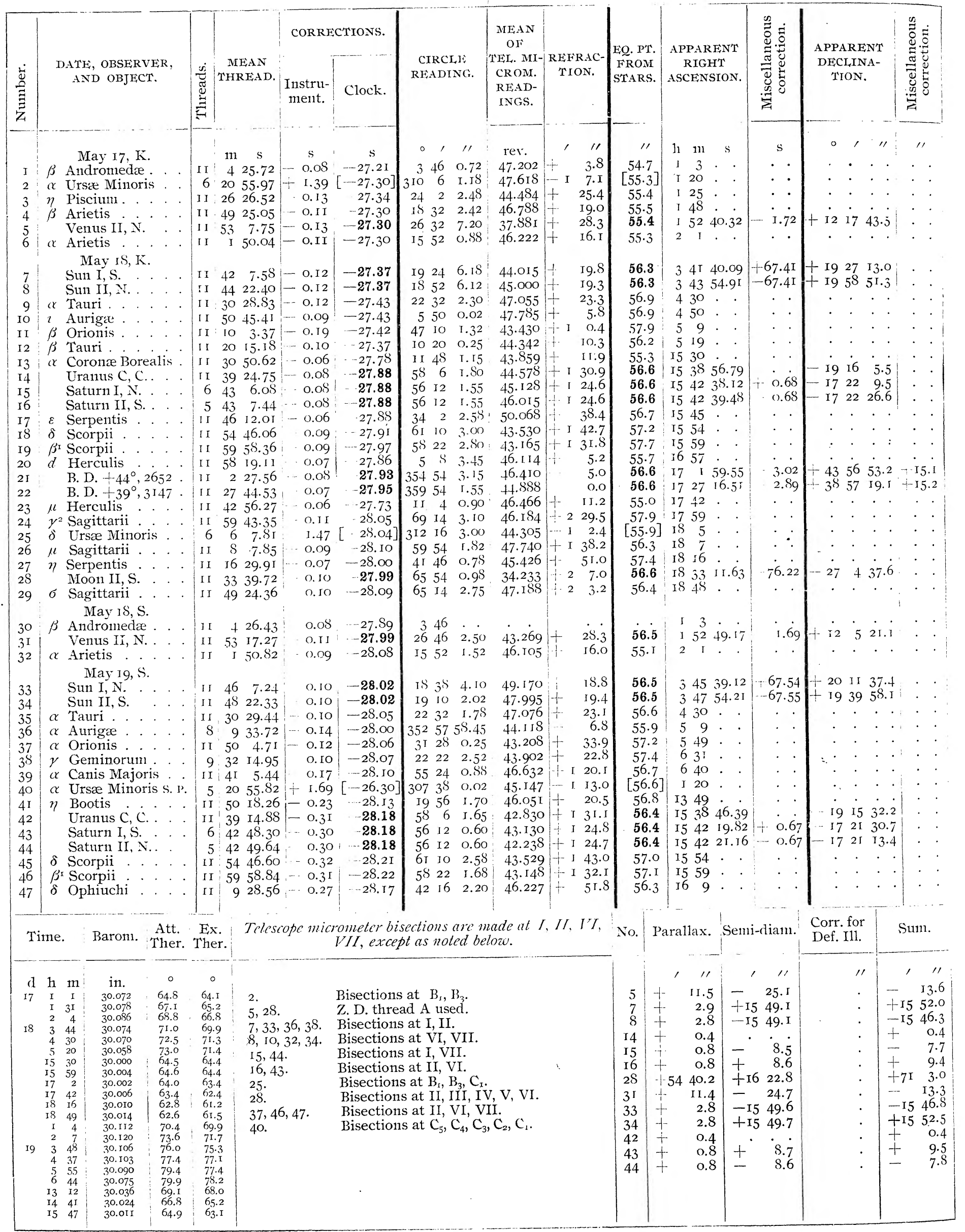




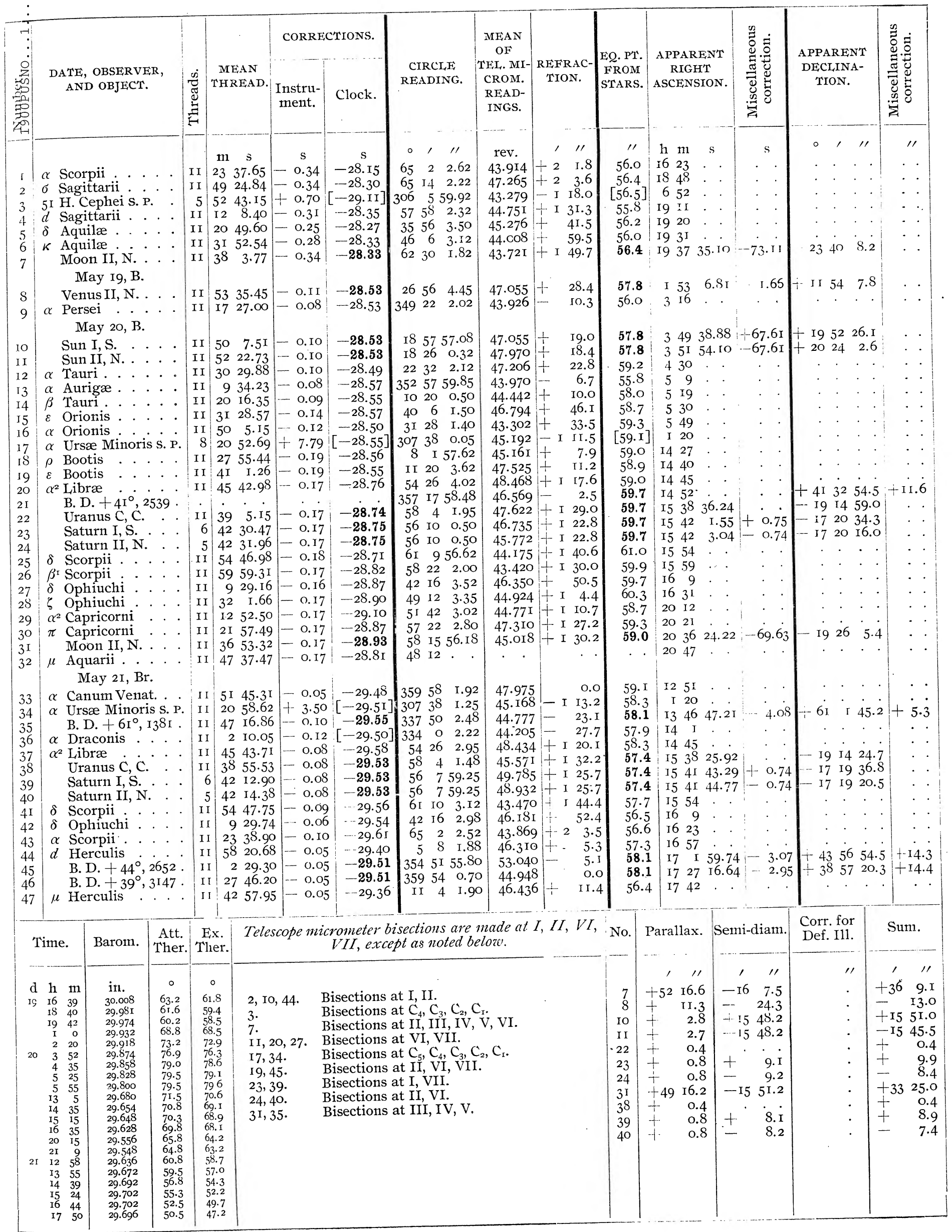




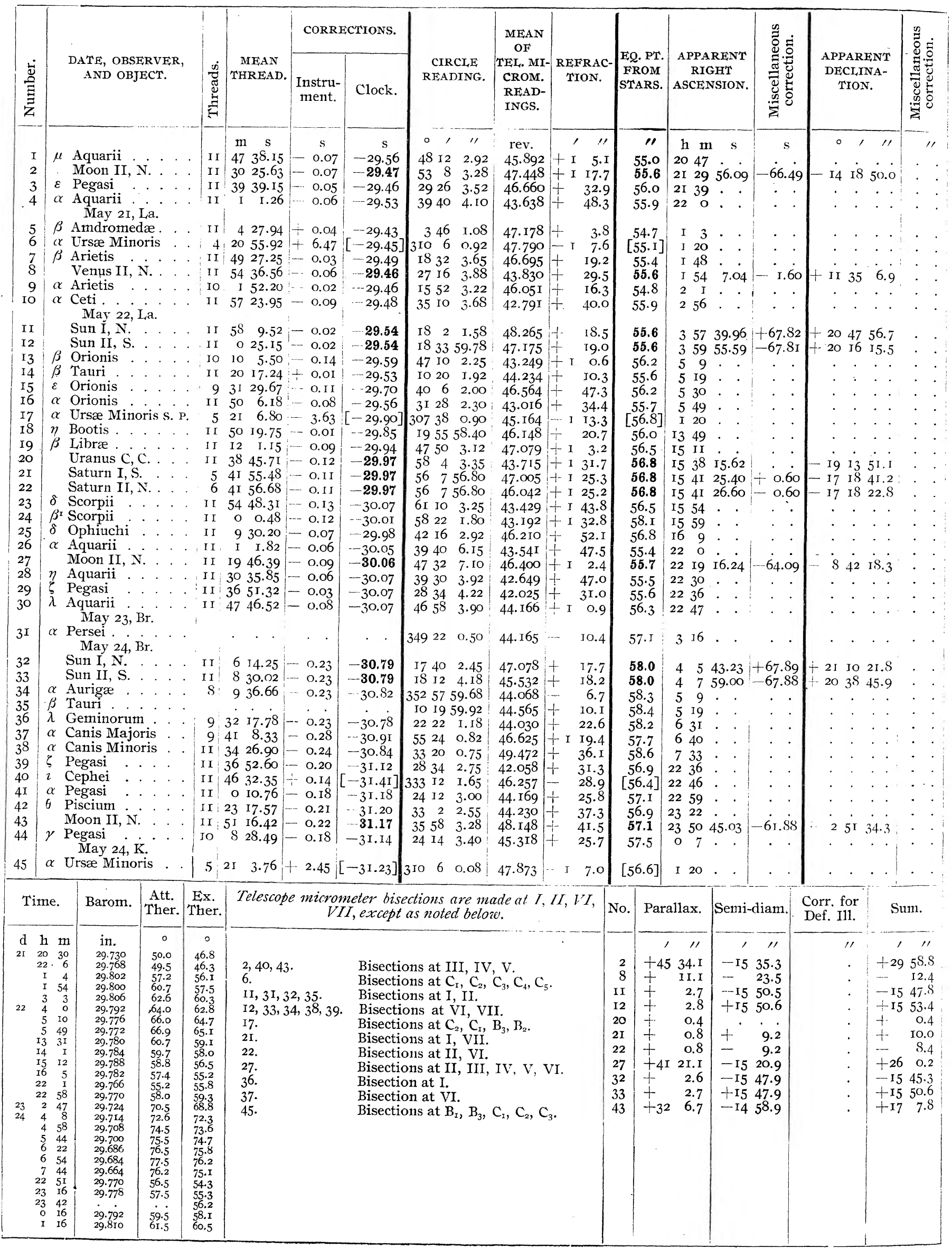



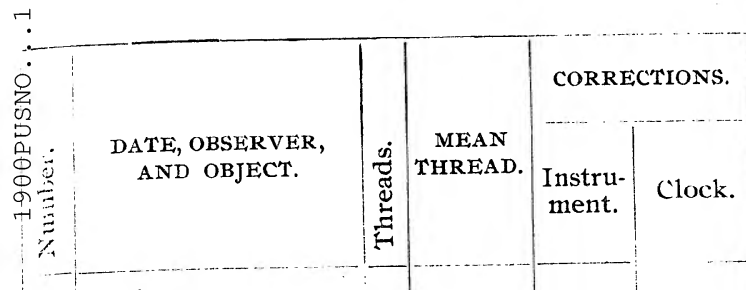

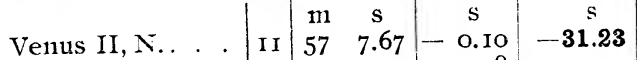

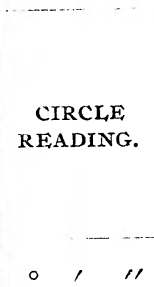
\begin{tabular}{|c|c|} 
MEAN & \\
OF & . \\
TEL.MI- & REFRAC- \\
CROM. & TION. \\
READ- & \\
INGS. & \\
\hline & \\
\hline reV. &, \\
34.671 & + \\
\hline & 29.7 \\
\hline 6.224 & 16.2
\end{tabular}

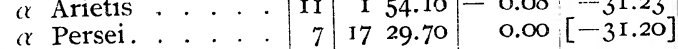
May 25, K.

Sun I, N.

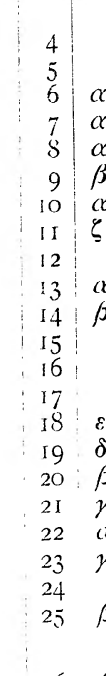




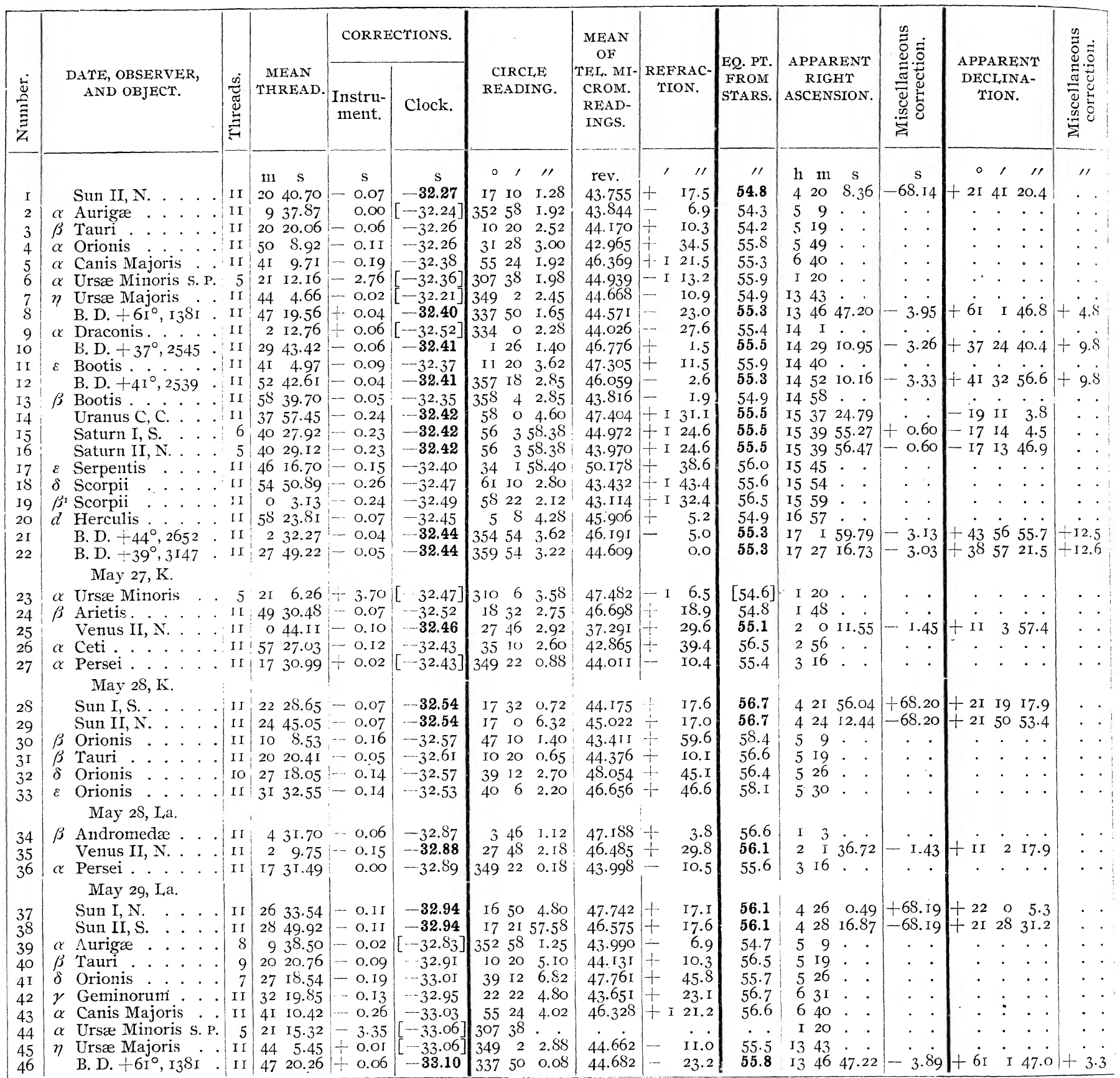

\begin{tabular}{|c|c|c|c|c|c|c|c|c|c|c|}
\hline Time. & Barom. & $\begin{array}{l}\text { Att. } \\
\text { Ther. }\end{array}$ & $\begin{array}{l}\text { Ex. } \\
\text { Ther. }\end{array}$ & $\begin{array}{l}\text { Telescope microl } \\
\text { VI }\end{array}$ & $\begin{array}{l}\text { meter bisections are made at } I, I I, V I, \\
I, \text { except as noted below. }\end{array}$ & No. & Parallax. & Semi-diam. & $\begin{array}{l}\text { Corr. for } \\
\text { Def. Ill. }\end{array}$ & Sum. \\
\hline 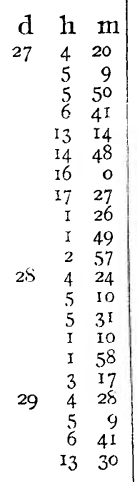 & 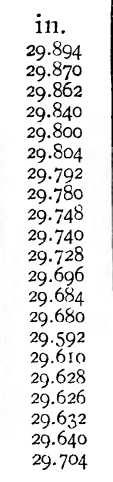 & 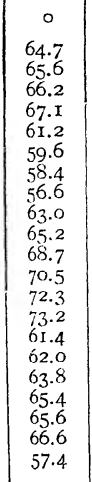 & $\begin{array}{l}\circ \\
63.4 \\
64.4 \\
64.0 \\
6.5 \\
65.5 \\
6.1 \\
59.9 \\
57.5 \\
55.9 \\
63.9 \\
64.4 \\
67.2 \\
69.2 \\
71.2 \\
71.2 \\
59.9 \\
59.3 \\
61.8 \\
62.2 \\
62.2 \\
62.9 \\
63.8 \\
55.8\end{array}$ & $\begin{array}{l}\text { I, 29, 34, 36, } 38 . \\
3 . \\
6 . \\
7,28,37,39,45 . \\
\text { S, } 9,46 . \\
\text { I5. } \\
\text { I6. } \\
23 . \\
25 .\end{array}$ & $\begin{array}{l}\text { Bisections at VI, VII. } \\
\text { Bisections at I, II, VI. } \\
\text { Bisections at } \mathrm{C}_{1}, \mathrm{C}_{2}, \mathrm{C}_{3}, \mathrm{C}_{4}, \mathrm{C}_{5} . \\
\text { Bisections at } \mathrm{I} \text {. II. } \\
\text { Bisections at III, III, V, VI. } \\
\text { Bisections at II, VII. } \\
\text { Bisection at VII. } \\
\text { Bisections at } \mathrm{D}_{1}, \mathrm{D}_{3} . \\
\text { Z. D. thread } \mathrm{A} \text { used. }\end{array}$ & $\begin{array}{r}\mathrm{x} \\
14 \\
15 \\
16 \\
25 \\
28 \\
29 \\
35 \\
37 \\
38\end{array}$ & $\begin{array}{rr} & 11 \\
+ & 2.6 \\
+ & 0.4 \\
+ & 0.8 \\
+ & 0.8 \\
+ & 10.2 \\
+ & 2.6 \\
+ & 2.5 \\
+ & 10.1 \\
+ & 2.5 \\
+ & 2.6\end{array}$ & 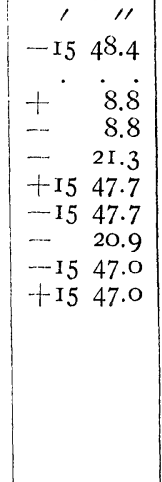 & $\begin{array}{l}\prime \prime \\
. \\
.\end{array}$ & $\begin{array}{rr} & 11 \\
-15 & 45.8 \\
+ & 0.4 \\
+ & 9.6 \\
- & 8.0 \\
- & 11.1 \\
+ \text { r5 } & 50.3 \\
-15 & 45.2 \\
- & 10.8 \\
-15 & 44.5 \\
+15 & 49.6\end{array}$ \\
\hline
\end{tabular}




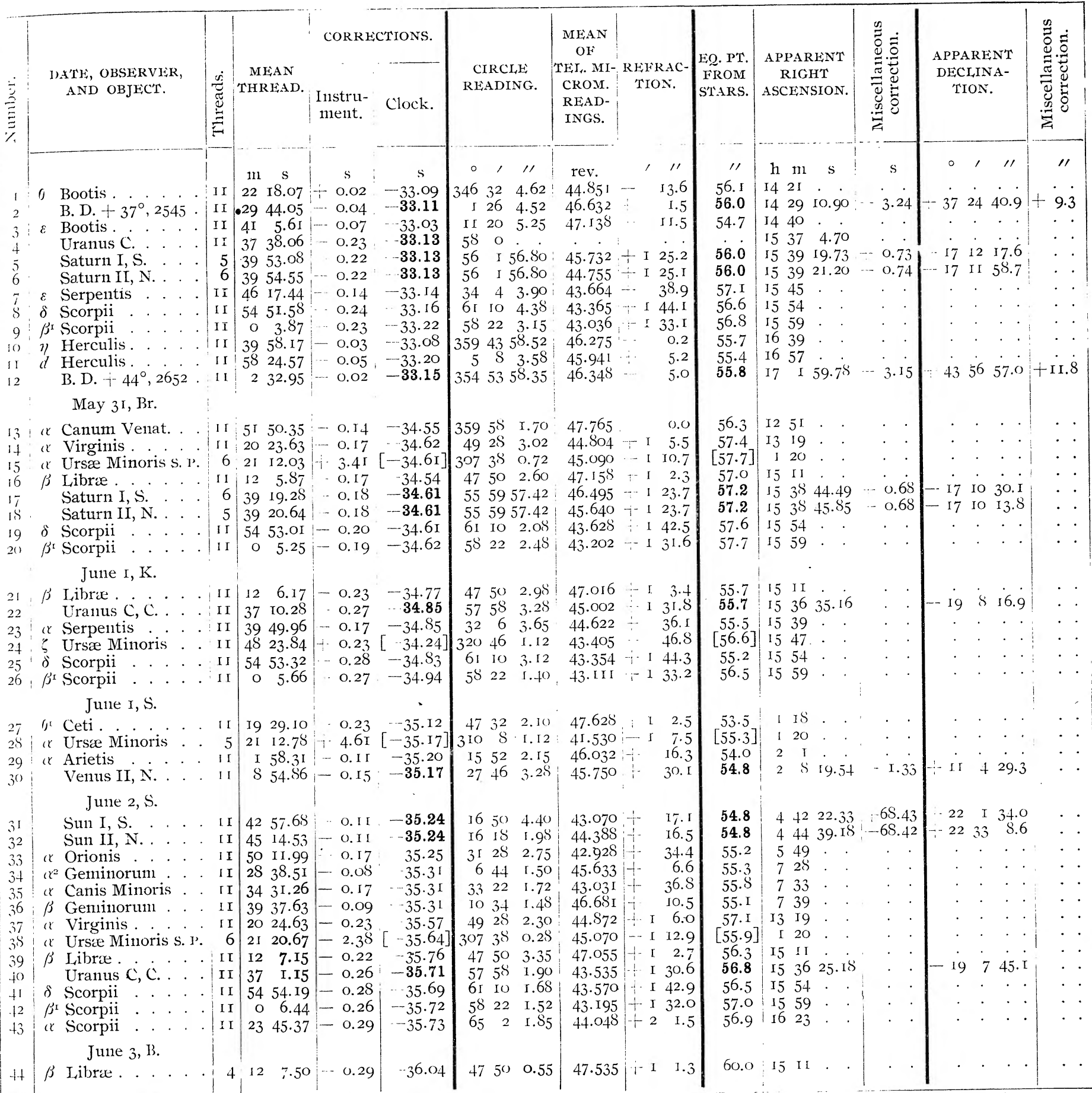

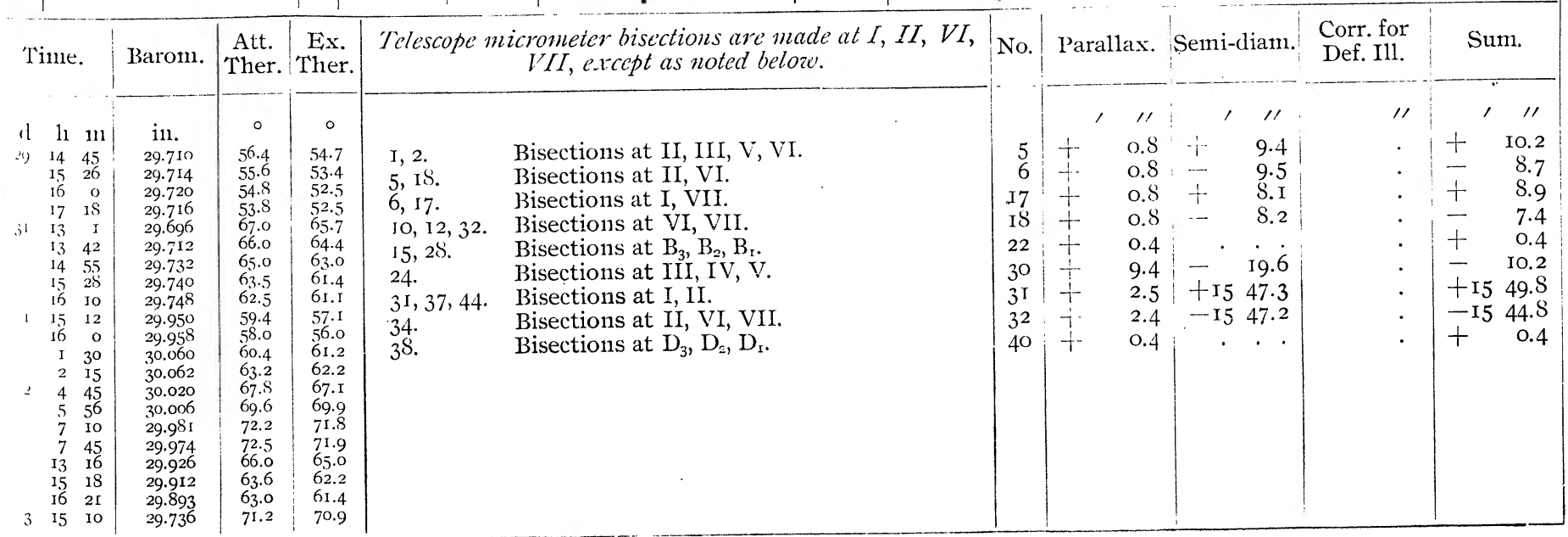




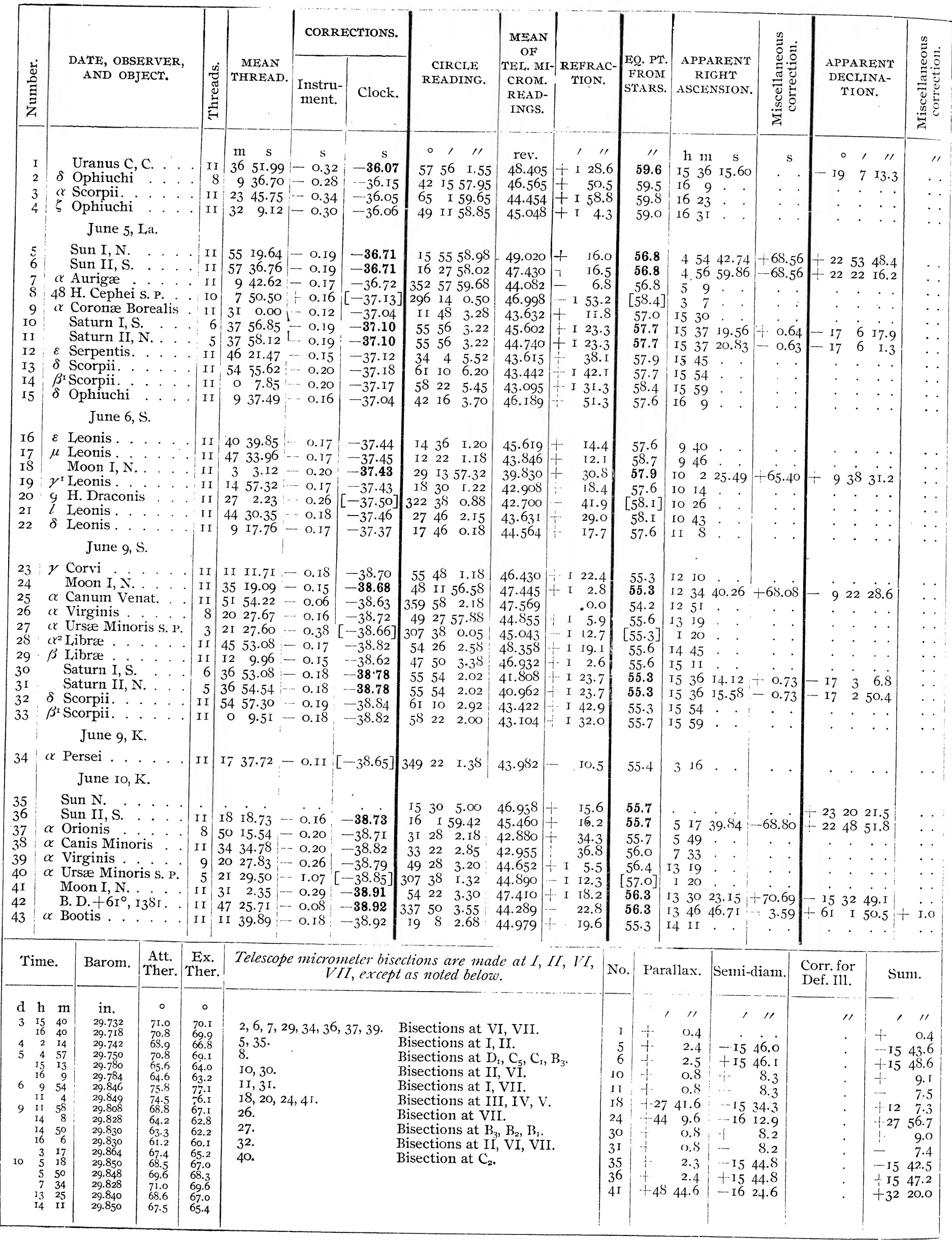




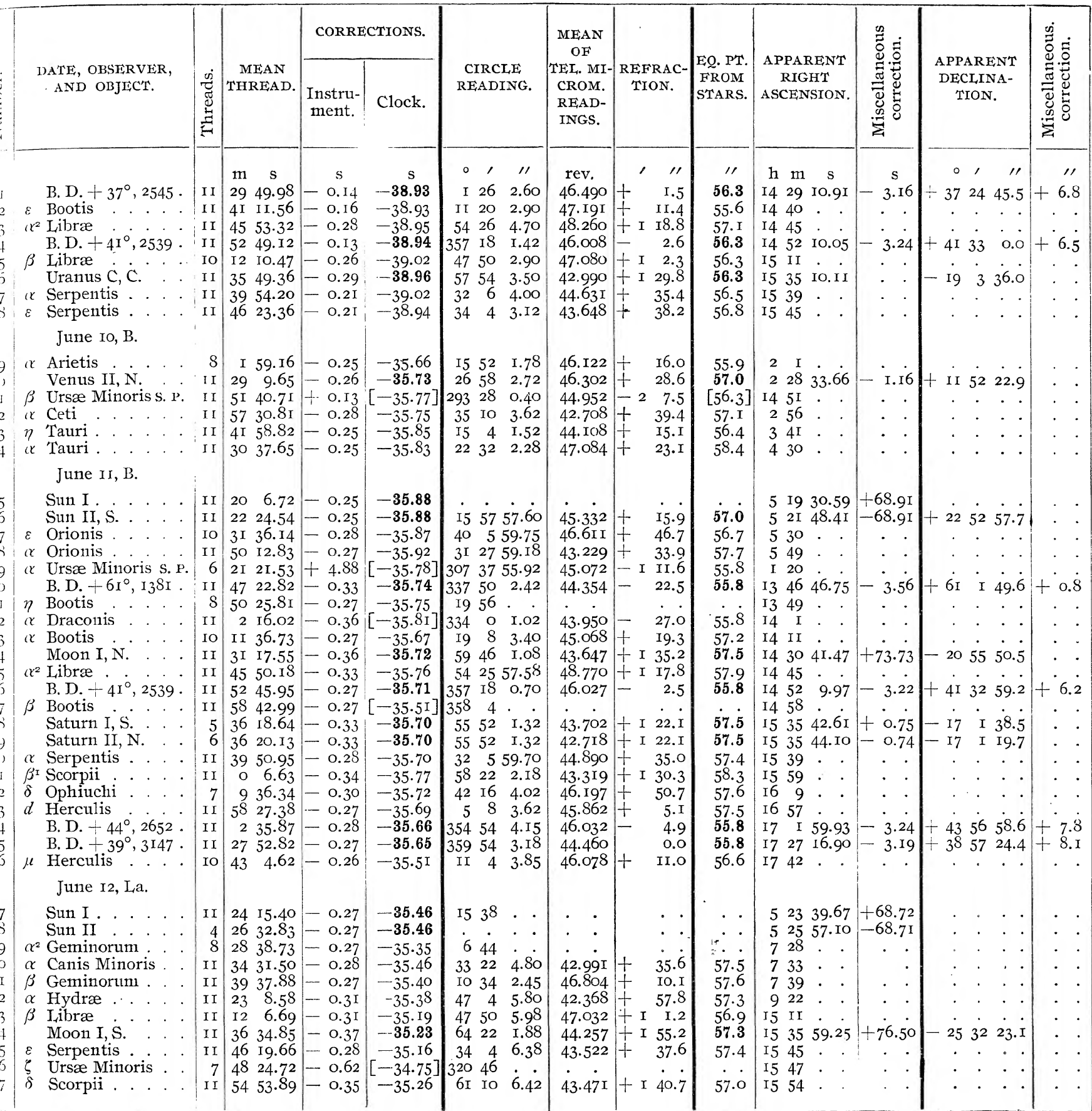

\begin{tabular}{|c|c|c|c|c|c|c|c|c|c|}
\hline Time. & Barom. & $\begin{array}{l}\text { Att. } \\
\text { Ther. }\end{array}$ & $\begin{array}{c}\text { Ex. } \\
\text { Ther. }\end{array}$ & $\begin{array}{c}\text { Telescope micrometer bisections are made at } I, I I, V I, \\
V I I, \text { except as noted below. }\end{array}$ & No. & Parallax. & Semi-diam. & $\begin{array}{l}\text { Corr. for } \\
\text { Def. I11. }\end{array}$ & Sum. \\
\hline 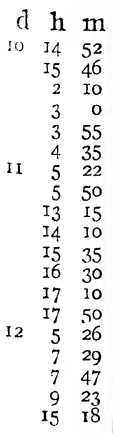 & $\begin{array}{l}\text { in. } \\
29.852 \\
29.848 \\
29.884 \\
29.894 \\
29.890 \\
29.884 \\
29.866 \\
29.860 \\
29.754 \\
29.754 \\
29.752 \\
29.742 \\
29.726 \\
29.704 \\
29.673 \\
29.644 \\
29.638 \\
29.612 \\
29.632\end{array}$ & $\begin{array}{c}\circ \\
65.9 \\
65.9 \\
67.0 \\
72.0 \\
74.2 \\
73.8 \\
73.8 \\
74.8 \\
75.0 \\
72.2 \\
71.0 \\
70.0 \\
69.8 \\
69.0 \\
68.2 \\
80.0 \\
82.0 \\
83.4 \\
84.0 \\
70.8\end{array}$ & \begin{tabular}{c|}
$\circ$ \\
64.3 \\
64.3 \\
67.7 \\
69.6 \\
71.1 \\
72.1 \\
73.5 \\
73.9 \\
71.1 \\
70.1 \\
68.6 \\
68.3 \\
67.5 \\
67.1 \\
79.6 \\
82.0 \\
82.9 \\
84.3 \\
69.7
\end{tabular} & $\begin{array}{ll}3,16,42 . & \text { Bisections at VI, VII. } \\
\text { II. } & \text { Bisections at } C_{5}, C_{4}, C_{3}, C_{2}, C_{1} . \\
\text { I2. } & \text { Bisections at I, VI, VII. } \\
\text { I9. } & \text { Bisections at } C_{3}, C_{2}, C_{1} . \\
22 . & \text { Bisections at I, II. } \\
23,32 . & \text { Bisections at I, II, VII. } \\
24 . & \text { Bisections at III, IV, V. } \\
25,26,30,35 . & \text { Bisections at II, VI, VII. } \\
28 . & \text { Bisections at I, VII. } \\
29 . & \text { Bisections at II, VI. } \\
44 . & \text { Bisections at II, III, IV, V, VI. }\end{array}$ & $\begin{array}{r}6 \\
10 \\
16 \\
24 \\
28 \\
29 \\
44\end{array}$ & \begin{tabular}{rr} 
& \multicolumn{1}{r}{} \\
$+\quad$ & 0.4 \\
$+\quad$ & 8.0 \\
+ & 2.4 \\
+ & 17.5 \\
+52 & 0.8 \\
+ & 0.8 \\
+ &
\end{tabular} & 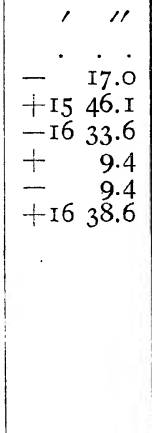 & $\begin{array}{l}11 \\
: \\
\cdot \\
\cdot \\
\cdot \\
\cdot \\
\cdot\end{array}$ & \begin{tabular}{lr}
\multicolumn{1}{r}{11} \\
$+\quad$ & 0.4 \\
+ & 9.0 \\
+15 & 48.5 \\
+35 & 43.9 \\
+ & 10.2 \\
+ & 8.6 \\
+71 & 30.1
\end{tabular} \\
\hline
\end{tabular}

$6855-9$ 


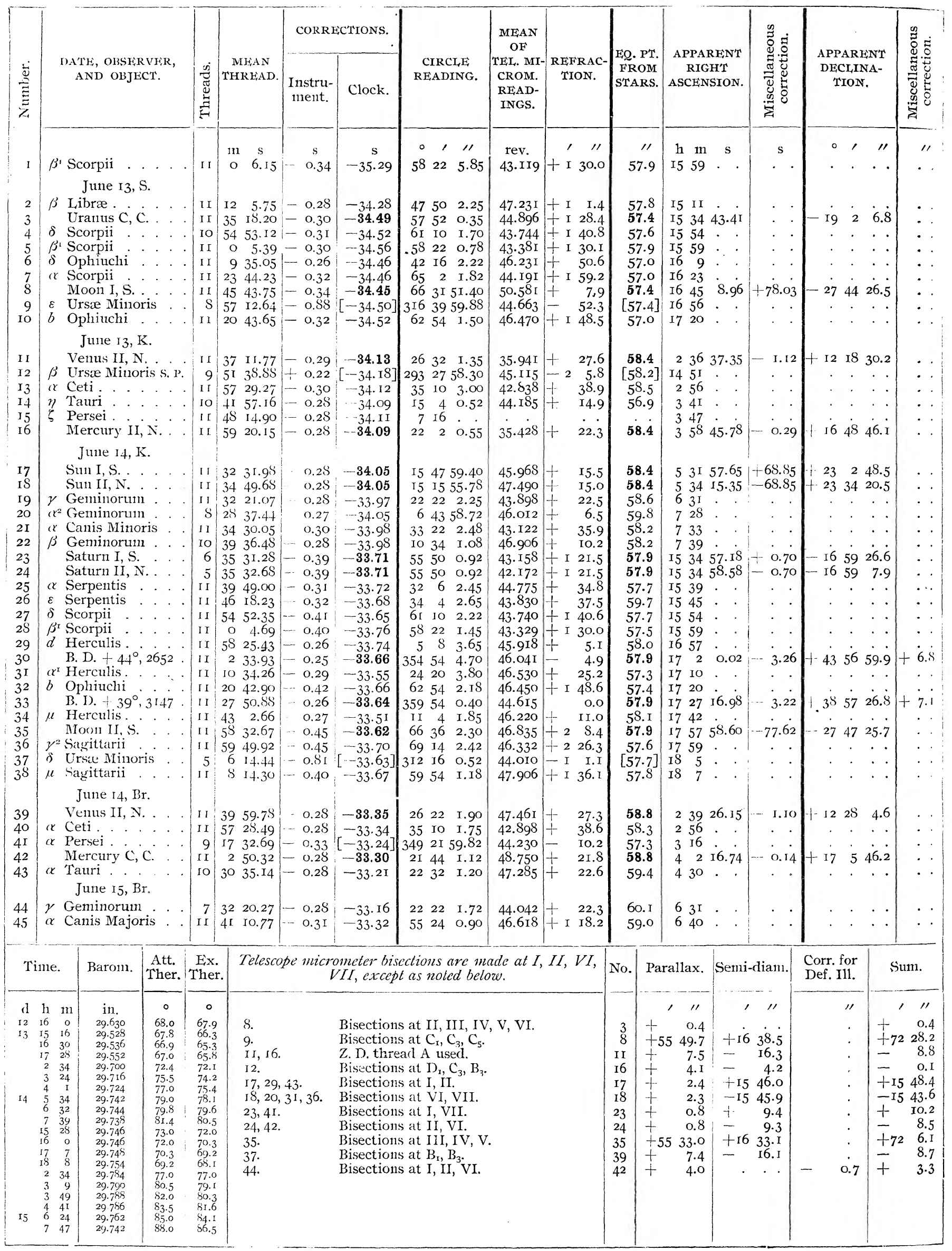




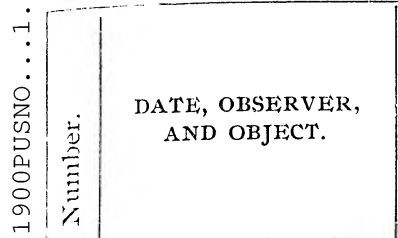

June $15, S$.

2 Orionis.

Sun I, N. . . . . II $4048.94-0.26-32.49$

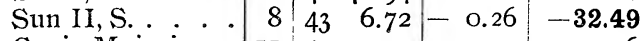

5 \& Canis Majoris. . II $4 \mathrm{I}$ 10.00 $-0.30-32.56$

6 \& 2 Canis vininoris . . II $3428.43-0.26-32.40$

7 $\beta$ Geminorum. . I I $3934.85-0.26-32.37$

June I8, $\mathrm{K}$.

Moon II, N. . . . I I $596.53-0.36-31.08$

9 a Aquarii . . . . . II I $4.03-0.3 \mathrm{I}$-3I.I7

IO 9 Aquarii .... . II II $58.35-0.34-3$ I.I I

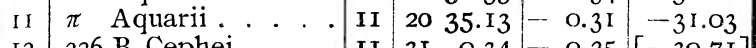

I2 226 B. Cephei . . . . II 3 I $0.34-0.25$ [ June I8, La.

I4 \& \&auri . . . . . . II $30 \begin{array}{llllllll}32.94 & -0.26 & -30.95 & 22 & 32 & 7.02\end{array}$

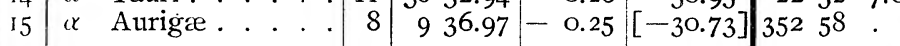

June I9, La.

Sun I, N. . . . . . I I 53 I5.90 5 - $0.25-30.91 \mid \begin{array}{llll}\text { I5 } & 8 & \text { I. } 22\end{array}$

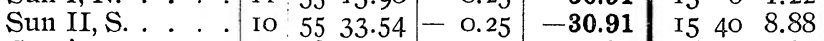

is $\alpha^{2}$ Geminorum Io $2834.22-0.25-30.84$

19 « Canis Minoris . . I I $34 \quad 26.93-0.27-30.89$

$\begin{array}{lllll}33 & 22 & 6.40\end{array}$

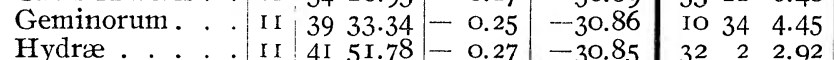

$22 \quad 2 \quad$ Ursæ Majoris . . II $52 \quad 42.29$ - 0.26 [-30.77] $35024 \quad 5.08$ June $20, \mathrm{~S}$.

$23 \lambda$ Aquarii

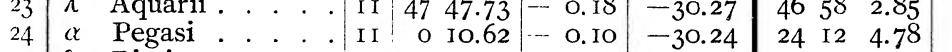

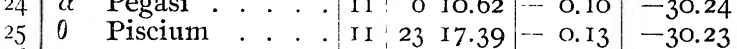

Moon II, N. . . . $\quad$ I I 34 I 2.54 - o. I5 -30.25

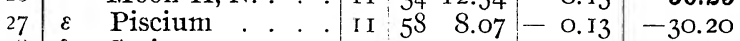

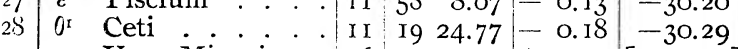
$29 \alpha \begin{gathered}\text { Ursæ Minoris . . } \\ \text { June 20, Br. }\end{gathered}$

$30 \quad \gamma$ Ceti $\ldots \ldots$ II 38 29.6I - o. I7 -30.25

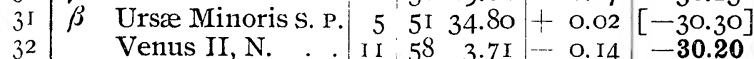

$33 \gamma$ Tauri..... II 1427.45 - o. I4 -30.23

34 Mercury C, C. . . I I 2929.82 - o.13 - 30.1

$35 \alpha$ Tauri..... 9 . 3032.01 - 0.14 -30.10 June 2I, Br.

Sun I, S.

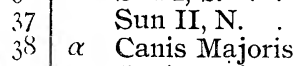

\begin{tabular}{l|ll|l|l}
. I I & I 34.07 & -0.12 & $\mathbf{- 3 0 . 1 8}$
\end{tabular}

\begin{tabular}{l|ll|l|r|}
11 & 1 & 34.07 & 0.12 & -30.18 \\
II & 3 & 5 I.98 & -0.12 & -30.18 \\
\hline & 4 & 7.65 & -0.23 & -30.25
\end{tabular}

II 4 I $\quad 7.65=0.23-30.25$

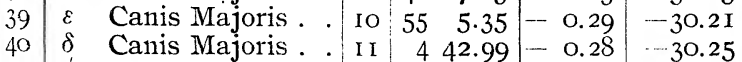

4I $\alpha^{2}$ Geminorum.

$43 \beta$ Geminorum . I I 34 26.09 - o.16 -30 . I5 $\begin{array}{lll}33 & 2 & 3.70\end{array}$

$\begin{array}{llll}37 & 58 & 3.40\end{array}$

$3 \mathrm{I} 3 \mathrm{O}$ 4.15

$\begin{array}{lll}47 & 32 & 2.30\end{array}$

$\begin{array}{lll}36 & 2 & 3.58\end{array}$

\section{8}

25 I8 3.60

$\begin{array}{lll}23 & 28 & 4.80 \\ 19 & 44 & 2.55\end{array}$

$\begin{array}{llr}\text { I9 } & 44 & 2.55 \\ 22 & 31 & 57.38\end{array}$

$\begin{array}{lll}5 & 40 & 1.82\end{array}$

$\begin{array}{rrr}15 & 8 & 0.32 \\ 55 & 24 & 2.22\end{array}$

$\begin{array}{lll}55 & 24 & 2.22 \\ 67 & 38 & 3.20\end{array}$

$\begin{array}{llll}65 & \cdot 2 & 3.00\end{array}$

$\begin{array}{rrr}6 & 44 & 2.25 \\ 33 & 22 & 1.85\end{array}$

$\begin{array}{lll}33 & 22 & 1.85 \\ \text { IO } & 34 & \text { I. } 42\end{array}$

\begin{tabular}{|c|r|}
\hline MEAN & \\
OF & \\
TEL. MI- & REFRAC \\
CROM. & TION. \\
READ- & \\
INGS. & \\
\hline & \\
rev. &,
\end{tabular}

$44.345-\quad 6.6$
$43.395+\quad 58.3$

$45.880+\quad 14.8$

$44.175+15.3$

$43.266+35.5$

$46.960+\quad$ IO.I

34.475 t. I 7.0

$43.565+47.0$

$44.470+$ I I. I

$48.440+44.3$

$46.27 \mathrm{I}-42.3$

44.08 I + I 0.8

$46.766+22.9$

..

$46.732+14.9$

$44.6 \mathrm{I} 2+\quad 15.4$
$45.695+\quad 6.5$

$42.902+$

$46.725+$

$48.080+34$

$\begin{array}{r}46.790-\quad 9.2 \\ \hline\end{array}$

$43.940+\mathrm{I} \quad \mathrm{I} .2$

$43.842+25.7$

$43.954+37.2$

$43.859+34.5$
$46.039+35.0$

$47.555+\mathrm{r} 2.3$

48.025 - I 7.3

$45.8 \mathrm{ro}+4 \mathrm{I} .3$

$45.252+26.9$

$44.900+24.6$

$44.130+20.3$

$47.192+23.5$

$43.842+\quad \mathrm{I} 5.8$

$45.360+\quad 15.3$

$46.194+$ I 21.3

$47.979+215.7$

$48.4 \mathrm{OI}+2$ O.I

$45.762+$

$43.025+$

$46.422+$

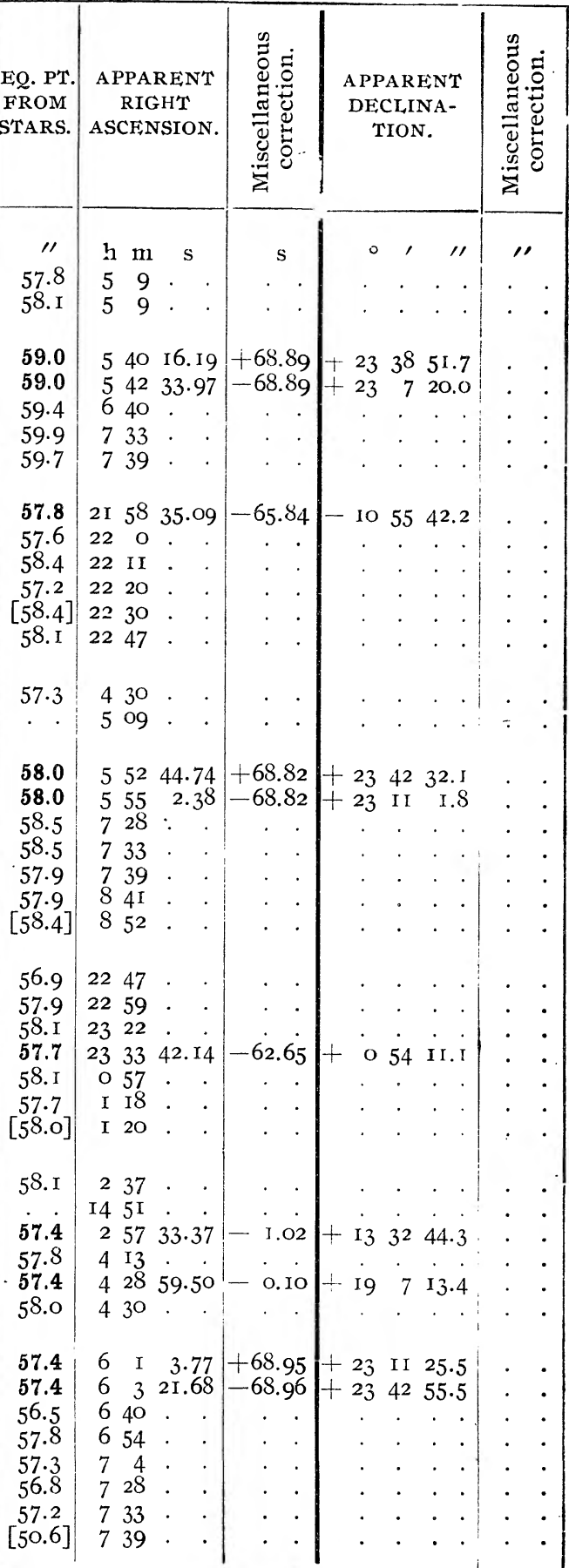

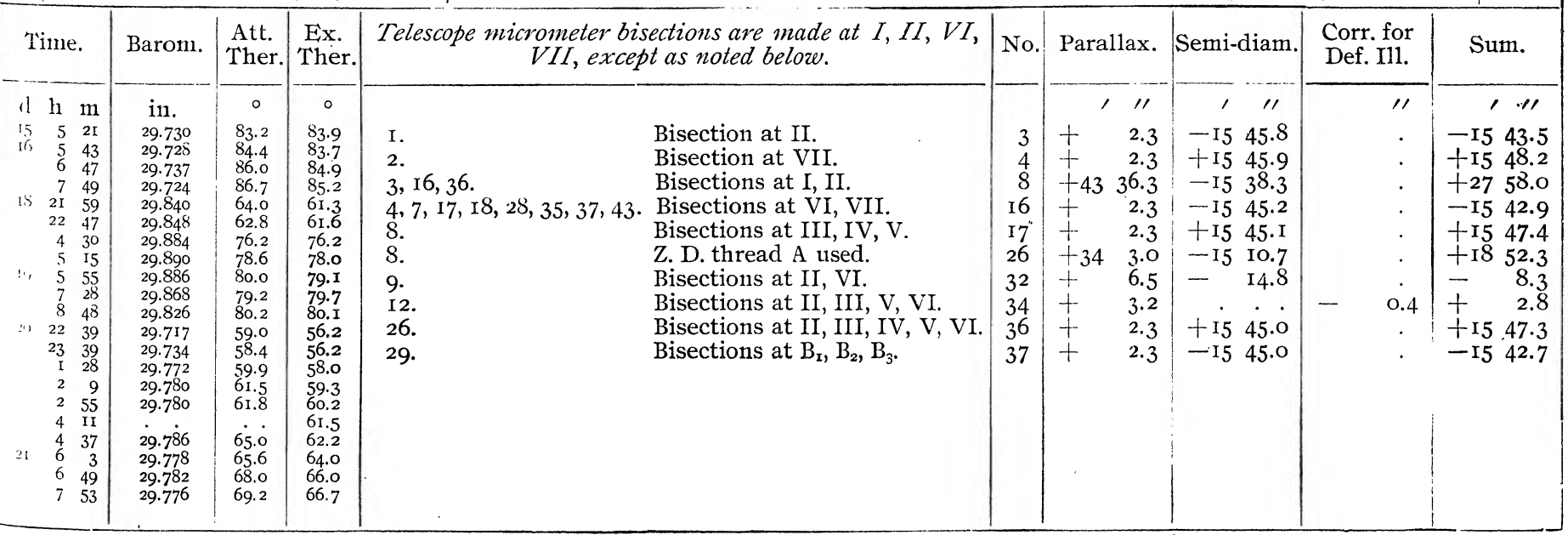




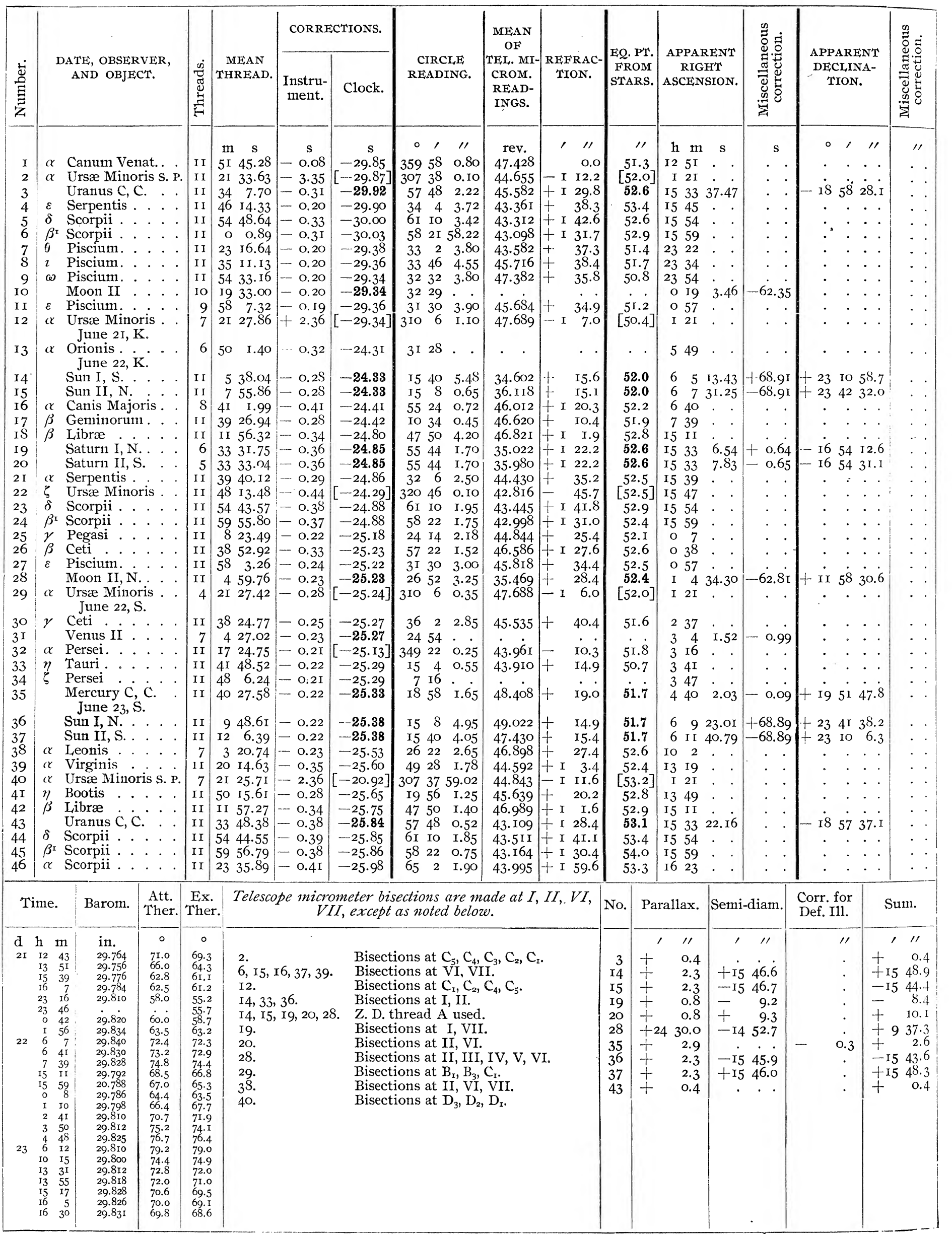




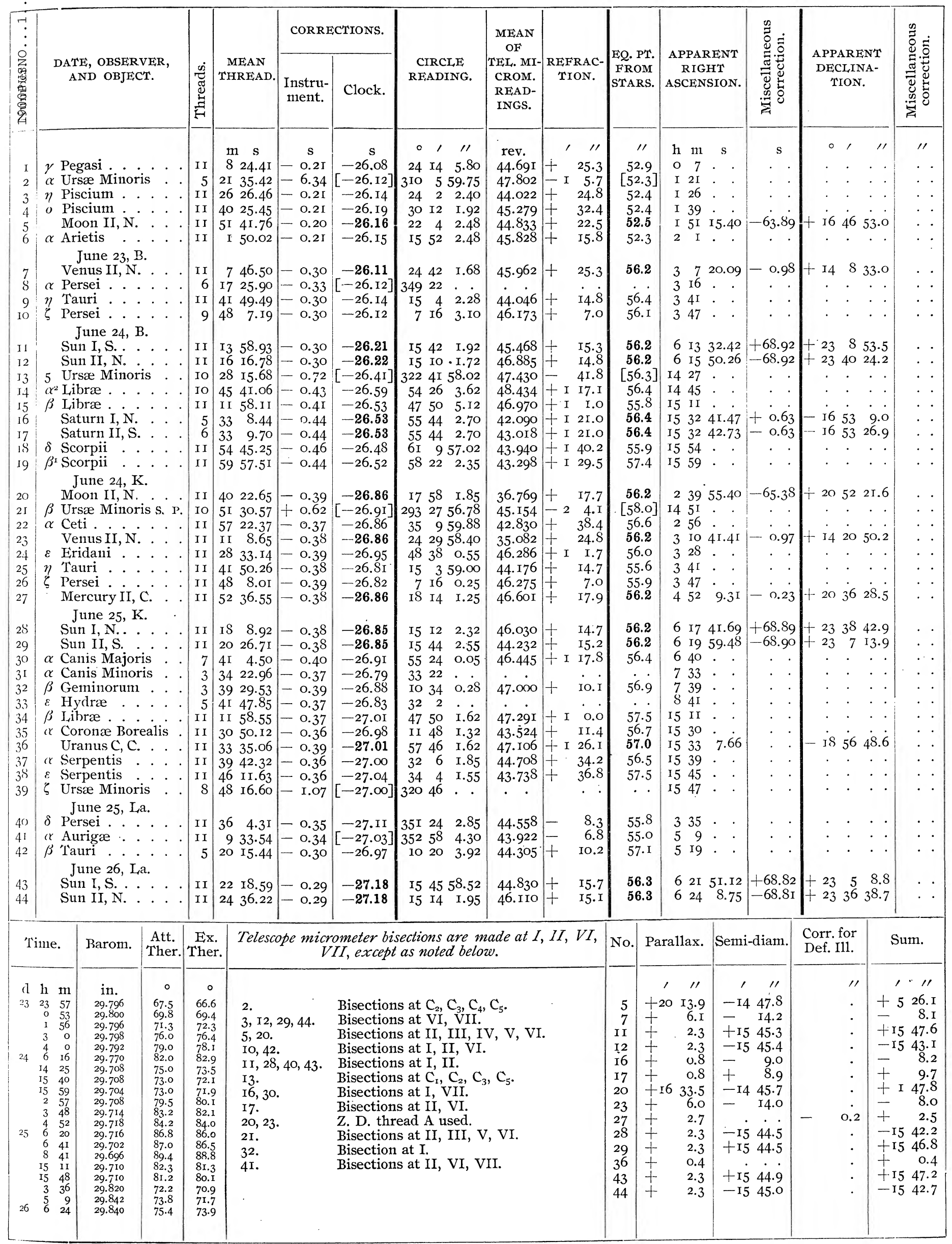




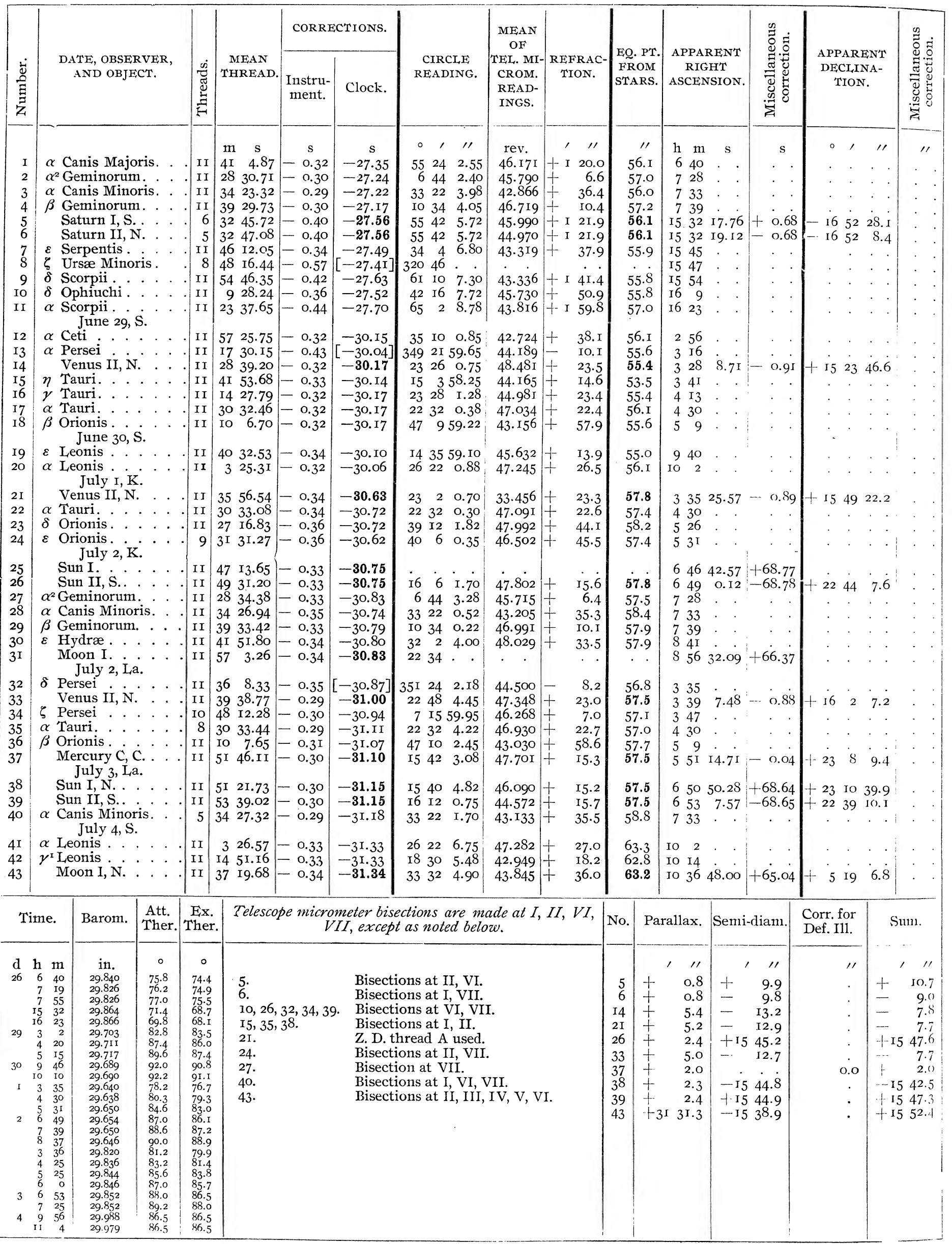




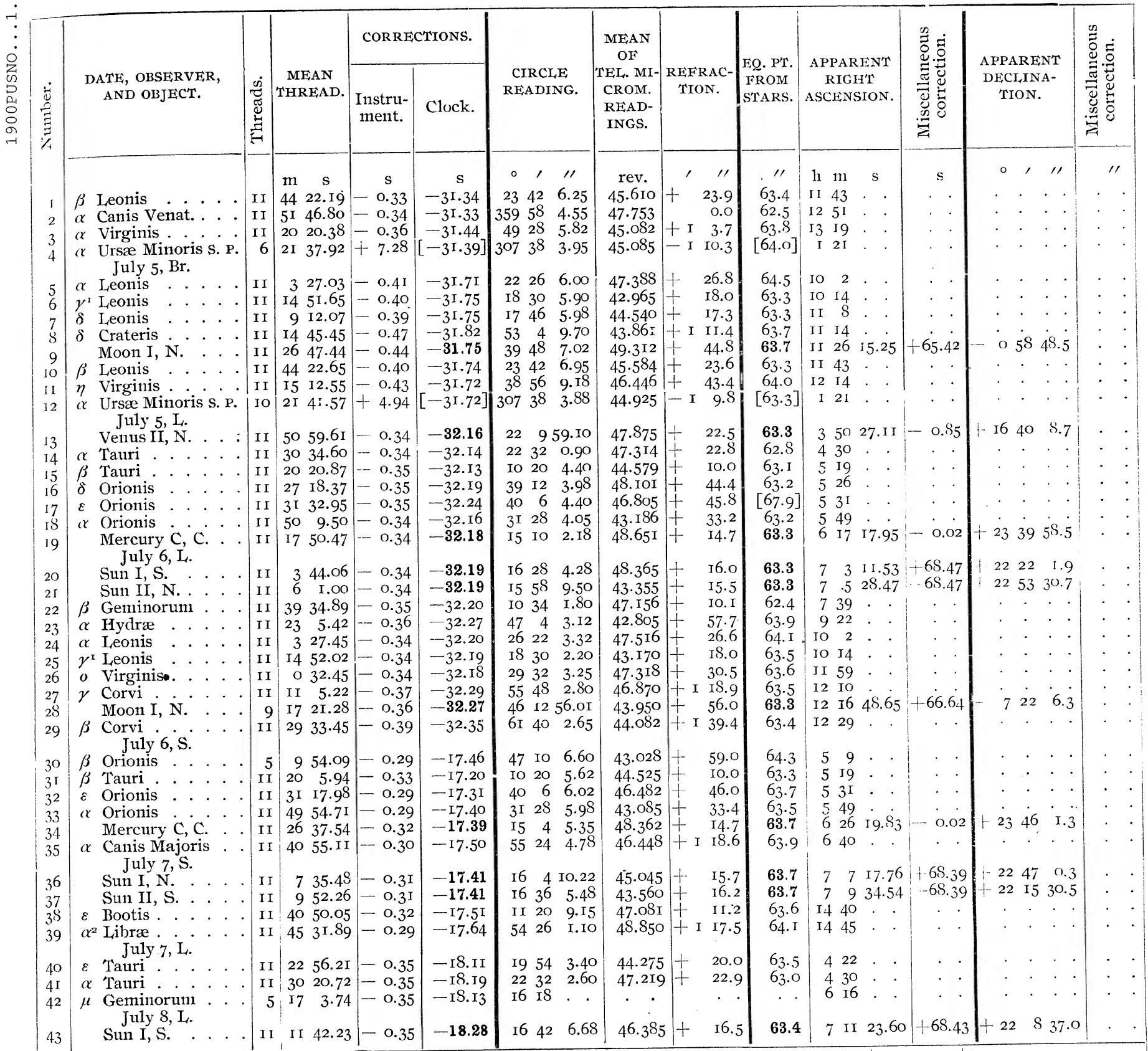

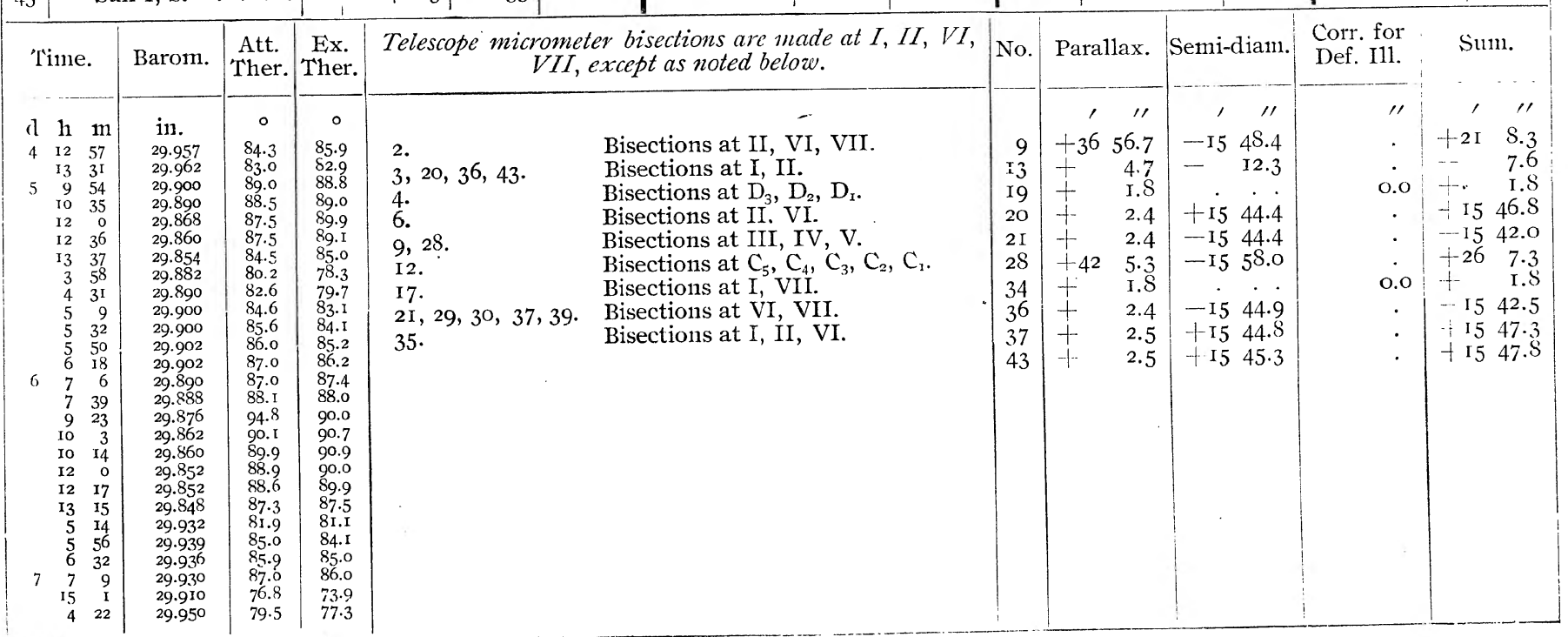




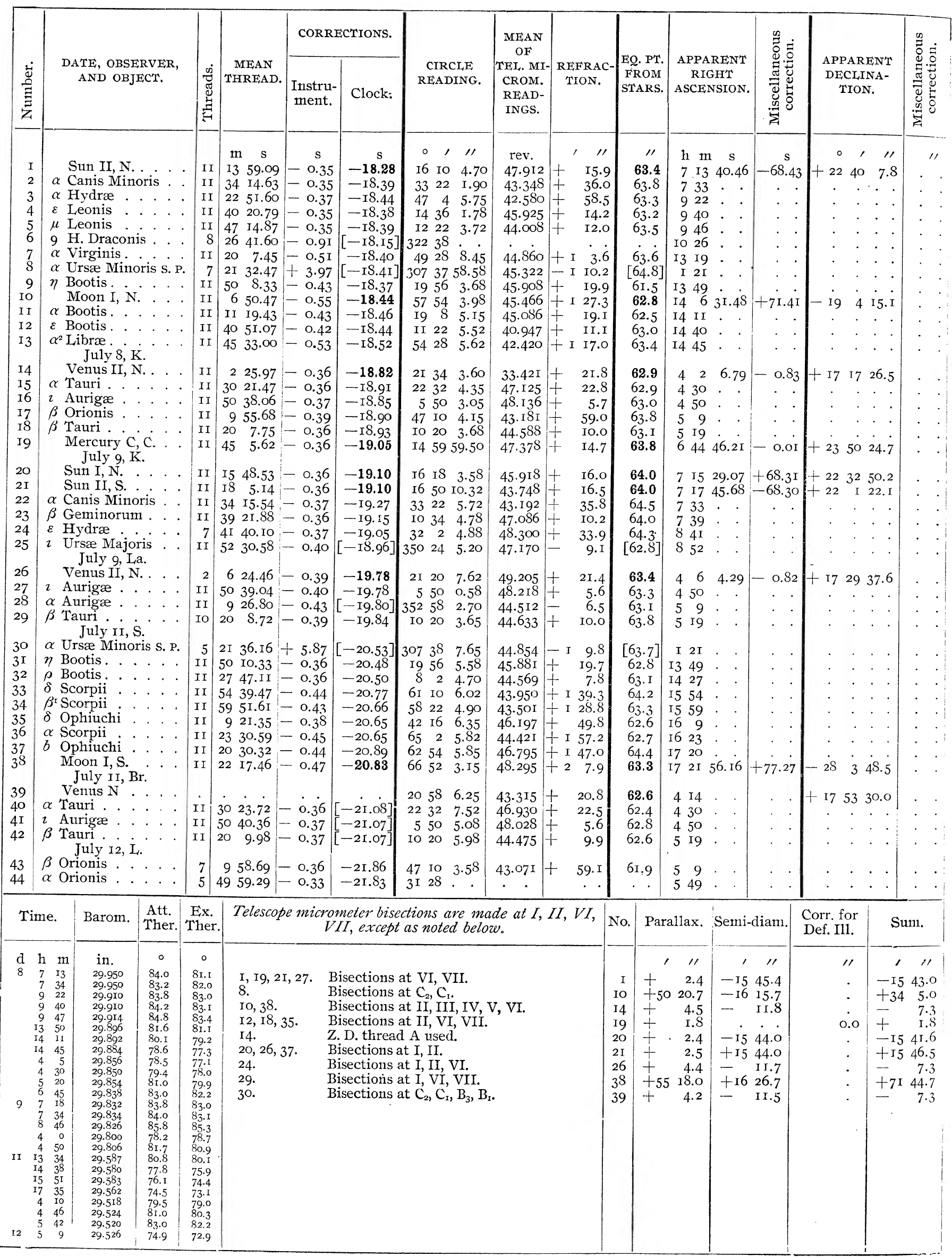




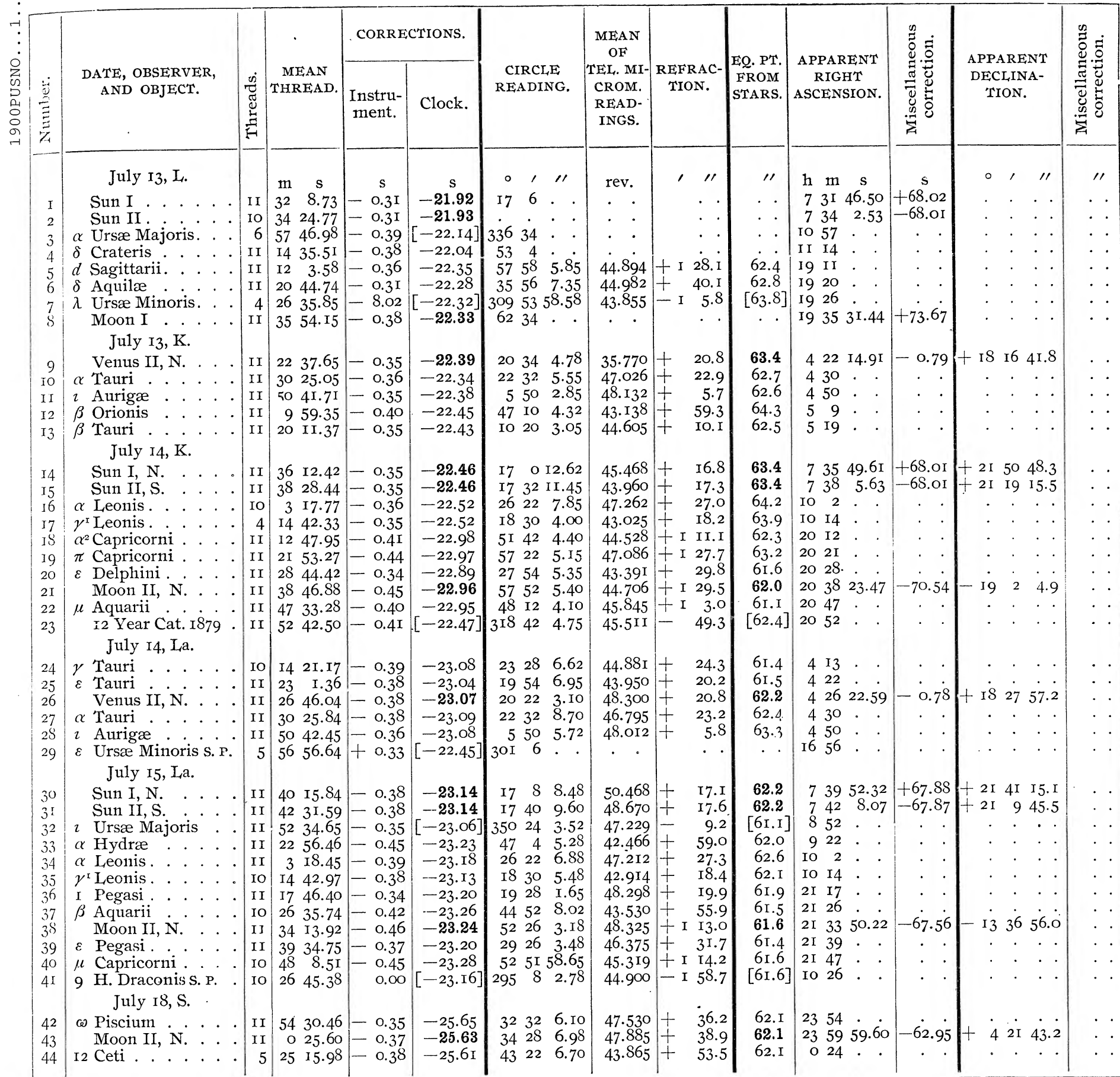

\begin{tabular}{|c|c|c|c|c|c|c|c|c|c|c|}
\hline Tine. & Barom. & $\begin{array}{l}\text { Att. } \\
\text { Ther. }\end{array}$ & $\begin{array}{l}\text { Ex. } \\
\text { Ther. }\end{array}$ & Telescope mic & $\begin{array}{l}\text { meter biseciions are made at } I, I I, V I \text {. } \\
I I, \text { except as noted beloze. }\end{array}$ & No. & Parallax. & Semi-diam. & $\begin{array}{l}\text { Corr. for. } \\
\text { Def. I11. }\end{array}$ & Sum. \\
\hline $\begin{array}{rrr}\mathrm{d} & \mathrm{h} & \mathrm{m} \\
\mathrm{I} 3 & 7 & 34 \\
& 19 & 12 \\
& 4 & 22 \\
& 5 & 20 \\
14 & 7 & 38 \\
& 10 & 3 \\
& 20 & 12 \\
& 20 & 58 \\
& 4 & 14 \\
& 5 & 5 \\
15 & 7 & 42 \\
& 8 & 52 \\
& 9 & 45 \\
& 10 & 14 \\
& 21 & 17 \\
& 22 & 22 \\
18 & 0 & 5 \\
& 1 & 8\end{array}$ & $\begin{array}{c}\text { in. } \\
29.530 \\
29.528 \\
29.648 \\
29.660 \\
29.684 \\
29.668 \\
29.800 \\
29.816 \\
29.932 \\
29.942 \\
29.956 \\
29.948 \\
29.954 \\
29.952 \\
29.922 \\
29.928 \\
30.080 \\
30.083\end{array}$ & $\begin{array}{c}\circ \\
80.2 \\
72.0 \\
74.0 \\
76.0 \\
79.0 \\
81.8 \\
68.0 \\
66.8 \\
72.8 \\
74.6 \\
78.5 \\
81.8 \\
81.8 \\
83.6 \\
71.2 \\
69.8 \\
68.3 \\
68.8\end{array}$ & $\begin{array}{c}\circ \\
79.2 \\
09.4 \\
72.0 \\
74.2 \\
78.1 \\
80.1 \\
65.3 \\
64.3 \\
70.8 \\
72.9 \\
77.4 \\
80.1 \\
79.8 \\
80.0 \\
67.8 \\
67.2 \\
66.1 \\
66.1 \\
66.9\end{array}$ & $\begin{array}{l}7 . \\
9 . \\
\text { I } 4,30,44 . \\
\text { I5, I } 7,3 \mathrm{I}, 37 \\
2 \mathrm{I}, 38,43 \\
23 . \\
27 . \\
4 \mathrm{I} .\end{array}$ & $\begin{array}{l}\text { Bisections at } \mathrm{C}_{2}, \mathrm{C}_{3}, \mathrm{C}_{4} \text {. } \\
\text { Z. D. thread A used. } \\
\text { Bisections at I, II. } \\
\text { Bisections at VI, VII. } \\
\text { Bisections at II, III, IV, V, VI. } \\
\text { Bisections at II, III, V, VI. } \\
\text { Bisections at II, VI, VII. } \\
\text { Bisections at V, IV, III. }\end{array}$ & $\begin{array}{r}9 \\
\text { I4 } \\
\text { I5 } \\
21 \\
26 \\
30 \\
31 \\
38 \\
43\end{array}$ & 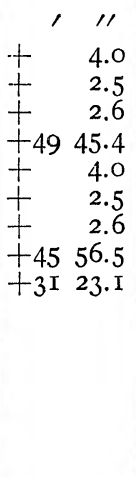 & 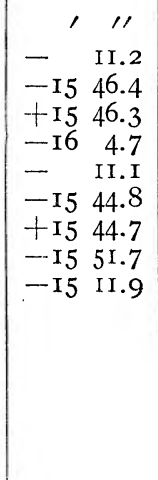 & $\begin{array}{l}\prime \prime \\
\cdot \\
\cdot \\
\cdot \\
\cdot \\
\cdot \\
\cdot \\
\cdot \\
\cdot \\
\cdot \\
\cdot\end{array}$ & 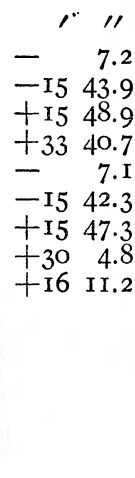 \\
\hline
\end{tabular}




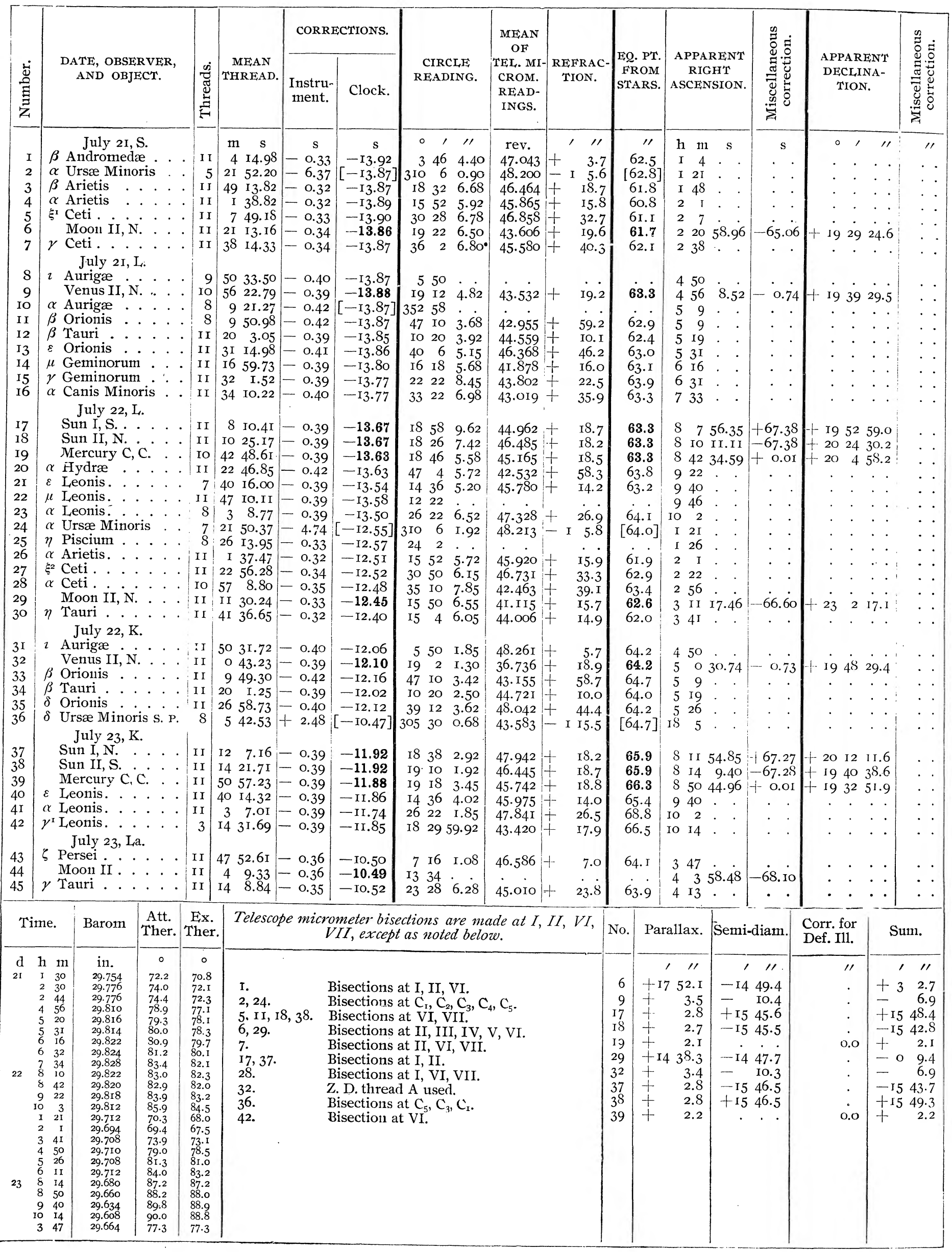




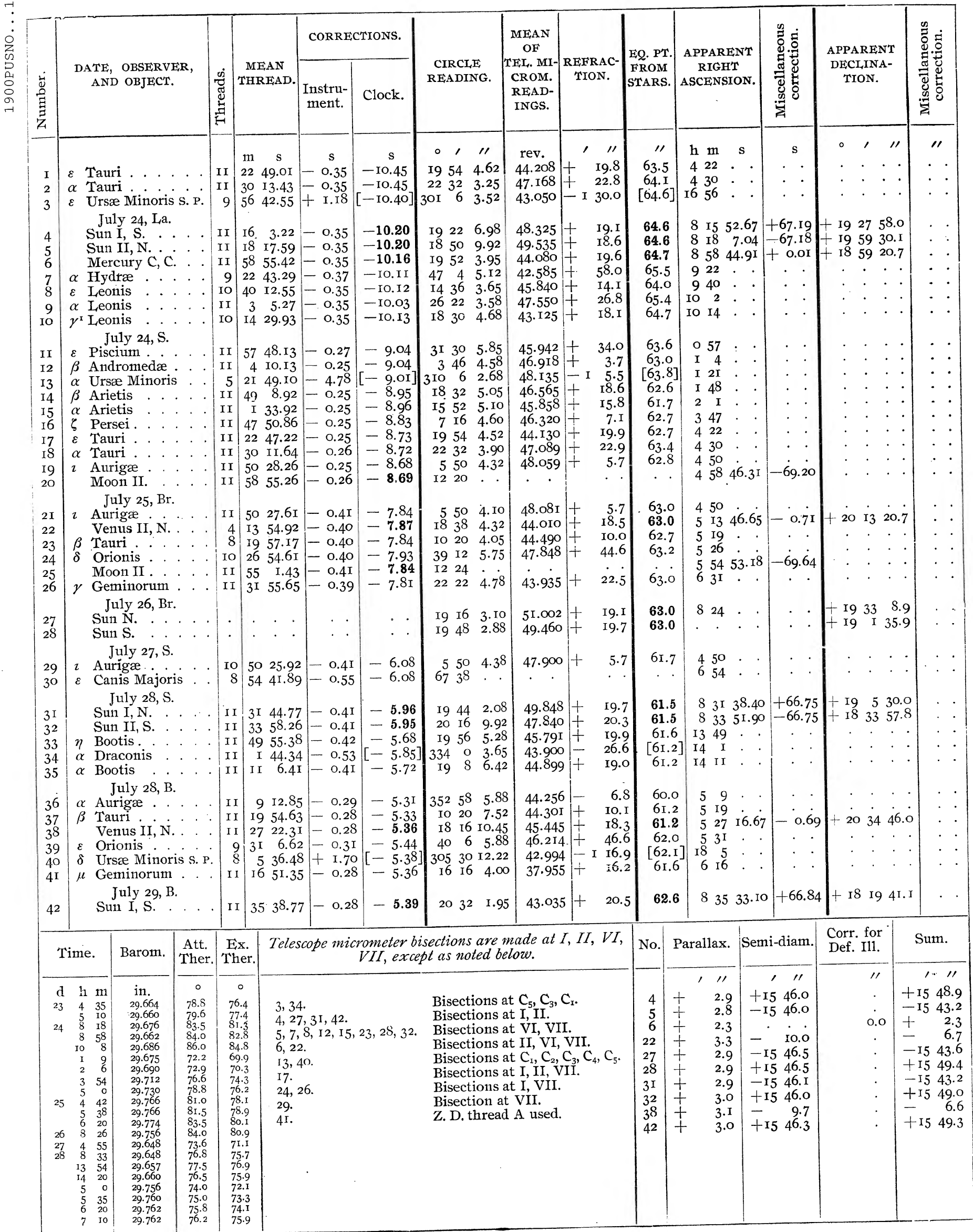




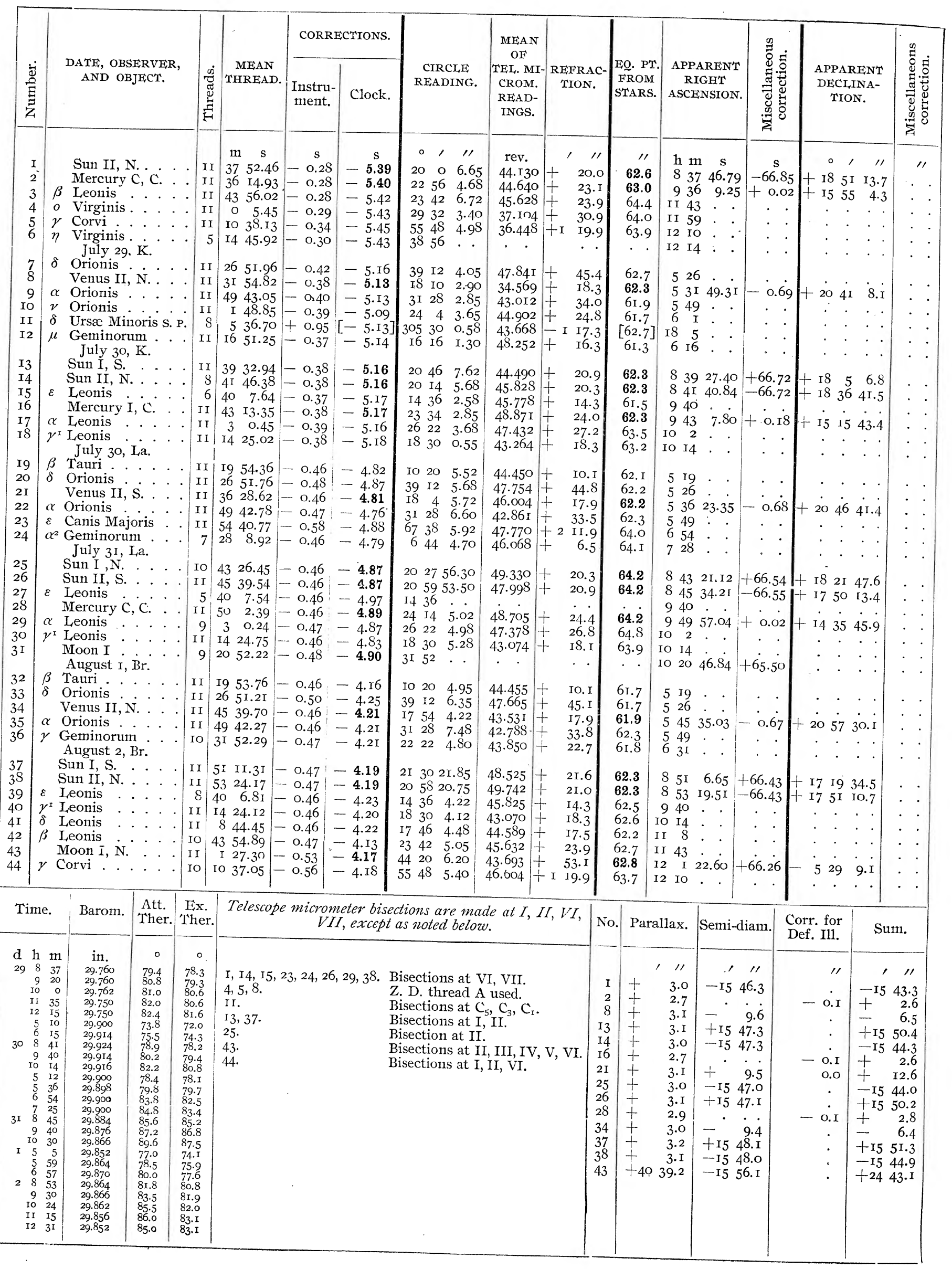




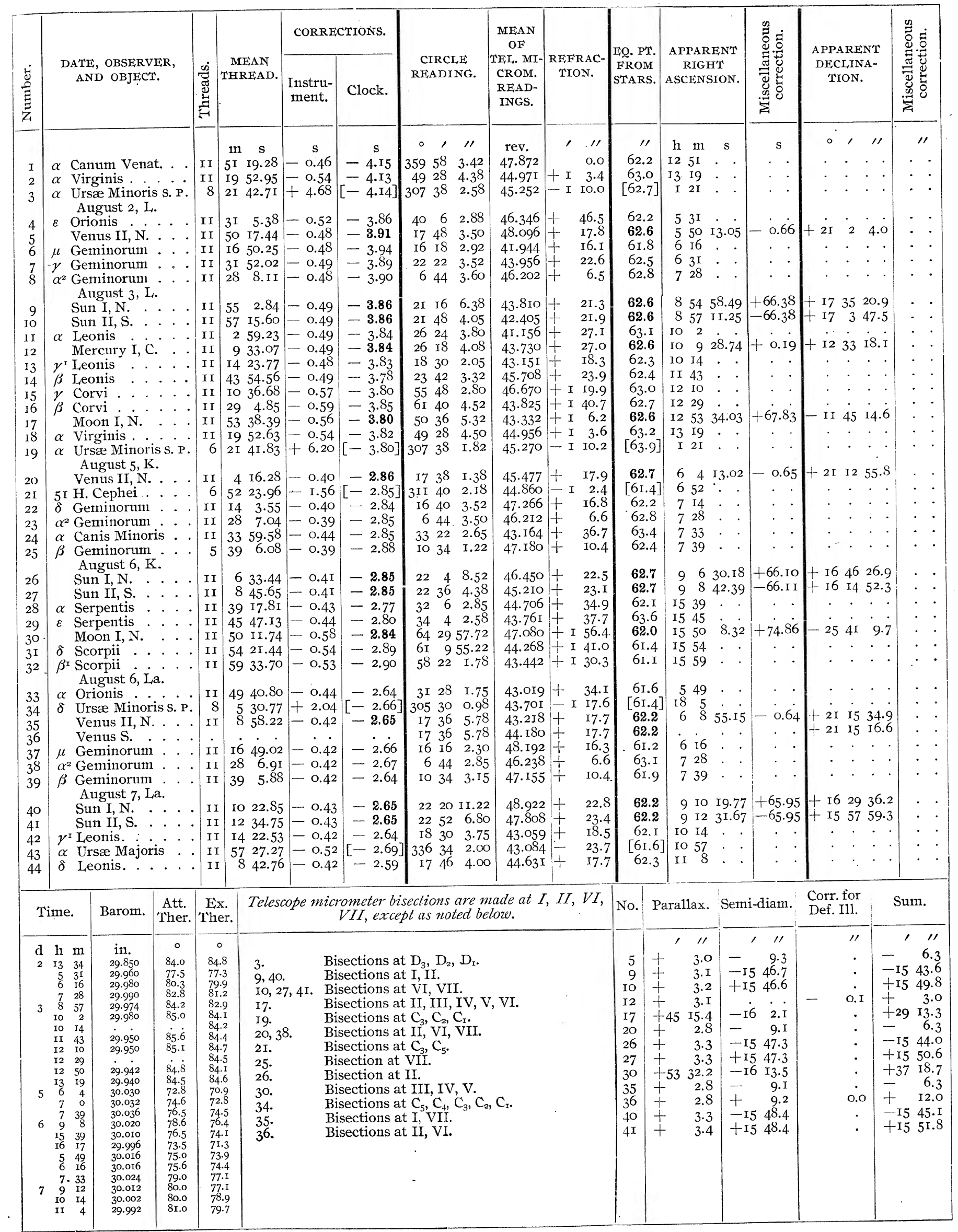




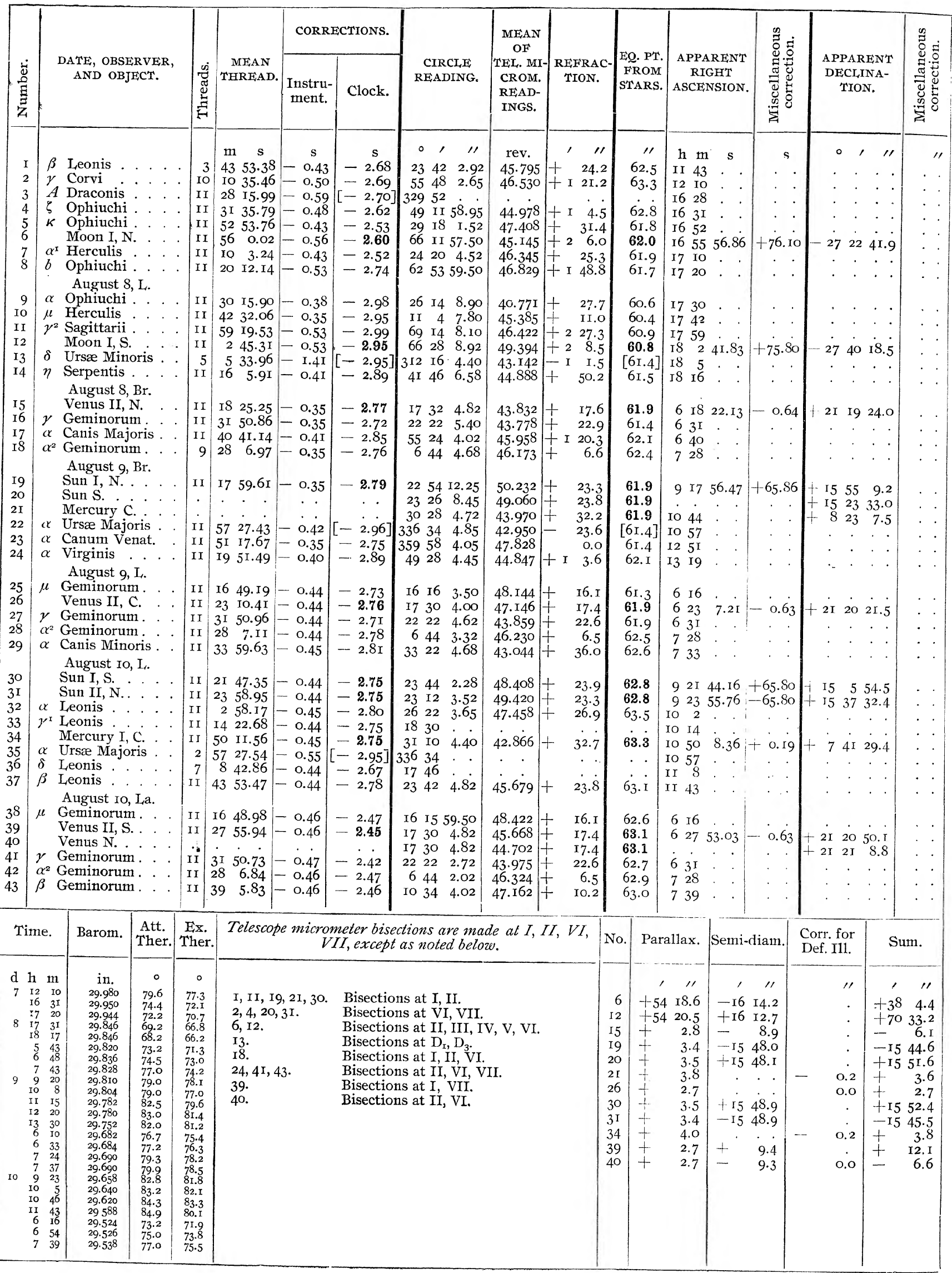




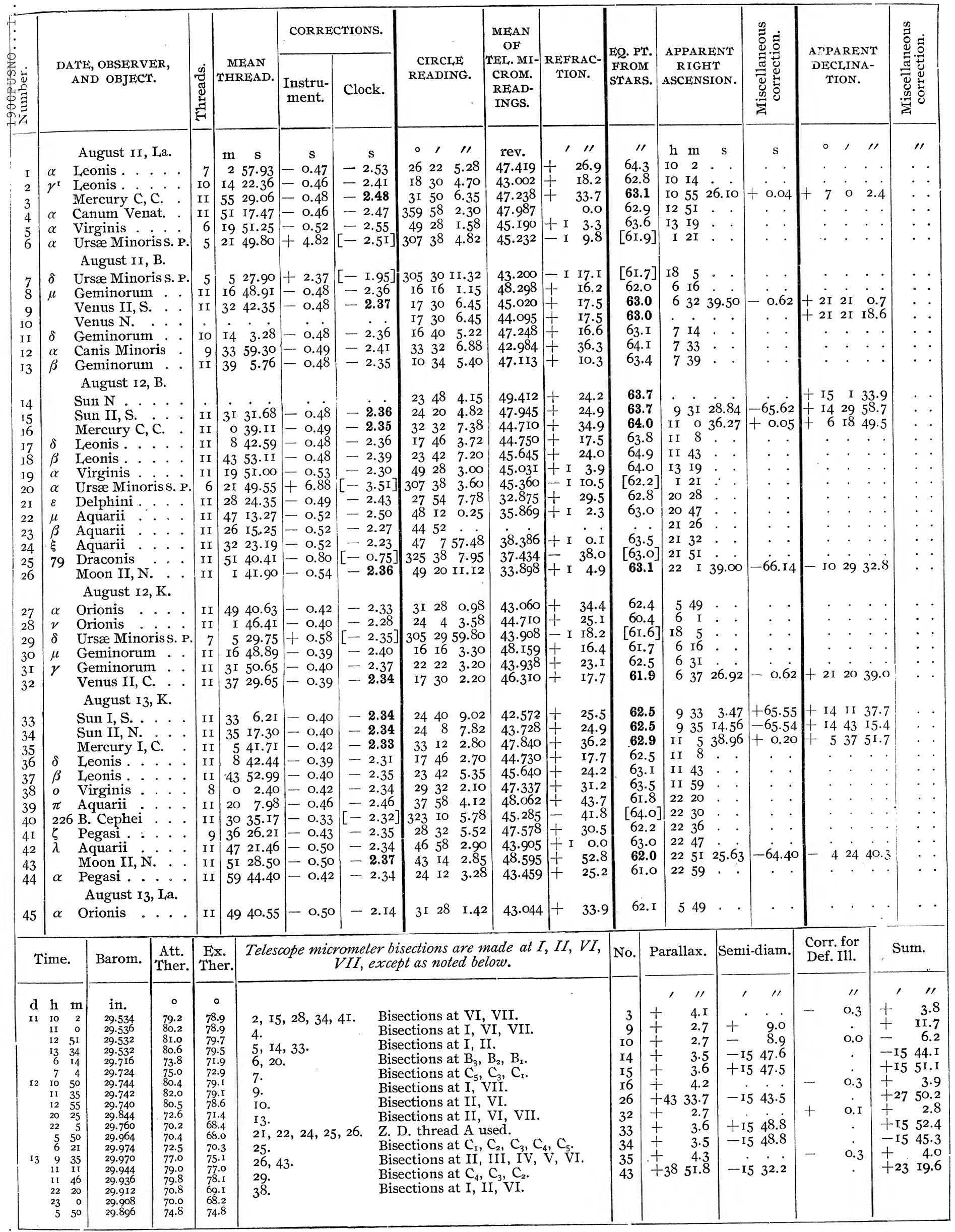




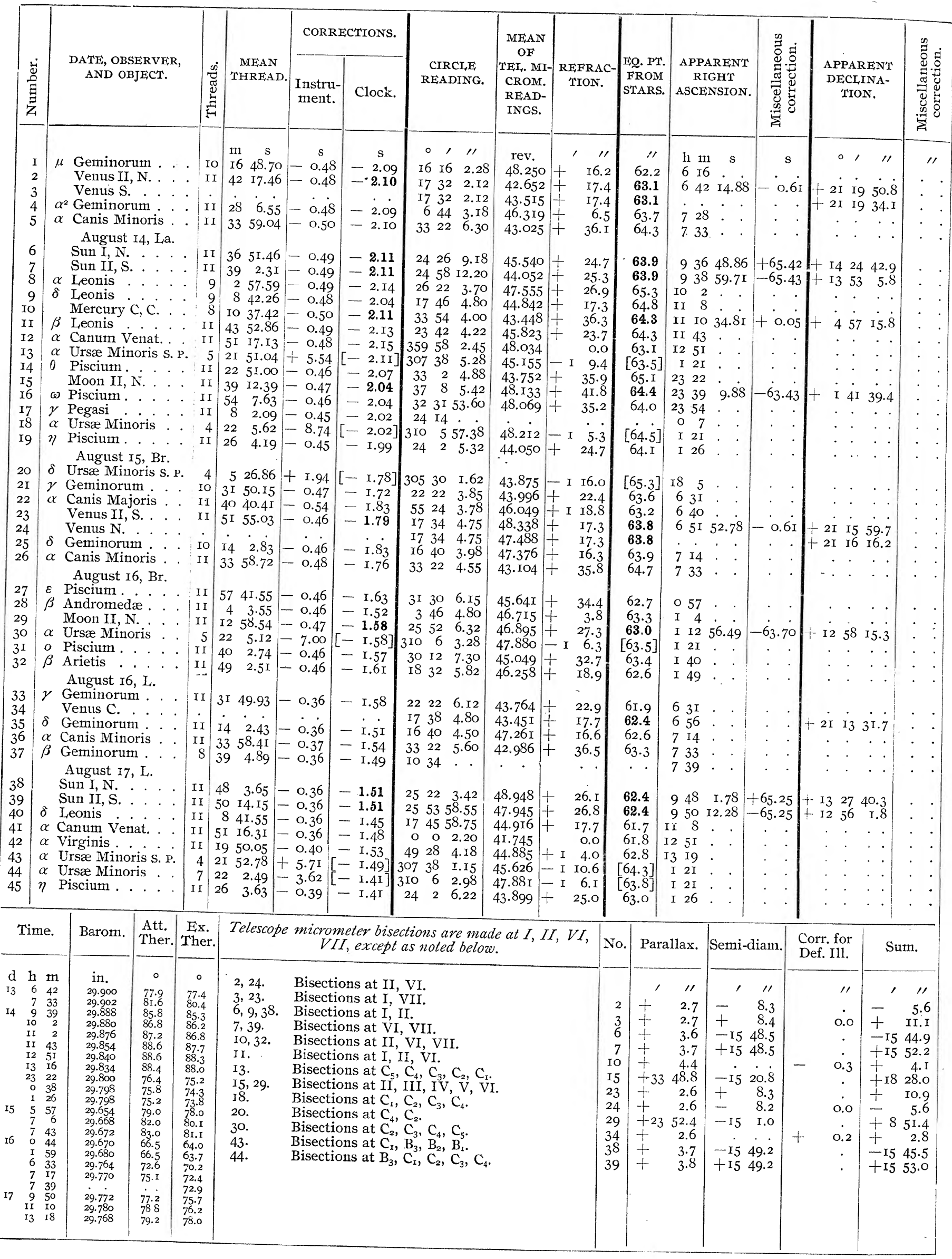




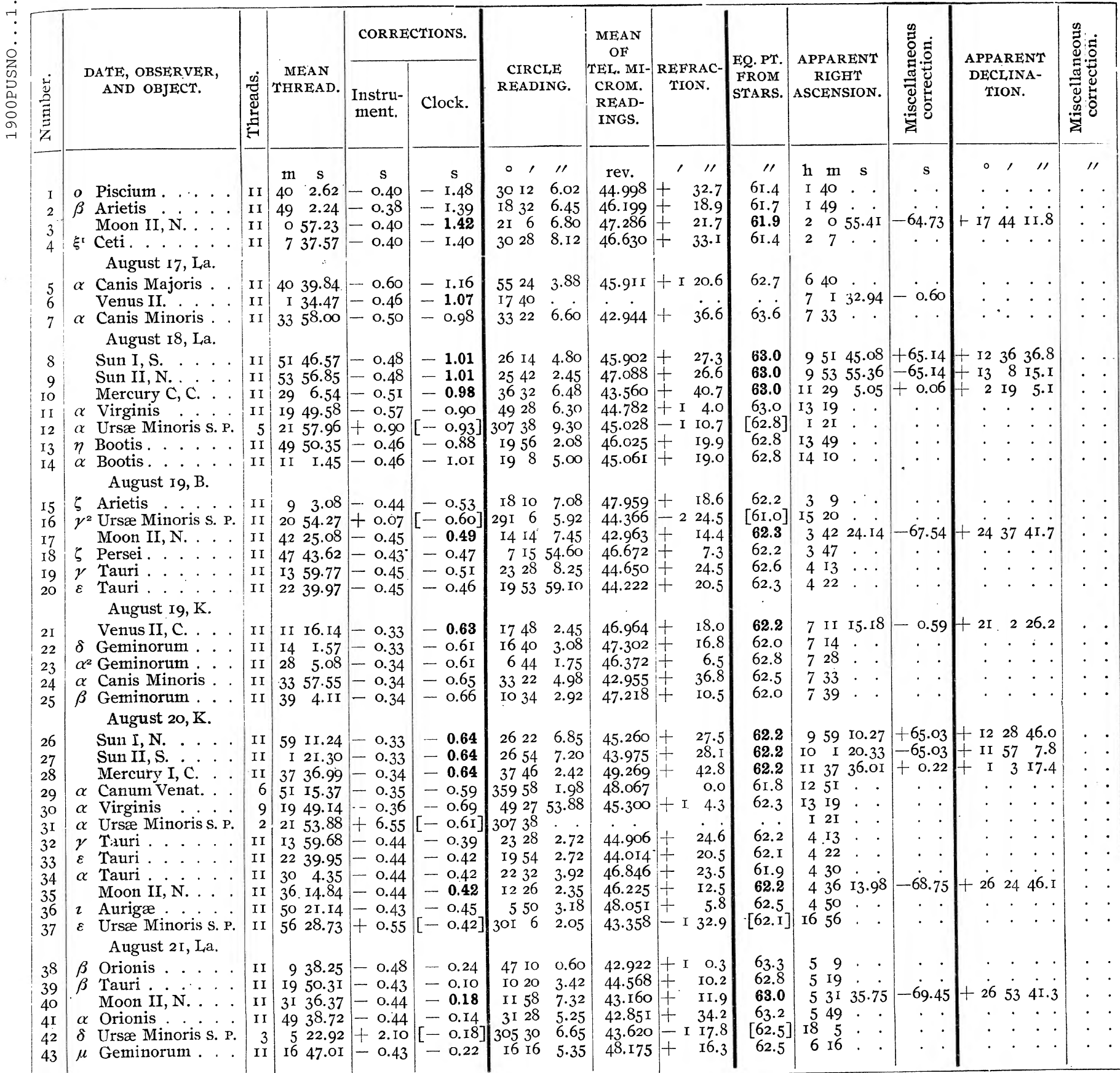

\begin{tabular}{|c|c|c|c|c|c|c|c|c|c|c|c|}
\hline Time. & Barom. & $\begin{array}{l}\text { Att. } \\
\text { Ther. }\end{array}$ & Ex. & Telescope mi & $\begin{array}{l}\text { crometer bisections are made at } I, I I, V I, \\
V I I ; \text { except as noted below. }\end{array}$ & No. & Parallax. & Semi-diam. & $\begin{array}{l}\text { Corr } \\
\text { Def }\end{array}$ & & Sum. \\
\hline $\begin{array}{rrr}\mathrm{d} & \mathrm{h} & \mathrm{m} \\
\mathrm{I} 7 & \mathrm{I} & 28 \\
& 2 & 9 \\
& 6 & 4 \mathrm{I} \\
& 7 & 43 \\
\mathrm{I} 8 & 9 & 54 \\
& \mathrm{II} & 29 \\
& \mathrm{I} 3 & 28 \\
& \mathrm{I} & 28 \\
19 & 3 & 0 \\
& 3 & 55 \\
& 4 & 25 \\
& 7 & 7 \\
& 7 & 37 \\
20 & 10 & 1 \\
& 11 & 34 \\
& 12 & 56 \\
& 13 & 26 \\
& 4 & 17 \\
& 4 & 50 \\
21 & 5 & 10 \\
& 6 & 12\end{array}$ & $\begin{array}{l}\text { in. } \\
29.812 \\
29.814 \\
29.868 \\
29.880 \\
29.856 \\
29.844 \\
29.816 \\
29.812 \\
29.824 \\
29.840 \\
29.852 \\
29.906 \\
29.916 \\
29.920 \\
29.910 \\
29.900 \\
29.900 \\
29.944 \\
29.950 \\
29.800 \\
29.806\end{array}$ & $\begin{array}{l}\circ \\
69.1 \\
68.2 \\
77.6 \\
74.6 \\
77.5 \\
80.8 \\
80.0 \\
79.2 \\
66.0 \\
66.0 \\
66.8 \\
7.0 \\
73.0 \\
7.0 \\
77.2 \\
77.8 \\
79.0 \\
78.6 \\
67.4 \\
67.9 \\
68.7 \\
71.0\end{array}$ & $\begin{array}{c}\circ \\
67.4 \\
66.1 \\
71.2 \\
72.9 \\
75.3 \\
78.3 \\
78.5 \\
78.5 \\
63.4 \\
63.8 \\
64.4 \\
70.4 \\
71.1 \\
75.0 \\
76.7 \\
77.2 \\
77.4 \\
65.4 \\
65.9 \\
67.5 \\
69.7\end{array}$ & $\begin{array}{l}3,17,35,40 . \\
8,26,43 . \\
9,10,27,30 . \\
12 . \\
16 . \\
29 . \\
37 . \\
42 .\end{array}$ & $\begin{array}{l}\text { Bisections at II, III, IV, V, VI. } \\
\text { Bisections at I, II. } \\
\text { Bisections at VI, VII. } \\
\text { Bisections at } \mathrm{C}_{2}, \mathrm{C}_{\mathrm{I}} \text {. } \\
\text { Bisections at } \mathrm{C}_{5}, \mathrm{C}_{4}, \mathrm{C}_{3}, \mathrm{C}_{2}, \mathrm{C}_{1} \text {. } \\
\text { Bisections at I, II, VI. } \\
\text { Bisections at V,IV, III. } \\
\text { Bisections at } \mathrm{C}_{4}, \mathrm{C}_{3} \text {. }\end{array}$ & $\begin{array}{r}3 \\
8 \\
9 \\
10 \\
17 \\
21 \\
26 \\
27 \\
28 \\
35 \\
40\end{array}$ & 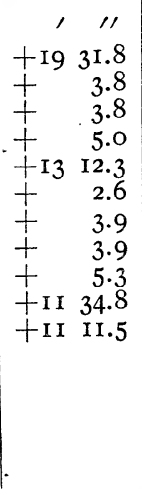 & $\begin{array}{rc}\prime & \prime \prime \\
-\mathrm{I} 4 & 54.2 \\
+\mathrm{I} 5 & 49.2 \\
-\mathrm{I} 5 & 49 . \mathrm{I} \\
. & . \\
-\mathrm{I} 4 & 48.6 \\
. & . . \\
-\mathrm{I} 5 & 49 . \mathrm{I} \\
+\mathrm{I} 5 & 49 . \mathrm{I} \\
- & .0 . \\
-\mathrm{I} 4 & 50.3 \\
-\mathrm{I} 4 & 54.9\end{array}$ & $\begin{array}{l}- \\
+ \\
-\end{array}$ & $\begin{array}{c}\prime \prime \\
\cdot \\
\cdot \\
\dot{0.4} \\
\dot{0} \\
0.2 \\
\cdot \\
\dot{0.5} \\
\cdot \\
\dot{0}\end{array}$ & 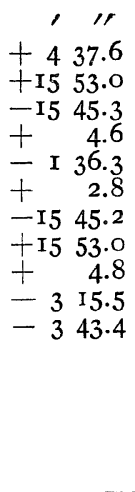 \\
\hline
\end{tabular}

$6855-10$ 


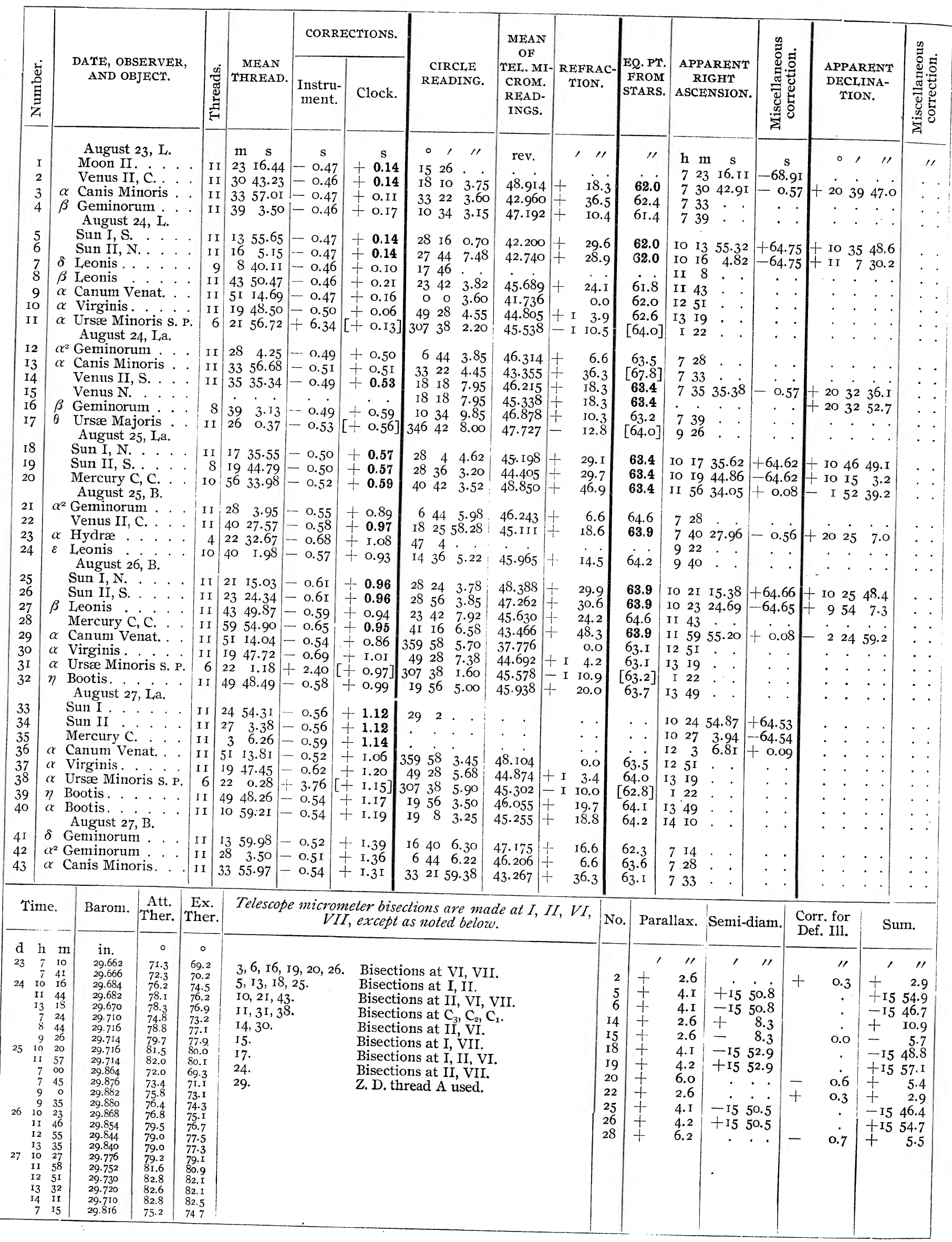


DATE, OBSERVER, AND OBJECT.

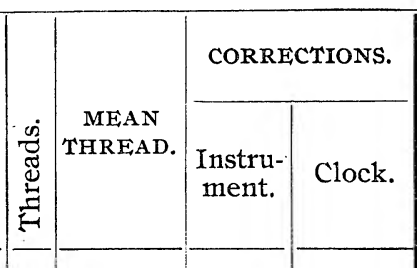

$\beta$ Geminorum . . . $\quad$ I I \begin{tabular}{cc|cc}
$\mathrm{m}$ & $\mathrm{s}$ & $\mathrm{s}$ & $\mathrm{s}$ \\
39 & 2.44 & $-0.5 \mathrm{I}$ & $+\mathrm{I} .38$
\end{tabular} Venus II, C. . . . I I 50 I $2.27-0.52+1.36$ August 28, B.

$\beta$ Leonis

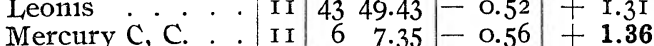

$6 \quad \alpha$ Canum Venat.

$\begin{array}{lll}6 & 7.35 & -0.56\end{array}$

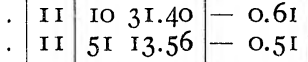

$\alpha$ Virginis

$8 \alpha$ Ursæ Minoris s. P. 72 I $59.06+5.40\left[\begin{array}{ll}+ & 1.39\end{array}\right]$ August 30, I

$9 \alpha^{2}$ Geminorum . . . I I $282.90-0.43+$ I.96

Io $\alpha$ Canis Minoris . . I I $3355.26-0.47+2.02$

$\beta$ Geminorum. . . I I 39 I.83 $-0.44+2.00$

Venus I, S. . . . $6{ }_{4} 48.15-0.45+\mathbf{2 . 0 0}$

Venus II, N. . $\quad . \quad \cdot \quad 5 \quad 448.94-0.45+\mathbf{2 . 0 0}$

August 3I, L.

Sun I, N. . . . I I $3927.50-0.47+2.06$

Sun II, S. . . . . I I 4 I $36.33-0.47+2.06$

Mercury I, C. . . I I I4 $2.79-0.50+2.09$

I6 $\alpha$ Mercury I

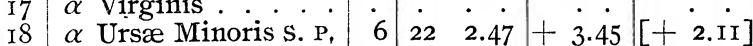

I9 Moon I . . . . I I $3054.01-0.55+2.11$

$20 \quad \eta$ Bootis...... I I $4947.17-0.45+2.12$

2I $\alpha$ Bootis. . . . . . I I IO 58.09-0.45 +2.16

$22 \rho$ Bootis. . . . . II $2723.74-0.44+2.10$ August 3I, La. $23 \alpha$ Leonis. . . . . .

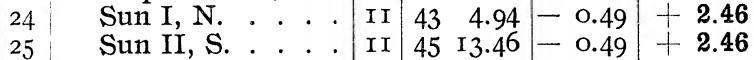

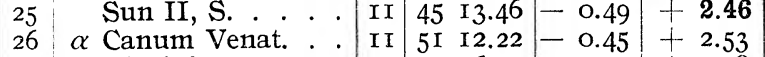

$27 \alpha$ Virginis..... II $1946.05-0.54+2.48$

$28 \alpha$ Ursæ Minoris s. P. 6 22 $3.07+3.25[+2.53]$

$29 \eta$ Bootis . . . . . . I I $4946.74-0.47+2.56$

30 $\alpha$ Bootis . . . . . . I I IO $57.73-0.47+2.53$

3I Moon I, N... . . II 29 I $4.38-0.6 \mathrm{I}+2.54$

$32 a^{2}$ Libræ. . . . . . . II 45 II.32 $-0.56+2.53$ September I, B.

33 Venus S..

34 Venus N. $\quad \cdot$ September 2, B.

35 Sun $\mathrm{S}$.

36 Sun $\mathrm{N}$

$37 \beta$ Corvi. . . . .

I $2857.92-0.52+2.79$

$39 \eta$ Bootis.... II $4946.35-0.46[+2.94$

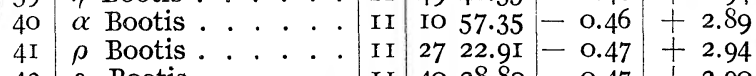

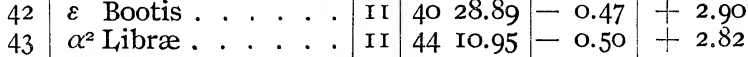

\begin{tabular}{c} 
CIRCLE \\
READING. \\
\hline 0,11
\end{tabular}

IO $34 \quad 5.90$

I8 $42 \quad 7.35$

$23 \quad 42 \quad 7.45$

$\begin{array}{lll}42 & \text { I6 } & 5.78 \\ 55 & 48 & 6.15\end{array}$

$\begin{array}{rrr}55 & 48 & 6.15 \\ 359 & 58 & 5.00\end{array}$

$\begin{array}{lll}49 & 28 & 7.12\end{array}$

$\begin{array}{lll}307 & 38 & 4.00\end{array}$

$\begin{array}{lll}6 & 44 & 4.48\end{array}$ \begin{tabular}{lll|l}
33 & 22 & 6.22 & 42.865
\end{tabular}

IO $34 \quad 6.00 \quad 47.095$ +

$\begin{array}{llll}19 & \text { I2 } & 6.65 & 46.310+\end{array}$

I9 I2 6.65

$\begin{array}{lll}30 & \mathrm{I} 2 & 2.72\end{array}$

$\begin{array}{llll}30 & 44 & 2.15 & 45.050 \\ 43 & .880\end{array}$

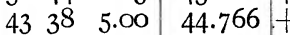

$\begin{array}{lll}49 & 28 & 4.75\end{array}$

$\begin{array}{lll}307 & 38 & \text { I. } 72\end{array}$

$\begin{array}{llll}54 & 46 & . & . \\ \text { I9 } & 56 & 5.35 & 45.965 \\ \text { I9 } & 8 & 5.85 & 45.048\end{array}$

$\begin{array}{rrrr}\text { I9 } & 8 & 5.85 & 45.048 \\ 8 & 2 & 5.72 & 44.499\end{array}$ +

$\begin{array}{lll}26 & 22 & 6.68\end{array}$

$\begin{array}{lll}30 & 34 & \text { I.80 }\end{array}$

\begin{tabular}{lrr|l}
$3 \mathrm{I}$ & 6 & 3.35 & $43.3 \mathrm{I} 8$
\end{tabular}

\begin{tabular}{lll|l}
359 & 58 & 2.10 & 48.166
\end{tabular}

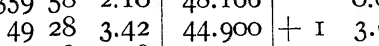

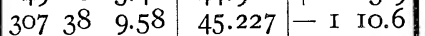

I9 $56 \quad 2.85 \quad 46.050$

I9. $82.70 \quad 45.240+\quad 19.0$

5940 I5.75 $47.738+$ I 33.4

$\begin{array}{lllll}54 & 26 & 3.82 & 48.701+\mathrm{I} & 16.5\end{array}$

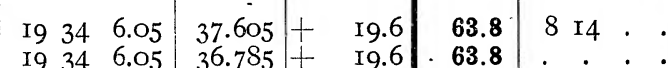

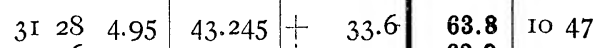

$30564.3544 .000+32.9$

6I $404.2543 .738+\mathrm{I} 4 \mathrm{I} . \mathrm{I}$

$30738 \quad 3.50$

I9 $56 \quad 5.80 \quad 45.934$ +

I9 $8 \quad 5.88 \quad 45.090+$

$\begin{array}{rrr}8 & 2 & 5.38 \\ \text { II } & 20 & 5.38\end{array}$

$\begin{array}{lll}54 & 26 & 6.32\end{array}$

$44.5 \mathrm{I2}+$

$48.558+$ I $I 6.7$

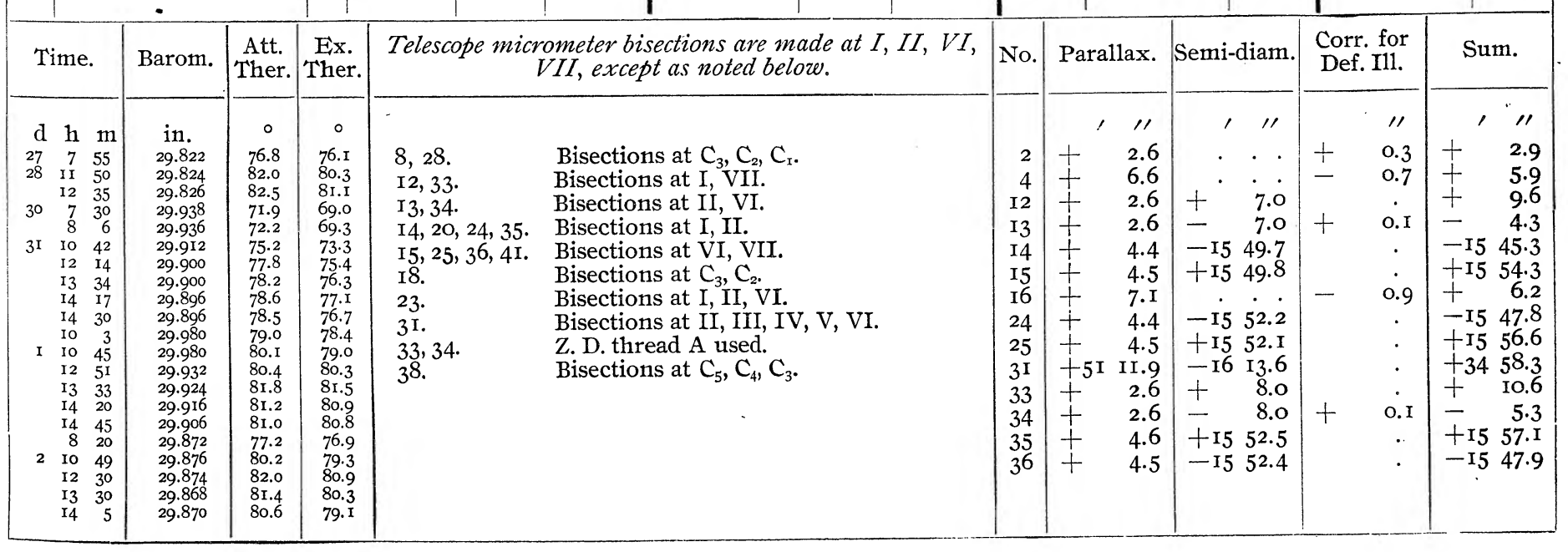




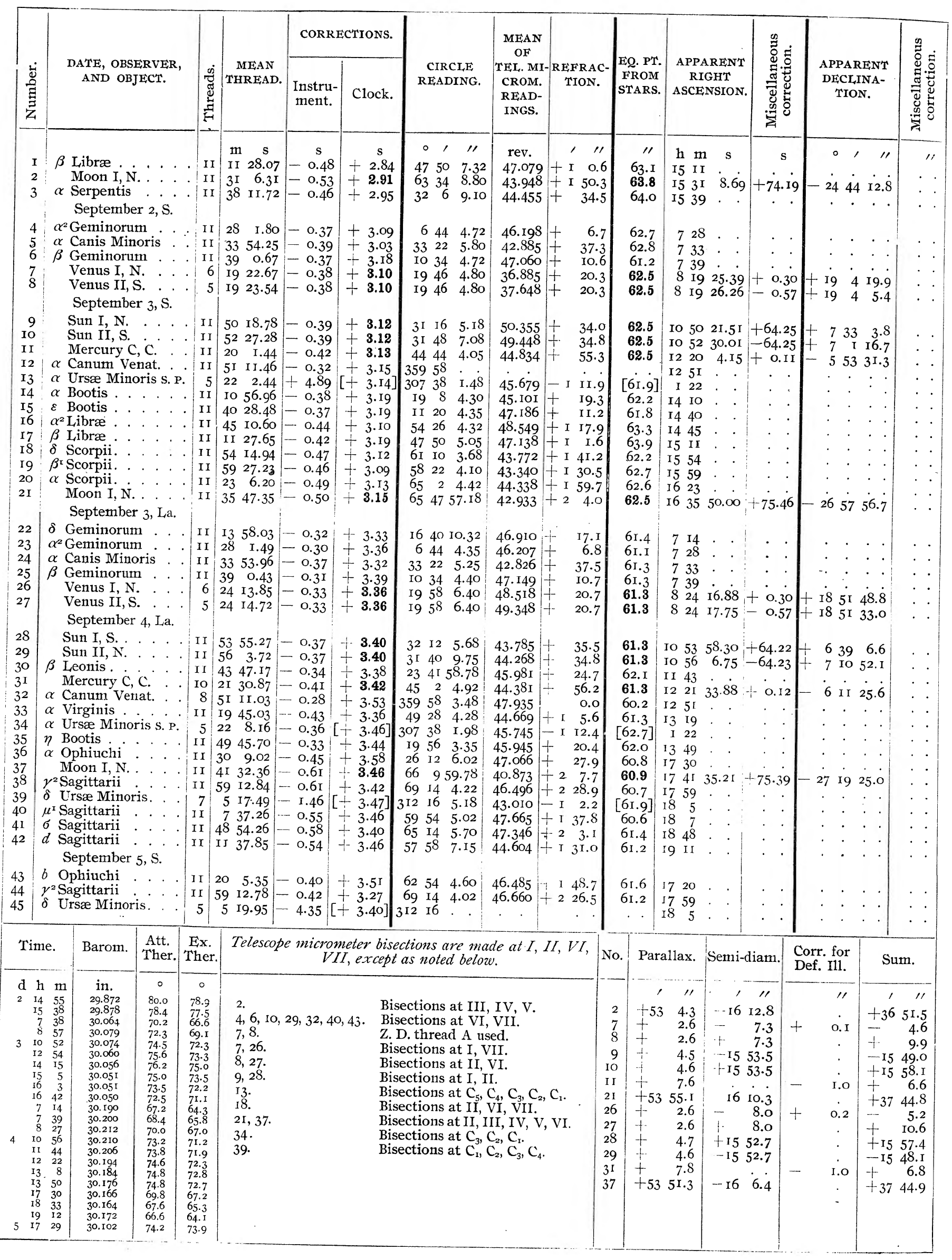




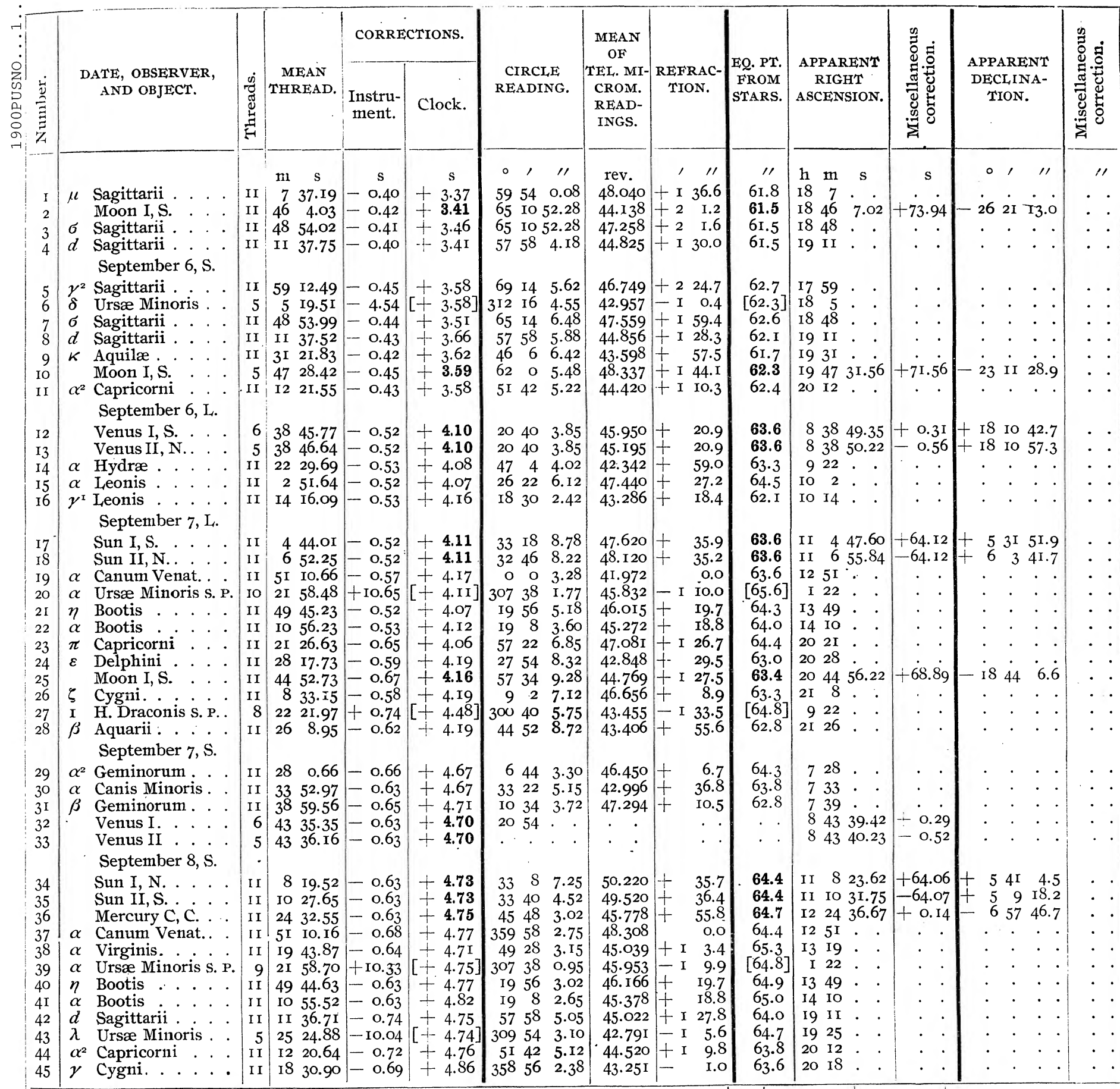

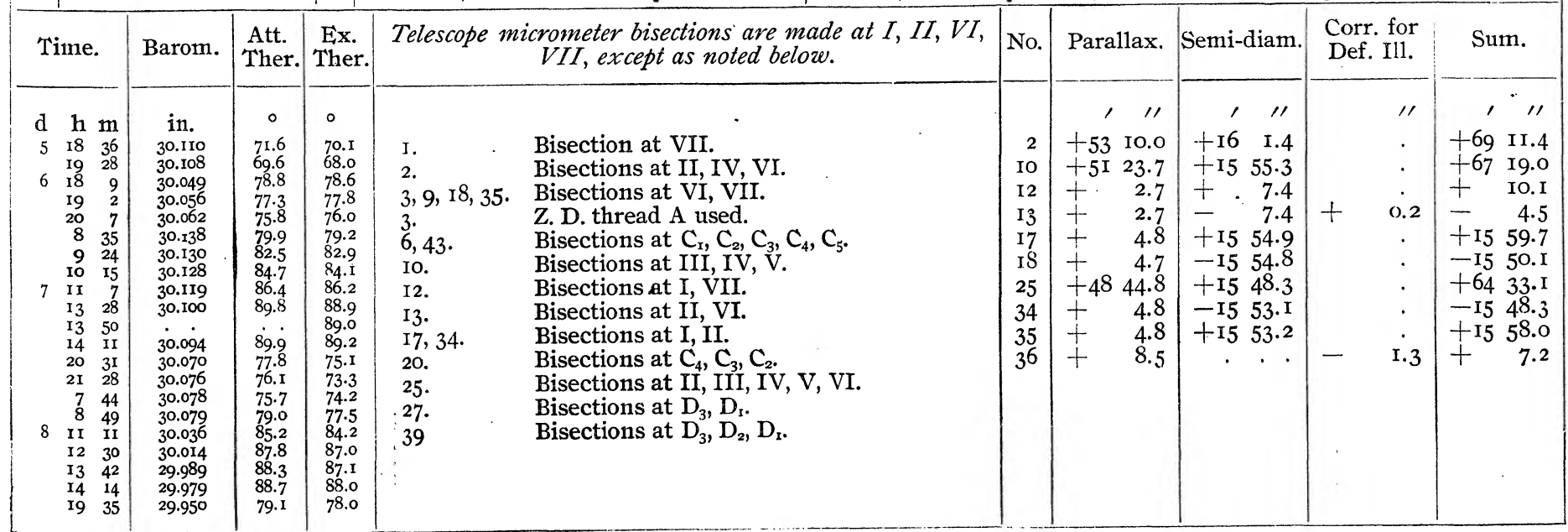




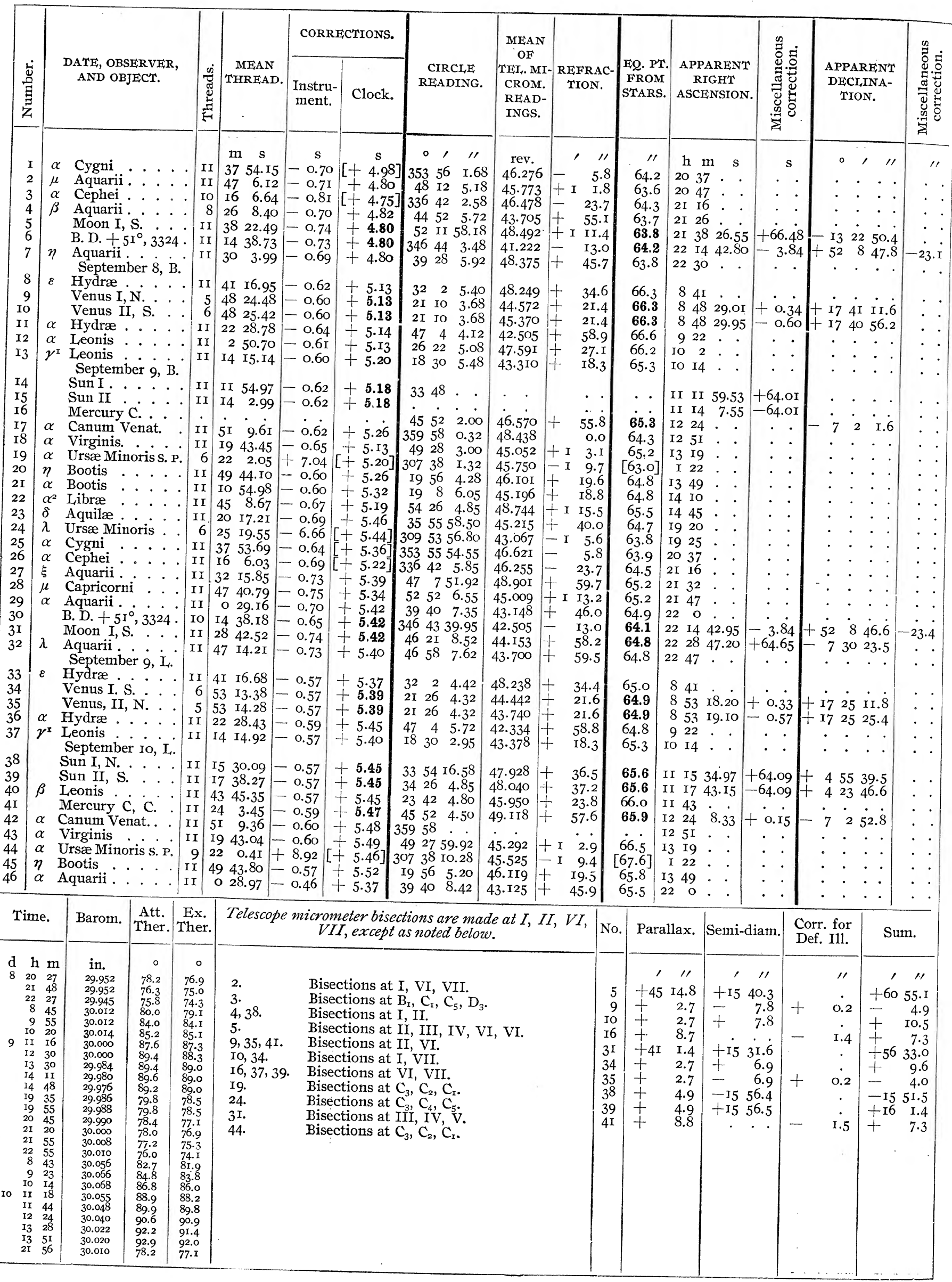




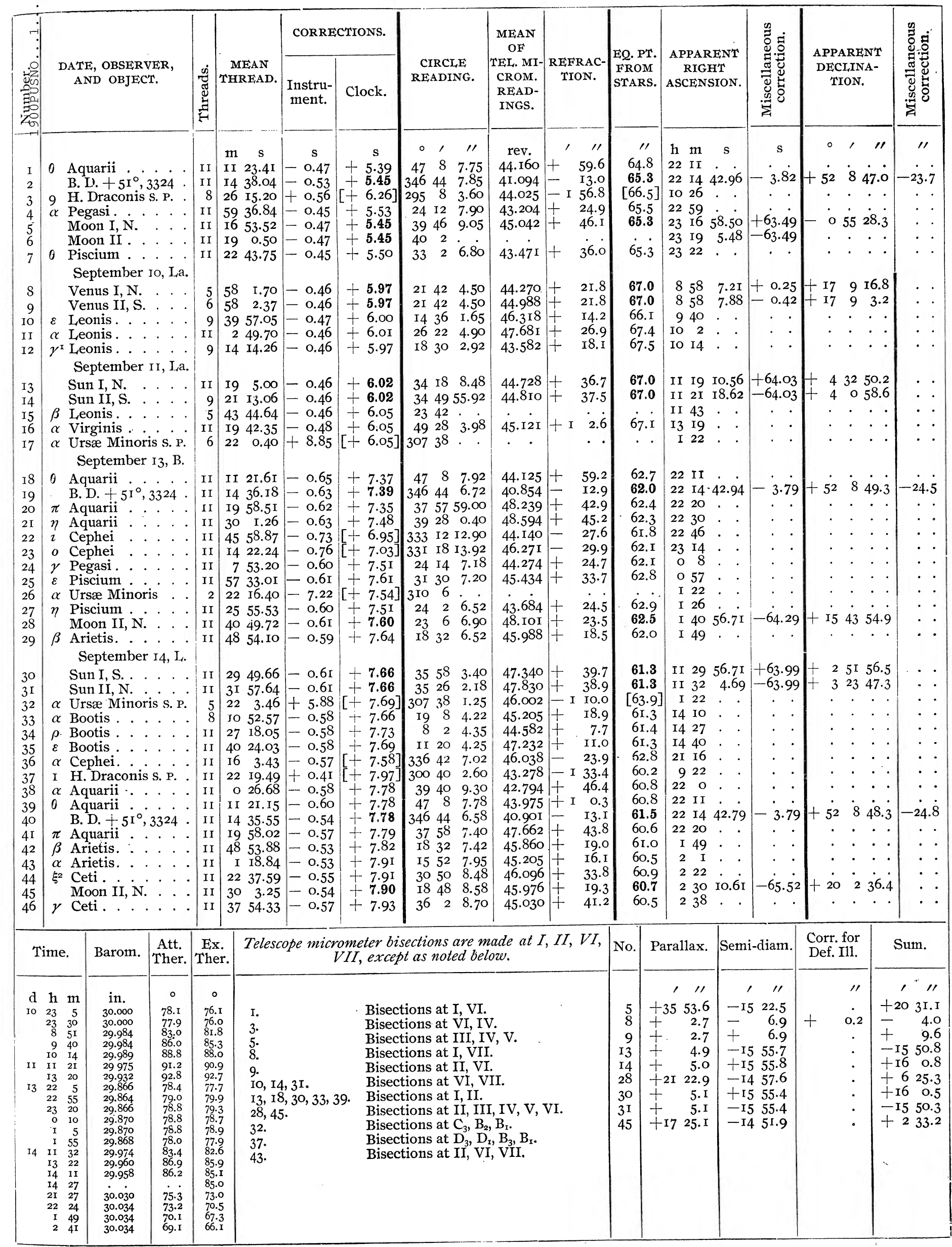




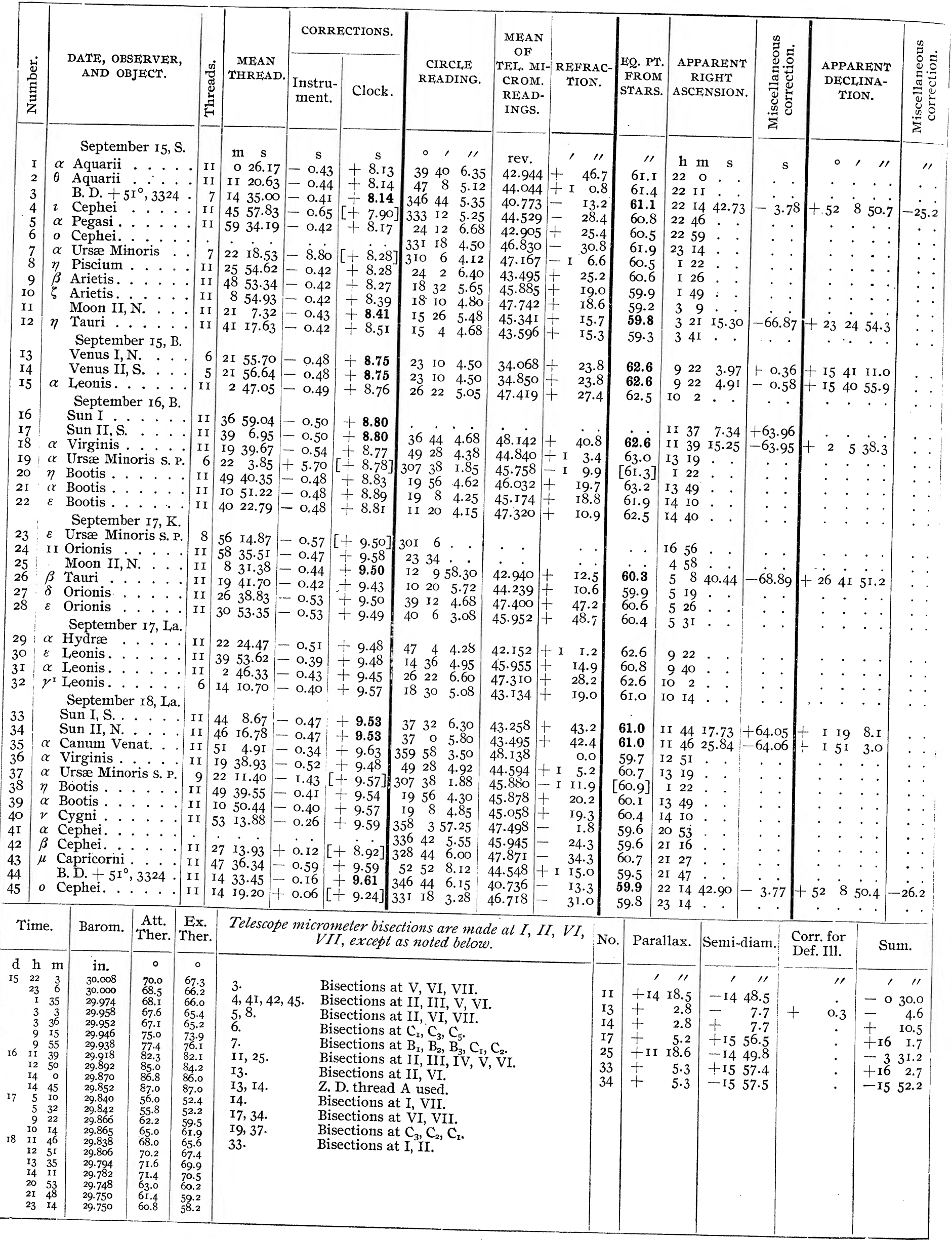




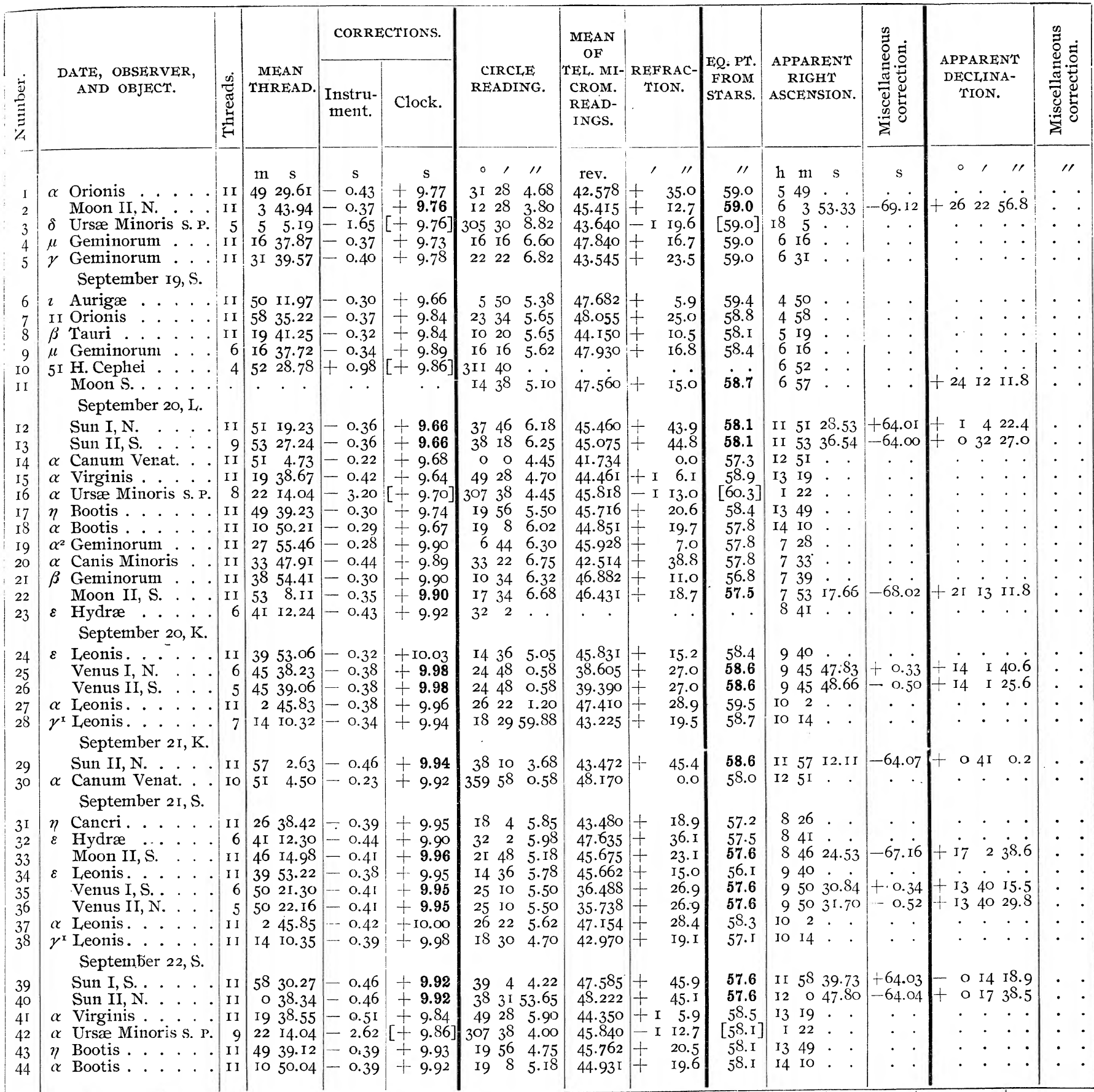

\begin{tabular}{|c|c|c|c|c|c|c|c|c|c|c|c|c|c|}
\hline \multicolumn{3}{|c|}{ Time. } & \multirow{2}{*}{$\frac{\text { Barom. }}{\text { in. }}$} & \multirow{2}{*}{$\frac{\text { Att. }}{\text { Ther. }}$} & \multirow{2}{*}{$\frac{\begin{array}{c}\text { Ex. } \\
\text { Ther. }\end{array}}{0}$} & \multicolumn{2}{|c|}{$\begin{array}{c}\text { Telescope micrometer bisections are made at I, II, VI, } \\
V I I, \text { except as noted below. }\end{array}$} & & Parallax. & Semi-diam. & \multicolumn{2}{|c|}{$\begin{array}{l}\text { Corr. for } \\
\text { Def. Ill. }\end{array}$} & Sum. \\
\hline $\mathrm{d}$ & & & & & & & & & 111 & 111 & & 11 & 111 \\
\hline 18 & & & 29.725 & 58.0 & 55.8 & $2,22,33$ & Bisections at II, III, IV, V, VI. & 2 & +II 40.I & -1454.9 & & - & -314.8 \\
\hline & & 32 & 29.730 & 58.6 & 56.3 & & Bisections at $B_{3}, B_{2}, B_{1}$. & II & +1348.6 & +152.9 & & • & $+285^{1.5}$ \\
\hline I9 & $\begin{array}{l}4 \\
5\end{array}$ & 57 & 9 & $\begin{array}{l}59.1 \\
58.0\end{array}$ & $\begin{array}{l}56.0 \\
55.2\end{array}$ & $9,12,39$. & Bisections at I, II. & 12 & $+\quad 5.4$ & - I5 57.7 & & - & - I5 52.3 \\
\hline & $\begin{array}{l}5 \\
7\end{array}$ & $\begin{array}{r}57 \\
5\end{array}$ & 29.782 & $\begin{array}{l}58.0 \\
59 \cdot 3\end{array}$ & $\begin{array}{l}55.2 \\
56.6\end{array}$ & $\begin{array}{l}\text { Y, } 12,390 \\
\text { II. }\end{array}$ & Bisections at III, IV, V. & 13 & 5.4 & + I5 57.7 & & • & +163.1 \\
\hline 20 & & 53 & 29.844 & 63.7 & 62.2 & $13,19,29,30,31,32,40$. & Bisections at VI, VII. & 22 & +1645.6 & + I5 I3.6 & & - & +3159.2 \\
\hline & I3 & $3 \mathrm{I}$ & & $\begin{array}{l}65.5 \\
65.8\end{array}$ & $\begin{array}{l}63.2 \\
63.4\end{array}$ & $\begin{array}{l}13,19,29,30,01,0^{2}, 400 \\
\text { I }\end{array}$ & Bisections at $\mathrm{C}_{3}, \mathrm{C}_{2}, \mathrm{C}_{\mathrm{x}}$. & 25 & $\begin{array}{r}7.9 \\
+\quad 2.9\end{array}$ & $-\quad 7.6$ & + & 0.2 & 4.5 \\
\hline & $\begin{array}{r}14 \\
7\end{array}$ & $\begin{array}{l}11 \\
25\end{array}$ & $\begin{array}{l}29.804 \\
30.070\end{array}$ & $\begin{array}{l}05.8 \\
48.2\end{array}$ & $\begin{array}{r}0.4 \\
46.2\end{array}$ & $22,25,26,35,36$ & hread A used. & 26 & 2.9 & 7.6 & & - & $+\quad 10.5$ \\
\hline & 7 & 57 & 30.076 & 50.1 & 48.0 & 25,36 & lons at I, VII. & 29 & 5.4 & - I5 58.0 & & . & $-I_{5} 52.6$ \\
\hline & $\begin{array}{r}9 \\
0\end{array}$ & 40 & 80 & $\begin{array}{l}54.2 \\
55.0\end{array}$ & 52.3 & 26,3 & ions at II, VI. & 33 & $+205^{2.2}$ & +1526.2 & & . & +36 I 8.4 \\
\hline $2 \mathrm{I}$ & $\begin{array}{l}\text { IO } \\
\text { II }\end{array}$ & $\begin{array}{l}14 \\
57\end{array}$ & & $\begin{array}{l}55.0 \\
57.8\end{array}$ & $\begin{array}{l}53.3 \\
56.4\end{array}$ & 28. & ion at VI. & 35 & 3.0 & $7 \cdot 3$ & & - & 10.3 \\
\hline & 12 & $-5 x$ & 30.048 & 59.8 & 58.3 & 34, & tions at II, VI, VII. & 36 & 3.0 & $7 \cdot 3$ & + & 0.3 & 4.0 \\
\hline & $\begin{array}{l}8 \\
9\end{array}$ & 32 & 29.966 & 57.5 & 55.2 & $\begin{array}{l}54,4 \pi \\
42 .\end{array}$ & Bisections at $\mathrm{D}_{3}, \mathrm{D}_{2}, \mathrm{D}_{1}$ & 39 & $5 \cdot 5$ & $+155^{8.7}$ & & . & +I6 4.2 \\
\hline & $\begin{array}{r}9 \\
\text { 10 }\end{array}$ & $\begin{array}{l}44 \\
17\end{array}$ & $\begin{array}{l}29.972 \\
29.968\end{array}$ & $\begin{array}{l}01.8 \\
63.3\end{array}$ & $\begin{array}{l}59.3 \\
60.7\end{array}$ & & & 40 & 5.5 & -1558.7 & & . & - I5 53.2 \\
\hline 22 & & & 29.930 & 65.6 & $64 \cdot 3$ & & & & & & & & \\
\hline & & & $\begin{array}{l}29.917 \\
29.900\end{array}$ & $\begin{array}{l}67.8 \\
67.9\end{array}$ & $\begin{array}{l}66.0 \\
66.2\end{array}$ & & & & & & & & \\
\hline
\end{tabular}




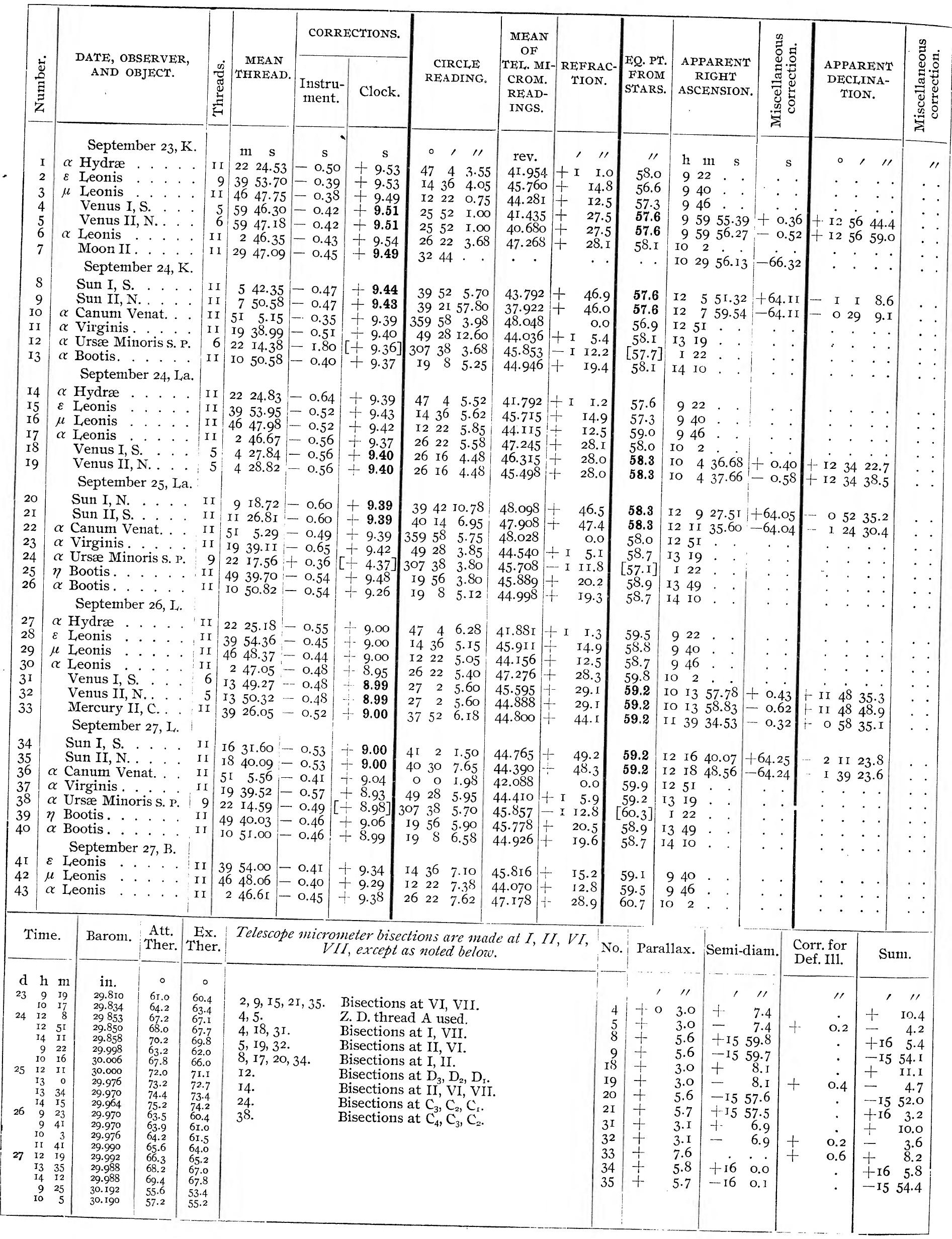




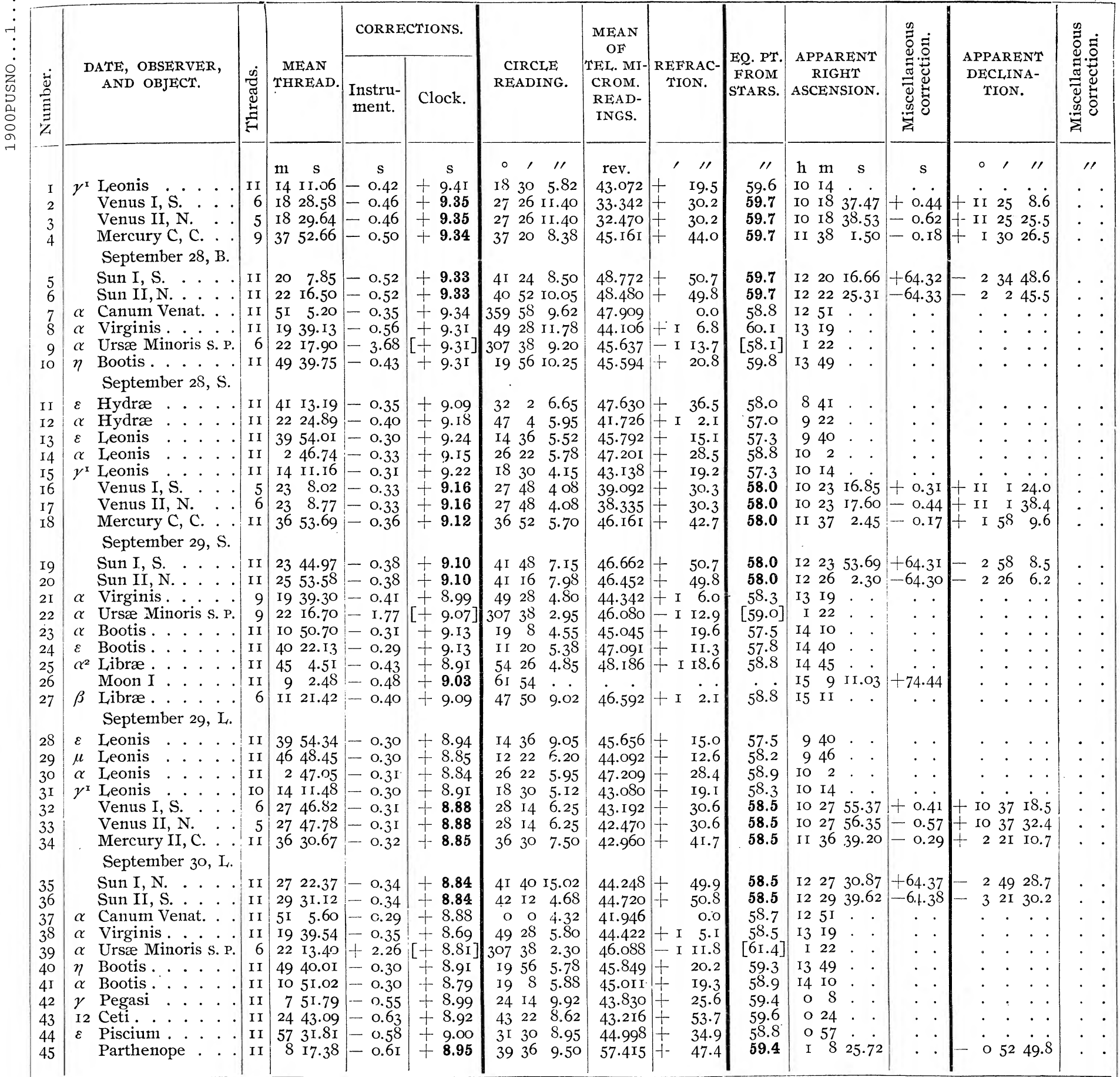

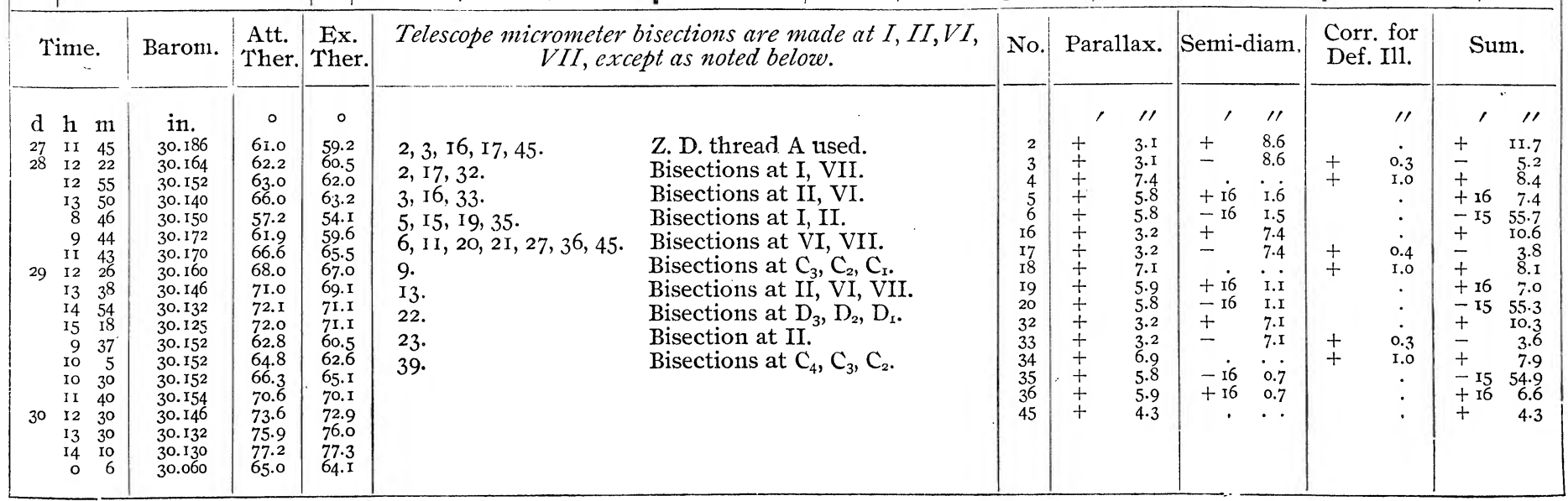




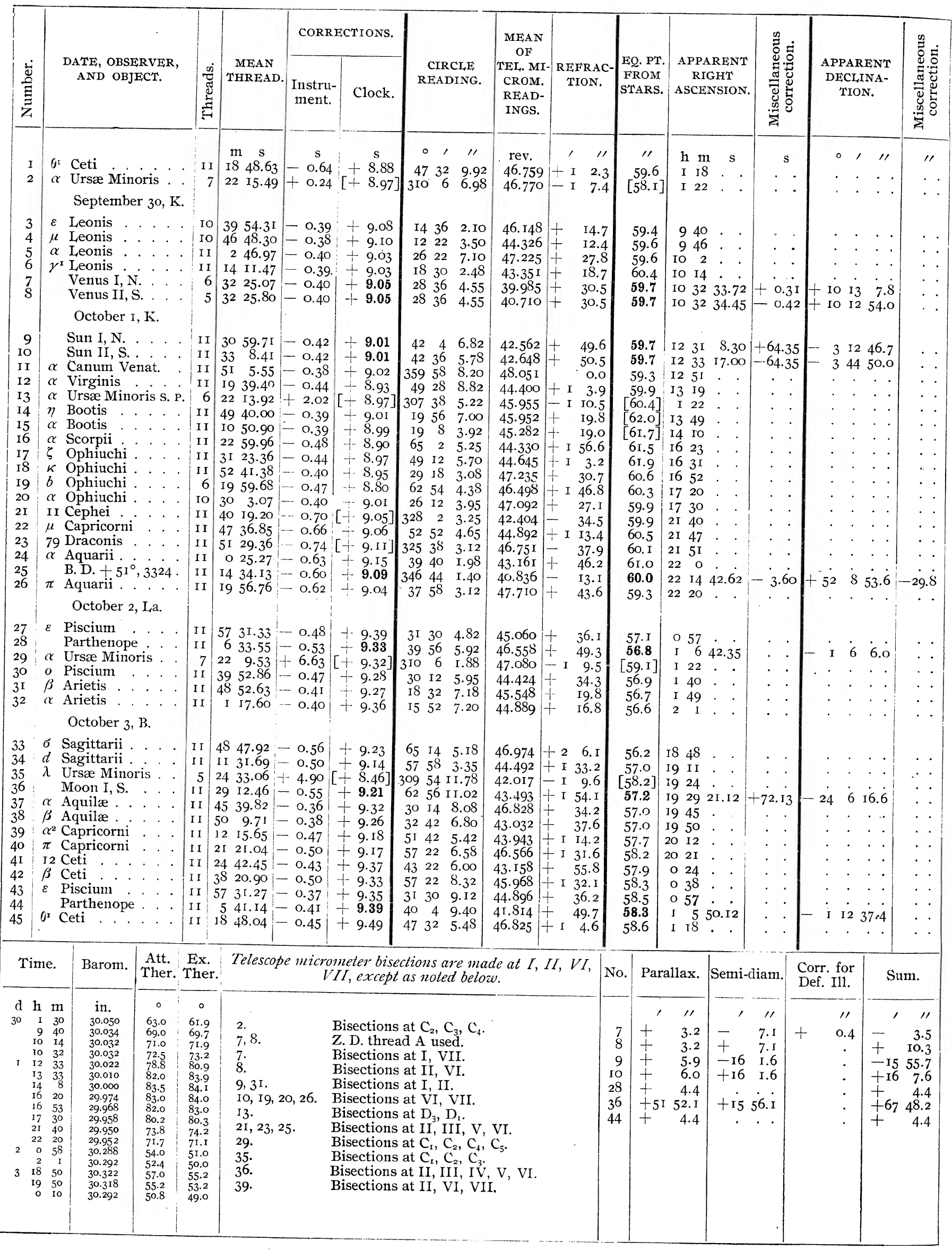




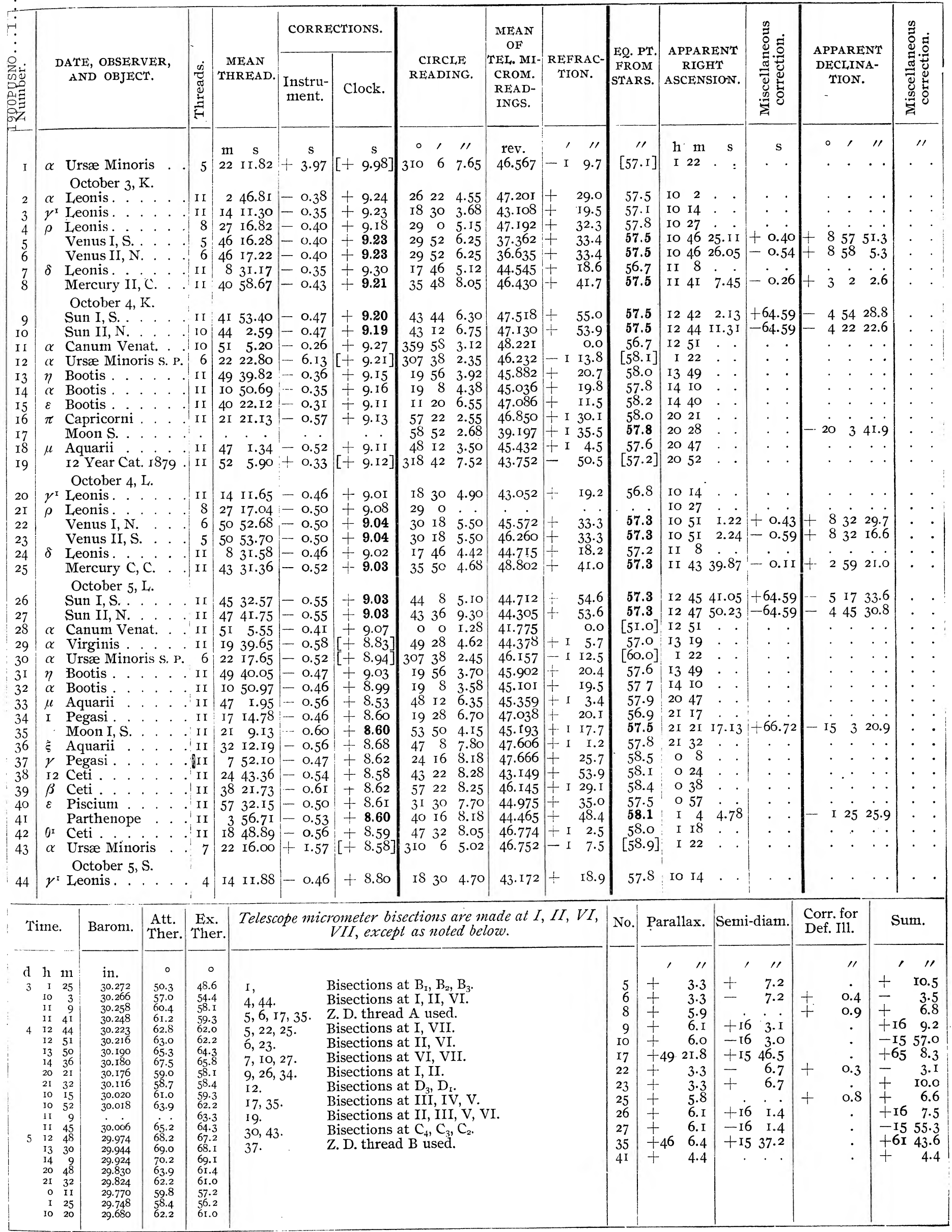




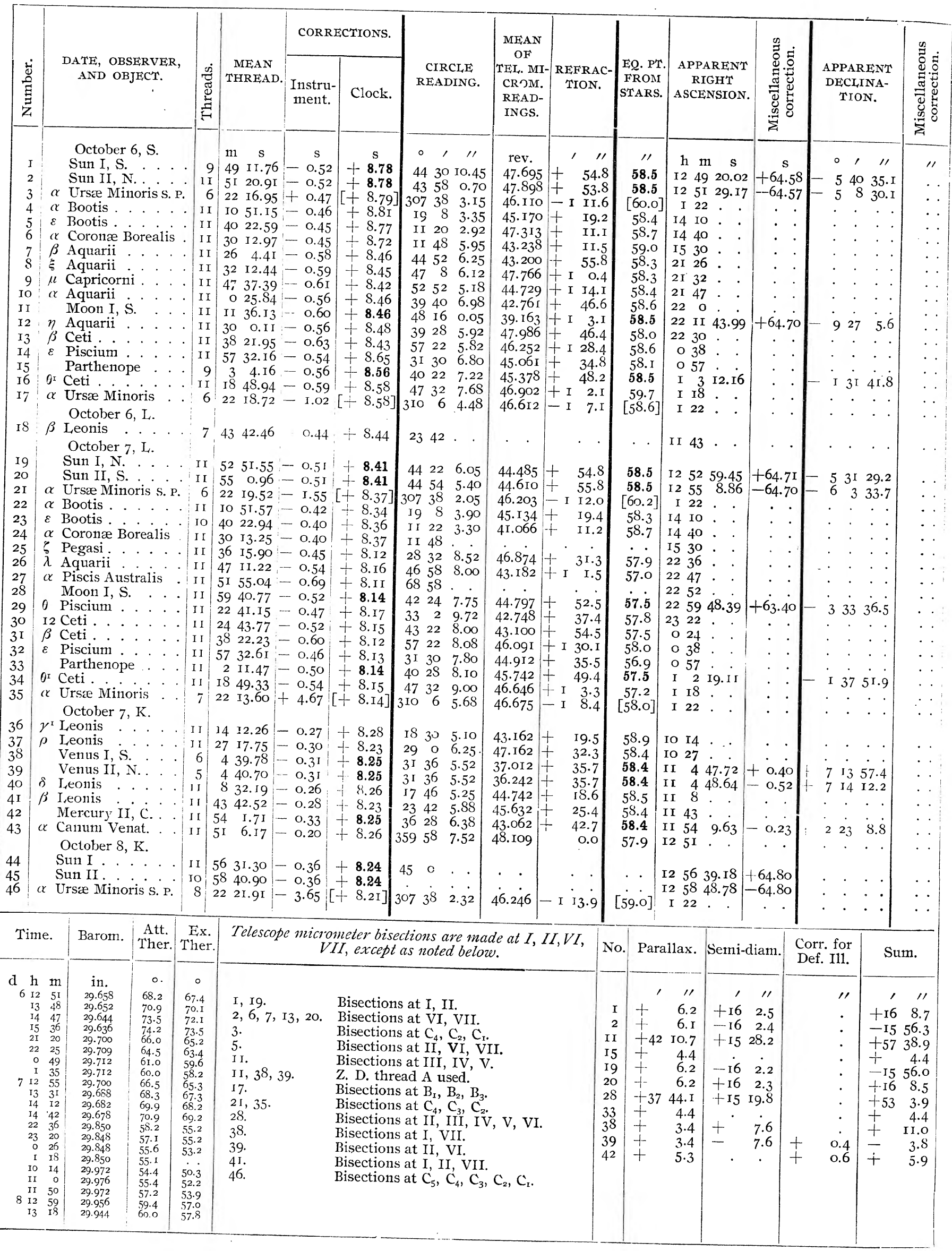




\begin{tabular}{|c|c|c|c|c|c|c|c|c|c|c|c|c|c|}
\hline \multirow[b]{2}{*}{ 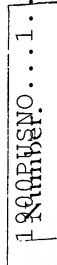 } & \multirow[b]{2}{*}{$\begin{array}{c}\text { DATE, OBSERVER, } \\
\text { AND OBJECT. }\end{array}$} & \multirow[b]{2}{*}{ 苞 } & \multirow[b]{2}{*}{$\begin{array}{l}\text { MEAN } \\
\text { THREAD. }\end{array}$} & \multicolumn{2}{|c|}{ CORRECTIONS. } & \multirow[b]{2}{*}{$\begin{array}{l}\text { CIRCIE } \\
\text { READING. }\end{array}$} & \multirow{2}{*}{$\begin{array}{c}\text { MEAN } \\
\text { OF } \\
\text { TEL. MI- } \\
\text { CROM. } \\
\text { READ- } \\
\text { INGS. }\end{array}$} & \multirow[b]{2}{*}{$\begin{array}{l}\text { REFRAC- } \\
\text { TION: }\end{array}$} & \multirow[b]{2}{*}{$\begin{array}{l}\text { EQ. PT. } \\
\text { FROM } \\
\text { STARS. }\end{array}$} & \multirow[b]{2}{*}{$\begin{array}{l}\text { APPARENT } \\
\text { RIGHT } \\
\text { ASCENSION. }\end{array}$} & \multirow{2}{*}{ 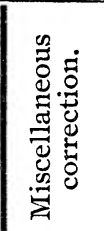 } & \multirow[b]{2}{*}{$\begin{array}{l}\text { APPARENT } \\
\text { DECLINA- } \\
\text { TION. }\end{array}$} & \multirow{2}{*}{ 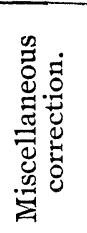 } \\
\hline & & & & $\begin{array}{c}\text { Instru- } \\
\text { ment. }\end{array}$ & Clock. & & & & & & & & \\
\hline & & & $\mathrm{m} \quad \mathrm{s}$ & $\mathbf{s}$ & & $\circ, 11$ & rev. & 111 & "1 & $\mathrm{h} \mathrm{m} \quad \mathrm{s}$ & $\mathrm{s}$ & 0,11 & 11 \\
\hline I & $\eta$ Bootis . & II & $\begin{array}{l}4940.68 \\
\end{array}$ & -0.27 & +8.20 & I9 $56 \quad 4.68$ & 45.886 & 20.8 & 58.2 & I3 49 & . & & \\
\hline 2 & $\alpha$ Bootis & II & I 10 5I.54 & -0.27 & +8.22 & I9 83.30 & 45.168 & I9.8 & 58.5 & 54 10 & $\cdot$ & & \\
\hline 3 & $\varepsilon$ Bootis $\begin{array}{l}\text { October } 8, \text { La. }\end{array}$ & II & I $40 \begin{array}{ll}40.85\end{array}$ & -0.24 & +8.28 & II 204.15 & 47.276 & II. 5 & 58.8 & I4 40 & • & . & . \\
\hline 4 & $\gamma^{\mathrm{I}}$ Leonis & II & \begin{tabular}{l|ll} 
I & I4 & I2.43
\end{tabular} & -0.38 & 8.24 & I8 $30 \quad 5.80$ & 42.952 & I9. I & 56.4 & IO I4 & & & \\
\hline 5 & Venus I, S. & 5 & $5 \quad 9 \quad 14.74$ & -0.44 & +8.18 & $\begin{array}{lll}32 & 2 & 6.02\end{array}$ & 48.920 & 35.4 & 57.3 & II $\quad 922.48$ & +0.32 & +64723.0 & \\
\hline 6 & Venus II, N. . & 6 & $9 \begin{array}{ll}15.48 \\
\end{array}$ & -0.44 & +8.18 & $\begin{array}{lll}32 & 2 & 6.02\end{array}$ & 48.235 & 35.4 & 57.3 & II 923.22 & -0.42 & +64736.1 & \\
\hline 7 & $\beta$ Leonis & II & I $43 \quad 42.77$ & -0.40 & +8.12 & $23 \quad 42 \quad 4.90$ & 45.685 & 24.7 & 58.0 & II 43 & & . & \\
\hline 8 & Mercury II, C. & II & I $\begin{array}{lll}58 & 20.03\end{array}$ & -0.46 & +8.15 & $\begin{array}{lll}36 & 48 & 4.90\end{array}$ & $44.33 I$ & $+\quad 4 \mathrm{I} .8$ & 57.3 & II $\begin{array}{lll}58 & 27.72\end{array}$ & -0.22 & †' $\quad 2 \quad 2 \quad 45.7$ & \\
\hline 9 & $\begin{array}{l}\alpha \text { Canum Venat. } \\
\text { October 9, La. }\end{array}$ & 5 & $\begin{array}{lll}5 & 5 \mathrm{I} & 6.44\end{array}$ & $-0.3 \mathrm{I}$ & +8.13 & $3595^{8} \cdot \ldots$ & & & & I2 $5 \mathrm{I}$ & 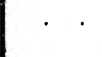 & 1 & . \\
\hline Io & Sun I, S. . . & I I & o 11.97 & -0.50 & +8.11 & $4540 \quad 4.30$ & 43.682 & 56.7 & 57.3 & I3 0 I 9.58 & +64.76 & 649 I 4.9 & \\
\hline II & , N. . . & II & $\begin{array}{ll}2 & 21.50\end{array}$ & -0.50 & +8.11 & 4588 4. 5 & 43.468 & 55.7 & 57.3 & I3 22 29.II & 64.77 & $\begin{array}{lll}6 & \text { I7 } & \text { II. } 7\end{array}$ & \\
\hline $\mathrm{I} 2$ & $\alpha$ Bootis & 7 & 7 Io 5 r.83 & -0.39 & +8.05 & $\begin{array}{lll}19 & 8 & 4.05\end{array}$ & 45.000 & 19.3 & 57. I & I4 Io & &.$\quad$. & \\
\hline 13 & $\varepsilon$ Boo & Io & $\begin{array}{lll}40 & 23.20\end{array}$ & -0.36 & $\begin{array}{l}+8.05 \\
\end{array}$ & II $20 \quad 9.40$ & 46.893 & II. 2 . & 56.7 & I4 40 & . & . & • \\
\hline 14 & $\beta$ Ursæ Minoris & II & I 5048.90 & -0.05 & $+7.89]$ & $\begin{array}{lll}324 & \text { I8 } & 9.05\end{array}$ & 43.087 & 39.6 & {$[56.4]$} & I4 5 O & . & . & • \\
\hline 15 & Borealis . & II & I $30 \quad \mathrm{I} 3.56$ & -0.36 & +8.00 & II $48 \quad 3.52$ & 74 & II. 6 & 57.5 & 1530 & . & . & \\
\hline 16 & $\alpha \operatorname{Serp}$ & . 10 & 396.15 & -0.44 & +8.03 & $\begin{array}{lll}32 & 6 & 3.7\end{array}$ & & 34.8 & $58 . \mathrm{I}$ & I5 39 & . & . & \\
\hline I7 & 79 Dra & II & I 5 I 29.53 & -0.18 & $+7.96]$ & $\begin{array}{lll}325 & 38 & 3.65\end{array}$ & 46.584 & 38.9 & 58.1 & $2 \mathrm{I} 5 \mathrm{I}$ & & . & \\
\hline 18 & $5^{\mathrm{I}^{\circ}}, 3324$ & i I & I I4 34.83 & -0.32 & 8.05 & $\begin{array}{lll}346 & 43 & 31.87\end{array}$ & $42 . \mathrm{I} 3 \mathrm{I}$ & 13.5 & 57.1 & 22 I4 42.56 & 3.45 & +52855.4 & 3 I. 6 \\
\hline 19 & o Ceph & II & 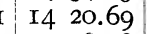 & -0.23 & {$[+7.80]$} & $33 \mathrm{I}$ I8 6.35 & 06 & $3 \mathrm{I} .4$ & $55 \cdot 7$ & 2314 & & . & \\
\hline 20 & $\omega$ Pisci & II & $535^{8.18}$ & -0.50 & +8.00 & $\begin{array}{lll}3^{2} & 32 & 7.50\end{array}$ & 46.612 & 36.8 & 57.4 & 2354 & & . & . \\
\hline $2 \mathrm{I}$ & $\gamma$ Pegas & II & 752.69 & -0.46 & +8.03 & $24 \mathrm{I} 4 \quad 7.90$ & 48 & 26.0 & $57 . \mathrm{I}$ & o 8. & & & \\
\hline 22 & I2 Ceti & II & $\begin{array}{ll}1 & 24 \\
1 & 43.95\end{array}$ & -0.54 & +8.00 & $\begin{array}{lll}43 & 22 & 8.38\end{array}$ & 50 & 54.6 & 56.9 & O 24 & & & . \\
\hline 23 & Moon & II & $\mathrm{I} \mid \begin{array}{ll}33 & 3.4 \mathrm{I}\end{array}$ & -0.5 & +8.06 & $\begin{array}{lll}30 & \text { I8 } & 3.98\end{array}$ & & & 57.0 & o $33 \quad 10.97$ & +62.99 & +83242.9 & \\
\hline 24 & Partl & II & I $\quad 026.30$ & -0.53 & +8.06 & $\begin{array}{llll}40 & 40 & 3.42\end{array}$ & 82 & 49.8 & 57.0 & $\mathrm{I} O 33.83$ & . & I 4952.7 & . \\
\hline 25 & $\alpha$ Ursæ Minoris & 7 & 722 II.II & +2.18 & {$[+\mathrm{I} 3.4 \mathrm{I}]$} & 310 $6 \begin{array}{ll}3.45\end{array}$ & 46.740 & - I 8.6 & 57.6 & I 22. & . . & $. \quad . \quad$. & \\
\hline $\begin{array}{l}26 \\
27\end{array}$ & $o$ Piscium . & II & $\begin{array}{l}1954.05 \\
\end{array}$ & -0.48 & +8.19 & $\begin{array}{lll}30 & \mathrm{I} 2 & 5.42\end{array}$ & 44.436 & $+\quad 33.9$ & 56.5 & I 40 & . & . & . \\
\hline 28 & $\beta$ Ceti & II & $\begin{array}{lll}38 & 22.98\end{array}$ & -0.5 & +7.32 & $\begin{array}{lll}57 & 22 & 4\end{array}$ & $\dot{I}$ & f- I 27.2 & 60.7 & o 38 & & & \\
\hline 29 & Parthenope . & II & $\begin{array}{lll}58 & 42.15\end{array}$ & $-0.4 \delta$ & +7.28 & $\begin{array}{lll}40 & 52 & 4.68\end{array}$ & 44.584 & 48.4 & 59.9 & O 5848.95 & & $-\begin{array}{llll}- & 2 & \text { I } & 22.9\end{array}$ & \\
\hline 30 & $\beta$ Ari & Io & $48 \quad 54.79$ & -0.43 & +7.24 & I8 $32 \quad 6.92$ & 45.612 & I 8.8 & 59. I & I 49. & & & \\
\hline $3 \mathrm{I}$ & $\begin{array}{l}\text { Moon II. } \\
\text { October I2, Br. }\end{array}$ & II & I I I 3.68 & -0.45 & $\begin{array}{r}+7.29 \\
\end{array}$ & $2046 \ldots$ & & & | & 2 II I0.52 & -64.87 & . & \\
\hline 32 & $\gamma$ Pegasi . . . & I I & 75 & $-0.3^{8}$ & +7 & $24 \mathrm{I} 4$ & & & & & • & • & \\
\hline 33 & $\beta \mathrm{Ce}$ & II & 3822.99 & -0.44 & & $\begin{array}{lll}57 & 22 & 6.6\end{array}$ & 46.445 & I 28.2 & 61.5 & о 38 & & & \\
\hline 34 & Parthenope & II & 5 & -0.40 & +7.26 & $4058 \quad 6.6$ & 43.026 & 49. I & 60.1 & o 5757.15 & & $26 \quad 655.4$ & \\
\hline 35 & $\alpha$ Ursæ Minoris & 5 & 52230.18 & -4.97 & {$[+2.19]$} & 3io $6 \quad 5$. 10 & 46.644 & - I 6.8 & {$[60.1]$} & I 22 & & $. \quad . \quad$. & \\
\hline $3^{6}$ & $\gamma$ Ceti & I I & $37 \quad 55.33$ & -0.39 & +7.30 & $\begin{array}{lll}36 & 2 & 4.9\end{array}$ & & $4 \mathrm{I} \cdot 3$ & & $23^{8}$ & & . & \\
\hline 37 & Moon II, N. . & I I & I 30.54 & $-0.3^{8} \mid$ & +7.22 & I6 $46 \quad 5.90$ & 44.572 & 17.2 & 60.1 & $\begin{array}{lll}3 & \text { I } & 37.38\end{array}$ & -66.20 & $+22 \quad 5 \quad 7.5$ & \\
\hline $3^{8}$ & $\varepsilon$ Eridani. . . . & II & $\left|\begin{array}{ll}28 & 2.07\end{array}\right|$ & -0.42 & +7.13 & $\begin{array}{lll}48 & 38 & 5.8\end{array}$ & 45.496 & + I 4.6 & 59 & 328. & 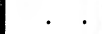 & & \\
\hline 39 & $\eta$ Tauri & II & $\begin{array}{lll}4 \mathrm{I} & \mathrm{I} 9.54\end{array}$ & -0.37 & +7.28 & $15 \quad 4 \quad 4.18$ & 43.542 & 15.4 & 6o.I & $34 \mathrm{I}$ & . & . & . \\
\hline 40 & $\zeta$ Persei. $\quad$. & II & I $47 \quad 37.65$ & -0.37 & +7.13 & $\begin{array}{lll}7 & 16 & 2.58\end{array}$ & $45.84 \mathrm{I}$ & $7 \cdot 3$ & 60.3 & 347 & & . & . \\
\hline $4 \Gamma$ & $\mu$ Leonis . . . & II & 465 & -0.37 & -7.66 & $12 \quad 22 \quad 3.3$ & & 12.5 & $5^{8.0}$ & 946 & & • & • \\
\hline 42 & $\alpha$ Leo & c & 248.57 & -0.3 & 7.69 & $\begin{array}{lll}26 & 22 & 4.0\end{array}$ & 60 & 28.3 & 58.0 & IO 2 & & 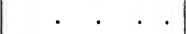 & \\
\hline 43 & $\gamma^{\mathrm{I}}$ Leonis & II & I4 I3.05 & $-0.3^{8}$ & +7.72 & I8 $30 \quad 3.5$ & 43.249 & I9.0 & 57.5 & IO $I_{4}$ & & . & \\
\hline 44 & $\rho$ Leoni & II & $27 \quad 18.49$ & -0.39 & +7.69 & 2903.6 & & $\mathrm{i}$ & & IO 27 & & & • \\
\hline 45 & Ver & 6 & $5 \mid 273$ I.I5 & -0.40 & +7.67 & $\begin{array}{lll}33 & 50 & 2.82\end{array}$ & 40.260 & 38.0 & 58.1 & II 2738.42 & +-0.35 & $+45^{8} 55.3$ & . \\
\hline 46 & Venus II, N. . & 5 & $5 \quad 2731.94$ & -0.40 & +7.67 & $\begin{array}{lll}33 & 50 & 2.82\end{array}$ & 39.648 & 37.9 & 58.1 & II 2739.21 & -0.44 & $+4597 . \mathrm{I}$ & . \\
\hline
\end{tabular}

\begin{tabular}{|c|c|c|c|c|c|c|c|c|c|}
\hline Time. & Barom. & Att. & Ex. & $\begin{array}{c}\text { Telescope micrometer bisections are made at } I, I I, V I, \\
V I I, \text { except as noted below. }\end{array}$ & No. & Parallax. & Semi-diam. & $\begin{array}{l}\text { Corr. for } \\
\text { Def. Ill. }\end{array}$ & Sum. \\
\hline 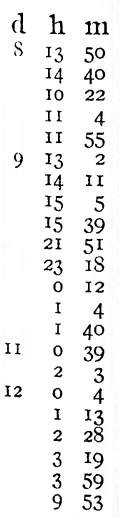 & $\begin{array}{l}\text { in. } \\
29.932 \\
29.966 \\
29.8280 \\
29.850 \\
29.868 \\
29.888 \\
29.876 \\
29.884 \\
29.880 \\
29.967 \\
29.980 \\
29.988 \\
29.992 \\
30.000 \\
29.732 \\
29.720 \\
29.930 \\
29.944 \\
29.956 \\
29.958 \\
29.964 \\
30.136\end{array}$ & $\begin{array}{c}\circ \\
61.5 \\
64.2 \\
59.0 \\
63.4 \\
68.0 \\
72.4 \\
73.6 \\
74.6 \\
73.8 \\
61.6 \\
58.4 \\
57.2 \\
55.6 \\
53.8 \\
68.0 \\
68.1 \\
65.5 \\
65.5 \\
64.5 \\
63.5 \\
63.5 \\
65.6\end{array}$ & $\begin{array}{c}\circ \\
59.3 \\
61.1 \\
58.5 \\
63.0 \\
68.8 \\
74.1 \\
73.9 \\
74.3 \\
73.5 \\
59.2 \\
56.4 \\
55.0 \\
53.3 \\
51.8 \\
67.1 \\
67.2 \\
64.2 \\
64.4 \\
63.0 \\
62.3 \\
62.3 \\
64.3\end{array}$ & $\begin{array}{ll}\text { 4, II. } & \text { Bisections at VI, VII. } \\
5,39,45 . & \text { Bisections at II, VI. } \\
\text { 6, 46. } & \text { Bisections at I, VII. } \\
\text { IO, I6. } & \text { Bisections at I, II. } \\
\text { I2. } & \text { Bisection at VII. } \\
\text { I3, 33. } & \text { Bisections at II, VI, VII. } \\
\text { I4, 37. } & \text { Bisections at III, IV, V. } \\
\text { I7, I9. } & \text { Bisections at III, C }, C_{5}, \text { V. } \\
\text { 23. } & \text { Bisections at II, III, IV, V, VI. } \\
\text { 25. } & \text { Bisections at } \mathrm{C}_{1}, \mathrm{C}_{3}, \mathrm{C}_{4} \cdot \\
\text { 35. } & \text { Bisections at } \mathrm{C}_{1}, \mathrm{C}_{2}, \mathrm{C}_{3}, \mathrm{C}_{4}, \mathrm{C}_{5} . \\
45,46 . & \text { Z. D. thread A used. }\end{array}$ & $\begin{array}{r}5 \\
6 \\
8 \\
\text { IO } \\
\text { II } \\
23 \\
24 \\
29 \\
34 \\
37 \\
45 \\
46\end{array}$ & $\begin{array}{lr} & 11 \\
+\quad & 3.4 \\
+\quad & 3.4 \\
+ & 5.2 \\
+ & 6.3 \\
+ & 6.3 \\
+27 & 43.5 \\
+ & 4.4 \\
+ & 4.4 \\
+ & 4.4 \\
+ & 30.5 \\
+ & 3.6 \\
+\quad & 3.6\end{array}$ & 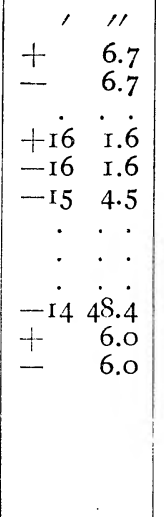 & $\begin{array}{c}0.3 \\
0.6 \\
\cdot \\
\cdot \\
\cdot \\
\cdot \\
\cdot \\
\cdot\end{array}$ & 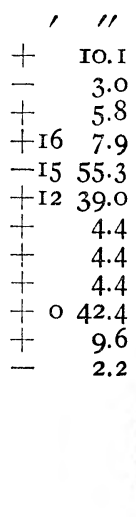 \\
\hline
\end{tabular}




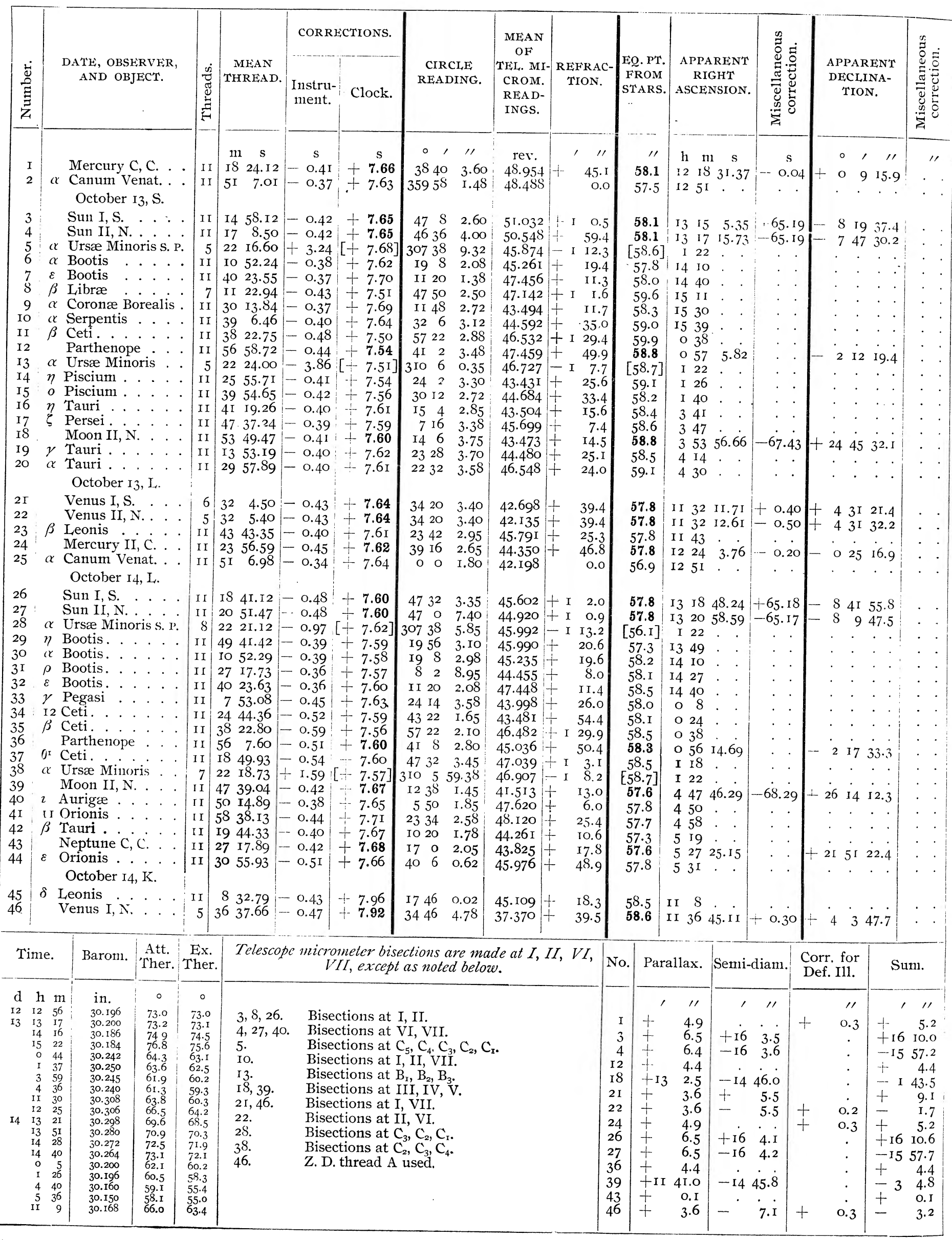




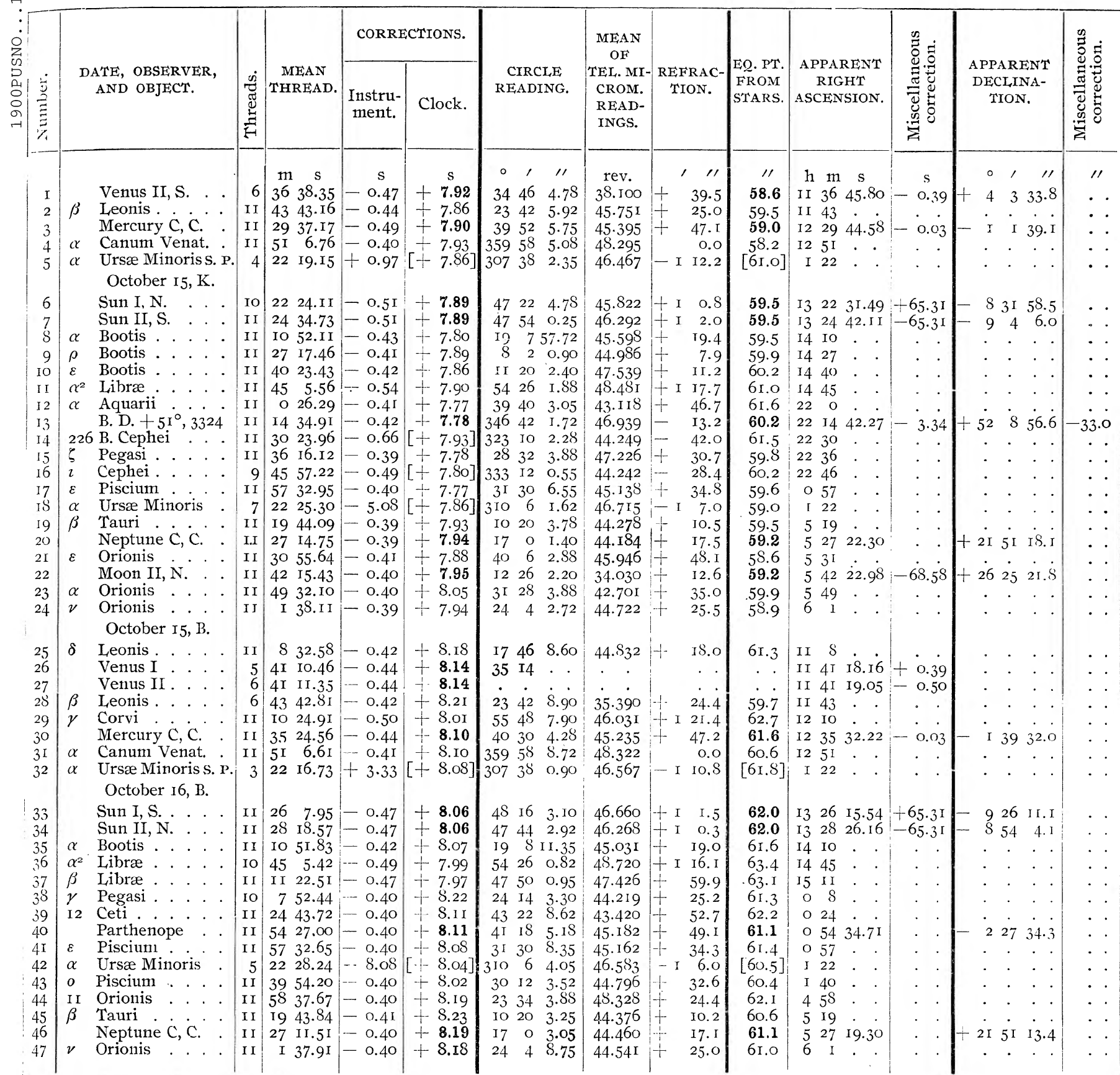

\begin{tabular}{|c|c|c|c|c|c|c|c|c|c|c|c|}
\hline Time. & Barom. & $\begin{array}{l}\text { Att. } \\
\text { Ther. }\end{array}$ & $\begin{array}{l}\text { Ex. } \\
\text { Ther. }\end{array}$ & Telescope $m$ & $\begin{array}{l}\text { crometer bisections are made at } I, I I, V I \text {, } \\
V I I \text {, except as noted below. }\end{array}$ & No. & Parallax. & Seni-diam. & & $\begin{array}{l}\text { for } \\
\text { I11. }\end{array}$ & Sum. \\
\hline d $\mathrm{h} \mathrm{m}$ & in. & $\circ$ & 0 & & & & 111 & ' !l & & 11 & , II \\
\hline 14 II 44 & 30.168 & 68.6 & 66.1 & I. & Bisections at II, VI. & I & 3.6 & 7.1 & & • & $+\quad 10.7$ \\
\hline $\begin{array}{rr}12 & 30 \\
13 & 7\end{array}$ & $\begin{array}{l}30.168 \\
30.164\end{array}$ & $\begin{array}{l}71.8 \\
73.3\end{array}$ & $\begin{array}{l}70.4 \\
72.2\end{array}$ & $\mathrm{I}, 22,28$ & Z. D. thread A used. & 3 & + & & + & 0.3 & 5.2 \\
\hline $\begin{array}{lll}15 & 13 & 25 \\
1 & 25\end{array}$ & 30.152 & 74.0 & $73 . \mathrm{I}$ & $4,7,28,34$ & Bisections at VI, VII. & 6 & 6.5 & $\begin{array}{ll}-16 & 3.7\end{array}$ & & . & -1557.2 \\
\hline I4 II & 30.125 & 75.6 & 75.2 & & Bisections at $D_{3}, D_{1}, C_{5}$. & 7 & 6.6 & $+16 \quad 3.7$ & & . & +1610.3 \\
\hline $\begin{array}{rr}14 & 45 \\
22 & 0\end{array}$ & $\begin{array}{l}30.0118 \\
30.060\end{array}$ & $\begin{array}{l}78.8 \\
70.2\end{array}$ & $\begin{array}{l}76.4 \\
68.9\end{array}$ & 6,33 & Bisections at I, II. & 20 & & & & . & + \\
\hline 2246 & 30.056 & 69.2 & 68.0 & $13,14,16$ & Bisections at II, III, V, VI. & 22 & + II 32.4 & -1448.0 & & . & -315.6 \\
\hline $\begin{array}{ccc}0 & 58 \\
0\end{array}$ & 30.026 & 66.5 & 65.3 & 18,42 & Bisections at $\mathrm{C}_{-1}, \mathrm{C}_{2}, \mathrm{C}_{3}, \mathrm{C}_{4}, \mathrm{C}_{5}$. & 30 & 4.9 & & + & 0.2 & 5. I \\
\hline & 30.018 & $\begin{array}{l}66.0 \\
61.8\end{array}$ & 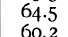 & 22. & Bisections at II, III, IV, V, VI. & 33 & 6.6 & $+16 \quad 3.5$ & & . & +16 IO.I \\
\hline $\begin{array}{rr}5 & 20 \\
6 & 2\end{array}$ & $\begin{array}{l}29.964 \\
29.960\end{array}$ & $\begin{array}{l}618 \\
61.5\end{array}$ & $\begin{array}{l}60.2 \\
59.5\end{array}$ & 32. & Bisections at $\mathrm{D}_{3}, \mathrm{D}_{2}, \mathrm{D}_{1}$ & 34 & 6.6 & $\begin{array}{ll}-16 & 3.5\end{array}$ & & . & - I5 56.9 \\
\hline II 0 & 29.996 & 72.0 & 70.1 & 36. & Bisections at II, VI, VII. & 40 & 4.4 & (5) & & . & $+\quad 4.4$ \\
\hline $\begin{array}{rr}12 & 0 \\
12 & 55\end{array}$ & 29.998 & 77.4 & 75.7 & & & 46 & &. & & . & + \\
\hline $\begin{array}{lll}16 & 12 & 55 \\
13 & 28\end{array}$ & $\begin{array}{l}29.990 \\
29.966\end{array}$ & $\begin{array}{l}80.0 \\
81.2\end{array}$ & $\begin{array}{l}77.1 \\
\text { So.9 }\end{array}$ & & & & & & & & \\
\hline $\begin{array}{ll}14 & 15 \\
15 & 10\end{array}$ & 29.940 & $\begin{array}{l}82.5 \\
86.6\end{array}$ & 83.1 & & & & & & & & \\
\hline $\begin{array}{rll}0 & 10 \\
0 & 10 & -1\end{array}$ & 29.870 & 71.0 & 69.9 & & & & & & & & \\
\hline $\begin{array}{rr}1 & 25 \\
5 & 0\end{array}$ & $\begin{array}{l}29.800 \\
29.820\end{array}$ & $\begin{array}{l}69.0 \\
68.8\end{array}$ & $\begin{array}{l}68.1 \\
70.1\end{array}$ & & & & & & & & \\
\hline 535 & 29.838 & 69.0 & 68.7 & & & & & & & & \\
\hline
\end{tabular}

$6855-11$ 


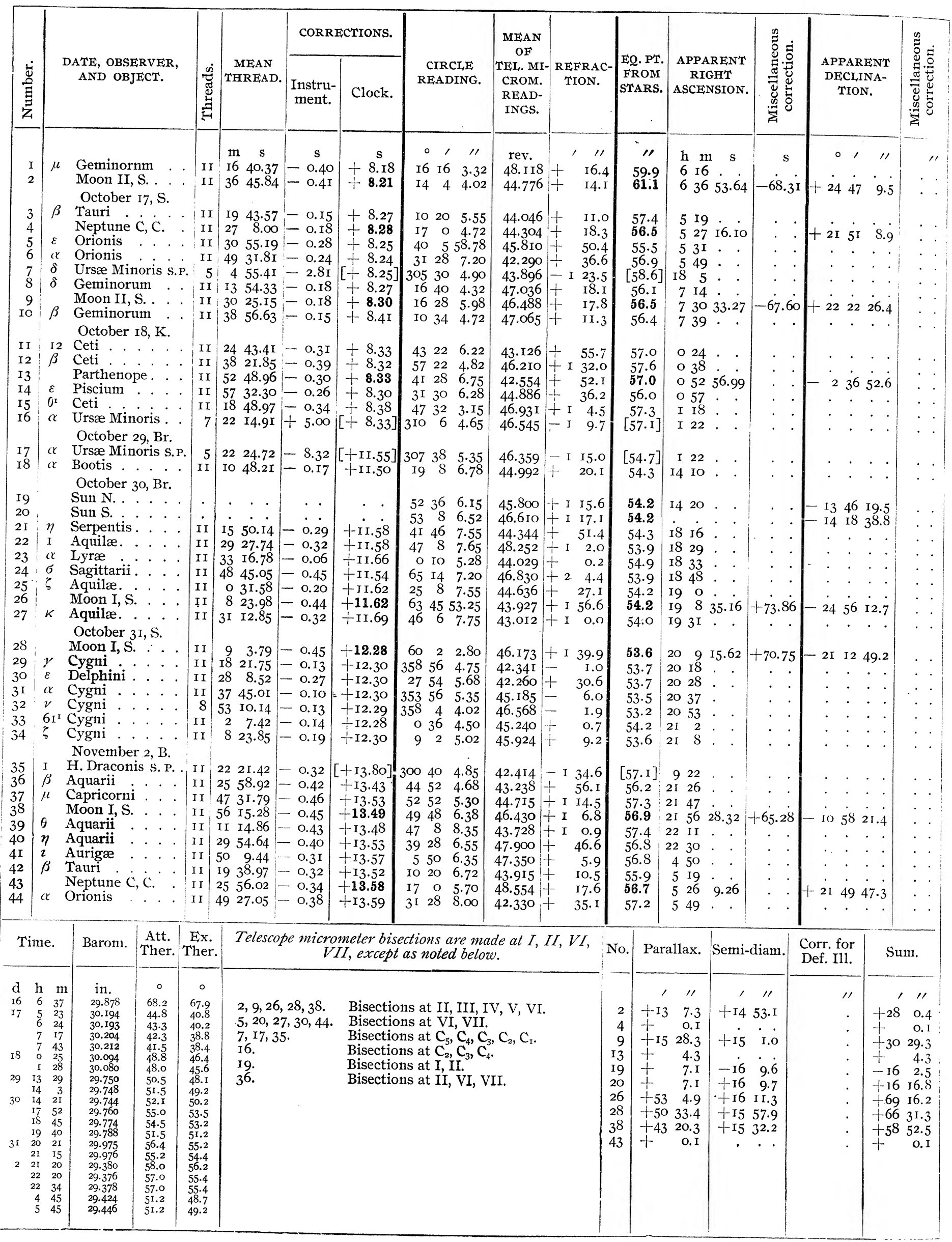




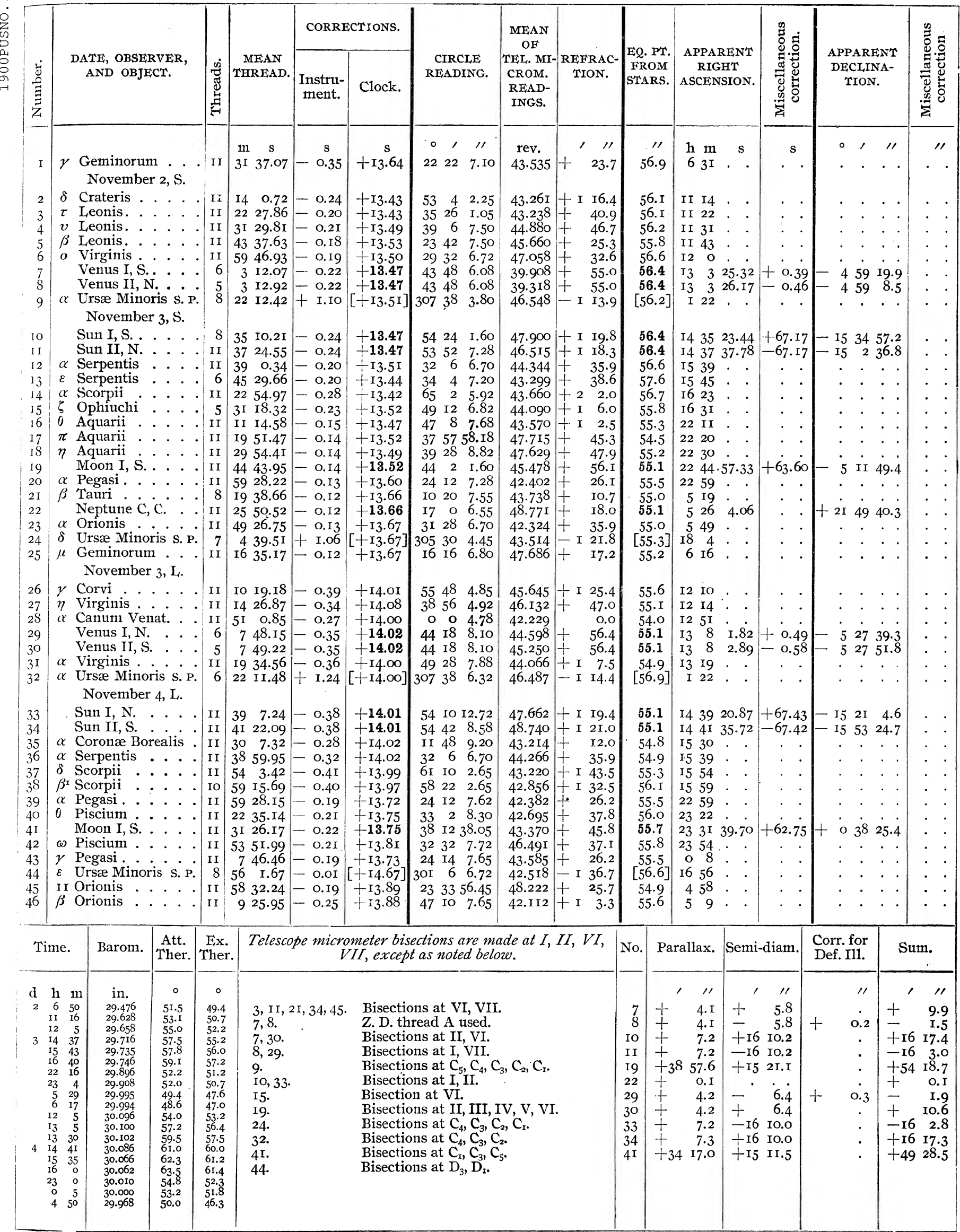




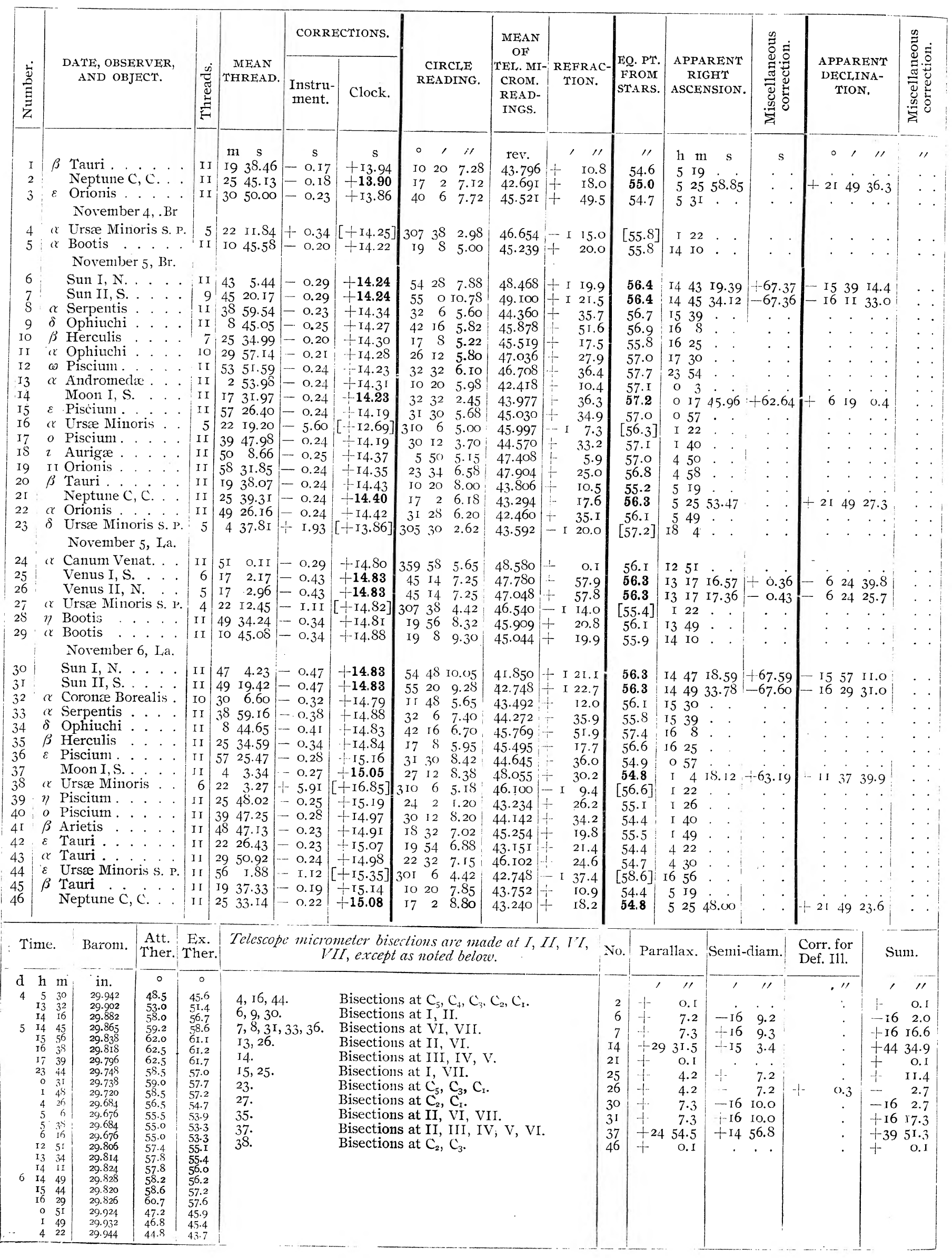




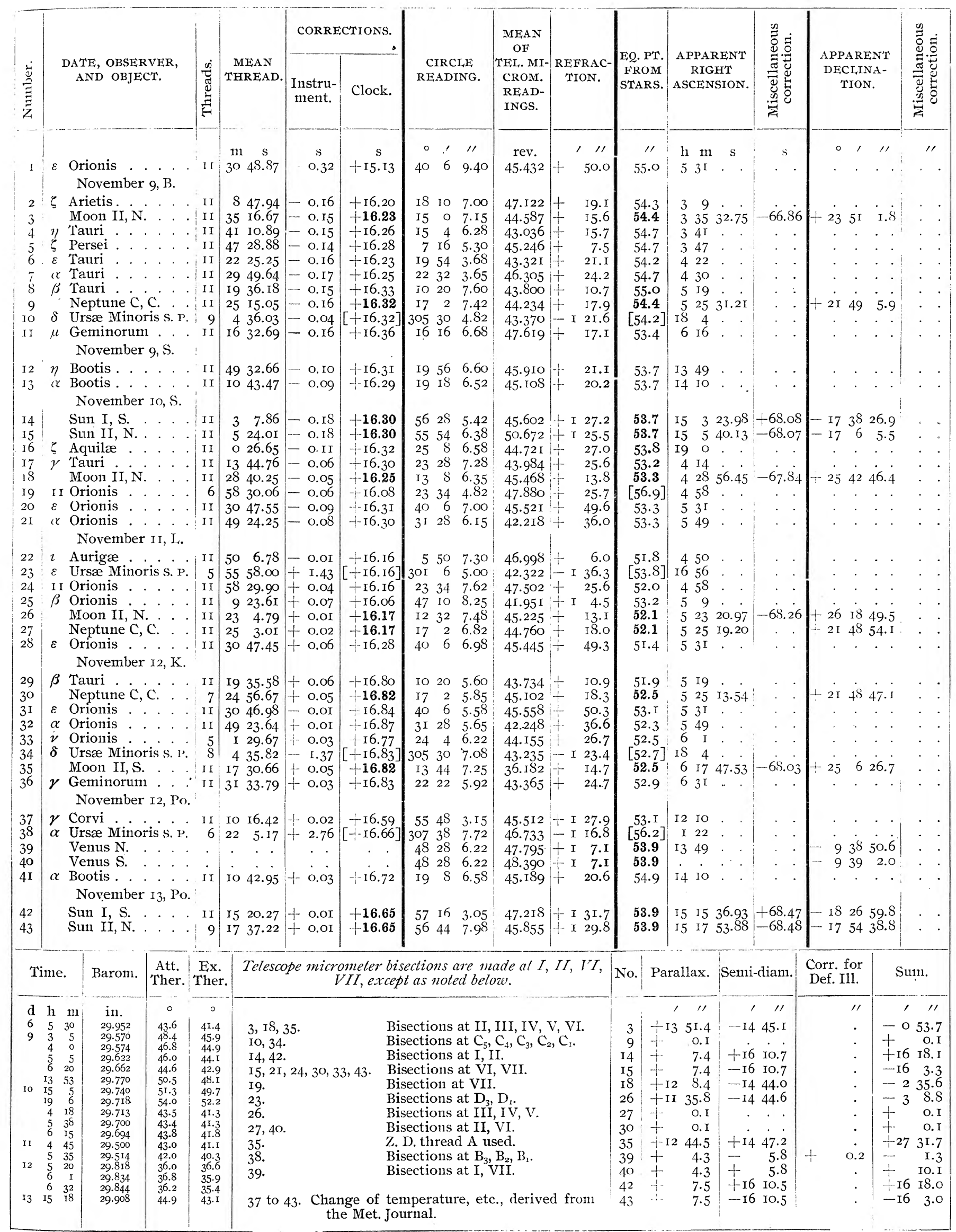




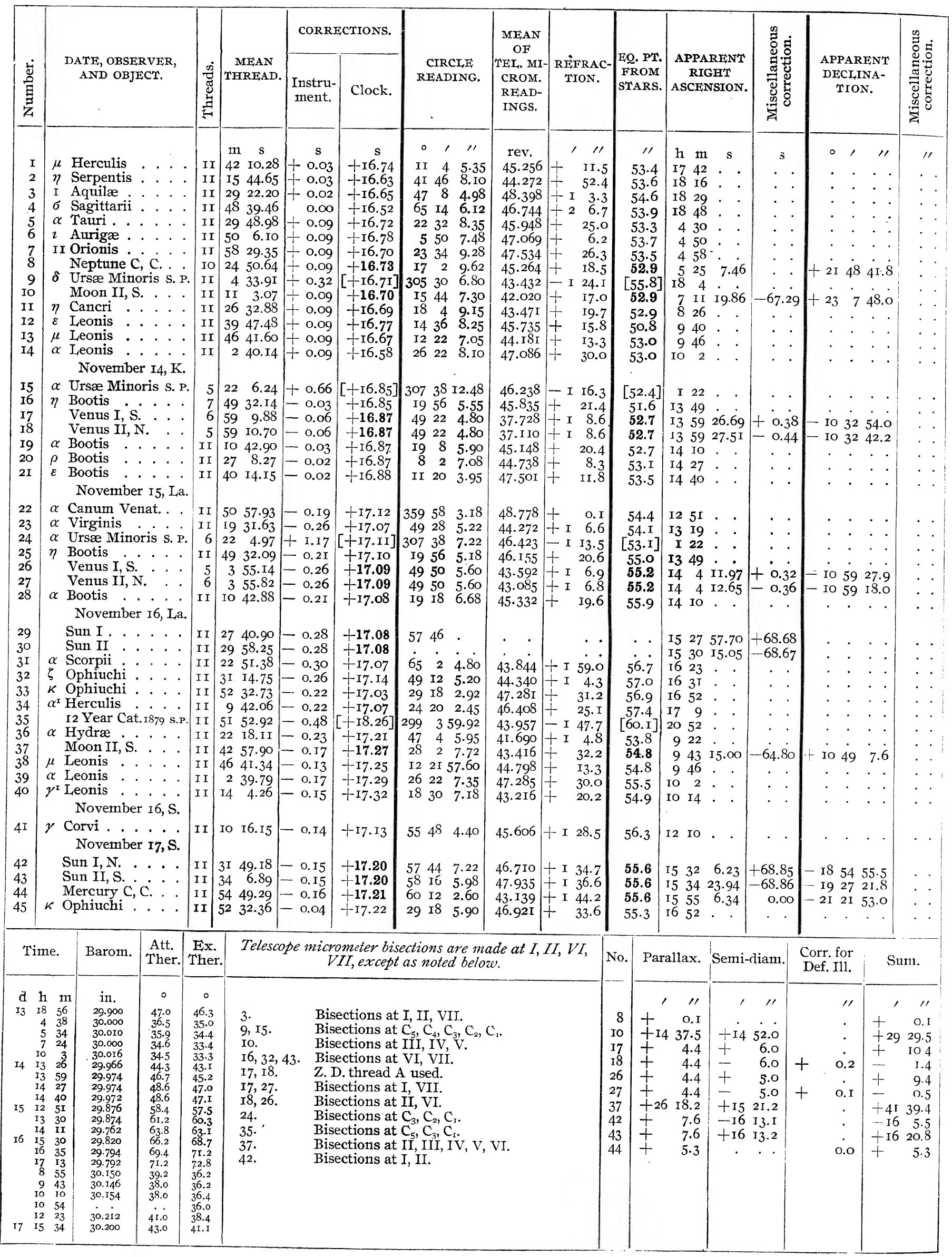




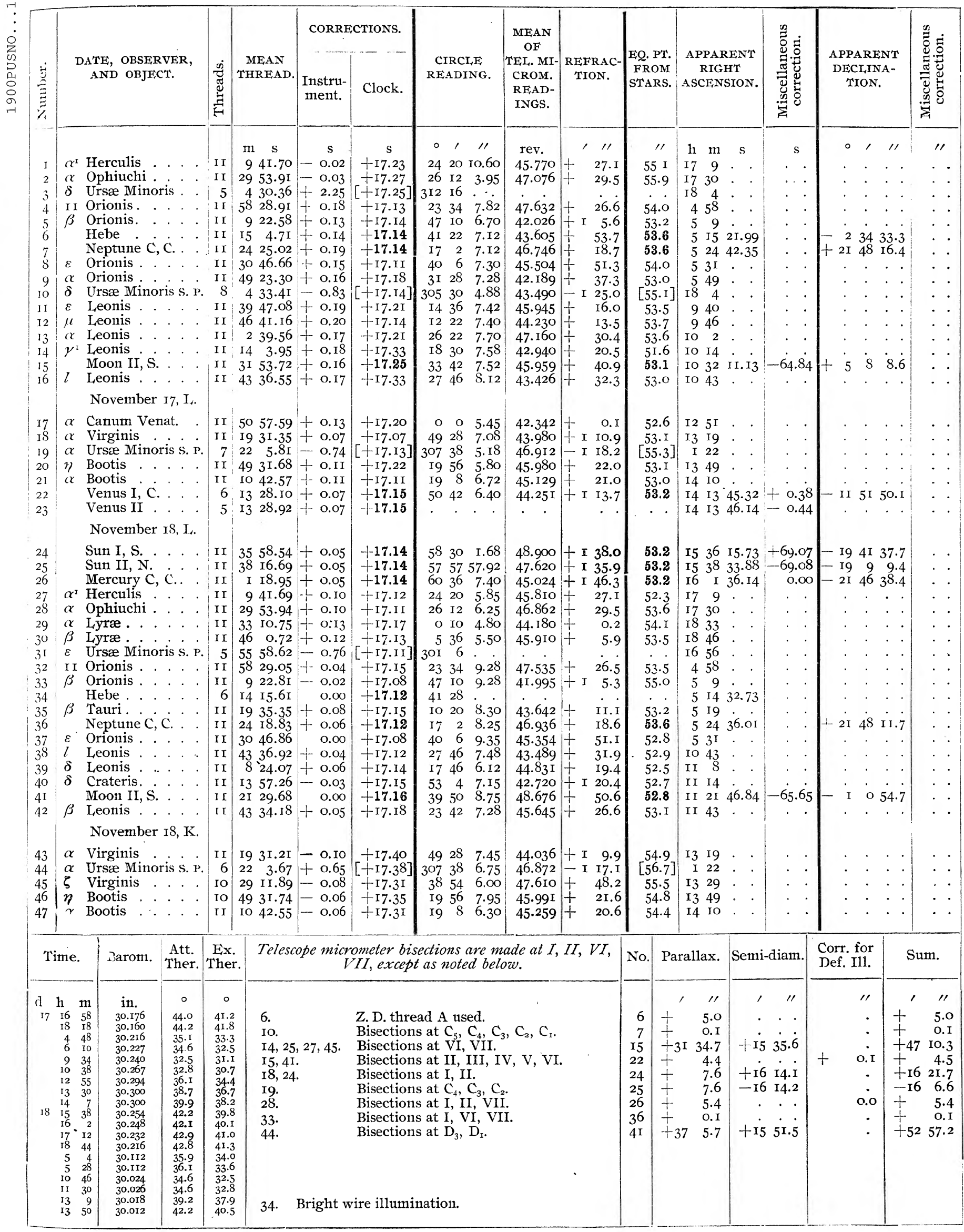




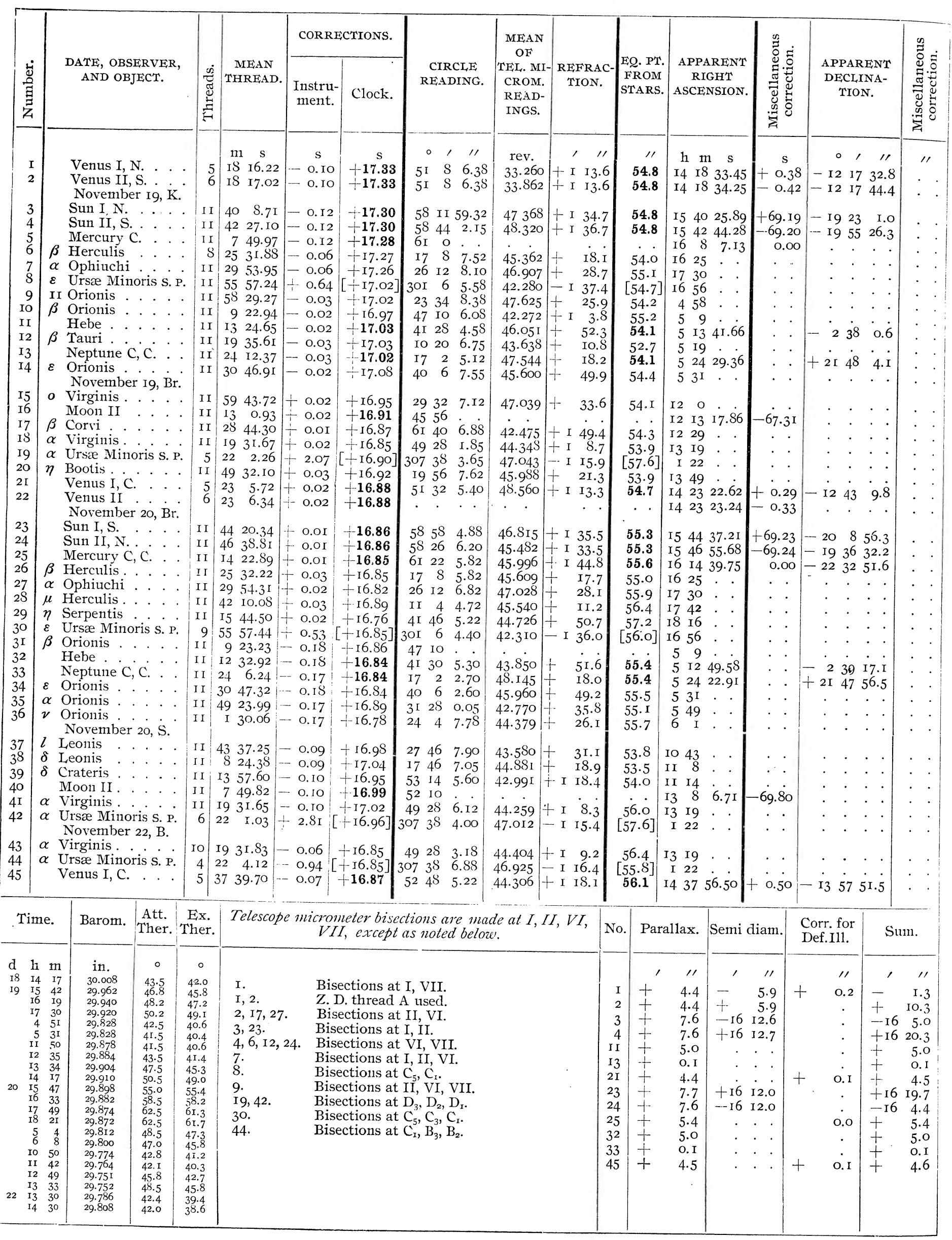




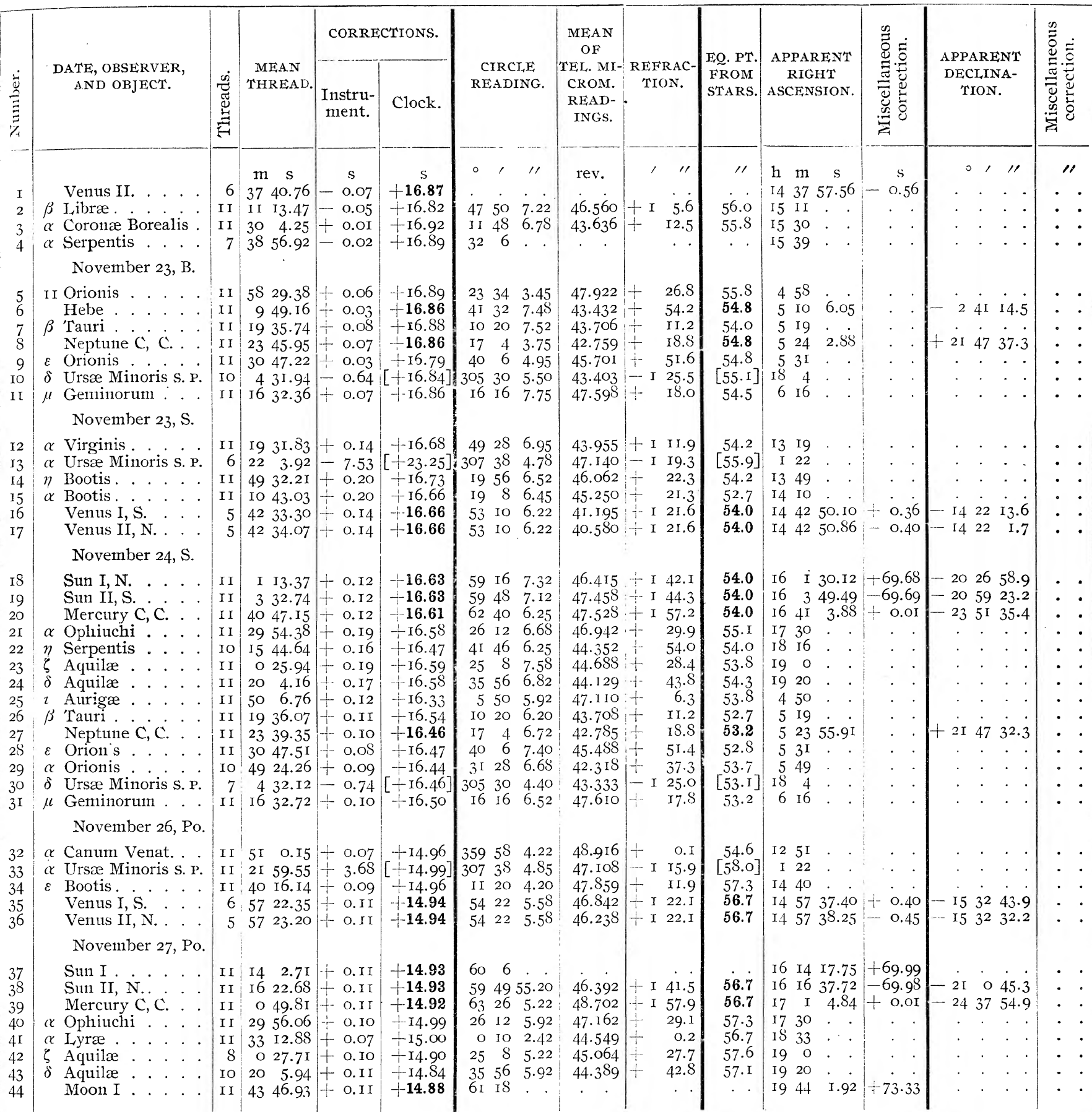

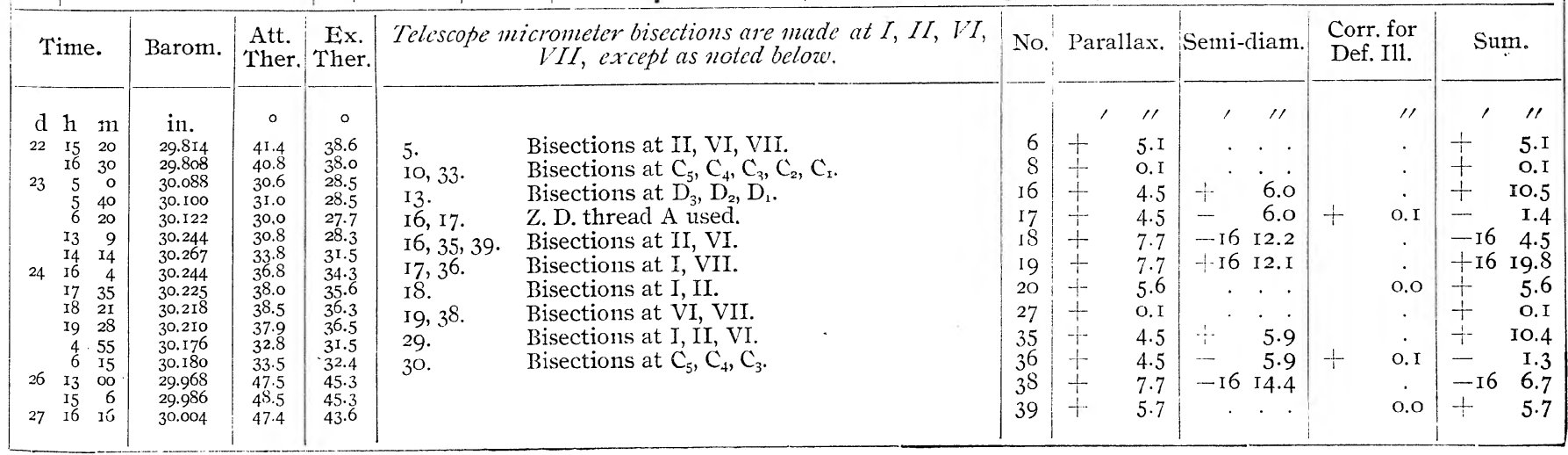




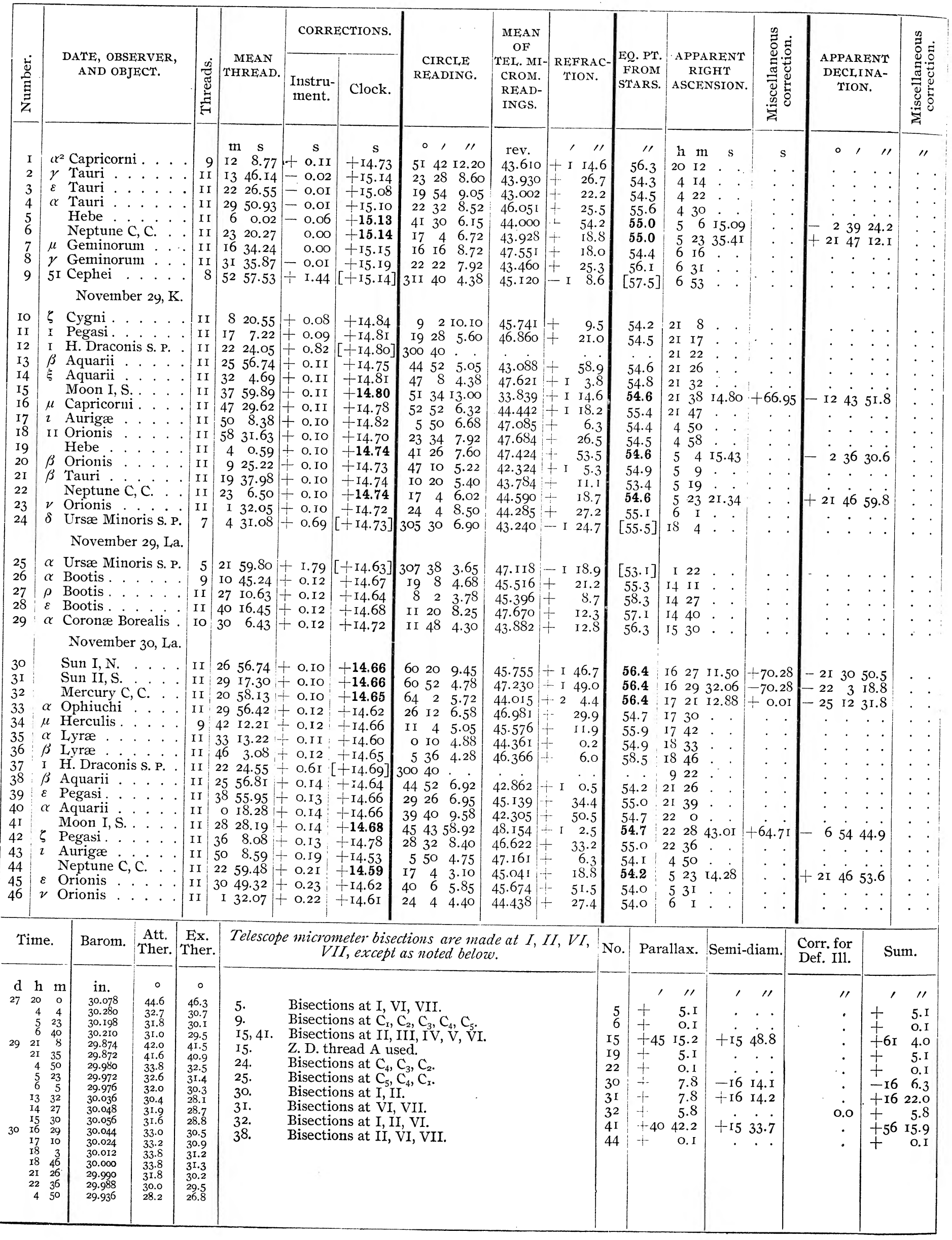




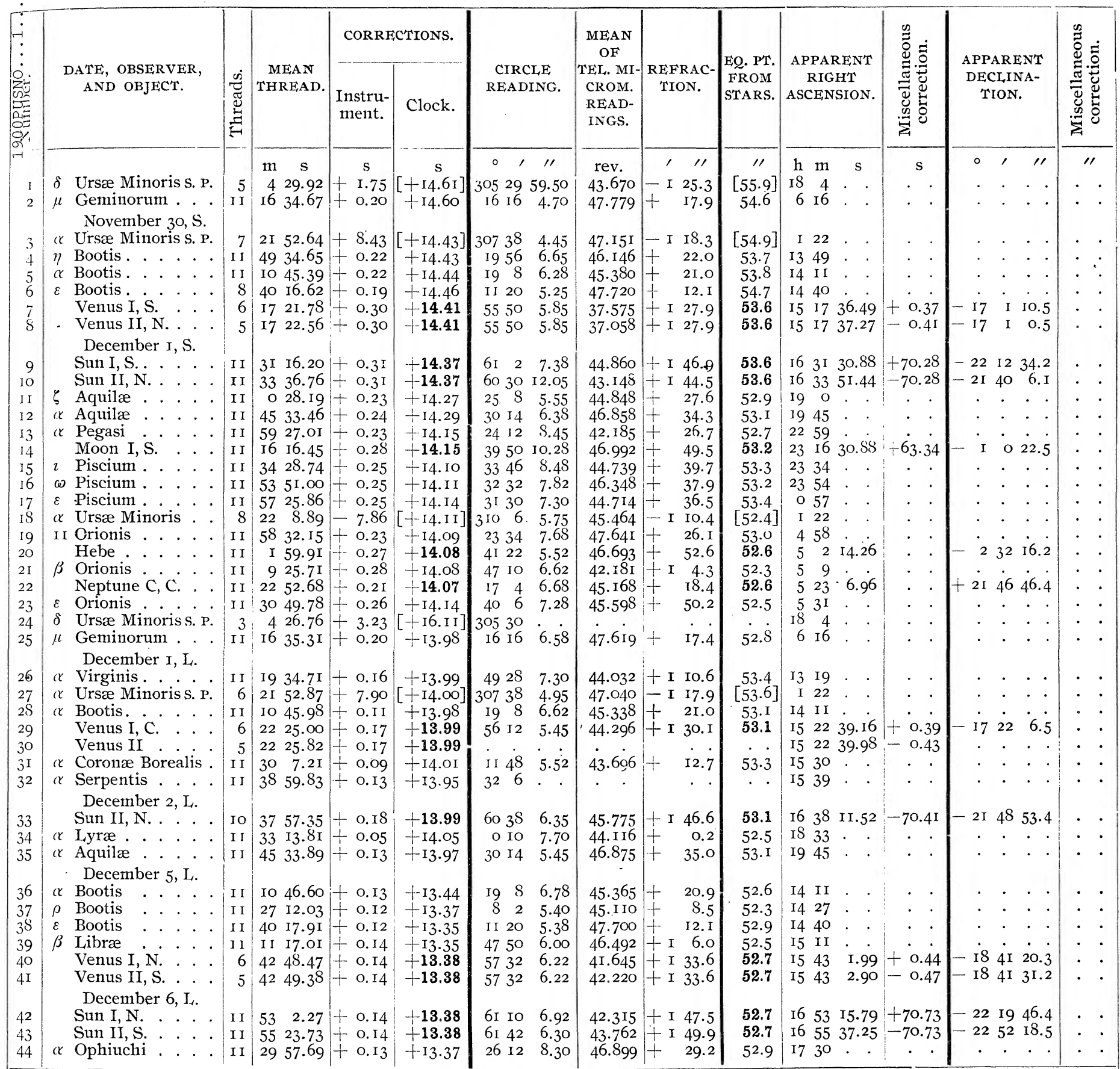

\begin{tabular}{|c|c|c|c|c|c|c|c|c|c|c|c|}
\hline Time. & Barom. & $\begin{array}{l}\text { Att. } \\
\text { Ther. }\end{array}$ & $\begin{array}{l}\text { Ex. } \\
\text { Ther. }\end{array}$ & Telescope & $\begin{array}{l}\text { micrometer bisections are made at } I, I I, V I, \\
\text { VII, except as noted below. }\end{array}$ & No. & Parallax. & Semi-diam. & & $\begin{array}{l}\text { for } \\
\text { I11. }\end{array}$ & Sum. \\
\hline $\mathrm{d} \quad \mathrm{h} \mathrm{m}$ & in. & $\circ$ & ० & & & & "1 & "1 & & "I & "I \\
\hline $\begin{array}{lrr}30 & 6 & 17\end{array}$ & 29.934 & 27.6 & 26.2 & I & Bisections at $\mathrm{C}_{0} \mathrm{C}_{-}$ & 7 & 4.6 & 5.0 & & & $+\quad .6 .6$ \\
\hline $13 \quad 26$ & 29.862 & 30.0 & 29.2 & & Bisections at $\mathrm{C}_{4}, \mathrm{C}_{3}, \mathrm{C}_{2}, \mathrm{C}_{5}$. & 8 & 4.6 & 5.0 & & 0.0 & 0.4 \\
\hline I4 43 & 29.850 & 35.0 & 34.4 & ${ }^{3 .} 8$ & $Z$ D thread A used $2, C_{1}$. & 9 & 7.8 & +16 I4.0 & & . & +1621.8 \\
\hline $\begin{array}{lll} & 15 & 26 \\
\text { I } & 16 & 34\end{array}$ & $\begin{array}{l}29.837 \\
29.805\end{array}$ & $\begin{array}{l}37.5 \\
40.0\end{array}$ & $\begin{array}{l}37.4 \\
39.4\end{array}$ & 7, 40. & Bisections at II, VI. & I0 & 7.8 & -. I6 I4. I & & . & - I6 6.3 \\
\hline $\begin{array}{r}196 \\
-9\end{array}$ & 29.738 & 43.6 & 43.0 & $8,4 \mathrm{I}$. & Bisections at I, VII. & I4 & $+355 \mathrm{I} . \mathrm{S}$ & $+\mathrm{I}_{5} 2 \mathrm{O} . \mathrm{I}$ & & . & +5111.9 \\
\hline $\begin{array}{ll}19 & 51 \\
22 & 54\end{array}$ & $\begin{array}{l}29.744 \\
29.794\end{array}$ & $\begin{array}{l}43.0 \\
39.5\end{array}$ & $\begin{array}{l}42.7 \\
39.4\end{array}$ & 9,42 . & Bisections at I, II. & 20 & 5.0 & . & & . & 5.0 \\
\hline I 34 & 29.840 & 38.5 & 37.2 & IO, 33,43 & Bisections at VI, VII. & 22 & O.I & • & & 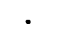 & O.I \\
\hline $4 \quad 42$ & 29.890 & 36.0 & 36.3 & I4. & Bisections at II, IIII, IV, V, VI. & 29 & 4.6 & & + & O.I & 4.7 \\
\hline $\begin{array}{ll}5 & 5 \mathrm{I} \\
6 & 25\end{array}$ & $\begin{array}{l}29.906 \\
29.928\end{array}$ & $\begin{array}{l}38.2 \\
39.4\end{array}$ & 39.4 & & Bisections at $\mathrm{C}_{1}, \mathrm{C}_{2}, \mathrm{C}_{3}, \mathrm{C}_{4}, \mathrm{C}_{5}$. & 33 & 7.8 & $-\mathrm{I} 6 \mathrm{I} 5 . \mathrm{I}$ & & 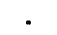 & -16 7.3 \\
\hline 1330 & 30.1 & 35.9 & 35.5 & 20. & Bisections at II, VI, VII. & 40 & 4.6 & 5.5 & & 0.0 & 0.9 \\
\hline $\begin{array}{ll}14 & 13 \\
\end{array}$ & 30.166 & $37 . \mathrm{I}$ & 35.5 & 27. & Bisections at $\mathrm{C}_{4}, \mathrm{C}_{3}, \mathrm{C}_{2}$. & $4 \mathrm{I}$ & 4.6 & $5: 4$ & & . & I0.0 \\
\hline $\begin{array}{lll}2 & 16 & 20 \\
2 & 38\end{array}$ & 30.576 & 39.0 & 38.5 & & & 42 & 7.9 & $-16 \mathrm{I} 6 . \mathrm{I}$ & & . & - I6 8.2 \\
\hline I9 47 & 30.158 & 42.0 & 39.4 & & & 43 & & $+16 \mathrm{I6.0}$ & & . & +1623.9 \\
\hline $\begin{array}{rrr}5 & \text { I4 } & 5 \\
& \text { I4 } & 38\end{array}$ & $\begin{array}{l}29.850 \\
29.870\end{array}$ & $\begin{array}{l}35.0 \\
36.0\end{array}$ & $\begin{array}{r}33.5 \\
35.0\end{array}$ & & & & & & & & \\
\hline $\begin{array}{ll}15 & 9\end{array}$ & 29.874 & 37.9 & 36.4 & & & & & & & & \\
\hline $\begin{array}{lll}6 & 15 & 40 \\
6 & 55\end{array}$ & $\begin{aligned} 29.880 \\
29.864\end{aligned}$ & $\begin{array}{l}38.9 \\
40.6\end{array}$ & $\begin{array}{l}37.4 \\
39.7\end{array}$ & & & & & & & & \\
\hline
\end{tabular}




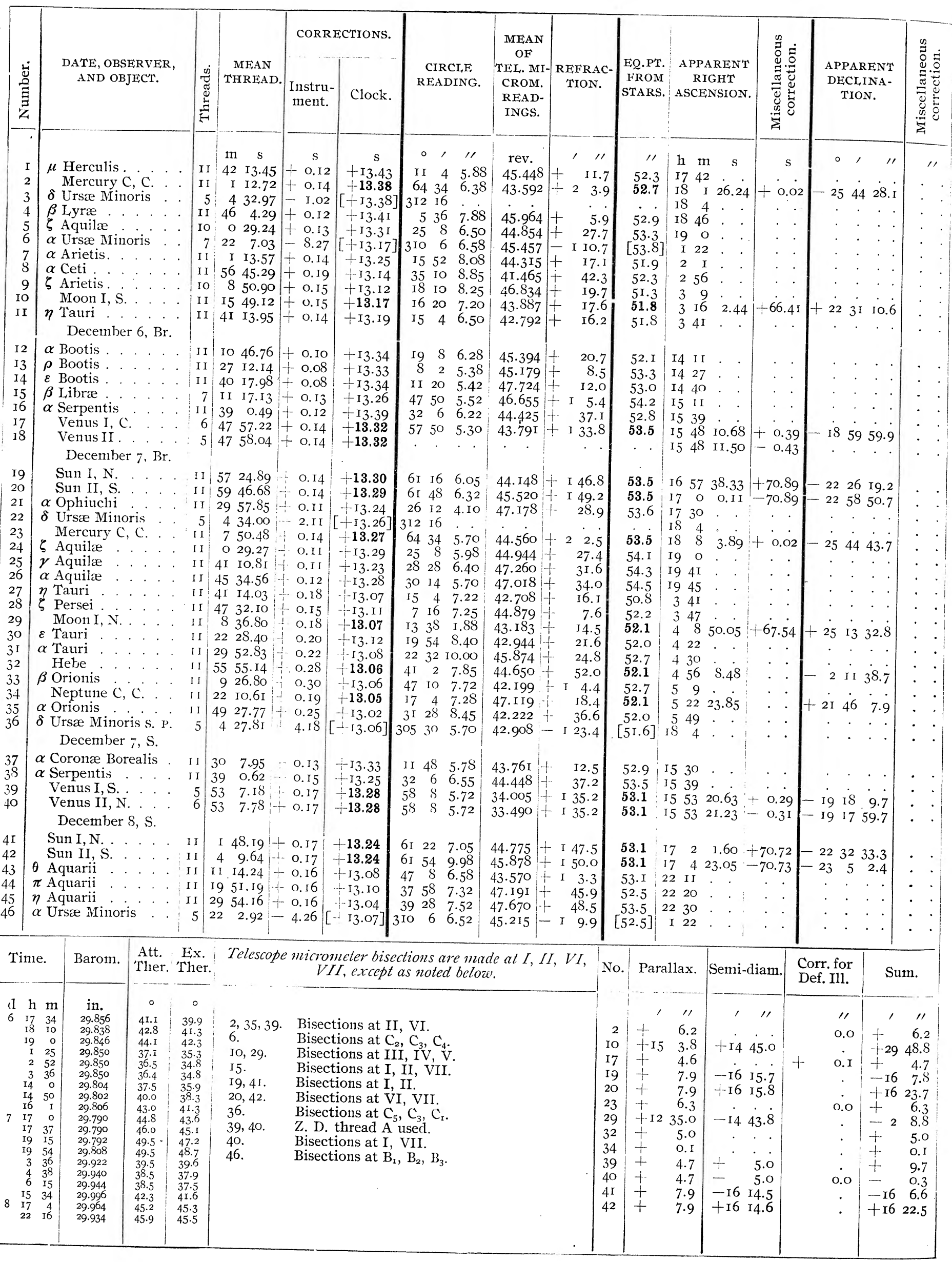




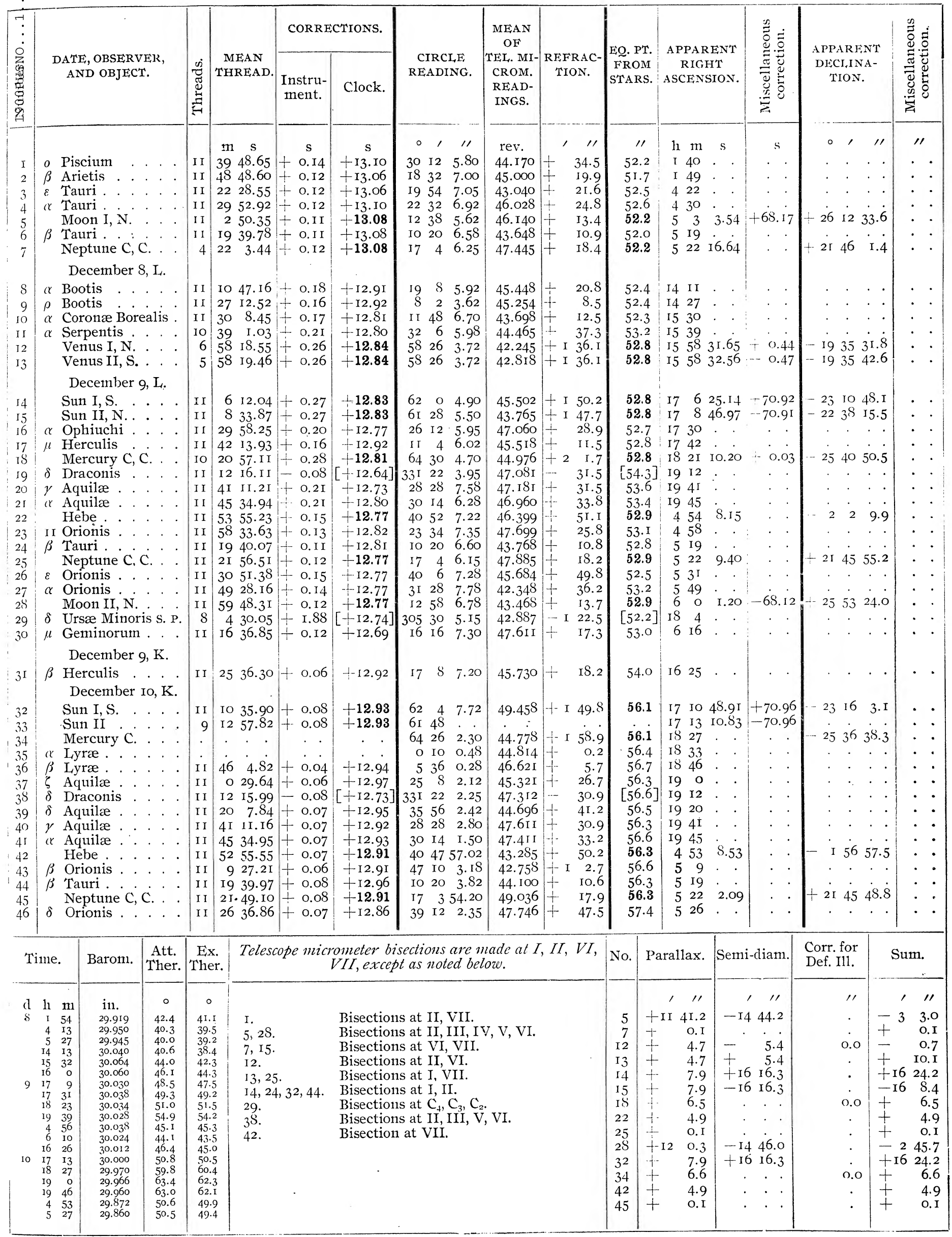




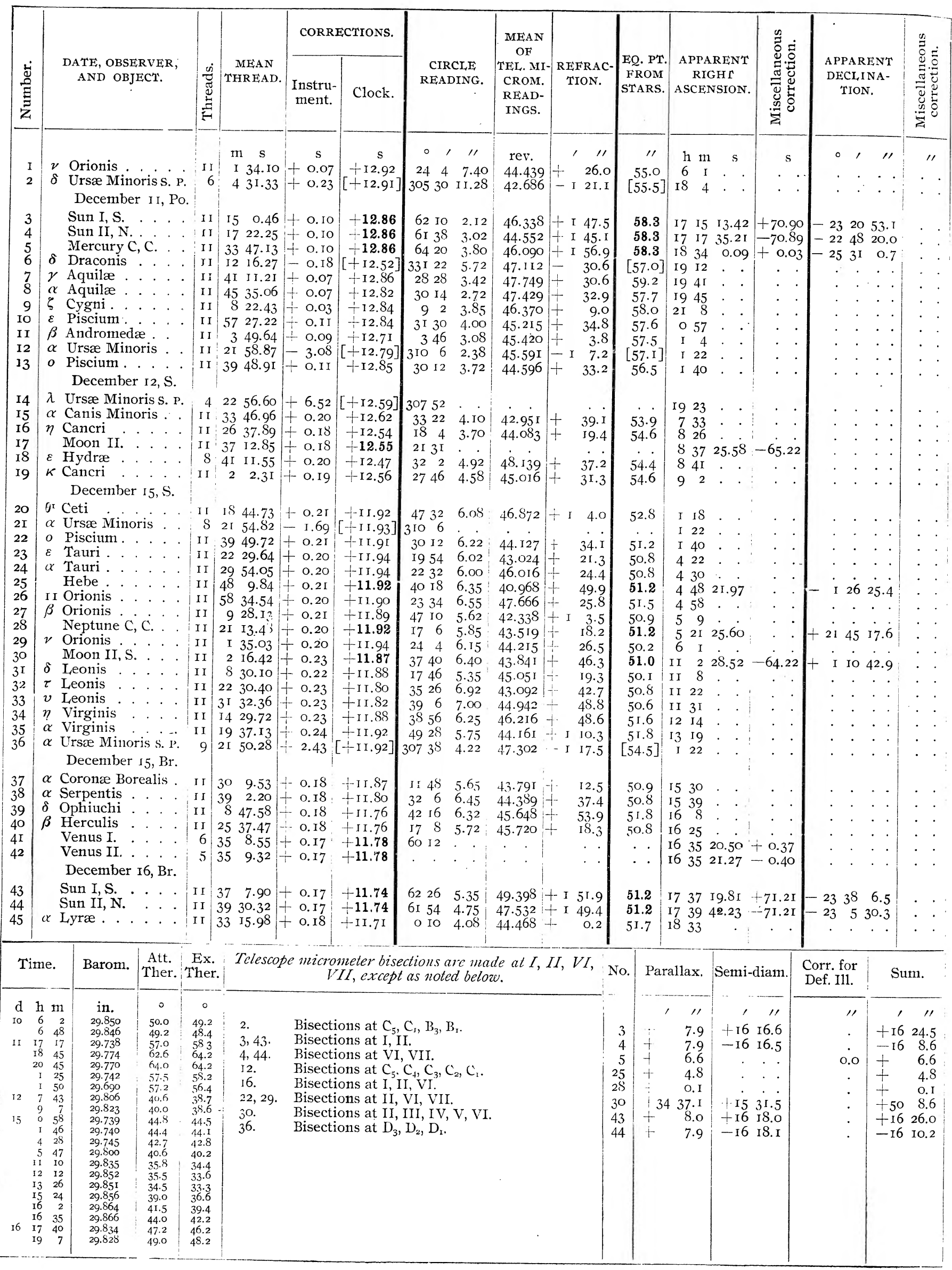




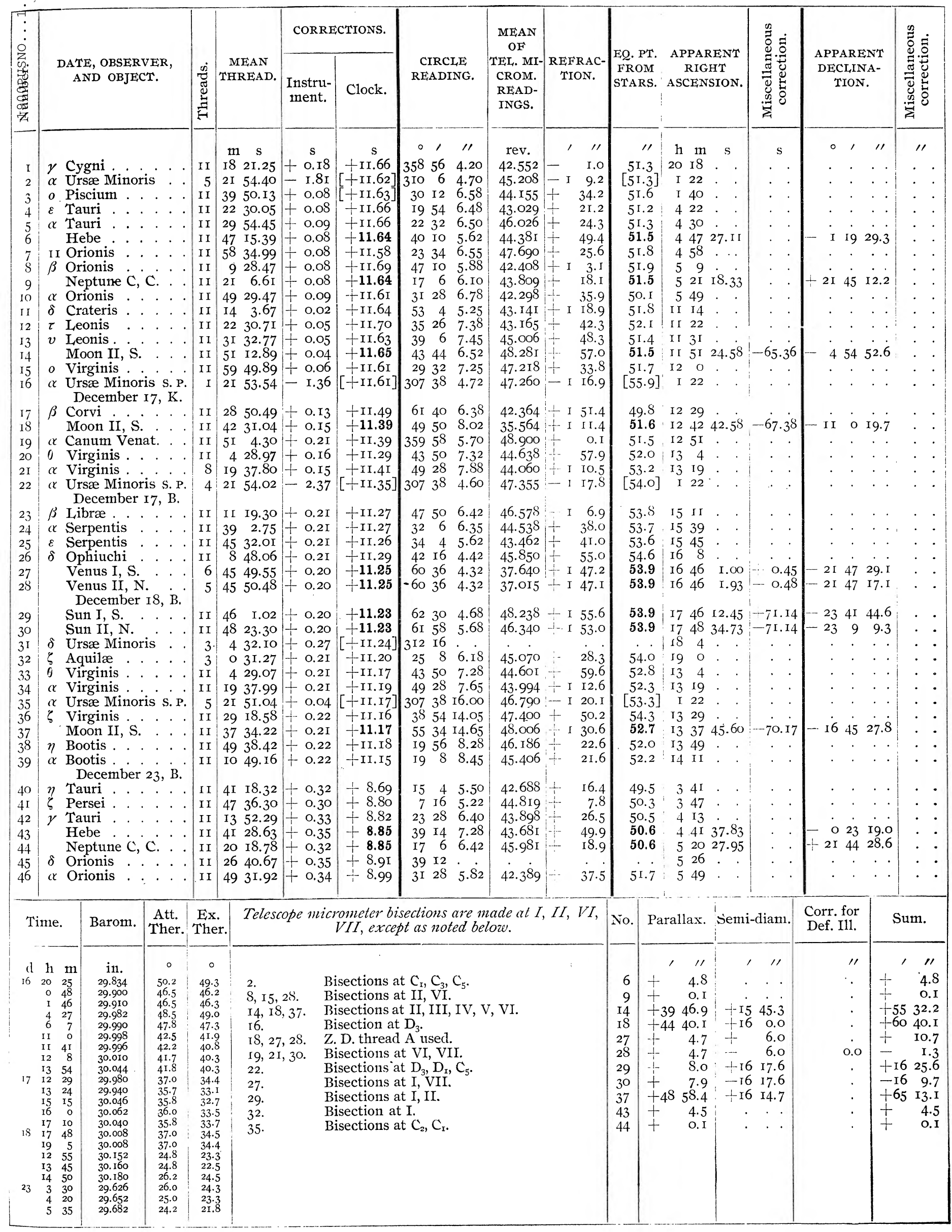




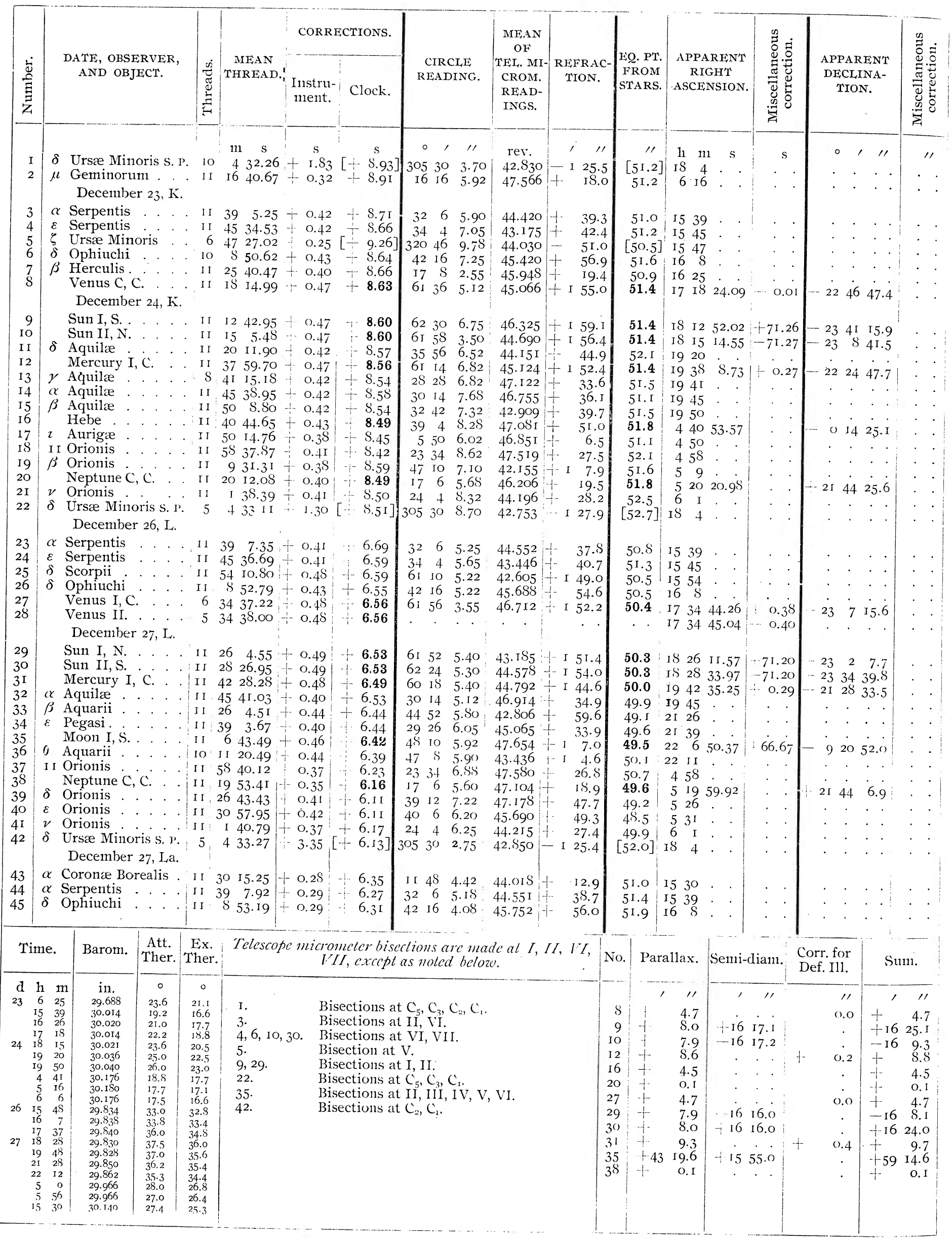




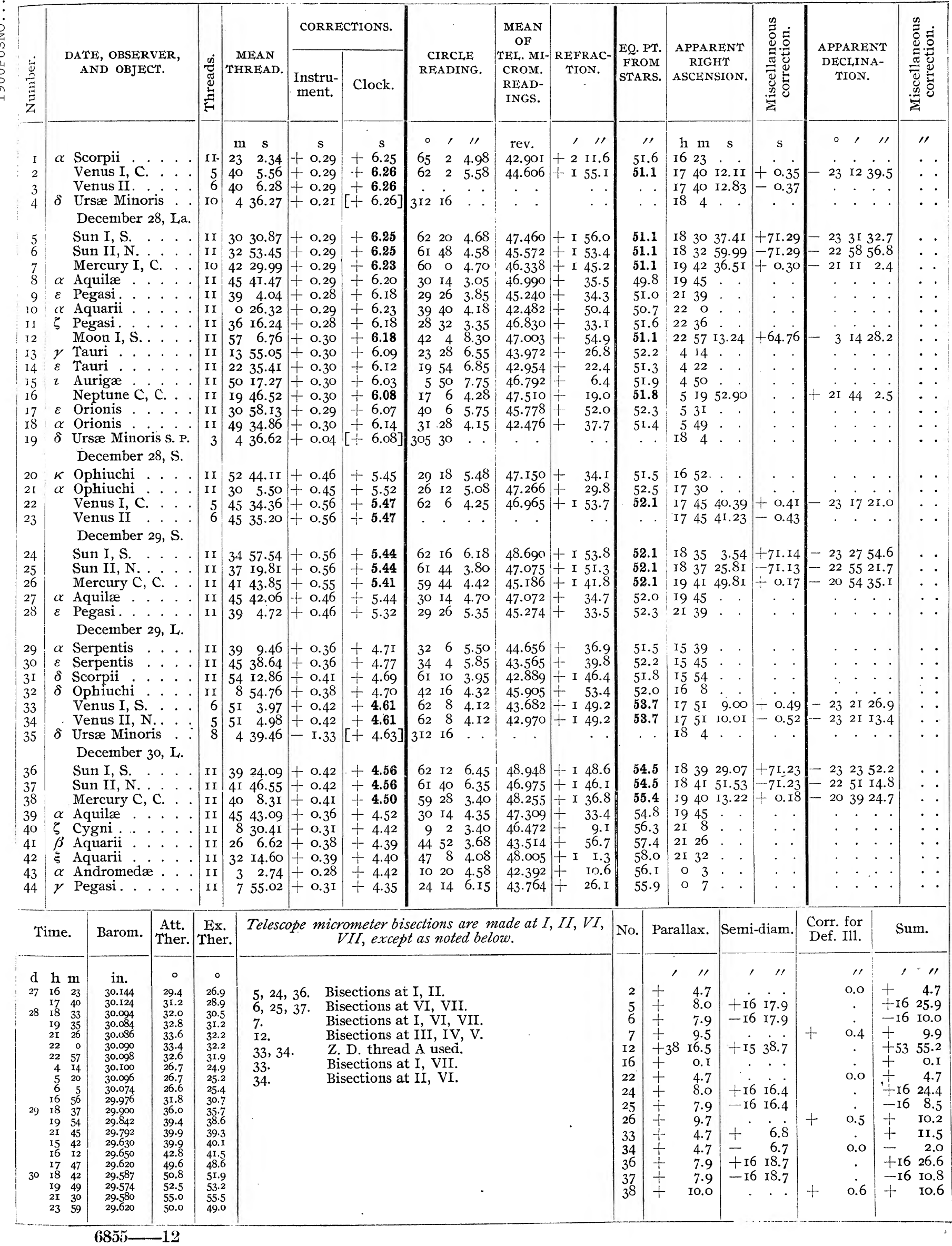




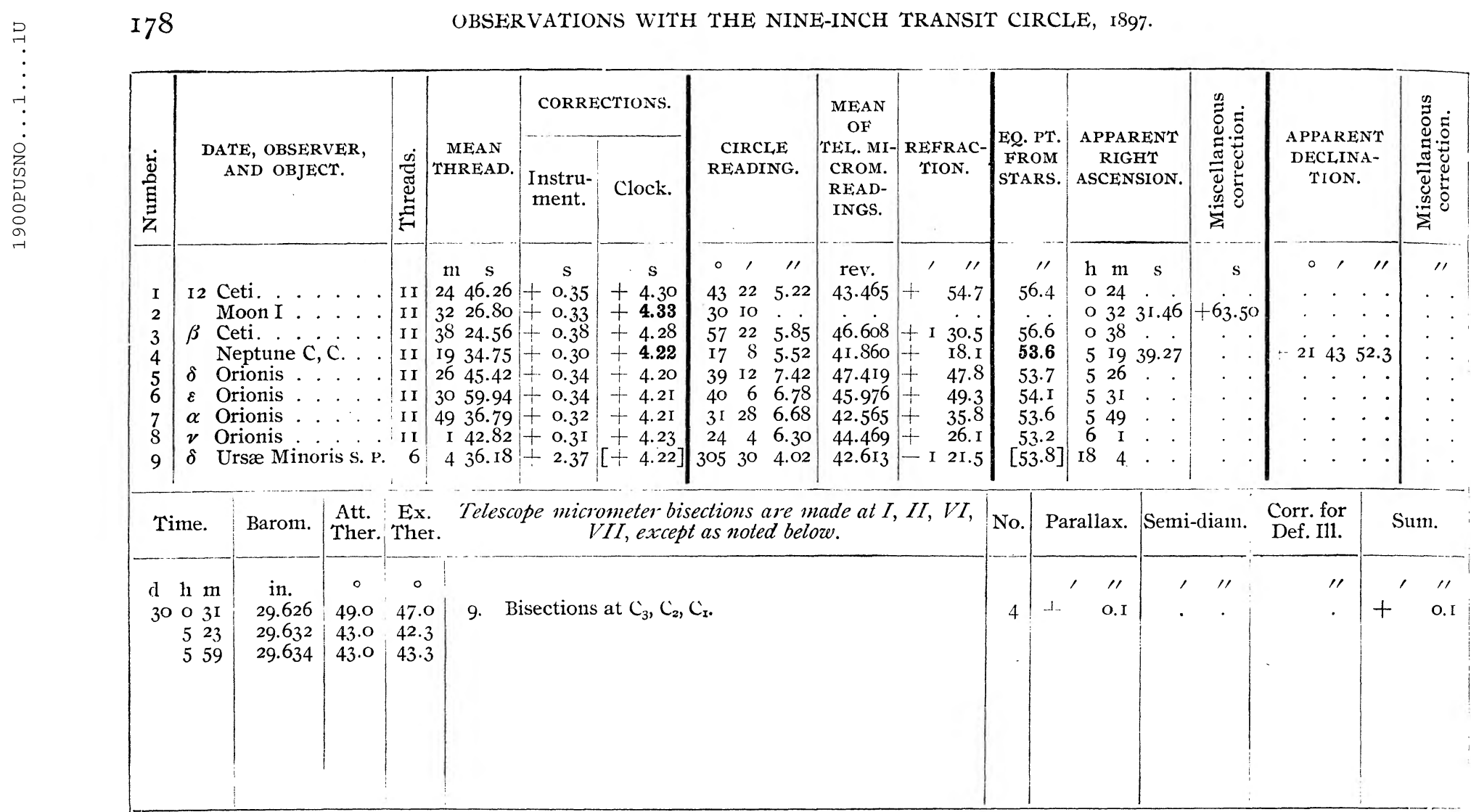




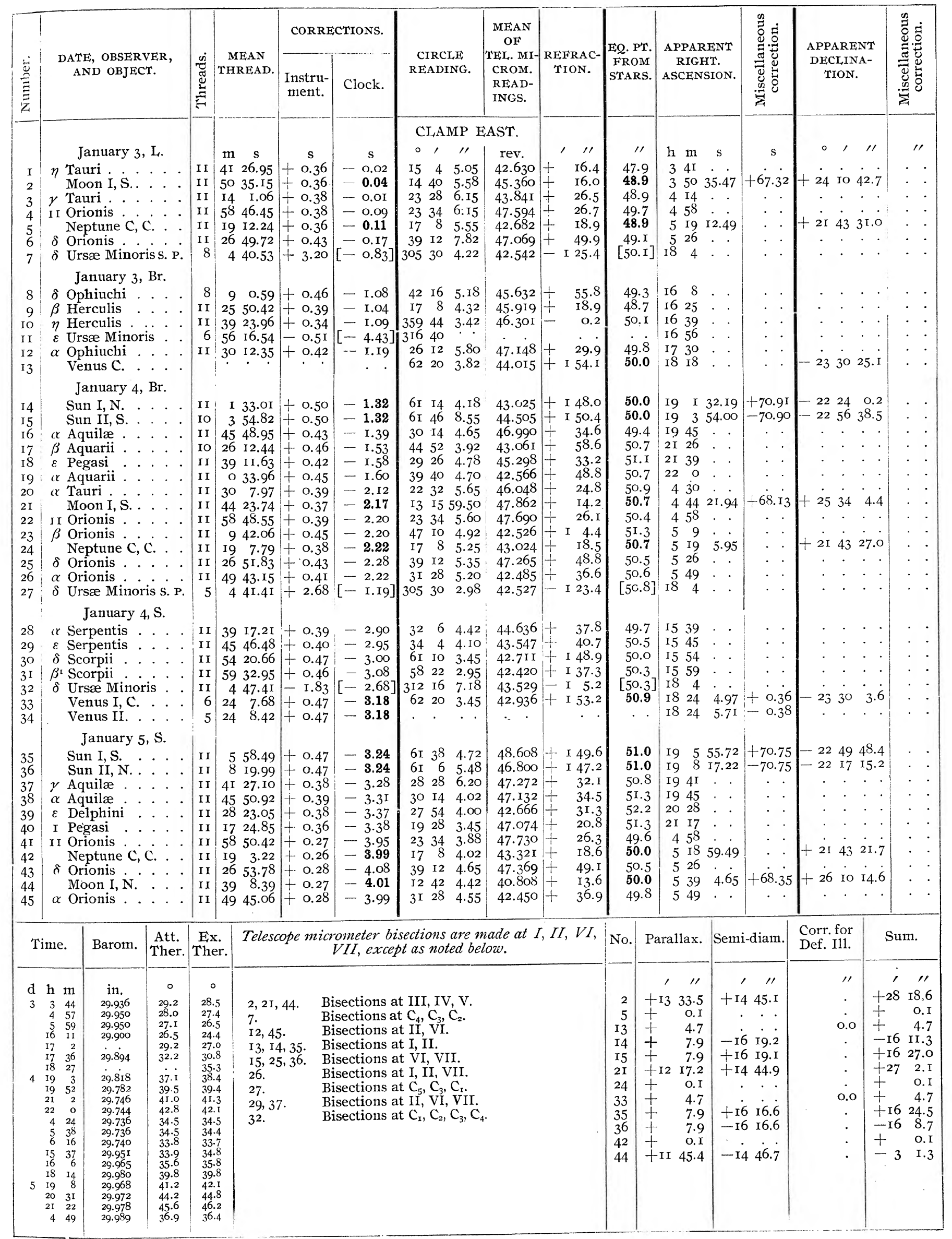




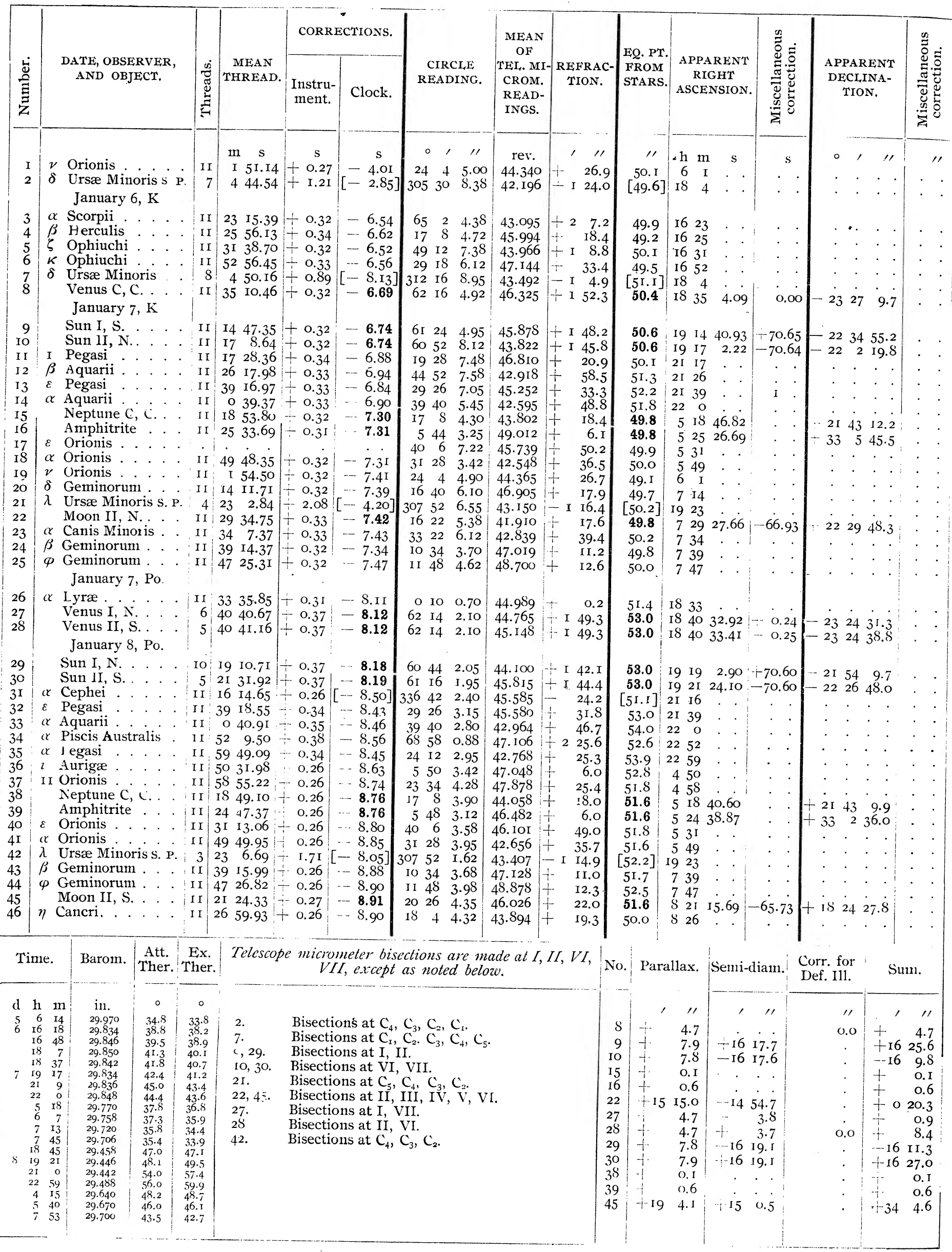




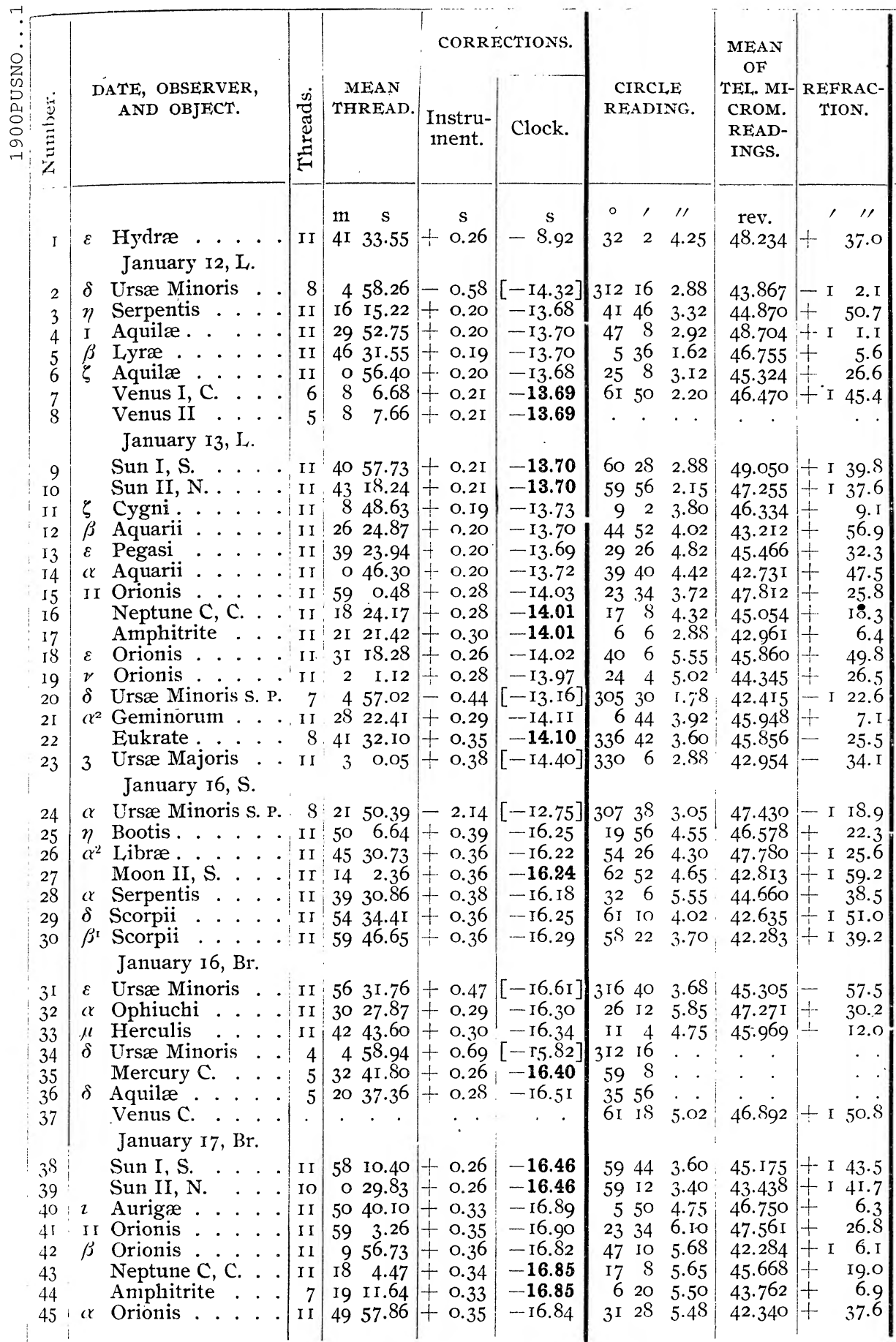

\begin{tabular}{|c|c|c|c|c|c|c|c|}
\hline $\begin{array}{l}\text { Q. PT. } \\
\text { FROM } \\
\text { TARS. }\end{array}$ & $\begin{array}{r}\text { APPA } \\
\text { RIG } \\
\text { ASCEN }\end{array}$ & $\begin{array}{l}\text { RENT } \\
\text { SHT } \\
\text { SSION. }\end{array}$ & 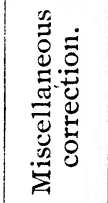 & $\begin{array}{r}\text { APP } \\
\mathrm{D}+\mathrm{T} \\
\mathrm{T}\end{array}$ & $\begin{array}{l}\text { PARI } \\
\text { CLII } \\
\text { ION }\end{array}$ & $\begin{array}{l}\text { ENT } \\
\text { NA- } \\
\text { T. }\end{array}$ & 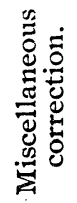 \\
\hline$\prime \prime$ & $\mathrm{h} \mathrm{ml}$ & $\mathrm{s}$ & $\mathbf{s}$ & & , & "I & 11 \\
\hline 5 I.o & $S_{4} \mathrm{I}$ & . & .1. & . & . & . . & \\
\hline$[52.8]$ & I8 4 & . & & . & . & . . & \\
\hline $52 . \mathrm{I}^{\mathrm{s}}$ & I8 I6 & . & & . & . & . & \\
\hline 52.I & I8 29 & . & . . & . & . & . . & \\
\hline $5 \mathrm{I} . \mathrm{I}$ & I8 46 & 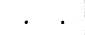 & & . & . & . . & \\
\hline 50.8 & I9 0 & & & 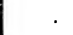 & . & & \\
\hline 51.6 & I9 7 & 53.20 & +0.49 & -23 & I & I. 6 & \\
\hline . . . & 197 & 54. I8 & -0.49 & $\cdot$ & $\cdot$ & $\cdot \cdot$ & \\
\hline 51.6 & I9 40 & 44.24 & +70.26 & $-2 I$ & 39 & 44.6 & \\
\hline 51.6 & I9 43 & 4.75 & -70.25 & $-2 I$ & 7 & 10.5 & \\
\hline $5 \mathrm{I} . \mathrm{I}$ & $2 I 8$ &. & . & . & . & . . & \\
\hline $5^{1} .5$ & 2I 26 & 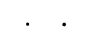 & . & . & . & . . & \\
\hline $5^{2}$ & 2I 39 & & . & . & . & . . & \\
\hline $5 \mathrm{I}$ & 220 & 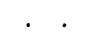 & . . & . & . & . . & . \\
\hline 50 & $45^{8}$ & & . & $\cdot$ & . & & \\
\hline 49 & $5 \mathrm{I} 8$ & I0.44 & . & $+2 \mathrm{I}$ & 42 & 48.0 & \\
\hline 48 & $52 \mathrm{I}$ & $7.7 \mathrm{I}$ & . & +32 & 45 & $4 \mathrm{I} .4$ & \\
\hline 45 & $53^{\mathrm{I}}$ & & . & . & . & . . & . \\
\hline 49 & $6 \quad I$ & & . & . & . & . . & . \\
\hline $5^{\mathrm{I}}$ & I8 4 & & . & . & . & . . & \\
\hline 49 & 728 & & . & & . & & \\
\hline 51.6 & $74 \mathrm{I}$ & IS 8.35 & . & +62 & 9 & I 8.4 & . \\
\hline 51.7 & 82 & . . & . & . & . & $\cdot \cdot$ & - \\
\hline$[49.9]$ & I $2 \mathrm{I}$ & & . & . & 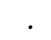 & . & - \\
\hline 49. & I 349 & & & . & . & . & . \\
\hline & I4 45 & & & 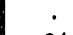 & . & & - \\
\hline 49 & I5 I3 & 46.48 & -73.76 & -24 & 2 & 9.8 & . \\
\hline 49 & I 539 & $\cdot$. & . & . & . & $\cdot \cdot$ & - \\
\hline 50 & I 554 & . . & . & . & . & $\cdot \cdot$ & - \\
\hline 49.7 & I5 59 & $\cdot \cdot$ & - & . & $\cdot$ & $\cdot \cdot$ & • \\
\hline$[52.9]$. & I6 56 & 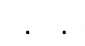 & - & & $\cdot$ & . & - \\
\hline 49. & 1730 & 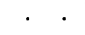 & 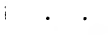 & . & . & $\cdot \cdot$ & • \\
\hline 50. & I 742 & 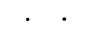 & . & . & . & . & - \\
\hline . . & I8 4 & . & & & . & $\cdot \cdot$ & - \\
\hline & IS 32 & 25.66 & 0.16 & & . & . & - \\
\hline 49.8 & $\begin{array}{l}1920 \\
1929\end{array}$ & 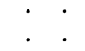 & . & -22 & 29 & $\dot{I S} . \dot{I}$ & : \\
\hline 49. & I9 & 54.20 & 69.72 & -20 & 54 & 36. & - \\
\hline & 200 & 13.63 & $-69.7 \mathrm{I}$ & -20 & 22 & 4.5 & . \\
\hline & 450 & 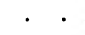 & . & & . & . & - \\
\hline & $45^{8}$ & & & & 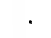 & . & 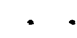 \\
\hline & 59 & & . & & 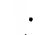 & . . & . \\
\hline & 517 & 47.96 & . & $+2 I$ & 42 & 34.4 & • \\
\hline & 5 I 8 & 55. I 2 & . & $+3^{2}$ & 31 & 20.4 & - \\
\hline 48.3 & 549 & $\cdot$ & • & & . & · & - \\
\hline
\end{tabular}

\begin{tabular}{|c|c|c|c|c|c|c|c|c|c|}
\hline 'Time. & Barom. & $\begin{array}{l}\text { Att. } \\
\text { Ther. }\end{array}$ & $\begin{array}{c}\text { Ex. } \\
\text { Ther. }\end{array}$ & $\begin{array}{c}\text { Telescope micrometer bisections are made at I, II, IT, } \\
\text { VII, except as noted below. }\end{array}$ & No. & Parallax. & Semi-diam. & $\begin{array}{l}\text { Corr. for } \\
\text { Def. Ill. }\end{array}$ & Sum. \\
\hline $\begin{array}{rrr}r & \mathrm{~h} & \mathrm{~m} \\
8 & 8 & 36 \\
12 & 18 & 19 \\
& 18 & 45 \\
& 19 & 5 \\
13 & 19 & 43 \\
& 21 & 29 \\
& 22 & 59 \\
& 4 & 59 \\
& 6 & 12 \\
& 7 & 58 \\
16 & 13 & 27 \\
& 14 & 35 \\
& 16 & 5 \\
& 17 & 5 \\
& 17 & 49 \\
& 19 & 23 \\
17 & 20 & 1 \\
& 4 & 42 \\
& 5 & 34\end{array}$ & $\begin{array}{l}\text { in. } \\
29.770 \\
29.618 \\
29.612 \\
29.614 \\
29.626 \\
29.674 \\
29.696 \\
29.810 \\
29.850 \\
29.874 \\
30.021 \\
30.025 \\
30.075 \\
30.108 \\
30.108 \\
30.104 \\
30.096 \\
30.128 \\
30.126\end{array}$ & $\begin{array}{c}\circ \\
42.0 \\
5.8 \\
57.9 \\
58.0 \\
58.2 \\
56.2 \\
5.1 \\
43.1 \\
41.8 \\
39.5 \\
29.7 \\
29.2 \\
30.2 \\
31.8 \\
32.5 \\
34.7 \\
34.4 \\
30.7 \\
29.5\end{array}$ & $\begin{array}{c}\circ \\
41.1 \\
48.1 \\
58.1 \\
58.9 \\
58.8 \\
48.9 \\
54.3 \\
53.4 \\
41.8 \\
40.1 \\
38.1 \\
27.6 \\
27.2 \\
28.0 \\
28.9 \\
29.8 \\
32.0 \\
32.2 \\
29.0 \\
28.1\end{array}$ & $\begin{array}{ll}2 . & \text { Bisections at } \mathrm{C}_{\mathrm{I}}, \mathrm{C}_{3}, \mathrm{C}_{5} . \\
9,38,43 . & \text { Bisections at I, II. } \\
\text { IO, 39, 44. } & \text { Bisections at VI, VII. } \\
\text { 20. } & \text { Bisections at } \mathrm{C}_{4}, \mathrm{C}_{3}, \mathrm{C}_{2} . \\
24 . & \text { Bisections at } \mathrm{D}_{2}, \mathrm{D}_{1}, \mathrm{C}_{5} . \\
\text { 27. } & \text { Bisections at II, III, IV, V, VI. } \\
\text { 30. } & \text { Bisections at II, VI, VII. } \\
\text { 37. } & \text { Bisections at I, II. } \\
& \\
& \\
\text { 22. } & \text { Bright wire illumination except at I and II. }\end{array}$ & \begin{tabular}{|r|}
7 \\
9 \\
IO \\
16 \\
17 \\
22 \\
27 \\
37 \\
38 \\
39 \\
43 \\
44
\end{tabular} & 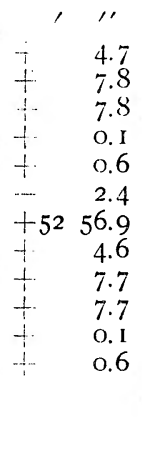 & 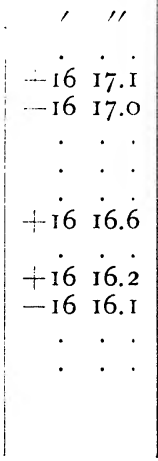 & $\begin{array}{l}11 \\
0.0 \\
\cdot \\
: \\
: \\
: \\
. \\
0.0 \\
: \\
: \\
\cdot\end{array}$ & 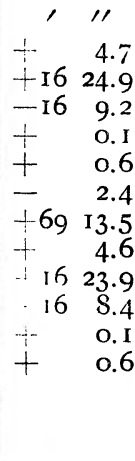 \\
\hline
\end{tabular}




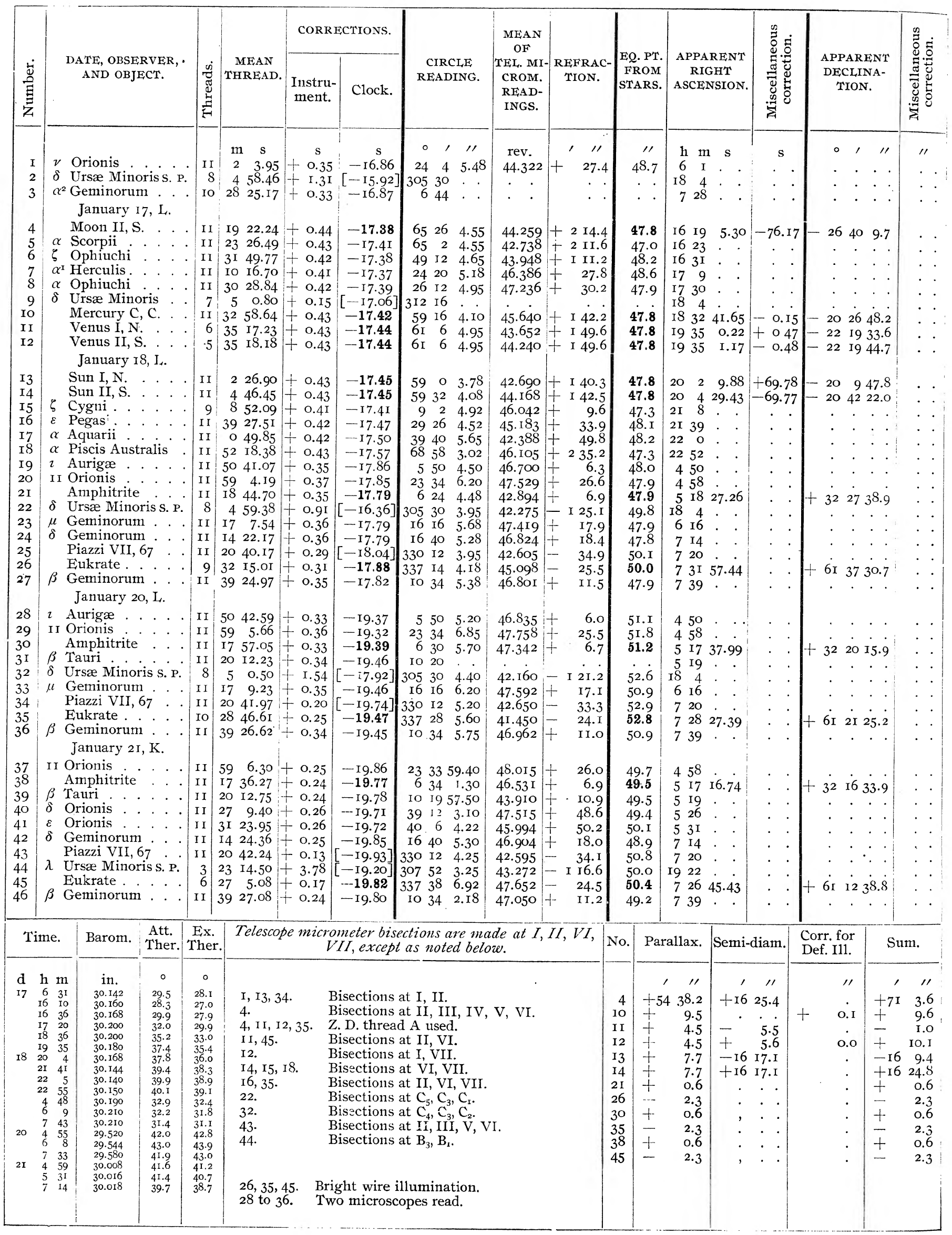




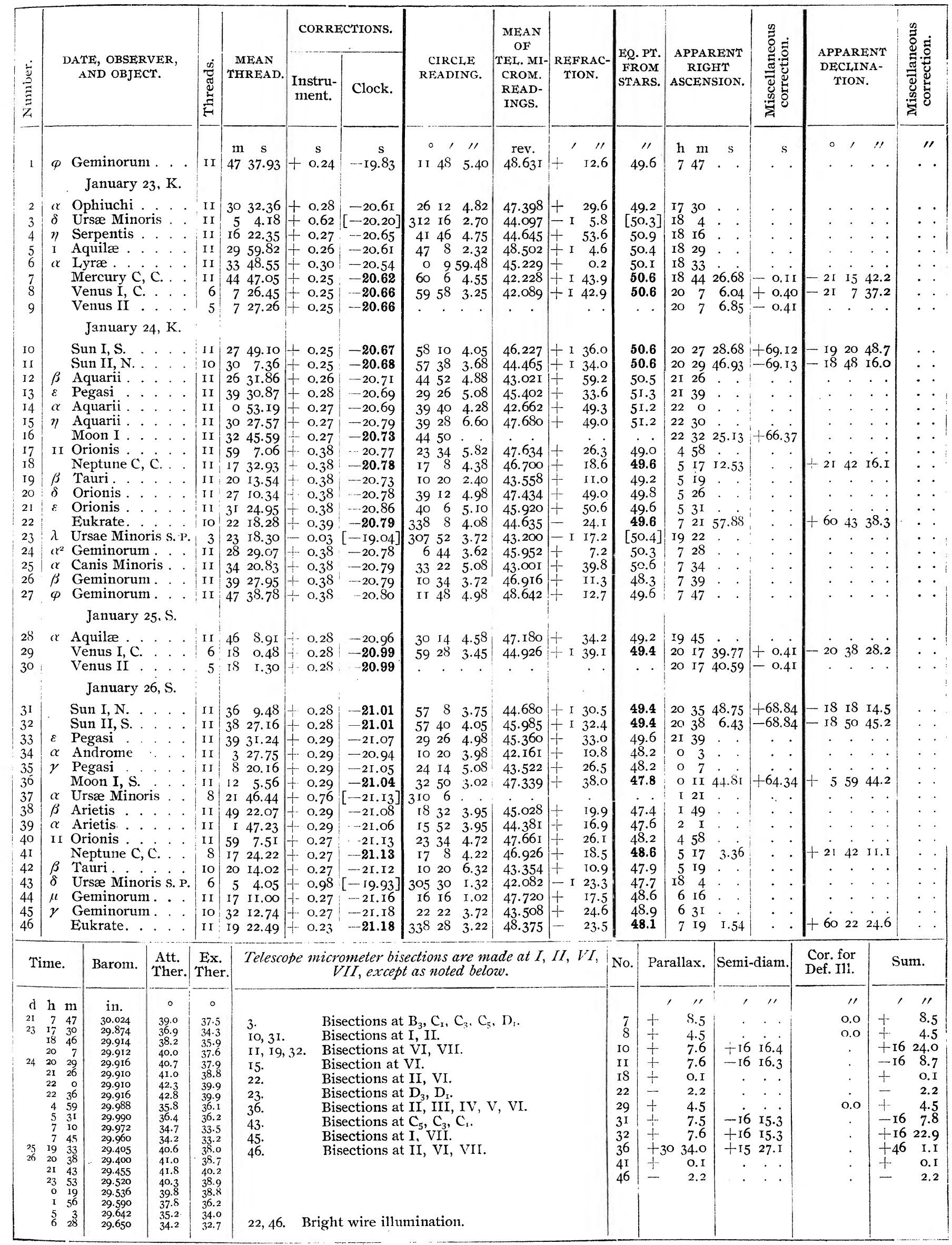




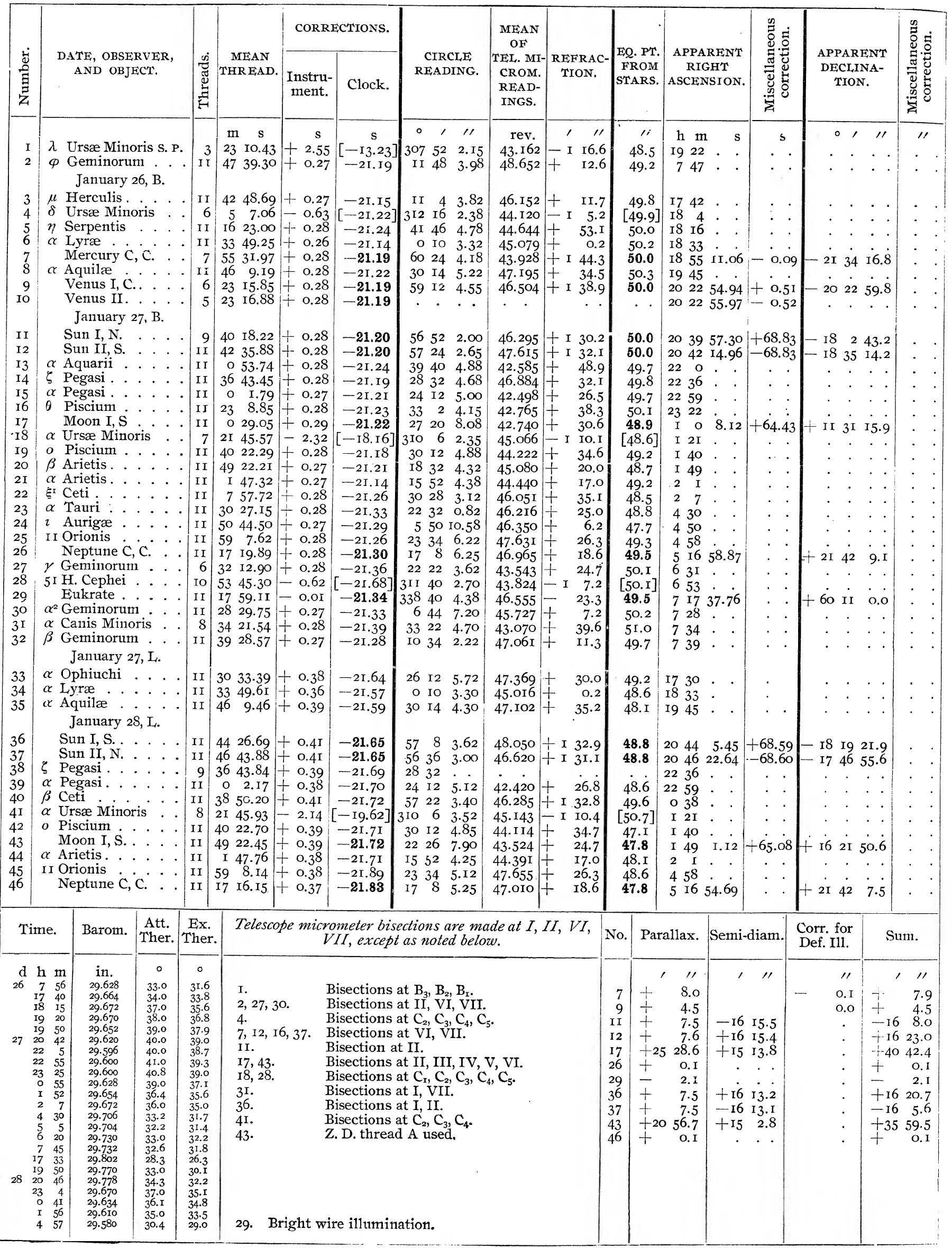




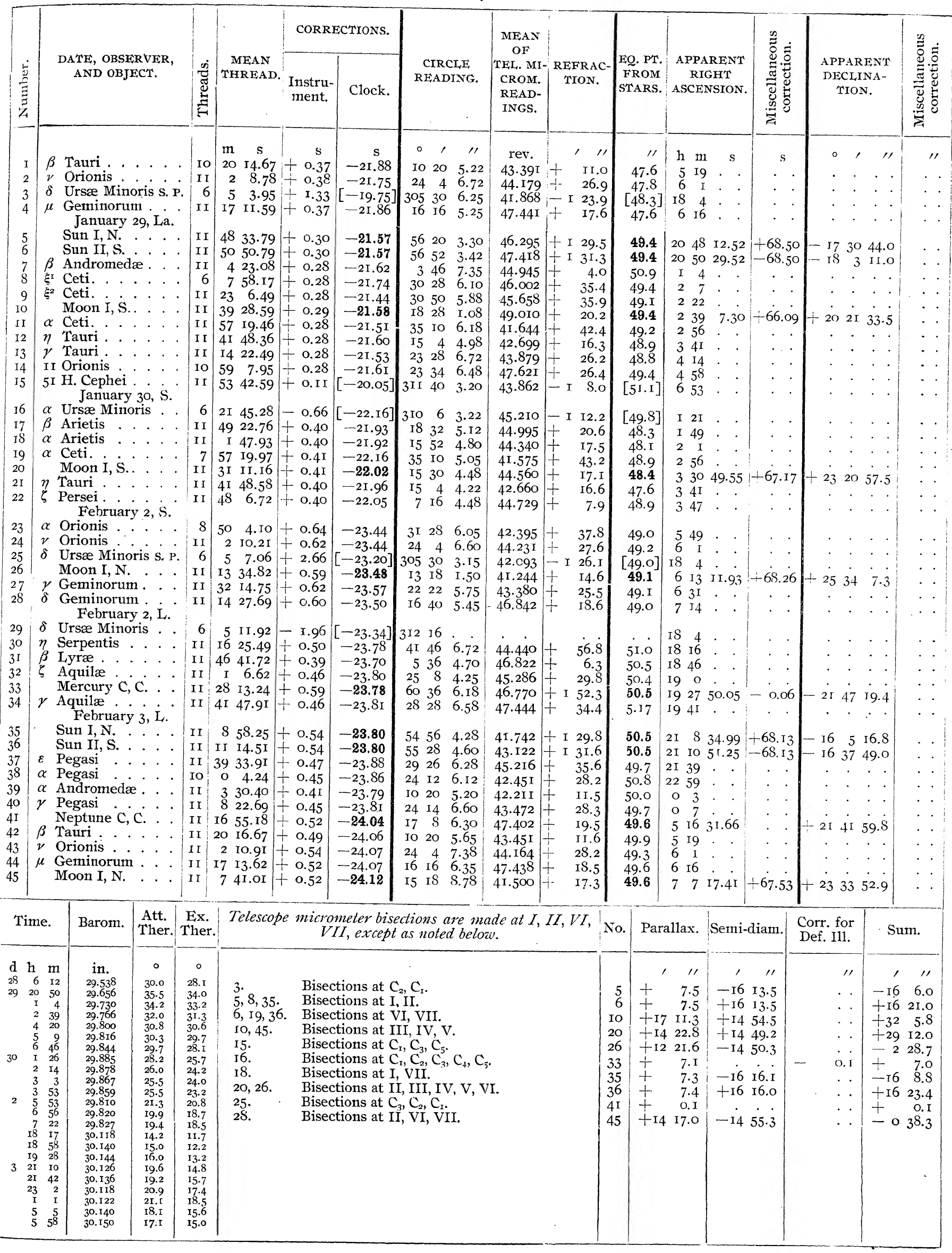




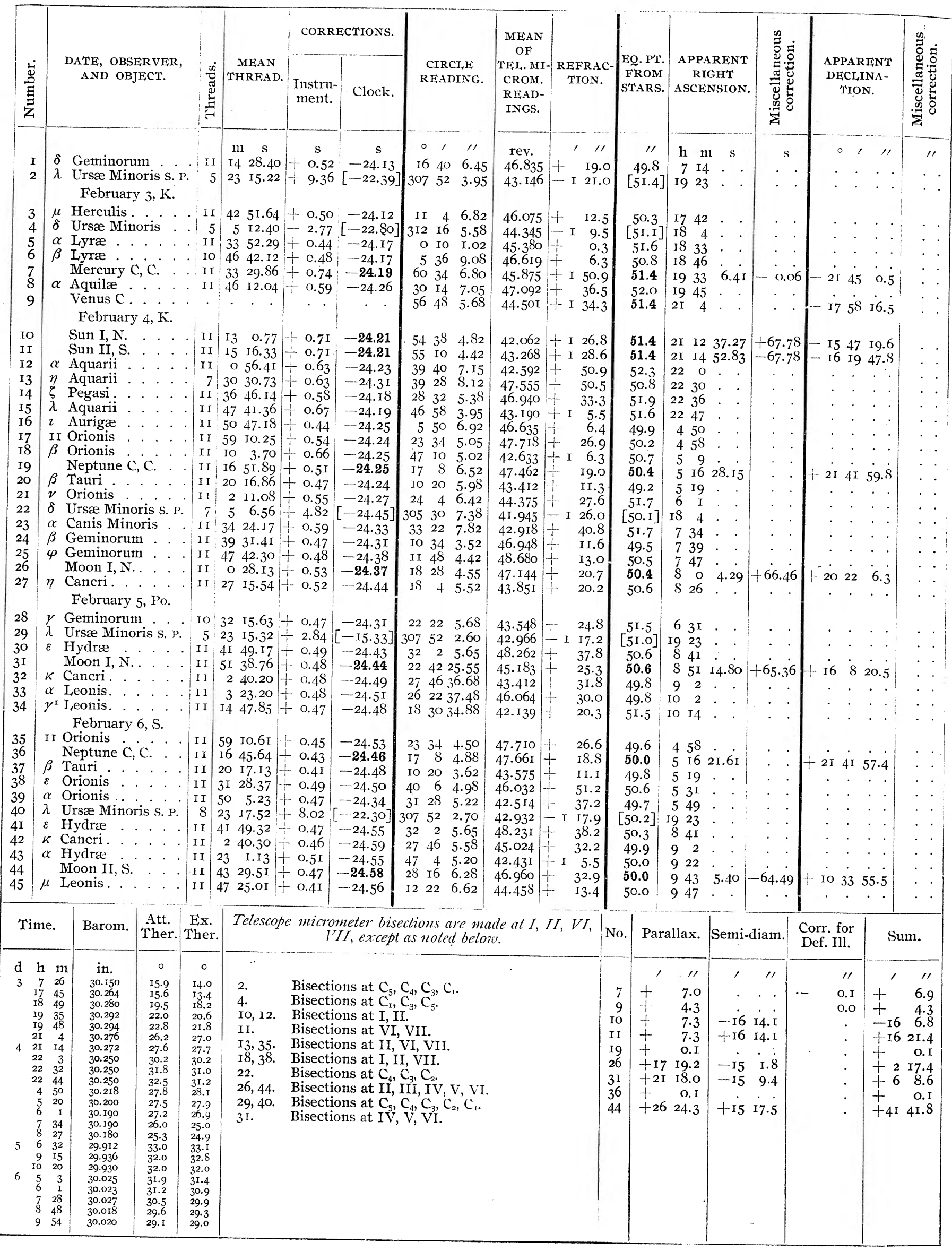




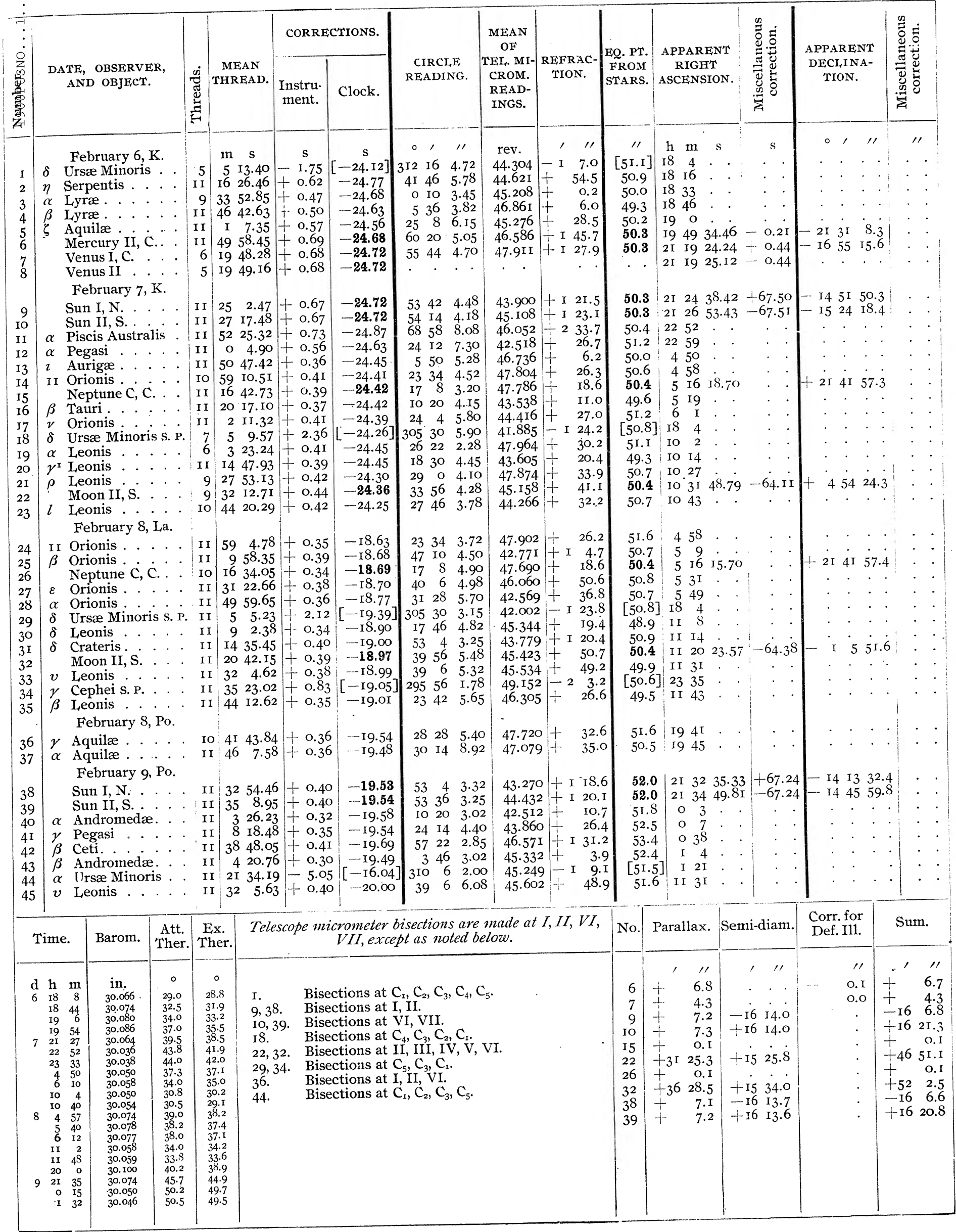




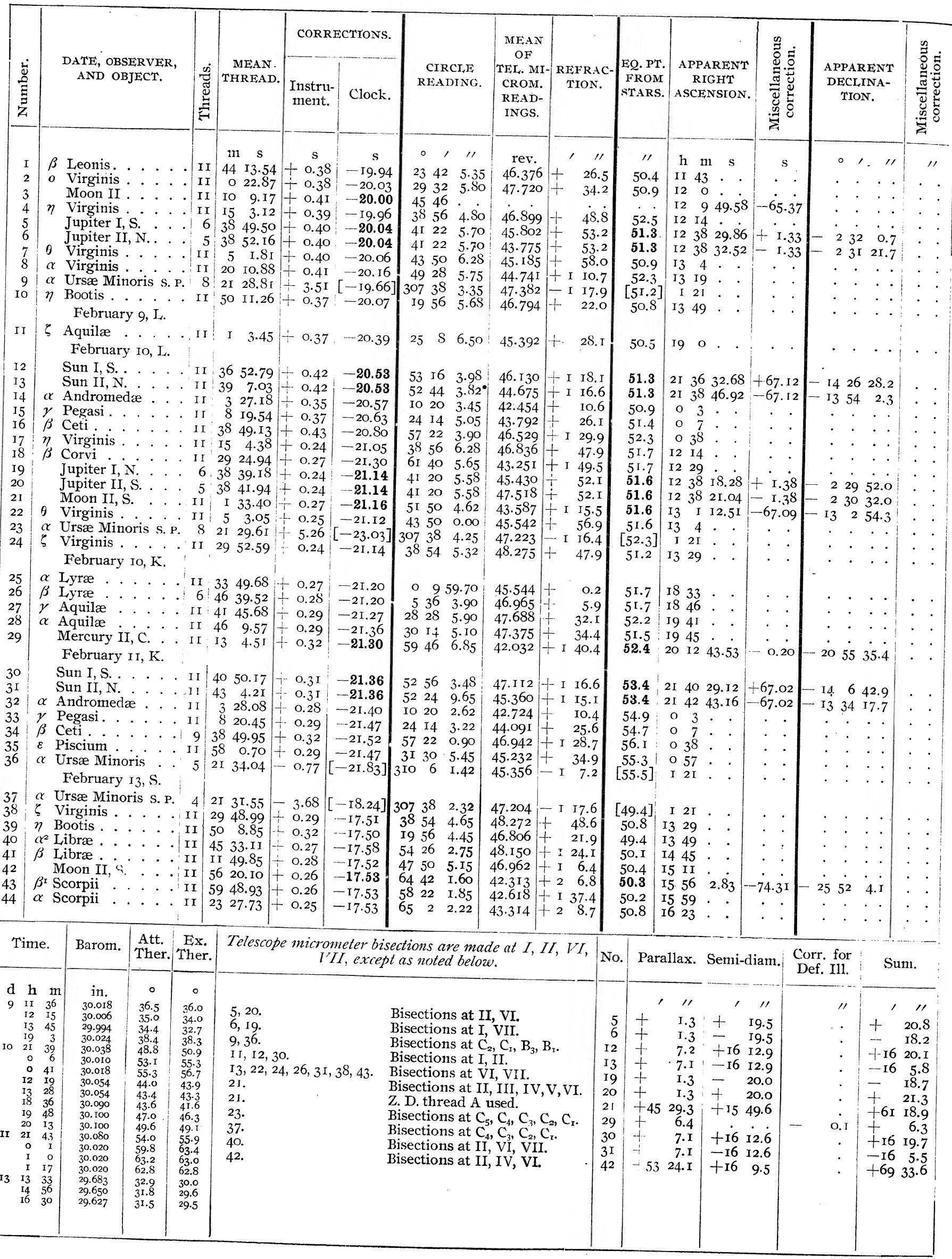




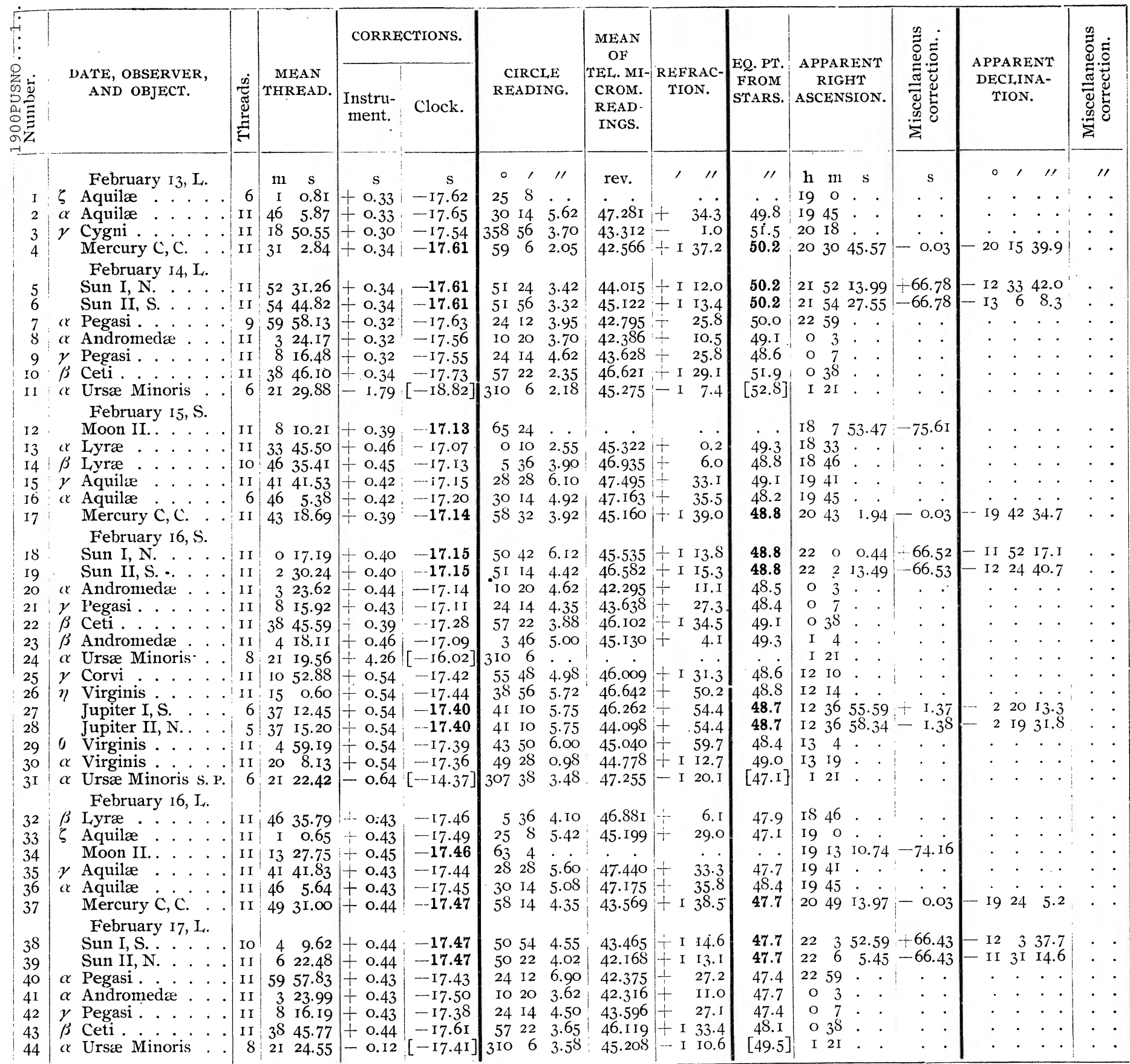

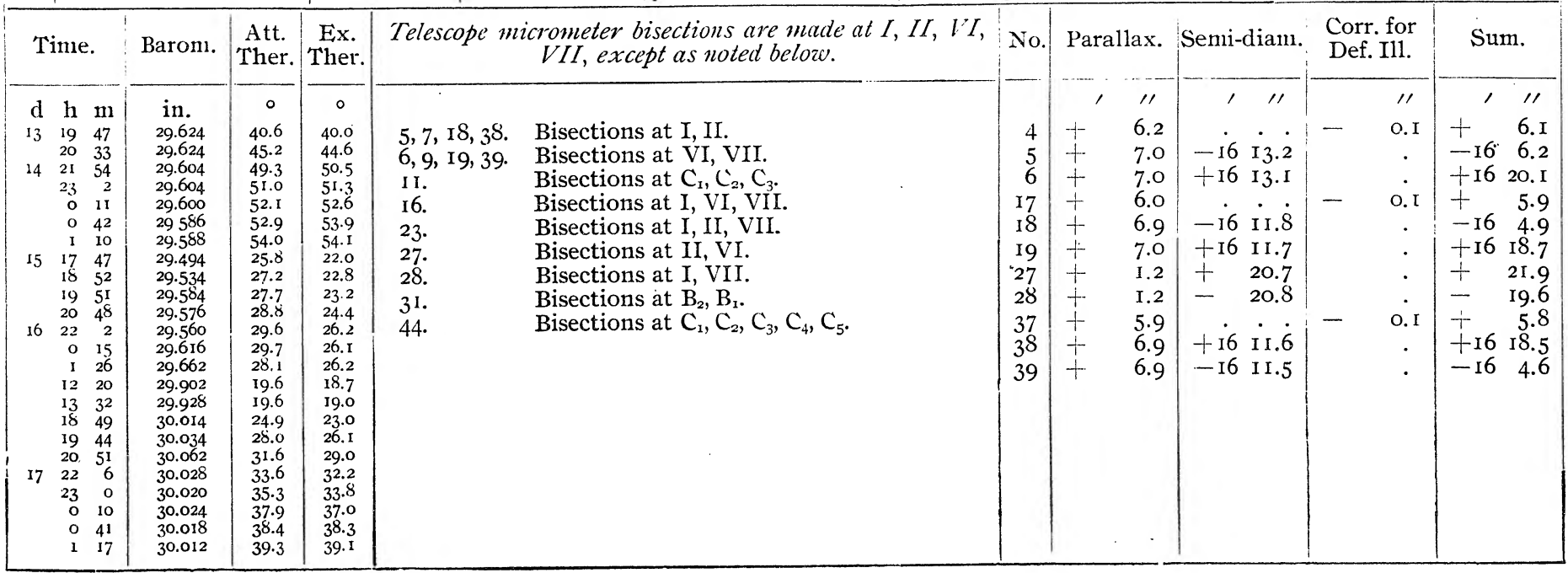




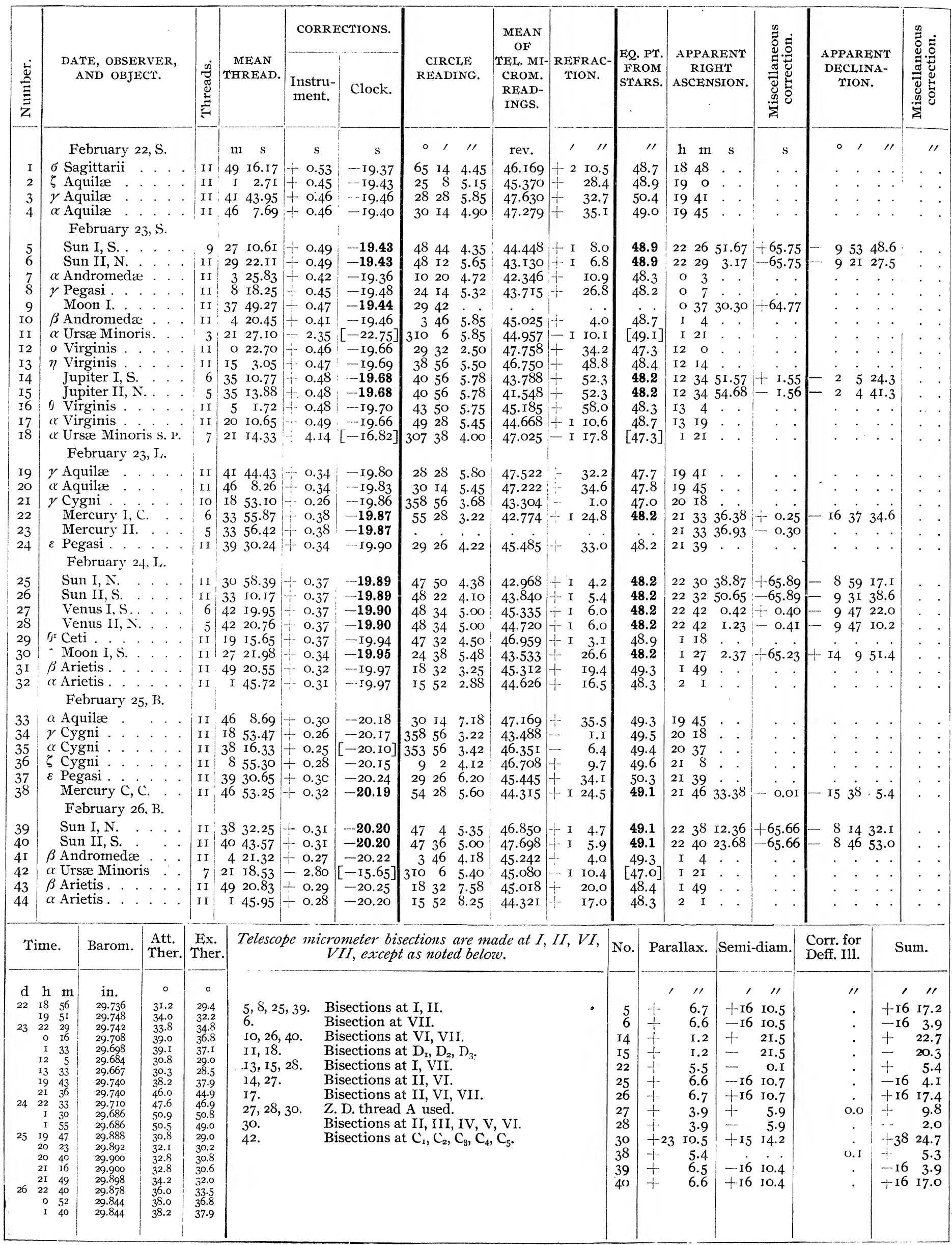




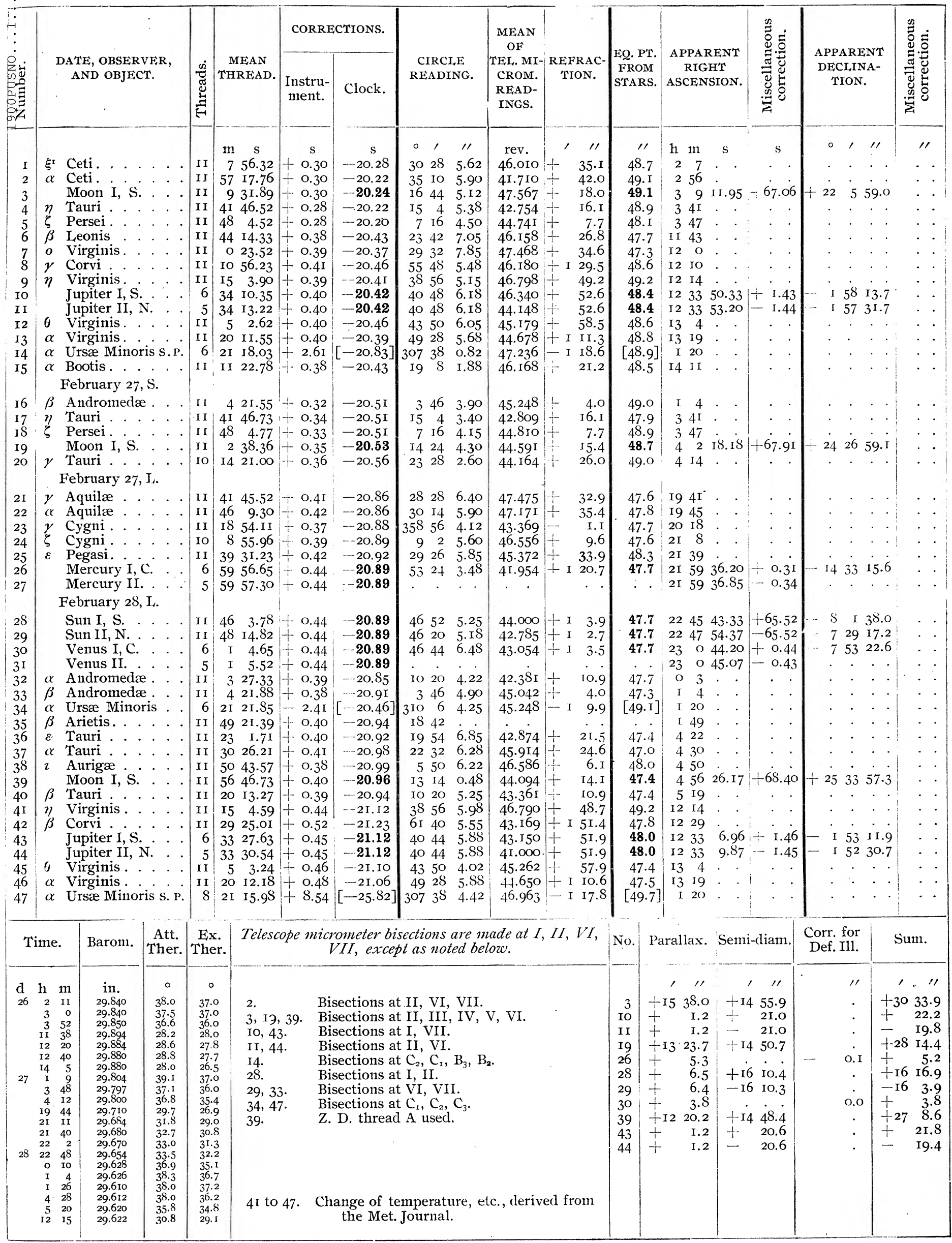




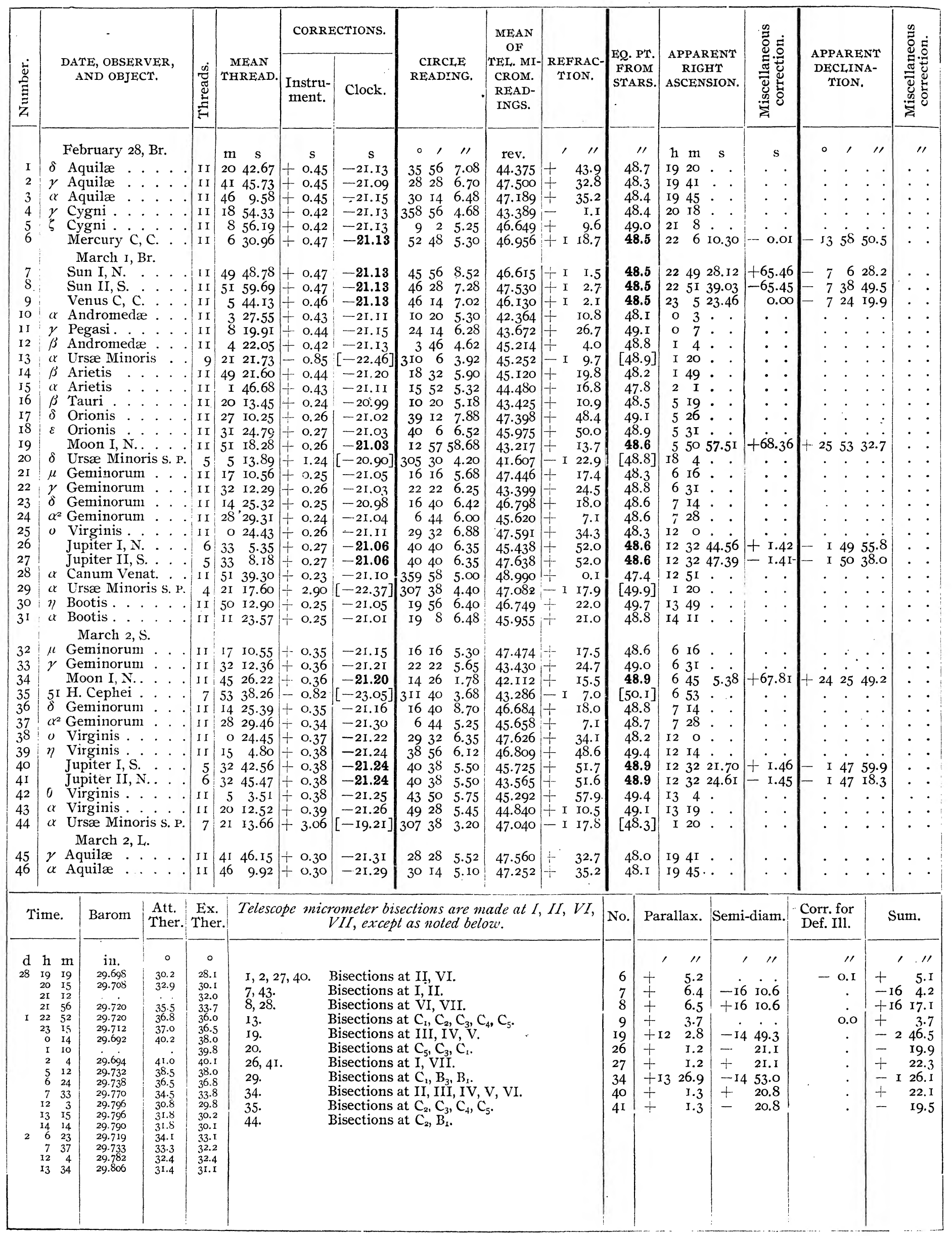




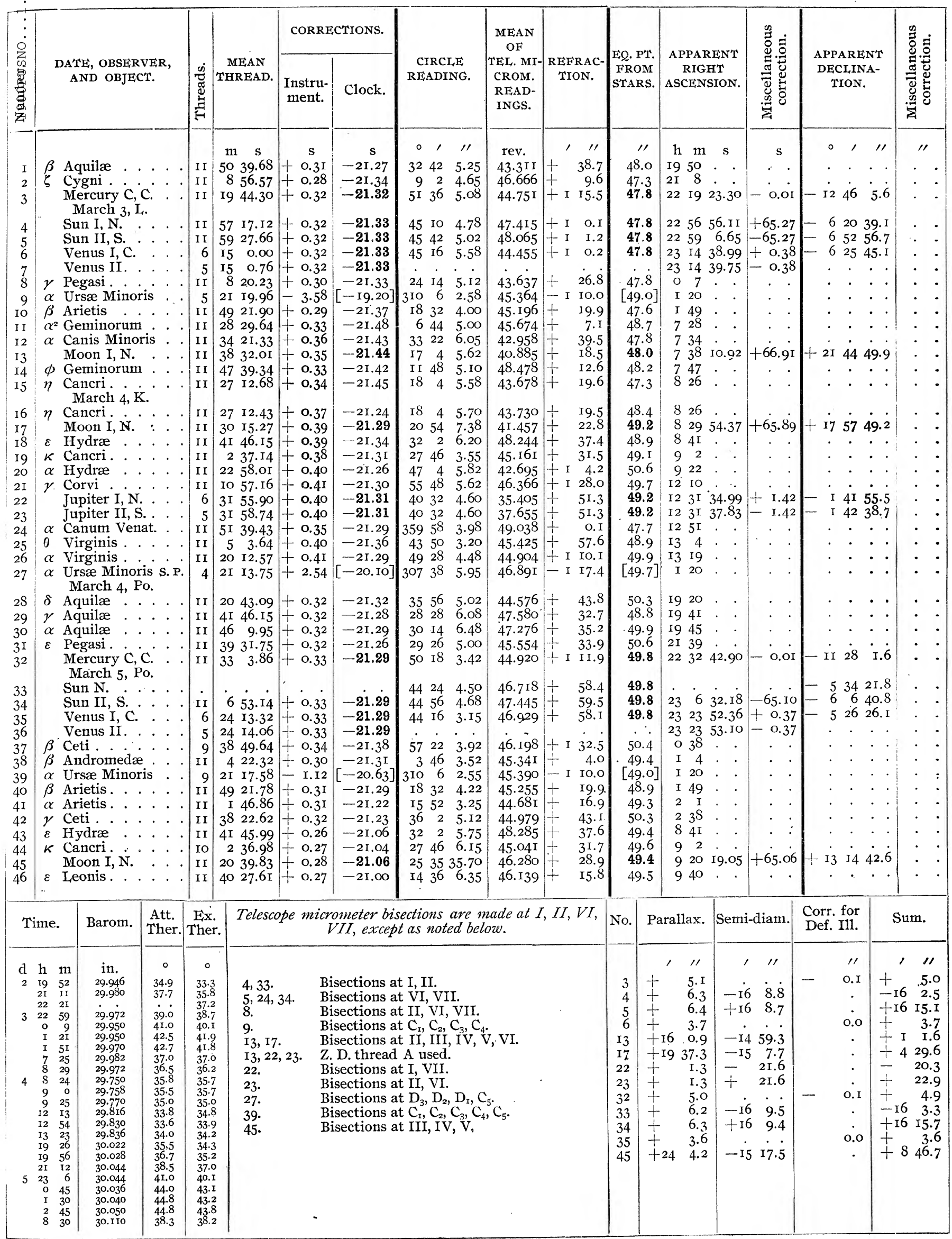




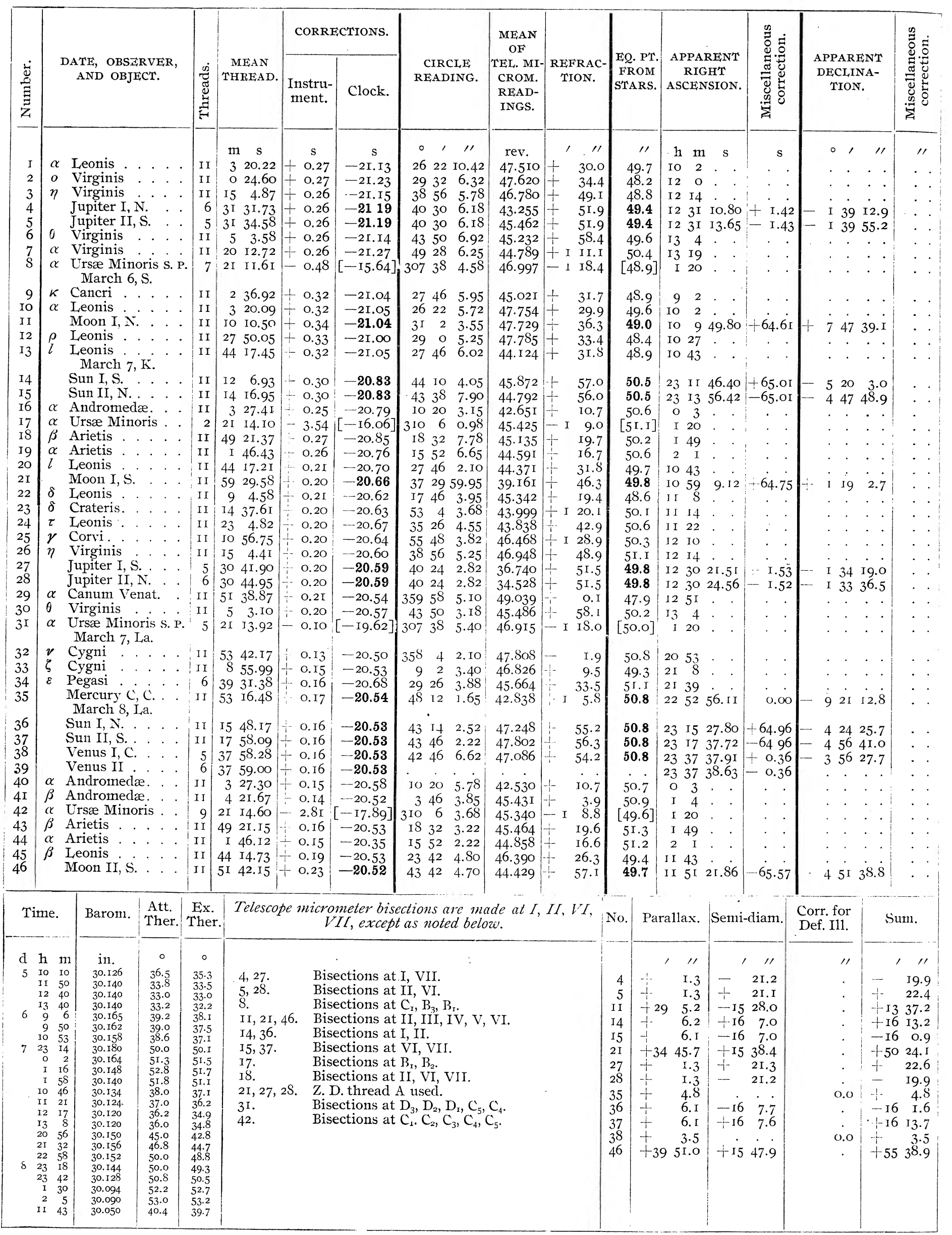




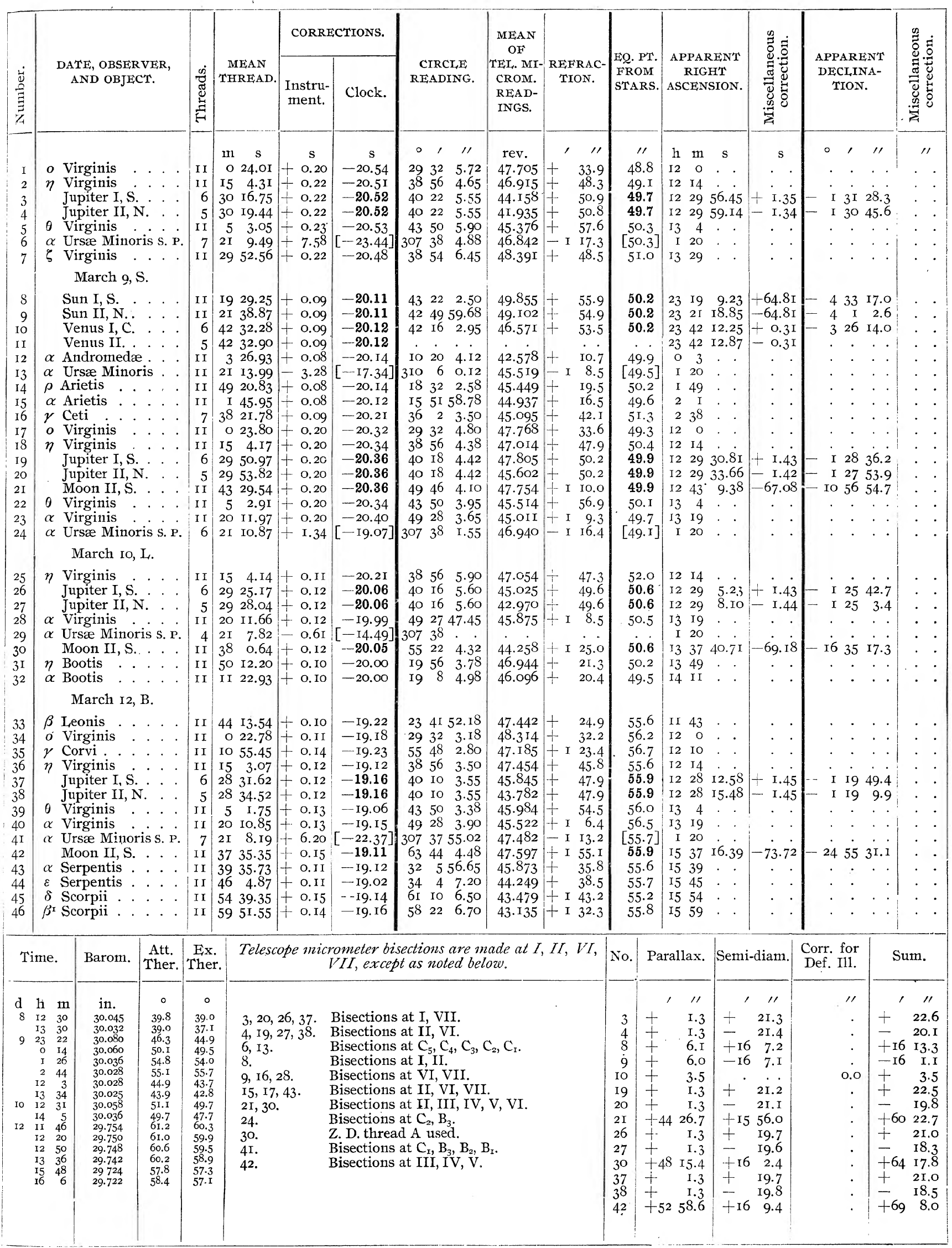




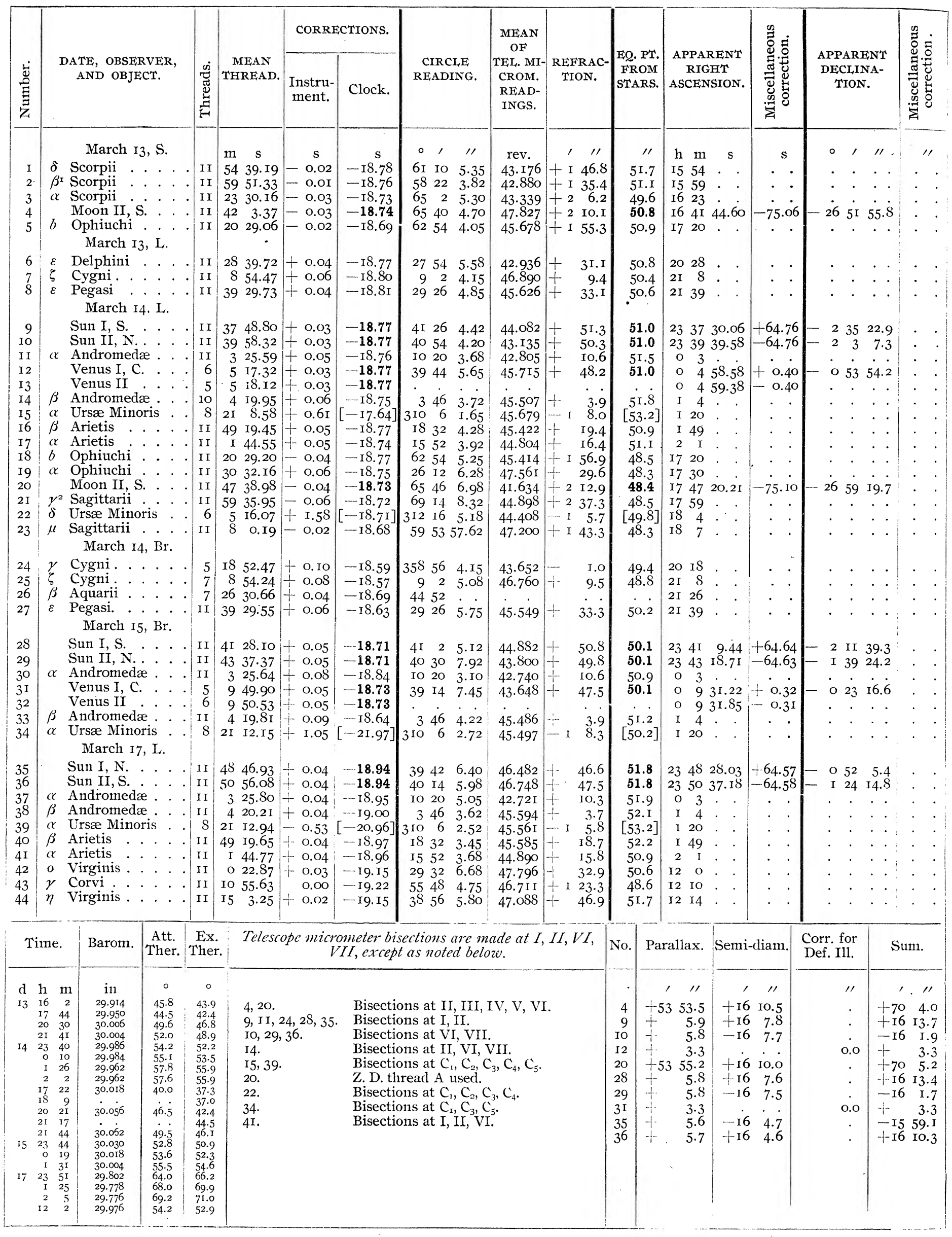




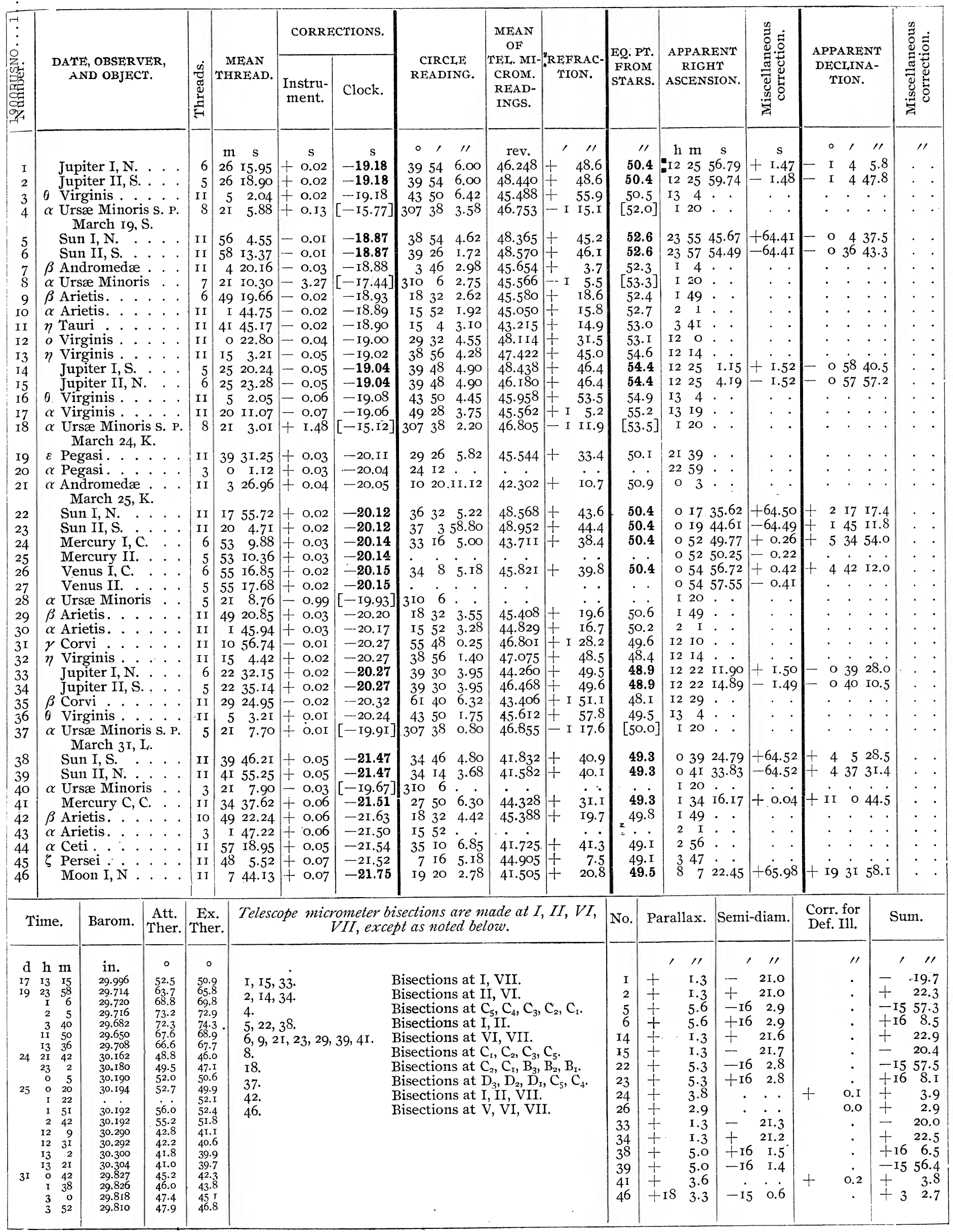




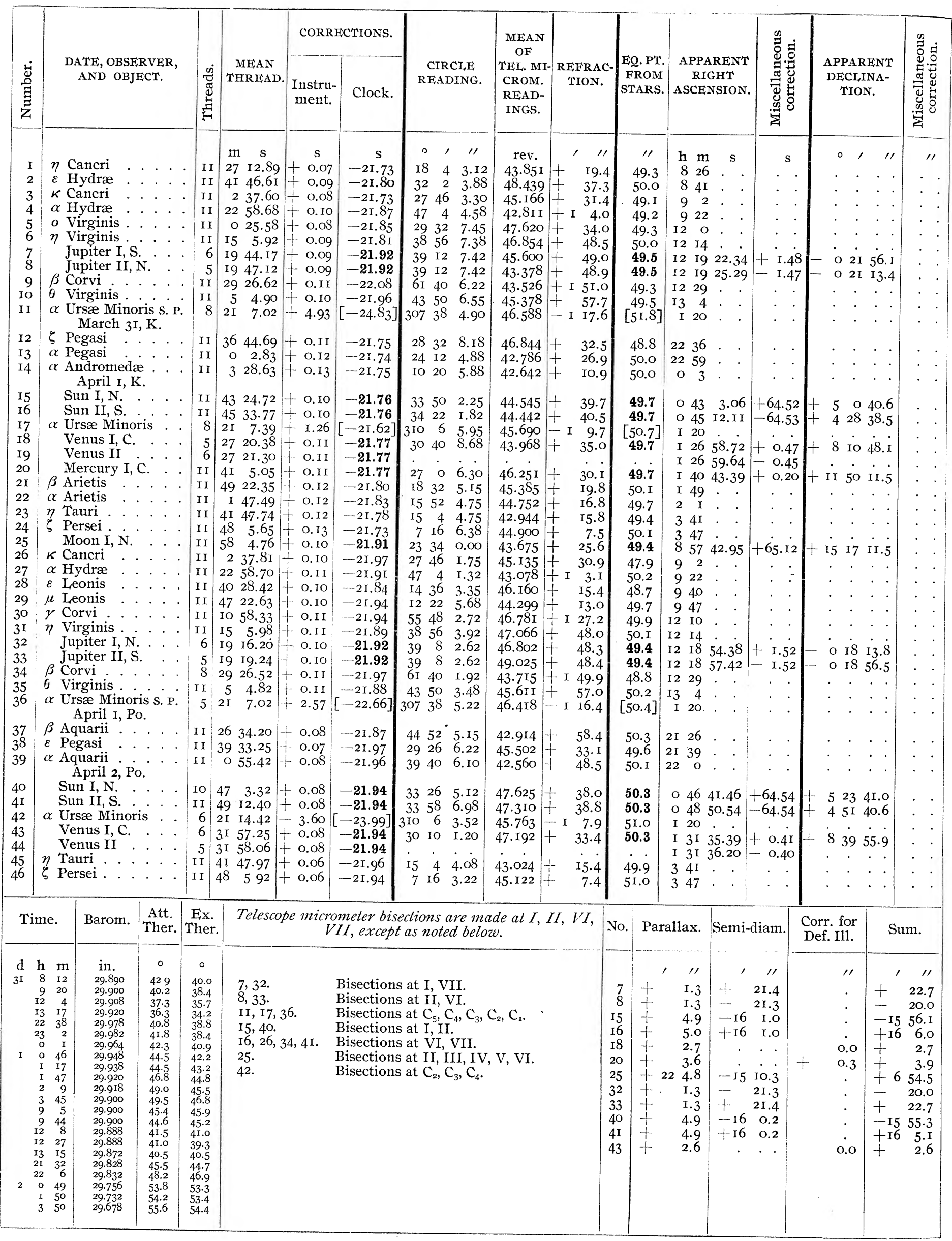




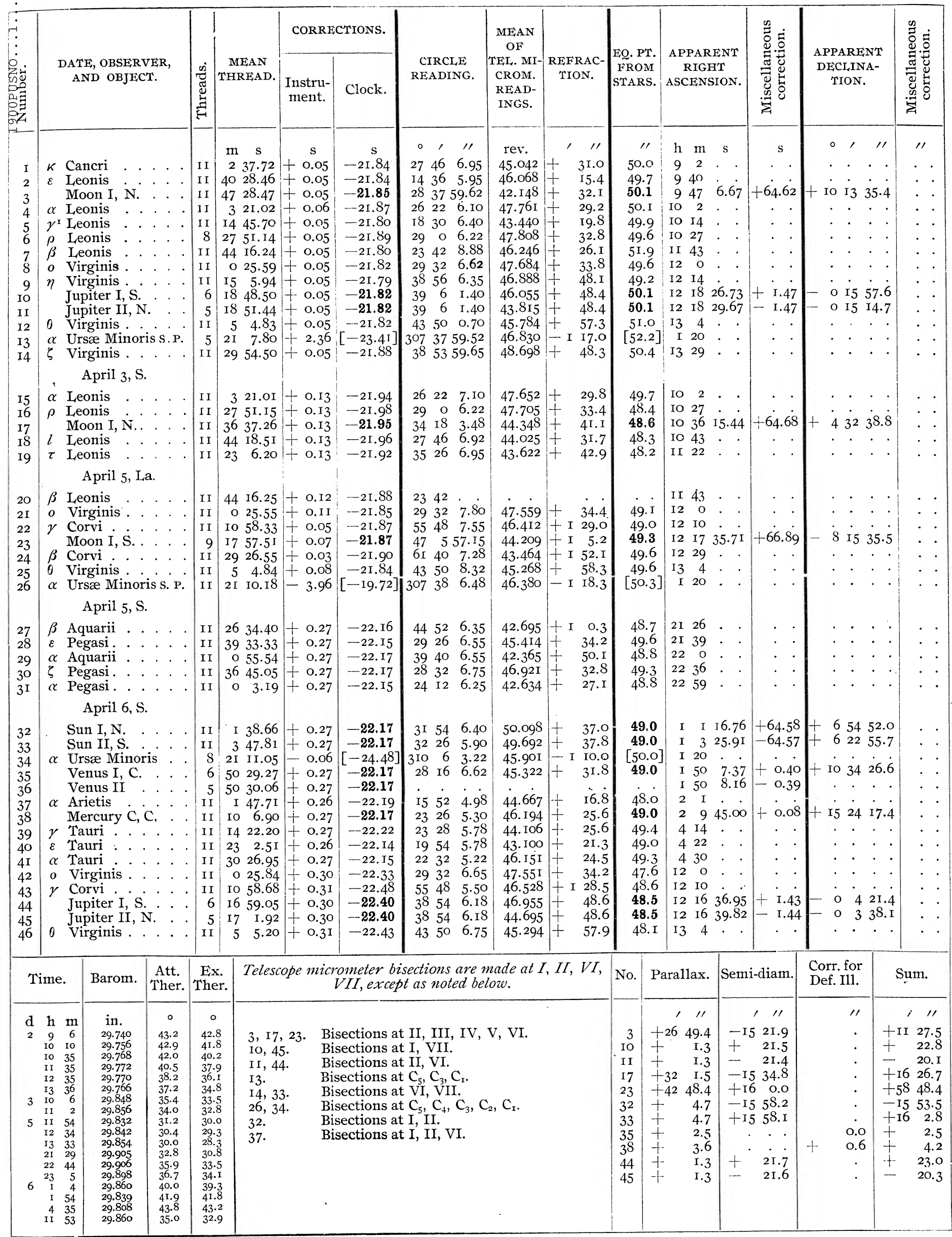




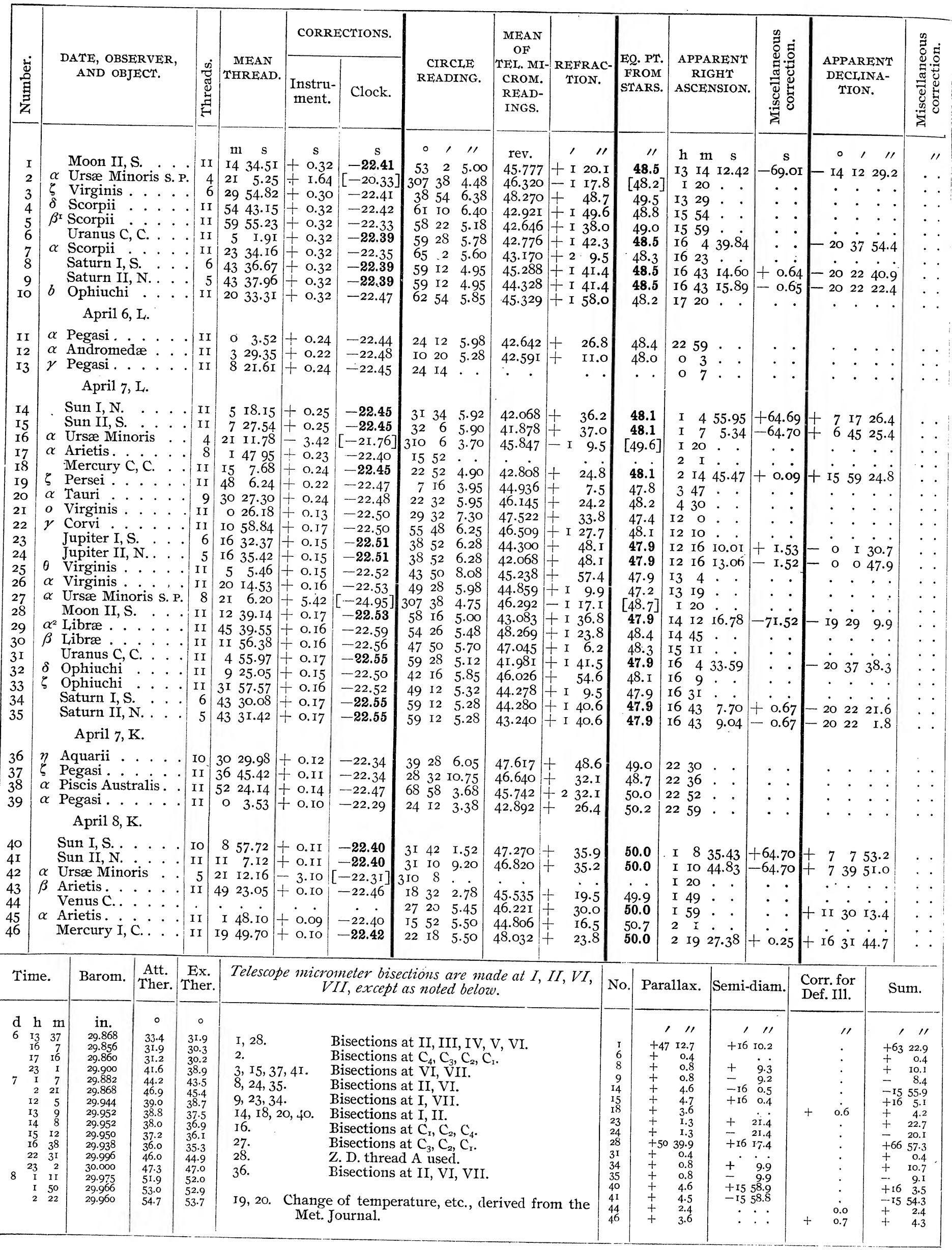




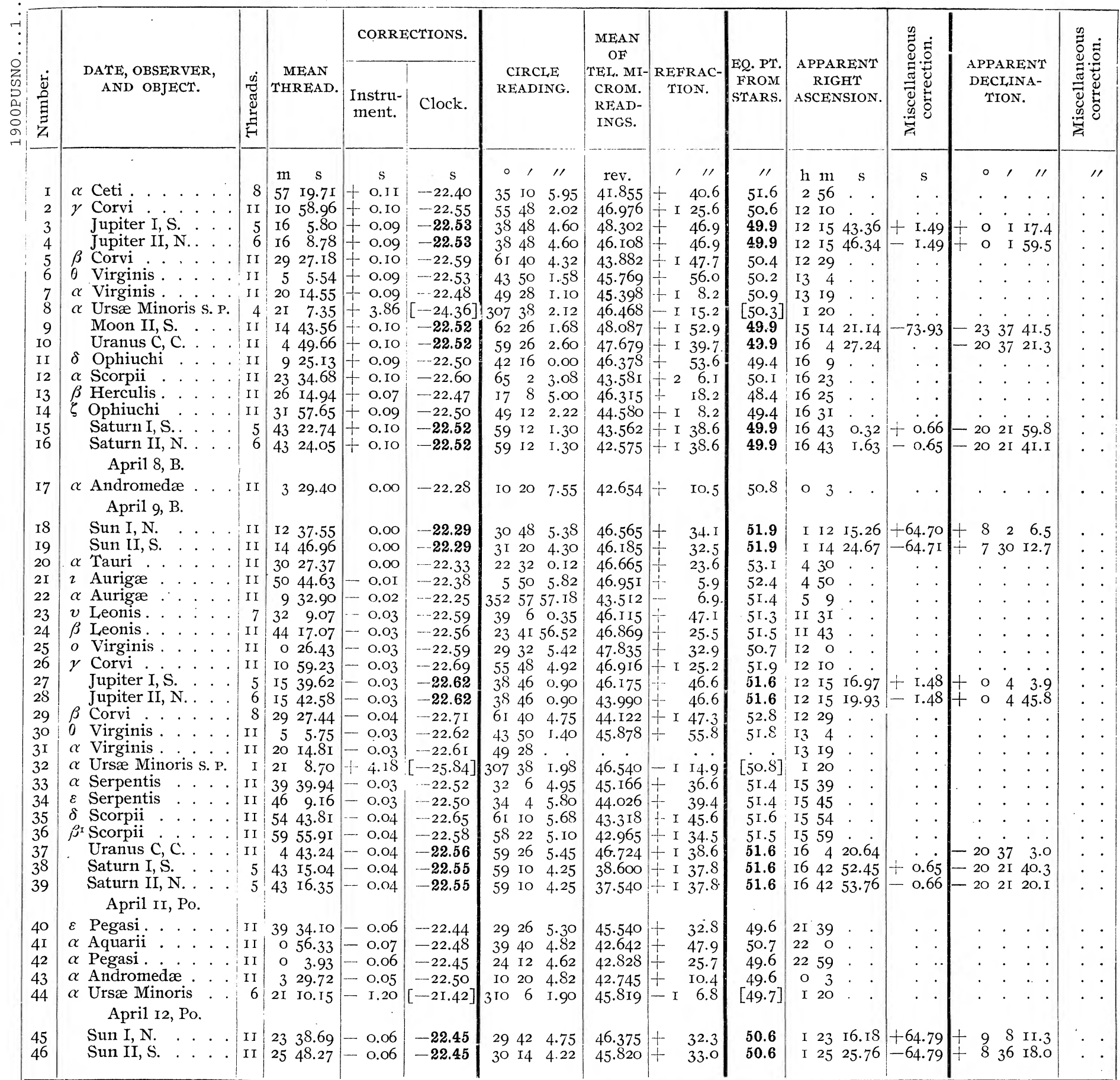

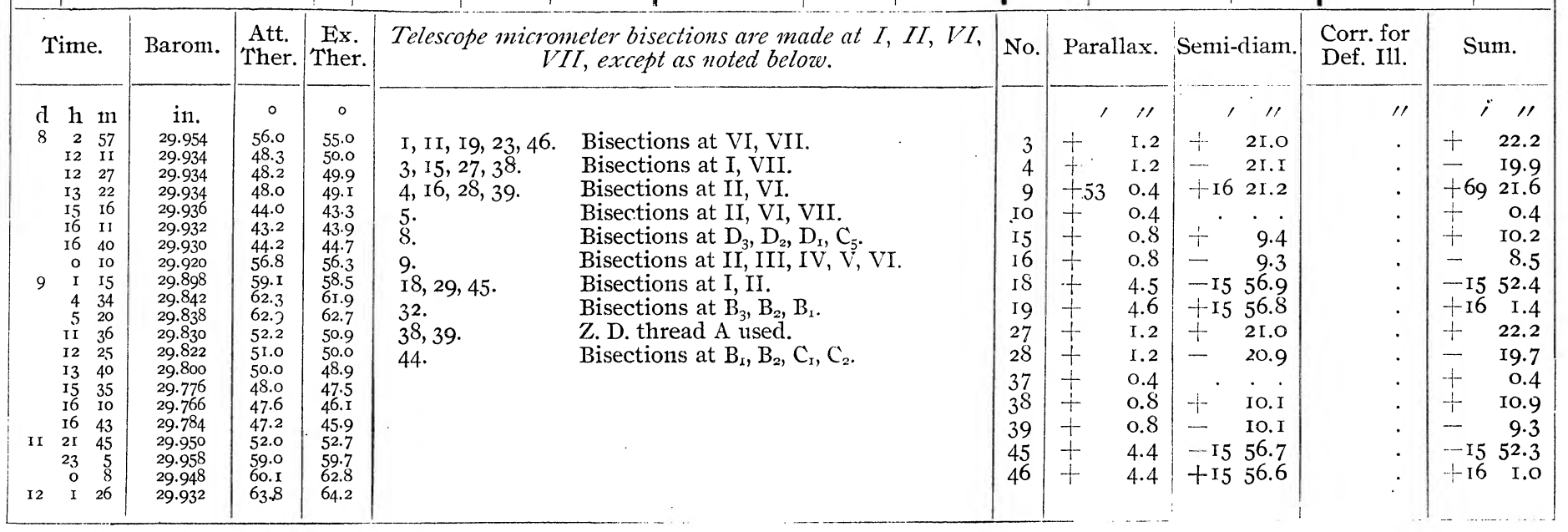




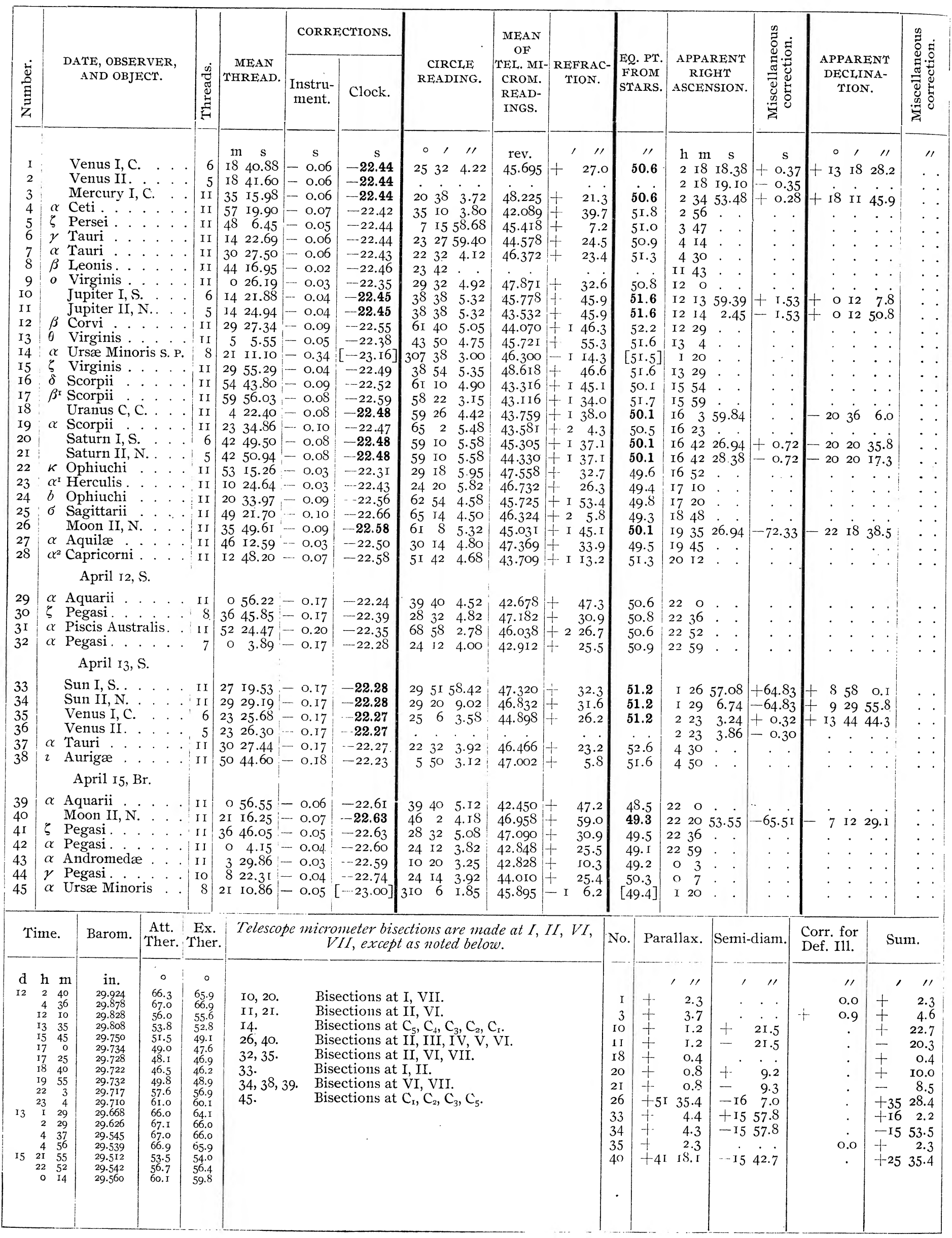




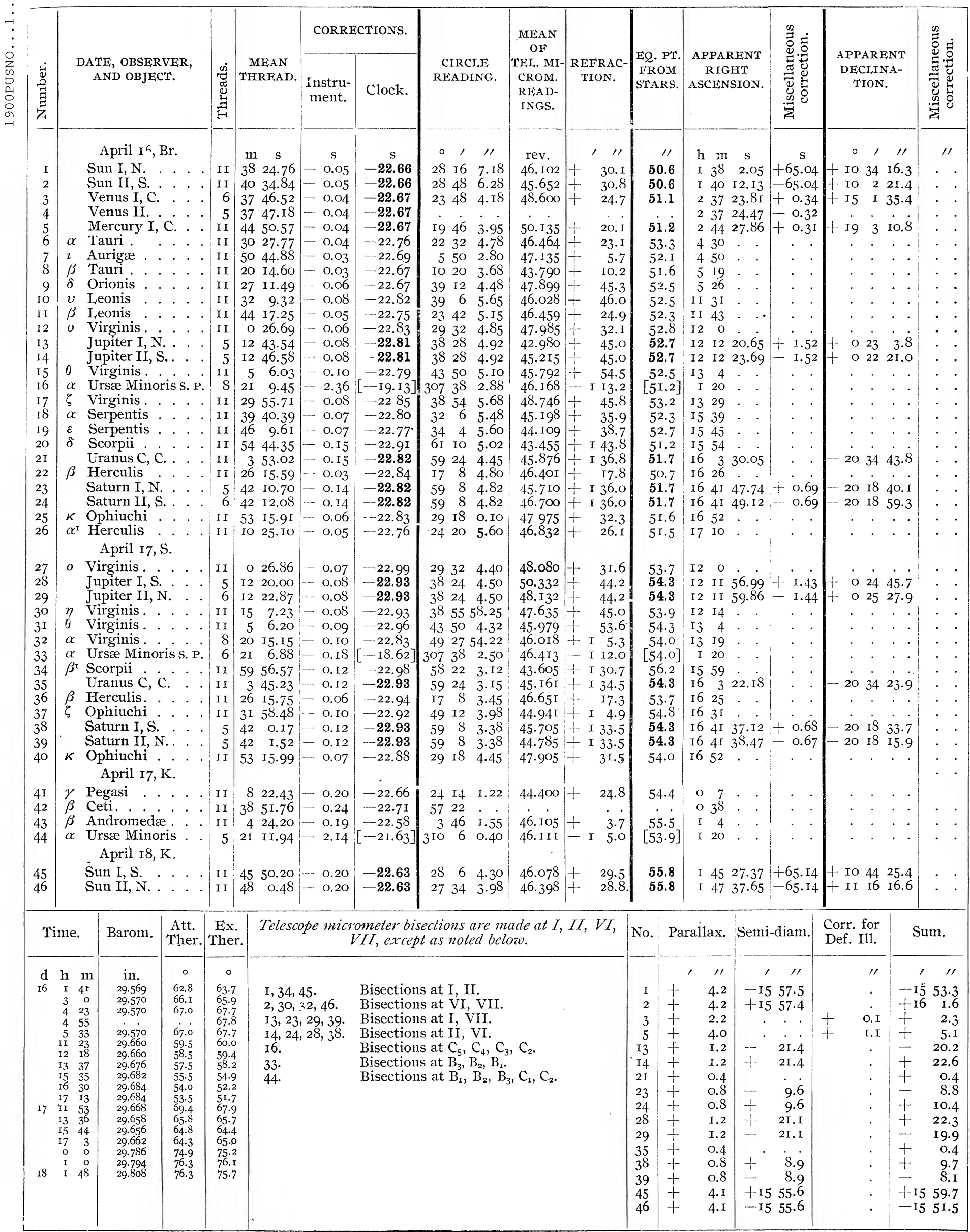




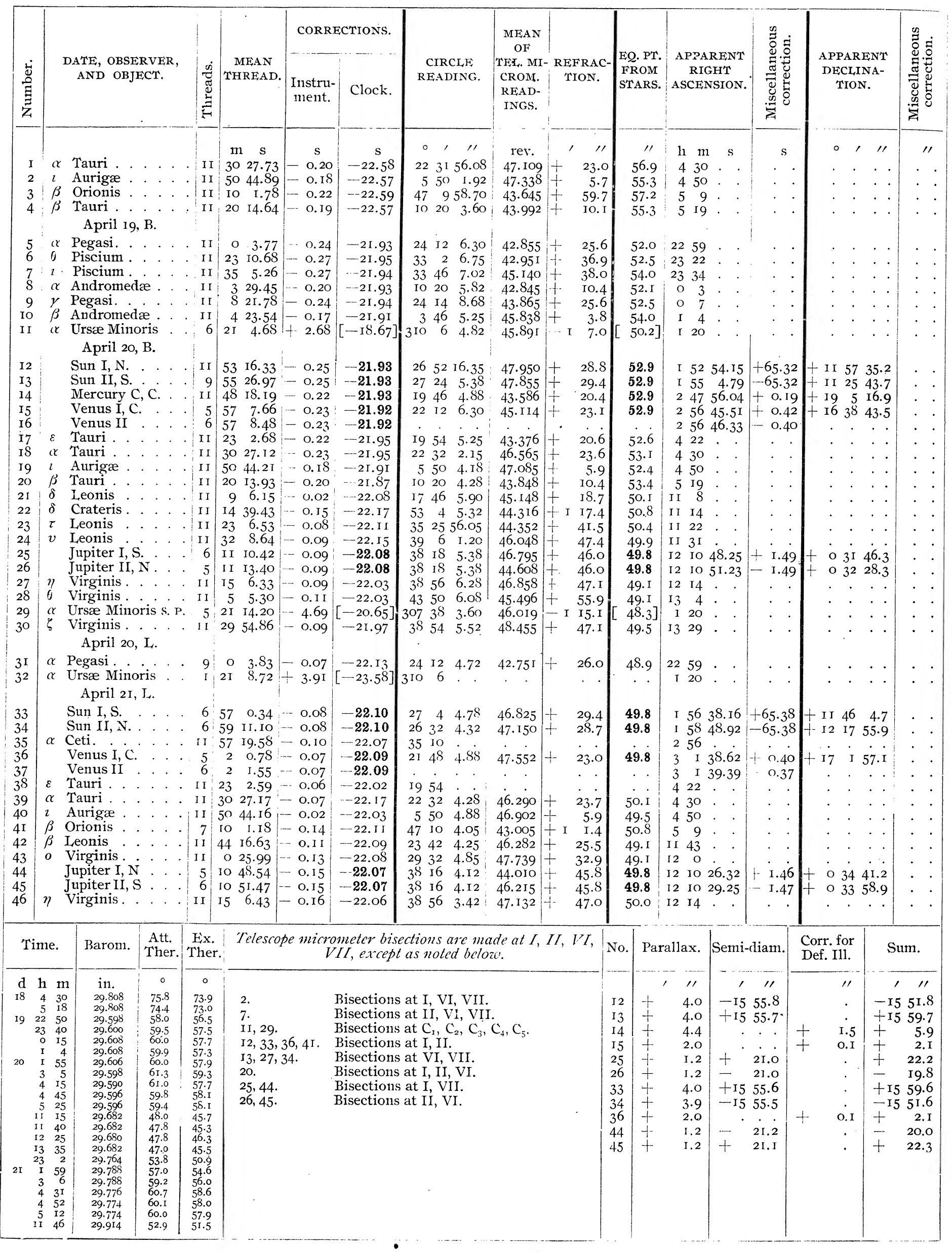




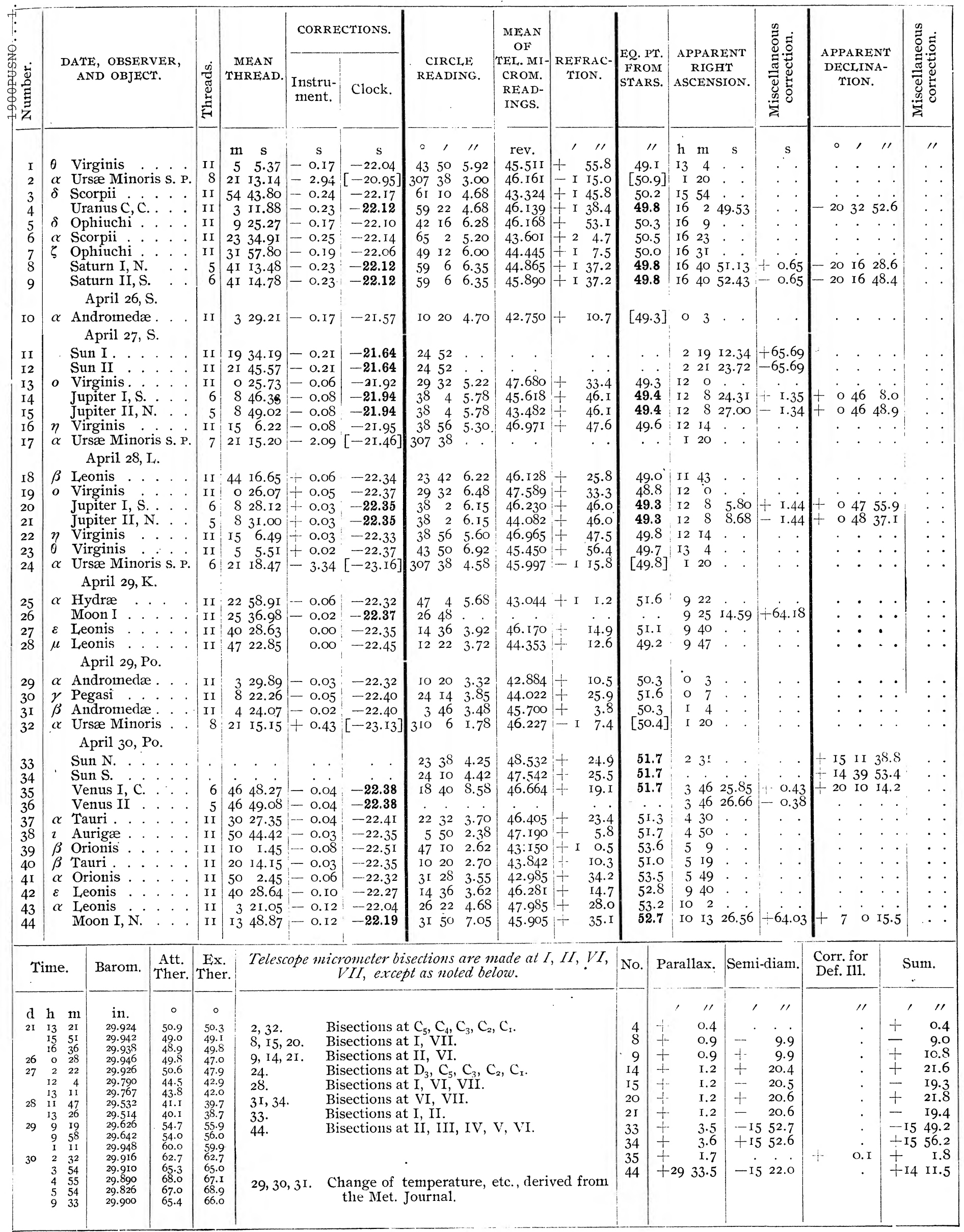




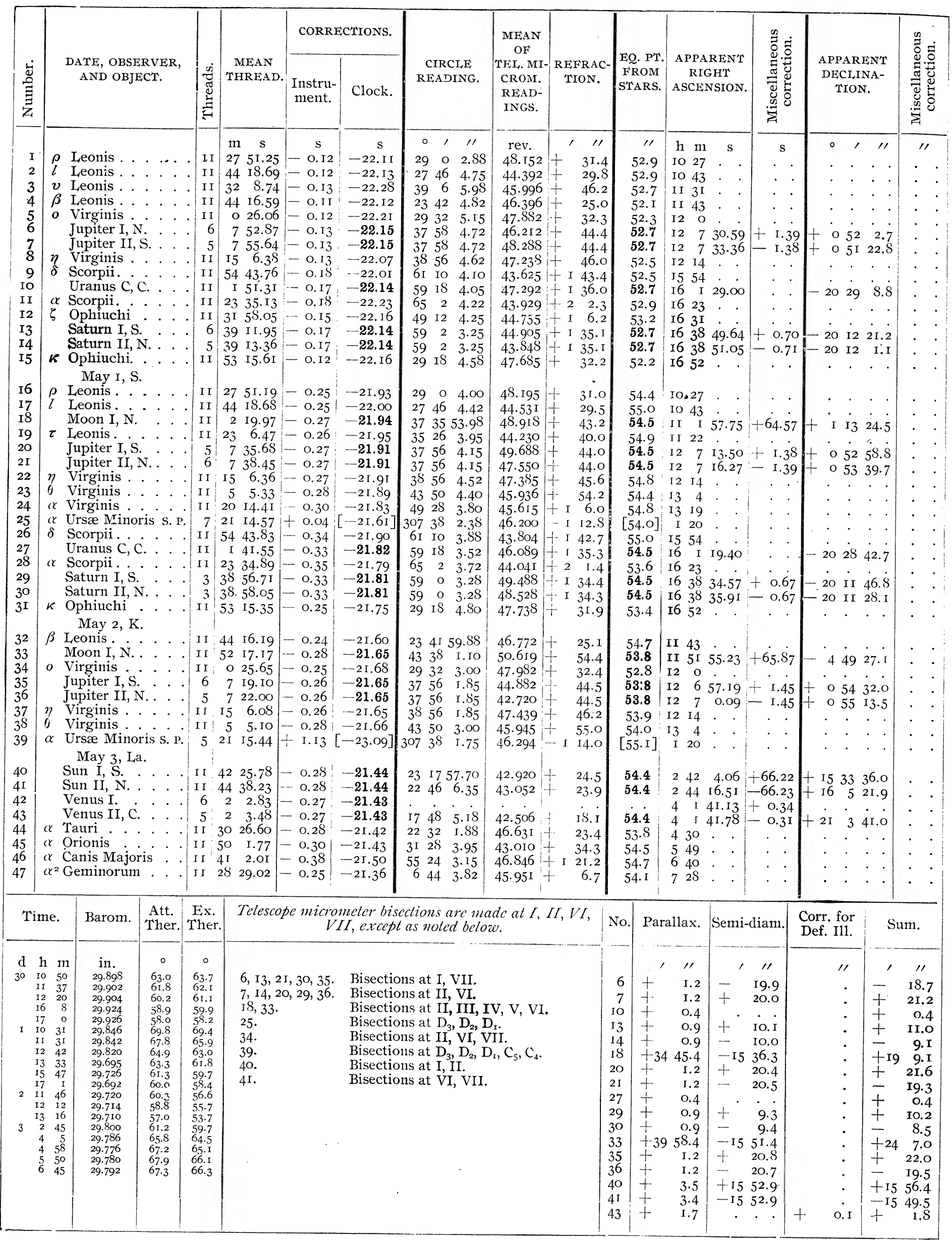




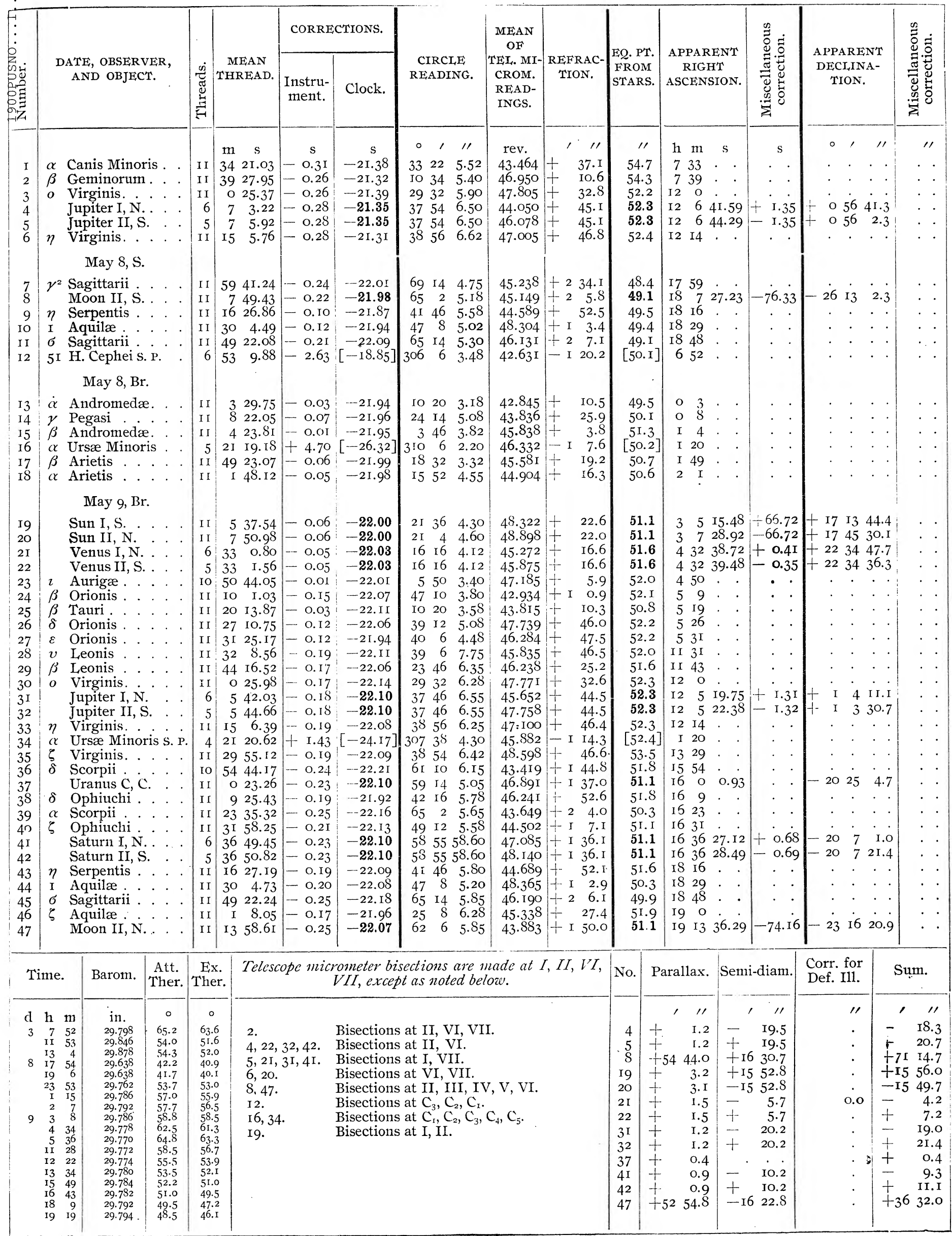




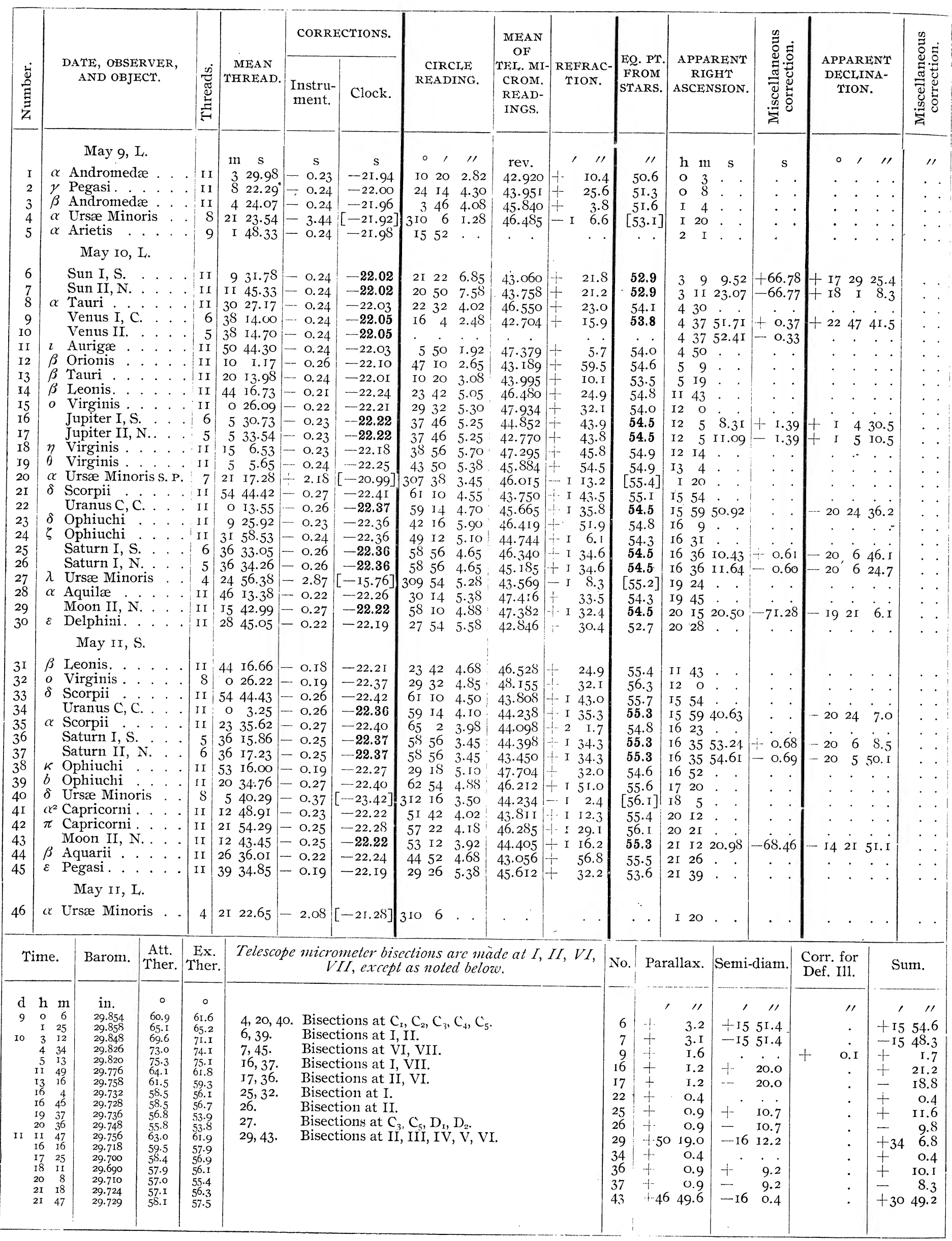




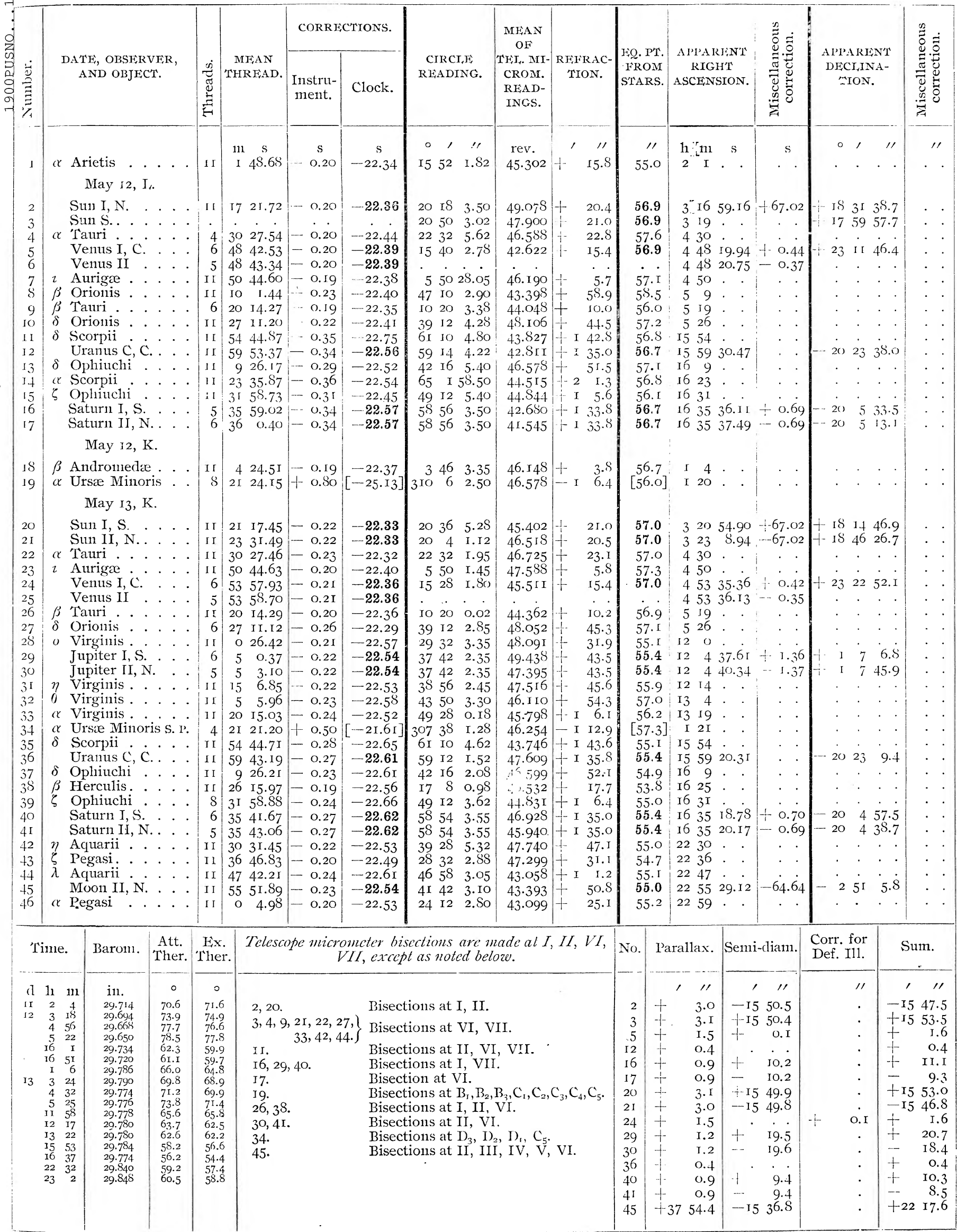

$(885-14$ 


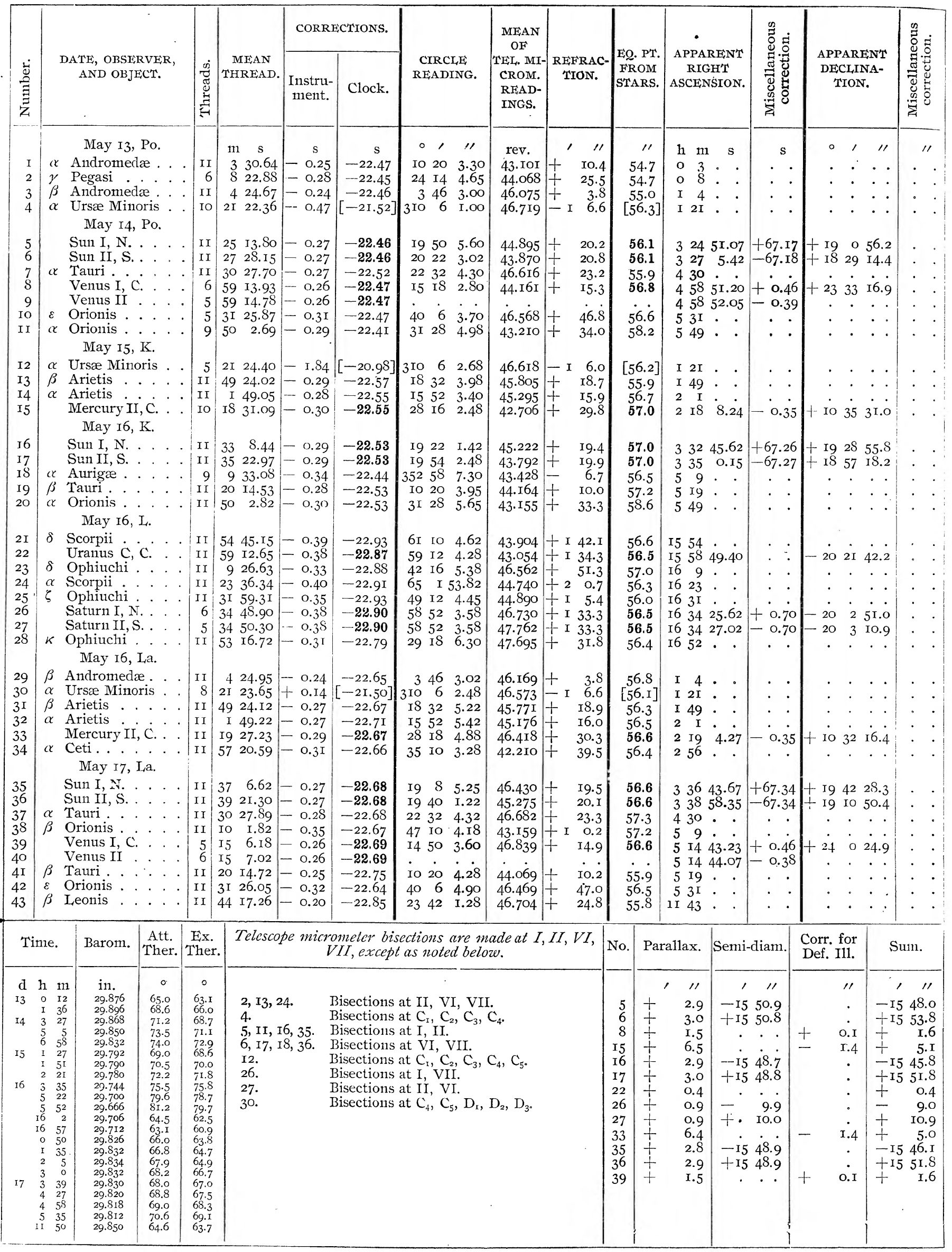




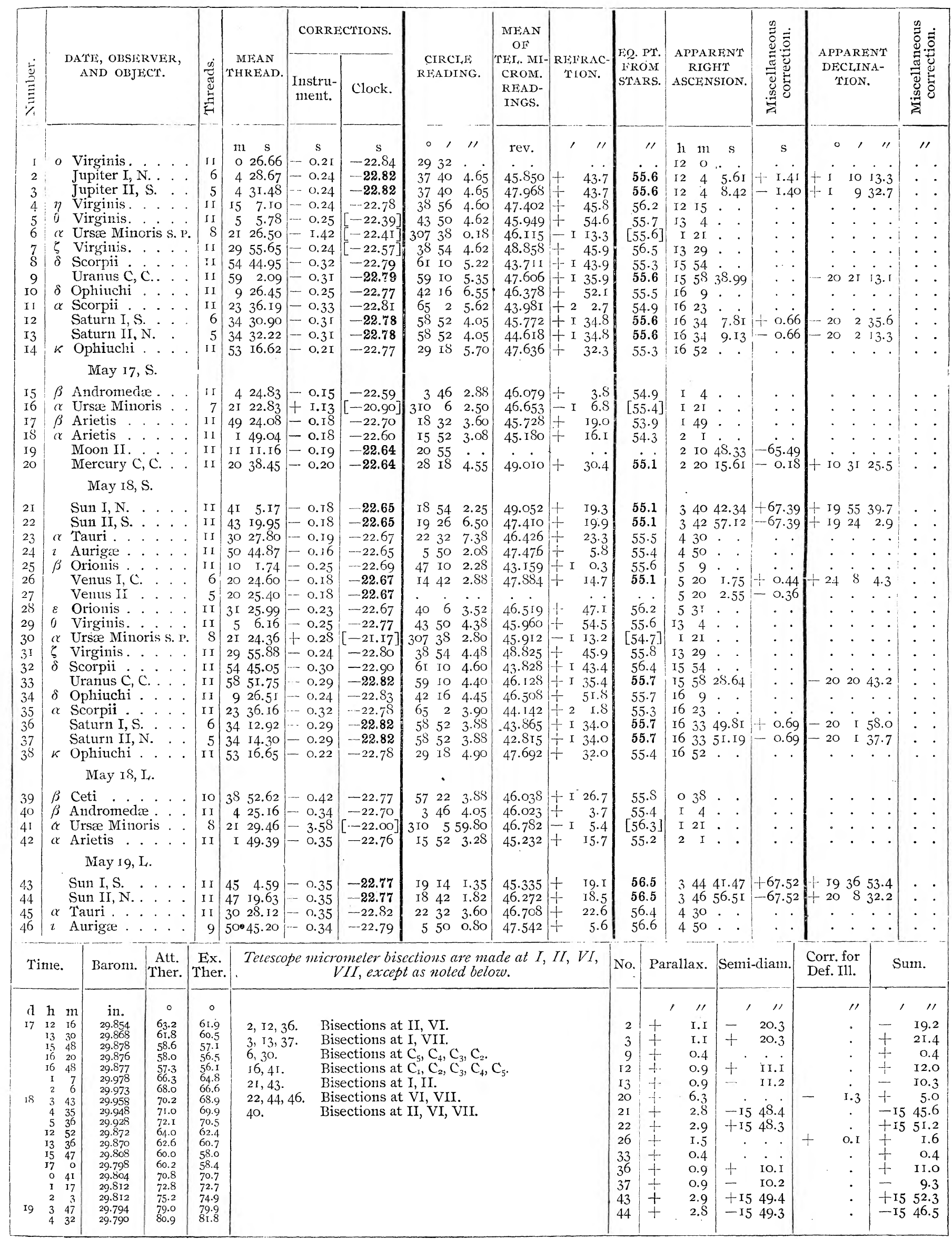




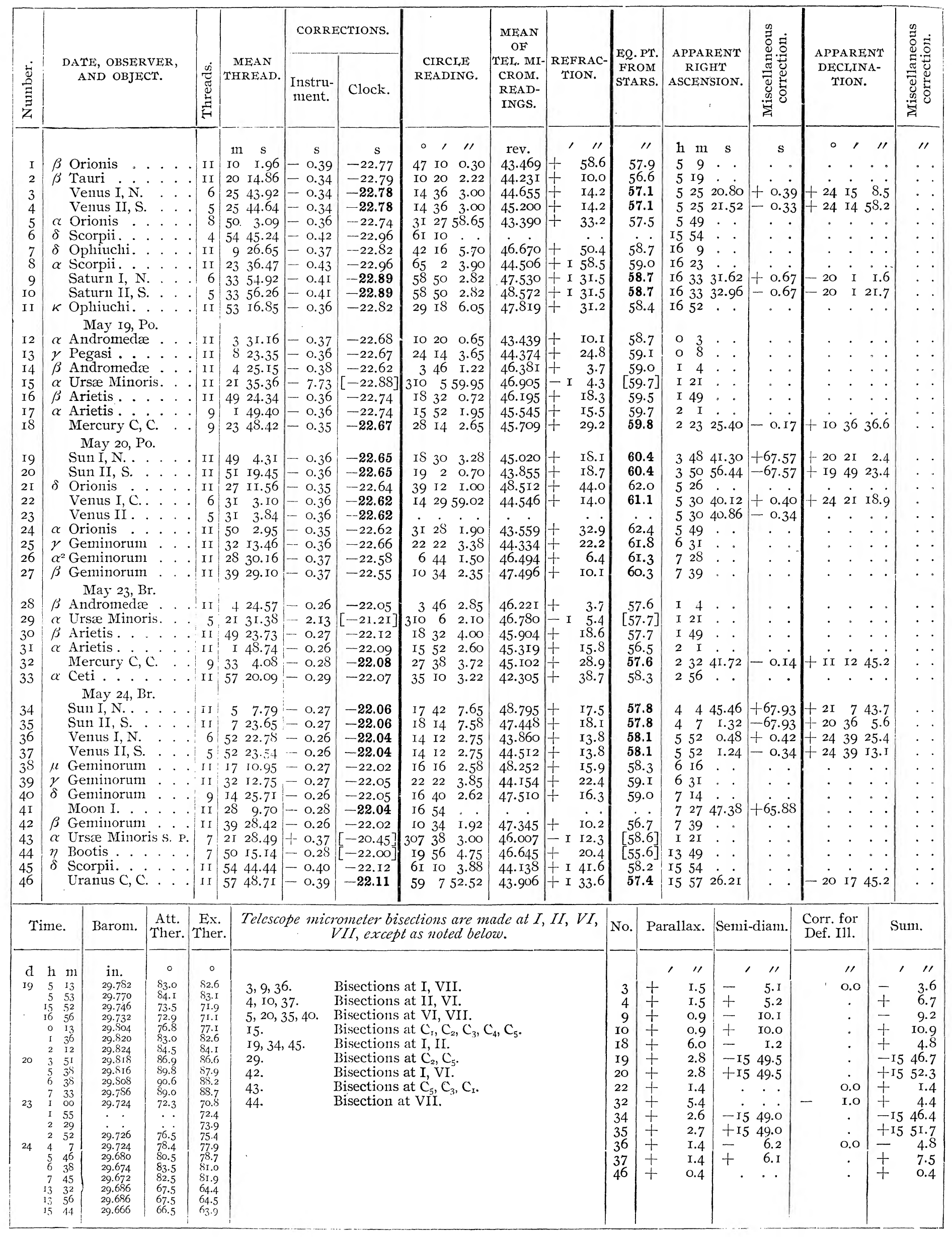




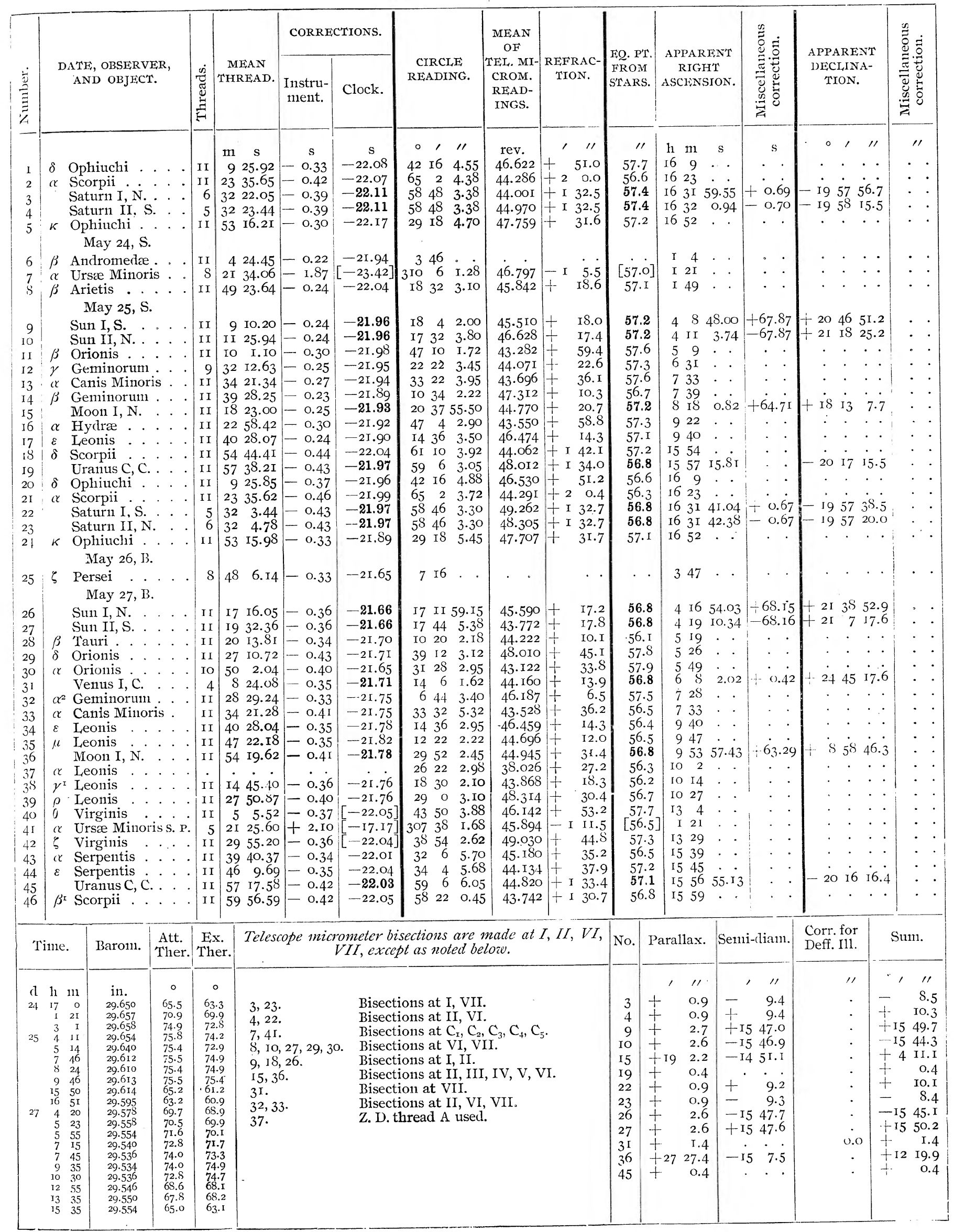




\begin{tabular}{|c|c|c|c|c|c|c|c|c|c|c|c|c|c|c|c|c|c|c|}
\hline \multirow[b]{2}{*}{ 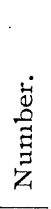 } & \multirow{2}{*}{\multicolumn{3}{|c|}{$\begin{array}{l}\text { DATE, OBSERVER, } \\
\text { AND OBJECT. }\end{array}$}} & \multirow[b]{2}{*}{ 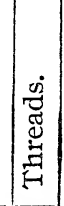 } & \multirow{2}{*}{$\begin{array}{c}\text { MEAN } \\
\text { THREAD. }\end{array}$} & \multicolumn{2}{|c|}{ CORRECTIONS. } & \multirow{2}{*}{$\begin{array}{c}\text { CIRCLE } \\
\text { READING. }\end{array}$} & \multirow{2}{*}{$\begin{array}{l}\text { MEAN } \\
\text { OF } \\
\text { TEL. MI- } \\
\text { CROM. } \\
\text { READ- } \\
\text { INGS. }\end{array}$} & \multirow{2}{*}{$\begin{array}{l}\text { REFRAC- } \\
\text { TION. }\end{array}$} & \multirow{2}{*}{\multicolumn{2}{|c|}{$\begin{array}{l}\text { EQ. PT. } \\
\text { FROM } \\
\text { STARS. }\end{array}$}} & & & & & & \\
\hline & & & & & & $\begin{array}{l}\text { Instru- } \\
\text { ment. }\end{array}$ & Clock. & & & & & & \begin{tabular}{|l} 
RIG] \\
ASCEN
\end{tabular} & ISION. & 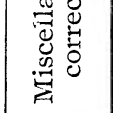 & $\begin{array}{l}\text { ECLIN } \\
\text { TION. }\end{array}$ & N. & 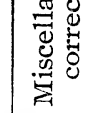 \\
\hline & & & & & $\mathrm{m} \quad \mathrm{s}$ & $\mathrm{s}$ & $\mathrm{s}$ & 0,11 & rev. & 11 & & "I & $\mathrm{h} \mathrm{m}$ & $\mathrm{s}$ & $\mathrm{s}$ & $\circ \quad$, & "I & "1 \\
\hline $\begin{array}{l}1 \\
2\end{array}$ & & $\begin{array}{l}\text { phiuchi } \\
\text { corpii. }\end{array}$ & $\therefore$ & $\mid \begin{array}{l}\text { II } \\
\text { I I }\end{array}$ & $\begin{array}{rr}9 & 25.9 \mathrm{I} \\
23 & 35.72\end{array}$ & $\left|\begin{array}{rr}-0.37 \\
-0.45\end{array}\right|$ & $\begin{array}{l}-22.00 \\
-22.07\end{array}$ & $\begin{array}{lll}42 & \text { I6 } & \text { I. } 22\end{array}$ & 46.740 & $+\quad 5$ I.O & & 6.9 & $\begin{array}{ll}16 & 9\end{array}$ & $\cdot \cdot$ & • & $\cdot \cdot$ & . $\cdot$ & . . \\
\hline 3 & & aturn I, $\dot{\mathrm{N}}$ & $\therefore:$ & $\begin{array}{r}11 \\
5\end{array}$ & 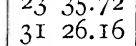 & $\left|\begin{array}{l}-0.45 \\
-0.42\end{array}\right|$ & $\begin{array}{l}-22.07 \\
-22.06\end{array}$ & $\begin{array}{rrr}65 & 2 & 4 \cdot 30 \\
5^{9} & 46 & 2.45\end{array}$ & $\begin{array}{l}44.336 \\
44.508\end{array}$ & $\begin{array}{r}\text { + I } 59.9 \\
\text { + I } 32.3\end{array}$ & & $\begin{array}{l}7.2 \\
7.1\end{array}$ & \begin{tabular}{|ll}
16 & 23 \\
I6 & $3 \mathrm{I}$
\end{tabular} & $3 . \dot{68}$ & $+\dot{0} \dot{6} 6$ & - ig 56 & 5.8 & $\cdot$ \\
\hline 4 & & $\begin{array}{l}\text { aturn II, S } \\
\text { May } 27, \mathrm{I}\end{array}$ & $\begin{array}{l}\text { S. . . . } \\
\text { Po. }\end{array}$ & 6 & 3127.48 & -0.42 & -22.06 & $\begin{array}{lll}58 & 46 & 2.45\end{array}$ & 45.530 & t I 32.3 & & 7.1 & $163 I$ & 5.00 & -0.66 & -19562 & 25.2 & . . \\
\hline 5 & $\beta$ & ndromeda & e. . . & I I & 424.72 & -0.34 & $-2 \mathrm{I} .99$ & 3460.05 & 46.294 & $+\quad 3.7$ & & 6.4 & I 4 & . & . & . & . . & . \\
\hline 6 & $\alpha$ & Mino & ris . . & IO & $2 \mathrm{I} \quad 3^{\mathrm{S} .24}$ & -5.36 & {$[-21.99]$} & $310 \quad 6 \quad 1.02$ & 46.786 & - I 5.2 & {$[56$} & $6.3]$ & I 2 I & . & . & . . & . . & . \\
\hline $\begin{array}{l}7 \\
8\end{array}$ & $\begin{array}{l}\beta \\
\alpha\end{array}$ & $\begin{array}{l}\text { rietis } \cdot . \\
\text { rietis }\end{array}$ & $\cdot \cdot \cdot$ & I0 & $\begin{array}{ll}49 & 23.79\end{array}$ & -0.34 & $-22 . \mathrm{OI}$ & I8 $32 \quad 2.22$ & $45.94 \mathrm{I}$ & $+\quad$ I8.5 & & 6.8 & I 49 & . & . & $\cdot \cdot$ & $\cdot \cdot$ & . \\
\hline 9 & & Iercury II, & $\dot{c}_{0} \cdot \dot{ }$ & $\begin{array}{l}11 \\
\text { I I }\end{array}$ & $\begin{array}{rr}\mathrm{I} & 45.86 \\
46 & 0.93\end{array}$ & {$\left[\begin{array}{r}-0.34 \\
-0.34\end{array}\right.$} & $\begin{array}{l}-22.03 \\
-22.04\end{array}$ & $\begin{array}{lll}\text { I5 } & 52 & 2.35 \\
26 & 32 & 3.48\end{array}$ & $\begin{array}{l}45.344 \\
46.395\end{array}$ & $\begin{array}{r}+\quad 15.7 \\
+\quad 27.4\end{array}$ & & $\begin{array}{l}6.9 \\
7.5\end{array}$ & $\begin{array}{rr}2 & I \\
2 & 45\end{array}$ & 38.55 & -0.27 & I2 I8 2 & $22 . \dot{I}$ & - \\
\hline IO & 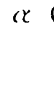 & $\begin{array}{l}\text { May } 2 S, \\
\text { Leti }\end{array}$ & $\begin{array}{l}\text {. . } \\
\text { Po. }\end{array}$ & 6 & $5720.2 \mathrm{~S}$ & -0.35 & -22.14 & 35 IO 3.32 & 42.260 & $+\quad 38.6$ & & 7.6 & 256 & 30.53 & & . & .. & . \\
\hline II & & un $I, N$. & & II & 2I 20.23 & -0.34 & -22.04 & I7 $\quad 2$ I.OO & 46.560 & $+\quad 16.8$ & 57 & 7.5 & 4205 & 57.85 & +68.15 & +21483 & 33.5 & \\
\hline I2 & & S. &.. & II & $2336.5 t$ & -0.34 & -22.04 & I7 $34 \quad 0.12$ & 45.070 & I7.3 & 57 & 7.5 & 423. & I4. 16 & -68.16 & +2 I 165 & 59.2 & . \\
\hline I3 & & ais . & . & $\mathrm{II}$ & IO I.25 & -0.37 & -22.04 & $47 \quad 959.22$ & 43. & 58.6 & & 3.0 & 59 &.. & & . . & . . & . \\
\hline I4 & $\varepsilon$ & iis & & $1 \mathrm{I}$ & $3125.5^{2}$ & -0.36 & -22.05 & $40 \quad 6 \quad 2.15$ & 46.668 & $+\quad 45.7$ & 57 & 7.5 & $53 \mathrm{I}$ & . & . $\cdot$ & . . & . . & . \\
\hline I5 & $\alpha$ & ri & & IO & $\begin{array}{ll}50 & 2.36\end{array}$ & -0.34 & -22 & $\begin{array}{lll}31 & 28 & \text { I. } 78\end{array}$ & 43.295 & + 33.2 & $5^{8}$ & 3.I & 549 & & & . . & & . \\
\hline $\begin{array}{l}\text { I6 } \\
\text { I7 }\end{array}$ & 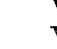 & $\begin{array}{l}\text { I, C. } \\
\text { II . }\end{array}$ & $\therefore$ & $\begin{array}{l}6 \\
5\end{array}$ & I3 44.93 & -0.34 & -22.04 & I4 4 I. 40 & 48.875 & $+\quad 13.6$ & 57 & 7.5 & $63_{3}$ & 22.55 & +0.35 & +24455 & 50.5 & \\
\hline I8 & $\alpha^{2}$ & oruii & & \begin{tabular}{r|r}
5 & \\
II
\end{tabular} & $\mid \begin{array}{ll}13 & 45.56 \\
28 & 29.50\end{array}$ & $\begin{array}{r}-0.34 \\
-0.34\end{array}$ & $\begin{array}{l}-22.04 \\
-22 . \mathrm{OI}\end{array} \mid$ & & & $6-1+1+2$ & & & 6132 & 23.18 & $-0.28 \mid$ & .. & . $\cdot$ & . \\
\hline I9 & $\alpha$ & Mino & ris . . & II & $\begin{array}{lll}213 & 29.50 \\
34 & 2 \mathrm{I} .58\end{array}$ & $\begin{array}{r}-0.34 \\
-0.35\end{array}$ & $\begin{array}{l}-22.01 \\
-22 . \mathrm{IJ}\end{array}$ & \begin{tabular}{rrr|}
6 & 44 & I. 45 \\
33 & 22 & 2.50
\end{tabular} & $\begin{array}{l}46.369 \\
43.760\end{array}$ & $\begin{array}{r}+ \\
+\quad 6.5 \\
+\quad 35.7\end{array}$ & $\begin{array}{l}5 s \\
57\end{array}$ & $\begin{array}{l}5.5 \\
7.0\end{array}$ & $\begin{array}{l}728 \\
733\end{array}$ & $\cdot \cdot$ & . & - $\cdot$ & $\cdot \cdot$ & - \\
\hline 20 & & inorum & 1.. . & II & $\begin{array}{ll}39 & 28.44\end{array}$ & -0.34 & -2 & $\begin{array}{lll}33 & & \\
\text { I0 } & 34 & 1.05\end{array}$ & $\begin{array}{l}43.700 \\
47.510\end{array}$ & $\begin{array}{r}35.7 \\
+\quad 10.2\end{array}$ & 57 & 7.0 & $\begin{array}{l}733 \\
739\end{array}$ &. & : &. &. & $\cdot$ \\
\hline $2 \mathrm{I}$ & $\alpha \mathrm{J}$ & eonis . . & . . & II & 320.96 & -0.33 & -22.08 & $\begin{array}{lll}26 & 22 & 3.25\end{array}$ & 48.326 & 27.0 & & 3.8 & IO 2 & . & . & . & $\therefore$ & . \\
\hline 22 & $\gamma^{x} I$ & is . & . & II & I4 45.66 & -0.32 & -22.07 & IS 30 1.02 & 44.015 & $+\quad \mathrm{I} 8.2$ & & 7.9 & IO I4 & . . & . & . &. & . \\
\hline 23 & $\beta I$ & is & . . . & II & 275 r.13 & -0.33 & --22 & $29 \quad 0 \quad 2.35$ & 48.439 & 30.2 & & 3.3 & Io 27 & & & . & .. & . \\
\hline 24 & & $I, N$ & . . & II ! & $4 \mathrm{I} 27.5 \mathrm{~S}$ & -0.35 & -22 & 35 I9 55.38 & 44.176 & $+\quad 38.6$ & 58 & 3.5 & Io $4 \mathrm{I}$ & 5.14 & +63.46 & $1+33 \mathrm{I}$ & 2.6 & \\
\hline 25 & & is . . & $\cdots$ & II & 96.03 & $-0.3^{2}$ & -22.08 & $\begin{array}{lll}17 & 46 & 3.15\end{array}$ & 45.544 & I 7.6 & & 7.4 & II 8 &. &.$\quad$. & .. & . . & . \\
\hline 26 & $\tau$ I & s. & $\therefore$. & II & 236.42 & -0.34 & -22.09 & $\begin{array}{lll}35 & 26 & 2.5\end{array}$ & 44.500 & $+\quad 3^{8.9}$ & 59 & 2. I & II 22. & & . & . & . & \\
\hline 27 & & rsæe Minor & ris s. I'. & 5 & $2 \mathrm{I} \quad 27.54$ & +4.52 & {$[-20.79]$} & 3073755.30 & $46.35^{8}$ & - I 10.9 & {$\left[5^{8}\right.$} & 3.6] & I $2 \mathrm{I}$ & & . & . & . & . \\
\hline 28 & $c x s$ & corpii . & . . . & II & 2335.75 & $-0.4 I$ & -22.13 & $\begin{array}{lll}65 & 2 & 4.08\end{array}$ & $44.5 \mathrm{I} 4$ & + I $5^{8.8}$ & 59 & 3 & 1623 & & & . & & . \\
\hline $\begin{array}{l}29 \\
30\end{array}$ & & $\begin{array}{l}\text { aturn I, N. } \\
\text { aturn II, s }\end{array}$ & $\therefore$. & 6 & $3 \mathrm{I} \quad 7.72$ & -0.39 & -22.13 & ; $5846 \quad 3.15$ & 42.675 & I I 3 r. 3 & 58 & 8.5 & I6 30 & 45.20 & +0.67 & - I9 552 & 28.9 & - \\
\hline 30 & & $\begin{array}{l}\text { aturn II, } \mathrm{S} \\
\text { May } 30, \mathrm{~N}\end{array}$ & S. & 5 & 3I 9.06 & -0.39 & -22.13 & $\begin{array}{llll}58 & 46 & 3.15\end{array}$ & 43.720 & t I $\quad 3$ I. & 58 & 3.5 & I6 30 & 46.54 & -0.67 & - I9 554 & 48.9 & \\
\hline $3 \mathrm{I}$ & $\gamma \mathrm{C}$ & orvi & . . & II & IO $5^{8.54}$ & -0.40 & 0 & 2.55 & 47.730 & 2 г.० & & & I2 & & & . & & \\
\hline 32 & $\eta \mathrm{V}$ & is & & II & I5 6.15 & -0.35 & -21 & 38563 & 0 & 44.5 & 58 & 3.8 & $\mathrm{I} 2 \mathrm{I} 4$ & & & & & \\
\hline 33 & I & $\mathrm{I}, \mathrm{N}$. & . . & II & I9 18.48 & -0.38 & -21.88 & 465955.90 & 45.766 & $59 . \mathrm{I}$ & 58 & 3.2 & I 2185 & 56.22 & +66.16 & 894 & 49.2 & \\
\hline 34 & $\beta \mathrm{C}$ & or & & II & 2926.84 & -0.42 & $-2 I$ & $\begin{array}{lll}51 & 40 & 3.75\end{array}$ & 9 & t I 42.0 & $5 \%$ & 7.9 & I 229 & & . . & . & . . & . \\
\hline 35 & $0 \mathrm{~V}$ & $\operatorname{ir}$ & & 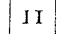 & $\begin{array}{ll}5 & 5.3 \mathrm{I}\end{array}$ & $\ldots-36$ & $-2 \mathrm{I} .87$ & $4350 \quad 3.20$ & 46.242 & $+\quad 53 . \mathrm{I}$ & 58 & 3.9 & 134 & & . & & & \\
\hline 36 & $\alpha U$ & Minor & ris s. 1 . & 5 & 2I 29.32 & +2.44 & {$[-\mathrm{I} 8.84]$} & $\begin{array}{lll}307 & 38 & 4.82\end{array}$ & 45. & - I I I 11.3 & {$\left[5^{8}\right.$} & 3.77 & I 21 & & . & . & . . & . . \\
\hline 37 & $\delta \mathrm{S}$ & co & . . . & II & 5444.28 & -0.42 & $-2 \mathrm{~L} .88$ & 61 IO 3.42 & 44.089 & + I 4 I. 4 & 57 & $7.8^{-1}$ & I5 54 & & . & . & & \\
\hline 38 & & S C, C & $\therefore .$. & II & $5^{6} 46.65$ & $-0.4 \mathrm{I}$ & -21 & $\begin{array}{lll}59 & 4 & 2.39\end{array}$ & 46.746 & t I 33.3 & 58 & 3.2 & 15562 & $24 \cdot 39$ & 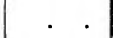 & $-20 \mathrm{I} 44$ & 48.4 & . \\
\hline 39 & $\beta^{\mathrm{x}} \mathrm{S}$ & : & . . . & I & 5956.49 & $-0.4 \mathrm{I}$ & -21.93 & $\begin{array}{lll}5^{8} & 22 & 0.05\end{array}$ & 43.846 & $+\mathrm{I} 30.7$ & $5^{8}$ & 3.4 & I5 59 & & . & r & . . & \\
\hline 40 & $\delta 0$ & chi & . . & It & & -0.36 & $2 \mathrm{I} .8 \mathrm{I}$ & $\begin{array}{lll}42 & 16 & 1.58\end{array}$ & 46.765 & $\mid-50.9$ & 57 & 7.9 & 169 & & & . & & \\
\hline $4 \mathrm{I}$ & $\alpha \mathrm{s}$ & 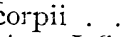 & & II & $23 \quad 35.46$ & -0.44 & -21 & $\begin{array}{lll}65 & 2 & 3.65\end{array}$ & 44.364 & † I 59.9 & 57 & & I6 23 & & & . . & 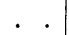 & \\
\hline 42 & & I, S. & $\therefore$ & 6 & 3030.08 & $-0.4 \mathrm{I}$ & -21 & $\begin{array}{lll}5 \mathrm{~S} & 44 & 2.20\end{array}$ & 46.098 & $\left|\begin{array}{ll}-1 & 32.3\end{array}\right|$ & 58 & 3.2 & 1630 & 7.82 & +0.72 & - 19543 & 34.8 & \\
\hline 43 & $\mathbf{S}$ & $\begin{array}{l}\text { Aturn II, N } \\
\text { May 3o, I }\end{array}$ & $\begin{array}{l}\text { s. . . } \\
\text { ata. }\end{array}$ & 5 & $3^{\circ} 31.52$ & $-0.4 \mathrm{I}$ & -21.85 & $\begin{array}{lll}58 & 44 & 2.20\end{array}$ & 44.982 & $+\mathrm{r} 32.2$ & 58 & & I6 30 & 9.26 & -0.72 & $\begin{array}{r}9 \\
-\end{array}$ & I3.4 & \\
\hline 44 & $\beta \mathrm{A}$ & ndromedx & & Ir & $\begin{array}{ll}4 & 24.49\end{array}$ & -0.27 & -21.74 & $\begin{array}{lll}3 & 46 & 3.3^{8}\end{array}$ & 46.146 & & 57 & & I 4 & & & . & & \\
\hline & & & ris . & II & $2 \times 3$ & -2.82 & {$[-18.8 \mathrm{I}]$} & $\begin{array}{lll}310 & 6 & 2.20\end{array}$ & 46.846 & - I 6.8 & {$[56$} & 5.4] & I $2 \mathrm{I}$ & & & . & & \\
\hline 16 & & is & & II & 4923.67 & -0.29 & -21.87 & I8 $32 \quad 4.22$ & 45.791 & $+\quad I 9.0$ & 56 & & I 49. & . & . & - . & $\cdot \cdot$ & . \\
\hline & me. & Barom. & Att. & $\begin{array}{l}\text { Ex. } \\
\text { Ther }\end{array}$ & Telescot & pe micro & $\begin{array}{l}\text { meter } \\
I I, \text { c.rc }\end{array}$ & ions are & $a$ & & No. & Para & rallax. & Semi- & -diamı. & $\begin{array}{l}\text { Corr. for } \\
\text { Def. I11. }\end{array}$ & & Sum. \\
\hline $\mathrm{d}$ & & in & & & & & & & & & & & & I &.$\prime \prime$ & 11 & & \\
\hline & $\begin{array}{rl}16 & 35 \\
1 & 10\end{array}$ & & & & 3 & 43. & & & & & & & & - & 9.7 & & & 8.8 \\
\hline & 26 & & & & & 2. $\quad \mathrm{Bi}$ & & vil. & & & 4 & & 0.9 & + & 9.7 & & & Io. 6 \\
\hline 28 & & & & & 6,45 & Bise & c & $C_{1}, C_{2}, C_{3} . C_{4}$. & & & 9 & & 4.8 & & & 0.8 & + & 4.0 \\
\hline 28 & $\begin{array}{ll}4 & 23 \\
6 & 0\end{array}$ & & & & & IBise & & II, VII. & & & II & & 2.5 & $-I_{5}$ & 547. & . & & 544.6 \\
\hline & 742 & & & & I I , 20. & $\mathrm{Bi}$ & & & & & & & 2.6 & -15 & $547 . \mathrm{I}$ & & + & 49.7 \\
\hline & 954 & & & & & Bis: & & VII. & & & 16 & & I. 4 & & & 0.0 & & I. 4 \\
\hline & $\begin{array}{ll}11 & 25 \\
13 & 36\end{array}$ & & & & I6. & Bisc & & v1. & & & 24 & & 19.6 & -15 & 519.6 & • & +1 & 0.0 \\
\hline & $\begin{array}{ll}3 & 30 \\
15 & 26\end{array}$ & & & & 24,33 . & Bis & & & & & 29 & 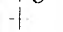 & 0.9 & - & IO.ก & . & & 9. I \\
\hline & & & 69 & & 27. & IBise & . & $\mathrm{C}_{\mathrm{I}}, \mathrm{B}_{3}, \mathrm{~B}_{2}$ & & & 30 & + & 0.9 & t- & I0.0 & & & 10.9 \\
\hline 30 & $\begin{array}{ll}12 & 5 \\
12 & 35 \\
3\end{array}$ & $\begin{array}{l}29.6018 \\
29,620\end{array}$ & 74. & 72.4 & 36. & Bise & ections at & $\mathrm{D}_{2}, \mathrm{D}_{1}, \mathrm{C}_{5}, \mathrm{C}_{4}$ & & & 33 & & 216.0 & -15 & 549.5 & . & +2 & 2625.5 \\
\hline & 13 il & & & & & & & & & & 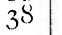 & & $0.2 .25-3$ & & & & & 0.4 \\
\hline & & & & & & & & & & & 42 & + & o. & + & 10.7 & . & + & I1.6 \\
\hline & 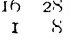 & 29.800 & & 61.3 3 & & & & & & & 43 & & 0.9 & - & J0.7 & & - & 9.8 \\
\hline
\end{tabular}




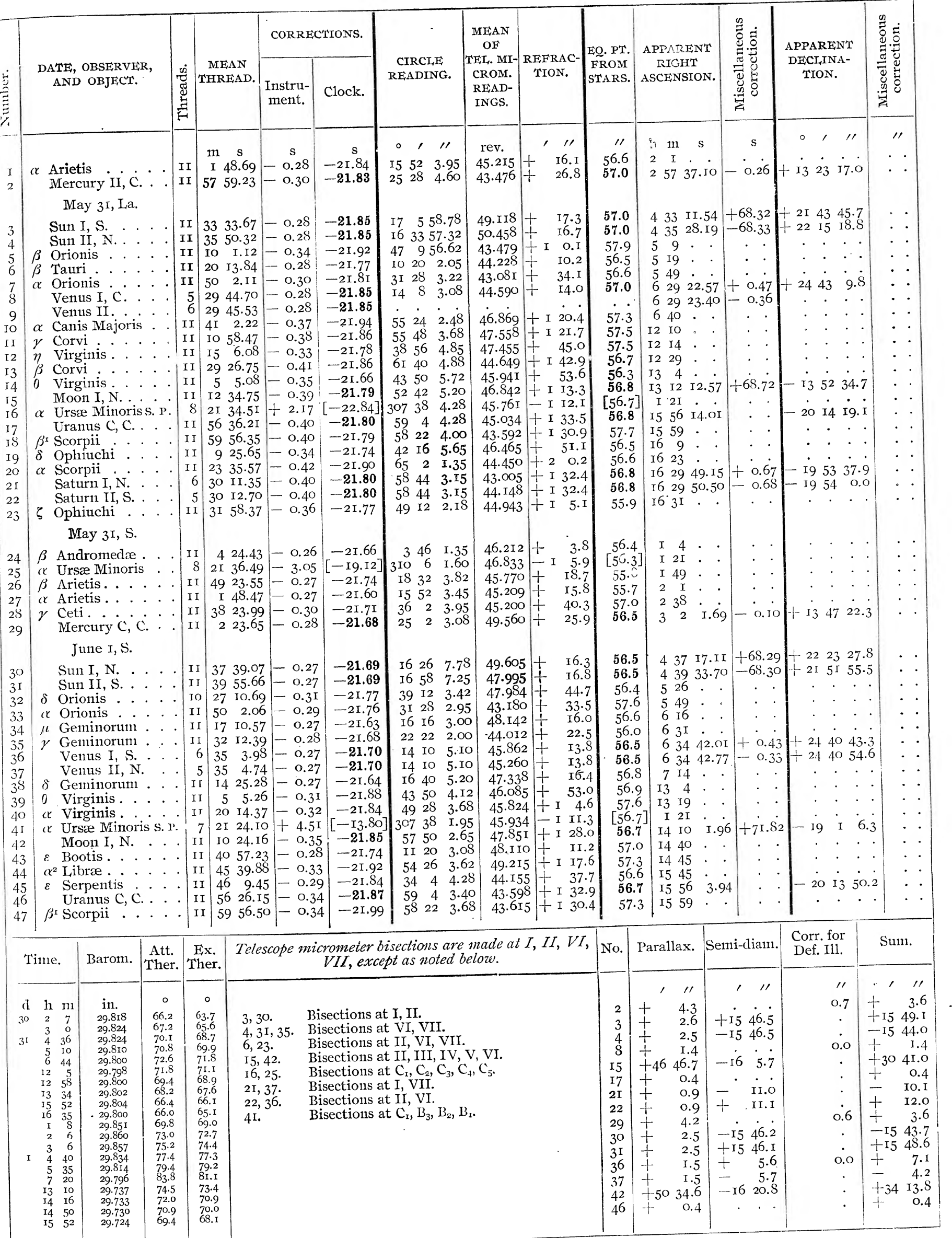




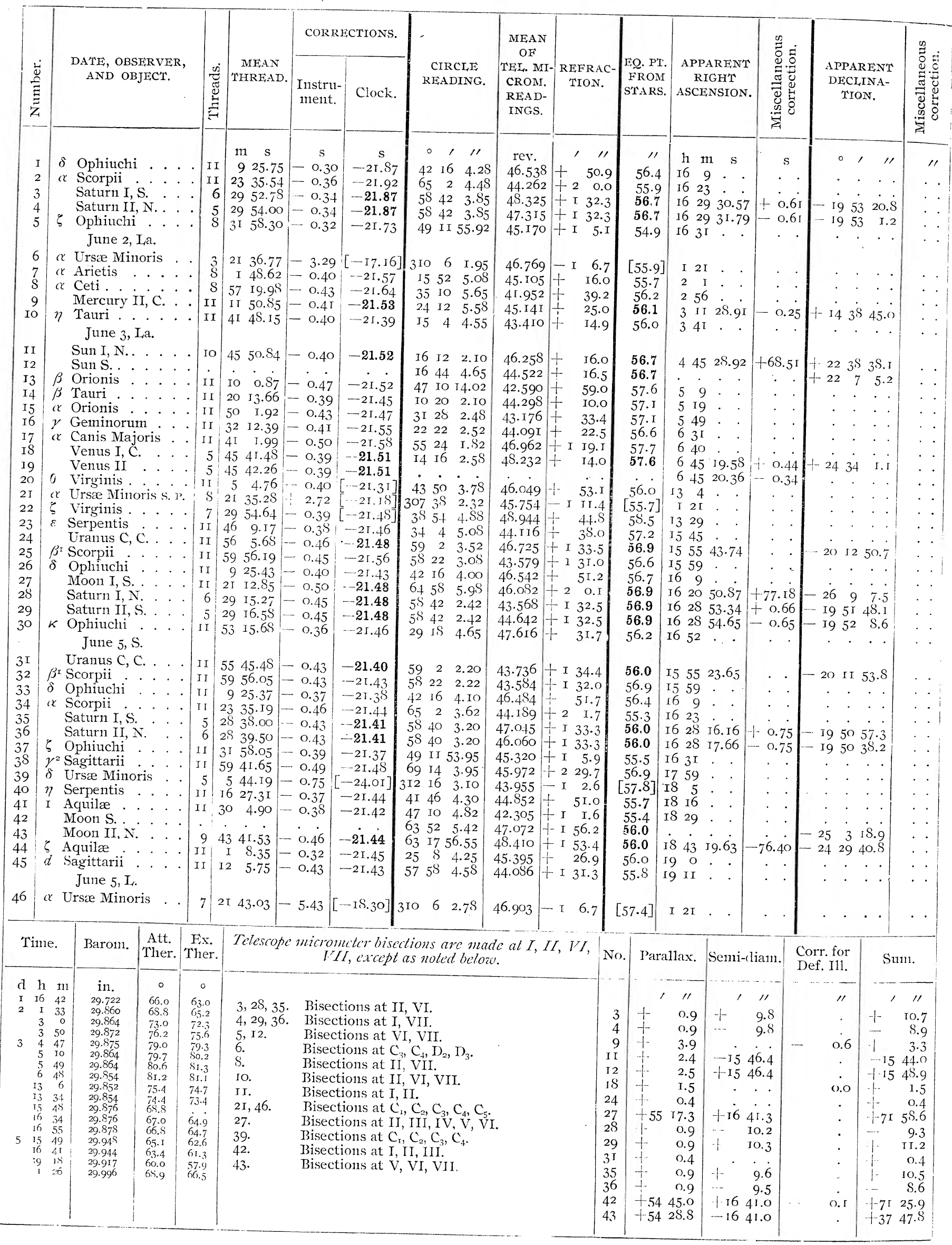




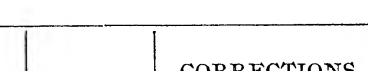

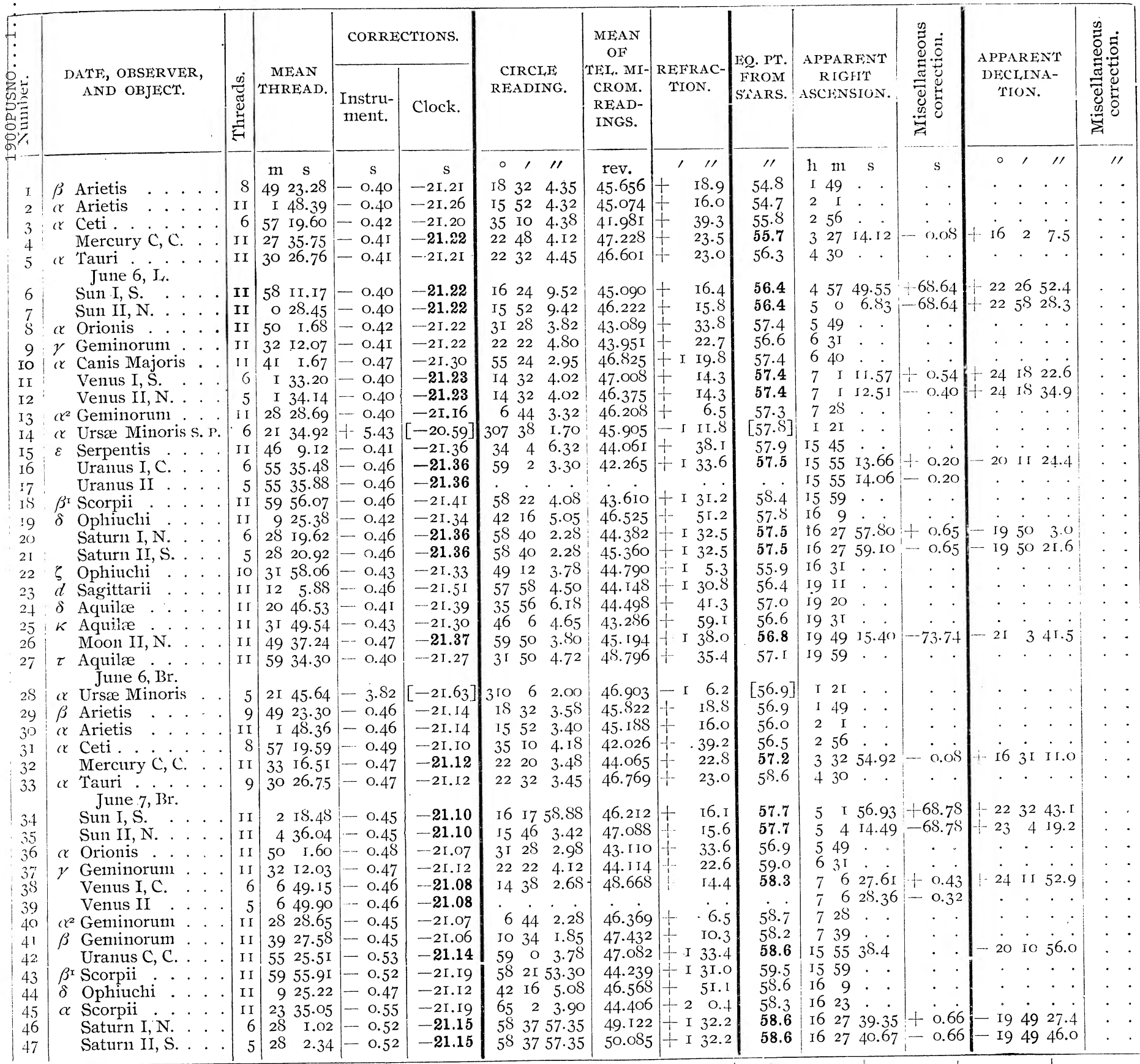




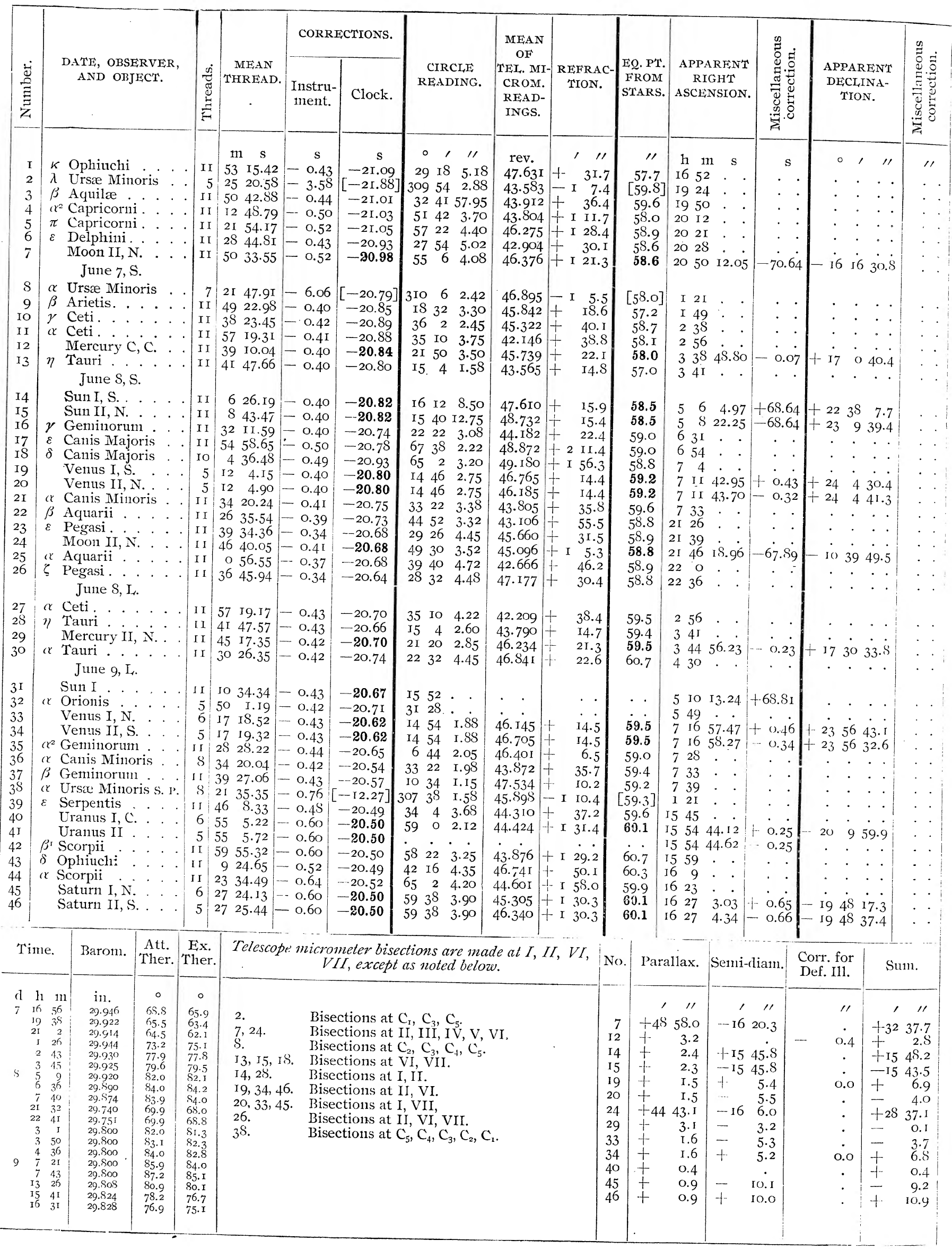




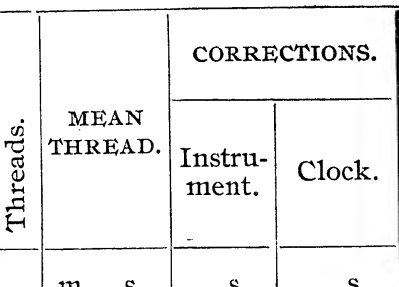

$\pi$ Aquarii . . . . . II 20 \begin{tabular}{lll|l}
27.69 & -0.50 & -20.45
\end{tabular}

$\eta$ Aquarii ..... . II 30 30.49

Moon II, N. . . . II $3^{8} 5^{8.5}$ I $-0.53-\mathbf{2 0 . 4 2}$

$\lambda$ Aquarii . . . . II 47 4I.I5 -0.54 - 20.40

$\alpha$ Pegasi ..

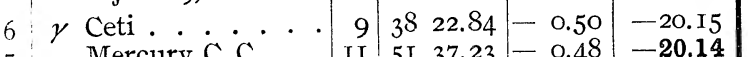

$$
\text { June io, B. }
$$

Sun I, N. . . . . . II I4 $42.05-0.47-\mathbf{2 0 . 1 4}$

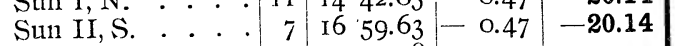

$\mu$ Geminorumi. . . IO I7 9.28 - 0.47 - 20.09

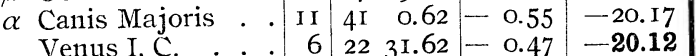

\begin{tabular}{llll|l|ll|l|l|} 
Venus I, C. &. &. &. & 6 & 22 & 31.62 & -0.47 & $-\mathbf{2 0 . 1 2}$ \\
Venus II &. &. &. & 5 & 22 & 32.44 & -0.47 & $\mathbf{2 0 . 1 2}$
\end{tabular}

$\alpha$ Canis Minoris . . I I 34 I9.67-0.49-20. Io

$\beta$ Geminorum . . . $\quad$ I $13926.67-0.47-20.14$

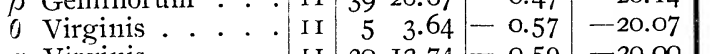

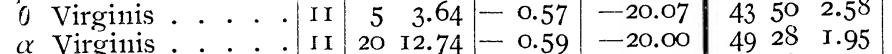

$\alpha$ Ursæ Minoris s. P. 6 2I 35.70 + 2.3I [-14.85] $3073^{8}$ r.55

$\zeta$ Virginis . . . . . II 29 \begin{tabular}{ll|l}
53.25 & -0.55 & -19.97 \\
$3^{8}$ & 53 & 56.55
\end{tabular}

$a$ Serpentis . . . . . I I $3938.55-0.53-19.95 \quad 3263.42$

$\varepsilon$ Serpentis . . . . I I $467.82-0.54-$ I 9.92

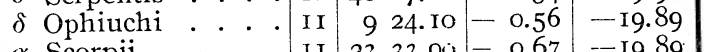

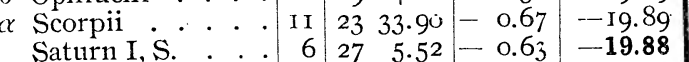

\begin{tabular}{lll|l|ll|l|l|} 
Saturn I, S. . . . . & 6 & 27 & 5.52 & -0.63 & -19.88 \\
Saturn II, N. . . . . & 5 & 27 & 6.90 & -0.63 & -19.88
\end{tabular}

$\zeta$ Ophiuchi . . . . I I 3 I $56.75-0.59-19.83$ June io, Po.

Mercury C. June I I, Po.

\begin{tabular}{llll|l|l|l|l} 
Sun I, N. . . . . & I I & IS & 50.44 & -0.44 & $\mathbf{- 2 0 . 0 5}$
\end{tabular}

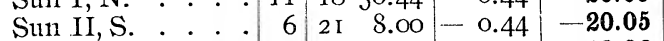

Venus I, C. . . . $62744.20-0.44$

Venus II ... . . $552744.88-0.44$

$\alpha$ Canis Minoris . . . $\begin{array}{r}5 \\ \alpha\end{array}$

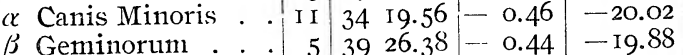

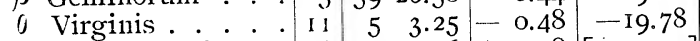

$\alpha$ Ursæ Minoris s. P. 8 2I $37.96+7.18$

$\zeta$ Virginis . . . . I I $2952.8 \mathrm{I}-0.47$

$\varepsilon$ Serpentis . . . . I I $467.37-0.46$

Uranus C, C.. . . . I I $5445.56-0.5^{2}$

$\beta^{\mathrm{r}}$ Scorpii . . . . I i $5954.49-0.52$

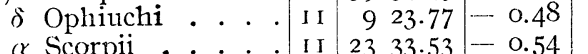

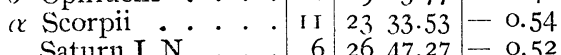

Saturn II, S. . . . . $55^{26} 48.74-0.5^{2}$

$\zeta$ Ophiuchi . . . . . I I 3 I 56.33 - 0.49

\begin{tabular}{lllll|l|rr|r}
$\omega$ & Piscium . . . . & I I & 54 & 26.65 & -0.46 \\
$c$ & Andromedac &. &. &. & I I & 3 & 28.95 & -0.46
\end{tabular} $\begin{array}{lll}34 & 4.3 .20 & 45.3 \mathrm{I} 8+ \\ 44.32 \mathrm{I}+ & 34.9\end{array}$

$\begin{array}{lll}42 \mathrm{I} 63.25 & 46.722+50.6\end{array}$

$\begin{array}{llll}65 & 2 & 3.35 & 44.559+\text { I } 59.1\end{array}$

58 38 2. IO

$\begin{array}{lll}58 & 38 & 2.10\end{array}$

$\begin{array}{lll}49 & \text { I2 } & 3.92\end{array}$

$20 \quad 20 \quad 0.95$

$-19.6 \mathrm{I}$

-19.54
-19.62

-19.74
-19.64

-19.64
-19.61

$-19.61$

-19.50
-19.62

-19.62
$-19.6 I$

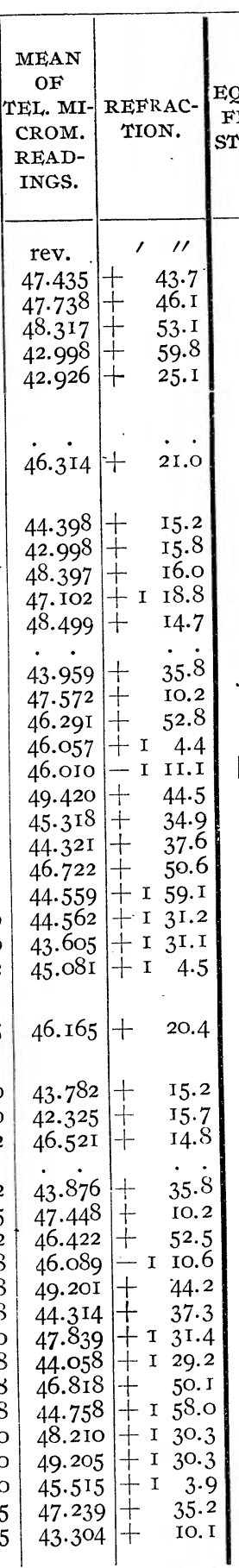

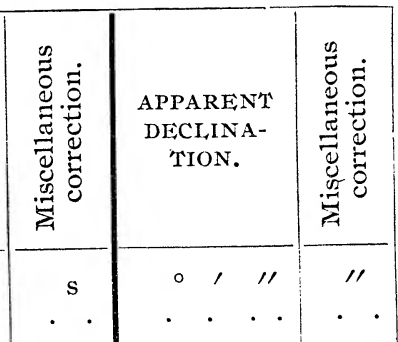

$58.0 \quad 22 \quad 30$

$\begin{array}{lllll}58.1 & 22 & 38 & 37.56 & -65.83\end{array}-44040.5$

$\begin{array}{llll}7.7 & 22 & 47 & .\end{array}$

8.0 2259 .

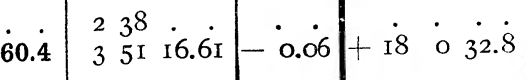

60.455 I4 $21.44+68.79+23$ I9 18.9

$\mathbf{6 0 . 4} 5$ I6 $39.02-68.79+224742.0$

$60.3 \quad 6 \quad 16$

$60.3 \quad 640$

60.4722 i $1.03+0.47+2348$ o. I

6. 722 I $1.85-0.35$

59.9

59.6 I3 4

60.0 I3 I9

[60.7] I $2 \mathrm{I}$

60.9 I3 29

58.6 I5 39

59.8 I5 45

$59.4 \quad 16 \quad 9$

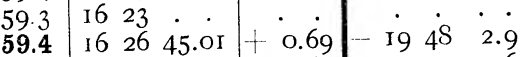

$\mathbf{5 9 . 4}$ I6 $2646.39-0.69-194744.6$

59.8 I6 $3 \mathrm{I}$

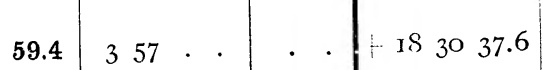

59.45 I $829.95+68.78+232327.7$

$59.4 \quad 52047.5 \mathrm{I}-68.78+225 \mathrm{I} 56.3$

$59.4+72723.80+0.39+233^{S} 39.5$

\begin{tabular}{c|ccc|c|}
.. & i & 27 & 24.48 & -0.29
\end{tabular}

$59.7 \quad 7333$

$59.2 \quad 7 \quad 39$.

60.9 I3 4

60.8 I3 29

59.9 I5 45

60.9 I5 $54 \quad 25.42$

62.2 I5 59

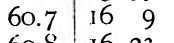

$60.8 \quad 16 \quad 23$

60.9 16 $2627.14+\dot{1} 4.0 .74-$ ig $47 \dot{9} \cdot \dot{5}$

60.9 16 $26 \begin{array}{lllllll}28.6 \mathrm{I} & -0.73 & - & -19 & 47 & 28.7\end{array}$

6 I.I $163 \mathrm{I}$

60.42354 .

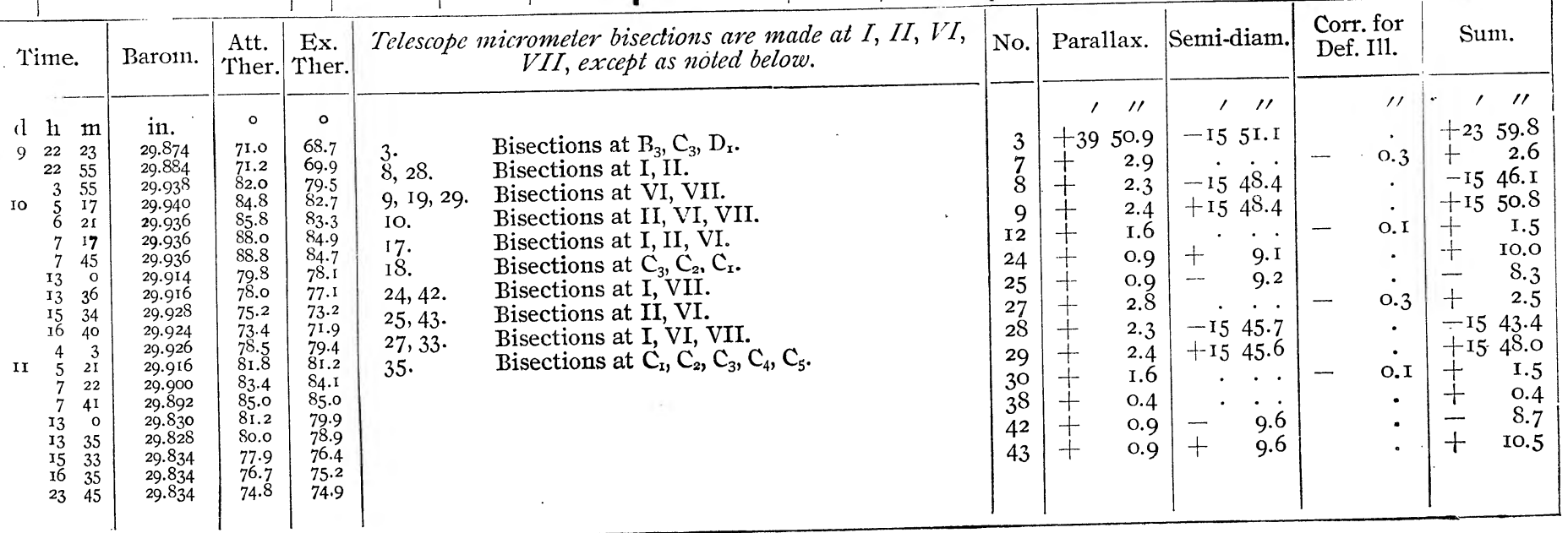




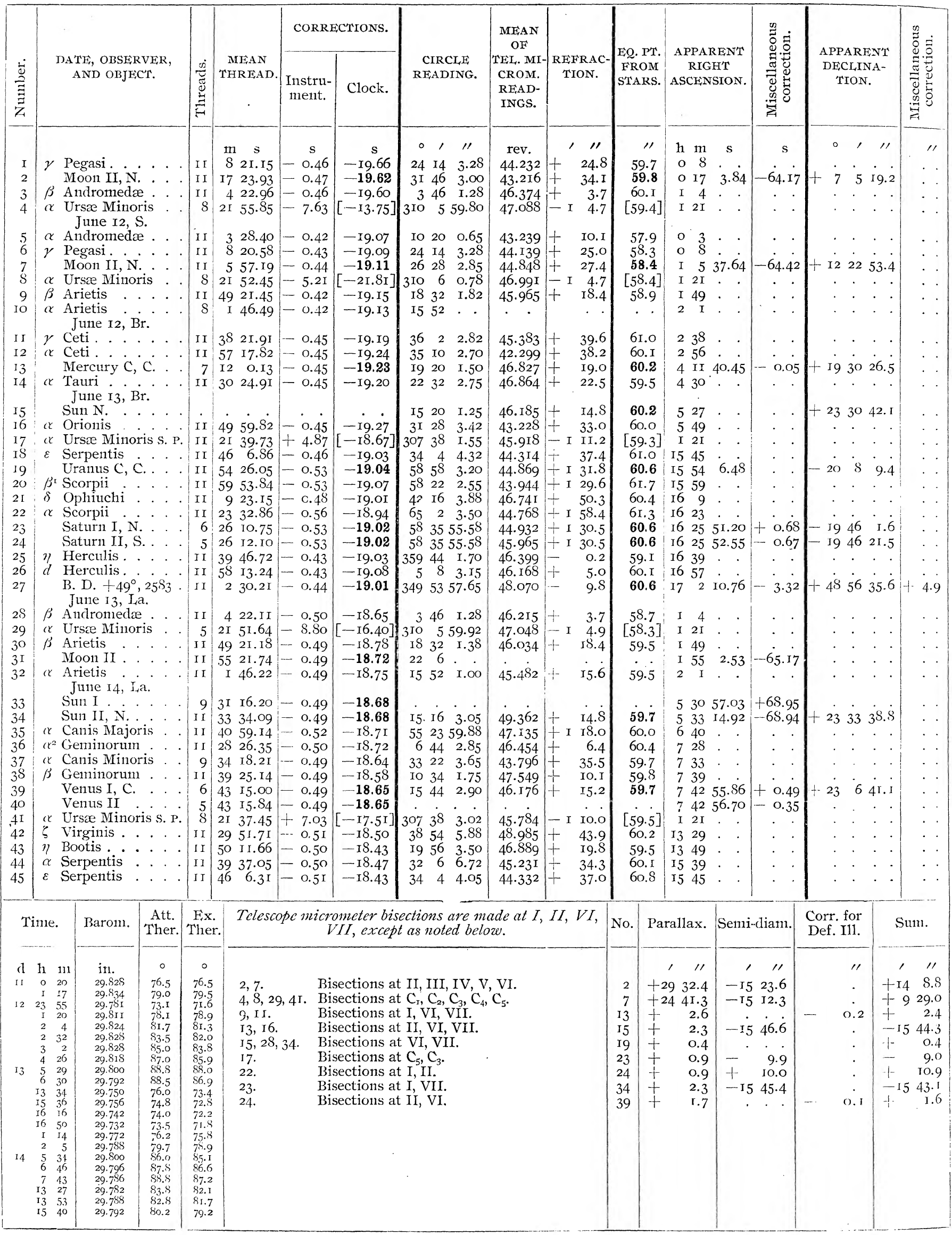




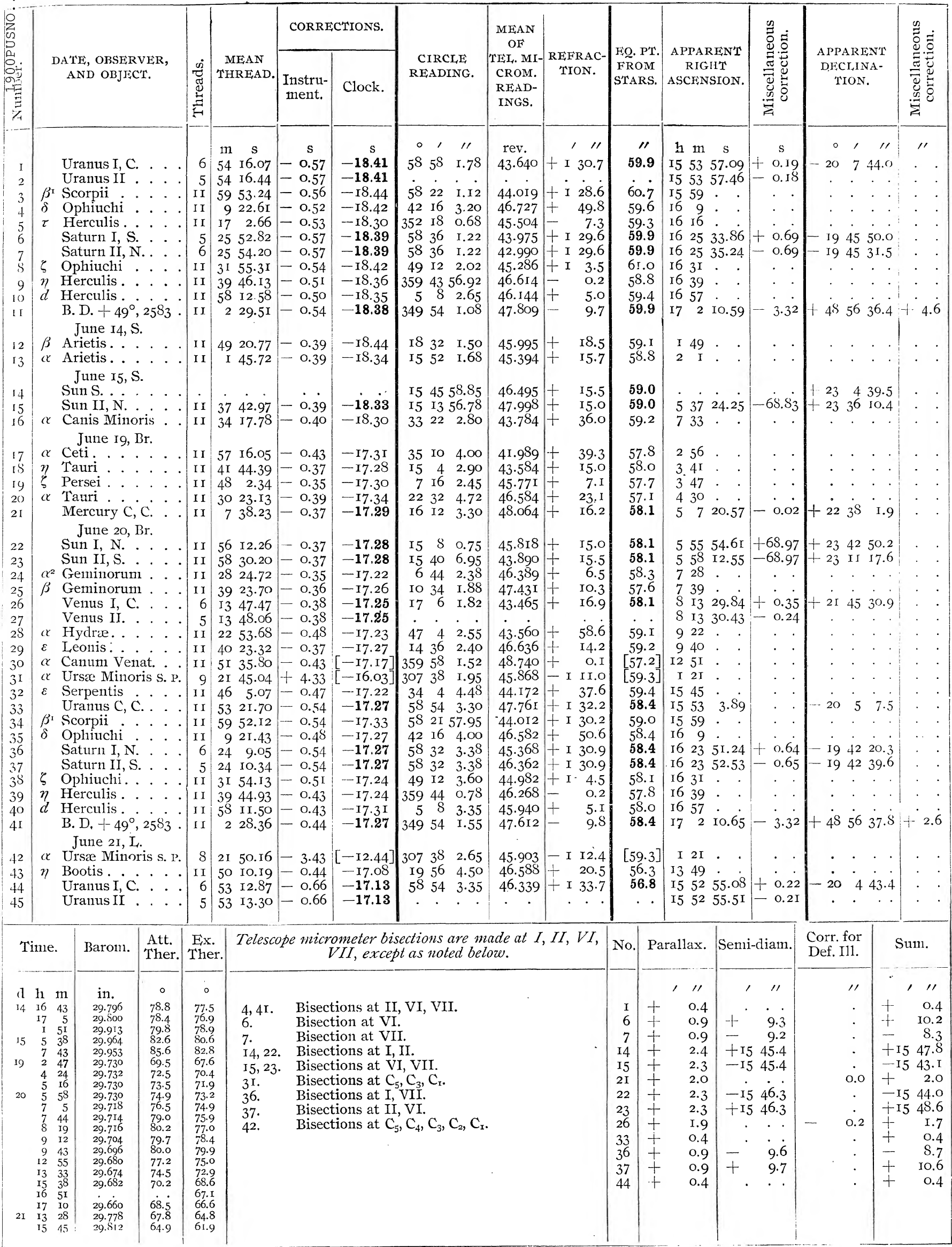




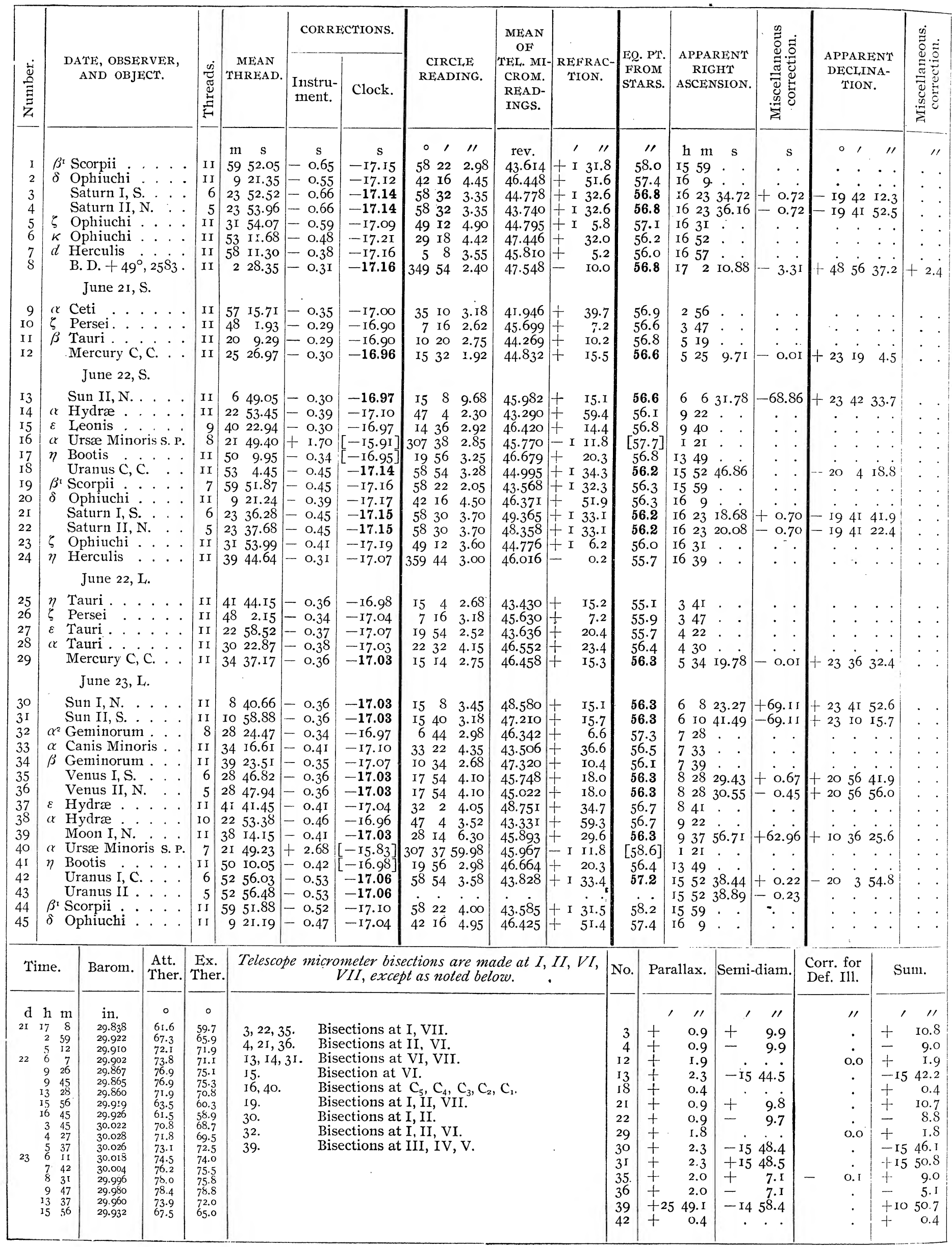




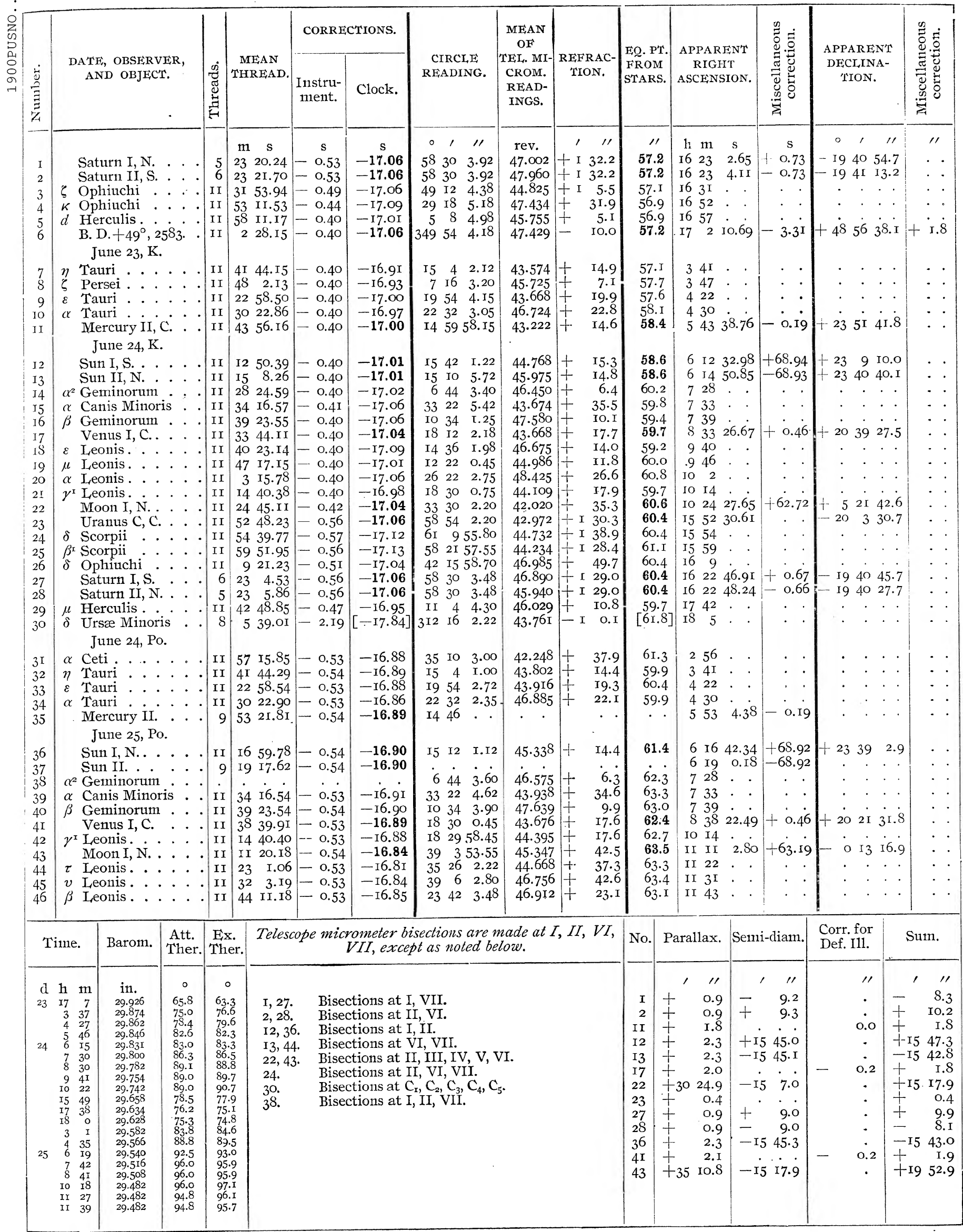




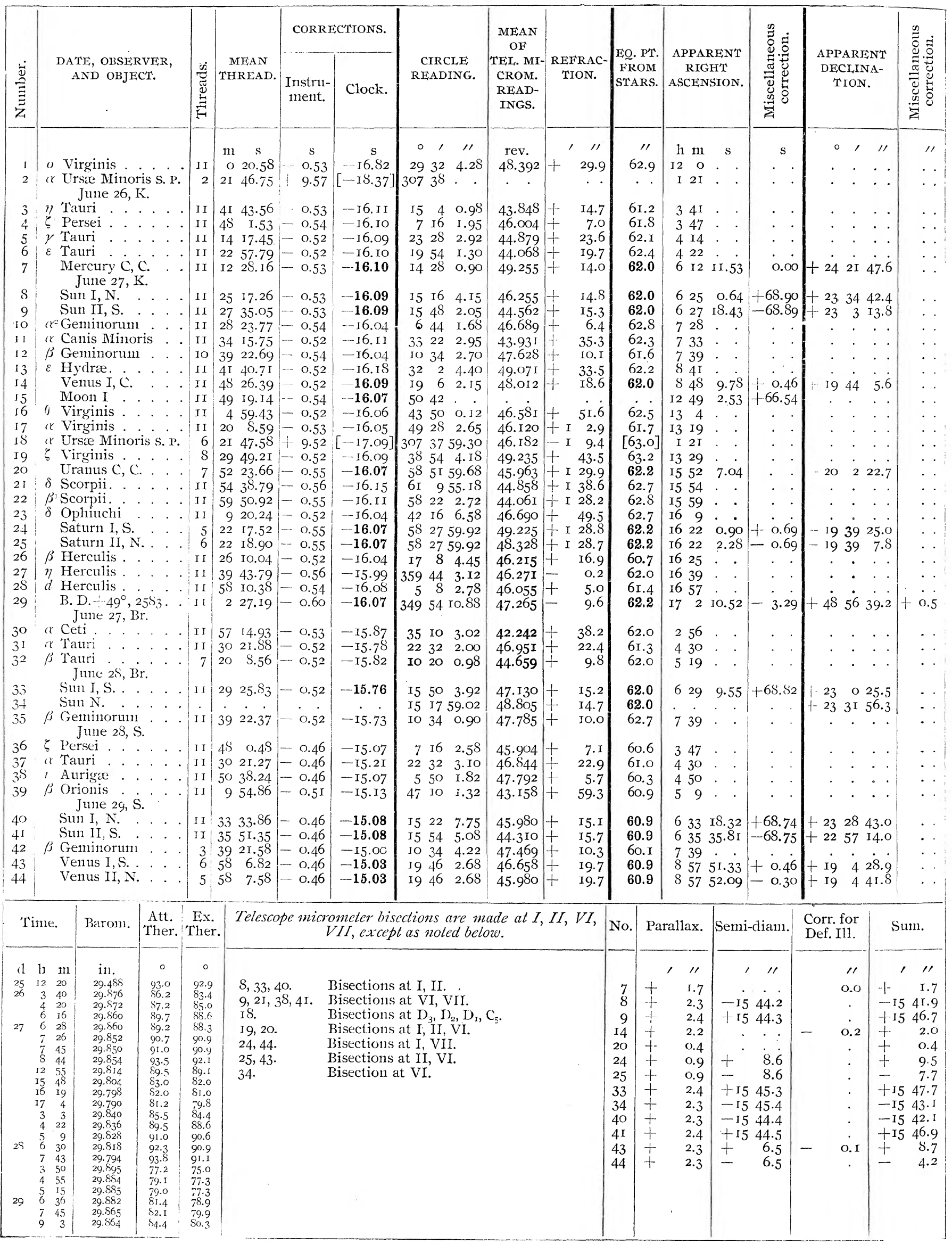




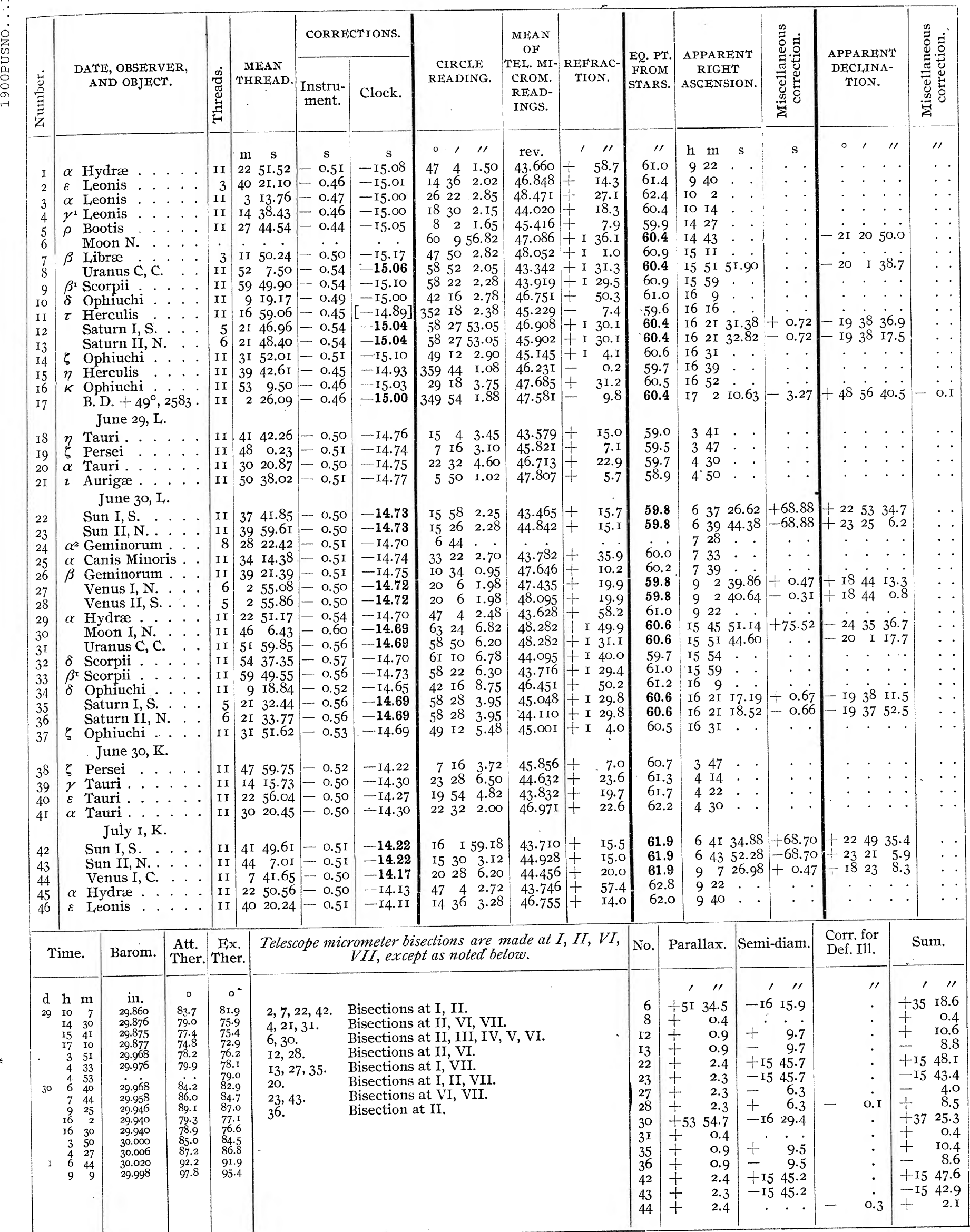

$6855-15$ 


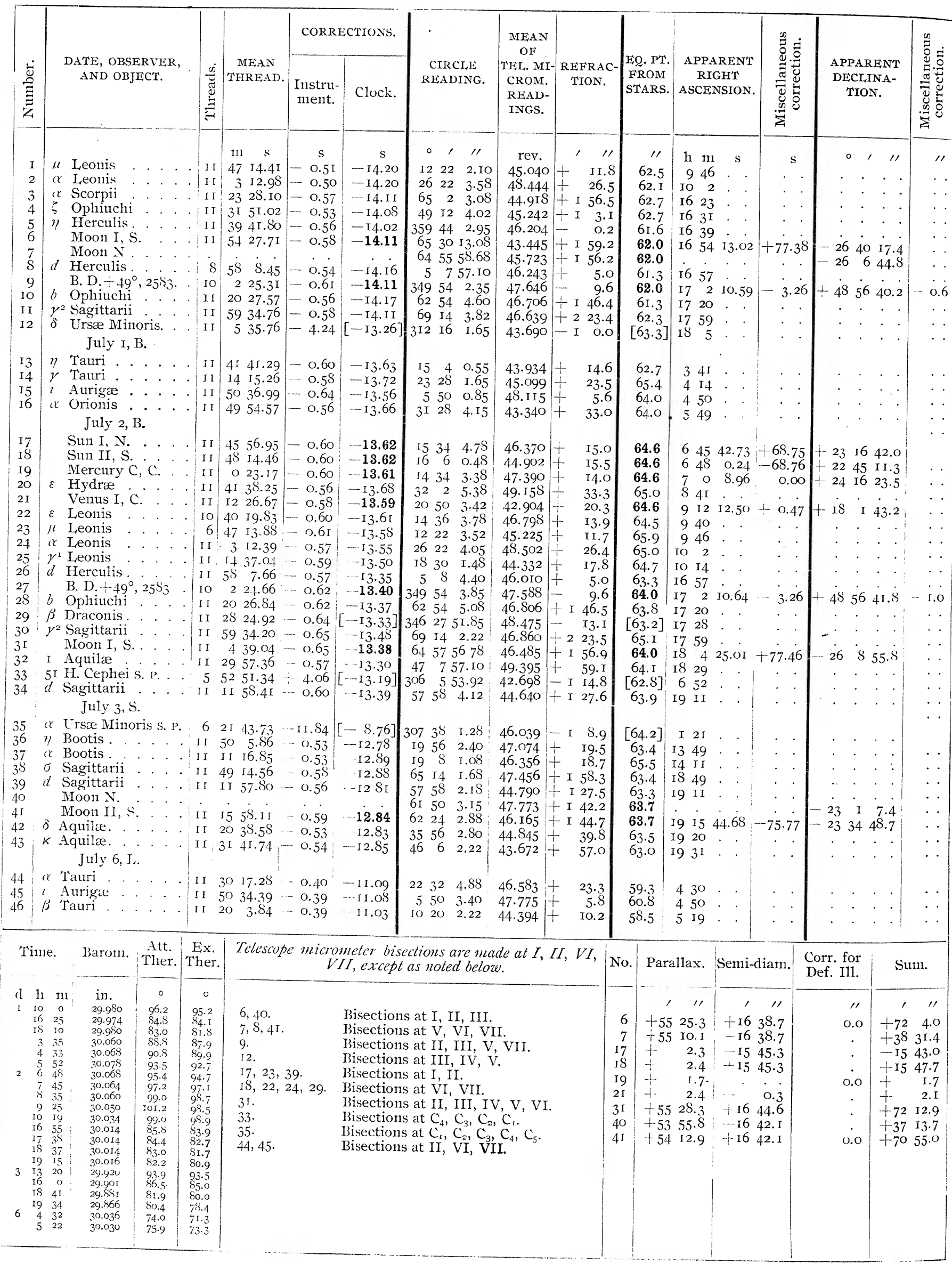




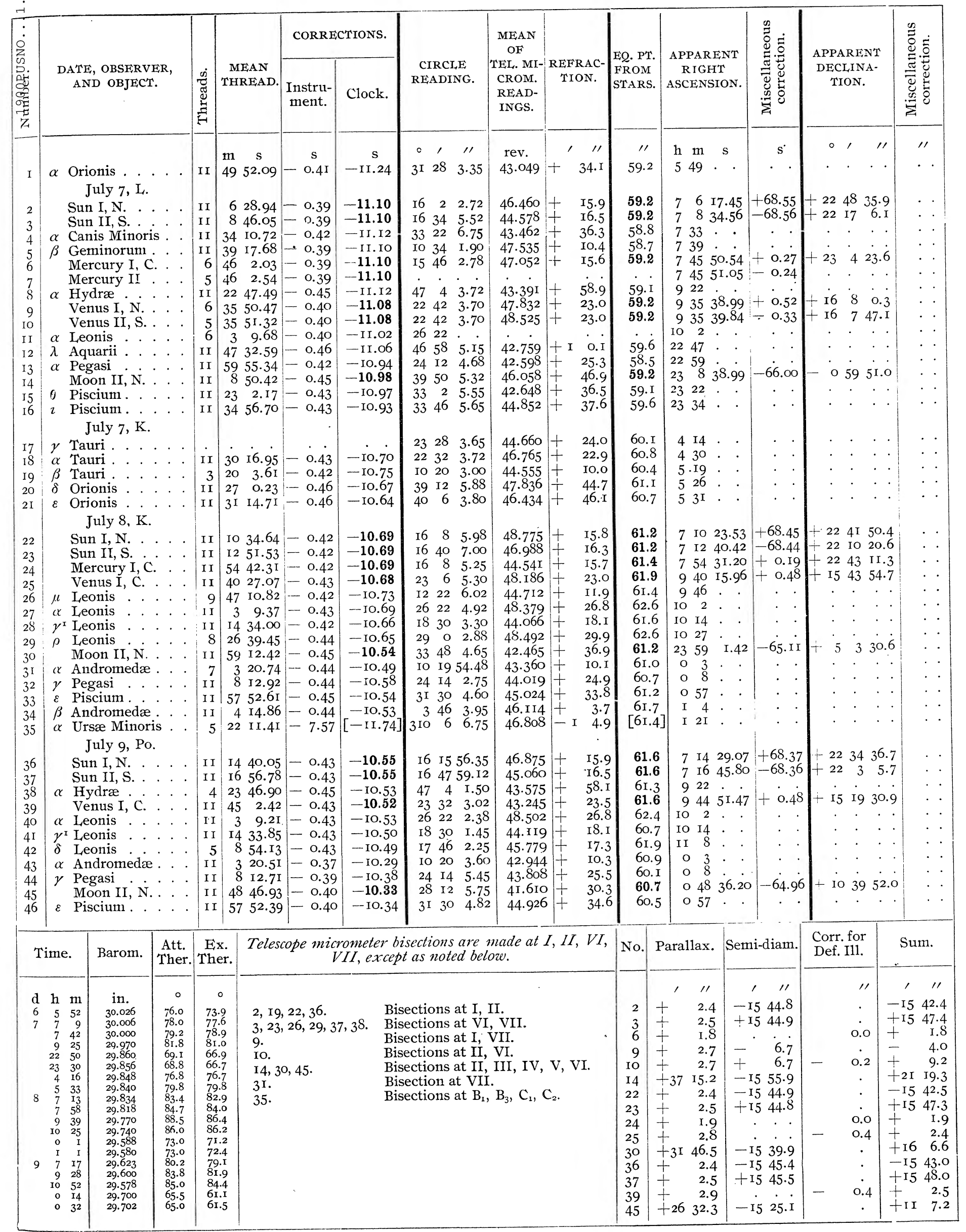




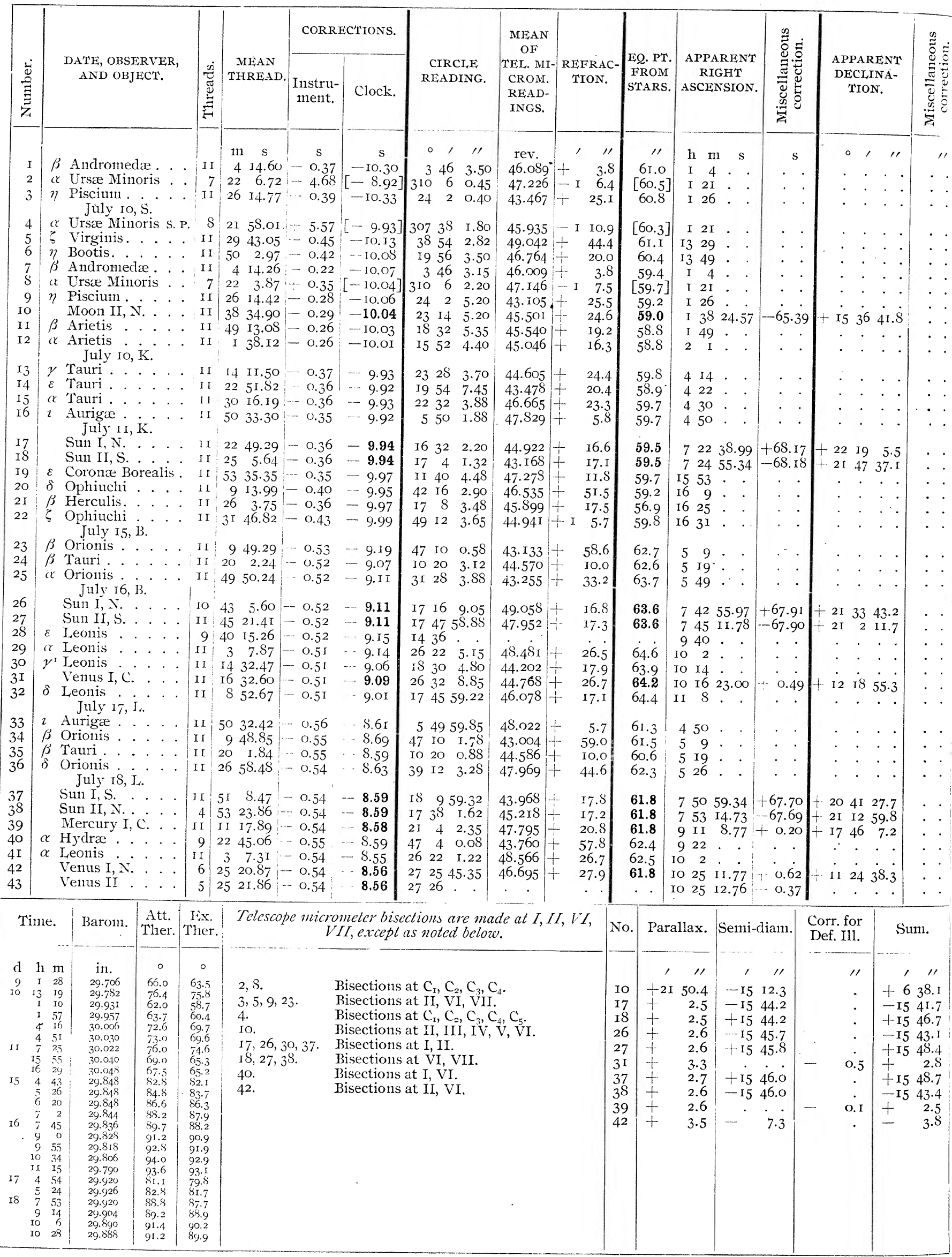




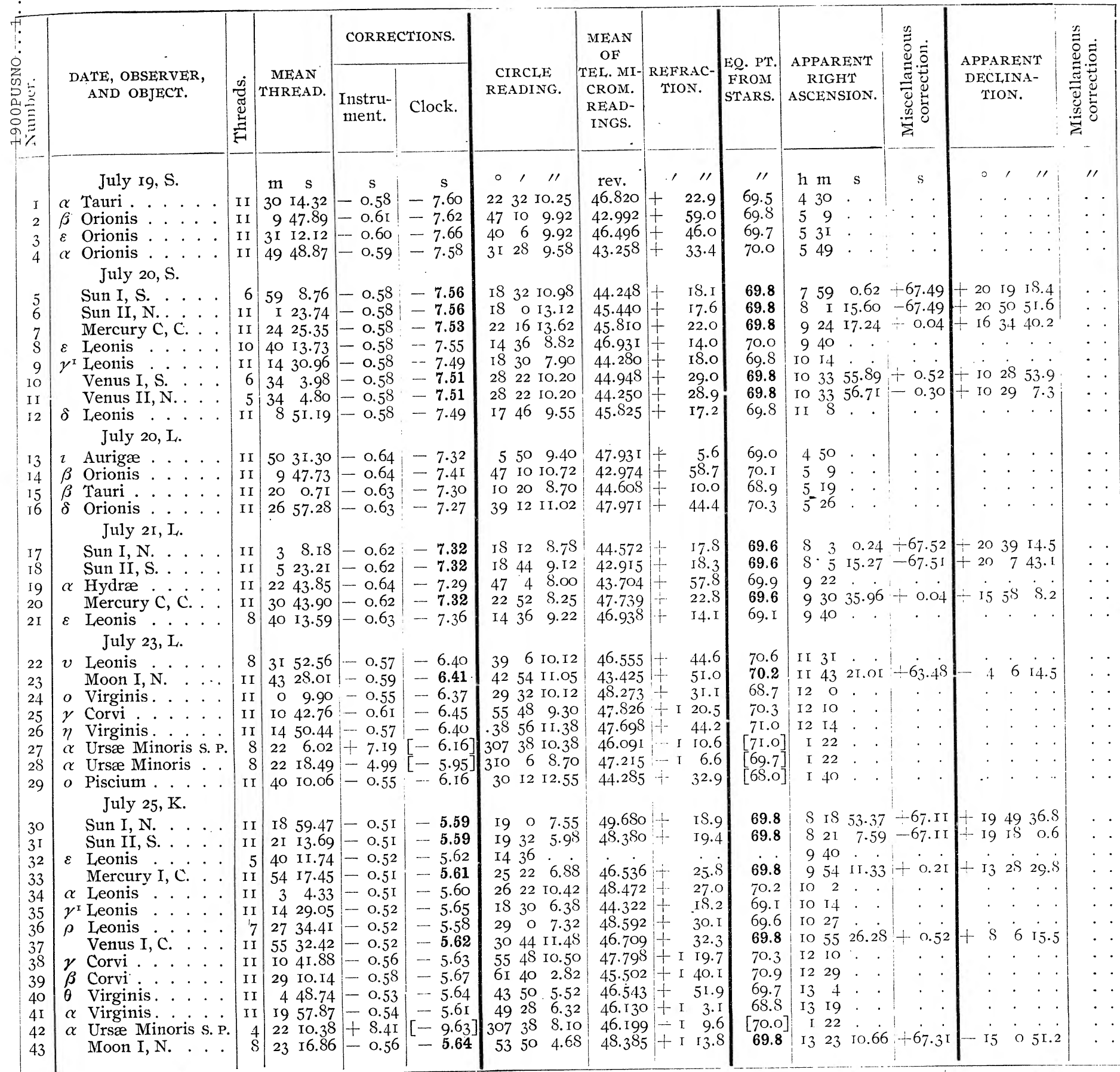

\begin{tabular}{|c|c|c|c|c|c|c|c|c|c|c|}
\hline Time. & Barom. & $\begin{array}{l}\text { Att. } \\
\text { Ther. }\end{array}$ & $\begin{array}{c}\text { Ex. } \\
\text { Ther. }\end{array}$ & $\begin{array}{r}\text { Telescope microme } \\
\text { VII, }\end{array}$ & $\begin{array}{l}r \text { bisections are made at } I, I I, I T I, \\
x c e p t \text { as noted below. }\end{array}$ & No. & Parallax. & Semi-diam. & $\begin{array}{l}\text { Corr. for } \\
\text { Def. I11. }\end{array}$ & Sum. \\
\hline 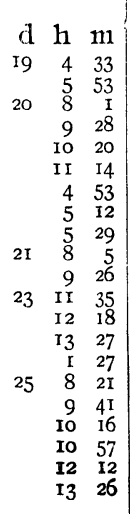 & $\begin{array}{c}\text { in. } \\
29.849 \\
29.854 \\
29.834 \\
29.831 \\
29.814 \\
29.811 \\
29.856 \\
29.862 \\
29.850 \\
29.832 \\
29.9900 \\
29.980 \\
29.976 \\
29.9998 \\
29.948 \\
29.920 \\
22.900 \\
29.886 \\
22.856 \\
29.830\end{array}$ & $\begin{array}{r}\circ \\
78.6 \\
83.8 \\
88.2 \\
91.8 \\
93.0 \\
90.8 \\
83.0 \\
\dot{8} .0 \\
88.3 \\
90.6 \\
83.2 \\
83.9 \\
82.7 \\
69.6 \\
83.5 \\
89.2 \\
85.8 \\
86.2 \\
85.0 \\
84.8\end{array}$ & $\begin{array}{c}\circ \\
77.6 \\
82.6 \\
86.8 \\
89.7 \\
90.8 \\
90.0 \\
82.2 \\
82.2 \\
83.9 \\
87.3 \\
88.1 \\
81.1 \\
81.3 \\
81.9 \\
66.9 \\
82.9 \\
84.7 \\
84.9 \\
85.3 \\
85.2 \\
86.6\end{array}$ & $\begin{array}{l}5, \mathrm{I} 7,2 \mathrm{I}, 3 \mathrm{O} . \\
6,7, \mathrm{I} 8,3 \mathrm{I}, 36,4 \mathrm{I} . \\
\text { IO. } \\
\text { II. } \\
23,43 . \\
24,40 . \\
27,28 . \\
42 .\end{array}$ & $\begin{array}{l}\text { Bisections at I, II. } \\
\text { Bisections at VI, VII. } \\
\text { Bisections at II, VI. } \\
\text { Bisections at I, VI. } \\
\text { Bisections at II, III, IV, V, VI. } \\
\text { Bisections at II, VI, VII. } \\
\text { Bisections at } \mathrm{C}_{5}, \mathrm{C}_{4}, \mathrm{C}_{3}, \mathrm{C}_{2}, \mathrm{C}_{\mathrm{r}} \text {. } \\
\text { Bisections at } \mathrm{D}_{3}, \mathrm{D}_{2}, \mathrm{D}_{1}, \mathrm{C}_{5} \text {. }\end{array}$ & $\begin{array}{r}5 \\
6 \\
7 \\
\text { IO } \\
11 \\
\text { I } 7 \\
\text { I8 } \\
20 \\
23 \\
30 \\
31 \\
33 \\
37 \\
43\end{array}$ & $\begin{array}{rr} & 11 \\
+ & 2.7 \\
+ & 2.7 \\
+ & 2.8 \\
+ & 3.6 \\
+ & 3.6 \\
+ & 2.7 \\
+ & 2.8 \\
+ & 2.9 \\
+ & 78 \\
+ & 7.5 \\
+ & 2.8 \\
+ & 2.9 \\
+ & 3.4 \\
+ & 4.0 \\
+ & 21.0\end{array}$ & 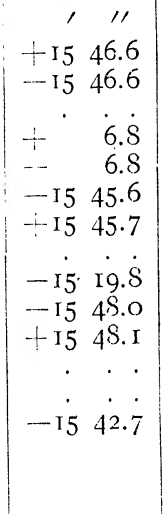 & $\begin{array}{c}-0.2 \\
-0.2 \\
\cdot \\
\cdot \\
-0.2 \\
\cdot \\
\cdot \\
\cdot \\
-0.3 \\
-0.7 \\
\cdot\end{array}$ & 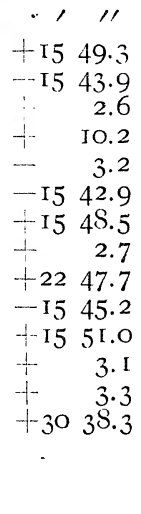 \\
\hline
\end{tabular}




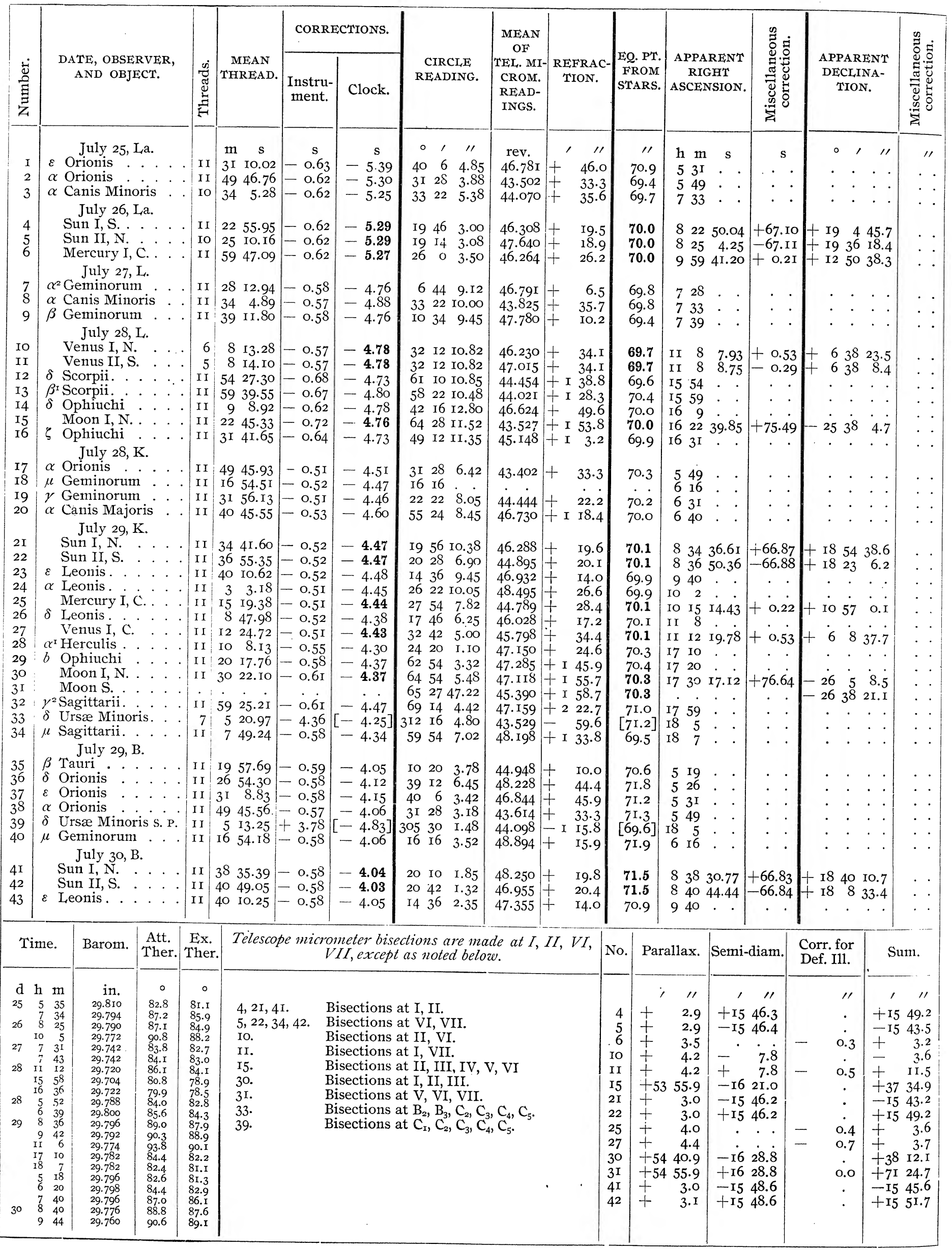




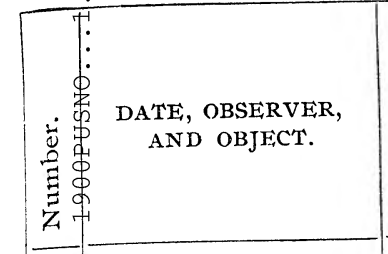

$\mu$ Leonis.

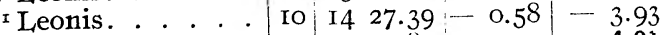

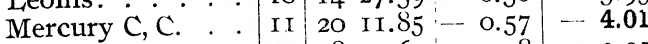

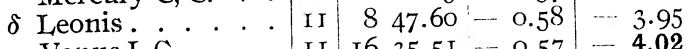

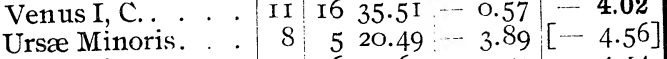

Serpent

Aquilæ

Moon I, S. .

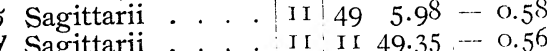
July 3I, L.

$\alpha$ Orionis...

$\gamma$ Geminorum

II 3 I $55.29 \quad-0.67$

$\alpha$ Canis Minoris

August I, L.

Sun I, S. . . . . . I I 46 21.65-0.67-3.36

Sun II, N. . . . . II $4834.75-0.67$

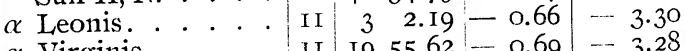

$\alpha$ Virginis . . . . II I9 55.62-0.69

\begin{tabular}{l|r|rr}
$\alpha$ Ursæ Minoris S. P. & 6 & 22 & $9.90+\mathrm{II} .42$ \\
$\alpha$ & {$[-$}
\end{tabular}

II 2 I $37.57-0.74-3.24$

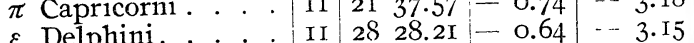

Moon I, N. . . . . II 47 4I.82 $-0.75-3.19$

Moon II

$\zeta$ Cygni.

Ursæ Minoris

\begin{tabular}{cc|c|c|c} 
August I, Br. & & & \\
$\alpha$ Orionis . . . . I I & 4944.57 & -0.56 & -3.00
\end{tabular}

$\gamma$ Geminorum $\cdots \cdot$ II 3I $^{2} 54.84-0.55 \mid-3.04$

\section{3}

34

34
35
36
37

36

38

40

$4 \mathrm{O}$

4

44 $\alpha$ Leonis.

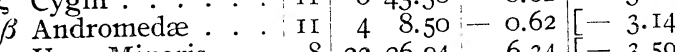

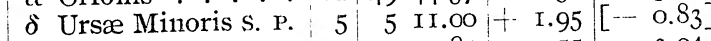

August $2, \mathrm{Br}$

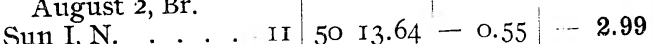

Sun II, S. . . . . . . $\quad$ II $5226.53-0.55$

$\alpha$ Leonis. . . . . . II 3 I. $77-0.55$

Mercury C. C. . . $103352.8 \mathrm{I}-0.56$

Venus I, N.

Venus S.

$\beta$ Leonis. . .

\begin{tabular}{l|l|ll|l|l} 
August 3, La. & & & & & \\
Sun I, N. . . . . & I I & 54 & 5.01 & -0.63 & -2.41
\end{tabular}

Sun II, S. . . . . . II 56 I7.78 $-0.63-2.41$

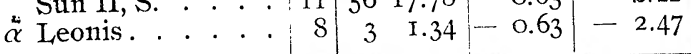

$\alpha$ Hydræ . . . . . II $22 \quad 39.57-0.60$

$\delta$ Leonis. . . . II $\$$ II $46.58-0.54$

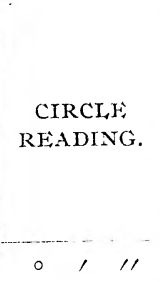

I2 $22 \quad 7.92$ $\begin{array}{lll}26 & 22 & 9.60\end{array}$

$28 \begin{array}{lll}32 & 3 & 9.55\end{array}$

I 7469.80

33 I $2 \quad 9.40$

$\begin{array}{rrr}3 \text { I } 2 & \text { I6 } & \$ .00 \\ 4 I & 46 & \text { I0. } 02\end{array}$

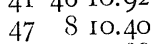

$\begin{array}{lll}63 & 55 & 57.88\end{array}$

65 It 6.55

$\begin{array}{lll}57 & 58 & 7.42\end{array}$

3I 2810.40

$22 \quad 22$ I2.I 5

$\begin{array}{llr}55 & 24 & 9.78 \\ 33 & 22 & \text { I0.45 }\end{array}$

2I I2 I 2.50

20409.38

2622 I0.52

4928 I0.20

$307 \quad 38 \quad 8.15$

5I 42 10.95

$\begin{array}{lll}57 & 22 & 9.95 \\ 27 & 54 & 19\end{array}$

$\begin{array}{rrr}27 & 54 & \text { II. } 20 \\ 55 & 6 & 2.75\end{array}$

$\begin{array}{rrr}55 & 6 & 2.75 \\ 55 & 22 & \end{array}$

$\begin{array}{lll}9 & 2 & 2.75 \\ 3 & 46 & 12.78\end{array}$

346 I 2.78

3IO $6 \begin{array}{rrr}3 & 7.65\end{array}$

$\begin{array}{rrr}31 & 28 & 10.28 \\ 305 & 30 & 8.18\end{array}$

$\begin{array}{rrrr}22 & 22 & \text { I0.30 } \\ \text { IO } & 34 & 9.55\end{array}$

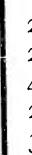

$20 \quad 56 \quad 4.22: 45.760$ +

2 I $28 \quad 4.58: 44.260$

$47 \quad 4 \quad 8.60: 43.562$

\begin{tabular}{lll|l|l|}
26 & 22 & 9.98 & 48.550
\end{tabular}

$\begin{array}{lll:l}30 & 22 & 9.25 & 47.184 \\ 17 & 46 & 9.65 & 45.992\end{array}$

\begin{tabular}{lll|l} 
I7 & 46 & 9.65 & 45.902
\end{tabular}

$34 \quad 42$ I0. 35

44.700

46.946

\begin{tabular}{lll|} 
2I & IO II.42 & 50.670 +
\end{tabular} \begin{tabular}{llll|l}
21 & 42 & 9.38 & 49.322 \\
26 & 22 & 4.62 & 48.846
\end{tabular} $\begin{array}{lllllll}34 & 42 & 10.35\end{array}$

45.572 -

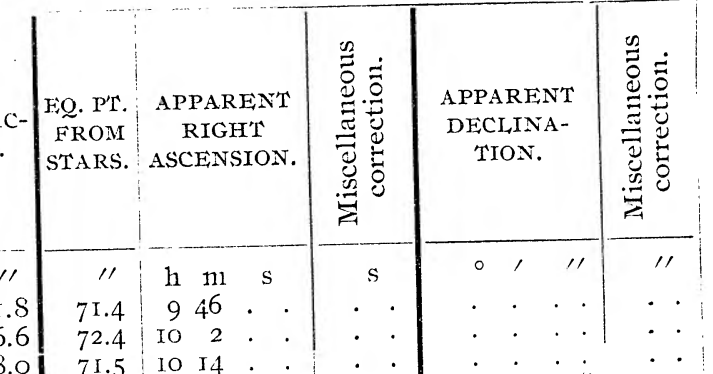

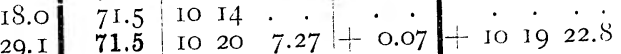

7 I. 7 II 8 .

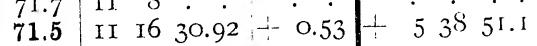

[69.6] Is 5

\begin{tabular}{l|l|l}
$7 \mathrm{I} .3$ & I8 & 16
\end{tabular}

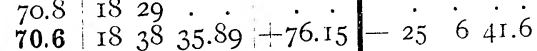

70.0 IS 49

\begin{tabular}{l|l|l}
70.4 & I9 II
\end{tabular}

\begin{tabular}{l|l|l}
69.3 & 5 & 49
\end{tabular}

\begin{tabular}{l|ll}
69.2 & 6 & $3 \mathrm{I}$ \\
70.5 & 6 & 40
\end{tabular}

\begin{tabular}{l|ll}
$70 . \mathrm{I}$ & 733
\end{tabular}

69.9 \& $46 \quad \mathrm{I} 7.62+66.55+\mathrm{I} 73840.6$

$69.9848 \quad 30.72-66.55+$ IS IO 15.9

70.5 IO 2

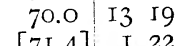

\begin{tabular}{c|cc}
71.4 & 1 & 22 \\
70.7 & $20 \mathrm{I} 2$ \\
71.1 & 20 & 21
\end{tabular}

$7 \mathrm{I} . \mathrm{I} 2 \mathrm{O} 2 \mathrm{I}$

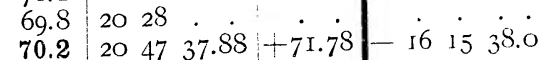

60
60

69.3218

\begin{tabular}{c|ccc}
70.2 & I & 4 \\
\hline 70.8 & 1 & 2 & 0
\end{tabular}

\begin{tabular}{l|ll}
70.5 & 5 & 49
\end{tabular}

\begin{tabular}{|c|rr}
{$\left[\begin{array}{l}70.7 \\
69.0\end{array}\right.$} & 6 & $3 \mathrm{I}$ \\
\hline 70.1 & 7 & 39
\end{tabular}

\begin{tabular}{l|l}
70.1 & 739
\end{tabular}

Telescope micrometer bisections are made at $I, I I, V I$,

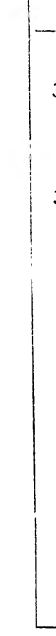

(⿸丆口
\begin{tabular}{c|c|c|} 
Ther. & Ther. & Telescope mincom \\
\hline
\end{tabular}

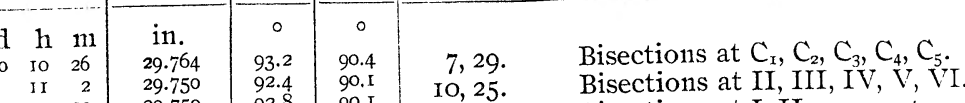

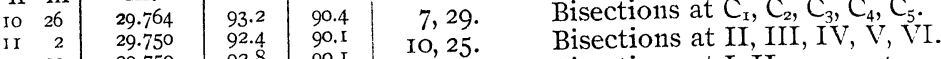

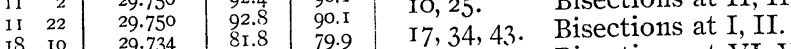

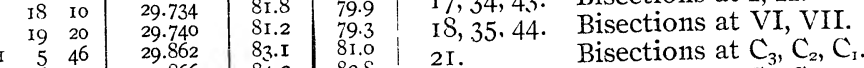

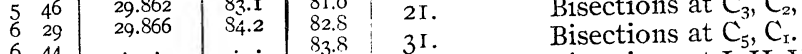

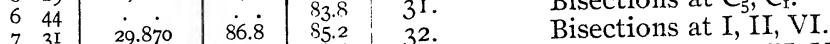

\begin{tabular}{ll|l|l|l|ll}
7 & $3 \mathrm{I}$ & 29.870 & 86.8 & 55.2 & 32. & Bisections at I, I, VI. \\
8 & 49 & 29.864 & 89.8 & 88.6 & 36. & Bisections at II, VI, VII
\end{tabular}

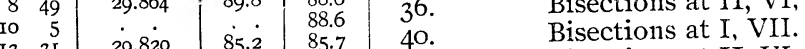

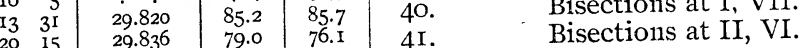

\begin{tabular}{ll|l|l|l|l}
$2 \mathrm{I}$ & 15 & 29.836 & 79.0 & 75.1 & $4 \mathrm{I}$.
\end{tabular}

\begin{tabular}{ll|l|l|l}
$\mathrm{I}$ & 27 & 29.850 & 75.4 & 72.8 \\
5 & 44 & 29.906 & $8 \mathrm{I} .5$ & 78.9
\end{tabular}

\begin{tabular}{ll|l|l|l}
5 & 44 & 29.906 & 81.5 & 78.9 \\
6 & 37 & 29.918 & 82.9 & 80.3
\end{tabular}

\begin{tabular}{ll|l|l|l|}
7 & 45 & 29.920 & 85.5 & 83.1 \\
8 & 52 & 29.914 & 86.8 & 84.7
\end{tabular}

\begin{tabular}{ll|l|l|l|}
8 & 52 & 29.914 & 86.8 & 84.7 \\
9 & 27 & 29.916 & 87.7 & 86.0
\end{tabular}

\begin{tabular}{rr|r|r|r}
9 & 27 & 29.916 & 87.7 & 86.0 \\
I0 & 38 & 29.902 & 90.6 & 87.6
\end{tabular}

\begin{tabular}{ll|l} 
II & 35 & 29.902 \\
& 29.884
\end{tabular}

II $47 \quad 29.880$

\begin{tabular}{lr|r}
10 & 5 & 29.840 \\
10 & 8 & 29.814 \\
\hline
\end{tabular}
$20.8 \quad 70.5$ S 50 ro. Io $+66.44+$ I 75454 . I

\begin{tabular}{l|l|lllllll}
$2 \mathrm{I} .4$ & 70.5 & $S$ & 52 & 22.99 & -66.45 & I 7 & 23 & I 9.8
\end{tabular}

\begin{tabular}{l|l|ll}
58.2 & 70.1 & 922
\end{tabular}

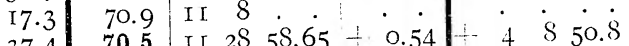

\begin{tabular}{l|l|lllllllll}
37.4 & 70.5 & I I & $2 S$ & 58.65 & 0.54 & 4 & $S$ & 50.8 \\
37.4 & 70.5 & & &. &. &. &. & 4 & $S$ & 34.1
\end{tabular}

23.7

7 I. 6 If 43 .

\begin{tabular}{llll|l|lll}
71.3 & 8 & 54 & 1.97 & +66.39 & -17 & 39 & $I_{3} .4$
\end{tabular}

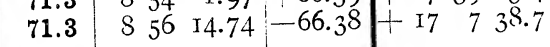

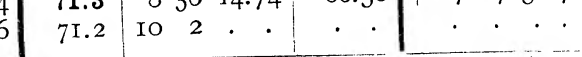

26.9 71.2 IO 2 .

$31.7 \quad 70.5$ IO $3349.30+0.07+$ \& 289.9

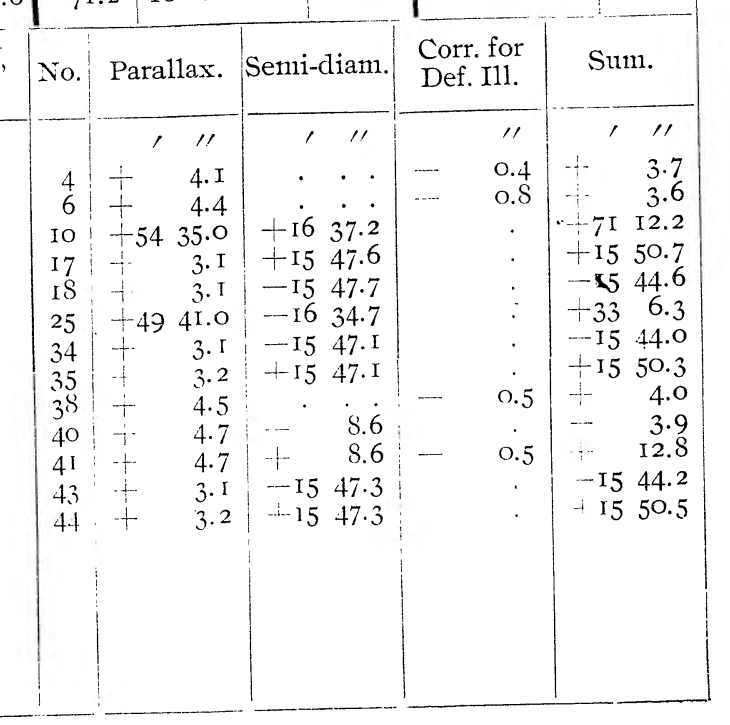




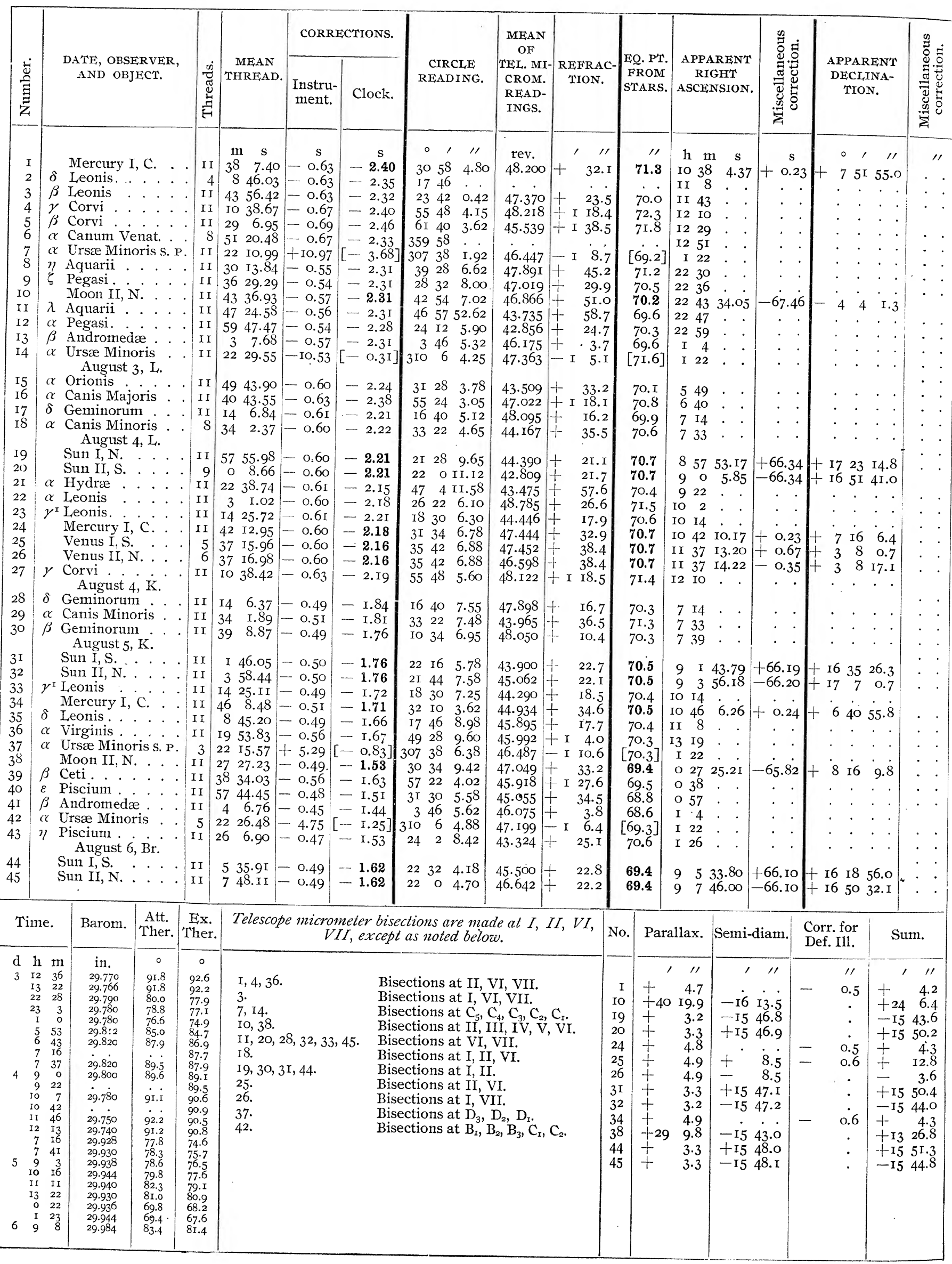




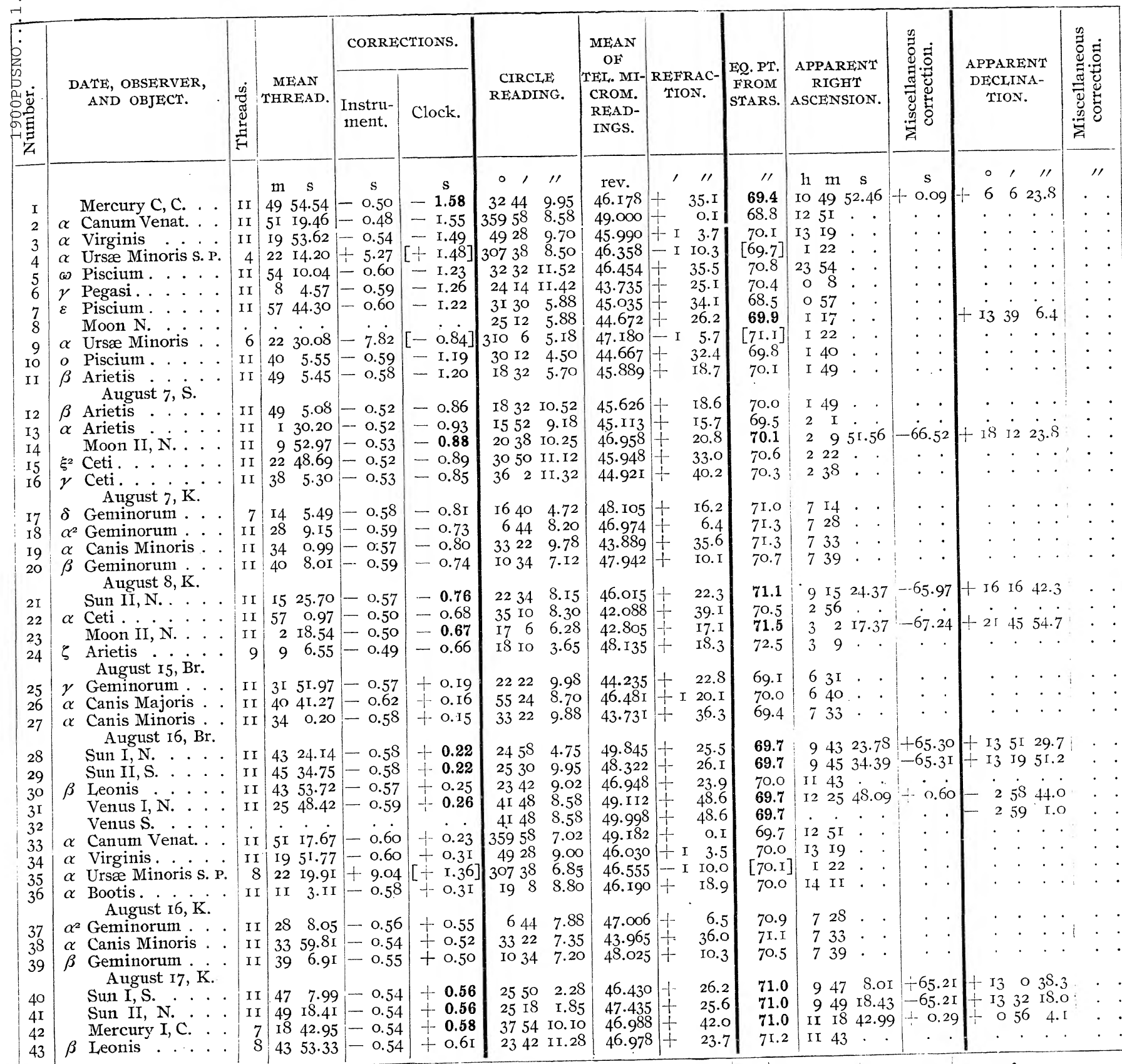

\begin{tabular}{|c|c|c|c|c|c|c|c|c|c|c|c|}
\hline Time. & Barom. & Att. & $\begin{array}{l}\text { Ex. } \\
\text { Ther. }\end{array}$ & $\begin{array}{r}\text { Telescope micro } \\
V\end{array}$ & $\begin{array}{l}\text { ter bisections are made at } I, I I, I T \text {, } \\
\text { except as noted below. }\end{array}$ & No. & Parallax. & Semi-diam. & $\begin{array}{l}\text { Cor } \\
\text { Def }\end{array}$ & & Sum. \\
\hline 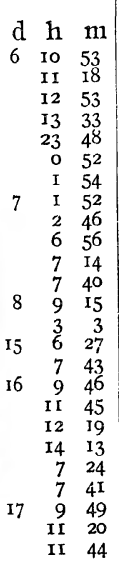 & $\begin{array}{c}\text { in. } \\
29.958 \\
29.948 \\
29.922 \\
29.910 \\
29.848 \\
29.840 \\
29.842 \\
29.778 \\
29.7783 \\
29.812 \\
29.808 \\
29.802 \\
29.810 \\
29.800 \\
29.996 \\
29.9944 \\
29.9980 \\
29.970 \\
22.960 \\
29.926 \\
29.896 \\
29.8900 \\
22.874 \\
29.854 \\
29.850\end{array}$ & $\begin{array}{c}0 \\
85.5 \\
85.5 \\
84.5 \\
83.8 \\
73.5 \\
73.0 \\
72.6 \\
76.2 \\
75.2 \\
85.2 \\
87.0 \\
88.0 \\
91.1 \\
73.8 \\
77.5 \\
81.5 \\
84.8 \\
87.0 \\
87.5 \\
86.0 \\
81.8 \\
83.0 \\
87.8 \\
88.8 \\
89.6\end{array}$ & \begin{tabular}{c|}
$\circ$ \\
83.8 \\
83.3 \\
83.7 \\
82.7 \\
71.9 \\
71.4 \\
71.2 \\
73.9 \\
73.9 \\
84.6 \\
85.6 \\
86.7 \\
89.7 \\
71.6 \\
76.5 \\
79.8 \\
83.1 \\
85.7 \\
85.2 \\
85.1 \\
80.8 \\
81.5 \\
86.1 \\
87.0 \\
87.6
\end{tabular} & $\begin{array}{l}\mathrm{I}, 3, \text { IO. } \\
4 . \\
8,14 . \\
9 . \\
\text { I3. } \\
\text { 17, 20, 21, 29, 4I. } \\
22,28,4 \mathrm{O}, 42,43 . \\
23 . \\
3 \mathrm{I} . \\
32 . \\
35 .\end{array}$ & $\begin{array}{l}\text { Bisections at II, VI, VII. } \\
\text { Bisections at } \mathrm{D}_{3}, \mathrm{D}_{2} . \\
\text { Bisections at II, III, IV, V, VI. } \\
\text { Bisections at } \mathrm{C}_{3}, \mathrm{C}_{4}, \mathrm{D}_{\mathrm{r}}, \mathrm{D}_{2} . \\
\text { Bisections at I, VI, VII. } \\
\text { Bisections at VI, VII. } \\
\text { Bisections at I, II. } \\
\text { Bisections at II, III, IV. } \\
\text { Bisections at I, VII. } \\
\text { Bisections at II, VI. } \\
\text { Bisections at } \mathrm{D}_{3}, \mathrm{D}_{2}, \mathrm{D}_{\mathbf{r}} .\end{array}$ & \begin{tabular}{|r|}
$\mathrm{I}$ \\
8 \\
$\mathrm{I} 4$ \\
$2 \mathrm{I}$ \\
23 \\
28 \\
29 \\
$3 \mathrm{I}$ \\
32 \\
$4 \mathrm{O}$ \\
$4 \mathrm{I}$ \\
42
\end{tabular} & \begin{tabular}{rr} 
& \multicolumn{1}{r}{11} \\
+ & $5 . \mathrm{I}$ \\
+23 & 58.9 \\
+19 & 32.9 \\
+ & 3.3 \\
+16 & 4.2 \\
+ & 3.7 \\
+ & 3.7 \\
+ & 6.1 \\
+ & 6.1 \\
+ & 3.8 \\
+ & 3.7 \\
+ & 7.0
\end{tabular} & 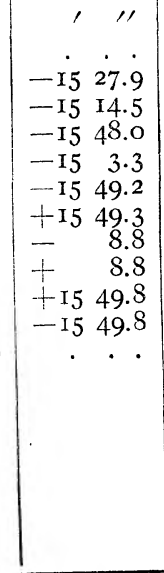 & - & $\begin{array}{c}1 \\
0.6 \\
. \\
: \\
. \\
. \\
. \\
0.6 \\
. \\
\text { I.I }\end{array}$ & 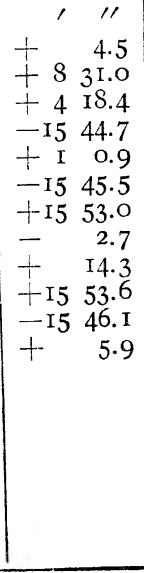 \\
\hline
\end{tabular}




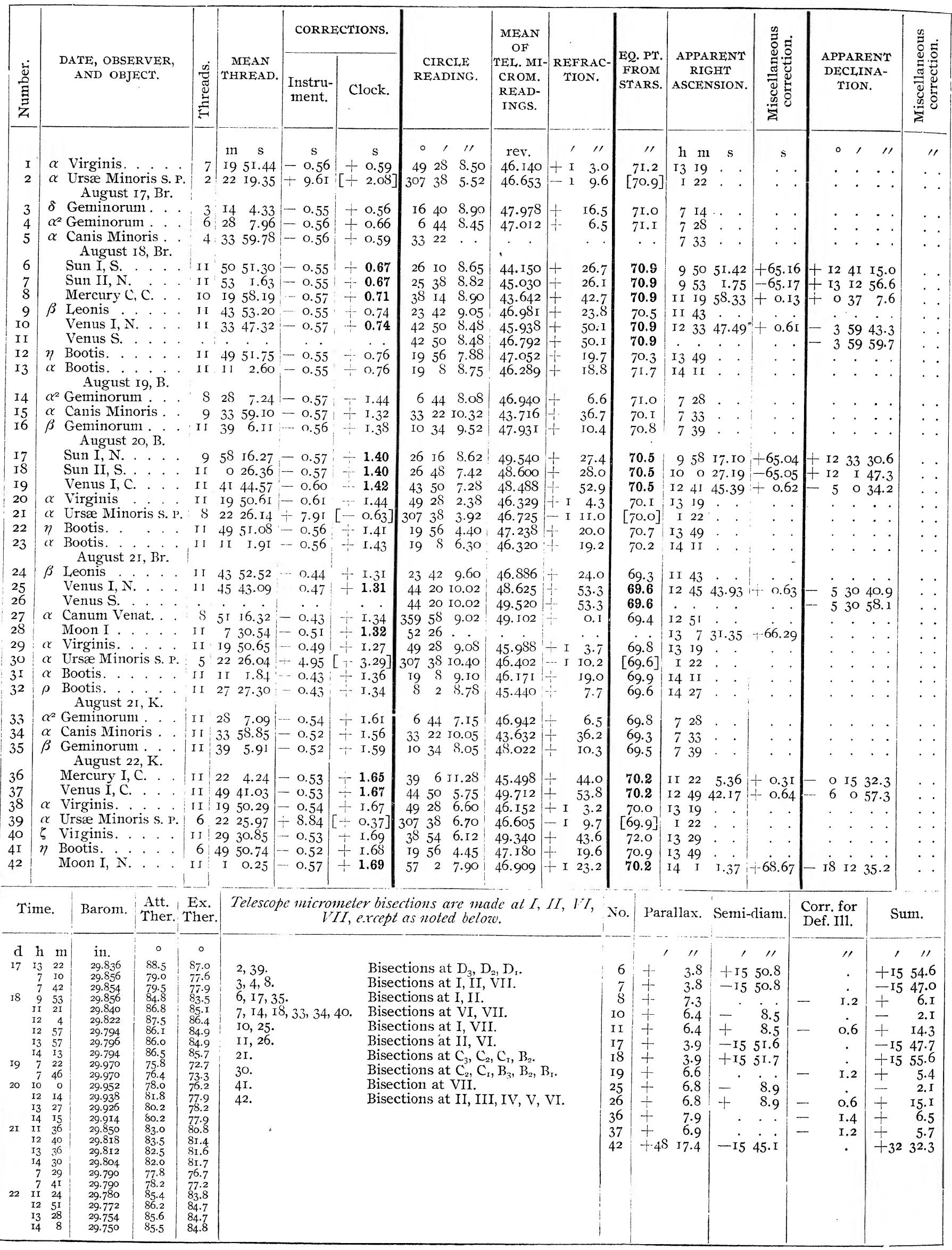




\begin{tabular}{|c|c|c|c|c|c|c|c|c|c|c|c|c|c|c|c|c|c|c|}
\hline \multirow[b]{2}{*}{ 蒿 } & \multirow{2}{*}{\multicolumn{3}{|c|}{$\begin{array}{l}\text { DATE, OBSERVER, } \\
\text { AND OBJECT. }\end{array}$}} & \multirow[b]{2}{*}{ 吾 } & \multirow[b]{2}{*}{$\left|\begin{array}{c}\text { MEAN } \\
\text { THREAD. }\end{array}\right|$} & \multicolumn{2}{|c|}{ CORRECTIONS. } & \multirow[b]{2}{*}{$\begin{array}{l}\text { CIRCLE) } \\
\text { READING. }\end{array}$} & \multirow{2}{*}{$\begin{array}{c}\text { MEAN } \\
\text { OF } \\
\text { TEI. MI- } \\
\text { CROM. } \\
\text { READ- } \\
\text { INGS. }\end{array}$} & \multirow[b]{2}{*}{$\begin{array}{l}\text { REFRAC- } \\
\text { TION. }\end{array}$} & \multirow{2}{*}{\multicolumn{2}{|c|}{$\begin{array}{l}\text { EQ. PT. } \\
\text { FROM } \\
\text { STARS. }\end{array}$}} & \multirow{2}{*}{\multicolumn{2}{|c|}{\begin{tabular}{|c|} 
APPARENT \\
RIGHT \\
ASCENSION.
\end{tabular}}} & \multirow[b]{2}{*}{ 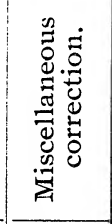 } & \multirow{2}{*}{\multicolumn{2}{|c|}{$\begin{array}{l}\text { APPARENT } \\
\text { DECLINA- } \\
\text { TION. }\end{array}$}} & \multirow[b]{2}{*}{ 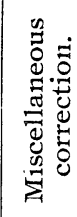 } \\
\hline & & & & & & $\begin{array}{l}\text { Instru- } \\
\text { ment. }\end{array}$ & Clock. & & & & & & & & & & & \\
\hline & & & & & $\mathrm{ml}$ & 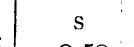 & & - ', 11 & rev. & ' I & & "I & $\mathrm{h} \mathrm{m}$ & $\mathrm{s}$ & 5 & $\circ \quad 1$ & " & 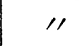 \\
\hline 2 & $\gamma^{2}$ & Sagittarii & & $\begin{array}{l}10 \\
\text { II }\end{array}$ & $\left|\begin{array}{cc}\text { II } & 0.36 \\
59 & 17.83\end{array}\right|$ & $\mid \begin{array}{l}-0.50 \\
-\quad 0.70\end{array}$ & $\begin{array}{l}2.84 \\
2.72\end{array}$ & $\begin{array}{lrr}19 & 8 & 5.22 \\
69 & 14 & 6.70\end{array}$ & $\begin{array}{l}46.423 \\
46.979\end{array}$ & $\begin{array}{r}18.9 \\
+\quad 224.3\end{array}$ & & $\begin{array}{l}71.0 \\
70.4\end{array}$ & I4 II & & & . & & \\
\hline 3 & $\begin{array}{ll}8 & \mathrm{C} \\
1 & \mathrm{~s}\end{array}$ & Ursæ Minc & pris & 5 & $\left|\begin{array}{rr}5 & 2.24 \\
5 & .88\end{array}\right|$ & -3.33 & {$\left[\begin{array}{l}-3.85 \\
-\end{array}\right]$} & ] ] $312 \quad 16$ & & & & & $\begin{array}{r}175 \\
185\end{array}$ & & & : & & \\
\hline $\begin{array}{l}4 \\
5\end{array}$ & $\mu \mathrm{S}$ & $\begin{array}{l}\text { Sagittar11 } \\
\text { Moon I, S. }\end{array}$ & & $\begin{array}{r}\text { II } \\
6\end{array}$ & $\begin{array}{r}7 \mathrm{r} .88 \\
\text { ro } \mathrm{I} 6.87\end{array}$ & $\begin{array}{l}-0.66 \\
-0.70\end{array}$ & $\begin{array}{r}+\quad 2.84 \\
+\quad 2.83\end{array}$ & $\left|\begin{array}{lll}59 & 54 & 3.48 \\
64 & 40 & 4.32\end{array}\right|$ & $\begin{array}{l}48.379 \\
49.015\end{array}$ & $\begin{array}{r}\text { I } 34.8 \\
+ \text { I } 56.0\end{array}$ & & $\begin{array}{l}71.8 \\
71.1\end{array}$ & \begin{tabular}{|rr} 
I8 & 7 \\
I8 & IO
\end{tabular} & $19.00-$ & $+75.2 \mathrm{I}$ & $-255 \mathrm{I}$ & & \\
\hline & $\eta \mathrm{s}$ & $\begin{array}{l}\text { Serpentis } \\
\text { August }\end{array}$ & $26, \mathrm{~B}$ & II & I6 3.38 & $-0.6 \mathrm{I}$ & $\begin{array}{r}+2.92 \\
+\end{array}$ & $\begin{array}{lll}4 \mathrm{I} & 46 & 2.08\end{array}$ & 45.439 & $\begin{array}{r}1 \\
+\quad 49.2\end{array}$ & & 71.2 & I8 16 & 19.00 & +75.21 & -25514 & 43.8 & \\
\hline$\stackrel{7}{8}$ & $\begin{array}{ll}\alpha & 0 \\
\beta & G\end{array}$ & $\begin{array}{l}\text { Canis Min } \\
\text { Geninorum } \\
\text { August }\end{array}$ & $\begin{array}{l}\text { oris. } \\
\text { on } \\
27, \mathrm{~B} \text {. }\end{array}$ & $\begin{array}{l}5 \\
9\end{array}$ & $\begin{array}{rr}33 & 57.37 \\
39 & 4.40\end{array}$ & $\left|\begin{array}{l}-0.55 \\
-0.53\end{array}\right|$ & $\begin{array}{l}+3.18 \\
+3.23\end{array}$ & $\begin{array}{lll}33 & 22 & 5 \cdot 38 \\
\text { IO } & 34 & \cdot\end{array}$ & 44.072 & $+\quad 36.9$ & & 7 r.o & $\begin{array}{ll}7 & 34 \\
7 & 39\end{array}$ & & - & : & & \\
\hline $\begin{array}{r}9 \\
10\end{array}$ & $\stackrel{S}{S}$ & $\begin{array}{l}\text { in } \mathrm{N} \text {. } \\
\text { un II, S. }\end{array}$ & & II & & -0.54 & 3.24 & $\begin{array}{lll}28 & 40 & 7.28\end{array}$ & 48.475 & +. 30.5 & & 70.6 & IO 24 & 2.07 & +64.62 & & & \\
\hline T & $\mathrm{N}$ & Iercury I, & & II & $\left|\begin{array}{rr}26 & 8.6 \mathrm{I} \\
\mathrm{I} 7 & 28.15\end{array}\right|$ & $\mid \begin{array}{l}-0.54 \\
-0.56\end{array}$ & $\begin{array}{l}3.24 \\
3.25\end{array}$ & $\begin{array}{lll}29 & 12 & 9.30\end{array}$ & 47.332 & $+\quad 31.2$ & & 70.6 & Io 26 & II. $3 \mathrm{I}$ & -64.62 & +938 & 6.6 & \\
\hline 16 & $\beta \mathrm{L}$ & eonis & & II & $\begin{array}{ll}1 & 20.15 \\
43 & 50.73\end{array}$ & $\begin{array}{l}-0.50 \\
-0.54\end{array}$ & $\begin{array}{l}3.20 \\
3.19\end{array}$ & $\begin{array}{rrr}39 & 6 & 9.28 \\
23 & 42 & 8.12\end{array} \mid$ & $\left|\begin{array}{l}47.070 \\
47.042\end{array}\right|$ & $\begin{array}{l}+\quad 45.2 \\
+\quad 24.4\end{array}$ & & $\begin{array}{l}70.6 \\
70.8\end{array}$ & \begin{tabular}{|lll} 
II & I \\
II & 43
\end{tabular} & $30.84-$ & +0.34 & -016 & 2.5 & \\
\hline I3 & $\alpha \mathrm{V}$ & $\begin{array}{l}\text { Jenus I, C. } \\
\text { Tirosinis }\end{array}$ & & II & \begin{tabular}{rr|}
9 & 26.94 \\
\end{tabular} & -0.58 & 3.27 & $\begin{array}{lll}47 & 20 & \text { Io. } 88\end{array}$ & 45.208 & $\begin{array}{ll}+ \\
+\end{array}$ & & 70.6 & 1399 & 29.63 & $+0 . \dot{6} 6$ & 8294 & $42 . \dot{\mathrm{I}}$ & \\
\hline $\begin{array}{l}14 \\
15\end{array}$ & $\eta \mathrm{B}$ & ootis. & & $\begin{array}{l}\text { II } \\
\text { II }\end{array}$ & I9 48.64 & -0.58 & $\div 3.32$ & $\begin{array}{lll}49 & 28 & 3.65\end{array}$ & $46.26 \mathrm{I} \mid$ & + I 4.9 & & $7 \mathrm{I} \cdot 3$ & 1319 & r & & 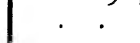 & & \\
\hline 16 & $a \mathrm{~B}$ & Bootis. : : & & II & $\mid \begin{array}{l}49 \\
\text { Io } 59.07 \\
\text { 5. }\end{array}$ & $\mid \begin{array}{l}-0.53 \\
-0.53\end{array}$ & $\begin{array}{l}3.30 \\
320\end{array}$ & $\begin{array}{lll}\text { I9 } & 56 & 4.18\end{array}$ & $\mid \begin{array}{l}47.148 \\
66.126\end{array}$ & $\begin{array}{l}+\quad 20.2 \\
+\quad 10.4\end{array}$ & & $\begin{array}{l}70.0 \\
70.0\end{array}$ & I3 49 & & & - & & 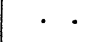 \\
\hline 17 & $\sigma \mathrm{S}$ & agittarii & & II & & -0.71 & $\begin{array}{r}3.29 \\
+3.18 \\
\end{array}$ & $\begin{array}{cccc}19 & \text { s } & 9.90 \\
65 & \text { I } & 1.28\end{array}$ & $\left.\mid \begin{array}{l}40.126 \\
47.176\end{array}\right]$ & $\begin{array}{l}+ \\
+2\end{array}$ & & $\begin{array}{l}7.0 \\
70.1\end{array}$ & $\begin{array}{ll}14 & \text { II } \\
\text { I8 } & 49\end{array}$ & & . & $\cdot$ & . & - \\
\hline 18 & $5 \mathrm{I} \mathrm{H}$ & I. Cephei & S. P. & 7 & 5253.05 & +2.54 & {$\left[\begin{array}{l}-2.8 \mathrm{I}] \\
-\end{array}\right.$} & ] & \begin{tabular}{|l|}
41.652 \\
1.11
\end{tabular} & $\begin{array}{lll}1 & -1 & 2.1 \\
-1 & 1.1\end{array}$ & & $67.9]$ & $\begin{array}{r}10049 \\
652\end{array}$ & & : & : & & \\
\hline $\begin{array}{l}19 \\
20\end{array}$ & $\begin{array}{ll}d \mathrm{~S} \\
\mathrm{M}\end{array}$ & & & . II & II 42.02 & -0.68 & +3.20 & $575^{8}$ го. 48 & 44.375 & $+\mathrm{I}$ & & 68.7 & $19 \mathrm{II}$ & & & & & \\
\hline $2 I$ & $\delta \mathrm{A}$ & $\begin{array}{l}\text { loon 1, S. } \\
\text { quilæ }\end{array}$ & & \begin{tabular}{l|l} 
II \\
II
\end{tabular} & I5 32.95 & -0.72 & -3.26 & 6.92 & 47.007 - & + I 46.9 & & 69.2 & 1915 & $35.49-$ & +74.08 & $-232 I$ & 0.7 & \\
\hline 22 & $\kappa \mathrm{A}$ & quilæ : & a. & II & 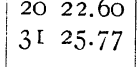 & $\mid$\begin{tabular}{|l|}
-0.66 \\
-0.63
\end{tabular} & $\begin{array}{r}3.30 \\
3.37 \\
3\end{array}$ & $\begin{array}{rrrr}35 & 55 & 56.62 \\
46 & 6 & \text { Io.82 }\end{array}$ & \begin{tabular}{|l|}
44.983 \\
43.216
\end{tabular} & $\begin{array}{l}+\quad 4 \mathrm{I} . \mathrm{O} \\
+\quad 58.8\end{array}$ & & 69.0 & $\begin{array}{l}1920 \\
1931\end{array}$ & & & & & \\
\hline $\begin{array}{l}23 \\
24\end{array}$ & $\mathrm{MI}$ & Ioon S. & & . & & & & $\begin{array}{llll}5 & 8 & 4\end{array}$ & & & & & & & & $-\mathrm{I}-\mathrm{I} \mathrm{I}_{42} 2$ & & \\
\hline $\begin{array}{l}24 \\
25\end{array}$ & $\varepsilon \mathrm{D}$ & Delphini & & 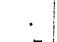 & & & & $\begin{array}{l}4.45 \\
0.12\end{array}$ & 42.328 & $\begin{array}{r}\quad 30.8 \\
+\quad 29.8\end{array}$ & & 67.3 & 2028 & & & 19142 & $\begin{array}{l}20.2 \\
. \quad .\end{array}$ & : \\
\hline $\begin{array}{l}25 \\
26\end{array}$ & $\mu \mathrm{A}$ & $\begin{array}{l}\text { quarii } \\
\text { ygni. }\end{array}$ & & $\begin{array}{r}7 \\
\mathrm{II}\end{array}$ & $\left|\begin{array}{rr}47 & 10.85 \\
2 & 20.46\end{array}\right|$ & $\begin{array}{l}-0.60 \\
-0.52\end{array}$ & $\begin{array}{r}+3.39 \\
+\quad 3\end{array}$ & $\begin{array}{lll}48 & 12 & 9.40\end{array}$ & 44.828 & $+\mathrm{I} 3.0$ & & 67.72 & 2047 & & & . & . . & \\
\hline 27 & $\begin{array}{l}6 \mathrm{I}^{x} \mathrm{C} \\
\mathrm{I} P \mathrm{P}\end{array}$ & $\begin{array}{l}\text { ygni. } \\
\text { egasi. }\end{array}$ & & II & 1723.22 & $\begin{array}{r}-0.52 \\
-\quad 0.54\end{array}$ & $\begin{array}{r}3.5^{2} \\
3.52\end{array}$ & 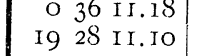 & $\begin{array}{l}45.125 \\
46.63 \mathrm{I}\end{array}$ & $\begin{array}{r}+\quad 0.7 \\
+\quad 20.0\end{array}$ & & $\begin{array}{ll}68.4 & 2 \\
67.2 & 3\end{array}$ & $\begin{array}{ll}2 I & 2\end{array}$ & & . & $\cdot$ & & \\
\hline & $\delta \mathrm{G}$ & $\begin{array}{l}\text { August } 2 \\
\text { eminorur. }\end{array}$ & $n \ldots$ & II & & & & & $\begin{array}{c}40.05^{1} \\
178.5\end{array}$ & & & $07.33^{2}$ & 2117 & & & & & \\
\hline & $\alpha^{2} \mathrm{G}$ & ei & & II & $28 \quad 5.39$ & -0 & $\begin{array}{r}3.49 \\
-\quad 3.47 \\
\end{array}$ & $\begin{array}{rrr}6 & 44 & 9.000\end{array}$ & $\begin{array}{l}47.835 \\
46.910\end{array}$ & $\begin{array}{r}+\quad 16.7 \\
+\quad 6.6\end{array}$ & & 69.1 & $\begin{array}{ll}7 & 14 \\
7 & 28\end{array}$ & & $r$ & & & \\
\hline $\begin{array}{l}30 \\
31\end{array}$ & $\begin{array}{ll}\alpha \\
\beta \\
\beta\end{array}$ & $\begin{array}{l}\text { anss Mino } \\
\text { eminorun }\end{array}$ & & $\begin{array}{l}\text { II } \\
\text { II }\end{array}$ & $\begin{array}{lll}33 & 57.23 \\
30 & \end{array}$ & $\begin{array}{r}-0.49 \\
-0.48\end{array}$ & +3.33 & 3322 I0. 22 & 43.678 & $+\quad 36.5$ & & 69.6 & 734 & & & & & \\
\hline 2 & $\propto \mathrm{H}$ & ydræ & & IO & $\begin{array}{rr}39 & 4.10 \\
22 & 33.21\end{array}$ & $\begin{array}{r}-0.48 \\
-0.5 \mathrm{I}\end{array}$ & $\begin{array}{r}3.50 \\
3.54 \\
\end{array}$ & $\begin{array}{rrr}\text { IO } & 34 & 9.32 \\
47 & + & 9.70\end{array}$ & $\begin{array}{l}47.88 \mathrm{I} \\
43.372 \mathrm{H}\end{array}$ & $\begin{array}{r}+\quad 10.4 \\
+\quad 58.9\end{array}$ & & 68.8 & $\begin{array}{ll}739 \\
0222\end{array}$ & $\therefore$ & & & & - \\
\hline & $\mathrm{Su}$ & $\begin{array}{l}\text { August } \\
\text { in I, S. }\end{array}$ & $\mathrm{Br}$ & II & & & $\begin{array}{r}5.34 \\
+\quad 3.49\end{array}$ & $\begin{array}{l}47+9.70 \\
30\end{array}$ & $\begin{array}{l}43.372 \\
47.318+1\end{array}$ & $\begin{array}{r}+50.9 \\
+\quad 35\end{array}$ & & 70.3 & & & & & & \\
\hline & Su & an II, N. . & & II & & -0.48 & $\begin{array}{r}3.49 \\
+\quad 3.49 \\
\end{array}$ & $\begin{array}{lll}30 & 16 & 11.98 \\
29 & 44 & 8.52\end{array}$ & $\begin{array}{l}47.318 \\
48.202\end{array}$ & $\begin{array}{r}+\quad 3 I .8 \\
+\quad 31.2\end{array}$ & & $\begin{array}{l}70.2 \\
70.2\end{array}$ & IO 345 & 58.04 & +64.44 & +834 & & \\
\hline & $\beta \mathrm{Le}$ & eonis & & 9 & 4350 & -0.48 & $\begin{array}{r}-52 \\
+\end{array}$ & $\begin{array}{lll}23 & 42 & 9.15\end{array}$ & 47.024 & $\begin{array}{r}01.2 \\
+\quad 23.9\end{array}$ & & 70.8 & $\begin{array}{ll}10 & 51 \\
\text { II } & \end{array}$ & & & $\begin{array}{l}+ \\
\end{array}$ & 50.4 & \\
\hline & $\begin{array}{ll}\alpha \mathrm{Ca} \\
\mathrm{Ve}\end{array}$ & Ven & & II & $5 \mathrm{I} \mathrm{I}$ & -0 & +3. & $\begin{array}{lll}359 & 58 & 7.60\end{array}$ & $49.268+$ & 0.1 & & 59.7 & $\begin{array}{l}1255 \\
1251\end{array}$ & & & & & \\
\hline & $\begin{array}{l}\mathrm{Ve} \\
\mathrm{Ve}\end{array}$ & $\begin{array}{l}\text { I, S. } \\
\text { N. }\end{array}$ & & II & $21 \quad 16.53$ & $-0.5 \mathrm{I}$ & +3.52 & $\begin{array}{lll}48 & 46 & 8.65\end{array}$ & $50.212+$ & $\begin{aligned} &+ 1.6 \\
&\end{aligned}$ & & 1.11 & 1325 & $19.54+$ & +0.69 & $957 \mathrm{I}$ & 7 & \\
\hline & $\measuredangle \mathrm{Ur}$ & rsæ Minor & ris s. p. & 7 & 2229 & +6.92[ & 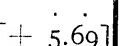 & 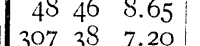 & $\begin{array}{l}49.190 \\
466.727\end{array}$ & $\begin{array}{l}+ \text { I } \\
\end{array}$ & & 1.1 & & & & $95^{6} 5$ & 57.1 & \\
\hline & $\eta$ Bo & oo & & II & 4948 & $-0.4 S^{2}$ & $\begin{array}{r}3.091 \\
+\quad 3.52\end{array}$ & I9 $56 \quad 8.28$ & $\begin{array}{l}40.727 \\
47.136-7\end{array}$ & $\begin{array}{l}-1 \\
+\quad 106\end{array}$ & & $0.5]$ & $\begin{array}{ll}1 & 22 \\
I\end{array}$ & & & & & \\
\hline $\begin{array}{l}4 \\
4\end{array}$ & (r) BC & bo & & II & 1059 & -0 & $\begin{array}{r}0.02 \\
+\quad 3.50\end{array}$ & $\begin{array}{ll}19 & 8.18\end{array}$ & $\begin{array}{l}4.3 \mathrm{I} 4 \mathrm{~T} \\
46.3\end{array}$ & $\begin{array}{l}+19.0 \\
+\quad 18.8\end{array}$ & & \begin{tabular}{l|l}
1.7 & 1 \\
\end{tabular} & 13 & & & . & & \\
\hline $\begin{array}{l}4 \\
4 \\
4\end{array}$ & 50 & पas & & II & 043 & -0 & 3.47 & 258 IO & $45.069+$ & & & \begin{tabular}{l|l}
2.1 & 1 \\
1.6 & 1
\end{tabular} & $\begin{array}{ll}14 & 11 \\
19 & 0\end{array}$ & & & & . & \\
\hline $\begin{array}{l}4 . \\
4 \\
4\end{array}$ & $\begin{array}{ll}d & \mathrm{Sa} \\
\lambda & \mathrm{Ur}\end{array}$ & $\operatorname{gin}$ & & II & $\begin{array}{lll}\text { II } & 4 \mathrm{r} .7 \mathrm{I}\end{array}$ & -0.61 & $3.4 \mathrm{I}$ & $575^{8}$ 10.18 & $44.569+$ & + 1227.8 & & 9.6 I & I9 II & & & . & . & \\
\hline & $\begin{array}{ll}\lambda & \mathrm{Ur} \\
y & \mathrm{Ac}\end{array}$ & $\begin{array}{l}\text { sa Minor } \\
\text { quilæ.. }\end{array}$ & & $\begin{array}{r}5 \\
I\end{array}$ & $\mid \begin{array}{lll}24 & 3 \mathrm{I} .98 \\
4 \mathrm{I} & 25.49\end{array}$ & $-9.06[-$ & {$\left[\begin{array}{c}+ \\
-\end{array}\right.$} & 3095 & 42.387 & $-\mathrm{I}$ & & I.o] & 19 & & & & & \\
\hline & & & & & 4125.49 & -0.54 & $3.5^{2}$ & 2828 II.15 & $47.280+$ & $\begin{array}{l}+\quad 29.9 \\
+\quad 1\end{array}$ & & \begin{tabular}{l|l}
1.0 & I \\
\end{tabular} & $194 \mathrm{I}$ & & & & & \\
\hline $\mathrm{Ti}$ & me & Barom. & $\begin{array}{l}\text { Att. } \\
\text { Ther. }\end{array}$ & $\begin{array}{l}\text { Ex. } \\
\text { Ther. }\end{array}$ & $\begin{array}{ll}\text { r. } & \text { Teles }\end{array}$ & $e e_{l}^{e m i c r ~}$ & $\begin{array}{l}\text { mete } \\
I I, e .\end{array}$ & ins & $d e$ & & No. & Para & allax. & Semi-d & diam. & $\begin{array}{l}\text { Corr. for } \\
\text { Def. Ill. }\end{array}$ & & sum. \\
\hline & & & $\circ$ & & & & & & & & & & & & & & & \\
\hline & $\begin{array}{ll}14 & 13 \\
18 & 0\end{array}$ & $\begin{array}{l}\begin{array}{l}29.750 \\
29.746\end{array} \\
29\end{array}$ & $\begin{array}{l}88.2 \\
78.2\end{array}$ & $\begin{array}{l}81.7 \\
74.8\end{array}$ & & & & & & & & & & & & . & & \\
\hline & & 29.750 & 77.0 & 74.4 & & & & & $\mathrm{~V}$, & & & & & -155 & & & & \\
\hline & & $\begin{array}{l}\begin{array}{l}29.89 \\
29.90\end{array} \\
29\end{array}$ & $\begin{array}{l}71.8 \\
73.8\end{array}$ & $\begin{array}{l}68.3 \\
7.1\end{array}$ & 7,9 & 4. $33,>$ & & & & & IO & & & +155 & & & & \\
\hline & & $\begin{array}{l}29.900 \\
29.900 \\
20.004\end{array}$ & $\begin{array}{l}1.0 \\
73.2 \\
750\end{array}$ & $7_{71}^{71}$ & $\begin{array}{l}\text { IO, I } \\
\text { I8. }\end{array}$ & 5,34 . & & & & & II & & & & & r.9 & & \\
\hline & & 26 & & $\begin{array}{l}72.7 \\
72.9\end{array}$ & 28 & & & & & & $\begin{array}{l}13 \\
20\end{array}$ & & $\begin{array}{l}7.4 \\
4.2\end{array}$ & & & & & \\
\hline & & $\begin{array}{l}20 \\
20\end{array}$ & $\begin{array}{l}76.0 \\
75.0\end{array}$ & 73 & $2+2-1$ & & & & & & 23 & $\begin{array}{r}50 \\
+51\end{array}$ & & $\begin{array}{r}102 \\
+162\end{array}$ & 5 & . & & \\
\hline & & $\begin{array}{l}29.95 \\
29.95 \\
\end{array}$ & $\begin{array}{l}70.0 \\
68.0\end{array}$ & 64 & $2+2-1$ & & & & & & 33 & + & 4.4 & +155 & & . & & \\
\hline & & $\begin{array}{l}29.952 \\
30.010\end{array}$ & 66.2 & 63. & & & & & & & 34 & + & $4 \cdot 3$ & -155 & $5^{2.6}$ & & & \\
\hline & & $\begin{array}{l}30.00 \\
30.01 \\
20.91\end{array}$ & $\begin{array}{l}160.2 \\
6.0 \\
7.0\end{array}$ & 67. & 4 & & Bisecti & tions at & $\mathrm{C}_{4}, \mathrm{C}_{5}$ & & & & & t & 10.2 & 0.8 & & \\
\hline & & $\begin{array}{l}29.91 \\
29.92 \\
292\end{array}$ & $\begin{array}{l}75.6 .5 \\
76.3\end{array}$ & 75 & & & & & & & & & & & 10.2 & & & \\
\hline & if & $\begin{array}{l}29 . \\
29 . \\
29 .\end{array}$ & $\begin{array}{l}81 \\
84 \\
86\end{array}$ & $\begin{array}{l}79 \\
83 \\
85\end{array}$ & & & & & & & & & & & & & & \\
\hline & $\begin{array}{l}13 \\
14 \\
14\end{array}$ & & $\begin{array}{l}80 . \\
89 \\
88\end{array}$ & & & & & & & & & & & & & & & \\
\hline & & 29.902 & 78.2 & 76.7 & & & & & & & & & & & & & & \\
\hline
\end{tabular}




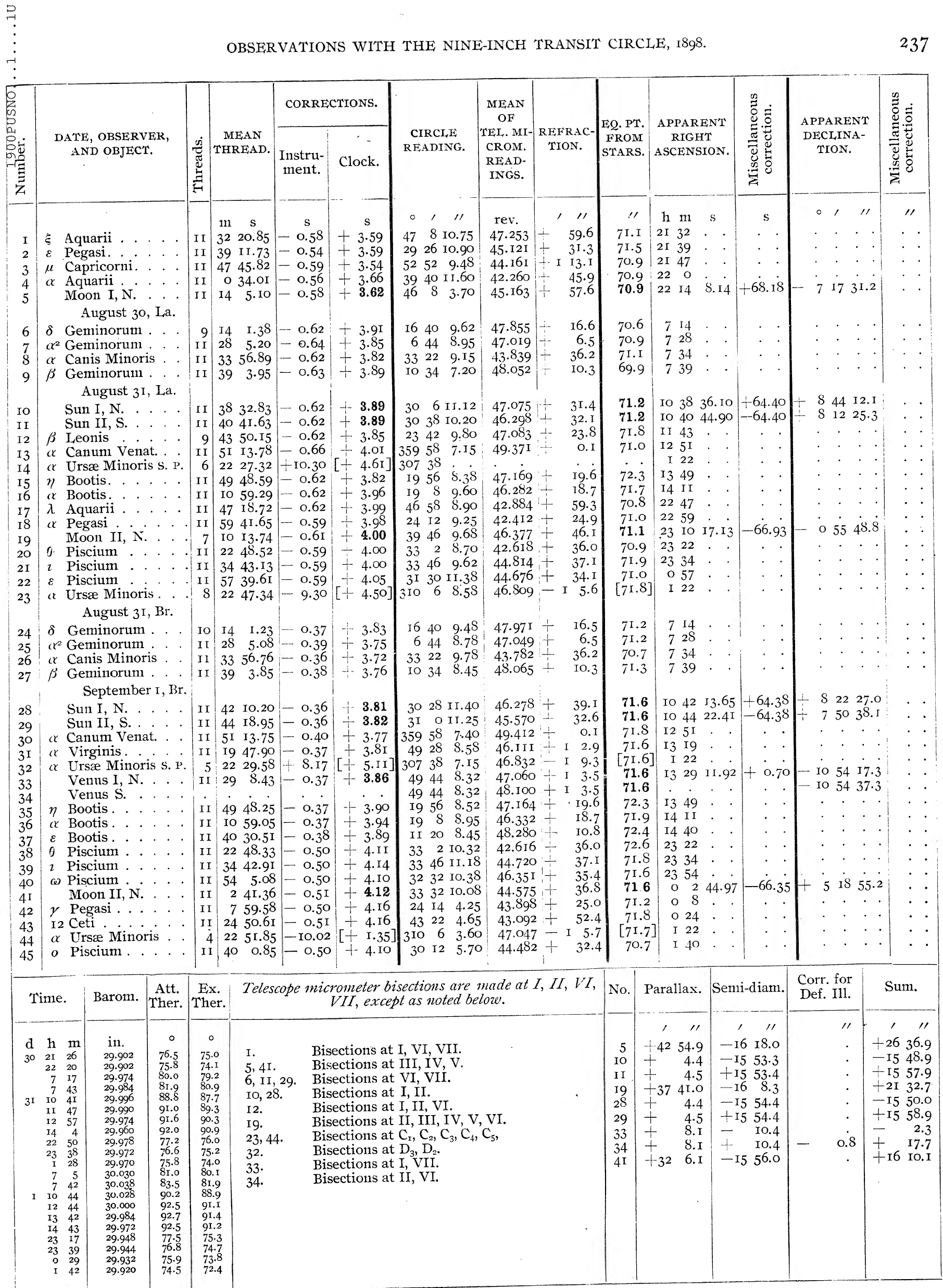




\begin{tabular}{|c|c|c|c|c|c|c|c|c|c|c|c|c|c|c|c|c|c|c|c|}
\hline \multirow[t]{2}{*}{$\begin{array}{c}\stackrel{+}{\Delta} \\
\stackrel{\Xi}{\Xi} \\
\Xi \\
\text { Z }\end{array}$} & \multicolumn{3}{|c|}{$\begin{array}{l}\text { DATE, OBSERVER, } \\
\text { AND OBJECT. }\end{array}$} & \multicolumn{2}{|r|}{ 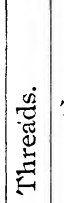 } & $\begin{array}{c}\text { MEAN } \\
\text { THREAD. }\end{array}$ & $\begin{array}{c}\text { Instru- } \\
\text { ment. }\end{array}$ & Clock. & $\begin{array}{c}\text { CIRCIE } \\
\text { READING. }\end{array}$ & $\begin{array}{c}\text { MEAN } \\
\text { OF } \\
\text { TEL. MI- } \\
\text { CROM. } \\
\text { READ- } \\
\text { INGS. }\end{array}$ & $\begin{array}{l}\text { REFRAC- } \\
\text { TION. }\end{array}$ & $\begin{array}{l}\text { EQ. PT. } \\
\text { FROM } \\
\text { STARS. }\end{array}$ & $\begin{array}{r}\text { APPAR } \\
\text { RIGI } \\
\text { ASCEN }\end{array}$ & $\begin{array}{l}\text { RENT } \\
\text { HT } \\
\text { SION. }\end{array}$ & 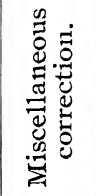 & $\begin{array}{r}\text { APPAI } \\
\text { DECL } \\
\text { TIO }\end{array}$ & $\begin{array}{l}\text { RE } \\
\text { LIN } \\
\text { ON. }\end{array}$ & TAT & 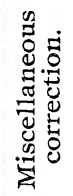 \\
\hline & & tember I & $\mathrm{K}$ & & & $\mathrm{m}$ & 3 & $\mathrm{~s}$ & 0,11 & rev. & 111 & $\prime \prime$ & $\mathrm{h} \mathrm{m}$ & $\mathrm{s}$ & $\mathrm{s}$ & & 1 & 11 & 11 \\
\hline I & $\delta \mathrm{G}$ & minorum & . & & $I x$ & I4 0.90 & -0.63 & +4.45 & $1640 \quad 8.52$ & 47.918 & I6.5 & 70.6 & $7 \mathrm{I} 4$ & . & . & . & . & . . & . . \\
\hline 2 & $\alpha^{2} \mathrm{G}$ & minorum & . . & & II & $28 \quad 4.62$ & -0.65 & +4.50 & $\begin{array}{lll}6 & 44 & 3.78\end{array}$ & 47.328 & 6.5 & 71.4 & 728 & &. & . & . & . . & . \\
\hline 3 & $\alpha \mathrm{C}$ & nis Mino & ris & & II & 3356.39 & -0.63 & $\begin{array}{r}+38 \\
+4.38\end{array}$ & $\begin{array}{lll}33 & 22 & 6.82\end{array}$ & 43.948 & 36.1 & 70.8 & 734 & . . &.$\quad$. & . & . & . . &. \\
\hline 4 & $\beta \mathrm{G}$ & $\begin{array}{l}\text { minorum } \\
\text { Septembe }\end{array}$ & $2, \mathrm{~K}$ & & II & 393.44 & -0.64 & +4.47 & IO 345.18 & 48.244 & $+\quad$ IO.3 & 71.4 & 739 & . . & $\cdot$ & . & . & .. &. \\
\hline 5 & & $n$ I. . & . . & & I I & $45 \quad 47.24$ & -0.63 & +4.51 & 3 I 6 & & . & . $\cdot$ & IO 45 & $5 \mathrm{I} .12$ & +64.32 & . & $\cdot$ & . & . \\
\hline 6 & & n II. . . & . . & & IO & 4755.88 & -0.63 & +4.51 & $3 I 6$. & & . & . . & Io 47 & 59.76 & $-64 \cdot 32$ & . & . & . . & . . \\
\hline 7 & $\alpha \mathrm{C}$ & num Ven & at. . & & I I & $5 \mathrm{I} \quad \mathrm{I} 3.24$. & -0.67 & +4.54 & $\begin{array}{lll}359 & 5^{8} & 4.55\end{array}$ & $49.5 \circ 5$ & O.I & 70.7 & $125 \mathrm{I}$ & . . &.$\quad$. & . & . &.$\quad$. & . . \\
\hline$\dot{S}$ & $\alpha \mathrm{V}$ & rginis . . & . . & & I & 1947.40 & -0.65 & $+4.5^{8}$ & $\begin{array}{lll}49 & 28 & \ldots\end{array}$ & & & . & I3 I9 & . . & . . & . & . &.$\quad$. & . . \\
\hline 9 & $\alpha \mathrm{U}$ & Sæ Minor & is $\mathrm{S} . \mathrm{P}$ & & 5 & $22 \quad 27.28$ & + IO. 30 & {$[+5.94]$} & $\begin{array}{lll}307 & 38 & 3.98\end{array}$ & 46.963 & - I 9.0 & {$[71.5]$} & I 22 & . . & . . & . & . & . . & . . \\
\hline IO & $\zeta \mathrm{V}$ & rginis . & . . & & I I & 2927.98 & -0.64 & +4.55 & ${ }_{38}^{8} 54$ 5. So & $49.46 \mathrm{I}$ & $\begin{array}{r}+\quad 43.3 \\
\end{array}$ & 72.5 & 1329 & . . & . . & . & . &.$\quad$. & . . \\
\hline II & & nus $\mathrm{I}, \mathrm{N}$. &. & & I I & 334.01 & -0.66 & $\begin{array}{r}+4.56 \\
+\quad 1\end{array}$ & 50 I2 5.40 & $48.27 \mathrm{I}$ & I I 4.3 & 71.3 & 1333 & $7 \cdot 9 \mathrm{I}$ & $+0.7 \mathrm{I}$ & - II 2 & 223 & 38.7 & . . \\
\hline I2 & $\eta \mathrm{Bc}$ & otis. . & . . & & II & 4947.82 & -0.63 & $+4.5^{8}$ & I9 $56 \quad 5.85$ & 47.265 & $\begin{array}{r}+ \\
+\quad 19.5 \\
\end{array}$ & 72.0 & I3 49 & .. & . . & . & . & . & . . \\
\hline $\mathrm{I} 3$ & I2 $\mathrm{Cc}$ & ti. . . . &. & & I I & 2450.28 & $--0.5 \mathrm{I}$ & +4.50 & $\begin{array}{lll}43 & 22 & 3.68\end{array}$ & 43.224 & 52.0 & 73.0 & O 24 & & & . & . & . & . . \\
\hline I4 & & on II, N. & . . & & I I & 544 I. I3 & $-0.5 \mathrm{I}$ & $\begin{array}{r}4.51 \\
+\quad 4.0\end{array}$ & 27443.80 & $48.47 \mathrm{I}$ & 29.0 & 72.2 & o 54 & 45.13 & -66.38 & $+I I$ & 55 & $55 . \mathrm{I}$ & . . \\
\hline 15 & $\varepsilon \quad \mathrm{Pi}$ & cium. & (2) & & II & 5739.12 & -0.50 & +4.49 & $\begin{array}{lll}3 \mathrm{I} & 3 \mathrm{O} & 4.02\end{array}$ & 45.056 & 33.8 & 70.8 & o 57 &.$\quad$. &.$\quad$. & . & . &. & . . \\
\hline 16 & $\alpha \mathrm{U}$ & sæe Minor & & & 5 & $22 \quad 45.84$ & $-10.3 \mathrm{I}$ & {$[+8.32]$} & $\begin{array}{lll}3 \text { Io } & 6 & 3.48\end{array}$ & 47.039 & - I $5 . I$ & {$[73 \cdot 3]$} & I 22 & . & . & . & . &.$\quad$. & . . \\
\hline 17 & $\eta \mathrm{Pi}$ & $\begin{array}{l}\text { cium . } \\
\text { Septembe }\end{array}$ & $2, \mathrm{~B}$. & & I I & $\begin{array}{ll}26 & \mathrm{I} .59\end{array}$ & -0.50 & +4.53 & $\begin{array}{rrr}24 & 2 & 7.35\end{array}$ & 43.276 & $\begin{array}{r}24.6 \\
+\quad 2\end{array}$ & 72.9 & I 26 & . . & $\cdot$. & . & . & .. & . . \\
\hline I8 & $\delta \mathrm{G}$ & minorum & . . & & I I & I4 0.49 & -0.59 & +4.85 & I6 $40 \quad 9.25$ & 48.049 & $\mathrm{I} 6.4$ & 72.2 & $7 \mathrm{I} 4$ & & . & . & • &.$\quad \cdot$ & . . \\
\hline 19 & $\alpha^{2} \mathrm{G}$ & norum & . & & II & $28 \quad 4.34$ & $-0.6 \mathrm{I}$ & $\begin{array}{r}4 \\
+\quad 4.77\end{array}$ & $\begin{array}{lll}644 & 8.75\end{array}$ & 47.134 & 6.5 & 72.6 & 728 & . . &.$\quad$. & . & . & . . & . . \\
\hline 20 & $\alpha \mathrm{C}$ & nis Mino & ris . & & I I & 3456.02 & -0.57 & +4.72 & $\begin{array}{lll}33 & 22 & 9.99\end{array}$ & 43.909 & 35.8 & 72.9 & 734 & . . & .. & . & . &. & . . \\
\hline $2 I$ & $\beta \mathrm{G}$ & minorum & . . & & II & 393.04 & -0.60 & $\begin{array}{r}+ \\
+4.85\end{array}$ & IO $34 \quad 5.30$ & 48.168 & 10.2 & 71.2 & 739 & . . &. & . & . &. & . . \\
\hline 22 & $\varepsilon \quad \mathrm{H}$ & $\begin{array}{l}\text { dræ } \\
\text { Septembe }\end{array}$ & r $3, \mathrm{~B}$. & & I I 4 & $4 \mathrm{I} 20.47$ & $-0.5^{8}$ & +4.77 & $\begin{array}{lll}32 & 2 & 10.75\end{array}$ & 49.205 & 33.9 & 73.5 & $84 \mathrm{I}$ & . . & $\cdot$. & . & . & . . & $\cdot \cdot$ \\
\hline 23 & Si & $\mathrm{n} \mathrm{I}, \mathrm{N}$ & . . & & I I & 4923.86 & --0.57 & +4.81 & 3 I II 49.22 & 47.252 & 32.4 & 73.0 & IO 49 & 28. 10 & +64.38 & +73 & 383 & $3 \mathrm{I} .4$ & . \\
\hline 24 & & n II, S. & & & I I 5 & 5I 32.62 & -0.57 & +4.81 & 3I 44 I0.40 & $45 \cdot 572$ & $33 . I$ & 73.0 & IO $5^{i}$ & 36.86 & $-64.3^{8}$ & +7 & 63 & 39.9 & . . \\
\hline 25 & $\alpha \mathrm{C}$ & num Ven & at. . & & I I 5 & 5I I2.95 & -0.64 & +4.79 & $\begin{array}{lll}359 & 58 & 5.62\end{array}$ & 49.586 & O.I & 73.1 & I2 $5 \mathrm{I}$ & &.$\quad$. & . & . &.$\quad$. & . . \\
\hline 26 & $\alpha \mathrm{U}$ & sæ Minor & is $\mathrm{S} . \mathrm{P}$ & & 62 & $22 \quad 25.78$ & +II.7I & {$[+6.72]$} & $\begin{array}{lll}307 & 38 & 5.98\end{array}$ & 46.910 & - I 8.6 & {$[72.6]$} & I 22 & & . & . & . & & . . \\
\hline 27 & & nus I, C. & . . & & II 3 & 3659.42 & -0.59 & $\begin{array}{l}4.84 \\
+4\end{array}$ & 50407.10 & 49. I 36 & + I 4.9 & $73.0^{\circ}$ & I3 37 & 3.67 & +0.72 & - II 5 & 505 & $55 \cdot 7$ & . . \\
\hline 28 & $\eta \mathrm{Bc}$ & otis... &. & 1 & I I 4 & $4947.5^{2}$ & -0.58 & +4.82 & I9 567.65 , & 47.325 & I9.4 & 74.2 & 1349 &. &. & . . & . & & . . \\
\hline 29 & $\alpha \mathrm{BC}$ & otis. . & & . & 3 & IO 58.29 & -0.58 & +4.88 & I9 8 S.I2 & 46.532 & I8.5 & 73.2 & I4 II & &. & . & . &.$\quad$. & . . \\
\hline 30 & $\rho \mathrm{B}$ & otis.. & & & II 2 & $27 \quad 23.74$ & $-0.6 \mathrm{I}$ & $\begin{array}{r}4.86 \\
+4.86\end{array}$ & $8 \quad 2 \quad 8.00$ & $45.75 \mathrm{I}$ & 7.6 & 73.7 & 1427 & . &. & . & . & . . & . . \\
\hline $3 \mathrm{I}$ & $\alpha \mathrm{U}$ & sæ Minor & is . & & 62 & 2244.97 & -9.78 & {$\left[+9.3^{8}\right]$} & 3 Io $\quad 6 \quad 8.25$ & 46.762 & - I $5 . I$ & {$[72.0]$} & I 22 & &. & . & . & . . & . . \\
\hline 32 & $\eta \mathrm{Pi}$ & cium . & . . & & I I 2 & 26 I.I 5 & -0.56 & +5.05 & $24 \quad 212.80$ & 42.925 & 24.6 & 71.7 & I 26 & &. & . & . & . . & . . \\
\hline 33 & o $\mathrm{Pi}$ & cium . . & . & & I I 3 & 3959.97 & -0.57 & $\begin{array}{r}5.10 \\
+\quad 5.10\end{array}$ & $\begin{array}{lll}30 & \text { I2 } & 9.95\end{array}$ & 44.295 & 32.1 & 70.8 & I 40 & & & . & &.$\quad$. & . . \\
\hline 34 & $\mathrm{M}$ & on II, N. & . . & & I I & 4659.25 & -0.57 & $\begin{array}{r}5 \\
+\quad 5.10 \\
\end{array}$ & $\begin{array}{lll}22 & 42 & 5.08\end{array}$ & 47.290 & $23 . I$ & 70.8 & I 47 & 3.78 & -66.83 & +16 & 82 & 24.0 & . . \\
\hline 35 & $\alpha \quad \mathrm{A}$ & ietis . & . . & & I I & I $25.0 \mathrm{I}$ & -0.56 & +5.10 & I5 $52 \quad 7.68$ & 45. IO & I5.7 & 70.5 & $2 \mathrm{I}$ &.$\quad$. & . . & . & . & . . & . . \\
\hline 36 & $\dot{\xi}^{\mathrm{r}} \mathrm{Ce}$ & eptember & $4, \mathrm{Br}$ & & II & 735.00 & -0.57 & $\begin{array}{r}5.14 \\
+\quad 5.14\end{array}$ & 30285.88 & 46.275 & 32.5 & 70.4 & 27 &. & . & - & . & . $\cdot$ & .. \\
\hline 37 & $\alpha \mathrm{Ar}$ & letis. . & . . & & . & . & & . & I5 $52 \quad 5.00$ & 45.155 & I5.8 & 70.8 & [ & ${ }^{*}$ & . & . & . & & . . \\
\hline 38 & M & on N. & & & & . & & • & I8 $38 \quad 4.78$ & 45. IO9 & 18.7 & 70.8 & 239 & & . & $+20 \mathrm{I}$ & I3 & 7.5 & . . \\
\hline 39 & $\alpha \mathrm{Ce}$ & ti : . . & $0^{\circ}$ & & & . & 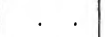 & . . & 35 Io 5.98 & $42.01 \mathrm{I}$ & 39.0 & $7 \mathrm{I} .2$ & 257 & & . & . & . &.$\quad \cdot$ & . . \\
\hline 40 & $\zeta A$ & $\begin{array}{l}\text { letis } \\
\text { eptembe }\end{array}$ & $5, \mathrm{~K}$ & & 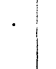 & . & 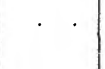 & 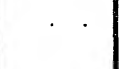 & I8 Io 5.05 & 47.772 & $+\quad 18.3$ & 70.4 & 39 & . & . & . & . &.$\quad \cdot$ & . . \\
\hline $4 \mathrm{I}$ & $\alpha \mathrm{Ce}$ & . . & . . & & II & 5655.46 & $-0.5^{2}$ & +5.65 & $\begin{array}{ll}35 & 957.20\end{array}$ & $42.45^{\mathrm{I}}$ & 39. I & 71.0 & 257 & & . & . & . & . & . . \\
\hline 42 & $\zeta \mathrm{Al}$ & etis. . &.$\quad$. & & II & 90.95 & -0.50 & $\begin{array}{r}5.8 \\
+\quad 5.82\end{array}$ & I8 Io 5.30 & $47.7 \mathrm{O} 2$ & 18.3 & 69.5 & 39 & & & . . & . & & . . \\
\hline 43 & $\mathrm{M}$ & on II, N. & . & & I I 3 & $34 \quad 4.49$ & $-0.5 \mathrm{I}$ & $\begin{array}{r}5.75 \\
+\end{array}$ & I5 $40 \quad 3.40$ & 48.003 & I5.6 & 70.2 & 334 & 9.73 & -68.08 & +23 I & IO 1 & I5.9 & . . \\
\hline 44 & $\eta \mathrm{Ta}$ & uri . . . & . . & & II & 4I 23.96 . & -0.49 & +5.73 & I5 357.75 & 44.085 & $+\quad 15.0$ & 69.9 & $34 \mathrm{I}$ & & . . &.$\quad$. & . &.$\quad$. & . . \\
\hline & ime. & Barom. & $\begin{array}{l}\text { Att. } \\
\text { Ther. }\end{array}$ & & $\begin{array}{l}\text { Ex. } \\
\text { Cher. }\end{array}$ & Telescof & pe micr & $\begin{array}{l}\text { veter b } \\
I, \text { exces }\end{array}$ & $\begin{array}{l}\text { tions are n } \\
\text { s noted bel }\end{array}$ & e at & $V I$ & No. & rallax. & Semi- & -diam. & $\begin{array}{l}\text { Corr. fo } \\
\text { Def. I11 }\end{array}$ & $\begin{array}{l}\text { or } \\
11 .\end{array}$ & & um. \\
\hline d & $\mathrm{h} \mathrm{m}$ & & 0 & & $\circ$ & & & & & & & & 11 & ' & 11 & & 11 & & 11 \\
\hline I & 716 & & $8 \mathrm{I} \cdot 3$ & & 79 & $I, 2 I, 2$ & & Bisectio & at VI & & & + & 8.2 & - & 10.3 & & . & & 2. I \\
\hline & $7 \quad 4 \mathrm{I}$ & & & & & 9,26 . & & Bisection & is at $\mathrm{D}_{3}, \mathrm{D}_{2}, \mathrm{D}_{\mathrm{I}}$ & & & I4 & 638.8 & -I5 & 42.3 & & . & $+I$ & 56.5 \\
\hline 2 & $\begin{array}{ll}\text { Io } & 51 \\
\text { I2 } & 53\end{array}$ & & $\begin{array}{l}89.4 \\
94.0\end{array}$ & & $\begin{array}{l}88.0 \\
91.7\end{array}$ & $\begin{array}{l}\text { 9. } 20 . \\
\text { I } 2 .\end{array}$ & & Bisection & $\mathrm{s}$ at II, VI, VI & & & $\begin{array}{l}14 \\
23\end{array}$ & $\begin{array}{r}5.5 \\
4.5\end{array}$ & $\begin{array}{r}15 \\
-15\end{array}$ & $\begin{array}{l}42.3 \\
55.7\end{array}$ & & . & -1 & $55^{\mathrm{I}} .2$ \\
\hline & 1347 & & $\begin{array}{l}94.0 \\
9 \mathrm{r} .7\end{array}$ & & 9 & I 4,34 , & 38,43 . & Bisection & is at II, III, IV & $\mathrm{J}, \mathrm{V}, \mathrm{VI}$ & & 24 & 4.6 & $\begin{array}{r}5 \\
+15\end{array}$ & 55.7 & & . & +1 & $\begin{array}{lll}6 & 0.3\end{array}$ \\
\hline & $0 \quad 27$ & & 77.0 & & 75.2 & I6. & & Bisection & is at $\mathrm{B}_{1}, \mathrm{~B}_{2}, \mathrm{~B}_{3}$ & $\mathrm{C}_{1}, \mathrm{C}_{2}$ & & 27 & 8.3 & & & - & {$[.5$} & & 6.8 \\
\hline & $\begin{array}{ll}I & 23 \\
7 & \text { IO }\end{array}$ & & $\begin{array}{l}75.8 \\
82.4\end{array}$ & & $\begin{array}{l}74.4 \\
81.5\end{array}$ & 23,29 . & & Bisection & is at I, II. & & & +2 & I 44.8 & -15 & 28.5 & & 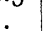 & & $6 \mathrm{x} 6.3$ \\
\hline & $\begin{array}{ll}1 & 10 \\
7 & 44\end{array}$ & 29.812 & $\begin{array}{l}2.4 \\
84.2\end{array}$ & & 83.5 & $3 \mathrm{I}$. & & Bisection & is at $\mathrm{B}_{3}, \mathrm{C}_{\mathrm{I}}, \mathrm{C}_{2}$ & & & 38 & 743.5 & $-\mathrm{I} 5$ & 15.7 & & . & + & 227.8 \\
\hline & 850 & & 88.4 & & 86.7 & & & Bisection & is at I, II, VI. & & & 43 & 4478 & -15 & 4.8 & & . & & o 17.0 \\
\hline 3 & $\begin{array}{ll}\text { IO } & 20 \\
\text { IO } & 52\end{array}$ & & $\begin{array}{l}91.0 \\
91.7\end{array}$ & & $\begin{array}{l}88.7 \\
90.7\end{array}$ & & & & & & & & & & & & & & \\
\hline 3 & $\begin{array}{ll}10 & 52 \\
12 & 44\end{array}$ & & 94 & & $\begin{array}{l}90.7 \\
93.1\end{array}$ & & & & & & & & & & & & & & \\
\hline & I3 4 & & 95 & & 93.3 & & & & & & & & & & & & & & \\
\hline & $14 \quad 35$ & 2 & 94 . & & 92.7 & & & & & & & & & & & & & & \\
\hline & $\mathrm{I}$ I2 & & 77. & & 74.7 & & & & & & & & & & & & & & \\
\hline & $\begin{array}{ll}2 & 13\end{array}$ & & 76 & & 74.1 & & & & & & & & & & & & & & \\
\hline 4 & I 59 & & 74.0 & & 71.9 & & & & & & & & & & & & & & \\
\hline & $\begin{array}{ll}2 & 4 \mathrm{r} \\
3 & \end{array}$ & & 73.8 & & 71.4 & & & & & & & & & & & & & & \\
\hline 5 & $\begin{array}{ll}3 & \text { II } \\
2 & 59\end{array}$ & 29.788 & $\begin{array}{l}73.5 \\
74.0\end{array}$ & & $\begin{array}{l}71.1 \\
71.8\end{array}$ & & & & & & & & & & & & & & \\
\hline
\end{tabular}




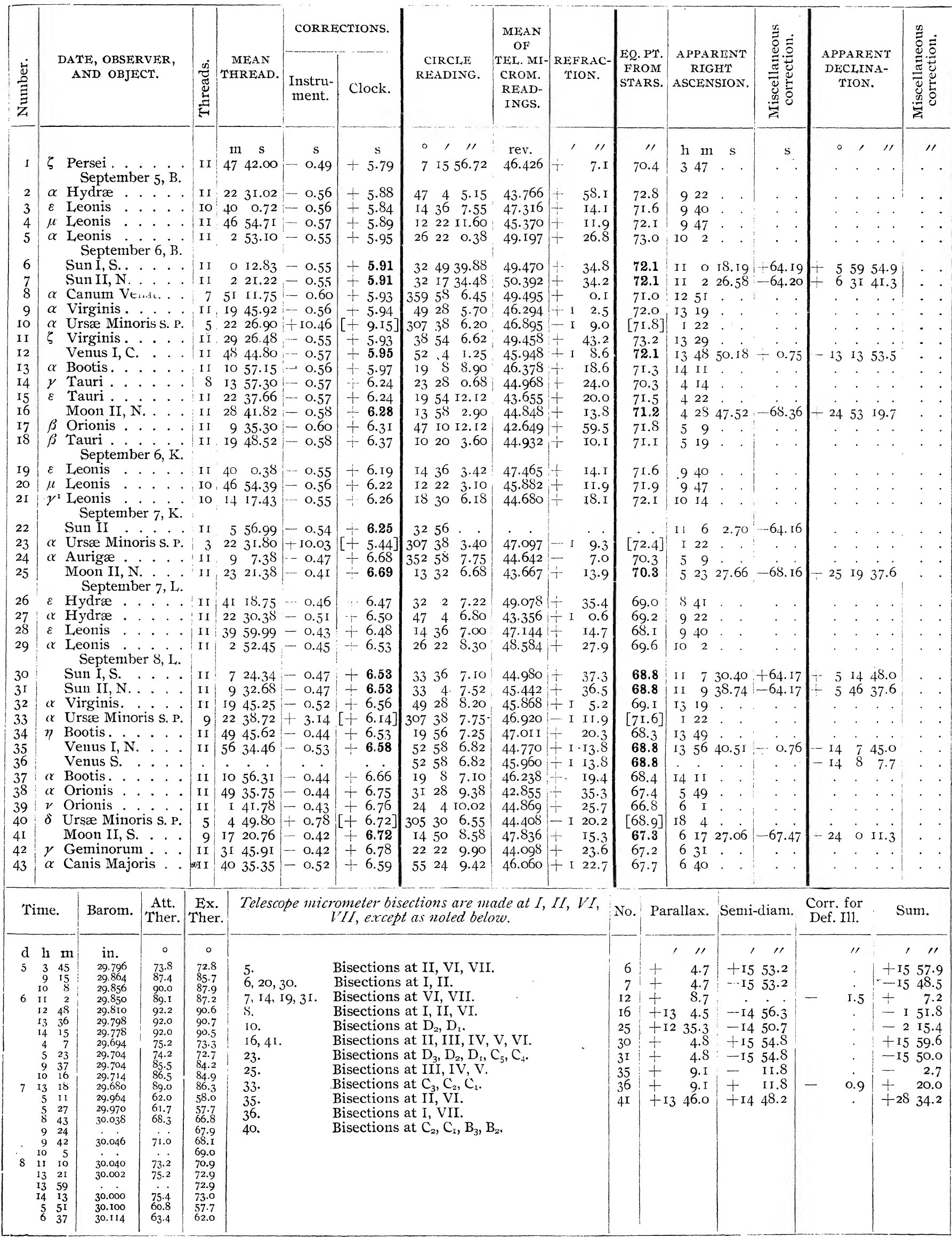




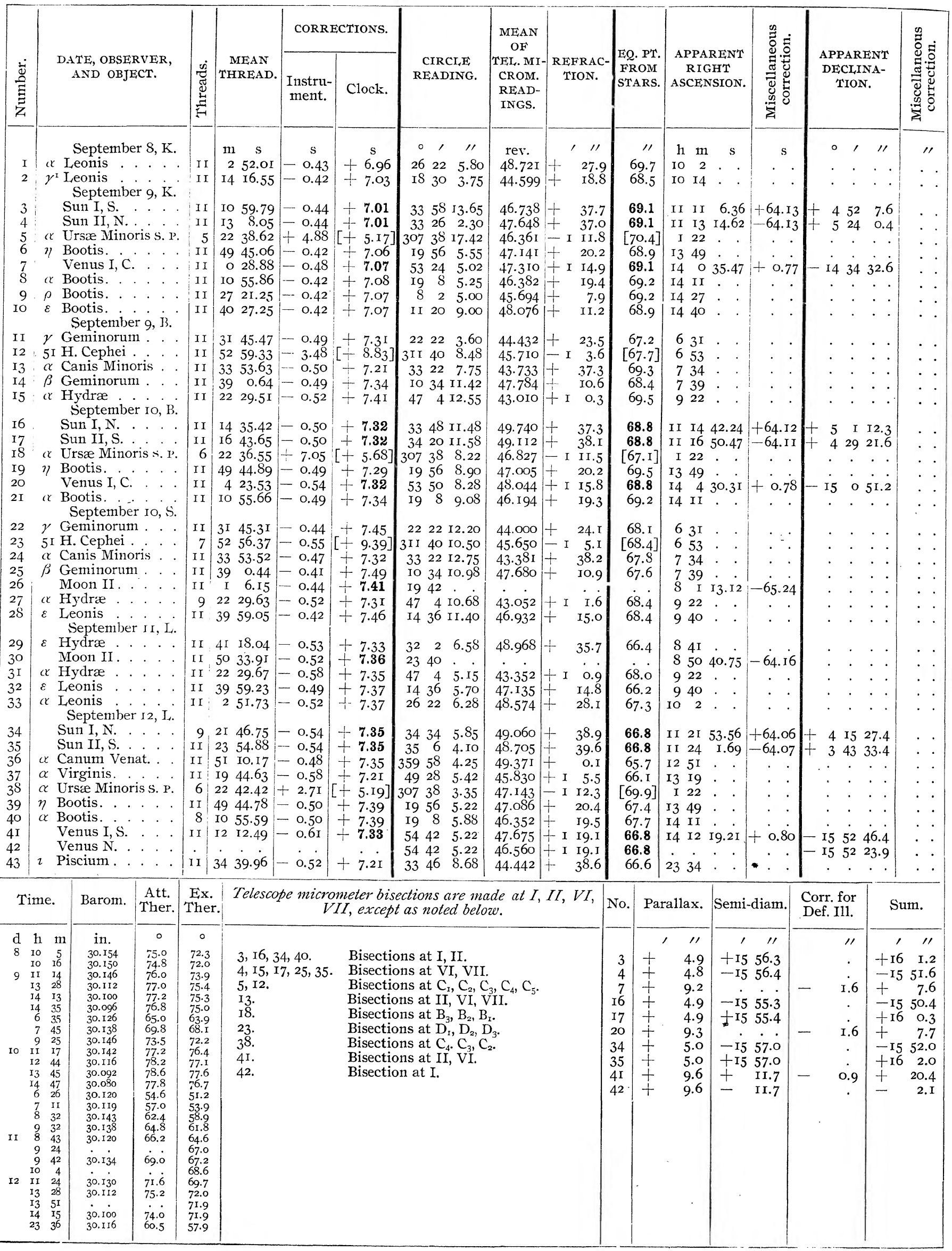




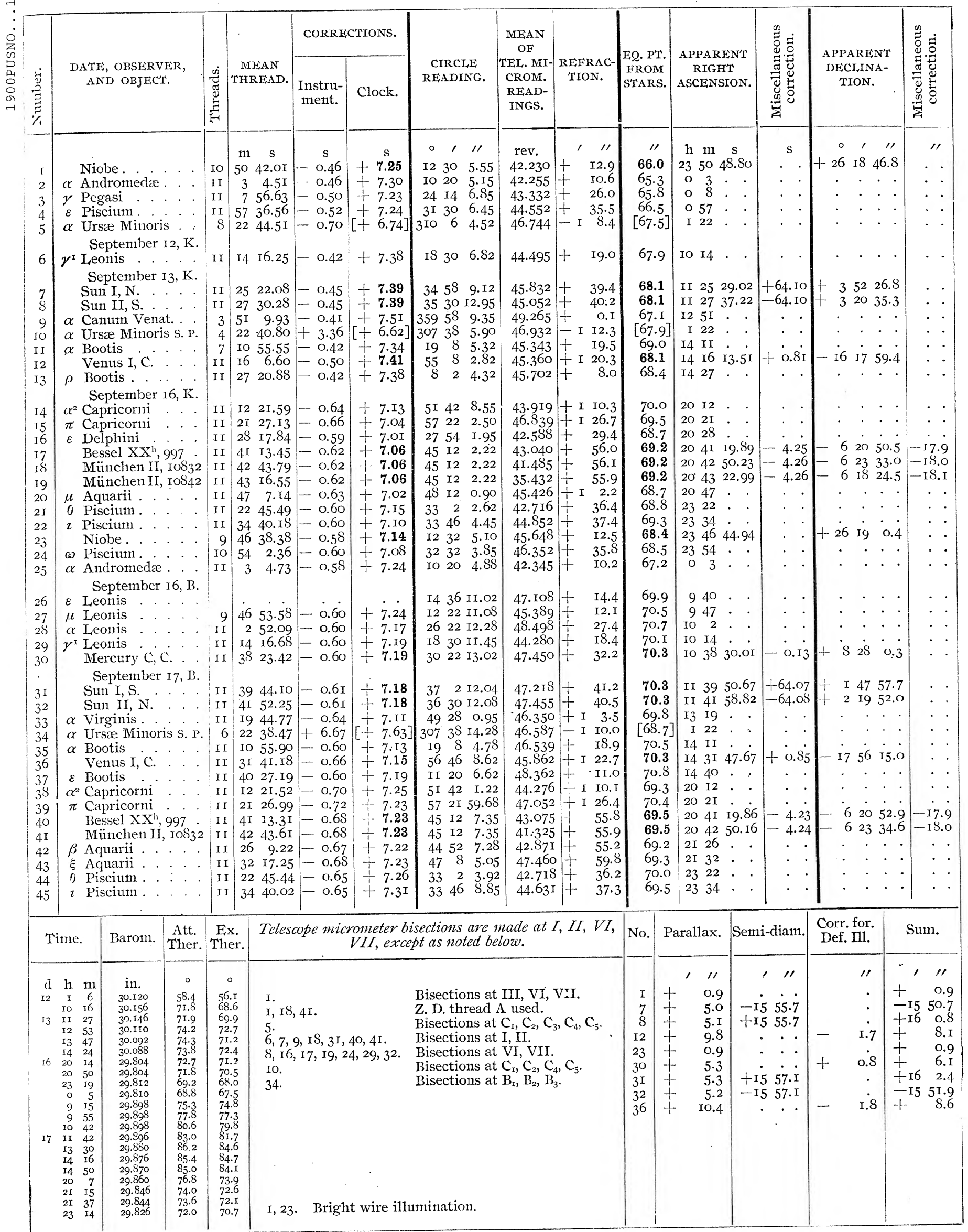

$6855-16$ 


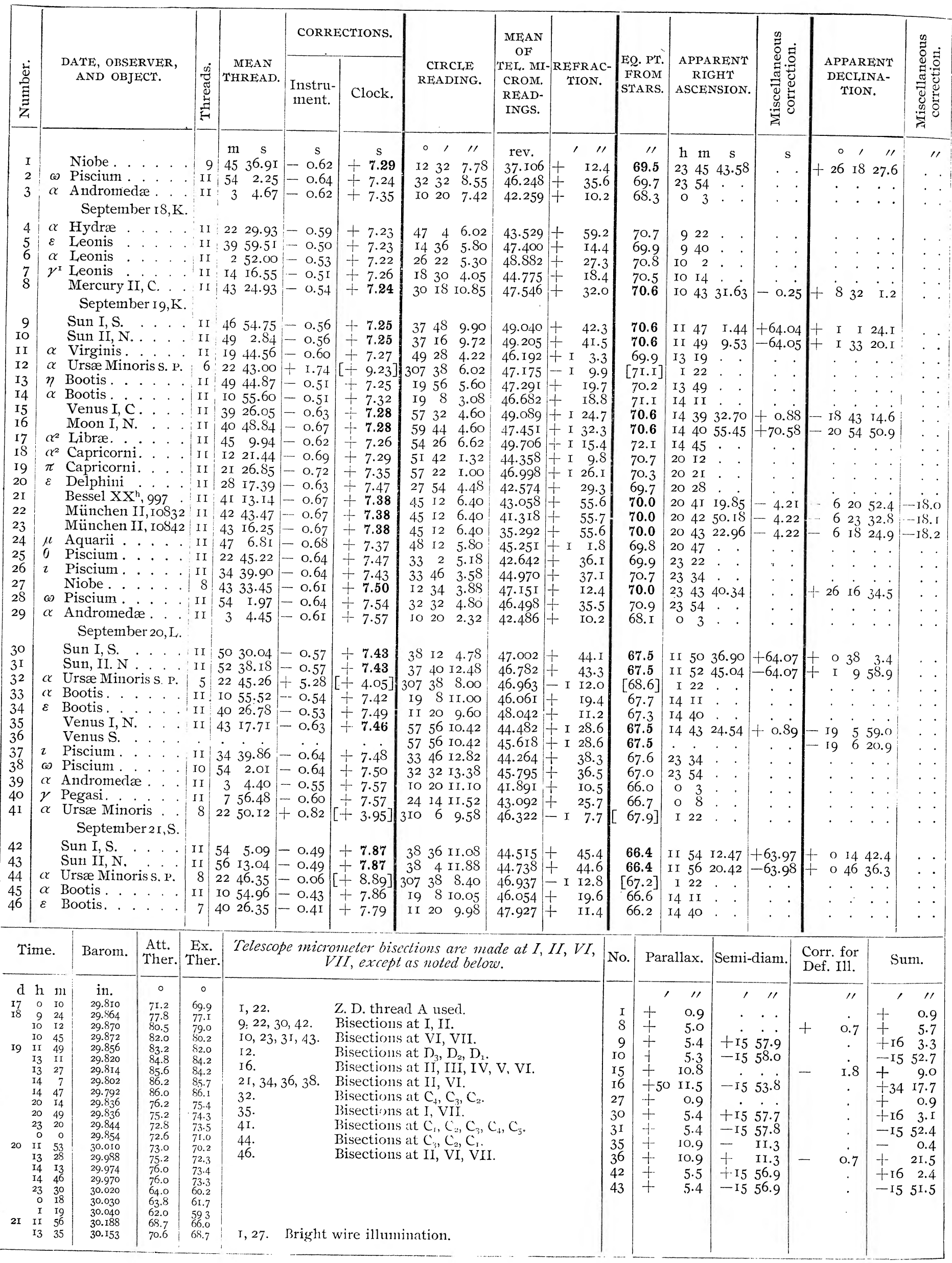




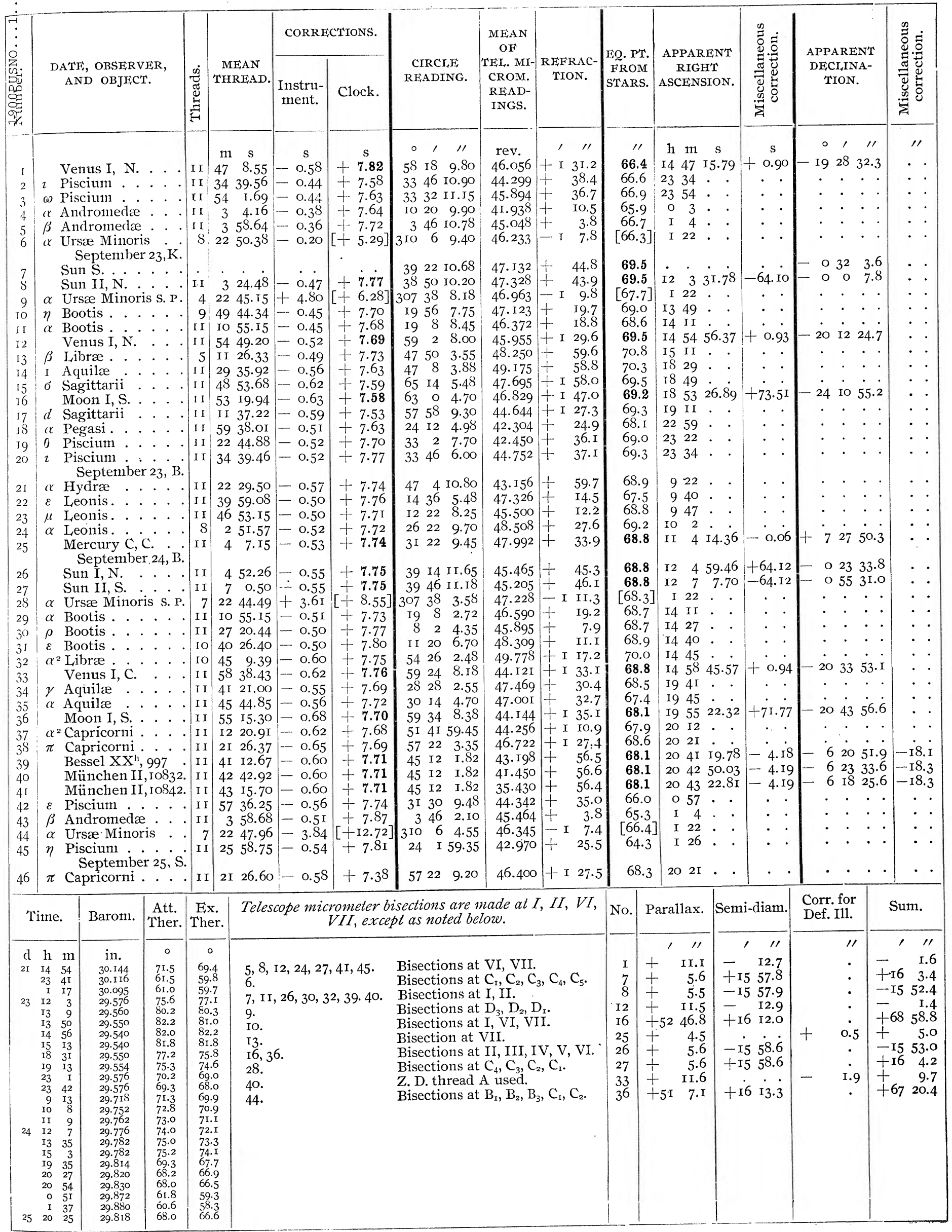




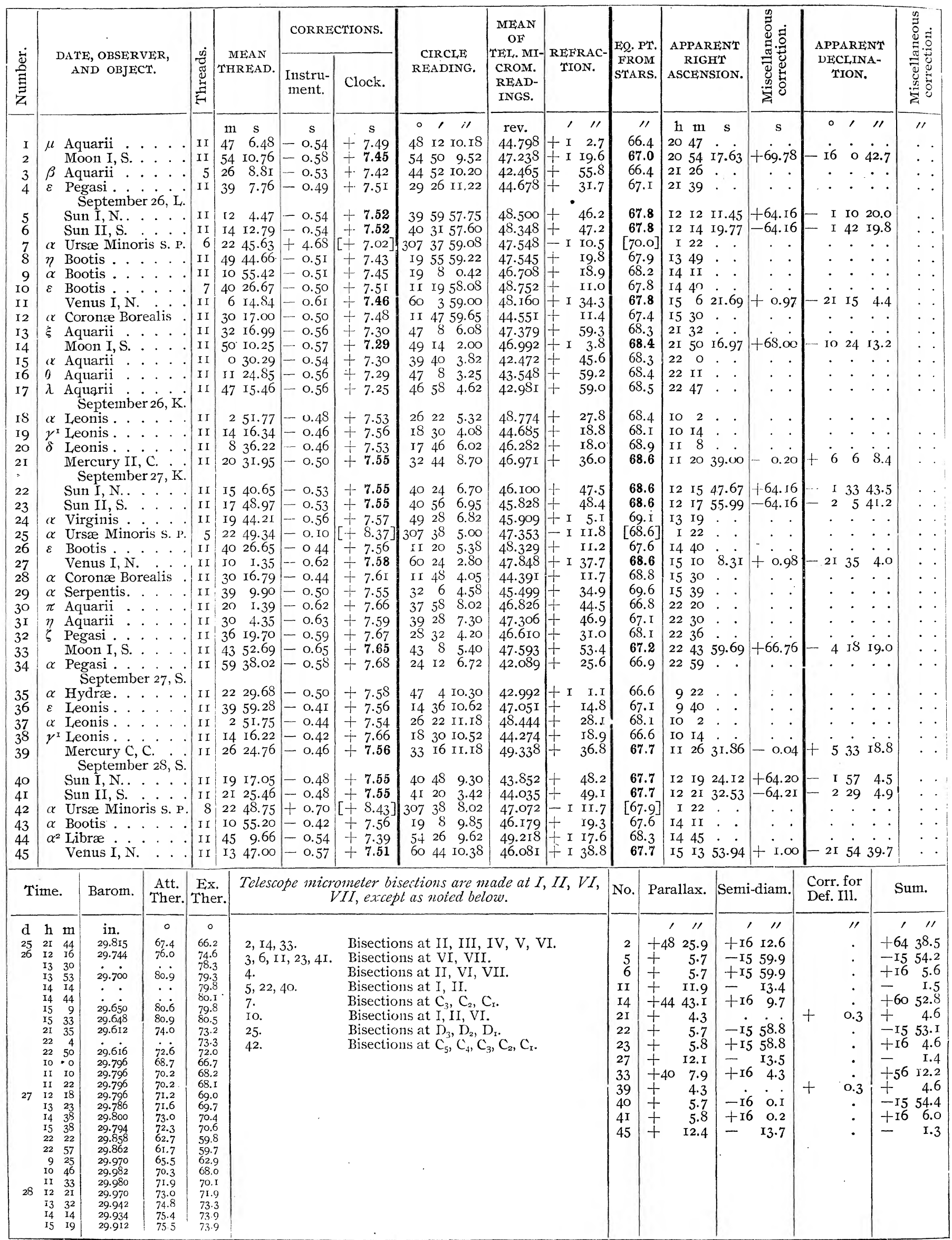




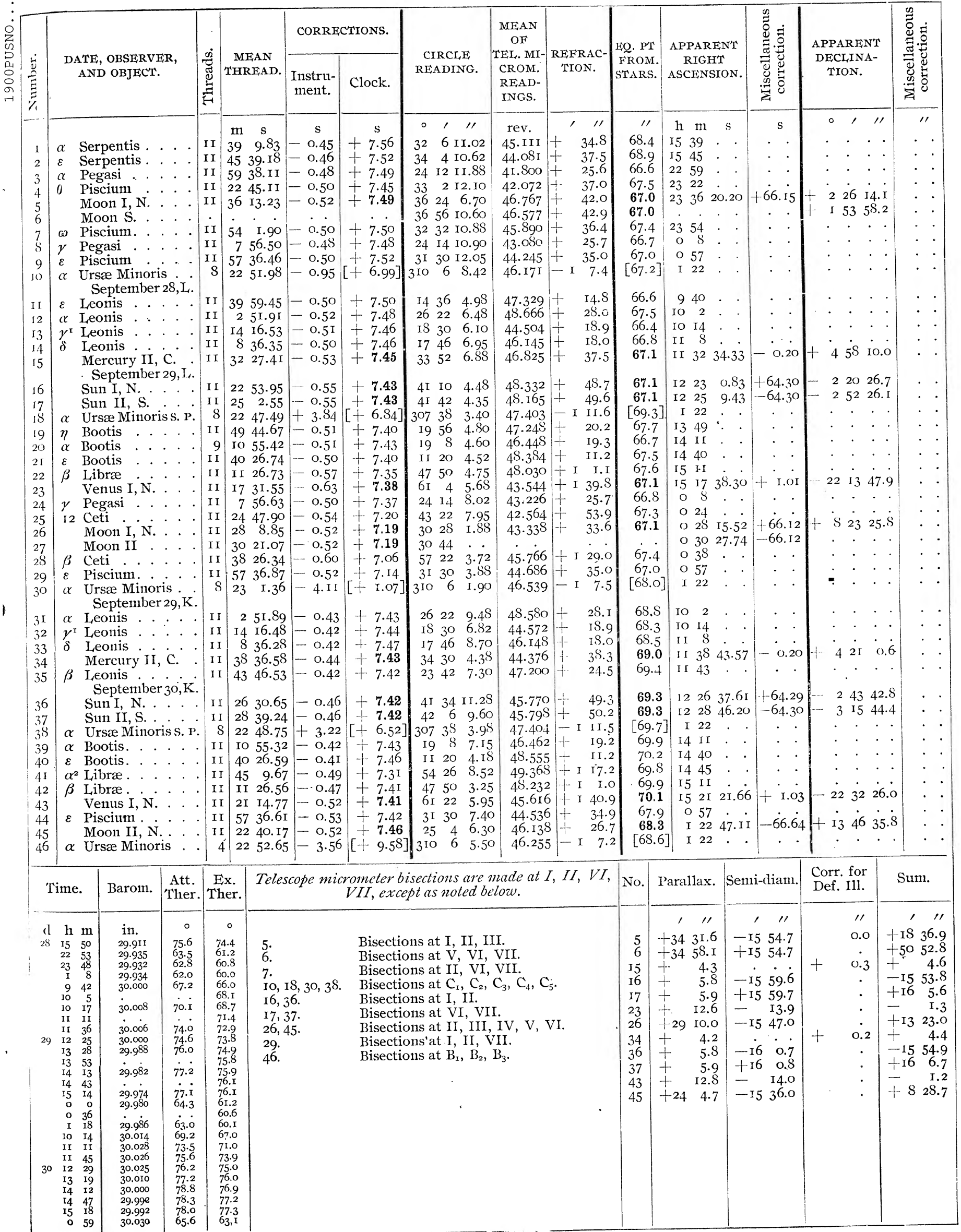




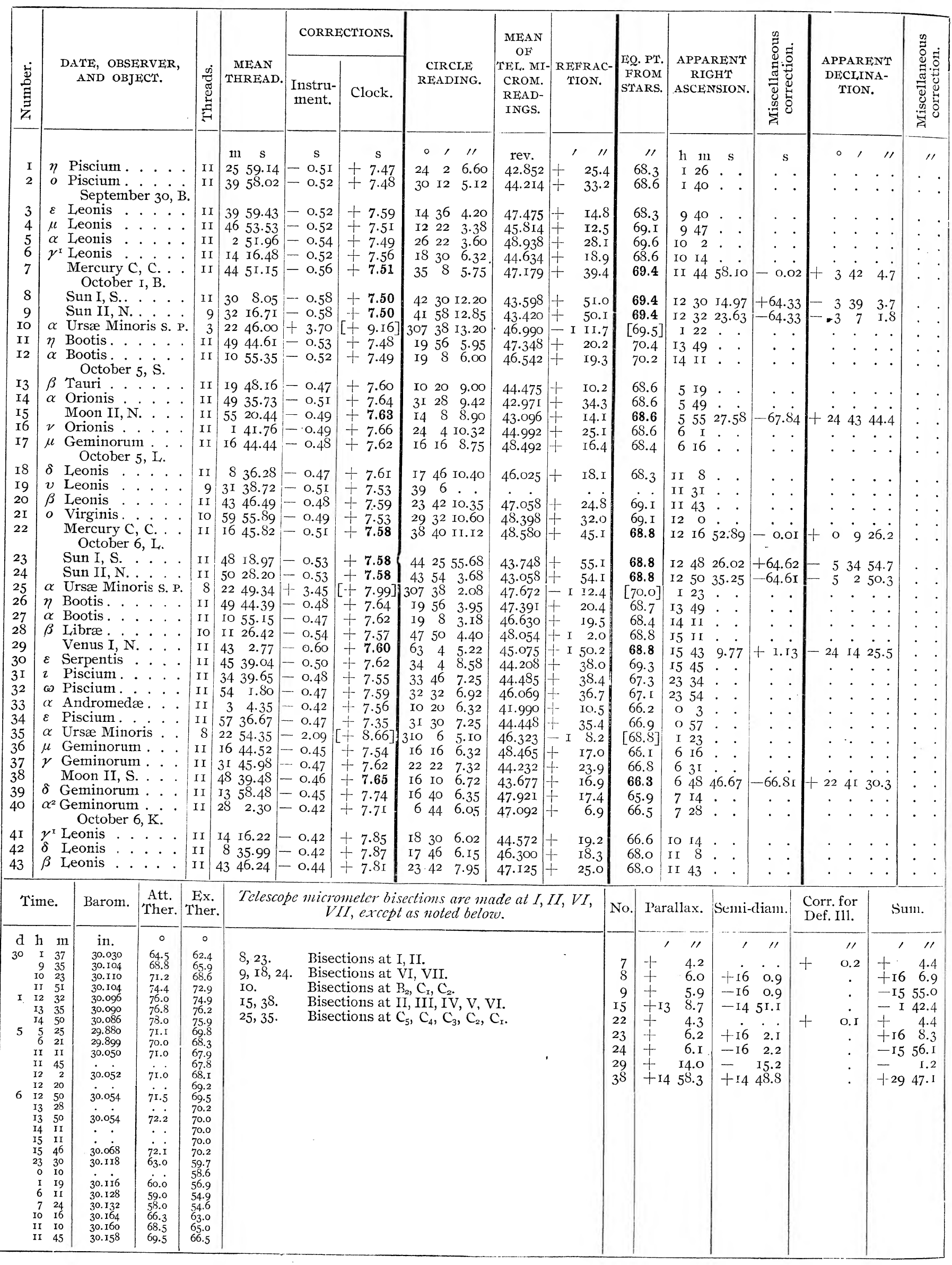




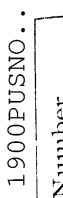
AND OBJECT.

\begin{tabular}{|c|c|c|c|}
\hline \multirow{2}{*}{ 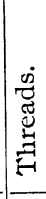 } & \multirow{2}{*}{$\begin{array}{l}\text { MEAN } \\
\text { THREAD }\end{array}$} & \multicolumn{2}{|c|}{ CORRECTIONS. } \\
\hline & & $\begin{array}{l}\text { Instru- } \\
\text { ment. }\end{array}$ & Clock. \\
\hline & $\mathrm{m} s$ & s & \\
\hline
\end{tabular}

October $7, \mathrm{~K}$

Sun I, N. . . . . II 5 I $57.96-0.50+7.82$

Sun II, S. . . . . . II $54 \quad 7.37-0.50+7.82$

$\alpha$ Ursæ Minoris s. P. $8{ }_{22} 5^{1} .72+0.69[+8.63]$

$\eta$ Bootis..... II $4944.10-0.43+7.88$

$\alpha$ Bootis . . . . II IO $54.83-0.42+7.89$

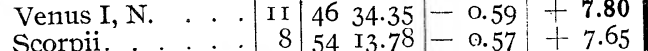

October $9, \mathrm{~L}$

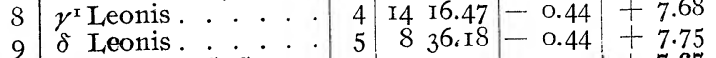

Mercury C, C. . . II $4222.19-0.5 \mathrm{I} \mid+\mathbf{7 . 6 7}$

October IO, L.

Sun I, N. . . . II $259.01-0.52+7.66$

Sun II, S. . . . . II $58.75-0.52+7.66$

$13 \alpha$ Ursæ Minoris S. P. 4 22 $5 \mathrm{r} .48+\mathrm{I} .25[+8.7 \mathrm{I}]$

I4 $\eta$ Bootis. ..... $99 \begin{array}{r}4944.33-0.44+7.65 \\ \text { IO } 55 . \text { II }-0.44+7.63\end{array}$

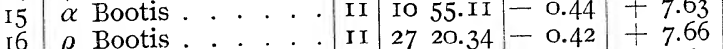

I6 $\rho$ Bootis...... II $2720.34=0.42+7.66$

I8 $\alpha$ Serpentis . . . . II $399.66-0.48+7.62$

I9 $\delta$ Scorpii. . . . II 54 I3.85 - $0.59+7.57$

20 Venus I, $\dot{N}$. . II $5656.35-0.6 \mathrm{I}+7.60$ October Io, $\mathrm{Br}$.

Moon'II . . . . II $545.34-0.44+7.54$

$22 \gamma^{\mathrm{x}}$ Leonis..... II I4 $16.60-0.40+7.54$

23 Mercury C, C. . II $4843.65-0.47+\mathbf{7 . 5 5}$

October II, Br.

24 Sun I, S. . . . . . II $640.25-0.48+7.55$

25 Sun II, N. . . . . II 8 II $80.07-0.48+7.55$

$26 \propto$ Bootis ...... II 1 I0 55.14 - o.40 + 7.56

$27 \varepsilon$ Bootis . . . . . II $4026.39-0.38+7.54$

Venus I, N.

October II, S.

$29 \gamma^{\mathrm{I}}$ Leonis.

Moon II . . II 53 II.87 $=0.52+7.61$

$\beta$ Leonis. . . . . II $4346.59-0.46+7.55$

Mercury C, C.

October I $2, \mathrm{~S}$

Sun I, N.

Sun II, S.

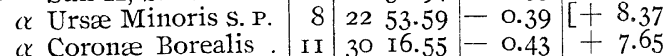

II 39 - $9.73-0.43+7.65$

Venus I N.

$\delta$ Ophiuchi

II $855.45-0.53+7.54$

$\alpha$ Scorpii. . . . . II $235.24-0.65+7.53$

$\varepsilon$ Piscium . . . $\quad$ II $5736.54-0.48+7.52$

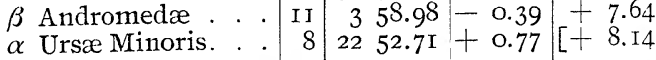

\begin{tabular}{|c|c|c|c|c|c|c|c|}
\hline $\begin{array}{c}\text { CIRCLE } \\
\text { READING. }\end{array}$ & $\begin{array}{c}\text { MEAN } \\
\text { OF } \\
\text { TEL. MI- } \\
\text { CROM. } \\
\text { READ- } \\
\text { INGS. }\end{array}$ & $\begin{array}{c}\text { REFRAC- } \\
\text { TION. }\end{array}$ & $\begin{array}{c}\text { EQ. PT. } \\
\text { FROM } \\
\text { STARS. }\end{array}$ & $\begin{array}{l}\text { APPARENT } \\
\text { RIGHT } \\
\text { ASCENSION. }\end{array}$ & 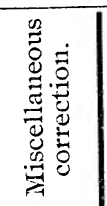 & $\begin{array}{l}\text { APPARENT } \\
\text { DECLINA- } \\
\text { TION. }\end{array}$ & 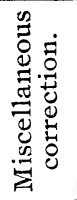 \\
\hline 0,111 & rev. & 111 & 11 & $\mathrm{~h} \quad \mathrm{~m} / \mathrm{s}$ & & 0,11 & "I \\
\hline 44 I6 ro. 48 & 45.768 & $+\quad 55.1$ & 68.0 & I2 $52 \quad 5.28$ & +64.70 & - 52549.0 & \\
\hline $\begin{array}{lll}44 & 48 & 6.88\end{array}$ & & $+\quad 56.2$ & 68.0 & I2 54 I 4.69 & $-64.7 \mathrm{I}$ & $-\quad 55752.8$ & \\
\hline $\begin{array}{lll}307 & 3^{8} & 8.08\end{array}$ & $47 \cdot 300$ & {$\left[\begin{array}{lll}-\mathrm{I} & \mathrm{I} & 2.7\end{array}\right]$} & {$[68.0]$} & I 23 & & & \\
\hline I9 $56 \quad 6.15$ & 47.282 & $+\quad 20.5$ & $68.7^{-}$ & I3 49 & & . & \\
\hline 1988.28 & 46.478 & +19.6 & 8.5 & I4 II : & & {$[-2420420$} & \\
\hline $\begin{array}{lll}63 & \text { I8 } & 4.55\end{array}$ & 49.001 & + I 5 I. 3 & 68.0 & $\mid \begin{array}{lll}\text { I5 } & 46 & 4 \mathrm{I} .56\end{array}$ & + I.I 4 & -242942.0 & \\
\hline 6 I 10 I0.75 & 44.110 & + I 41.6 & 68.3 & I5 54. & & - $\cdot$ & \\
\hline $\begin{array}{l}\text { I8 } 30 . \\
\text { I7 } 46 .\end{array}$ & & . & . & $\begin{array}{rrl}\text { IO } & \text { I4 } & . \\
\text { II } & 8 & .\end{array}$ & & - & \\
\hline $\begin{array}{lll}41 & 42 & 4.95\end{array}$ & 43.424 & $+\quad 50.2$ & 66.4 & $\begin{array}{lll}12 & 42 & 29.35\end{array}$ & 0.00 & $-\quad 25056.2$ & \\
\hline $\begin{array}{lll}45 & 24 & 6.68 \\
45 & 56 & 6.52\end{array}$ & 5 & 57.1 & 66.4 & I3 3 & +64.87 & $6342 \mathrm{I} . \mathrm{I}$ & \\
\hline $\begin{array}{rrr}45 & 56 & 6.52 \\
307 & 37 & 55.40\end{array}$ & 622 & $+\quad 58.2$ & 66.4 & I3 5 I 5.89 & -64.87 & $7627 . \mathrm{I}$ & . \\
\hline $\begin{array}{rrr}307 & 37 & 55.40 \\
19 & 56 & 5.72\end{array}$ & 48.016 & $-\mathrm{I} \quad \mathrm{I} 2.5$ & [68. I] & $\begin{array}{llll} & 1 & 23 & \end{array}$ & . & $\cdot \cdot \cdot \cdot$ & · \\
\hline $\begin{array}{lll}\text { I9 } & 56 & 5.72 \\
\text { I9 } & 8 & 5.40\end{array}$ & 95 & $+\quad 20.4$ & 66.0 & I3 49. & $\cdot$ & 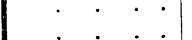 & \\
\hline $\begin{array}{rll}19 & 8 & 5.40 \\
8 & 2 & 4.50\end{array}$ & & $+\quad 19.5$ & 66.3 & I4 II . & . & $. \quad . \quad . \quad$. & • \\
\hline $\begin{array}{rrr}8 & 2 & 4.5 \\
\text { II } & 20 & 3.7\end{array}$ & $\begin{array}{l}45.835 \\
48.454\end{array}$ & $\left|\begin{array}{rr}+ & 8.0 \\
+ & \text { II. } 3\end{array}\right|$ & $\begin{array}{l}66.6 \\
65.9\end{array}$ & $\begin{array}{l}\text { I4 } 27 \\
\text { I4 } 40\end{array}$ & & 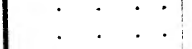 & \\
\hline $\begin{array}{lrl}11 & 6 & 3.70\end{array}$ & 45 & $\begin{array}{l}11.3 \\
+\quad 35.1\end{array}$ & $\begin{array}{l}0.9 \\
66.6\end{array}$ & $\begin{array}{l}1440 \\
1539\end{array}$ & . & . $\quad$. & \\
\hline $6 \mathrm{r}$ io 4.92 & 4 & + I $4 \mathrm{I} .5$ & 67.3 & I5 54 & & 35 T2 308 & \\
\hline $\begin{array}{lll}64 & 2 & 4.48\end{array}$ & 45.029 & + I 54.7 & 66.4 & I5 $57 \quad 3.34$ & $+\mathrm{I} .2 \mathrm{O}$ & $\begin{array}{lll}-25 & 12 & 30.8\end{array}$ & $\cdot$ \\
\hline 3 I 30 & & & & IO $55^{2.44}$ & -63.16 & & \\
\hline I8 $30 \quad 4.88$ & 44.625 & I9.0 & 67.0 & $10 \mathrm{I4}$ & & 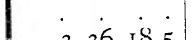 & \\
\hline $\begin{array}{lll}42 & 26 & 4.35\end{array}$ & 47.806 & 50.7 & 68.0 & I2 4850.73 & 0.00 & - 336 I8.5 & \\
\hline $\begin{array}{lll}46 & 18 & 5.70\end{array}$ & 49.888 & $+\quad 58.0$ & 68.0 & I3 $\quad 6 \quad 47.32$ & $+64.9 \mathrm{I}$ & - $7296 . x$ & \\
\hline 4546 II.I5 & 49.298 & $+\quad 5^{6.9}$ & 68.0 & I3 857.14 & $-64.9 I$ & -657 1.o & \\
\hline $198 \quad 5.22$ & $46.5^{89}$ & I9. 2 & 68.5 & I4 II & & $\cdot \cdot \cdot$ & - \\
\hline II $20 \quad 3.98$ & 48.578 & It II.I & 68.4 & I 440 & $1+22$ & -2525436 & \\
\hline $\begin{array}{lll}64 & \text { I4 } & 4.08\end{array}$ & 49.068 & t I 53.2 & 68.0 & I6 $\quad 025.62$ & +1.22 & -252543.6 & \\
\hline I8 $30 \quad 10.02$ & $44.45^{2}$ & $+\quad 18.9$ & $67 . \mathrm{I}$ & Io 14 & & & . \\
\hline 3656 & & & & I0 53 18.96 & $-63 \cdot 36$ & . & - \\
\hline $23 \quad 42$ II.9O & 46.978 & $\begin{array}{l}+24.7 \\
+\quad 52.6\end{array}$ & $\begin{array}{l}68.0 \\
67.8\end{array}$ & 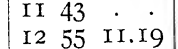 & o.0o & 4 2 1 I 34.6 & . \\
\hline $\begin{array}{lll}43 & \text { I2 } & 4.70\end{array}$ & $45 \cdot 388$ & $5^{2.0}$ & 06.0 & 125511.49 & & & \\
\hline 46 Io 4.80 & & 58.3 & $\begin{array}{l}67.8 \\
67.8\end{array}$ & $\begin{array}{lll}\text { r3 } & \text { 10 } & 29.10 \\
\text { 13 } & \text { 12 } & 38.98\end{array}$ & +64.94 & 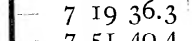 & \\
\hline $\begin{array}{lll}46 & 42 & 5.22\end{array}$ & & $+\quad 59.4$ & $\begin{array}{c}67.8 \\
{[67.3]}\end{array}$ & $\begin{array}{rrr}I_{3} & \text { I2 } & 38.98 \\
\text { I } & 23 & .\end{array}$ & -64.94 & $75 \mathrm{I} 40.4$ & - \\
\hline $3073^{8}$ II. 35 & 47.162 & - I 12.2 & {$\left[\begin{array}{c}{[67.3} \\
67.5\end{array}\right.$} & $\begin{array}{r}\text { I } 23 . \\
1530 .\end{array}$ & $\cdot$ & . & 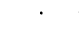 \\
\hline II 488.50 & 14.200 & III.7 & 67.5 & 1530 & & $\cdot$ & - \\
\hline $3^{2} 610.05$ & $45 \cdot 176$ & $+\quad 35$ & 67.9 & $\begin{array}{llll}15 & 39 & 8 & 0\end{array}$ & $+\mathrm{I} 24$ & $-253^{8} 26.9$ & . \\
\hline 6428 10.4 & 44.504 & +.56 & $\begin{array}{l}67.8 \\
68.6\end{array}$ & $\begin{array}{lll}16 & 3 & 45.16 \\
16 & 9 & .\end{array}$ & & & \\
\hline 42 I6 10.4 & 46.5 & 50. & $\begin{array}{l}68.6 \\
68.0\end{array}$ & $\begin{array}{rr}16 & 9 \\
16 & 23\end{array}$ & & . & \\
\hline 652 Io. & 4 & t 159.5 & $\begin{array}{l}68.0 \\
66.0\end{array}$ & $\begin{array}{rl}16 & 23 \\
\text { O } 57\end{array}$ & & • & . \\
\hline 3I 30 II.I2 & & $35 \cdot 4$ & $\begin{array}{l}66.0 \\
66.0\end{array}$ & $\begin{array}{l}\text { O } 57 \\
\mathrm{I} \quad 4\end{array}$ & & 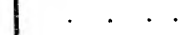 & \\
\hline $\begin{array}{rrr}3 & 46 & 4.80 \\
310 & 6 & 8.95\end{array}$ & $\begin{array}{l}45.159 \\
45.869\end{array}$ & $\begin{array}{ll}t & 3.9 \\
-I & S . I\end{array}$ & $\left.\begin{array}{c}60.0 \\
{[66.4]}\end{array}\right]$ & ] $] \begin{array}{rr}1 & 4 \\
1 & 23\end{array}$ & & 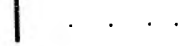 & . \\
\hline
\end{tabular}

43 a Ursa Mate

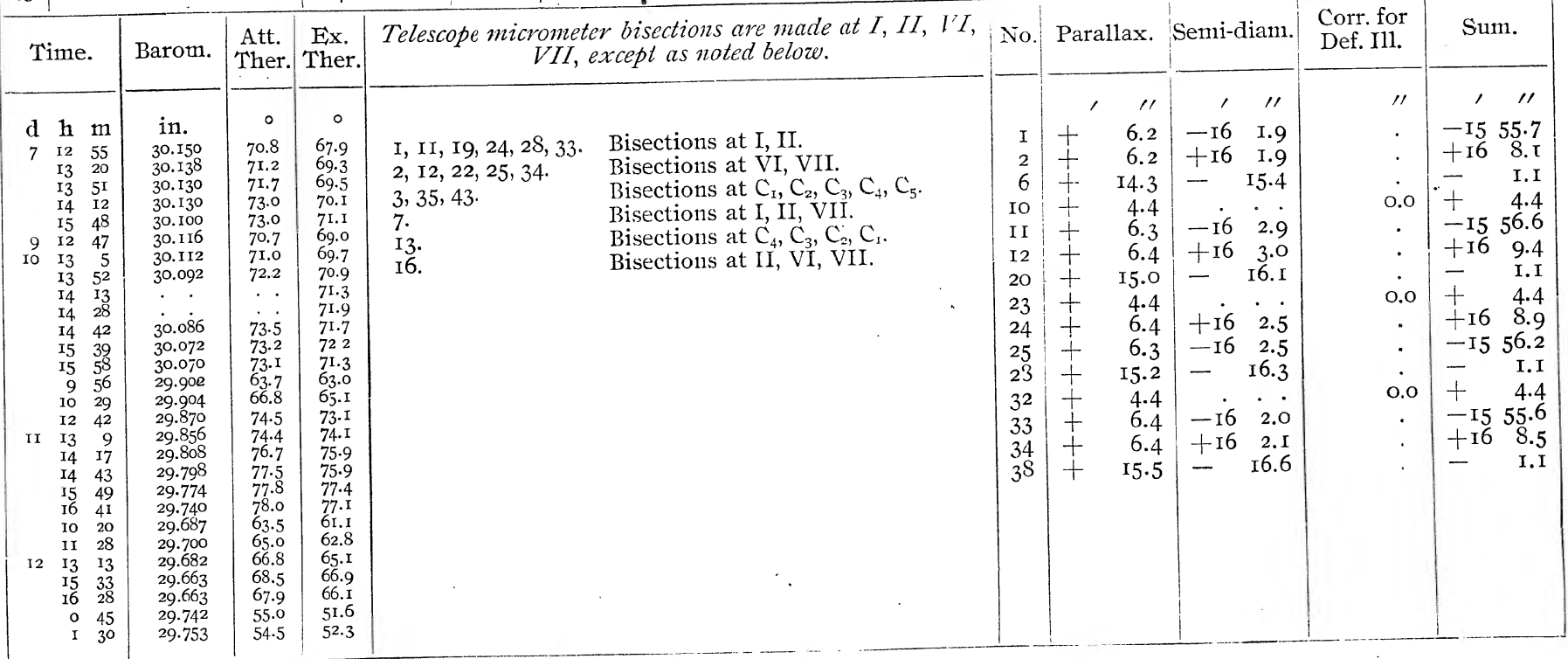




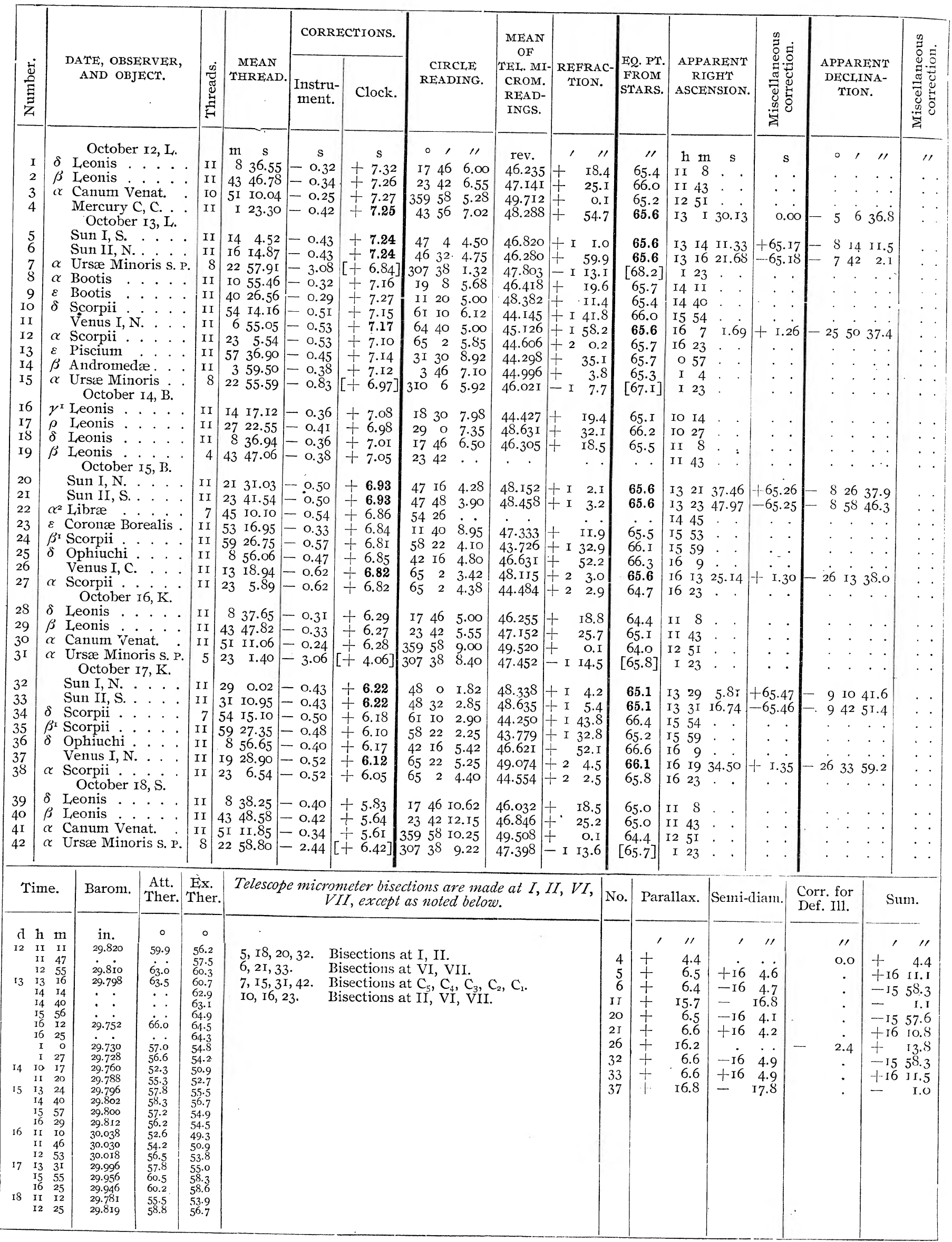




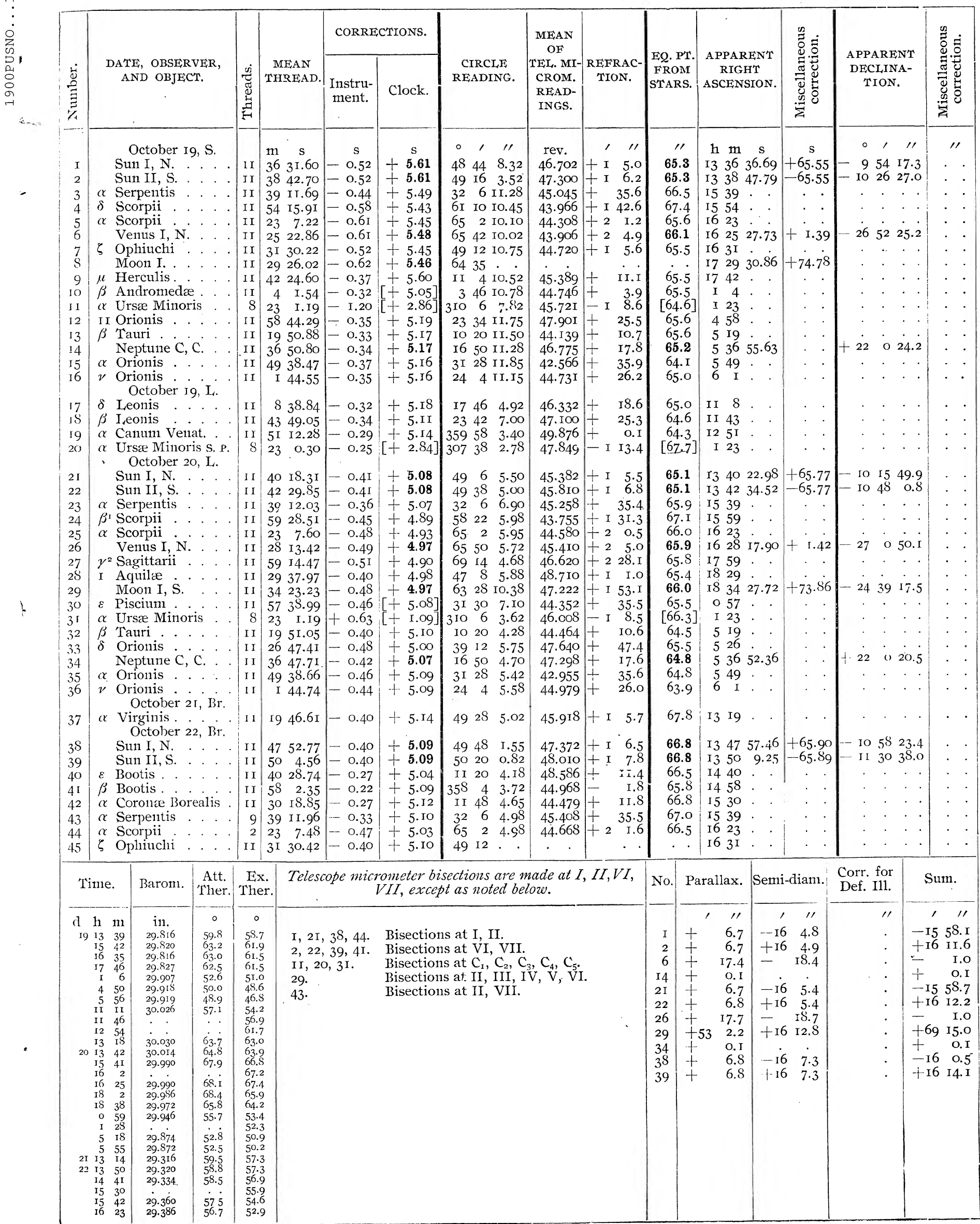




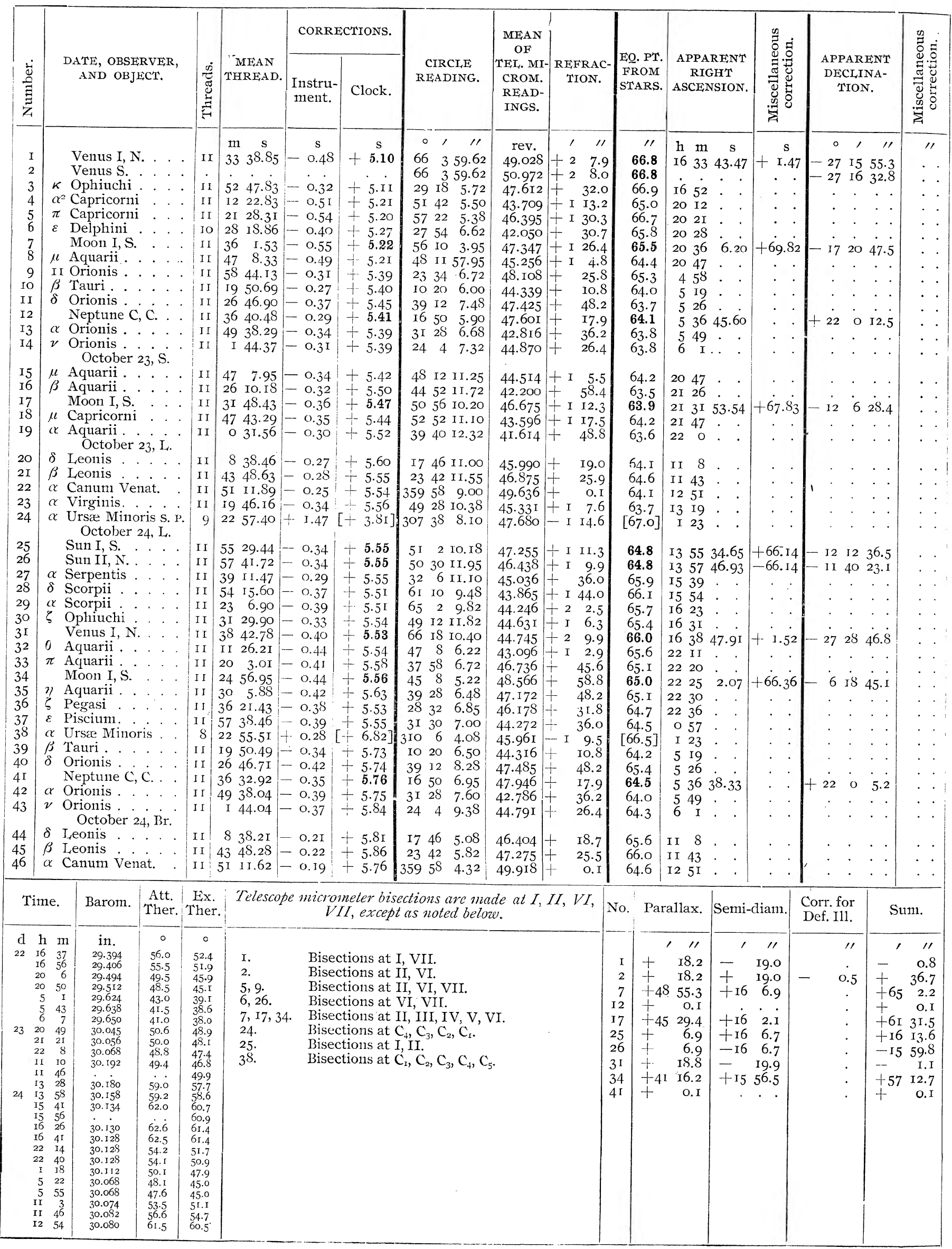




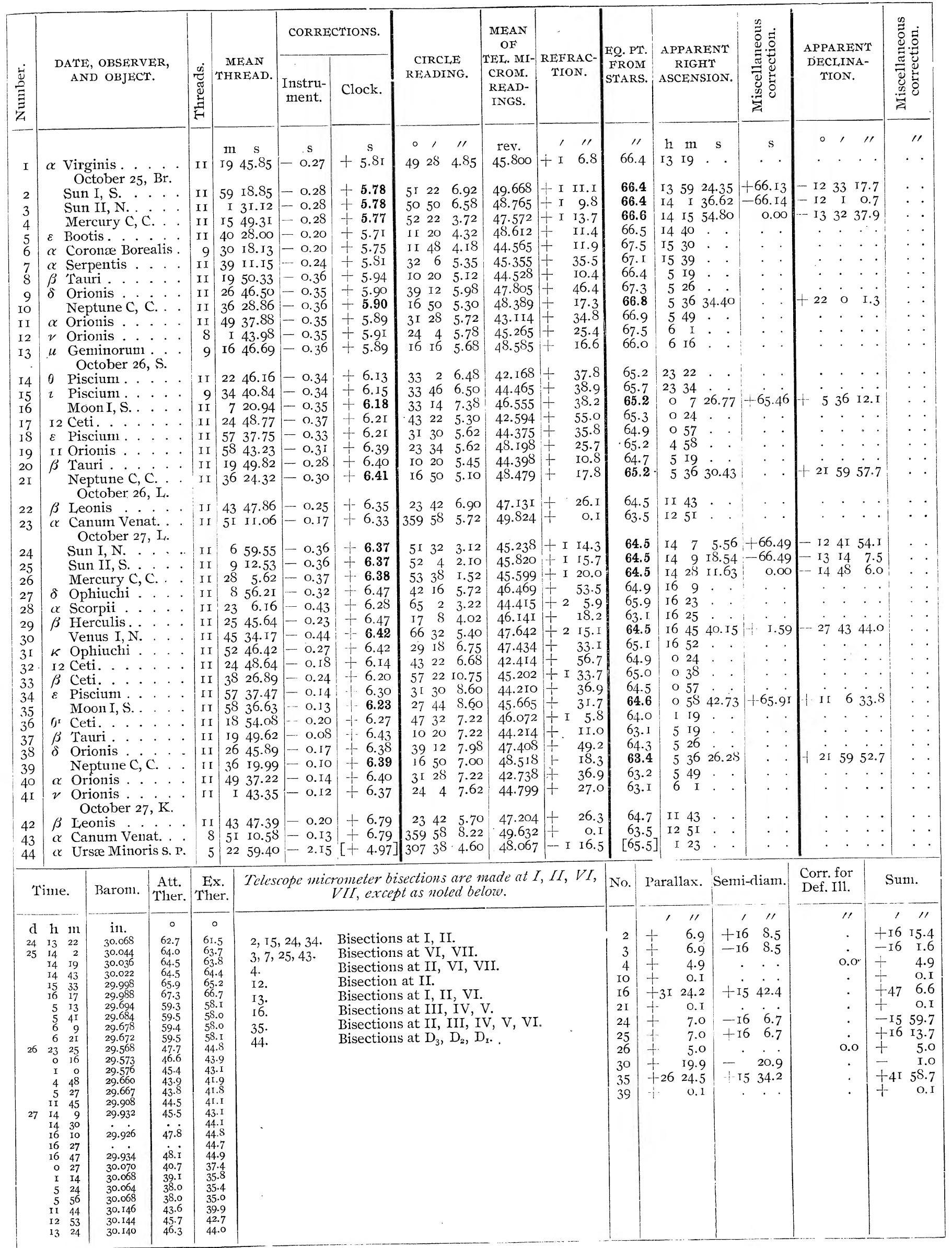




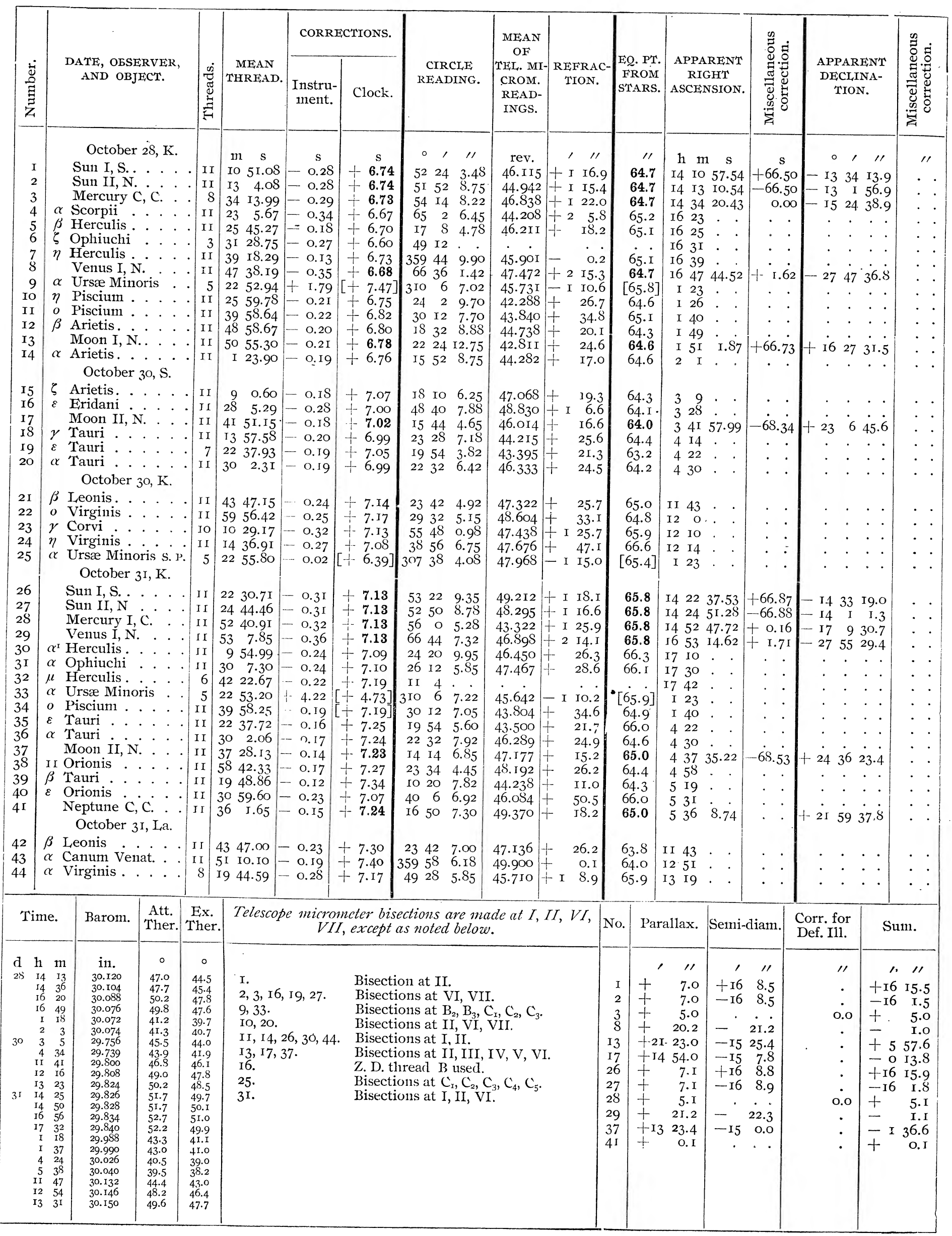




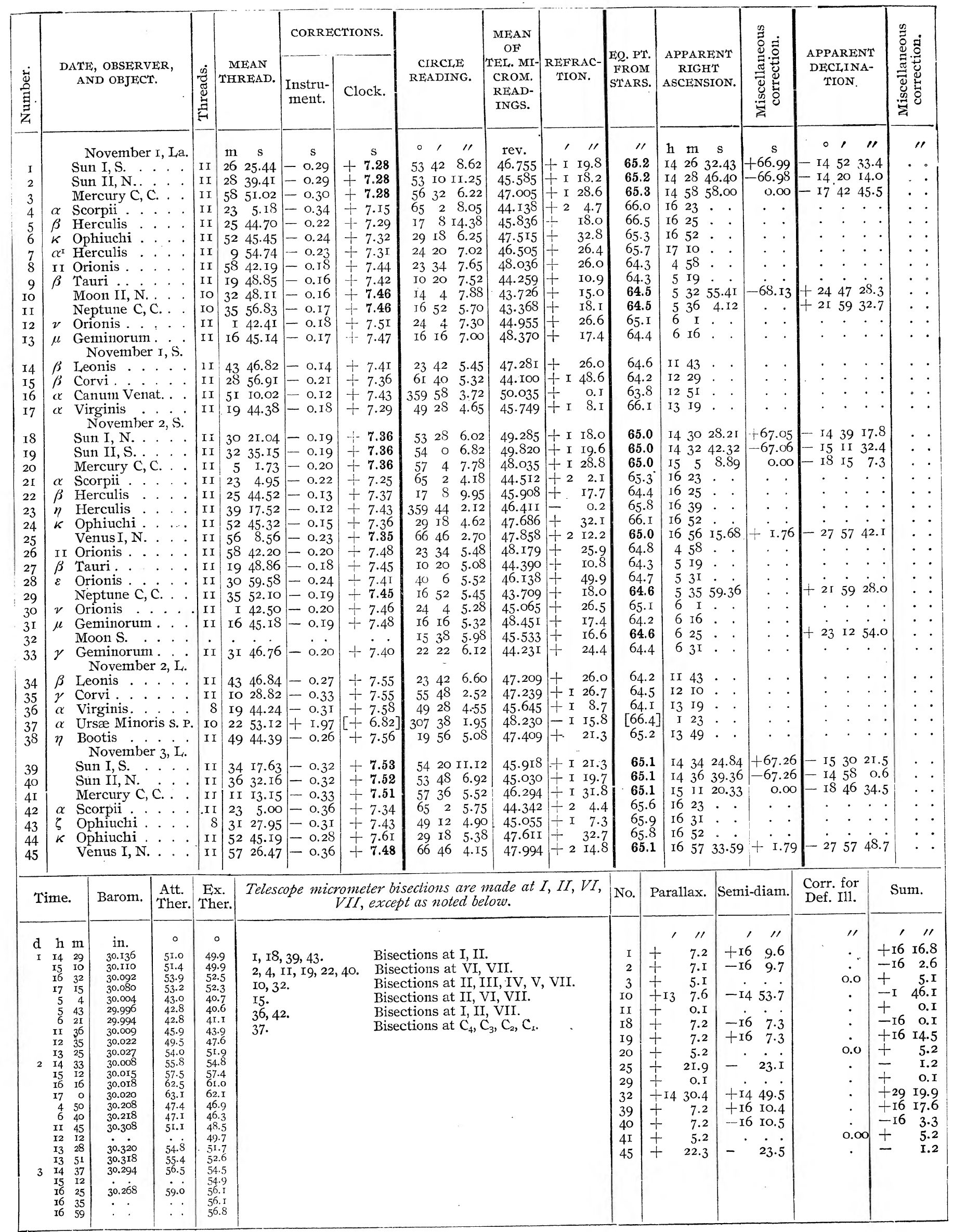




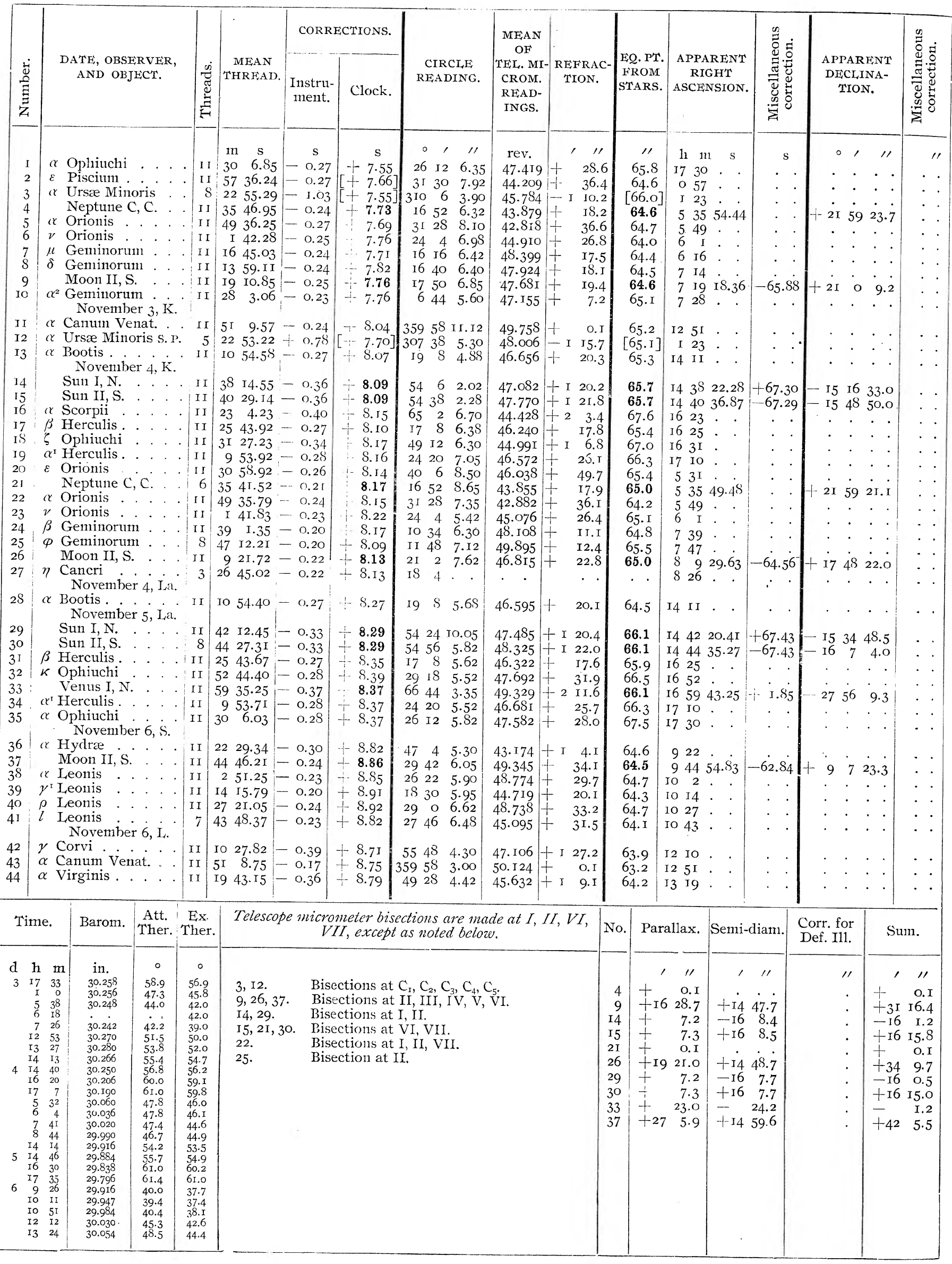




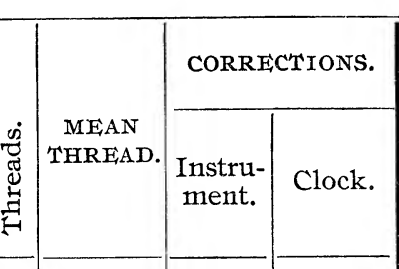

$\alpha$ Ursæ Minoris s. P. $\left.\quad 6 \mid \begin{array}{ccc}22 & 56.08 & -3.69 \mid[+8.32\end{array}\right]$ 307 $38 \quad 2.50$ $4943.19-0.24+8.79$ I9 564.22 November $7, \mathrm{~L}$

Sun I, N. . . . II 50 II.I $7-0.39+8.81$

Sun II, S. . . . . II $5226.67-0.39+8.81$

Mercury C, C. . . II $365.3 \mathrm{I}-0.42+\mathbf{8 . 8 4}$

$\delta$ Ophiuchi . . II $853.77-0.33+8.9$ I

$\beta$ Herculis . . . II $2543.16-0.23+8.8 \mathrm{I}$

$\kappa$ Ophiuchi . . . II $5243.86-0.28+8.92$

Venus I, N. . . . II I I $7.19-0.44+8.87$

$\alpha^{\mathrm{I}}$ Herculis. . . . II $953.2 \mathrm{I}-0.26+8.83$

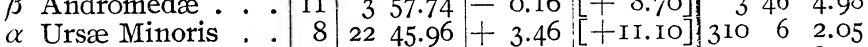

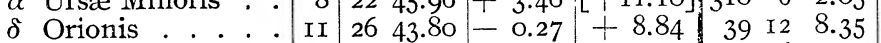

Orionis II $3058.26-0.28+8.89$

Neptune C, C. . . II $3525.13-0.20+8.91$

$\alpha$ Orionis . . . . II 49 35.12 $-0.25+8.90$

Orionis

November $7, \mathrm{Br}$.

$18 \alpha$ Canum Venat. . . $955^{\mathrm{I}} 8.58+0.03+8.34359586 .02$

I9 $\alpha$ Virginis. . . . . II I9 42.84 - o. I4 + I 8.90 49285.22

$20 \propto \alpha$ Bootis . . . . . II IO $53.60-0.04+8.87$ November 8 , Br.

Sun I, N. . . . . II 54 II.66 - 0.16 + 8.87 55 I 87.08

Sun II, S. . . . . II $5627.12-0.16+8.87$

$\beta$ Herculis. . . . . II $2542.93-0.03+8.84$

Ophiuchi ... II 3I $26.35-0.14+8.85$

Venus I, N. . . . II I I $38.74-0.22+8.87$

$\alpha$ Ophiuchi ... II 30 5.24 $-0.06+8.91$

November $\dot{8}, \dot{S}$.

$28 \rho$ Leonis .... II 2720.58 - o.I5 + 9.36

29 Leonis . . . . II 4347.80 - o.I4 + 9.36

$30 \quad \delta$ Leonis . . . . . II $834.96-0.12+9.38$

Moon II, S. . . . II I8 3 I.69-0.18 + 9.37

$\beta$ Leonis . . . II $4345.04-0.14+9.37$ $\begin{gathered}\alpha 3 \text { Ursæ Minoris S. P. } \\ \text { November 9, S. }\end{gathered} \quad 6 \quad 2250.37-2.65[$ [+12.26

Sun I, N. . . . . I I 58 I3.03 $-0.23+9.34$

Sun II, S. . . . . II 028.7 I $-0.23+9.34$

Mercury C, C. . . II $4836.13-0.24+9.33$

$\delta$ Ophiuchi... 7 . 8 53.2I $-0.18+9.32$

a Scorpii . . . . . II $232.99-0.26+9.26$

$\zeta$ Ophiuchi... II 3 I $25.99-0.2 \mathrm{I}+9.28$

Venus I, N. . . . II $\begin{array}{rr}3 & 0.16\end{array}-0.27+9.33$

Herculis... . . II 4220.18 - 0.IO +9.45

November Io, $\mathrm{K}$

$42 \alpha$ Virginis..... II I9 $42.02-0.39$ + 10.03

$43 \alpha$ Ursæ Minoris s. P. 5 22 $50.70-0.68[+9.37$

$44 \mid \eta$ Bootis . . . . . II $|4942.13|-0.3 \mathrm{I} \mid+9.99$

\section{$\begin{array}{rrr}55 & 50 & 9.80 \\ \mathrm{I} 7 & 8 & 4.60\end{array}$}

$\begin{array}{lll}49 & 12 & 8.38\end{array}$

$\begin{array}{lll}66 & 38 & 5.15 \\ 26 & 12 & 6.05\end{array}$

$\begin{array}{lll}26 & 12 & 6.05 \\ & 4 & 4\end{array}$

29 O II.IS

$\begin{array}{lll}27 & 46 & 5.98 \\ 17 & 6 & 6.35\end{array}$

$\begin{array}{lll}17 & 46 & 6.35\end{array}$

$\begin{array}{lll}23 & 42 & 6.25 \\ 3 & 4 & \end{array}$

$\begin{array}{llll}307 & 38 & 3.82\end{array}$

$\begin{array}{rrrr}55 & 34 & 17.85 \\ 56 & 6 & 9.00 \\ 60 & 24 & 4.75\end{array}$

$\begin{array}{lll}60 & 24 & 4.75 \\ 42 & 16 & 4.65\end{array}$

$\begin{array}{lll}65 & 2 & 5.35\end{array}$

$\begin{array}{lll}49 & \text { I2 } & 4.48 \\ 66 & 34 & 3.55\end{array}$

$\begin{array}{lll}66 & 34 & 3.55\end{array}$

II 4 3.98

\begin{tabular}{lll}
49 & 28 & 5.40 \\
\hline 07 & 38 & 9.48
\end{tabular}

$\begin{array}{lll}307 & 38 & 9.48\end{array}$

I9 $56 \quad 7.68$ $\begin{array}{lll}40 & 22 & 6.58 \\ 2 & 4 & 4\end{array}$

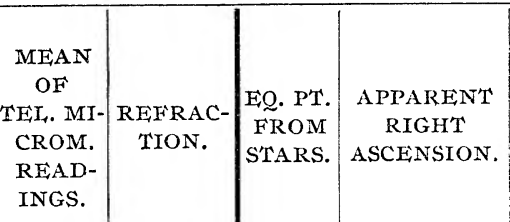

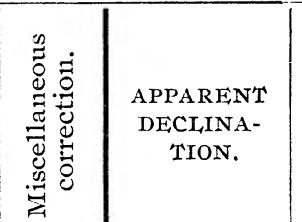

苟

rev., $1 / \quad \mathrm{h} \mathrm{m} \mathrm{s}$

48.3 I0 - I I6.2 [66.6] I 23

$47.419+21.563 .9$ I3 49

$46.385+$ I 24.0

64.0 I4 50 I9.59 +67.75 - i6 Iо 3 1.0

$\mathbf{6 4 . 0}$ i4 52 35.09 -67.75 - I6 42 5 5 I.2

64.0 I5 36 I3.73+0.0I -204255.2

$46.850+$ I 39.7

$46.405+53.3$

46.III

$47.491+32.9$

$47.660+215.2$

$46.478+26.5$

$44.792+$

45.802 - I 10.5

$47.484+49.0\left[\begin{array}{ccc}65.8 & 526 \\ 64.8 & 5\end{array}\right.$

$45.982+50.5633531$

$44.674+\quad 18.2 \quad 63.7 \quad 53533.94$

$42.722+36.7 \quad 63.1 \quad 549$

44.770

$50.054+\quad$ o. I 64.6 I2 $5 \mathrm{I}$

$45.688+\mathrm{I} \quad 8.5 \quad 65.4$ I3 19

$46.728+20.2$

$44.702+$ I 23.7

65.4 i4 $5420.37+67.73-$ - 627256.2

$45.425+\mathrm{I} 25.4$
$46.352+\mathrm{I} 2.8$

$44.87 \mathrm{I}+\mathrm{I} r .5$

$44.040+2$ Ir. 9

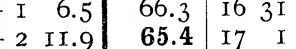

\begin{tabular}{l|l|l|l}
$47.479+28.2$ & 65.6 & I 30 \\
$45.884+\quad$ II. & 65.6 & I 742
\end{tabular}

$48.532+32.8 \quad 64.5$ 10 27

45.I8I + 31.I 64.4 10 43

$46.43 \mathrm{I}+\mathrm{I9.0} 64.4$ II 8 .

$49.04 \mathrm{I}+50.2 \quad 64.6$ II I8 $40.88-63.47-$ I 3247.3

$47.329+25.9 \quad 64.7$ in 43 .

$47.832+$ I 24.0

[65.3] I 22.

65.6 I4 $5822.14+67.84-16 \quad 45 \quad 7.0$

$\begin{array}{llllllllll}65.6 & \text { I5 } & 0 & 37.82 & -67.84 & - & \text { I } 7 & \text { I } 7 & 26.2\end{array}$

47.484 + I 40.7

$46.718+52.0$

$\mathbf{6 5 . 8}$ I5 4845.22 - 0.01 - 2 I 354.8

66.0 I6 9 .

\begin{tabular}{ll|l|ll}
$44.475+2$ & 2.1 & 66.2 & 16 & 23
\end{tabular}

$45.067+\cdot \mathrm{I} \quad 6 . \mathrm{I} \quad 66 . \mathrm{I}$ I6 $3 \mathrm{I}$

$44.295+2$ 10.7 66.1 ir $720.22+1.97-274432.0$

$45.906+$ I1.2 65.7 I7 42

45.735 + I $8.4 \quad 66.6$ I3 19

48.048 - I $15.4[$ [66.7 $]$ I 22

$47.475+2$ I.3 67.3 I3 49

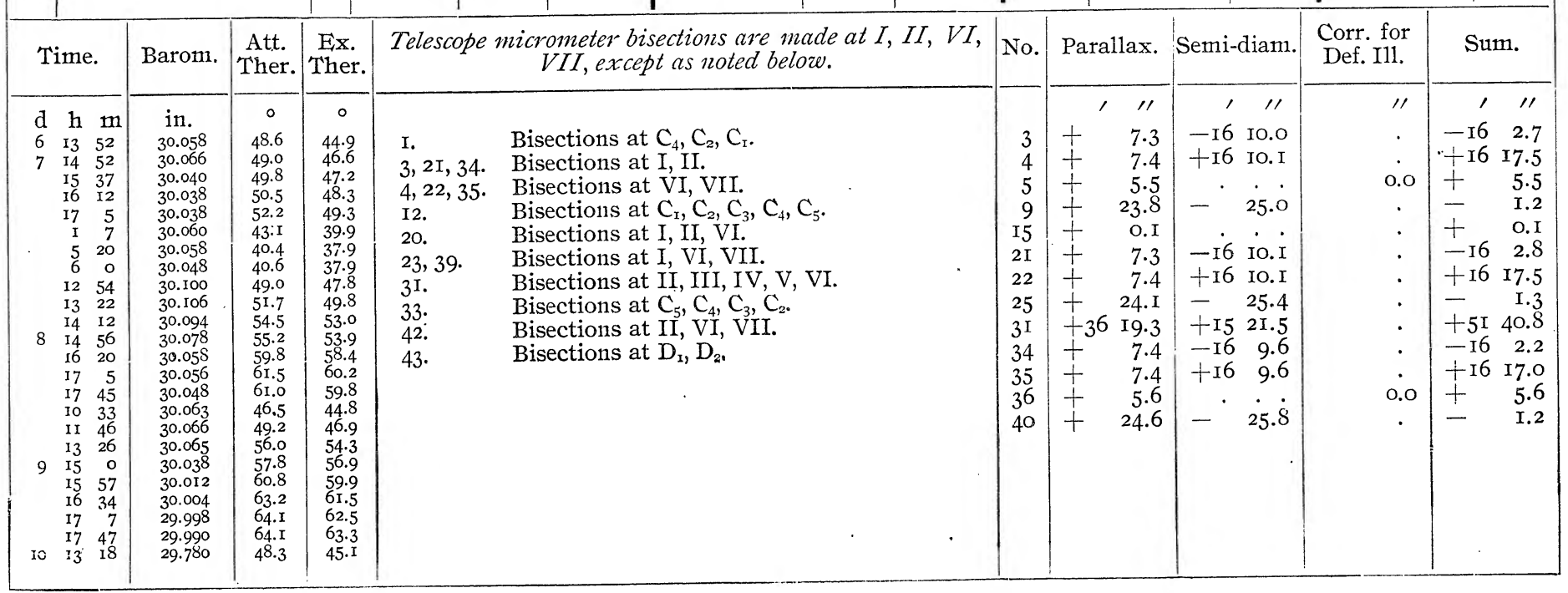




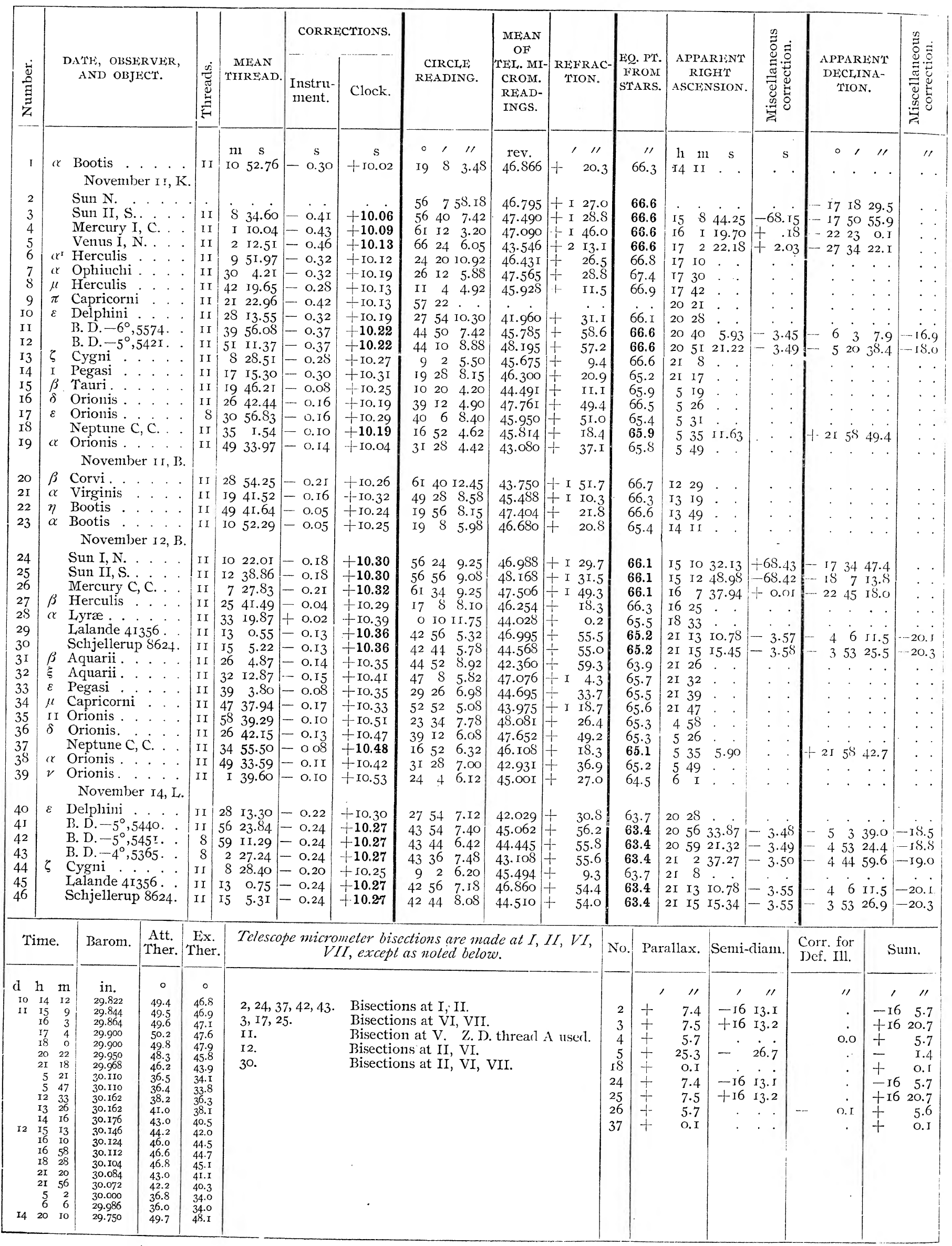




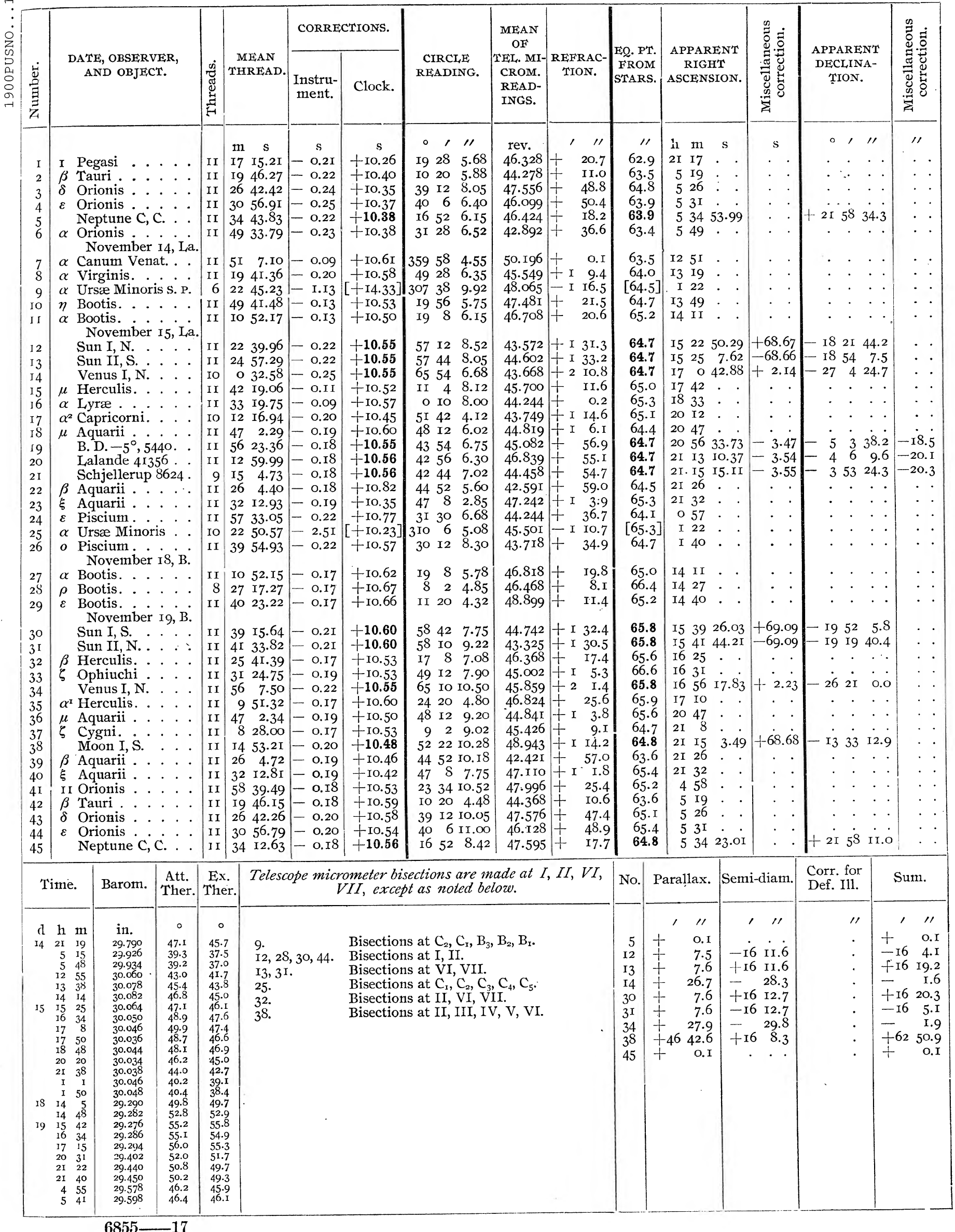




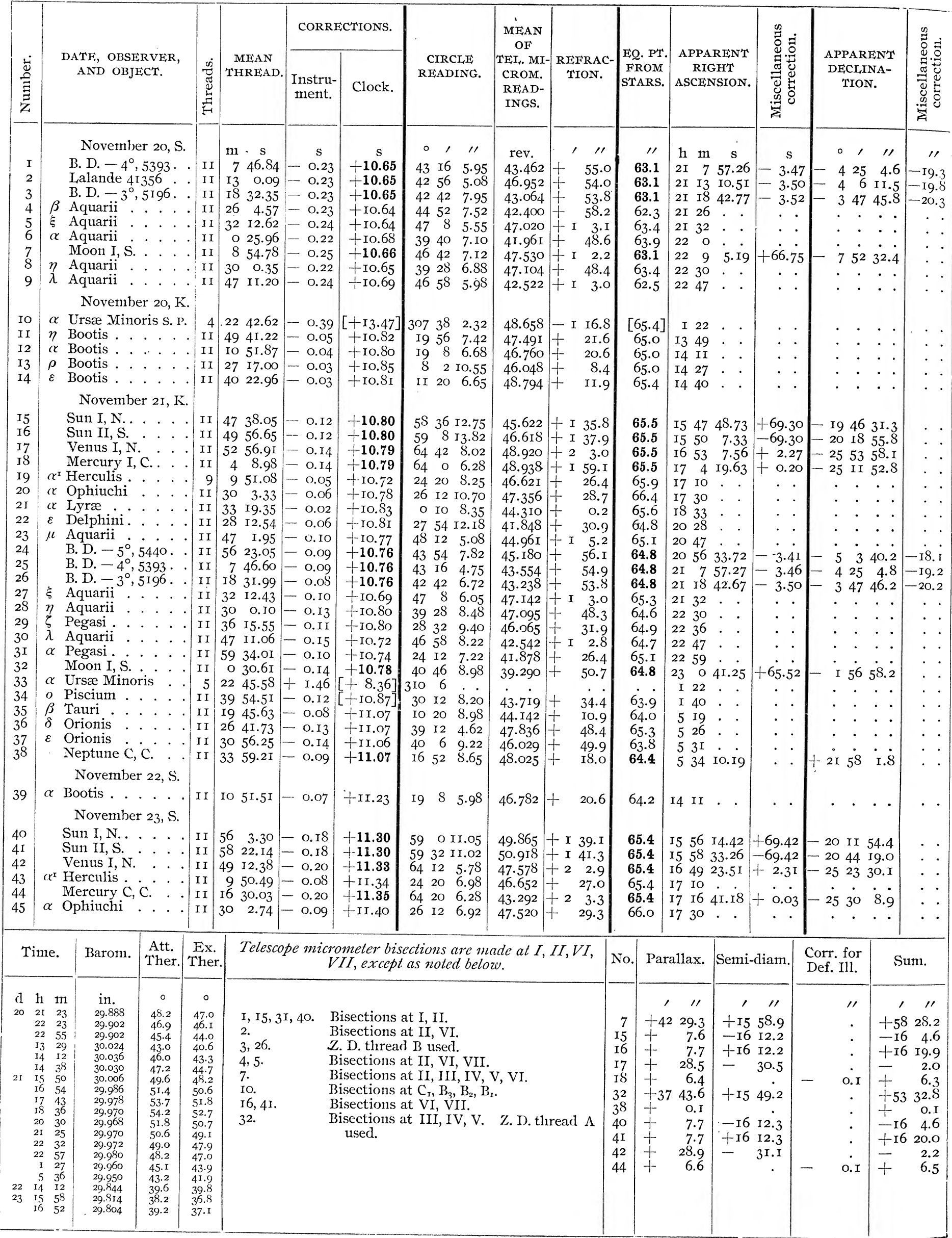




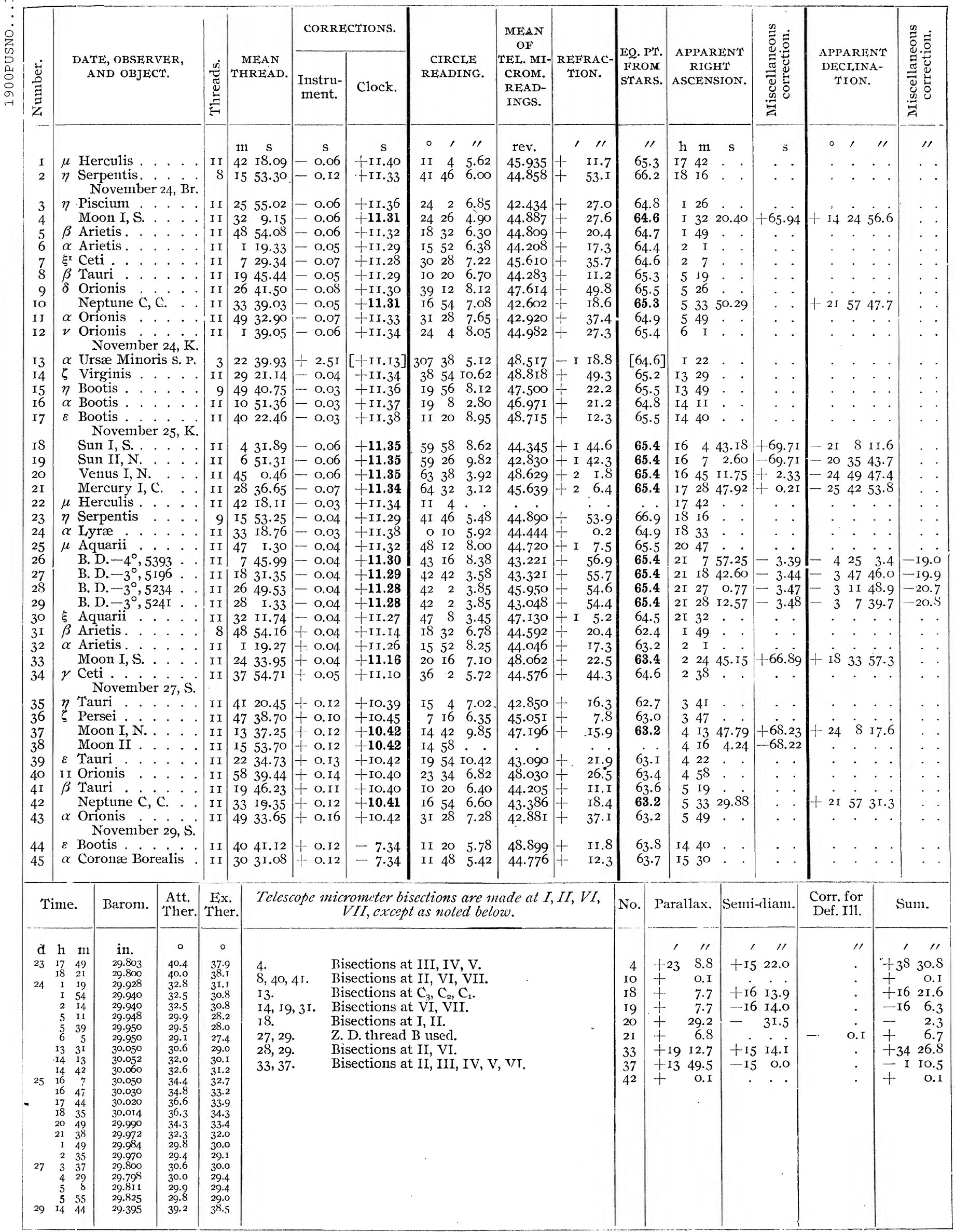




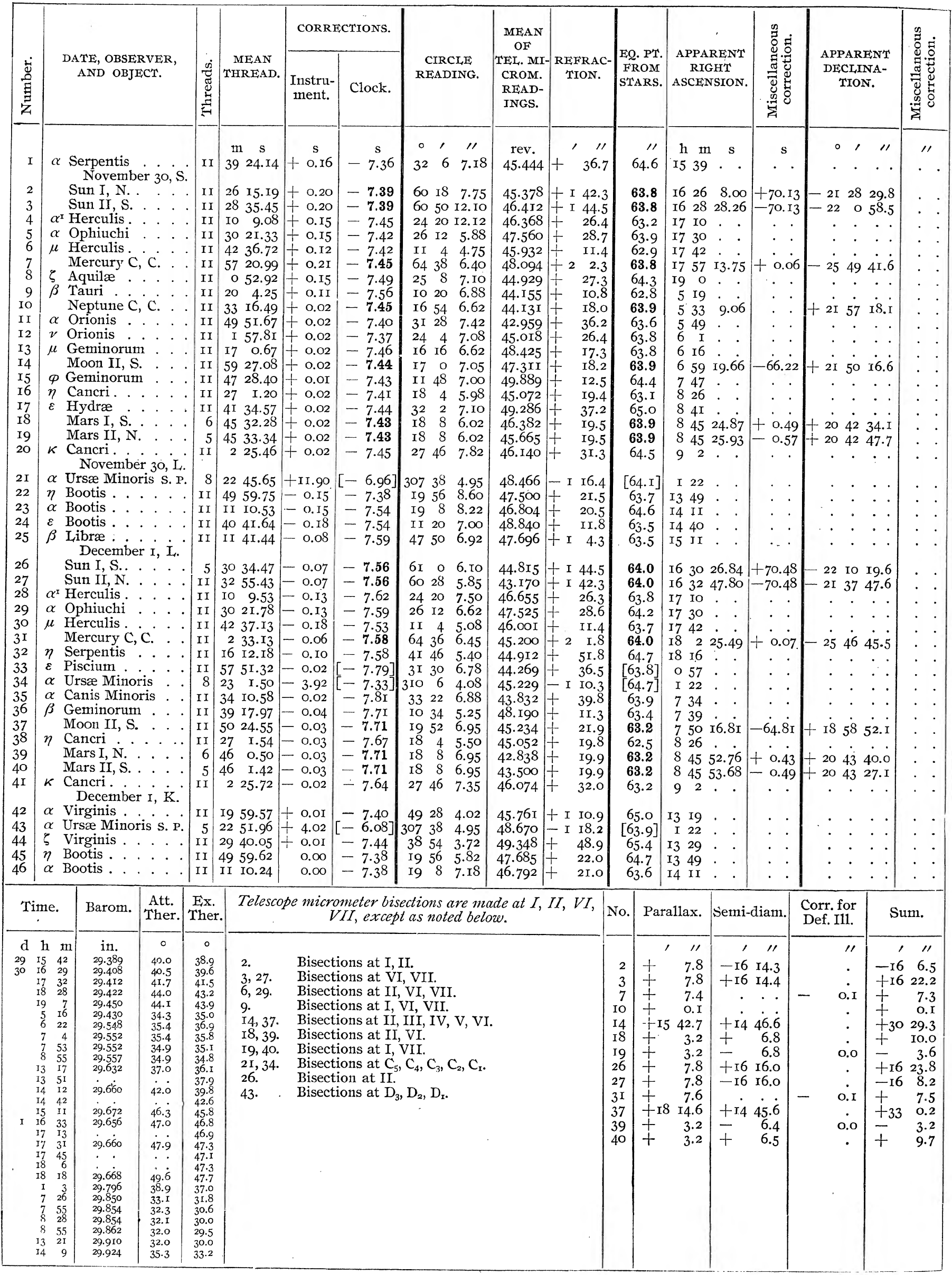




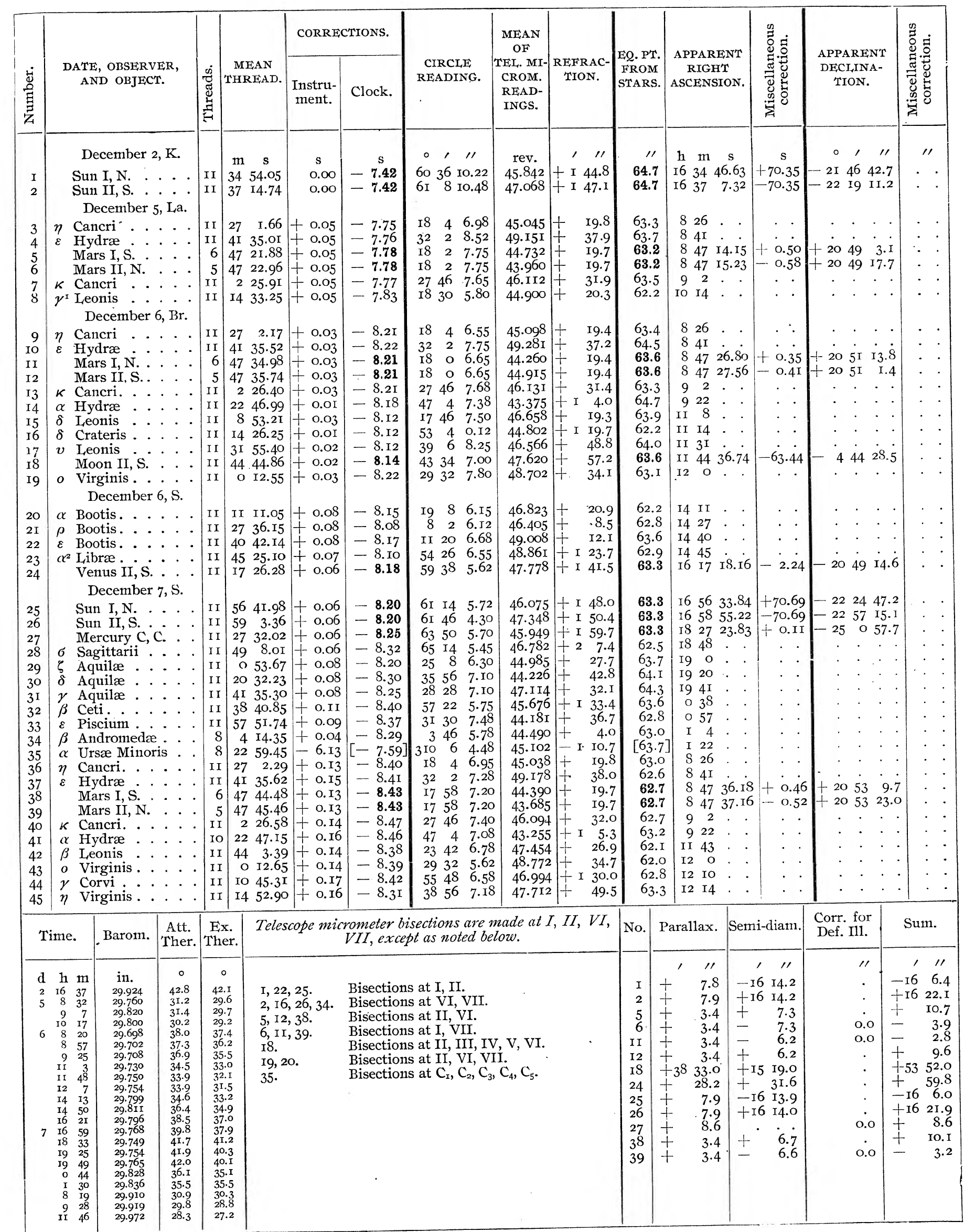




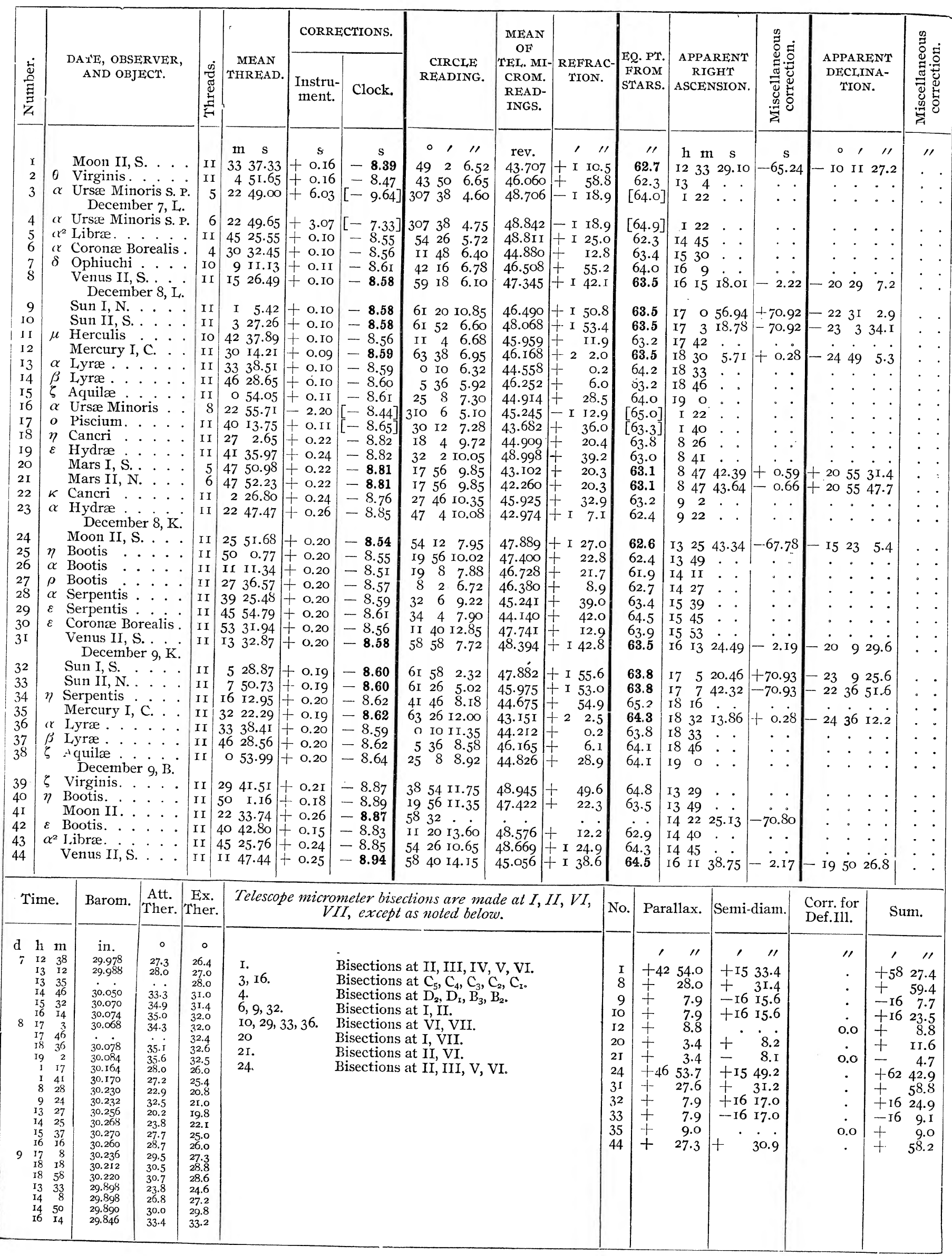




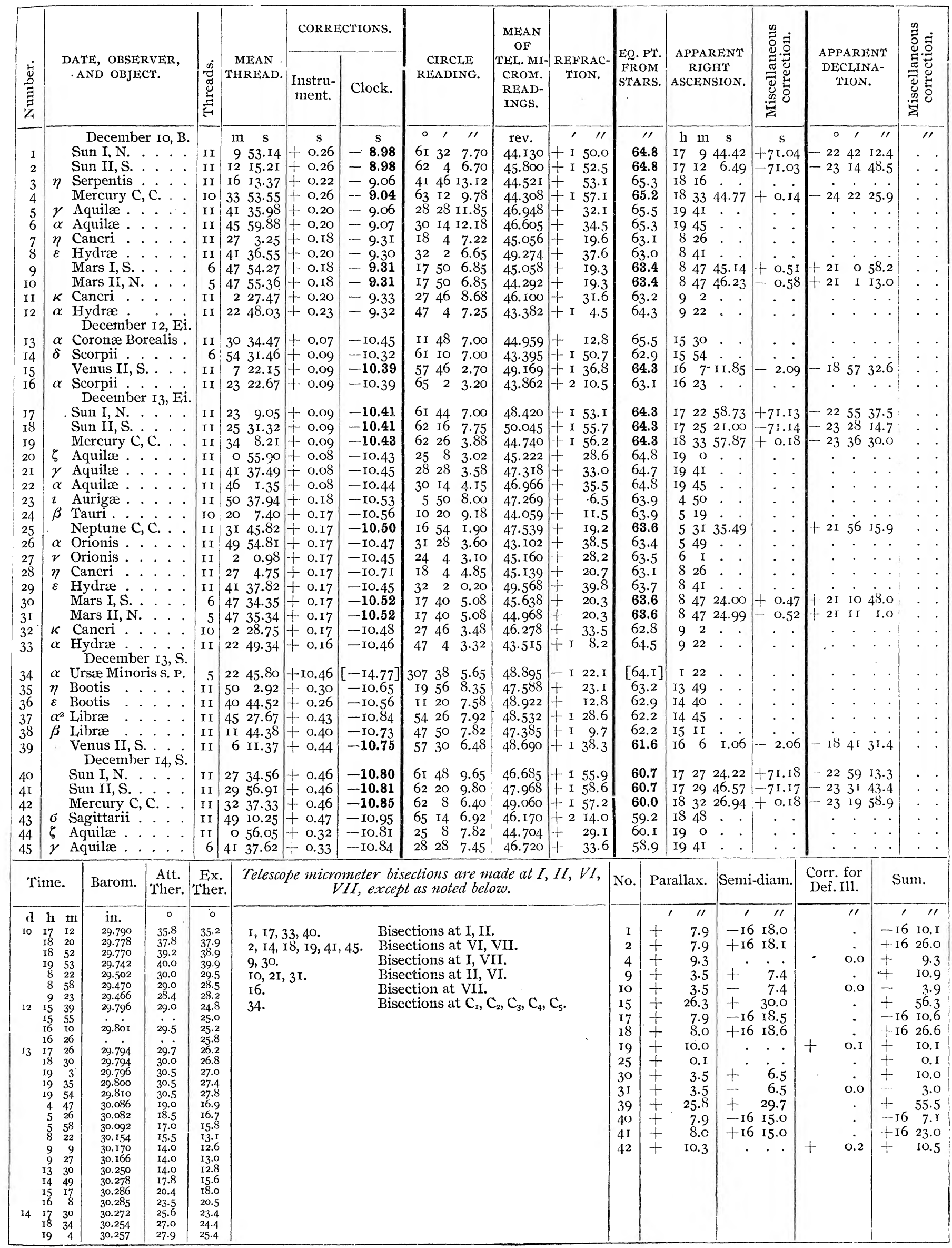




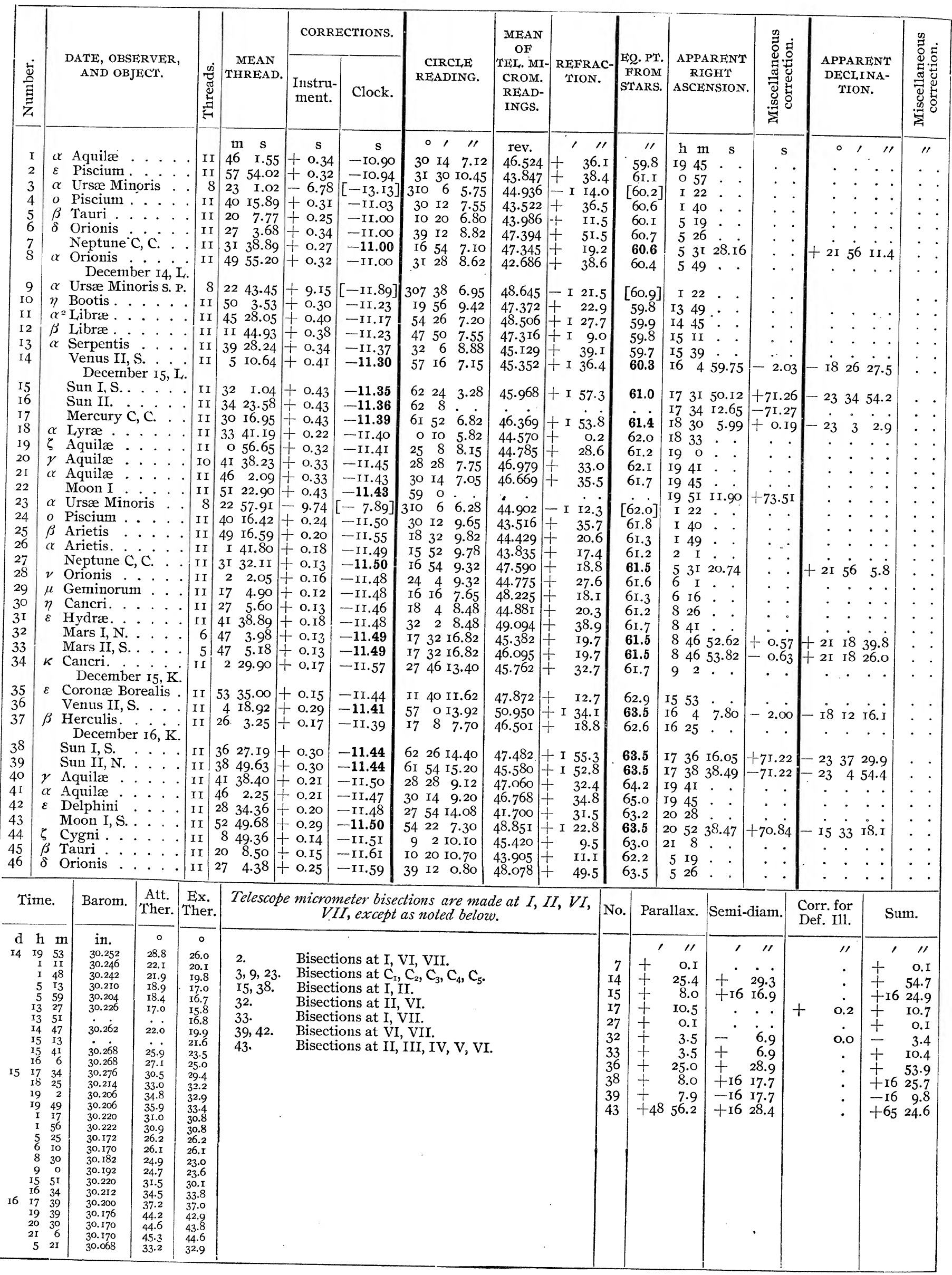




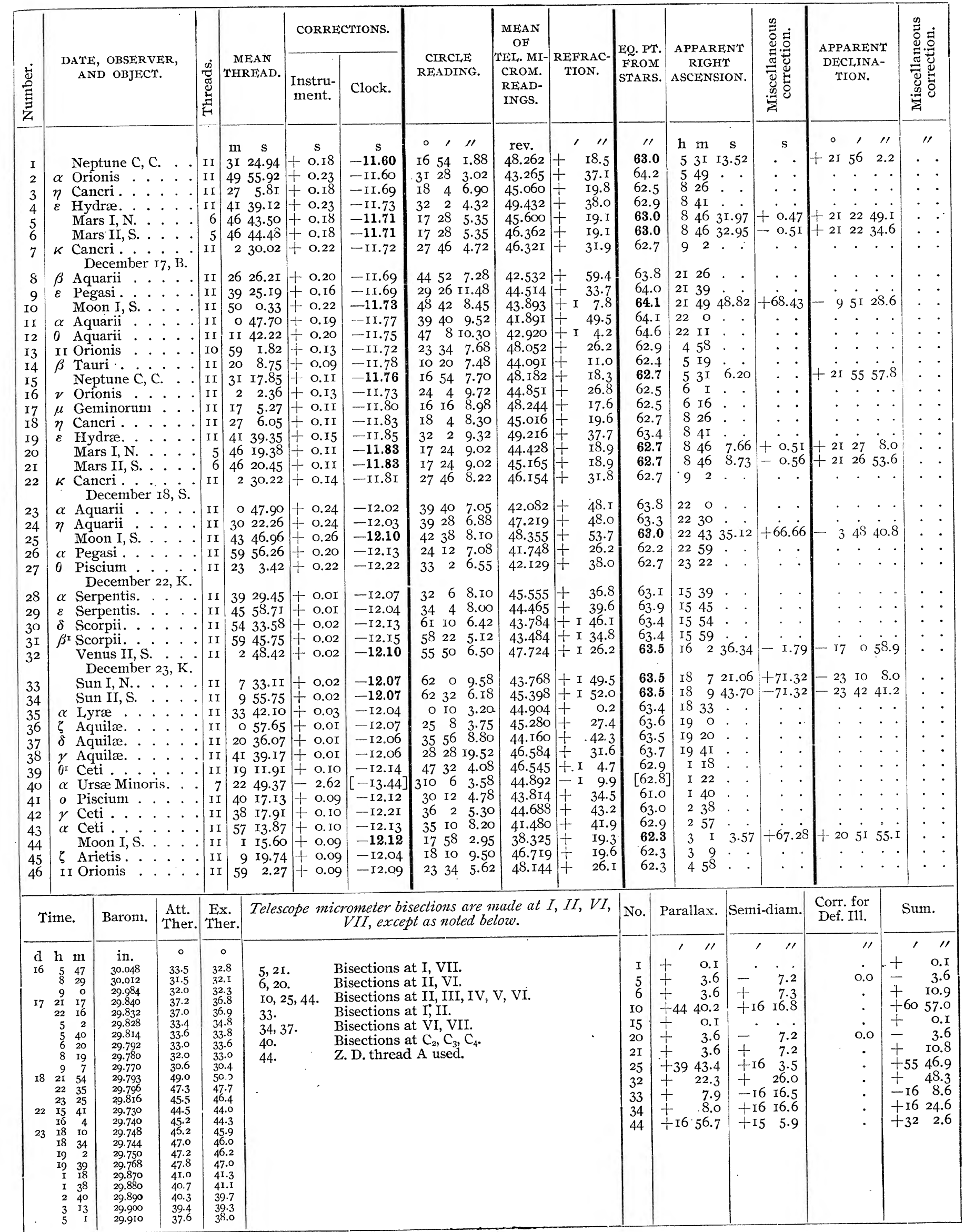




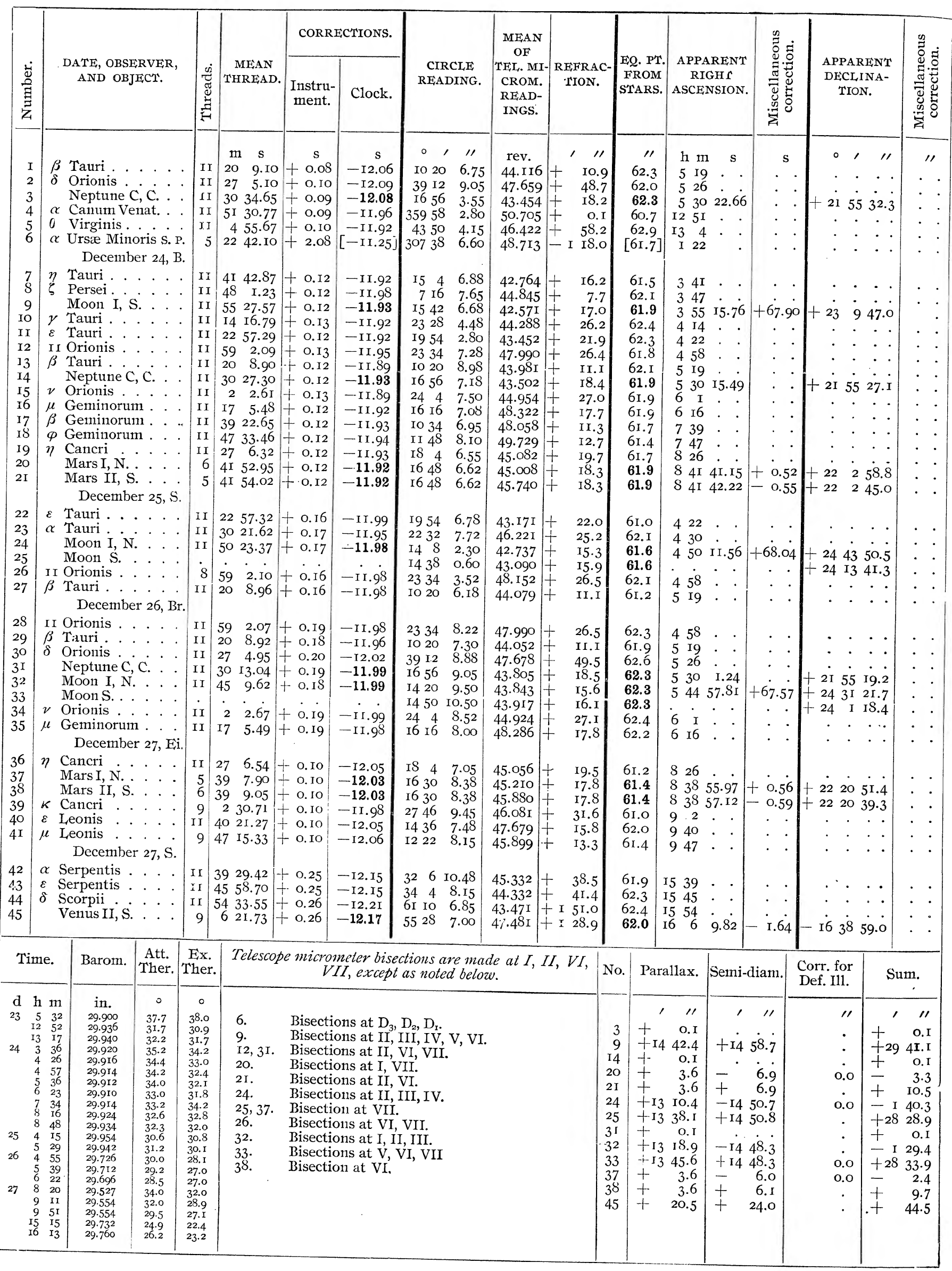




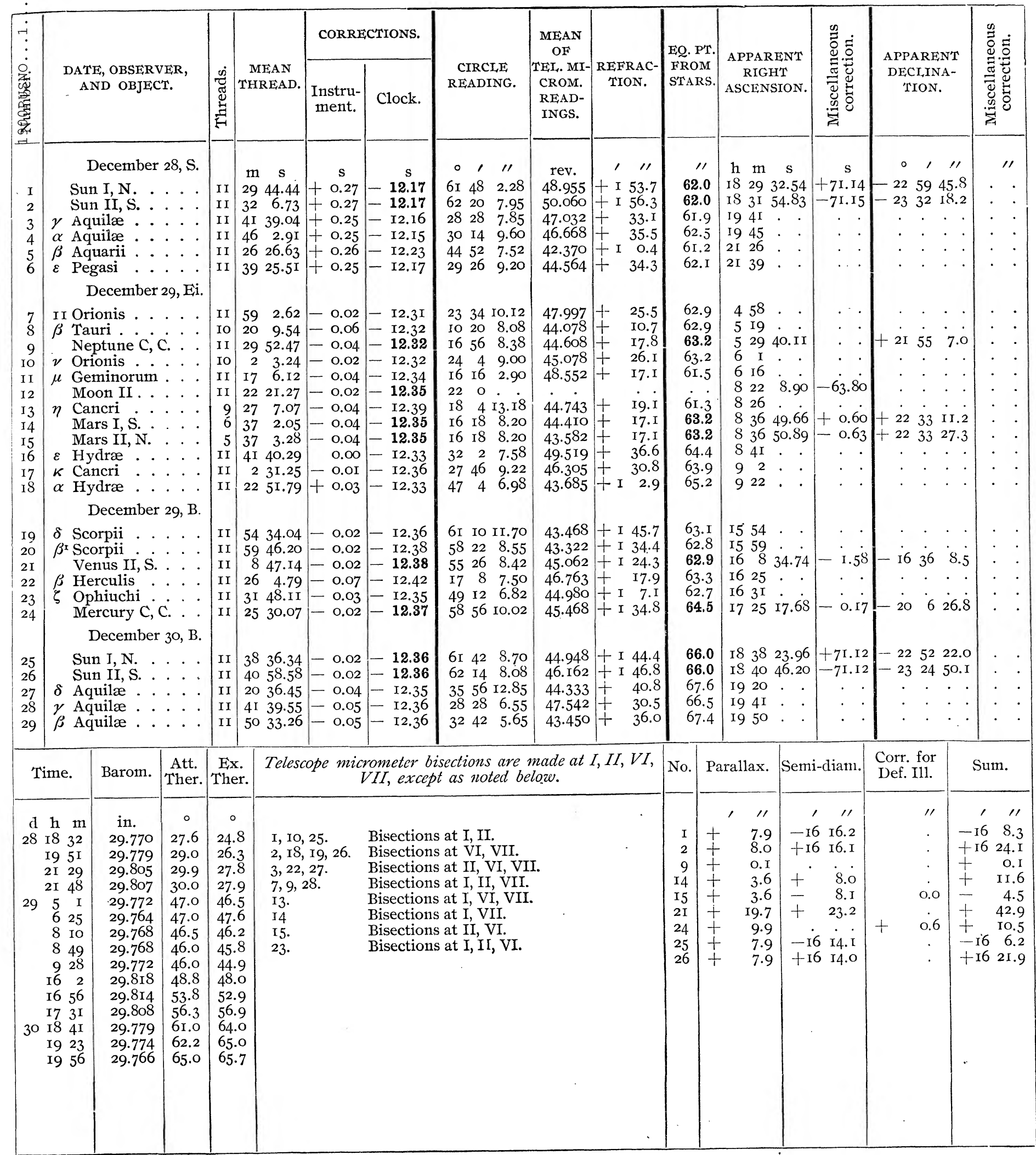




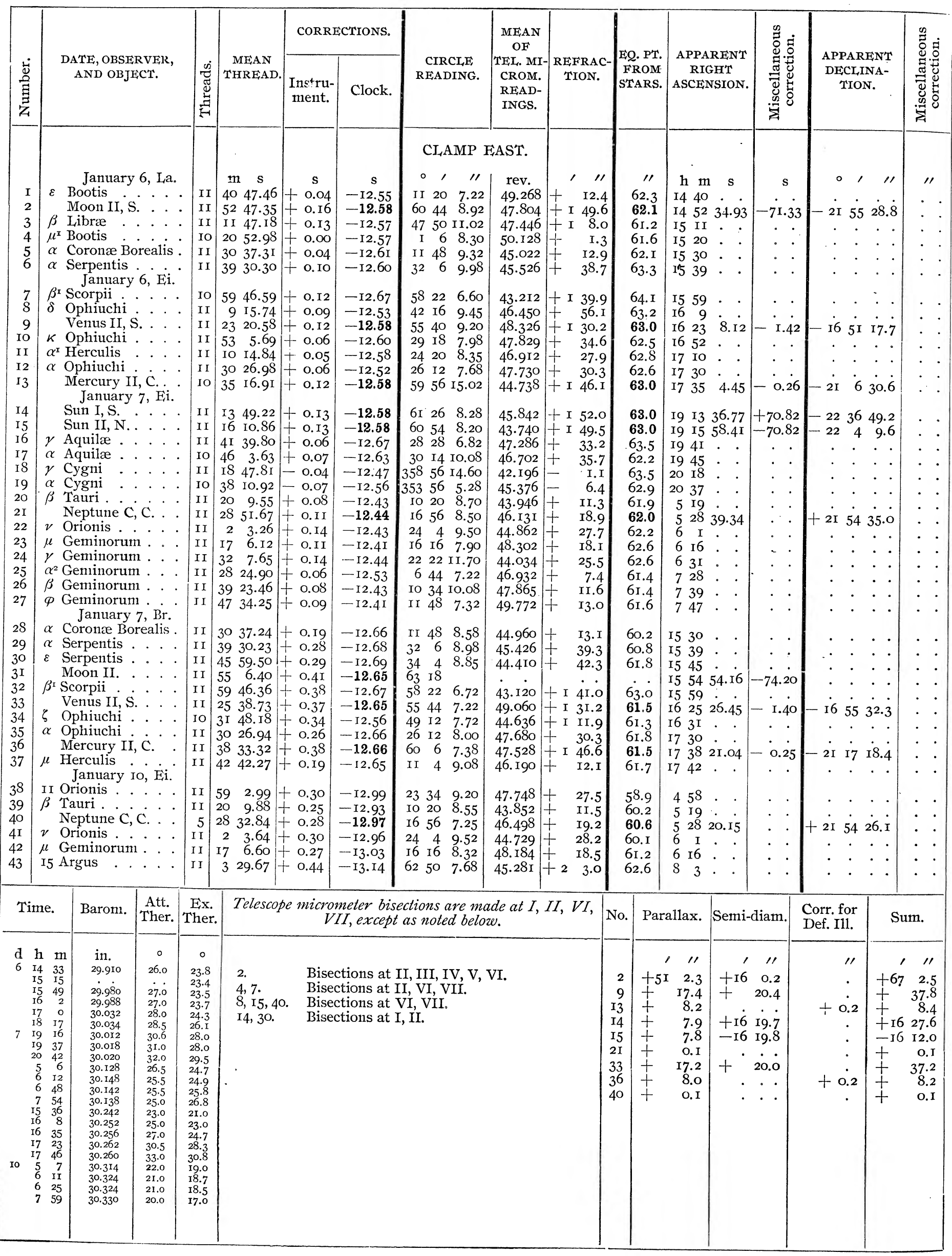




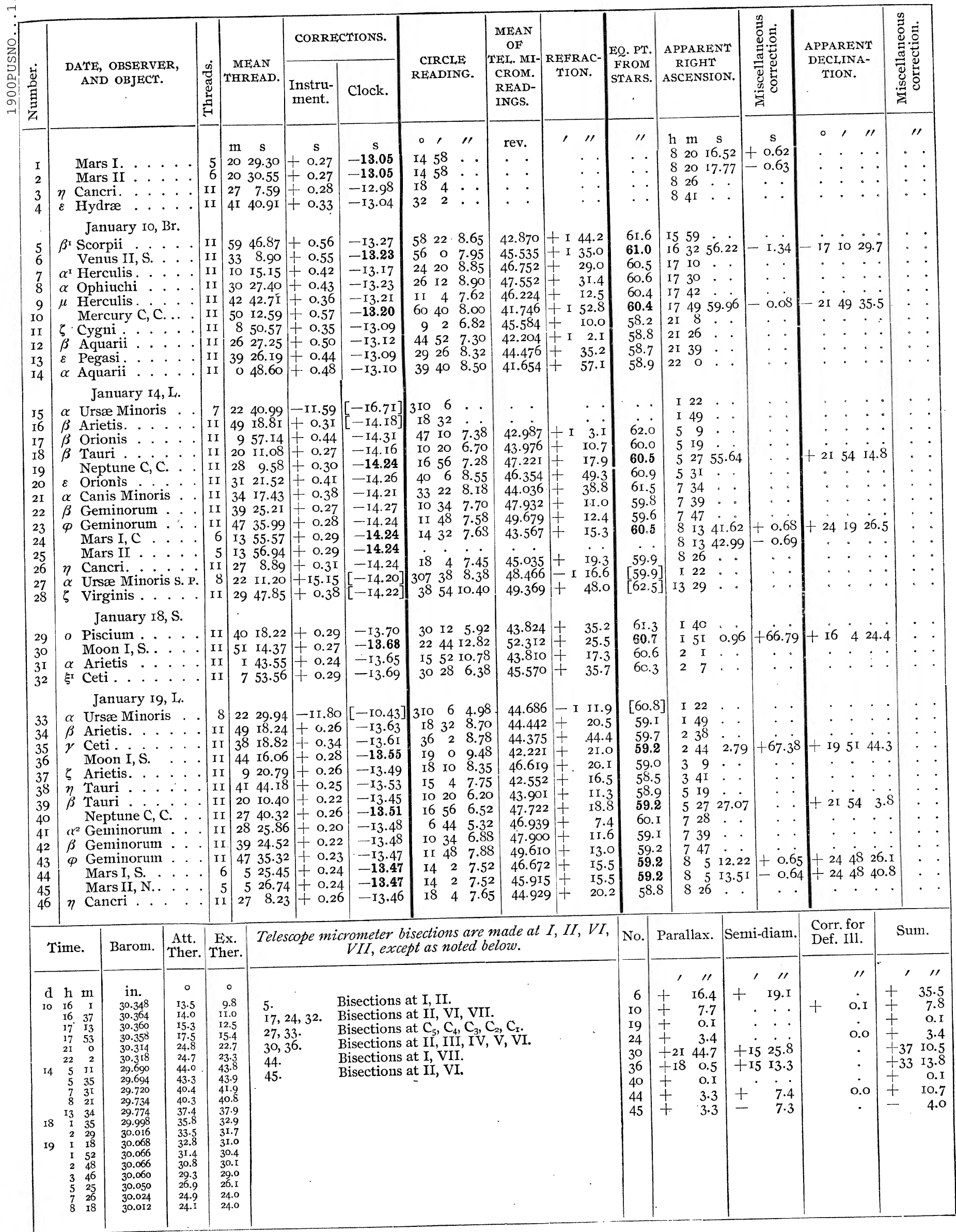




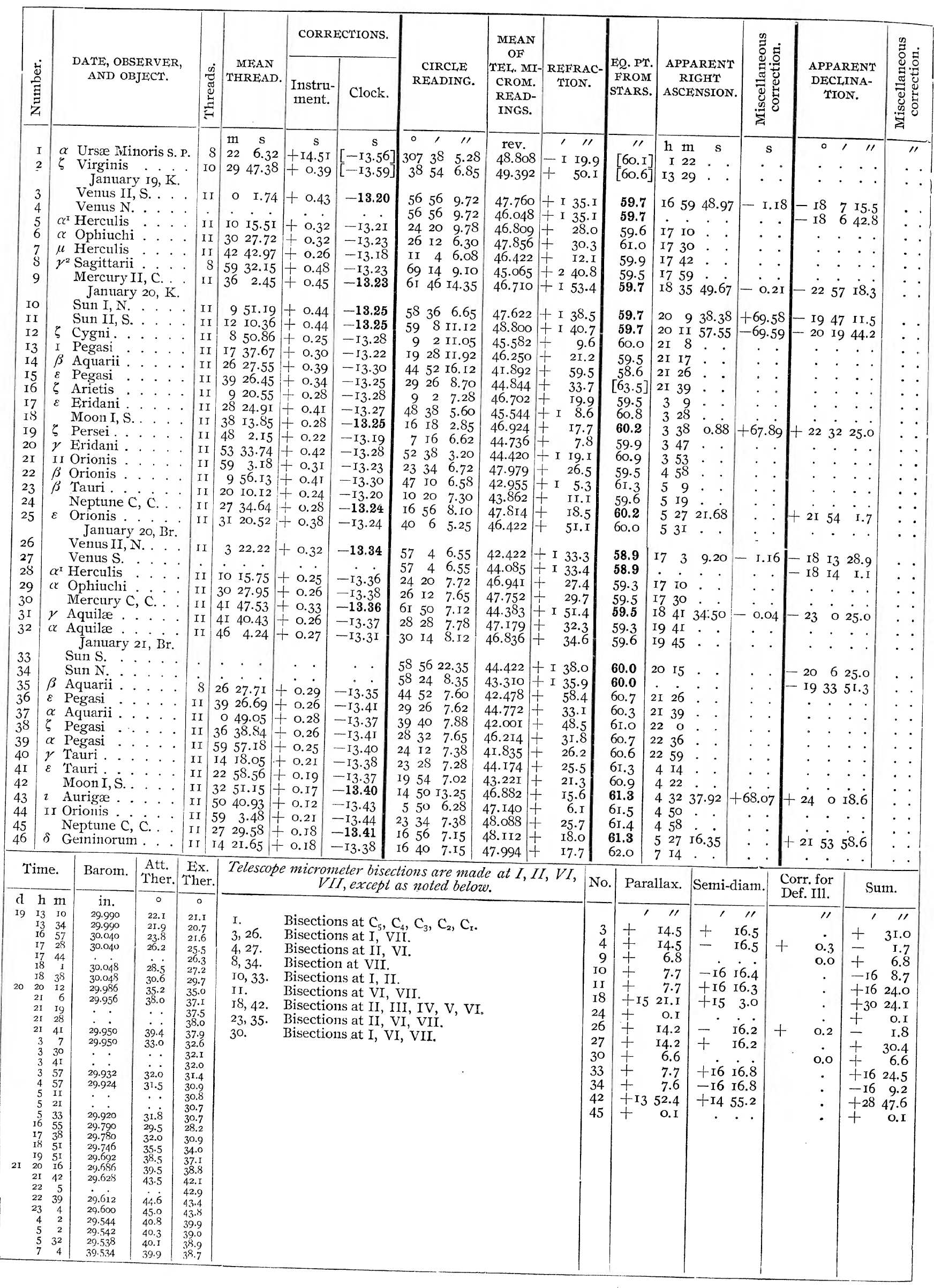




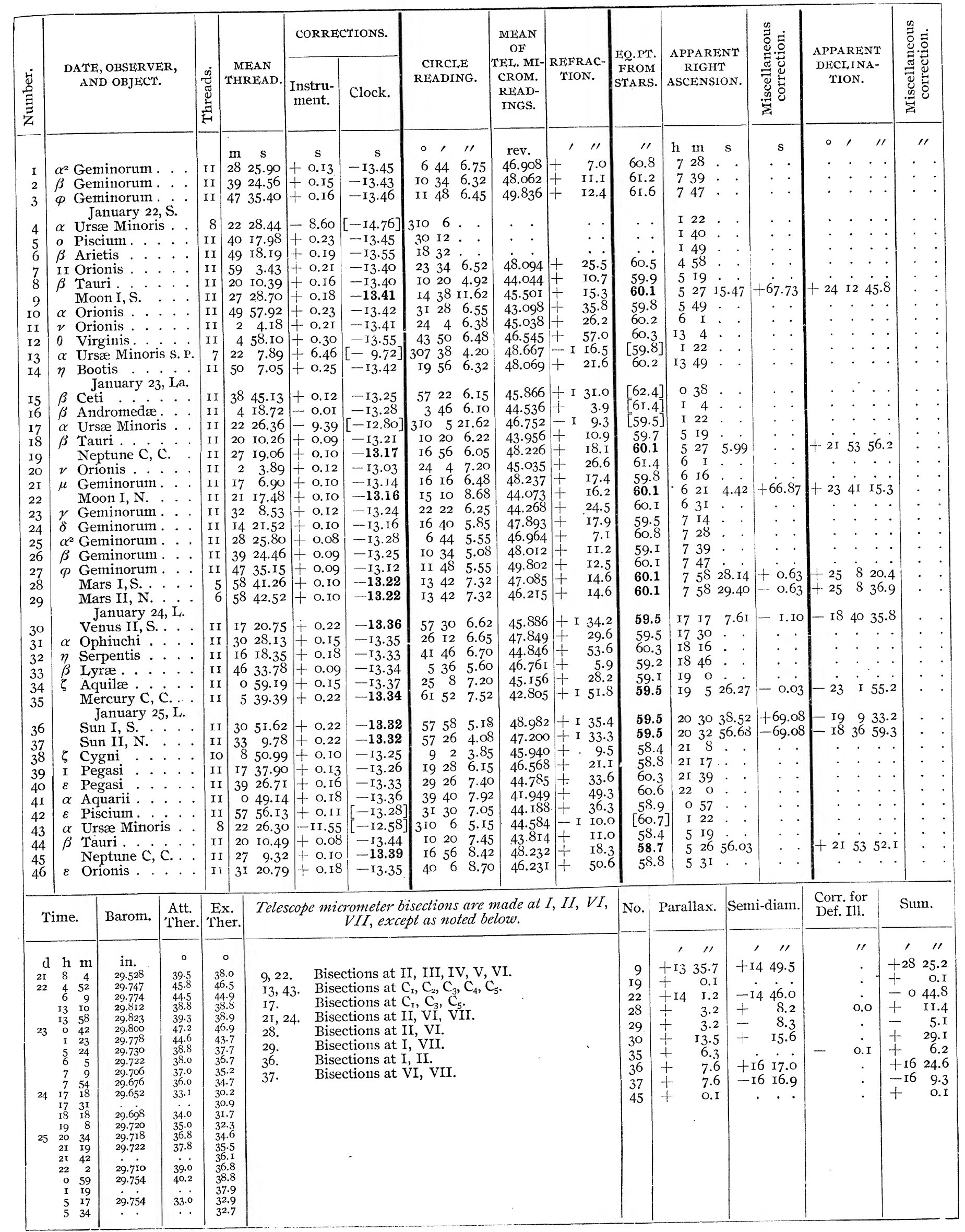




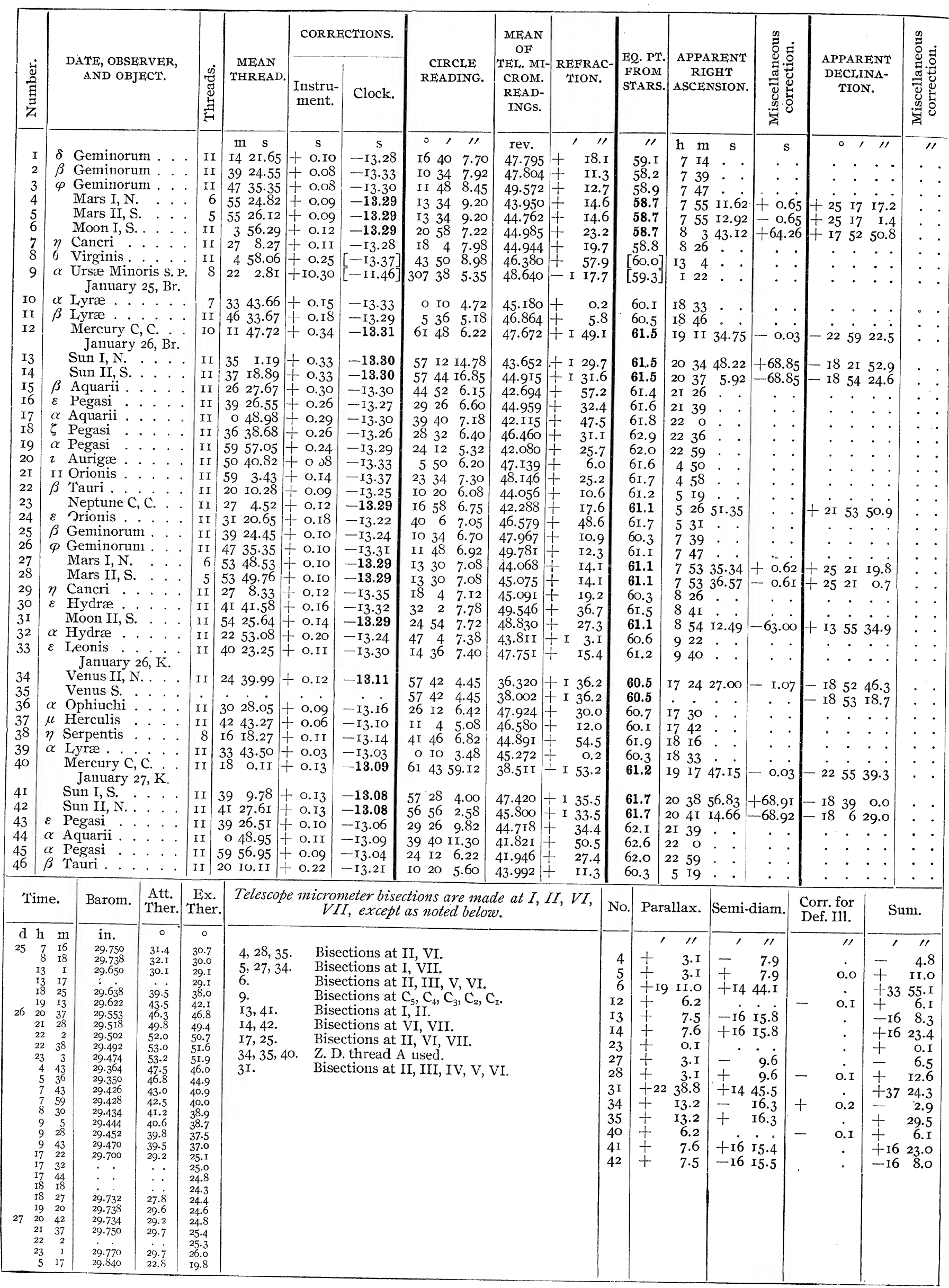




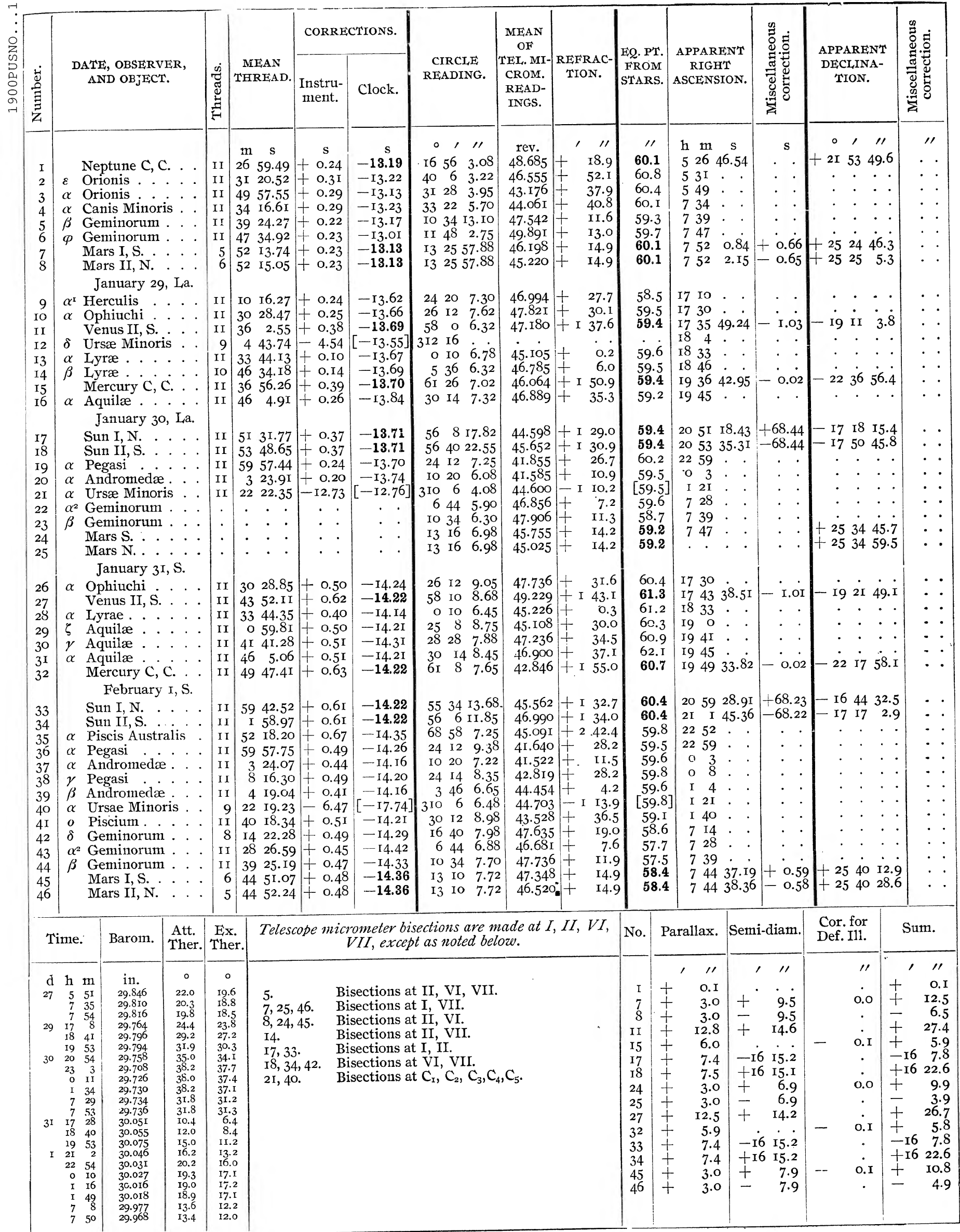




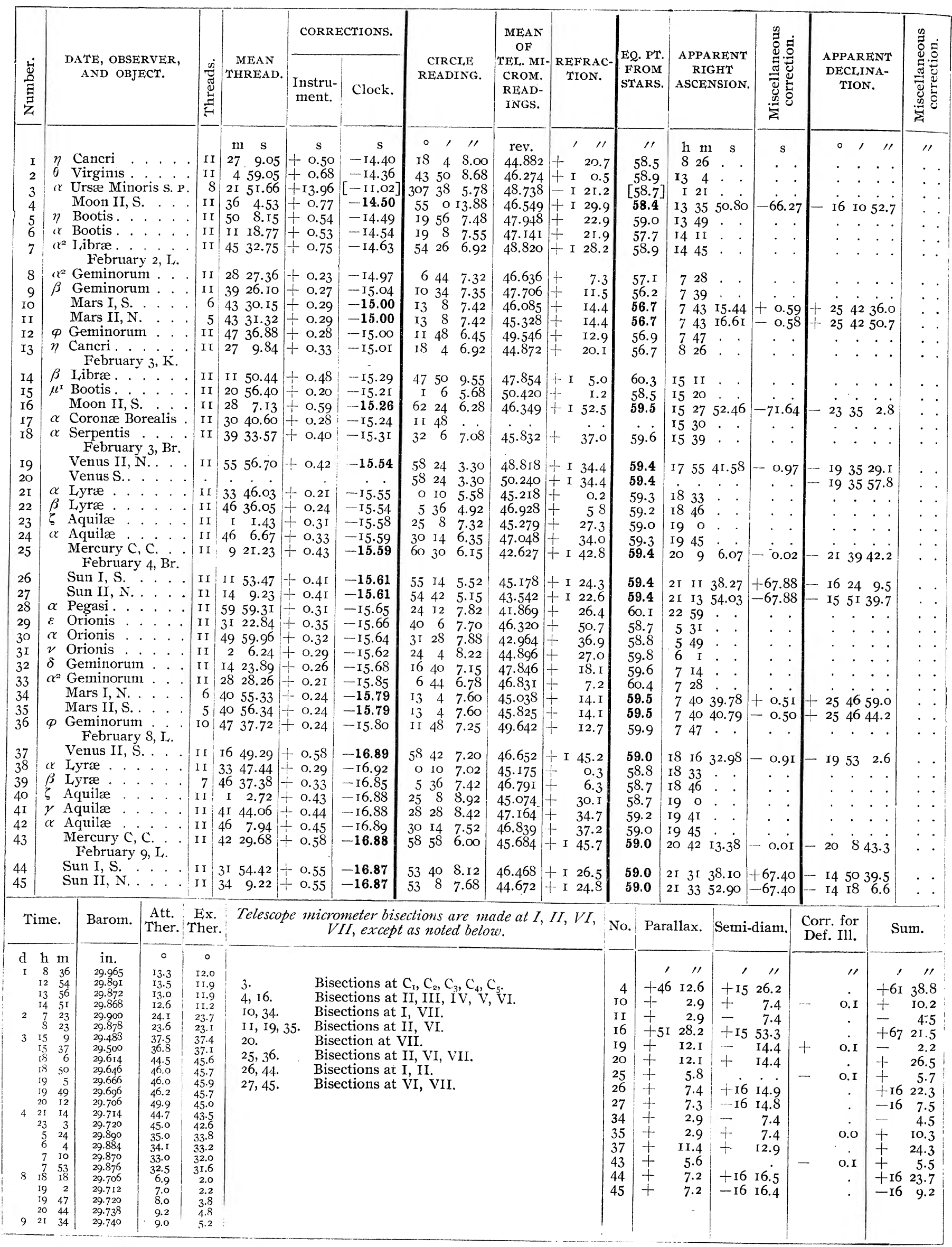




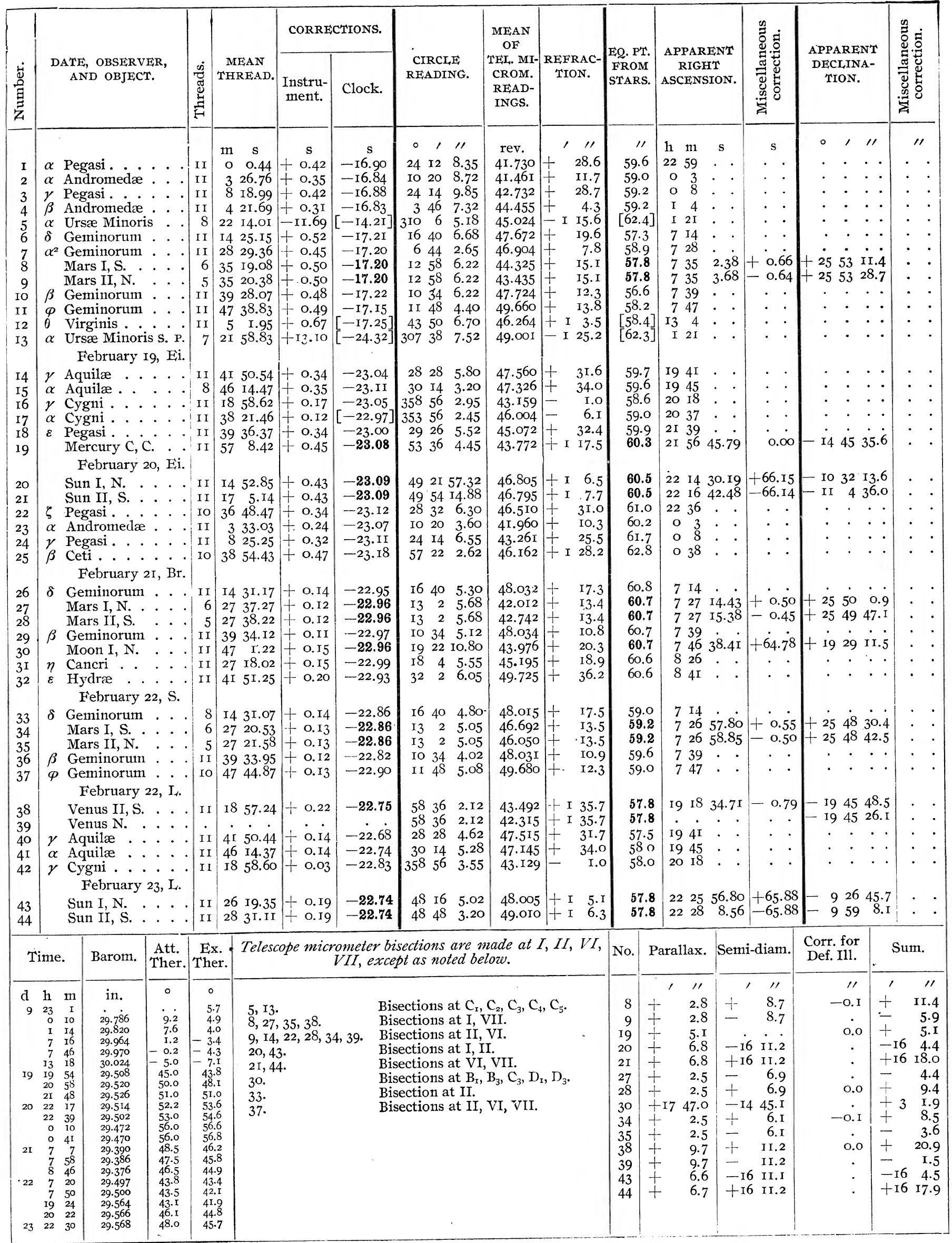




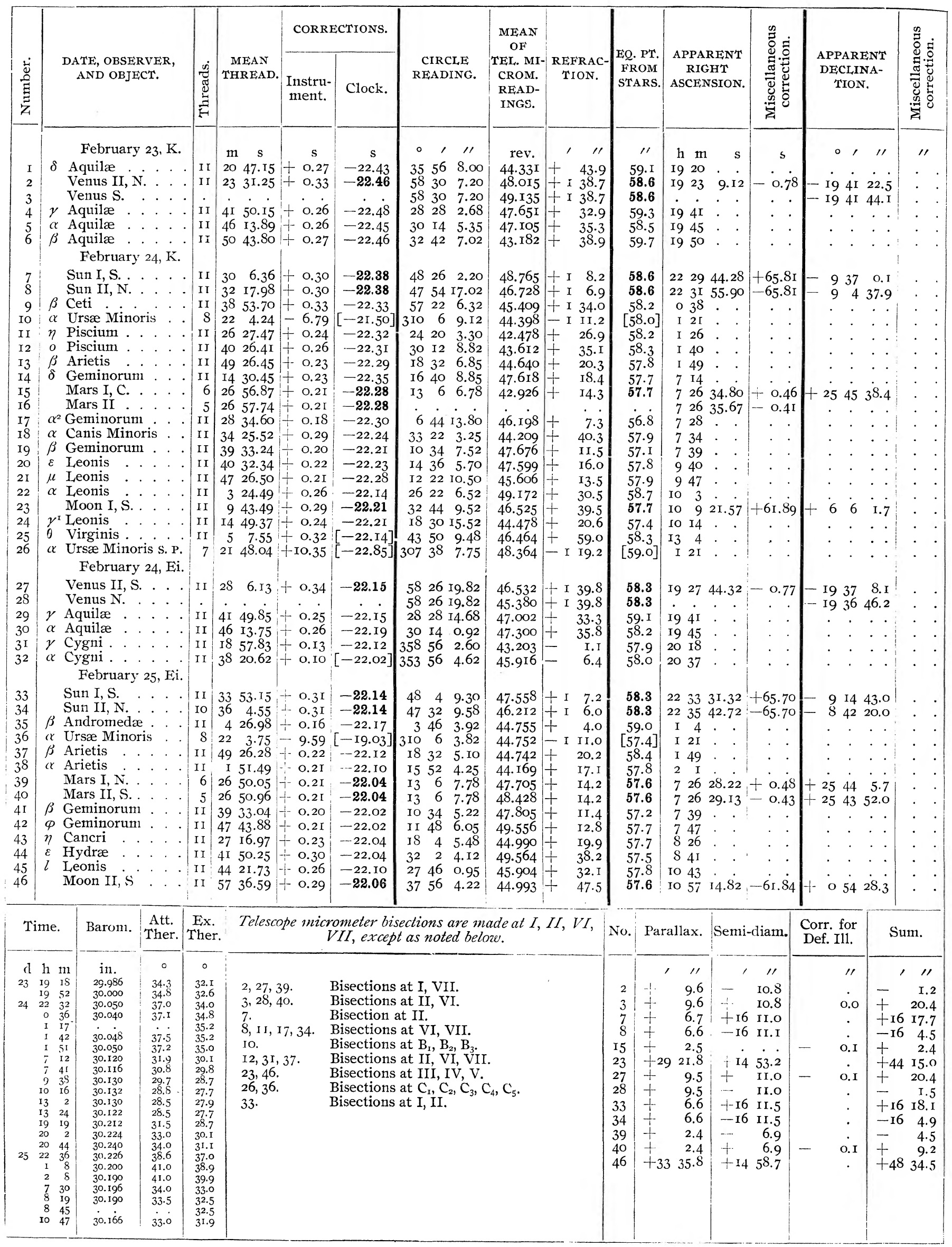




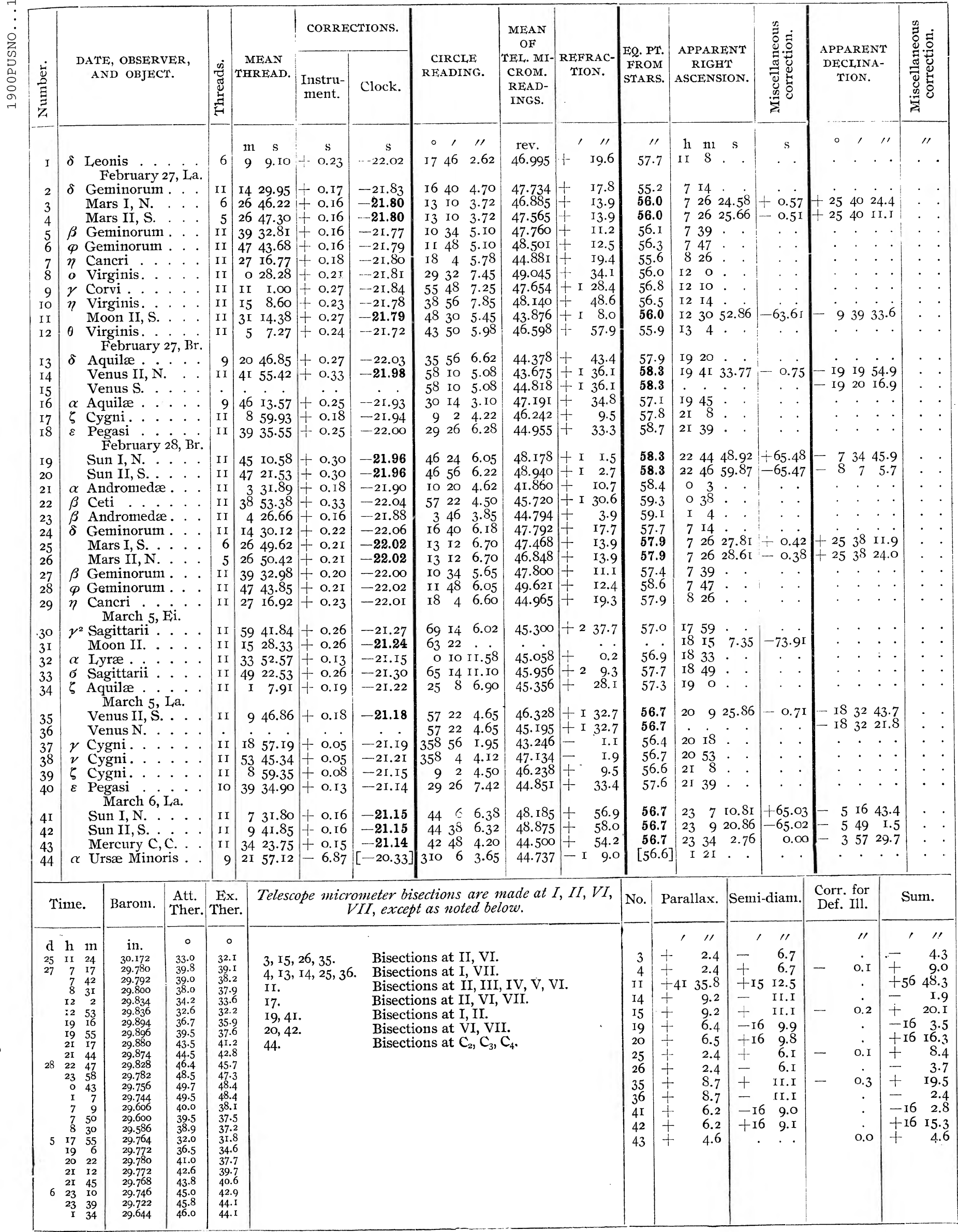




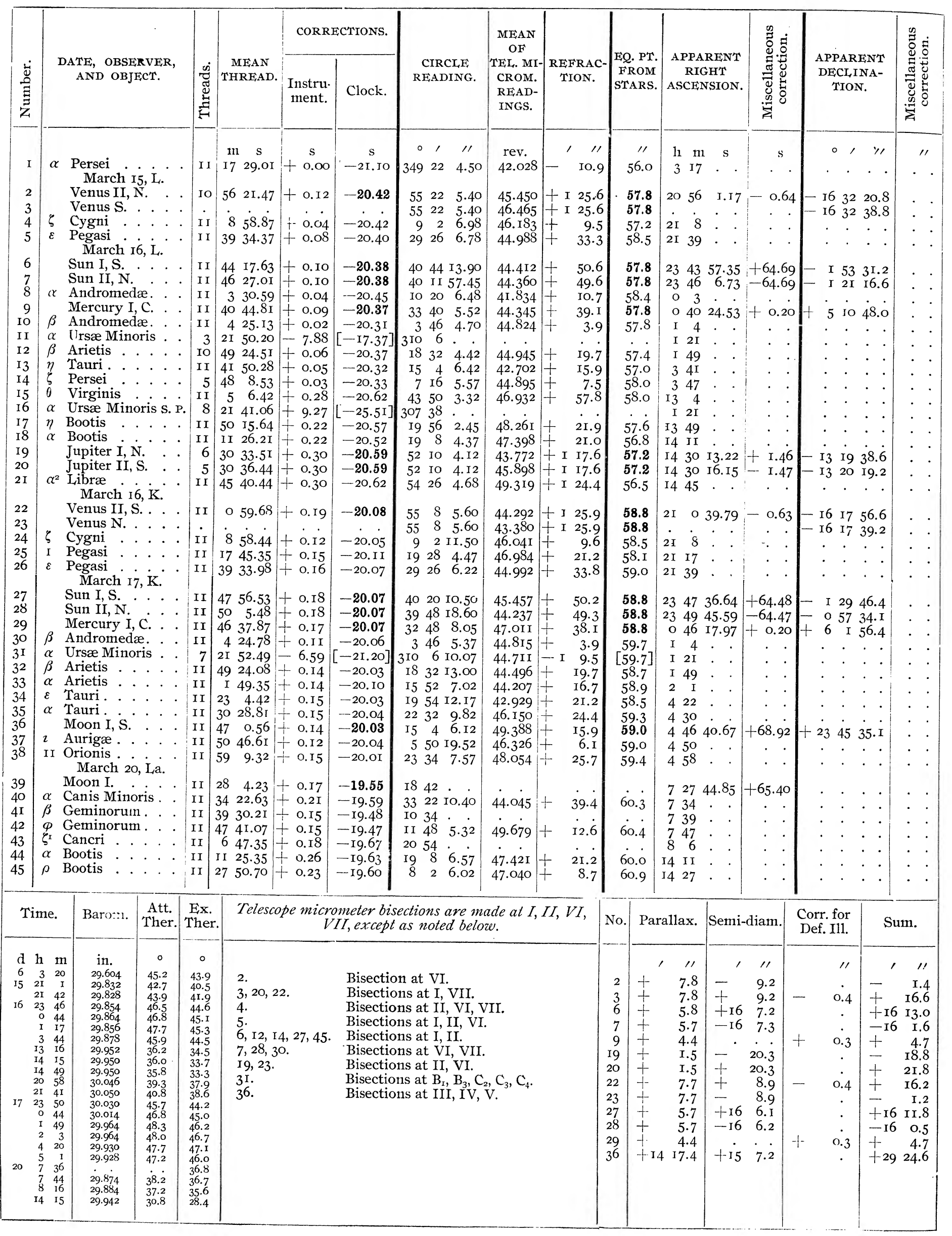




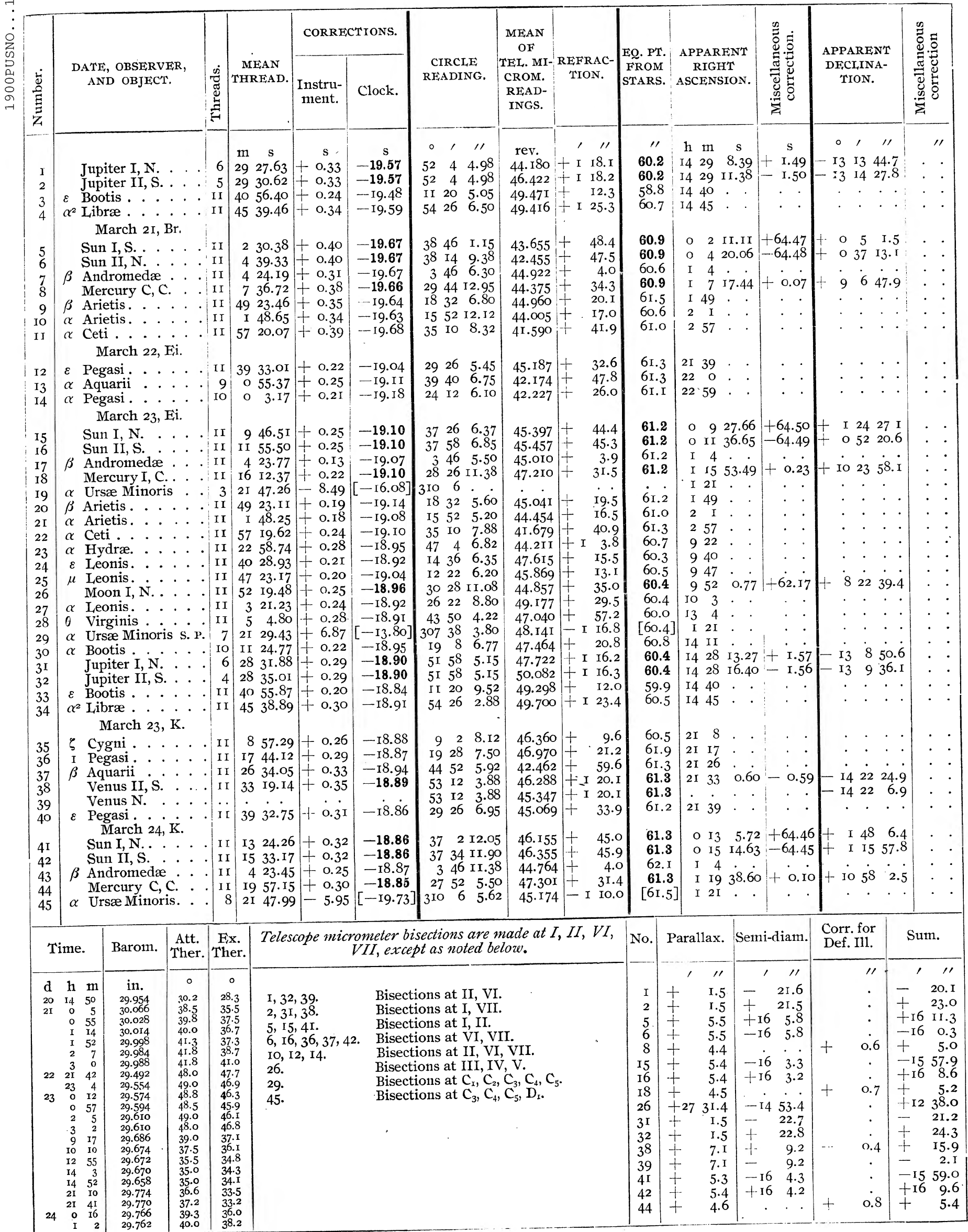




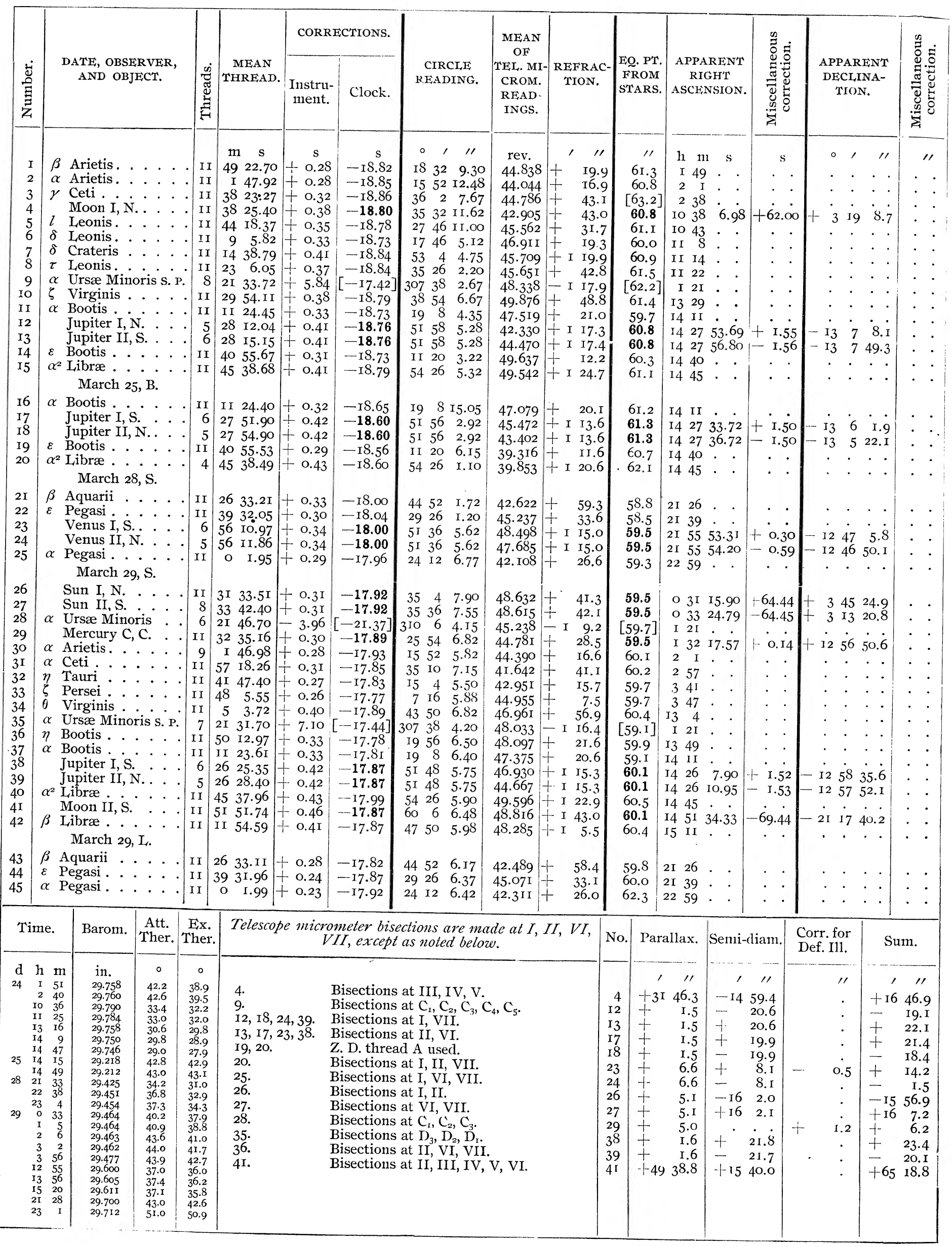




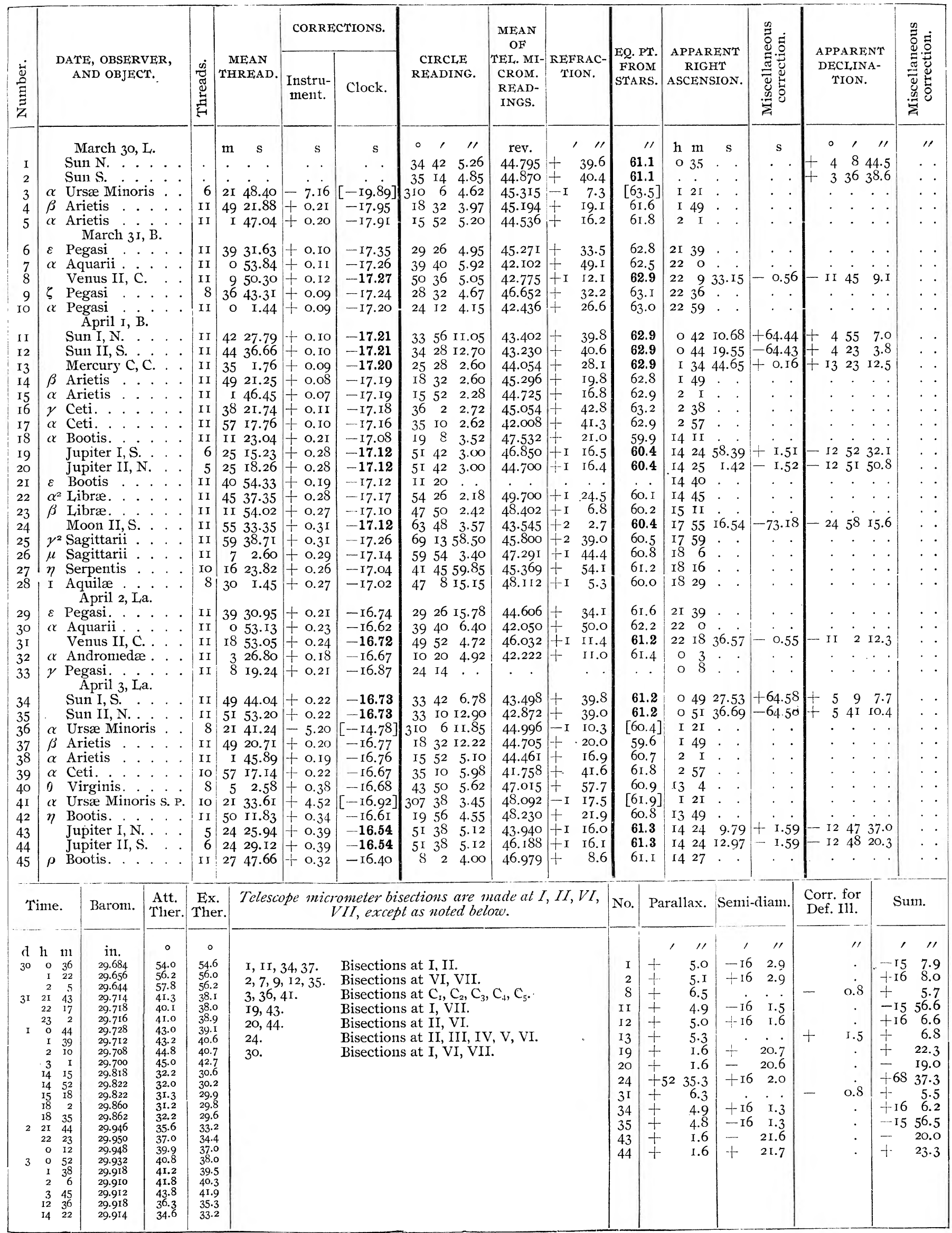




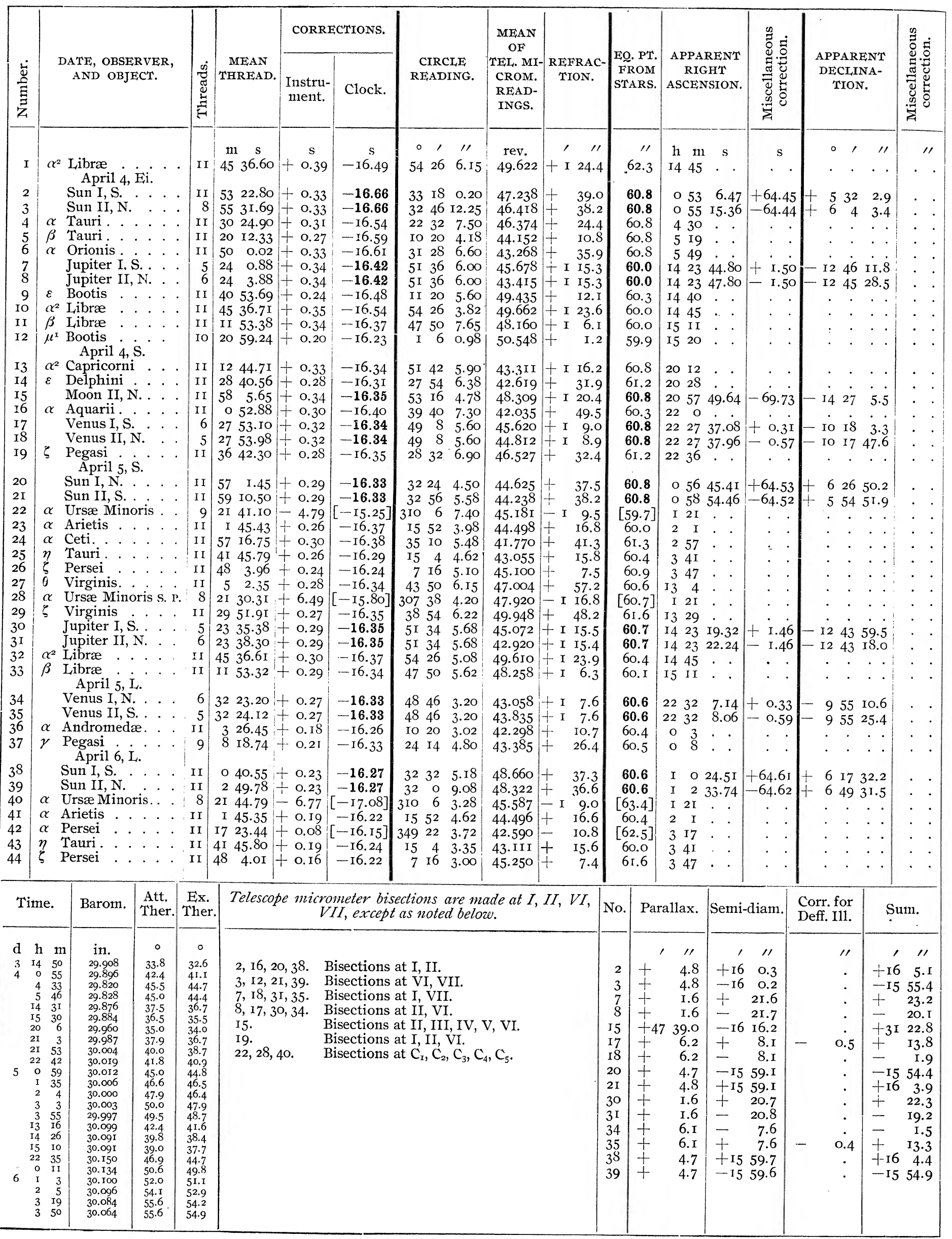




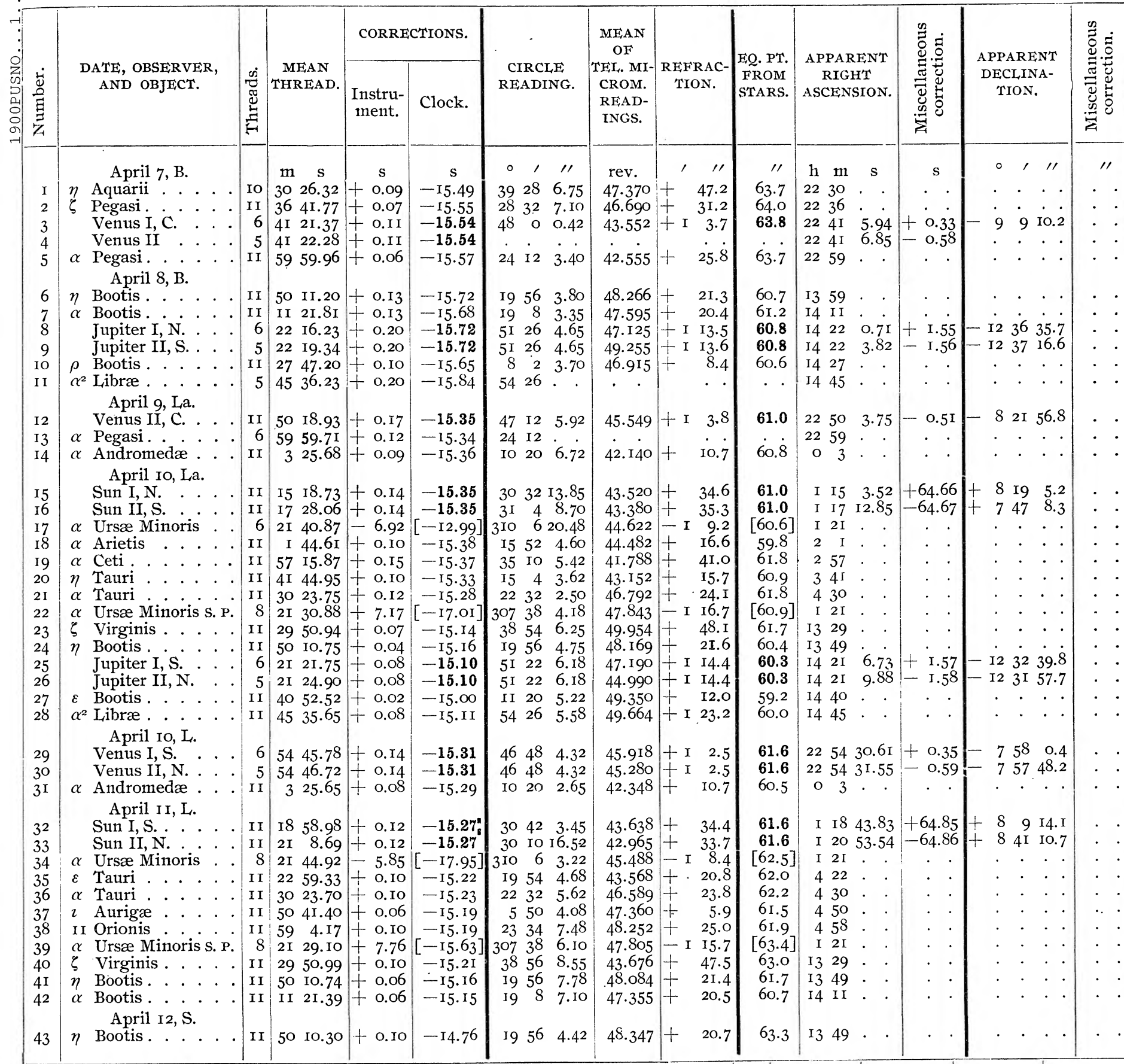

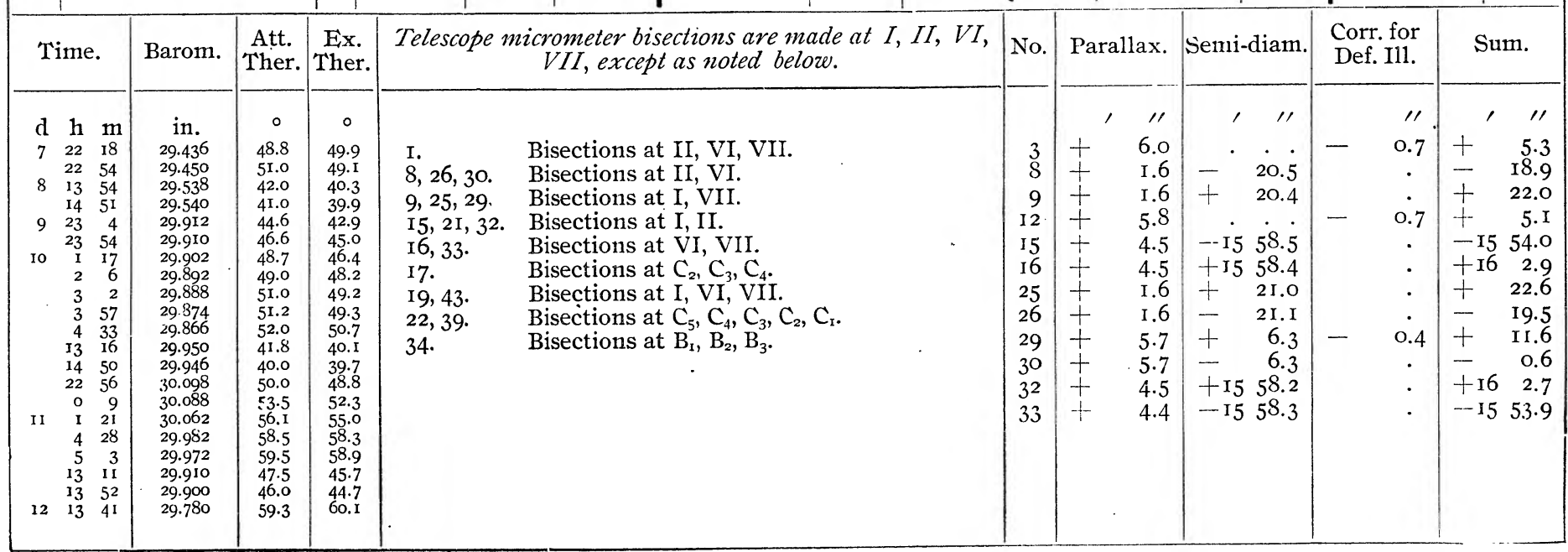




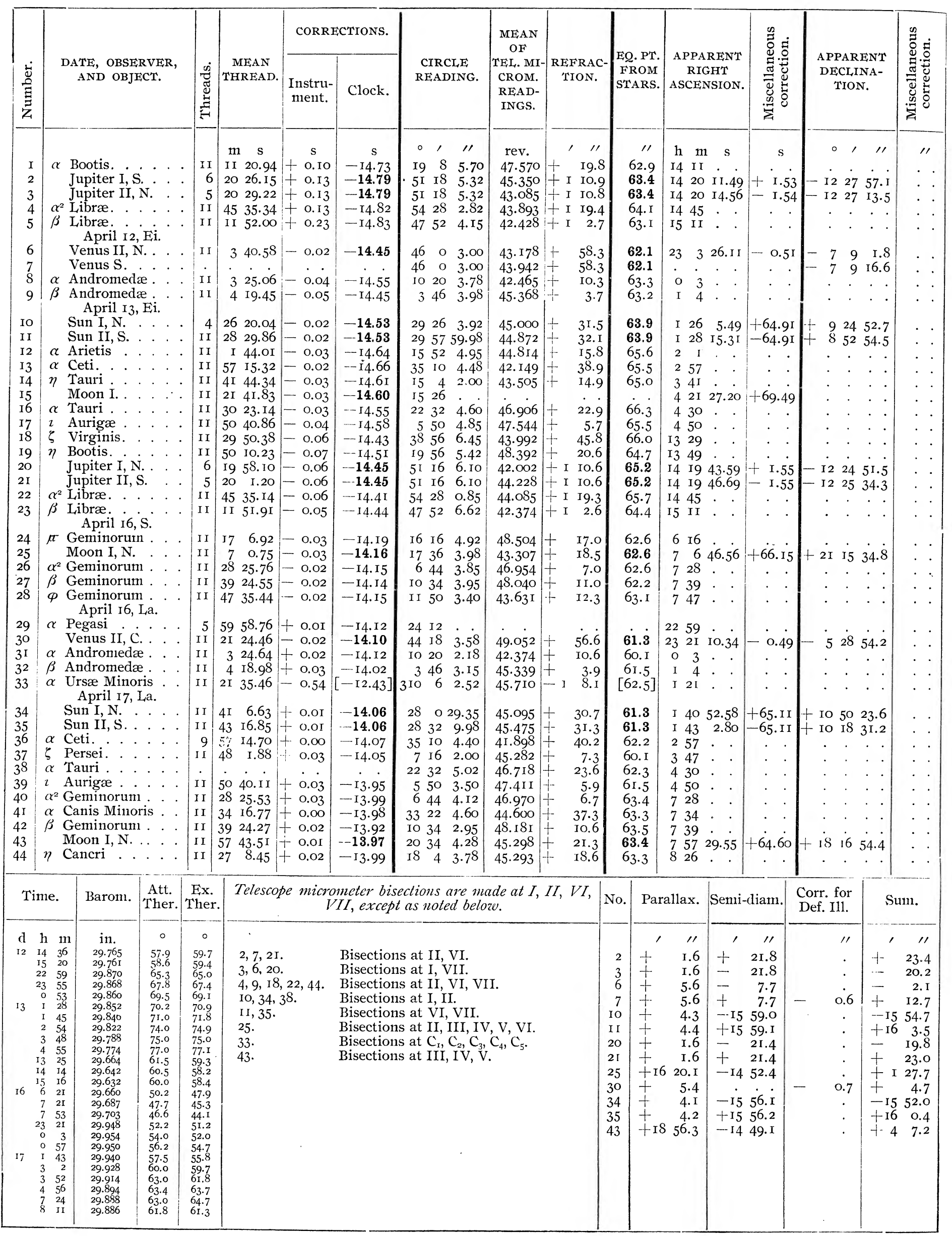




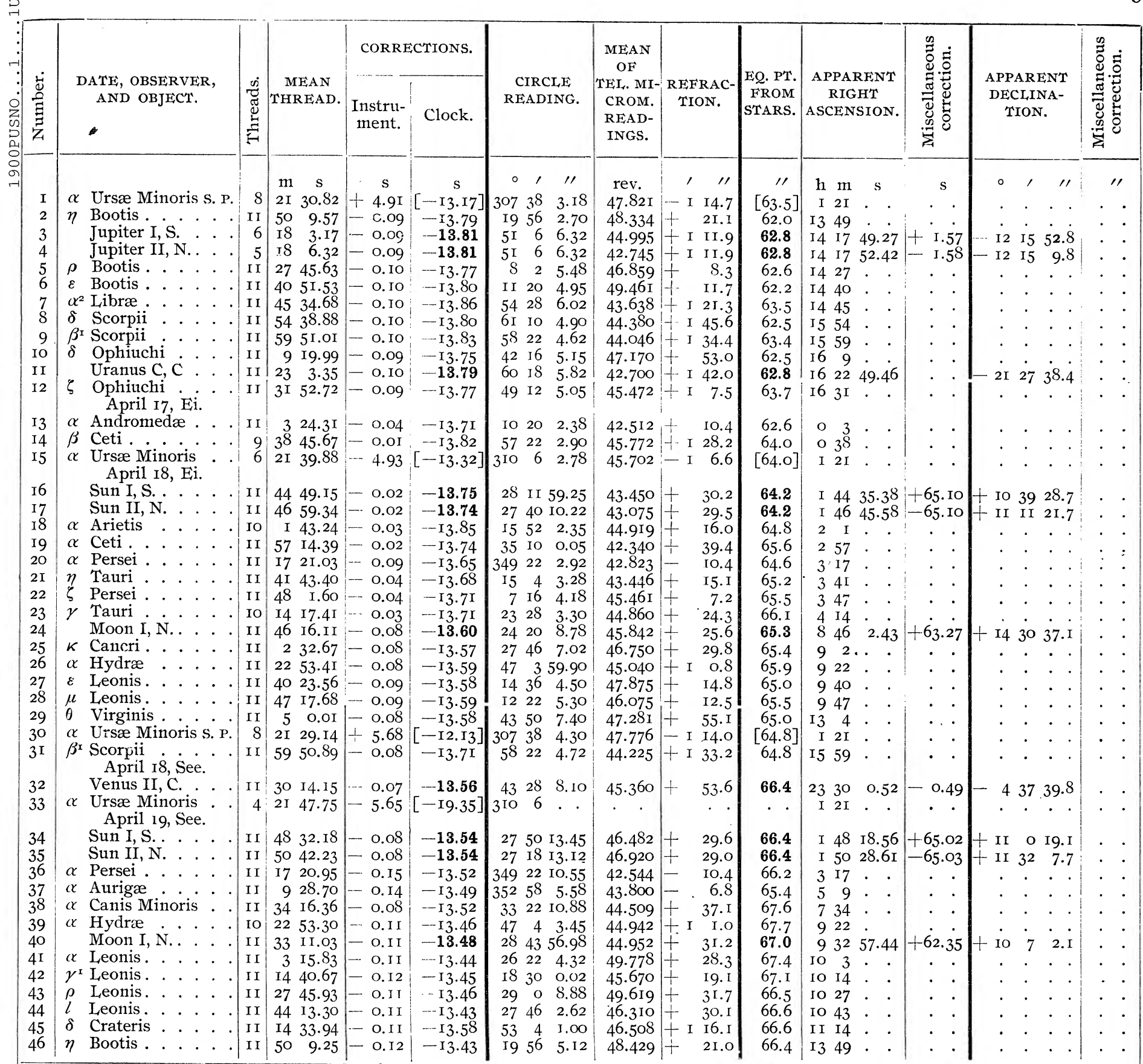

\begin{tabular}{|c|c|c|c|c|c|c|c|c|c|c|c|c|}
\hline \multicolumn{3}{|c|}{ Time. } & Barom. & $\begin{array}{l}\text { Att. } \\
\text { Ther. }\end{array}$ & $\begin{array}{l}\text { Ex. } \\
\text { Ther. }\end{array}$ & Telescope & $\begin{array}{l}\text { micrometer bisections are made at } I, I I, V I \text {, } \\
V I I, \text { except as noted below. }\end{array}$ & No. & Parallax. & Semi-diam. & $\begin{array}{l}\text { Corr. for } \\
\text { Def. I11. }\end{array}$ & Sum. \\
\hline$d$ & h & & in. & $\circ$ & $\circ$ & & & & 11 & "1 & "I & , \\
\hline & I3 & & 29.892 & 52.0 & $5^{1.6}$ & I, 30. & Bisections at $\mathrm{C}_{1}, \mathrm{C}_{2}, \mathrm{C}_{3}, \mathrm{C}_{4}, \mathrm{C}_{5}$. & 3 & I. 6 & 21.5 & . & 23. I \\
\hline & $\begin{array}{l}14 \\
14\end{array}$ & & $\begin{array}{l}29.8900 \\
29.888\end{array}$ & $\begin{array}{l}51.2 \\
51.6\end{array}$ & $\begin{array}{l}50.9 \\
50.1\end{array}$ & & Bisections at I, VII. & 4 & I.6 & $\ldots \quad 2 \mathrm{I} .5$ & . & $-\quad 19.9$ \\
\hline & & & 29.886 & 50.2 & 48.9 & & Bisections at II, VI. & II & 0.4 & 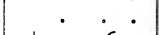 & . & $+\quad 0.4$ \\
\hline & 16 & & 29.872 & 49.8 & 48.4 & & Bisections at VI, VII. & 16 & 4. I & +1556.5 & . & +160.6 \\
\hline & $\begin{array}{l}0 \\
\mathrm{I}\end{array}$ & & $\begin{array}{l}29.960 \\
29.950\end{array}$ & $\begin{array}{l}62.7 \\
66.0\end{array}$ & $\begin{array}{l}62.5 \\
66.1\end{array}$ & I 5. & Bisections at $\mathrm{C}_{1}, \mathrm{C}_{2}, \mathrm{C}_{5}$. & $\mathrm{I} 7$ & 4. I & - I5 56.5 & 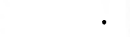 & -1552.4 \\
\hline I8 & I & & 29.934 & 67.5 & 68.0 & I6. 34. & Bisections at I, II. & 24 & +2213.8 & -I4 48.8 & . & +725.0 \\
\hline & 2 & & 29.936 & 68.0 & 68.9 & I 7,35 . & Bisections at VI, VII. & 32 & 5.2 & & -0.7 & $+\quad 4.5$ \\
\hline & & & $\begin{array}{l}29.922 \\
29.910\end{array}$ & 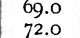 & $\begin{array}{l}69.9 \\
70.8\end{array}$ & & Bisections at II, VI, VII. & 34 & 4. I & + I5 54.2 & . & +1558.3 \\
\hline & & 20 & 29.900 & 72.0 & $7 \mathrm{~T} . \mathrm{O}$ & 23. & Bisections at I, II, VII. & 35 & 4.0 & -I5 54.3 & • & - I5 50.3 \\
\hline & & 52 & 29.870 & 65.8 & 63.9 & 24 . & Bisections at $B_{1}, C_{1}, C_{5}, D_{3}$. & 40 & +26 I.6 & -I4 51.5 & . & +II IO.I \\
\hline & & $\begin{array}{l}54 \\
59\end{array}$ & $\begin{array}{l}29.878 \\
29.854\end{array}$ & $\begin{array}{l}64.0 \\
58.5\end{array}$ & $\begin{array}{l}62.2 \\
55.8\end{array}$ & 40. & Bisections at $B_{1}, B_{3}, D_{1}, D_{3}$. & & & & & \\
\hline & & $\begin{array}{l}24 \\
49\end{array}$ & $\begin{array}{l}29.830 \\
29.818\end{array}$ & $\begin{array}{l}57.0 \\
56.0\end{array}$ & $\begin{array}{l}54.7 \\
53.9\end{array}$ & & & & & & & \\
\hline & & 32 & 29.864 & 64.8 & 63.8 & & & & & & & \\
\hline 19 & $\begin{array}{l}\mathrm{I} \\
\mathrm{I}\end{array}$ & $\begin{array}{l}34 \\
5 \mathrm{I}\end{array}$ & $\begin{array}{l}29.874 \\
29.874\end{array}$ & $\begin{array}{l}68.8 \\
69.2\end{array}$ & $\begin{array}{l}68.3 \\
68.5\end{array}$ & & & & & & & \\
\hline & & $\begin{array}{l}17 \\
20\end{array}$ & $\begin{array}{l}29.854 \\
29.848\end{array}$ & $\begin{array}{l}70.6 \\
70.2\end{array}$ & $\begin{array}{l}69.7 \\
69.6\end{array}$ & & & & & & & \\
\hline & & 34 & 29.872 & 67.4 & 66.2 & & & & & & & \\
\hline & & 54 & 29.872 & $\begin{array}{l}64.0 \\
6.8\end{array}$ & 62.5 & & & & & & & \\
\hline & & $\begin{array}{l}53 \\
32\end{array}$ & $\begin{array}{l}29.002 \\
29.888\end{array}$ & $\begin{array}{l}\begin{array}{l}1.0 \\
6 \mathrm{I} .2\end{array} \\
\end{array}$ & $\begin{array}{l}00.2 \\
59.1\end{array}$ & & & & & & & \\
\hline & & 20 & 29.886 & 59.5 & 57.2 & & & & & & & \\
\hline & & 45 & 29.890 & $5^{0.3}$ & 54.5 & & & & & & & \\
\hline
\end{tabular}




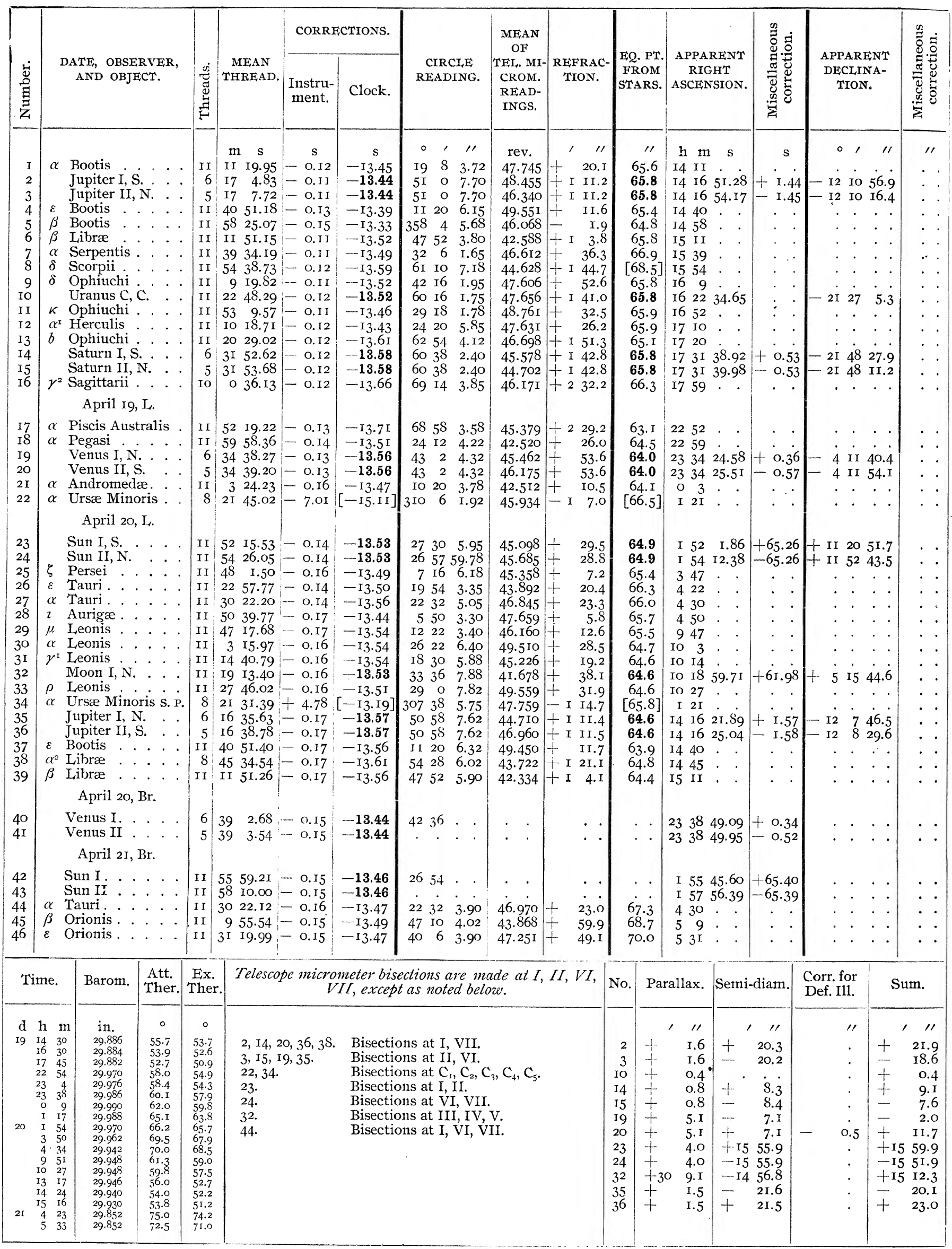




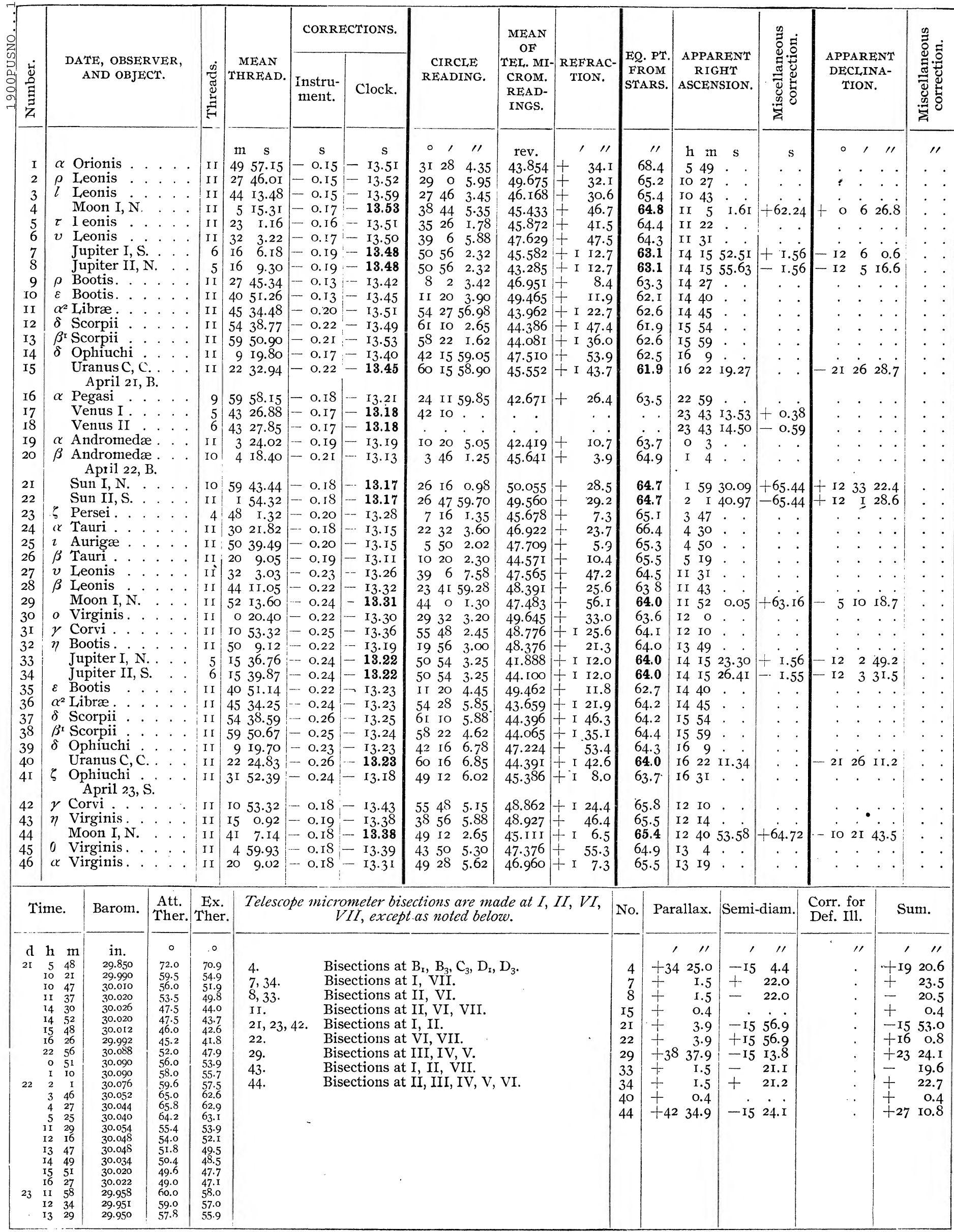




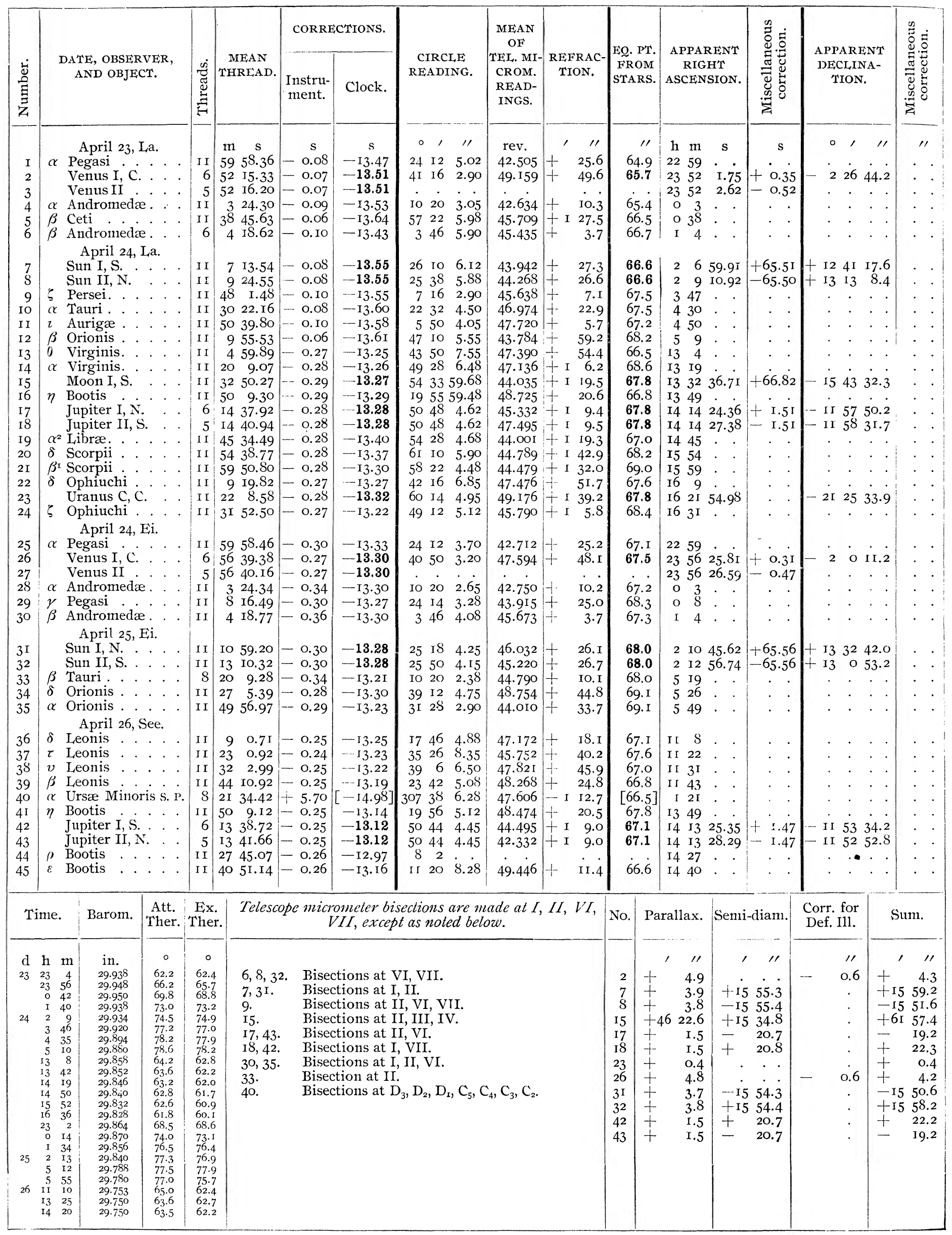




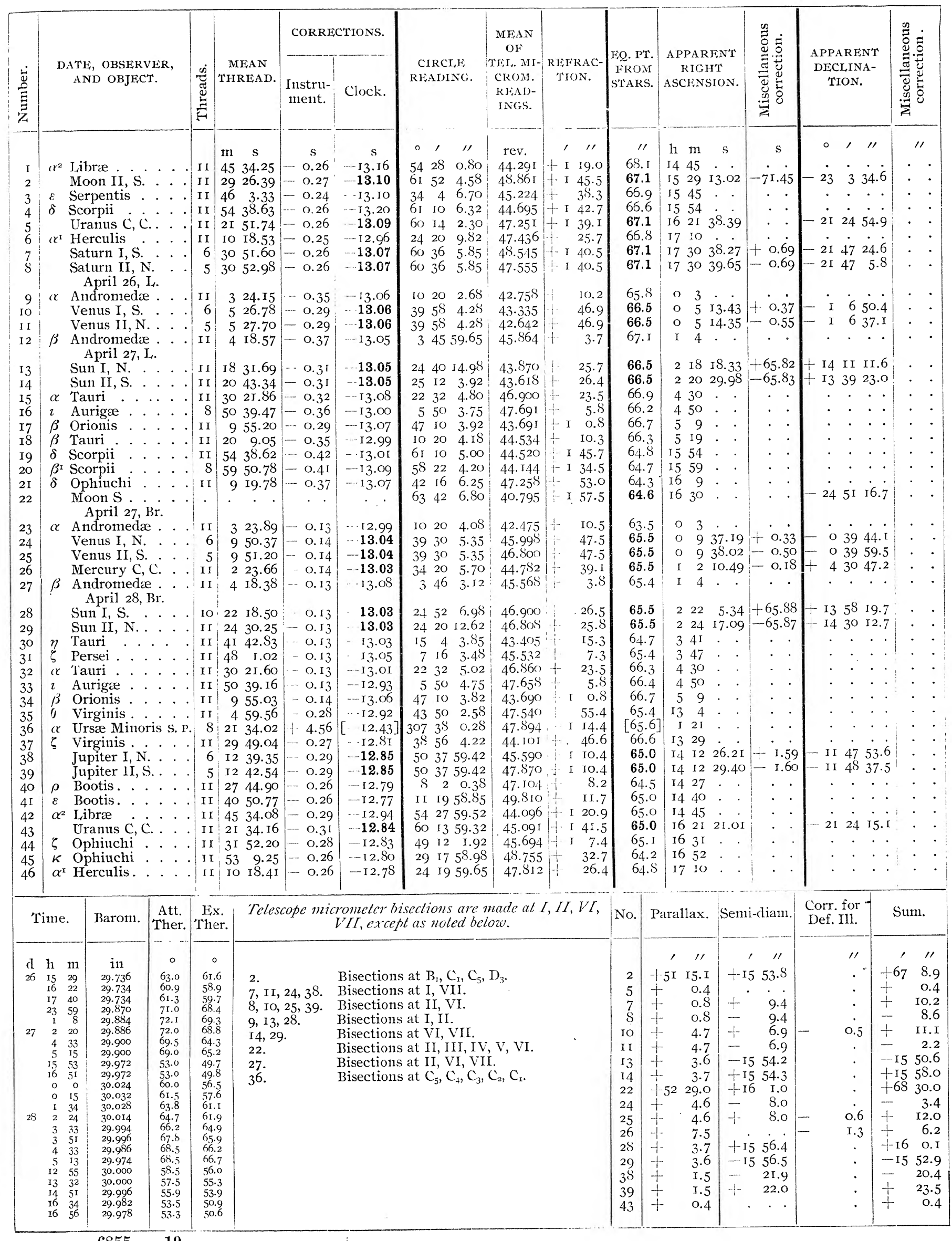

$6855-19$ 


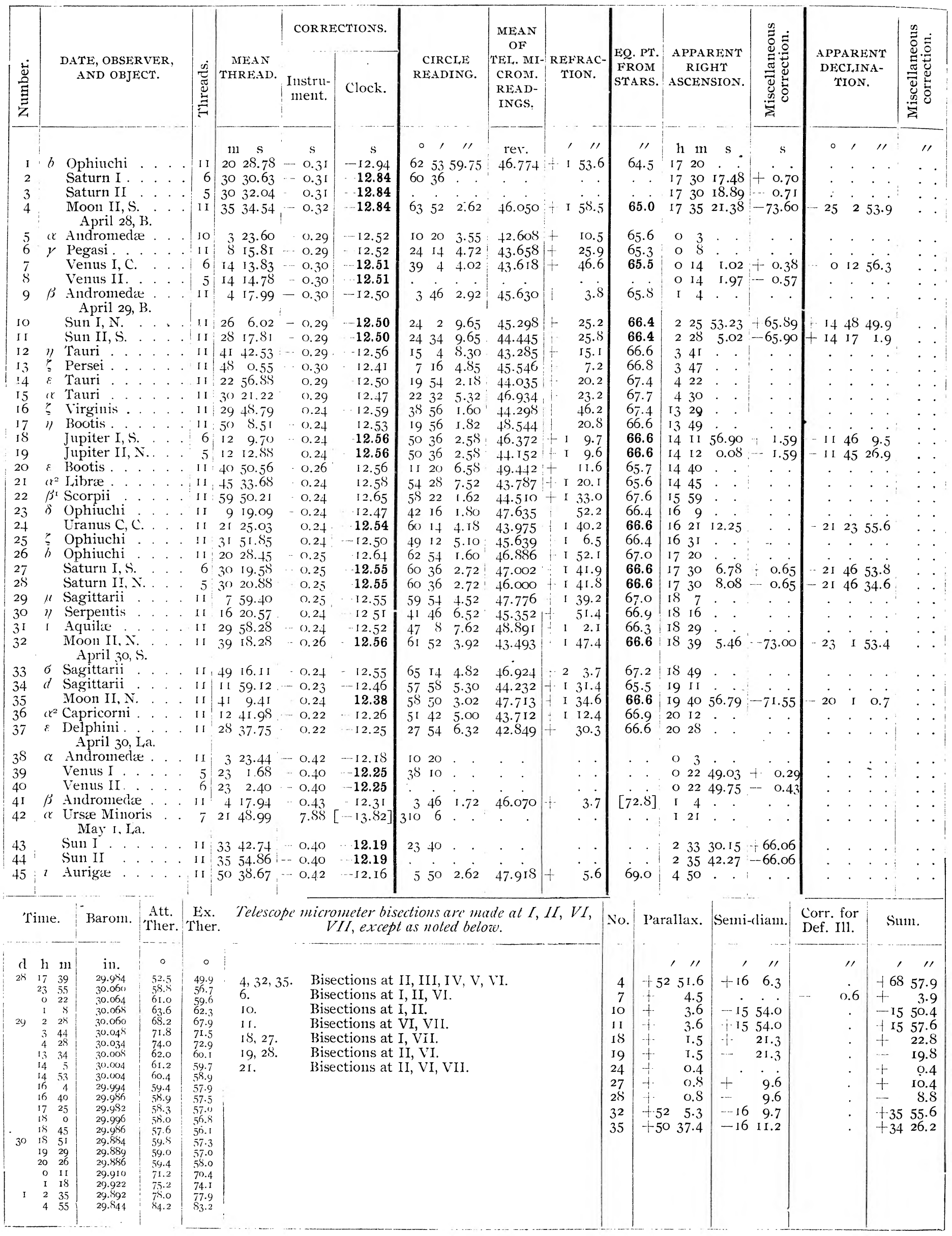




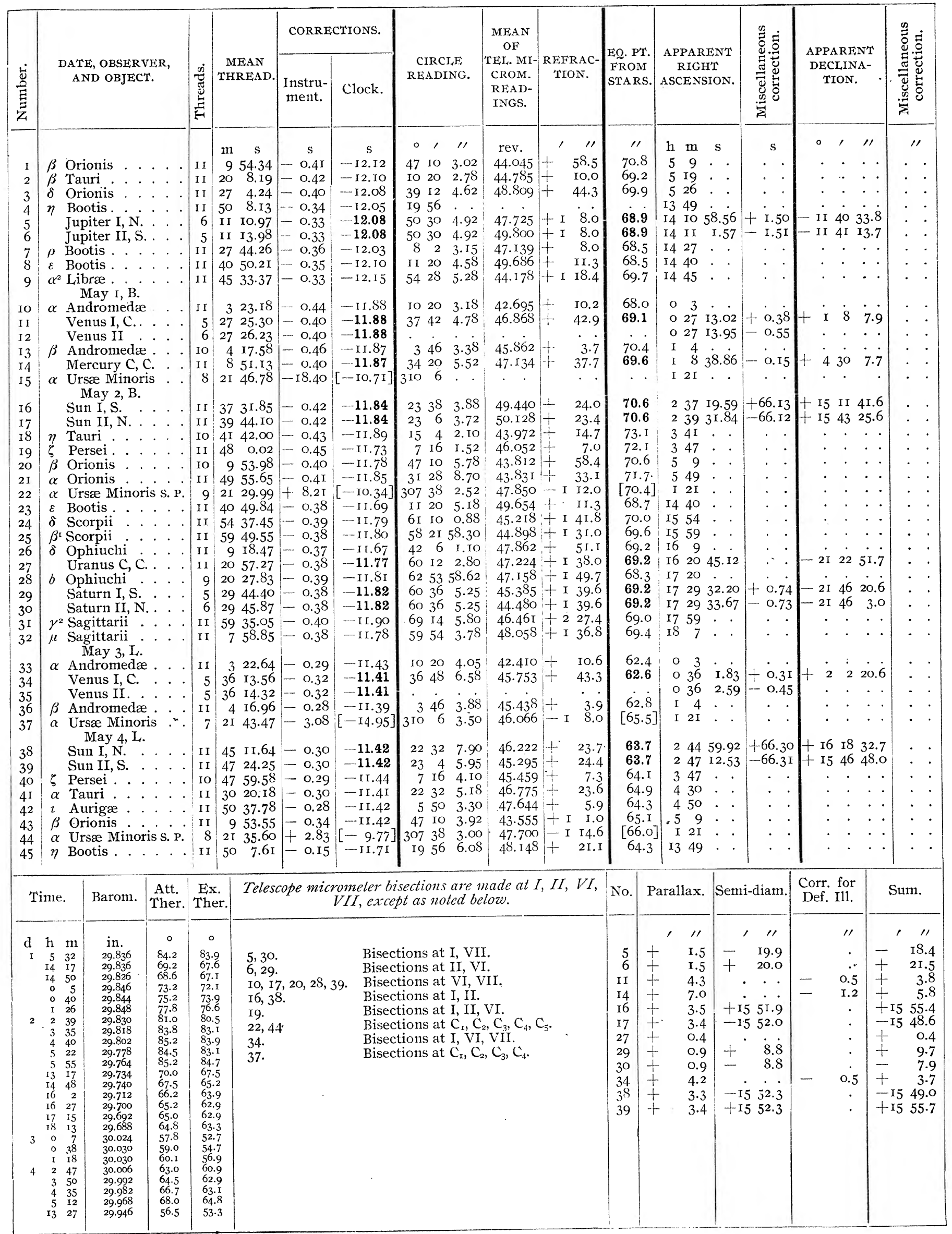




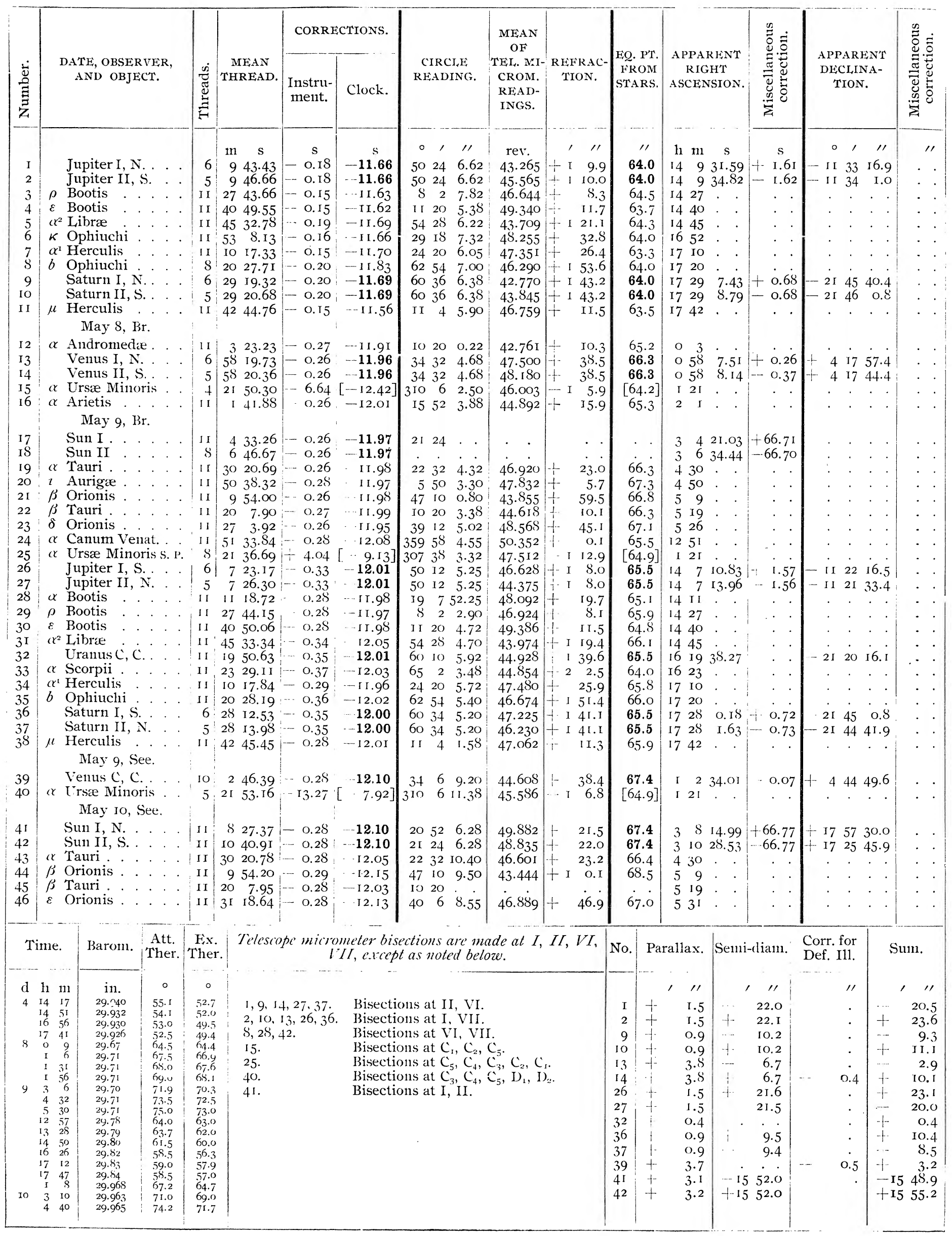




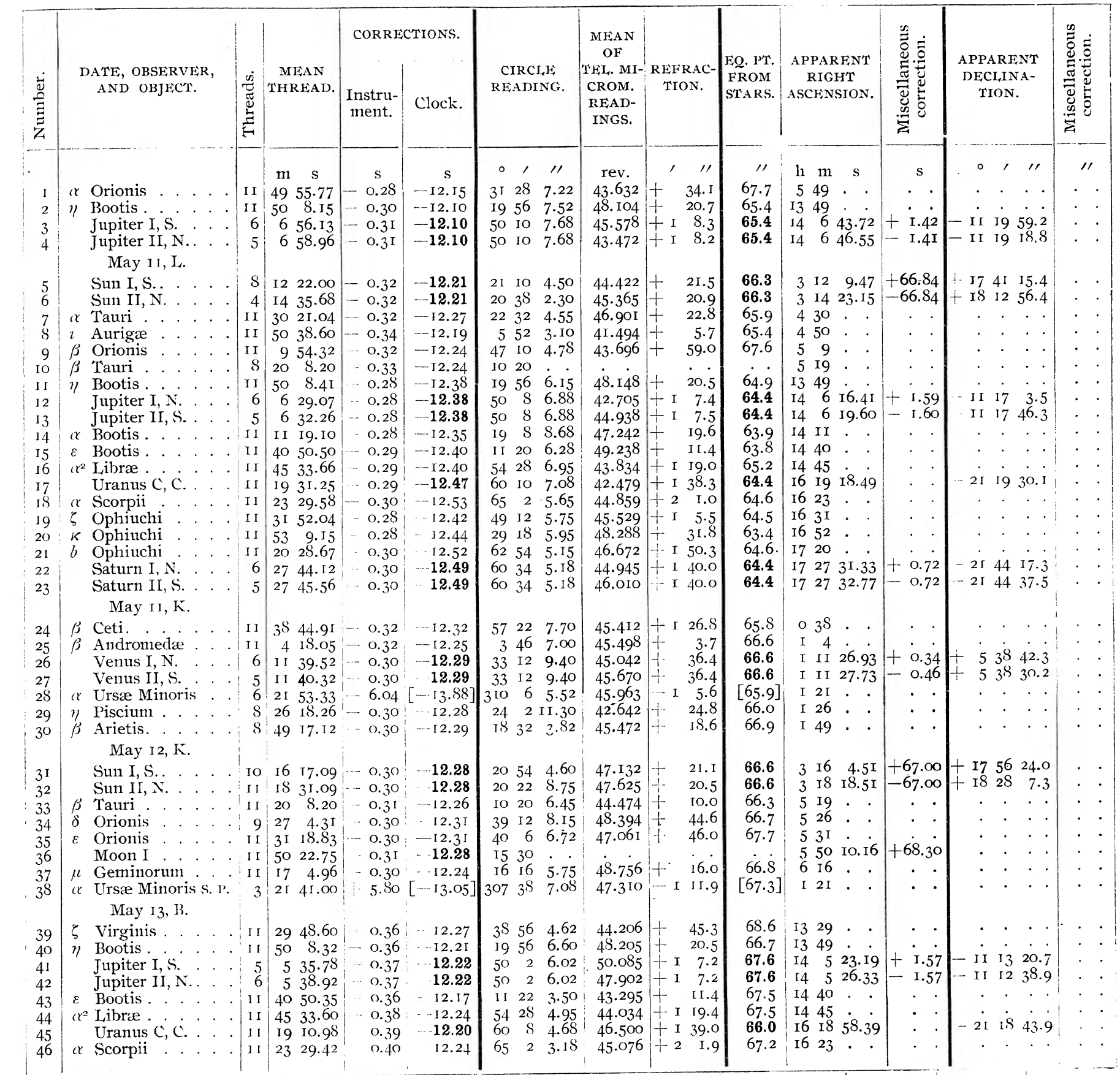

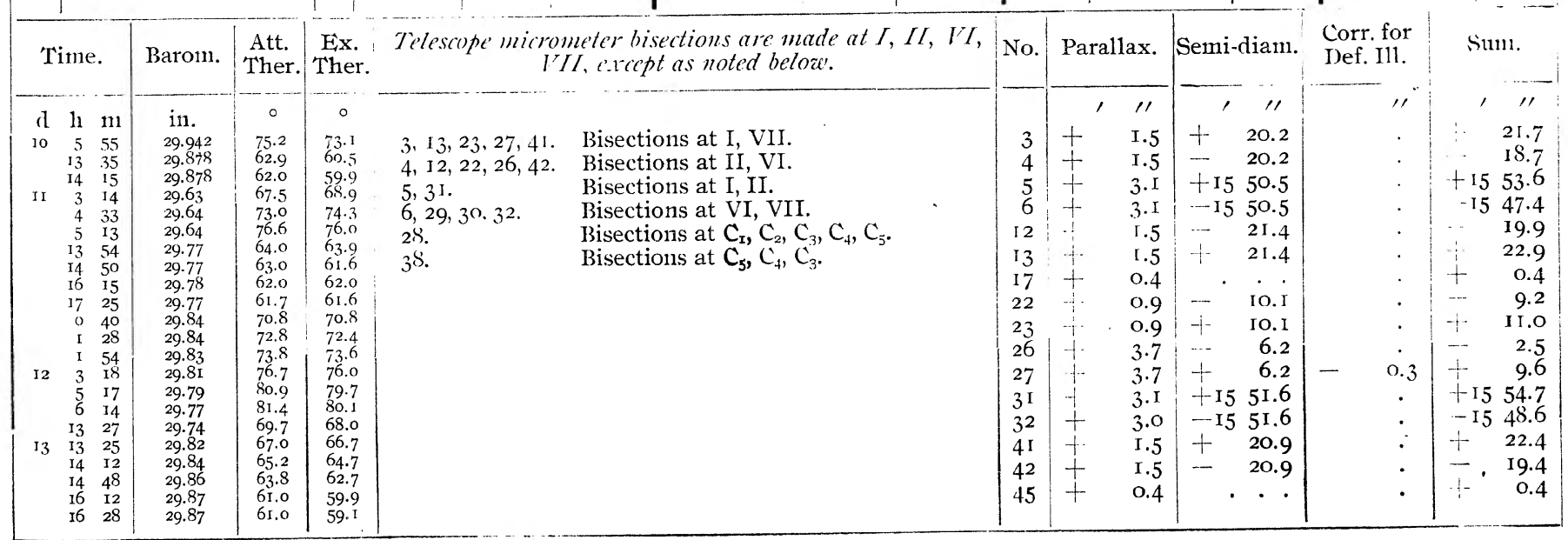




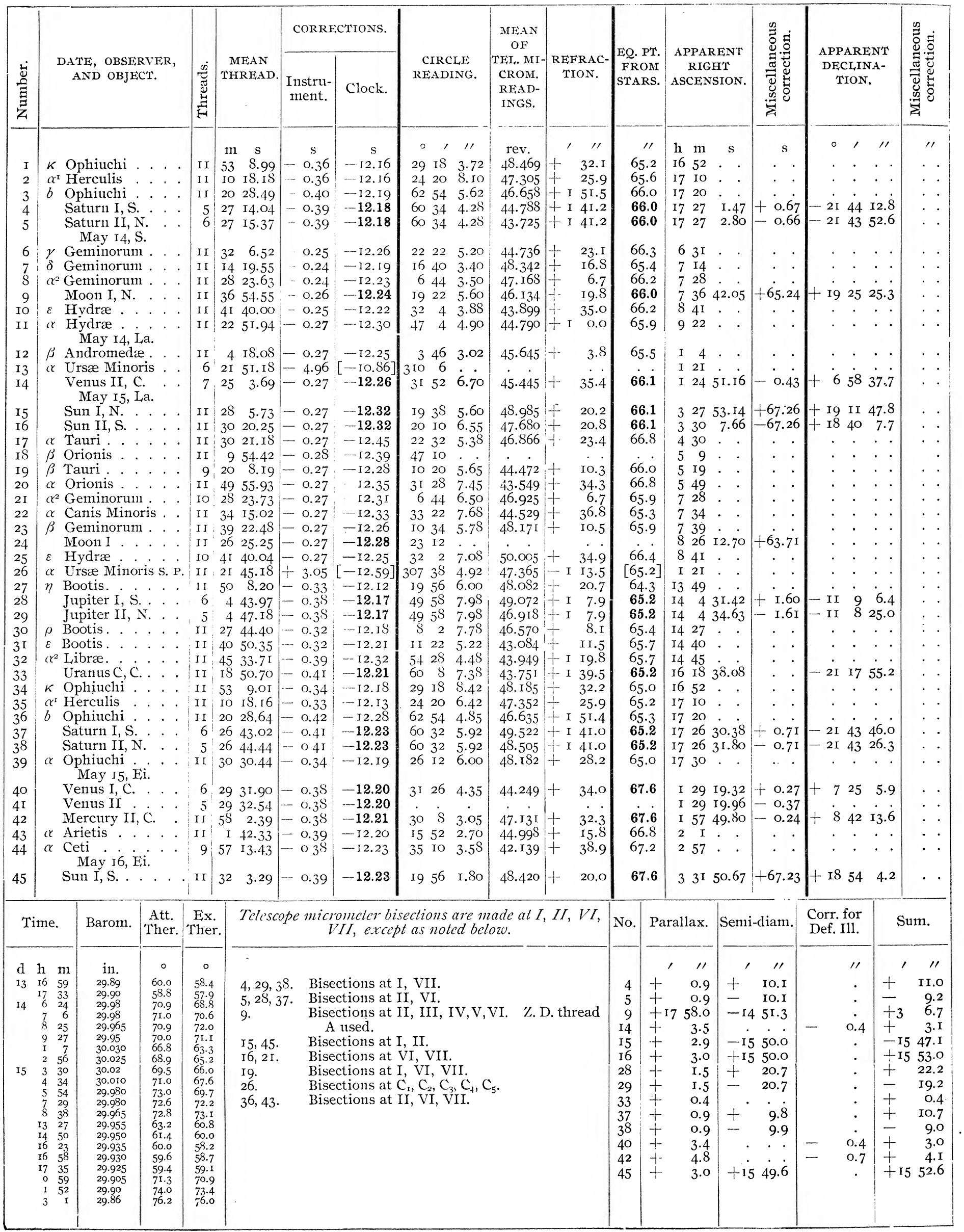




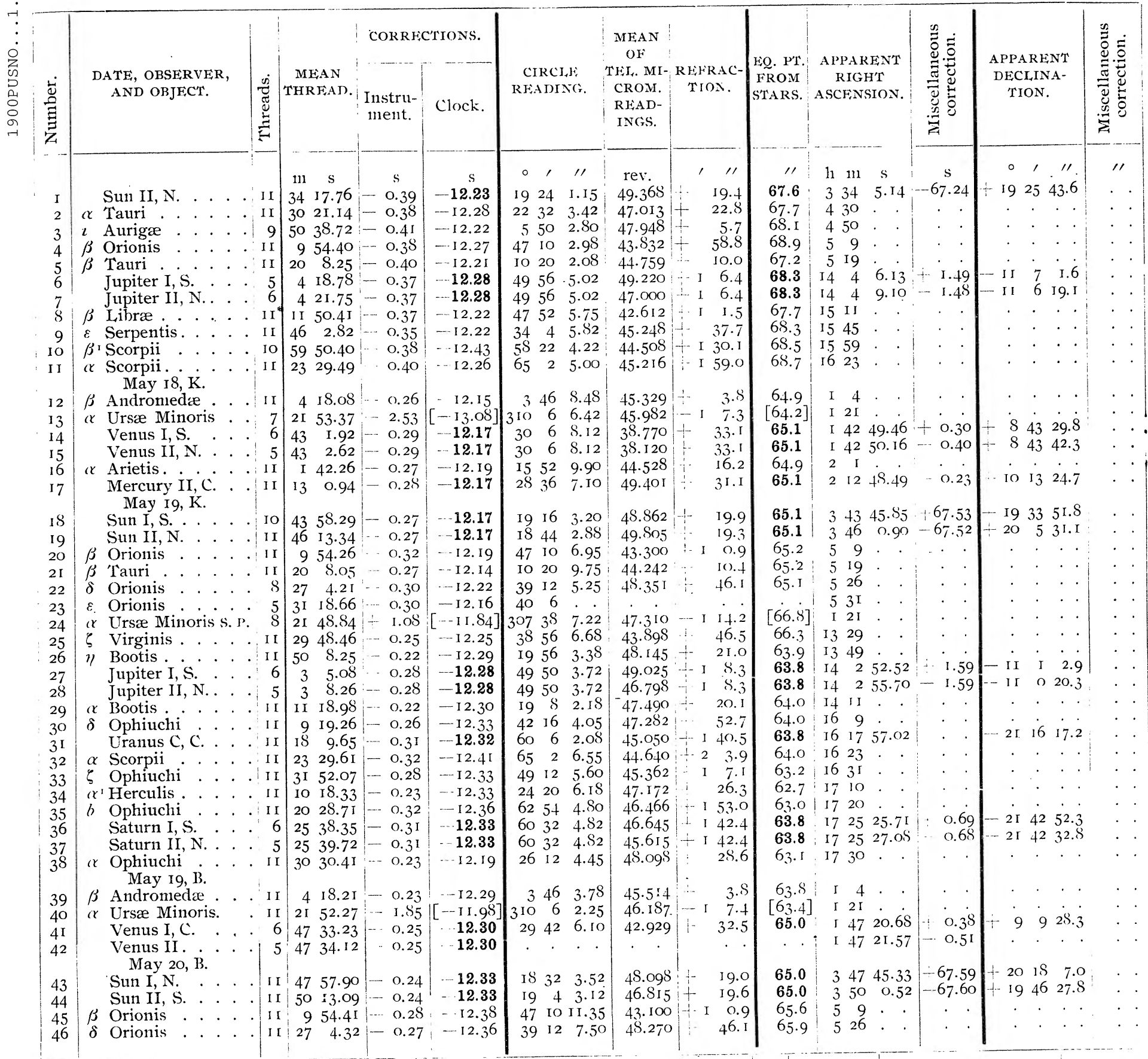

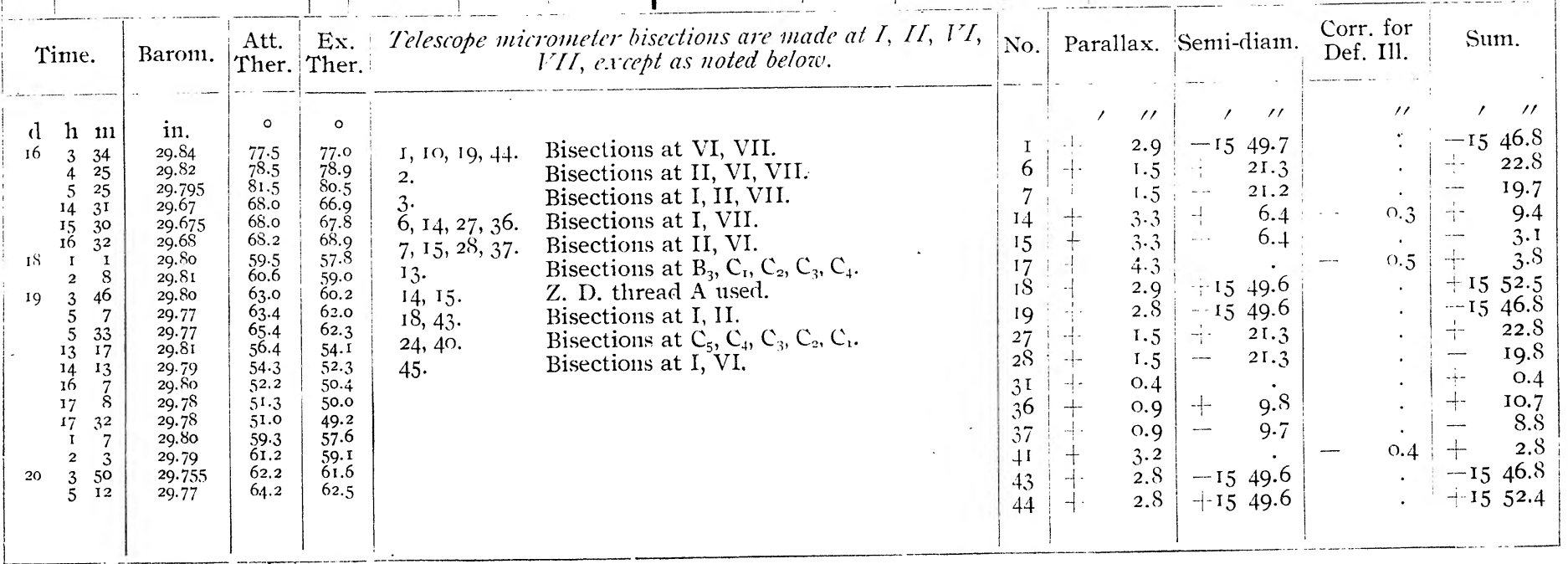




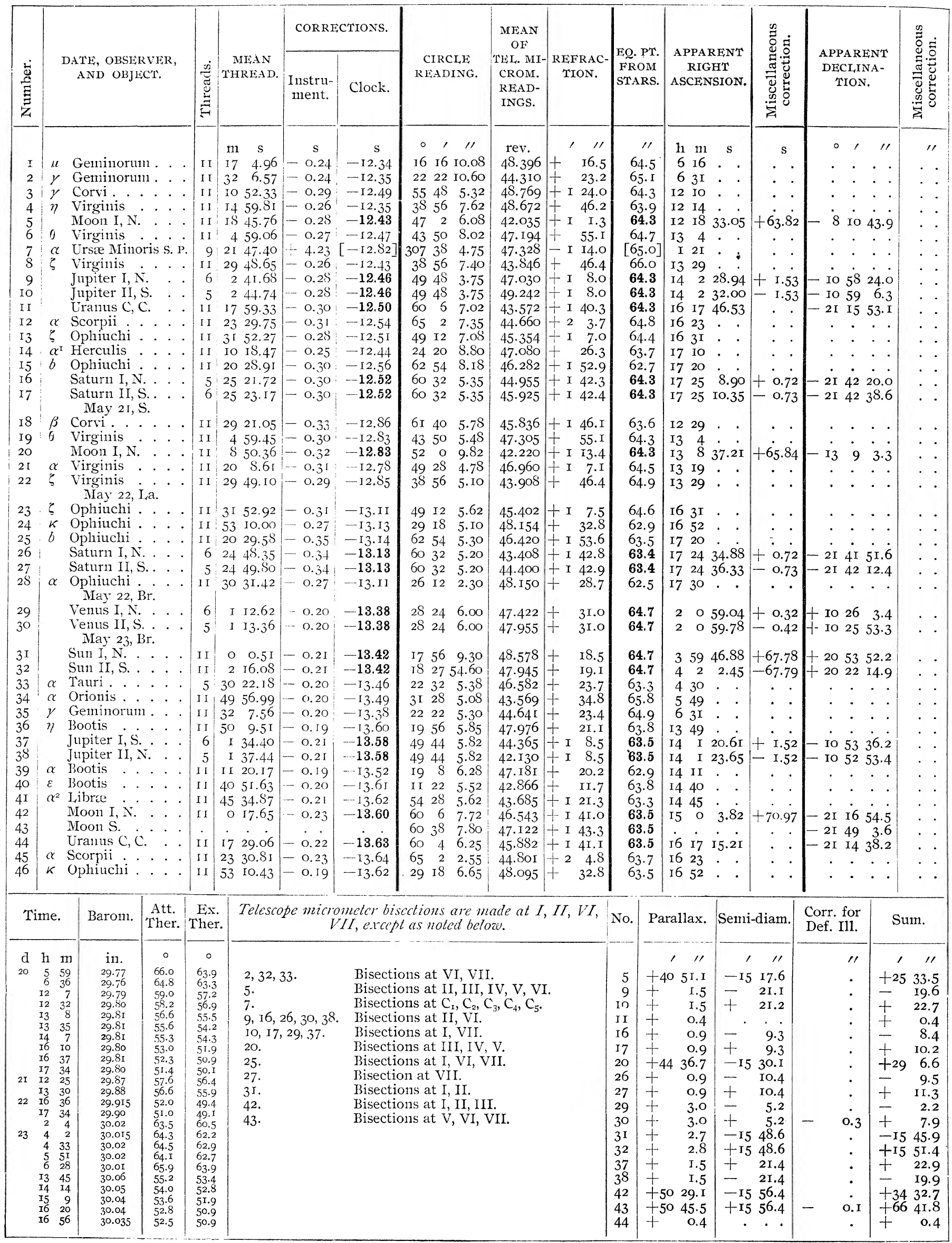




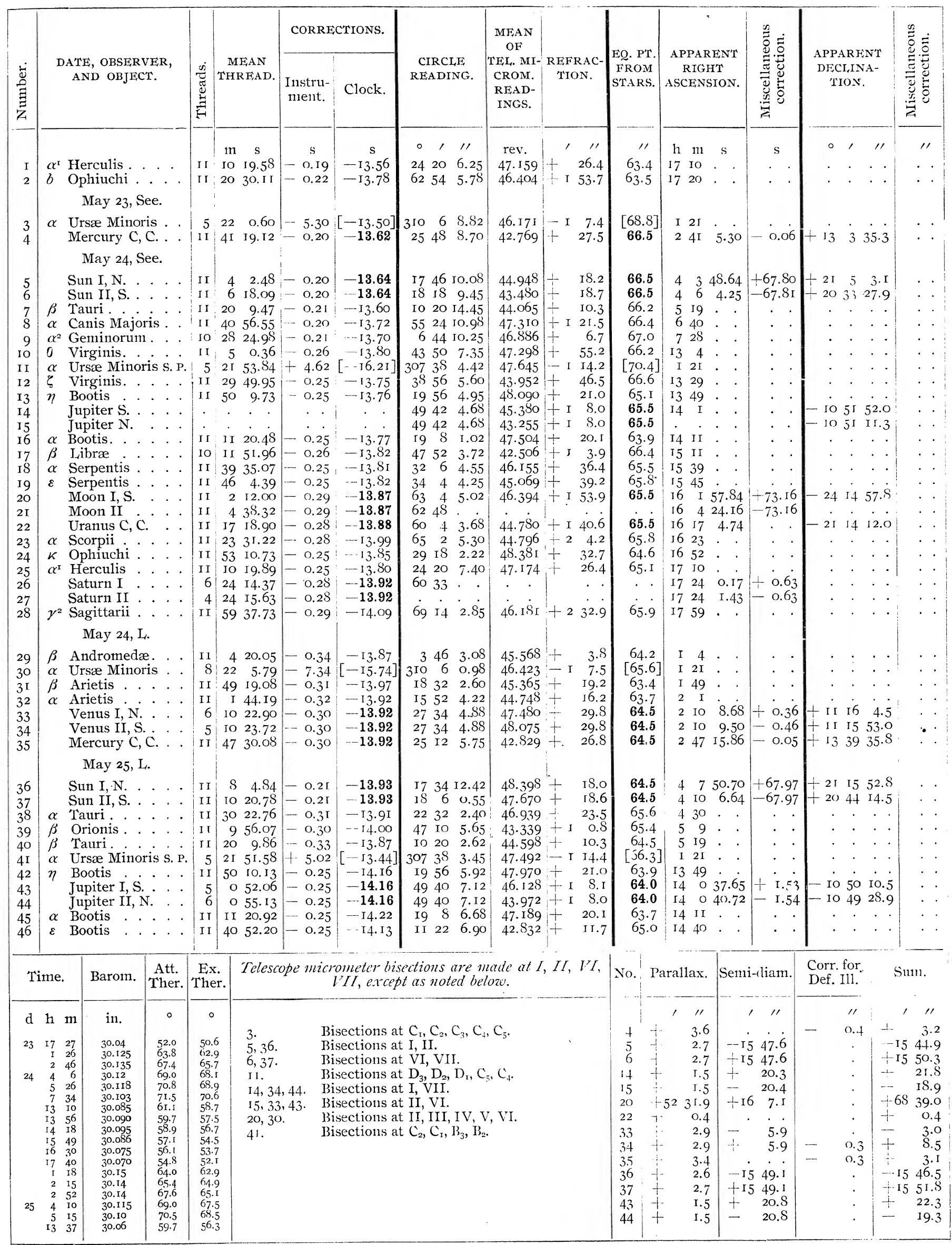




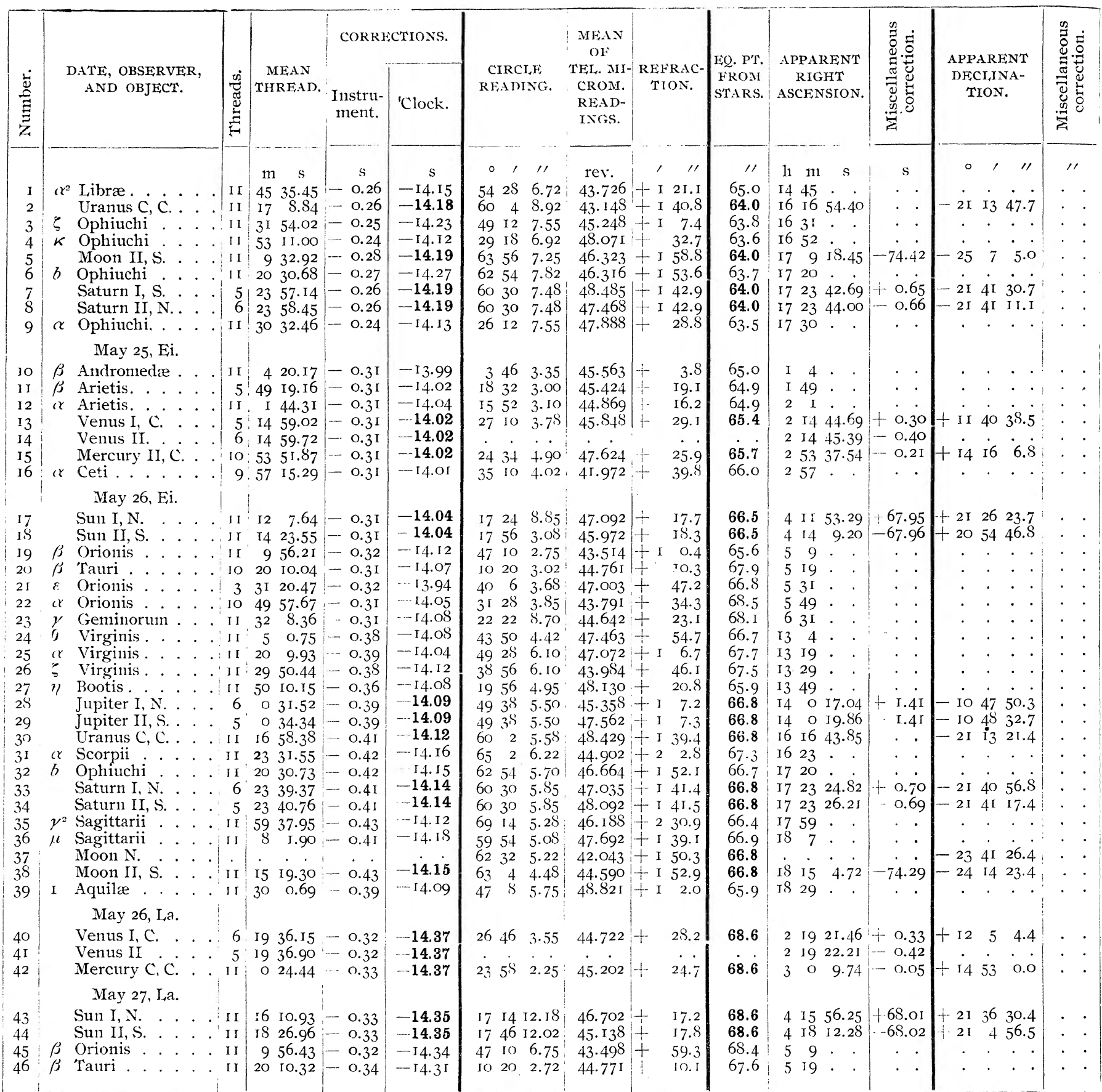

Time. Barom. Att. Ex. Telescope micrometer bisections are made at $I, I I, I T$, No. Iarallax. Semi-dian

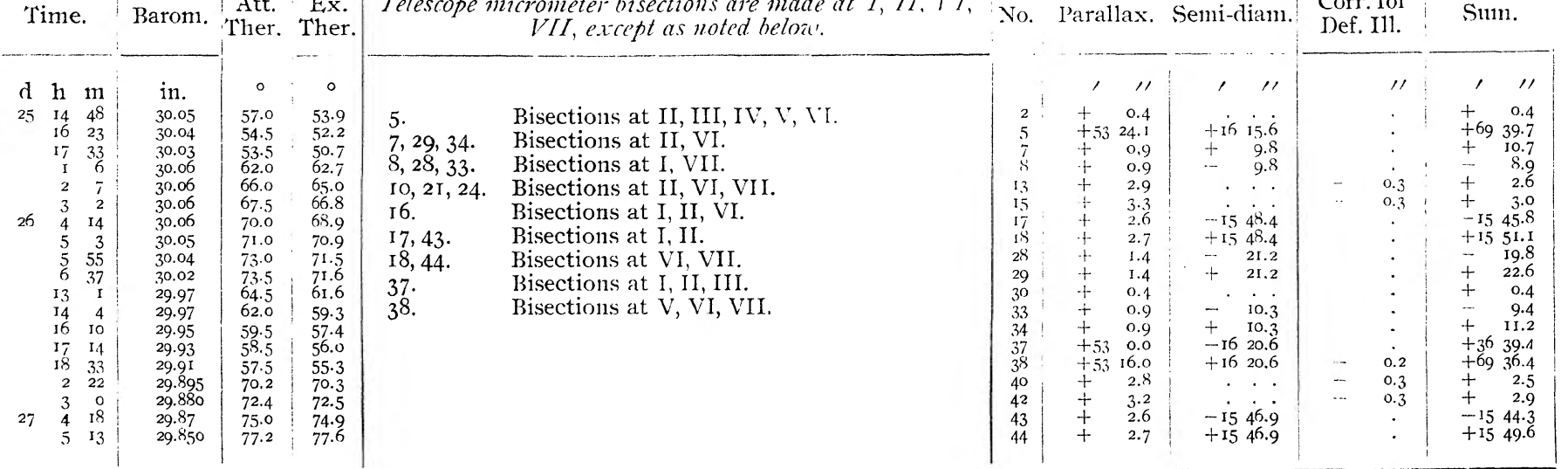




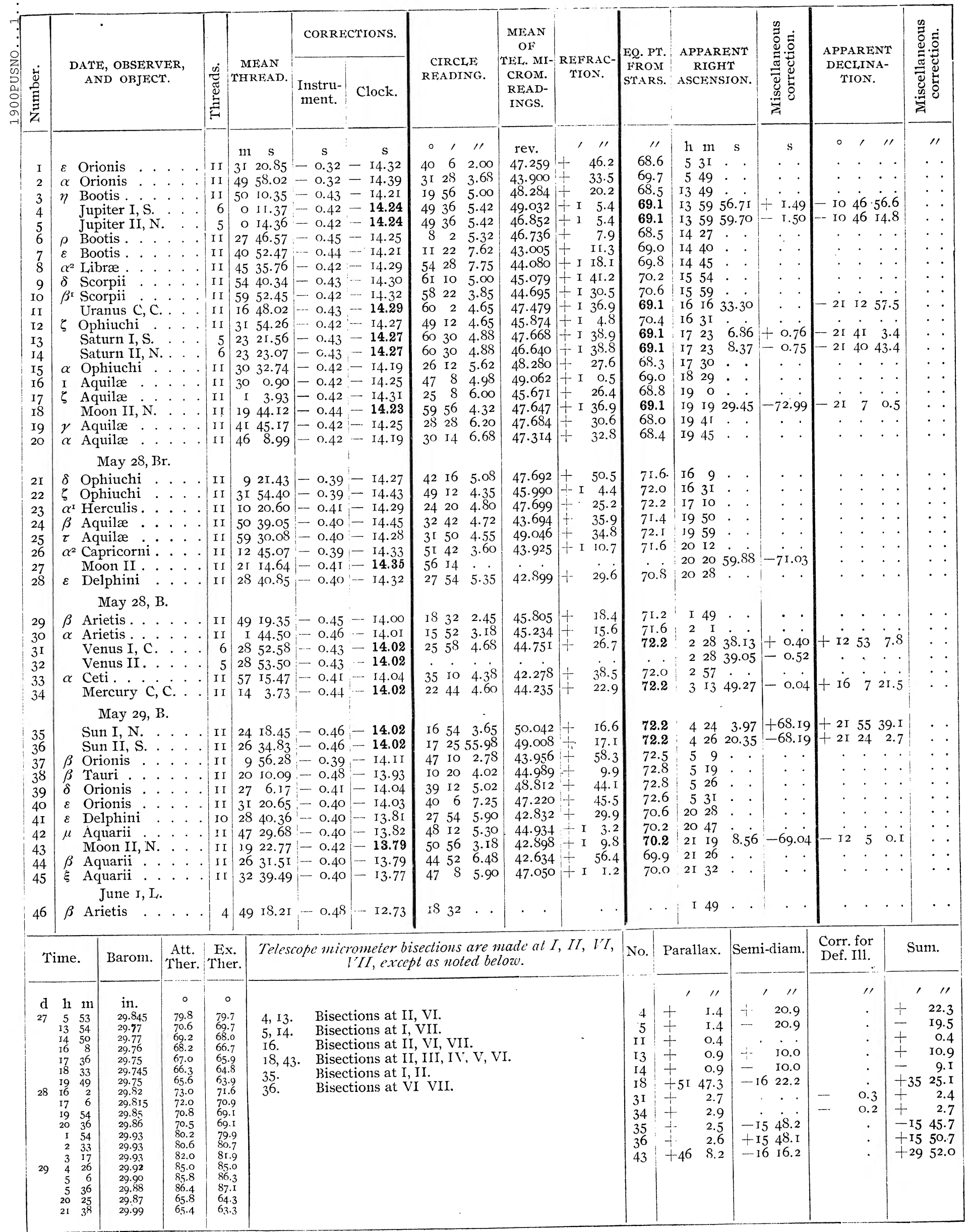




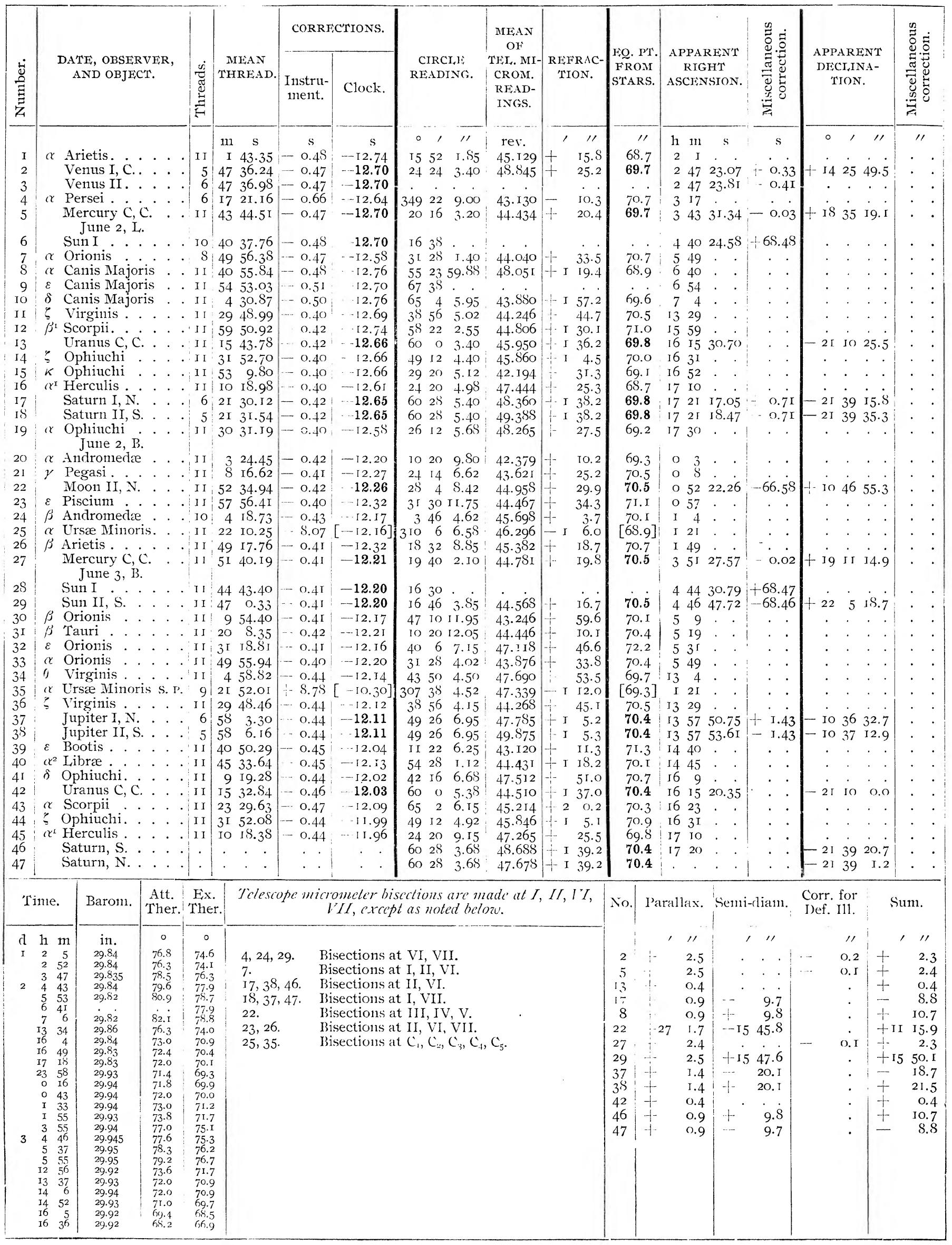

